\title{
Fast Beam Studies of Free Radical Photodissociation
}

\author{
Douglas Robert Cyr \\ Ph. D. Thesis \\ Department of Chemistry \\ University of California \\ and \\ Chemical Sciences Division \\ Lawrence Berkeley Laboratory \\ University of California \\ Berkeley, CA 94720
}

November 1993

This work was supported in part by the Director, Office of Energy Research, Office of Basic Energy

Sciences, Chemical Science Division of the U.S. Department of Energy under contract No. DE-AC03-76SF00098, and in part by the National Science Foundation under Grant No. CHE 91-08145. 


\title{
Fast Beam Studies of Free Radical Photodissociation
}

Copyright @ 1993

by

\author{
Douglas Robert Cyr
}

The U. S. Department of Energy has the right to use this thesis for any purpose whatsoever including the right to reproduce all or any part thereof. 


\author{
Abstract \\ Fast Beam Studies of Free Radical Photodissociation \\ by \\ Douglas Robert Cyr \\ Doctor of Philosophy in Chemistry \\ University of Califormia at Berkeley \\ Professor Daniel M. Neumark, Chair
}

The photodissociation of free radicals is studied in order to characterize the spectroscopy and dissociation dynamics of the dissociative electronic states in these species. To accomplish this, a novel method of radical production, based on the photodetachment of the corresponding negative ion, has been combined with a highly complementary form of photofragment translational spectroscopy. The optical spectroscopy of transitions to dissociative states is determined by monitoring the total photofragment yield as a function of dissociation photon energy. Branching ratios to various product channels, internal energy distributions of the fragments, bond dissociation energies, and the translational energy-dependent photofragment recoil angular distributions are then determined at selected excitation energies. A detailed picture of the dissociation dynamics can then be formulated, allowing insight concerning the interactions of potential energy surfaces involved in the dissociation.

After an introduction to the concepts and techniques mentioned above, the experimental apparatus used in these experiments is described in detail.

The basis and methods used in the treatment of data, especially in the dissociation dynamics experiments, are then put forward. 
A brief illustration of the capabilities of the experiment is then given, using as examples results from the the initial photodissociation studies completed on the prototypical test systems of $\mathrm{O}_{2}$ and the $\mathrm{N}_{3}$ radical.

An investigation of the spectroscopy and dissociation dynamics of the NCO radical following excitation of the $\tilde{B}^{2} \Pi \leftarrow \tilde{X}^{2} \Pi$ electronic transition is then detailed. Measurements of the photodissociation cross section as a function of dissociation wavelength show that even the lowest vibrational levels of the $\tilde{B}^{2} \Pi$ state predissociate. Analysis of fragment kinetic energy release reveals that the spin-forbidden $\mathrm{N}\left({ }^{4} S\right)+\mathrm{CO}$ $\left({ }^{1} \Sigma^{+}\right)$products are exclusively produced until $20.3 \mathrm{kcal} / \mathrm{mol}$ above the origin, at which point the spin-allowed $\mathrm{N}\left({ }^{2} D\right)+\mathrm{CO}$ product channel becomes energetically accessible. The spin-allowed channel dominates above this threshold. By determining the location of this threshold we obtain a new $\Delta_{f} H_{(298 K)}^{o}$ for NCO of $30.5 \pm 1 \mathrm{kcal} / \mathrm{mol}$, several $\mathrm{kcal} / \mathrm{mol}$ lower than the previously accepted value.

A photodissociation study of the nitromethyl radical, $\mathrm{CH}_{2} \mathrm{NO}_{2}$, is then reported. Two major dissociation product channels are observed at each of three dissociation wavelengths investigated in the range from 240 to $270 \mathrm{~nm}$, and are identified as (I) $\mathrm{CH}_{2} \mathrm{NO}_{2} \rightarrow \mathrm{CH}_{2} \mathrm{NO}+\mathrm{O}$ and (II) $\mathrm{CH}_{2} \mathrm{NO}_{2} \rightarrow \mathrm{H}_{2} \mathrm{CO}+\mathrm{NO}$. No evidence is found for simple $\mathrm{C}-\mathrm{N}$ bond fission to give (III) $\mathrm{CH}_{2} \mathrm{NO}_{2} \rightarrow \mathrm{CH}_{2}+\mathrm{NO}_{2}$. Translational energy and angular distributions are presented for the two observed channels. The translational energy distribution of channel (I) peaks at only $5-8 \mathrm{kcal} / \mathrm{mol}$, while the distribution for channel (II) peaks at $\sim 60 \mathrm{kcal} / \mathrm{mol}$. The angular distributions for both channels are largely isotropic. The nature of the electronic excitation and dissociation dynamics are considered at length. The upper state in the electronic transition is assigned to the $1^{2} \mathrm{~B}_{1}$ state, and the data is found to be consistent with both dissociation processes occurring primarily on excited state surfaces. 
This thesis is dedicated

to my wife

Lejla

and my daughters

Ana Katarina and Jelena Kristina 


\section{Table of Contents}

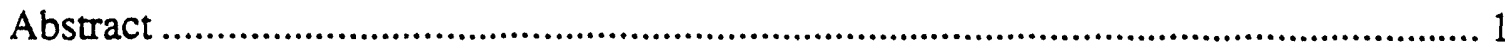

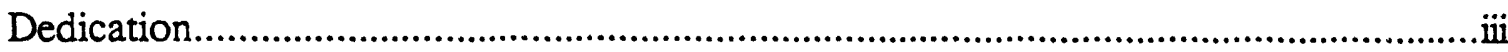

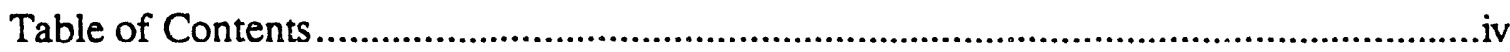

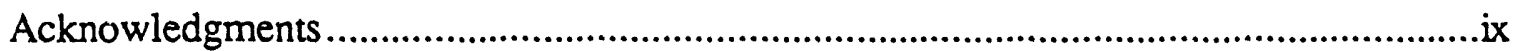

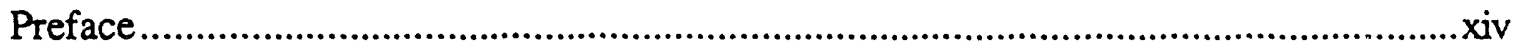

1 Fast Radical Beam Photofragment Translational Spectroscopy - An Introduction...... 1

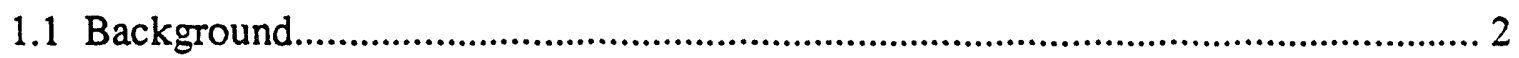

1.2 Fast Radical Beam Photofragment Translational Spectroscopy .................................. 4

1.3 Photodissociation Spectroscopy and Dynamics ……................................................ 8

1.3.1 The Spectroscopy of Dissociative Excited States....................................... 8

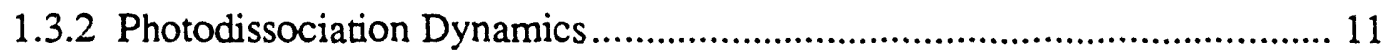

1.3.3 Excited State Potential Energy Surfaces ................................................ 15

1.3.4 Photofragment Recoil Angular Distributions .......................................... 16

1.4 Photodetachment as a Source of Free Radicals ................................................. 18

1.4.1 Photodetachment Considerations ……..................................................... 21

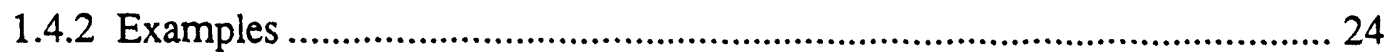

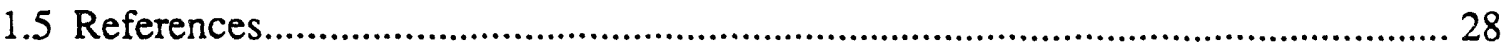

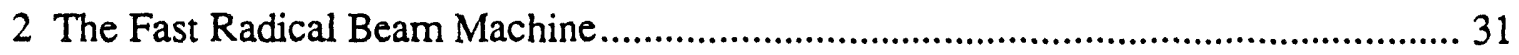

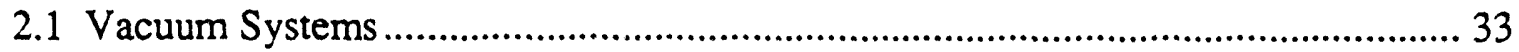

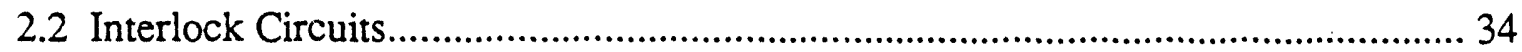

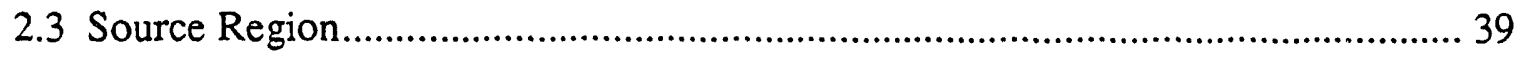

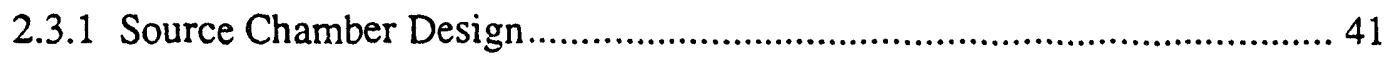

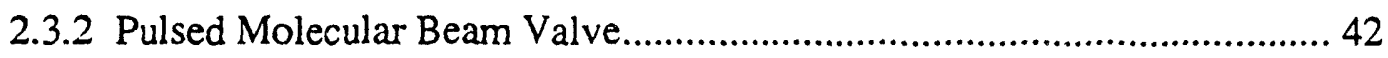




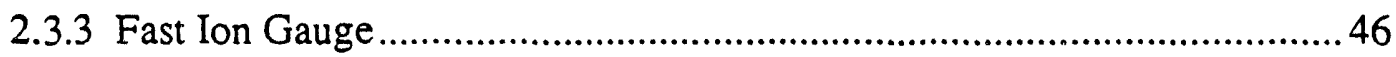

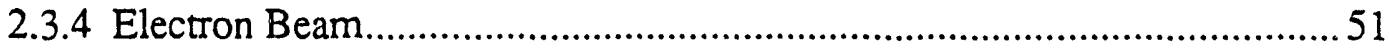

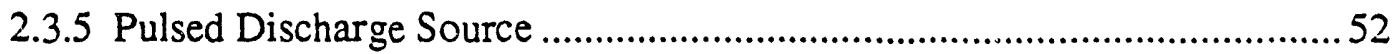

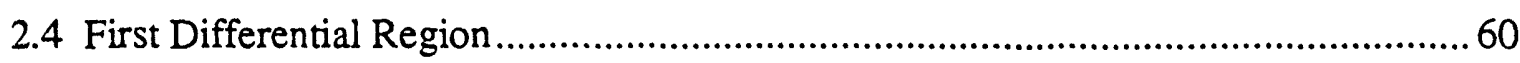

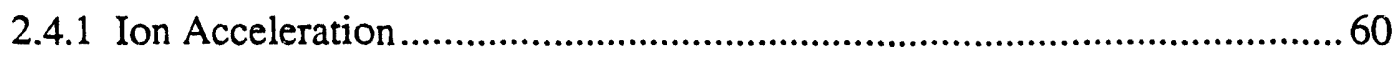

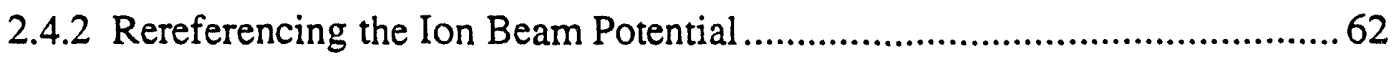

2.4.2.1 Details of the 'Potential Switch' Rereferencing Circuit ..................66

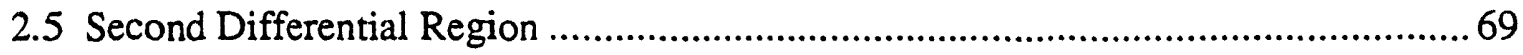

2.5.1 Beam Modulation Mass Spectrometer ……........................................... 71

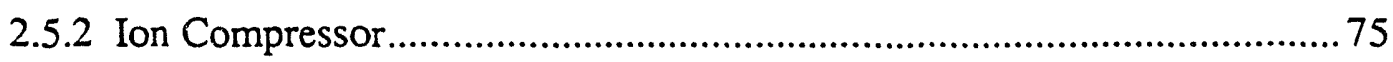

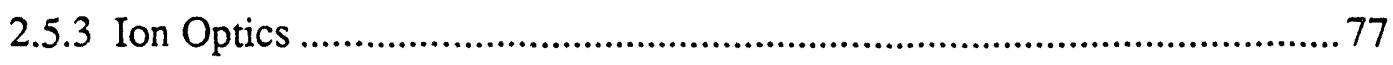

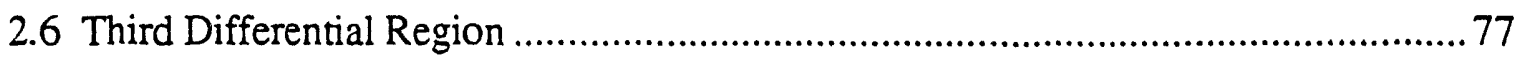

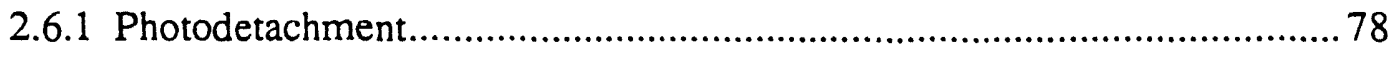

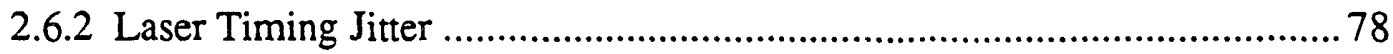

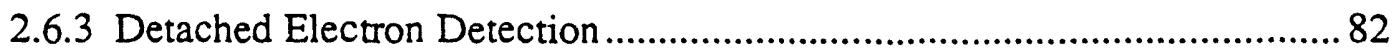

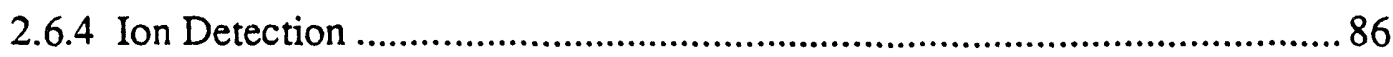

2.6.4.1 Ion Time-of-Flight Mass Spectroscopy:

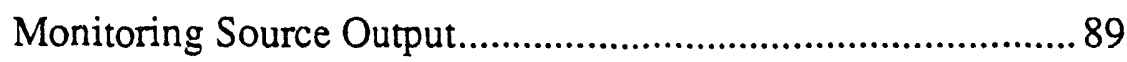

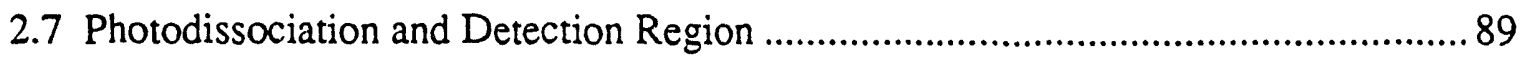

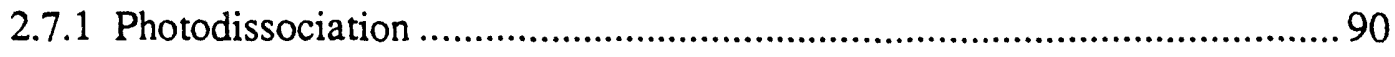

2.7.2 Acquiring Photodissociation Laser Timing.............................................. 90

2.7.3 Monitoring Photodissociation Laser Power.............................................. 93

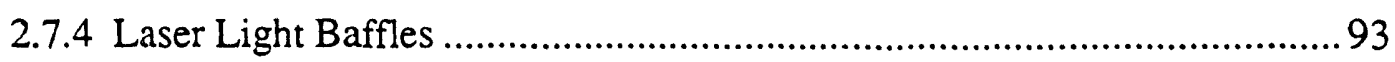

2.7.5 Total Photodissociation Cross Section Detector ……………….................. 95

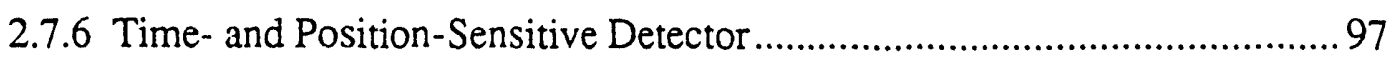

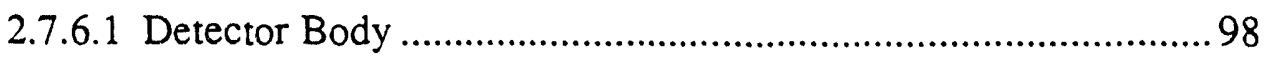

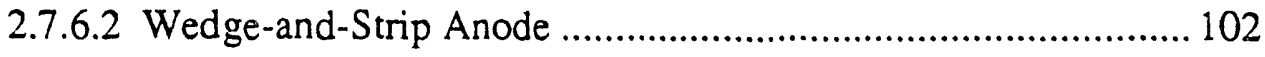




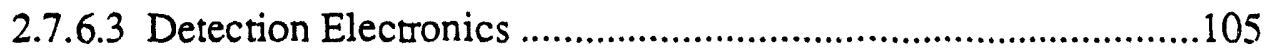

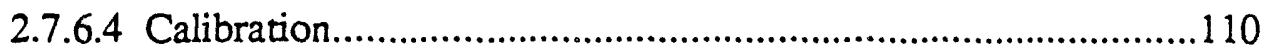

2.7.6.5 Translational Energy Resolution Considerations ........................113

2.8 Experimental Timing Sequence...........................................................................115

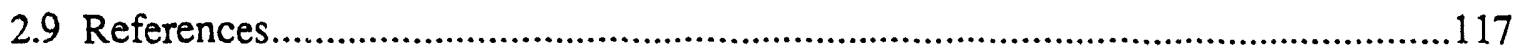

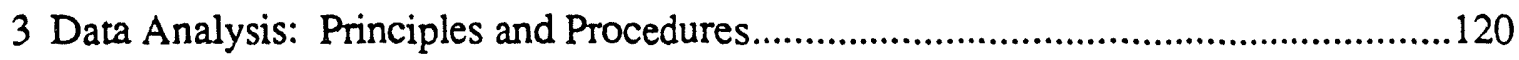

3.1 Time-of-Flight Distributions: Monte Carlo Forward Convolution............................120

3.2 Analysis of Time- and Position-Sensitive Detector Data..........................................123

3.2.1 Derivation of the Kinematic Equations Involved in Determining the Center-of-Mass Recoil Angle, Kinetic Energy Release and Mass Fraction for a Dissociation Event.........................................................124

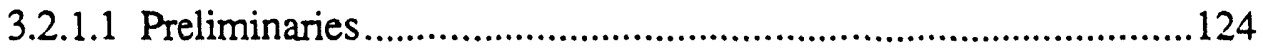

3.2.1.2 Center-of-Mass Recoil Angle..................................................128

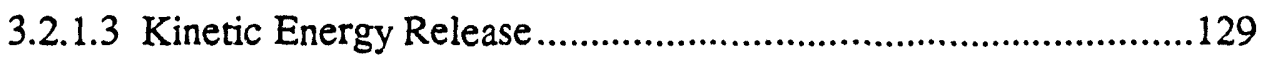

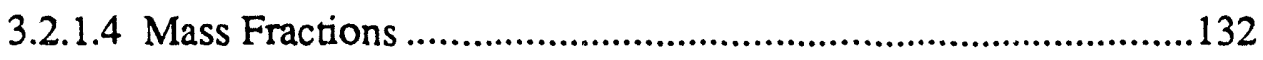

3.2.2 Raw Translational Energy and Recoil Angle Distributions ........................135

3.2.3 Normalization: The Detector Acceptance Function .................................136

3.2.4 Final Determination of the Angle-Integrated Translational Energy

Distribution, $P\left(E_{T}\right)$, and the Energy Dependent Anisotropy

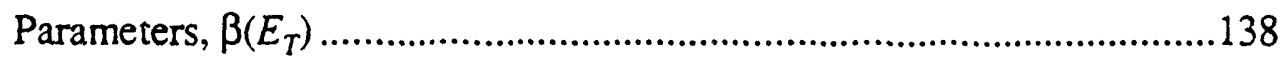

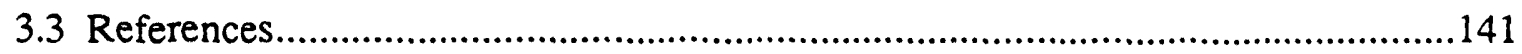

4 Photodissociation Studies of $\mathrm{O}_{2}$ and $\mathrm{N}_{3}$ : Examples of FRBM Capabilities .................142 
4.1 Photodissociation Cross Sections in the $B^{3} \Sigma_{u}^{-}\left(v^{\prime}\right) \leftarrow X^{3} \Sigma_{g}^{-}\left(v^{\prime \prime}\right)$

Schumann-Runge Bands of $\mathrm{O}_{2}$.

4.2 TPS Detection of $\mathrm{O}_{2}$ Dissociation:

Resolving Correlated Spin-Orbit States of the $\mathrm{O}^{3} \mathrm{P}_{\mathrm{j}}, \mathrm{O}{ }^{3} \mathrm{P}_{\mathrm{j}}$ Products ...................... 146

4.3 Total Photodissociation Cross Sections of the $\mathrm{N}_{3}$ Free Radical............................... 152

4.4 TPS Detection of $\mathrm{N}_{3}$ Radical Dissociation:

'Mode Specific' Effects in the N Atom Electronic State Branching Ratio ................ 158

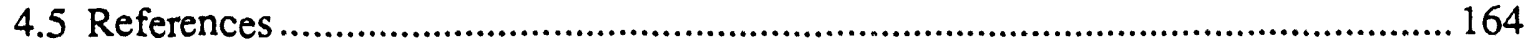

5 Photodissociation of the NCO Free Radical............................................................. 165

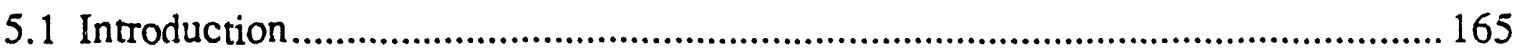

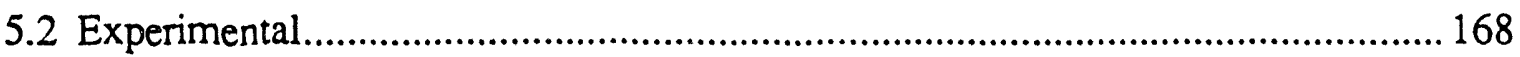

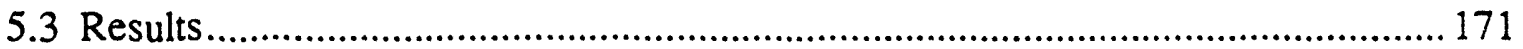

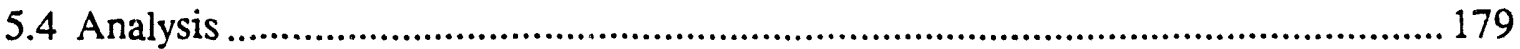

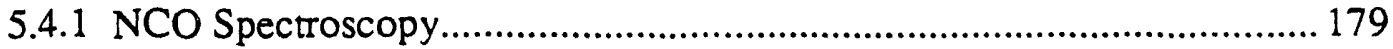

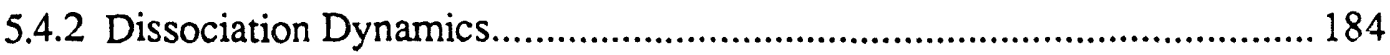

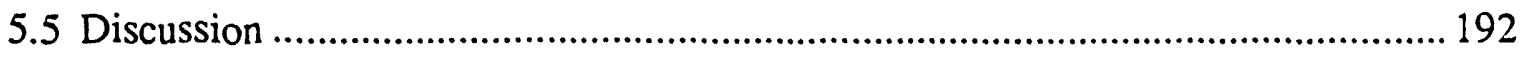

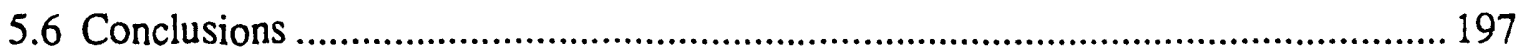

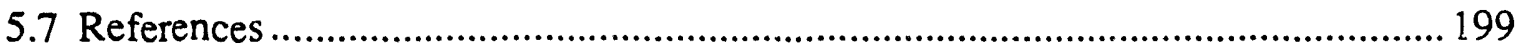

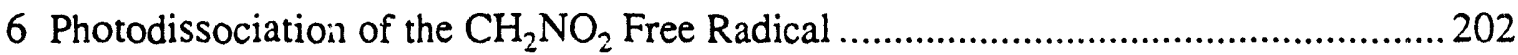

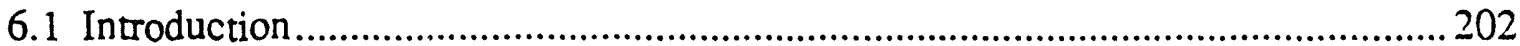

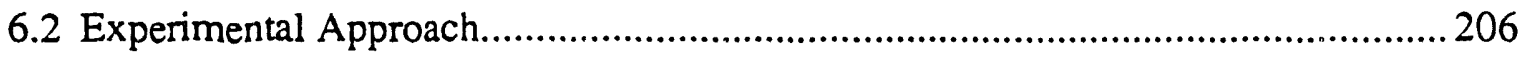

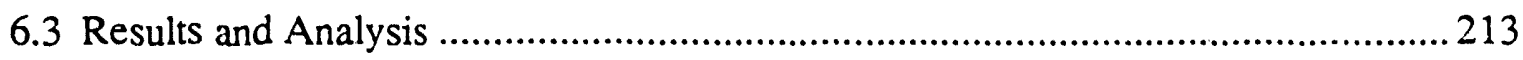

6.3.1 Identification of Photodissociation Channels .........................................214

6.3.2 Translational Energy and Angular Distributions .....................................217

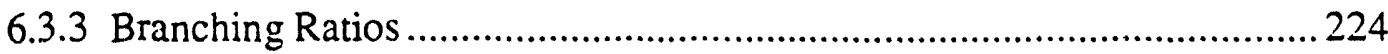




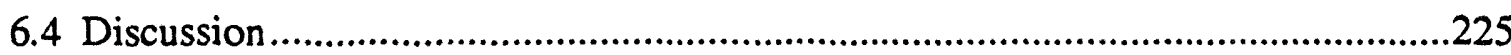

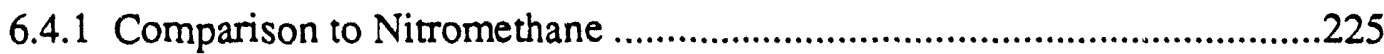

6.4.2 Nature of the Initially Excited Electronic State ........................................228

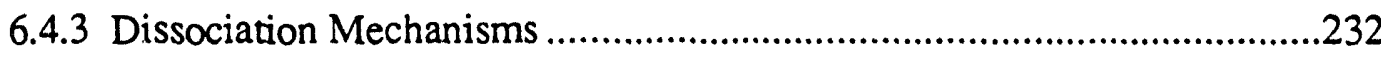

6.4.3.1 Possible Role of Ground State Statistical Dissociation ................232

6.4.3.2 $\mathrm{CH}_{2} \mathrm{NO}_{2} \rightarrow \mathrm{H}_{2} \mathrm{CO}+\mathrm{NO}$ Dissociation Mechanism....................241

6. ‘.3.3 $\mathrm{CH}_{2} \mathrm{NO}_{2} \rightarrow \mathrm{CH}_{2} \mathrm{NO}+\mathrm{O}$ Dissociation Mechanism.....................247

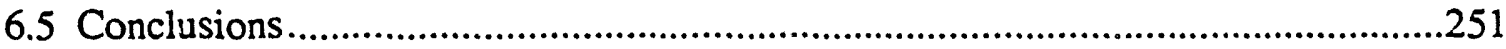

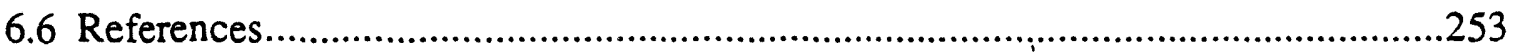

Appendix A FRBM - The Ion Mass Spectrum and Total Photodissociation Cross

Section Experiment Control and Data Acquisition Program

.256

Appendix B TPS - The Control and Data Acquisition Program for

Photodissociation Dynamics Experiments Involving Time- and

Position-Sensitive Detection .

.382 


\section{Acknowledgments}

Much credit for the success of this experiment has to go to those I have been fortunate to work with in the last five years. Above all, Professor Daniel Neumark deserves recognition as the originator of this ambitious and rewarding project, and my thanks for being a superb research advisor. Dan has managed to foster an atmosphere within the group that is both ambitious and confident, while at the same time very supportive and unified. In addition, Dan actively encourages his students to pursue ideas of their own; as just one example, this encouragement lead to the development of our pulsed discharge negative ion source.

Looking back, I feel very fortunate to have been at the right place at the right time, having joined the Neumark Group soon after the free radical photodissociation proposal received funding. As I surveyed the completely empty laboratory that was D-21 Latimer, I felt very excited and eager. But how was I, a naive, inexperienced, first-year graduate student, going to build, trouble-shoot and operate such a complex experiment? The answer, of course, was that I would not do it alone. Through every step of the building process Dan was always accessable, providing clever ideas to solve difficult problems as well as a reasonable amount of patience and understanding when my inexperience occasionally showed through. Not long into the project, Ricky Metz and then Bob Continetti signed on, bringing not only enthusiasm but also a wealth of knowledge, which they always took time to confer to me.

Ricardo Metz had a major role in building the machine; he was spirited away from the operational fixed-frequency photoelectron spectrometer in the early stages of construction. Ricky was very multi-talented and managed to keep a hand in explaining transition state data from his old machine even as he worked on the radical project. $\mathrm{He}$ taught me the basics of photoelectron spectroscopy, taking me under his wing when we slipped across the hall and spent a few weeks on his old stomping grounds, using the 
fixed-frequency photoelectron spectrometer to scout for negative ions suitable for use as radical precursors.

Dr. Robert Continetti joined the experiment at a critical point, when the major building was nearly complete and the first experiments were only a few months away. Initially, he provided some critical technology (piezo valve and fast ion gauge) and 'hard experiment' experience that surely made a world of diffierence. Later, Bob played a leading role in developing and implementing other essential components, including the time-and-position sensitive detector. As the first postdoc on the experiment, Bob lead by example, working long hours and giving his all. I have no doubt he will be successful in his position on the faculty at UC San Diego.

As a new member of the FRBM team, David Osborn had an ideal philosophy, striving to learn as much as possible while enthusiastically doing whatever was asked, all the while growing more independent. Dave patiently waited for his opportunity to lead a project, knowing that he would inherit an experiment with all major wrinkles ironed out, in part due to his own hard work. I am fully confident that he is ready to help lead the machine into new and exciting areas in the next few years. He is also thanked for his (often thankless) work as system manager for the various Sun workstations since the departure of Steve Bradforth.

Dr. David Leahy (DaveBob) was a welcome addition, arriving to help fill the void left by the departure of Bob Continetti to San Diego. Although excellent in all regards, Dave has made major contributions to our time- and position-sensitive data analysis effort, which was functional but a somewhat underdeveloped area on his arrival. He has also been active in developing a better understanding of the remaining non-linearities in our time- and position-sensitive detector, thereby pushing our energy resolution to new limits. This progress allowed him to lead the way on a very comprehensive study of $\mathrm{O}_{2}$ predissociation. Because Dave hails (most recently) from Stanford, I have one parting message for him that I hope he will remember and cherish: GO BEARS! 
To the newest member of the FRBM team, Eric Ross, I wish you well. There is no doubt that with hard work you will carry on the tradition of success that has been established thus far.

I owe thanks to several former Neumark Group members who I did not work directly with, but definitely contributed to my graduate school experience in one way or another.

Theofanis Kitsopolous continually demonstrated that hard work, dedication and perseverance in the laboratory eventually brings rewards; he has been an excellent role model in this regard. His instigation of impromptu discussions on any and all topics (particularly scientific in nature) is sorely missed. I wish Theo continued success at his faculty position in Greece.

As well as being a fine scientist, Alexandra Weaver managed to maintain a sense of perspective, having outside interests resembling those of a real person! Alex was also a very patient and kind person, whose genuine concern for the well-being of others was refreshing and inspirational.

Sharing an office with Steven Bradforth was good fun. Since we both instinctively sensed the tack the other would take in a discussion, and happily provided the opposing viewpoint as a matter of course, there was never a dull moment. Steve was also a storehouse of information about various theoretical and computational matters. He deserves recognition for his efforts as Sun system manager while in the group.

In accepting an appointment at UBC following a postdoctoral term in the Neumark Group, Dr. Irene Waller showed that it is possible to return to a faculty position in Canada. I wish her much success in her work there.

The current members of the Neumark Group (in addition to those I work most closely with, who were acknowledged above), also deserve mention. Don Arnold and I were new graduate students together in the Fall of ' 88 , when we both joined the Neumark Group. In addition to his considerable success in the lab, Don breathed much needed life 
into the softball exploits of the Neumark Group. I wish him and his co-workers, Eun $\mathrm{Ha}$ Kim, Cangshan Xu and Esther de Beer, every success in the future. Caroline Amold has managed to maintain the success of the difficult threshold photoelectron experiment, as well as being a leading force in planning and executing many of the Group's social events. Caroline and her co-workers, Georg Reiser, Yuexing Zhoa, and Ivan Yourshaw, have my best wishes, as does Jeff Greenblatt, who recently joined the group to work on a planned fourth experiment.

Various staff members of the College of Chemistry have also contributed to make this project successful. The skill, dedication and responsiveness of the machine shop has been unrelenting, and all of the machinists and supervisors I have had the pleasure to work with have been extremely helpful and professional. Special thanks to George Weber, Chuck Souders, Eric Granlund, Larry Hull, Scott Bonilla, Bob Dunn and the rest of the machine shop staff. Instrument Repair, lead by Bob Cook, has been helpful, particularly in the building stages of the machine with advice on electrical wiring, etc. The Wood Shop, especially Norman Tom, also played a critical role during the building of the machine, provided sage advice, responsive service and plenty of Unistrut $\circledast$ !

The Neumark Group was especially lucky to gain the privilege of having Cheryn Gleibe as Dan's Administrative Assistant. Cheryn is exceptionally talented and her experience and intelligence allows her to cheerfully do the job of two people, in half the time!

Within the Lawrence Berkeley Laboratory Chemical Science Division we are fortunate to have Auben Winters as our effective advocate. Our connection to Lawrence Berkeley Laboratory has also allowed us to receive helpful advice at various times from Joe Katz, Oren Milgrome, and George Gabor.

Another resource to which we were fortunate to have access is Space Sciences Laboratory, a division of the UC Berkeley physics department. From them, we learned much about wedge-and-strip detectors. Without the help and advice of Dr. Oswald 
Seigmund and Joe Stock, developing our time- and position-sensitive detector would have taken much longer.

I wish to thank my Preliminary Exam committee, which consisted of Profs. Yuan Lee (Chair), Gabor Somerjai, John Porter and Marjory Olmstead (Physics). My thesis committee, consisting of Profs. Daniel Neumark (Chair), Yuan Lee and Jeffery Reimer (Chemical Engineering) also deserve thanks for their efforts in this capacity.

I would not have considered setting my sights as high as Beikeley if not for the encouragement of many people. While working at the Notre Dame Radiation Laboratory as part of an undergraduate co-op program, I was under the supervision of Dr. P.K. Das. A frequent collaborator was a distinguished professor from India, Prof. M.V. George. Both of these gentlemen freely offered encouragement and knowledge, and without trying explicitly to do so, raised my opinion of science and scientists a great deal. Prof. John Hepburn at the University of Waterloo continued this trend, and introduced me to the exciting world of Chemical Physics.

I wish to thank NSERC (Canada) for a 1967 Science and Engineering Fellowship. The research described in tinis thesis was supported by the Director, Office of Basic Energy Sciences, Chemical Sciences Division of the U. S. Department of Energy under Contract No. DE-AC03-76SF00098. Additional support was provided by the National Science Foundation under Grant No. CHE 91-08145.

I have much to be thankful for away from the laboratory as well. I am very lucky to have both the strong support for my professional goals as well as the refreshing counterbalance to science that comes from my family. During my time here at Berkeley my wife, Lejla, and I have had two children. Because of the sacrifices they have made for me during my graduate career, especially during the writing of this thesis, it is to these three that this thesis is dedicated. 


\section{Preface}

This thesis is an account of the photodissociation studies carried out using the Fast Radical Beam Machine to characterize the dissociative electronic states of several reactive free radicals.

Chapter 1 gives a general overview of the experimental program, including the motivation for our work, the approach that is used and the knowledge that is gained. The general technique of photofragment translational spectroscopy is introduced and the adaptations present in our experiments are then discussed. The principles of applying negative ion photodetachment as the source of our free radicals are also examined.

Chapter 2 describes the Fast Radical Beam Machine at length, as this thesis is the first detailed account of its construction and operation. Included is a description of a fast high voltage switch, which was designed and implemented as part of the construction process. This switch is the subject of a publication having Dr. Robert Continetti as principal author, appearing in Rev. Sci. Instrum. 63, 1840 (1992). In addition, a promising new source of negative ions, based on a pulsed discharge-free jet expansion is outlined within this chapter.

Chapter 3 provides the details of our data analysis procedures. Included is a short description of Monte Carlo forward convolution. This method of analysis is applied in time-of-flight distribution analysis of the type found in Chapter 5, and has been used as a consistency check of the more direct inversion of data from our dissociation dynamics experiments involving the time and position-sensitive detector. This direct inversion process is the subject of the remainder of the chapter, with a step-by-step description of the techniques involved in the analysis of experiments such as those described in Chapter 6 (see below).

In Chapter 4, examples of experimental results obtained in our studies of $\mathrm{O}_{2}$ and $\mathrm{N}_{3}$ are presented as an illustration of the capabilities of the machine. The $\mathrm{O}_{2}$ data shown is 
part of a data set which resulted in a letter to appear in Chemical Physics Letters and a full article to be submitted to the Journal of Chemical Physics, both of which will have Dr. David Leahy as the principal author. The $\mathrm{N}_{3}$ data have been published previously with Dr. Robert Continetti as the principal author; the relevant articles can be found in Chem. Phys. Lett. 182, 406 (1992) and J. Chem. Phys. 99, 2616 (1993).

Chapter 5 describes experiments characterizing the NCO free radical. The photofragment time-of-flight spectrum is analyzed to reveal the occurence of spinforbidden dissociation below the energetic threshold for spin-allowed dissociation. It is then shown that above the threshold for spin-allowed dissociation, the spin-allowed channel dominates. From the determination of this threshold, we are able to directly deduce a heat of formation for the NCO radical. These experiments resulted in the first full publication from this machine; published in J. Chem. Phys. 97, 4937 (1992).

Chapter 6 contains an account of our work on the nitromethyl radical $\mathrm{CH}_{2} \mathrm{NO}_{2}$. This radical dissociates in a manner completely different from that which might be expected based on the UV photodissociation behavior of nitromethane, $\mathrm{CH}_{3} \mathrm{NO}_{2}$. We observe the existence of two major dissociation channels, and propose excited-state mechanisms that are consistent with the experimental observations. This work was discussed in preliminary form in SPIE Proceedings 1858, 49 (1993) where the principal author was Dr. David Leahy, but a more detailed treatment, following Chapter 6, will appear in the December $1^{\text {st }} 1993$ issue of the Journal of Chemical Physics.

Appendices A and B contain the Control and Data Aquisition programs FRBM and TPS. 


\section{Chapter 1}

\section{Fast Radical Beam Photofragment Translational Spectroscopy - An Introduction}

In recent years there has been a growing interest in the characterization of reactive free radicals. These species have long attracted attention ${ }^{1}$ because of their universally acknowledged role as important intermediates in a variety of critical processes, such as many of those found in combustion or atmospheric chemistry. However, both the inability to easily generate such species purely and their high reactivity once made have historically hindered their study. For this reason free radicals are, in general, much less well characterized than stable, closed shell molecules.

Consequently, the thermodynamics and photochemistry of radicals is often illdefined. Many radicals have large uncertainties associated with even the most basic thermochemical information, such as their heats of formation. These quantities are vitally important if we are to fully understand and eventually model processes involving these radicals.

Further motivation for the investigation of the free radicals lies in the fact that the open shell nature of free radicals will result in a higher density of low lying electronic states. Because of the presence of these low lying electronic states we can expect a relatively rich spectroscopy and photochemistry for these species. This increased state density will also result in a higher probability of surface crossings following photoexcitation, which will of ten result in complex dissociation dynamics. The dynamics involved in such dissociation processes will be directly reflected in the nature of the resulting product final state distributions. In addition, this regime where strong interaction amongst various excited states is common can typically be accessed with relative ease in free radicals (the excitation energies required are usually amenable to visible or UV laser excitation). In this regard free radicals may represent model systems for study of these interactions. 
In cases where the difficulties associated with generating a suitable sample of free radicals have been overcome, spectroscopic techniques such as laser induced fluorescence (LIF), multiphoton ionization (MPI), ${ }^{2}$ and absorption spectroscopy have enjoyed success in the study of the bound states and molecular structure of free radicals. ${ }^{3}$

Dissociative states of free radicals, however, have received much less attention. A brief review of work completed thus far in this area is given in the next section. In the remainder of the chapter, our technique, fast beam photofragment translational spectroscopy, is outlined, followed by a consideration of what can be learned from photodissociation spectroscopy and dynamics experiments. The final section of the chapter discusses the method by which we produce our reactive free radicals for subsequent photodissociation, namely, photodetachment of the corresponding negative ion, and goes into some detail about the characteristics of this process relevant to our application.

\subsection{Background}

Lee and co-workers have completed a variety of photofragment translational spectroscopy studies involving the photodissociation of radicals. Specifically, they have studied the primary photodissociation of $\mathrm{CCl}_{3}, \mathrm{ClO}_{2}$, and $\mathrm{NO}_{3}$ radicals. The secondary dissociation of the $\mathrm{CCH}$ and HS radicals, observed following the primary dissociation of $\mathrm{C}_{2} \mathrm{H}_{2}$ and $\mathrm{H}_{2} \mathrm{~S}$, respectively, also received attention, although secondary dissociation experiments in general suffer from ill-defined initial state preparation of the radical via the primary dissociation process. Briefly, Hintsa et al. ${ }^{4}$ used photolytic generation of $\mathrm{CCl}_{3}$ from the precursor $\mathrm{CCl}_{4}$, followed by thermalization in a buffer gas and then molecular beam expansion. This allowed the study of $\mathrm{CCl}_{3}$ photodissociation, to form $\mathrm{CCl}_{2}+\mathrm{Cl}$.

Davis and $\mathrm{Lee}^{5}$ observed a mode specific effect in the photodissociation of $\mathrm{ClO}_{2}$; where at roughly the same photolysis energy concerted elimination of $\mathrm{O}_{2}$ is promoted by both bending excitation and combinations of symmetric and antisymmetric stretches. 
Additionally, Davis et al. ${ }^{6}$ directed a study at the photodissociation of the $\mathrm{NO}_{3}$ radical. Two separate dissociation channels were observed, forming $\mathrm{NO}+\mathrm{O}_{2}$ and $\mathrm{NO}_{2}+\mathrm{O}$ products. A heat of formation was found for the $\mathrm{NO}_{3}$ radical, by measuring the $\mathrm{O}-\mathrm{NO}_{2}$ bond dissociation energy, which is in good agreement with a recent value determined by Weaver et al. ${ }^{7}$. The height of the potential barrier involved in the $\mathrm{NO}_{3} \rightarrow \mathrm{NO}+\mathrm{O}_{2}$ channel was also determined. Wodtke and Lee ${ }^{8}$ used $193 \mathrm{~nm}$ light to photodissociate $\mathrm{C}_{2} \mathrm{H}_{2}$, observing secondary dissociation of the $\mathrm{CCH}$ in the process. Three electronic levels of $C_{2}$ are populated as a result of dissociation, with the lower ${ }^{1} \Sigma_{g}$ and ${ }^{3} \Pi_{v}$ electronic states having a greater amount of vibrational excitation than the ${ }^{1} \Pi_{u}$ electronic state. Secondary HS fragmentation was observed in a photodissociation study of $\mathrm{H}_{2} \mathrm{~S}$ by Continetti et al.,9 providing an improved heat of formation for the HS radical.

Coombe and co-workers ${ }^{10}$ performed photolysis experiments on $\mathrm{NCO}$ at $193 \mathrm{~nm}$, forming $\mathrm{CN}\left(X^{2} \Sigma^{+}\right)+\mathrm{O}\left({ }^{3} P\right)$ and monitoring the laser-induced fluorescence of the $\mathrm{CN}$ fragment.

Houston and co-workers ${ }^{11}$ have thoroughly investigated $\mathrm{HCO} / \mathrm{DCO}$ dissociation, observing interesting effects on predissociation lifetime, photofragment recoil distribution and $\mathrm{CO}$ product distributions brought about by pumping various $K$ levels of the upper $\tilde{A}\left({ }^{2} A^{\prime \prime}\right)$ state. These observations confirmed a dynamical curve crossing model developed by Dixon ${ }^{12}$ to describe the $\tilde{A}\left({ }^{2} A^{\prime \prime}\right)-\tilde{X}\left({ }^{2} A^{\prime}\right)$ Renner-Teller interaction. ${ }^{13}$

$\mathrm{Ng}$ and co-workers have investigated the dissociation of the $\mathrm{HS}^{\prime 4}$ and $\mathrm{CH}_{3} \mathrm{~S}^{15}$ radicals. They determine the $S\left({ }^{3} P\right) / S\left({ }^{1} D\right)$ branching ratios and the fine structure distribution for the $S\left({ }^{3} P_{j=2,1,0}\right)$ photoproducts following $193 \mathrm{~nm}$ photolysis for both radicals through $2+1$ resonance enhanced multiphoton ionization of the $S$ atoms.

Dagciigian and co-workers ${ }^{16}$ have observed the production of ND from the predissociation of the DNF radical. This molecule is subject to Renner-Teller interaction, between the $\tilde{A}\left({ }^{2} A^{\prime}\right)$ and $\tilde{X}\left({ }^{2} A^{\prime \prime}\right)$ states, just as is found for $\mathrm{HCO}$ (the state ordering is reversed here, however). Radiative lifetimes for various $\tilde{A}\left({ }^{2} A^{\prime}\right)$ excited levels are found, 
and considerable state-mixing is proposed to explain the photofragment excitation spectra, which also depend significantly on which ND rotational level is probed.

As can be seen by the preceding summary, clever methods exist through which dissociative states of free radicals can be investigated. However, with the notable exception of Lee's photofragment spectroscopy experiments, which use mass selected time-of-flight following electron bombardment ionization, these techniques lack broad generality, as they depend on the formation of a photoproduct that can be probed via a state-selective technique such as MPI or LIF. In addition, the limited number of studies involving reactive free radicals underscores the fact that a truly general source of reactive free radicals remains elusive.

It was the major goal of this project to develop a general technique for probing both the spectroscopy and the dynamics of dissociative electronic states, suitable for the study of reactive free radicals. To do this, we have developed a novel method of generating a pure, internally cold beam of free radicals for subsequent photodissociation.

The spectroscopy of the dissociative electronic states in a given radical is then mapped out by measuring the total photofragment flux as a function of excitation energy. To investigate the dynamics of a particular dissociating system, we utilize a coincidence version of photofragment translational spectroscopy that is adapted to our fast beam conditions (see below). This allows us to obtain detailed information about the disposal of energy within the various degrees of freedom in the photofragments. By exploiting the relationship between the product state distribution of the photofragments and the molecular potential energy surfaces sampled during the dissociation, we can learn about the potential energy surface(s) involved in the dissociation process.

\subsection{Fast Radical Beam Photofragment Translational Spectroscopy}

Photofragment translational spectroscopy is an extremely general, powerful tool for investigating molecular photodissociation dynamics. ${ }^{17.18}$ The original form of 
photofragment translational spectroscopy, demonstrated by Wilson ${ }^{19}$ in the late 60 's, involved the dissociation of a molecule of interest, followed by the measurement of the distribution of times taken for a particular type of photofragment to travel a well-defined distance to a detector, all in a collision-free environment. Since then, substantial improvements in both laser photolysis sources and the original experimental design ${ }^{17}$ have been made. In addition, variations of the basic experiment have appeared, perhaps most notably the ion-imaging technique of Chandler and Houston ${ }^{20}$ and the $\mathrm{H}$ atom time-offlight method developed by Welge and Ashfold. ${ }^{21}$

Our experiment involves quite a different application of photofragment transiational spectroscopy. It can be summarized as follows:

$$
\mathrm{AB}^{-} \stackrel{h v_{1}}{\longrightarrow} \mathrm{AB}+\mathrm{e}^{-} \stackrel{h v_{2}}{\longrightarrow} \mathrm{A}+\mathrm{B} .
$$

In Eqn. (1.1), as is true throughout this chapter, $A B$ refers to a generic free radical, not necessarily a diatomic, and therefore the products $\mathrm{A}$ and $\mathrm{B}$ may be polyatomic.

Due to their open shell nature, free radicals typically have positive electron affinities. Our experiment utilizes this property in order to generate a pure, well-characterized packet of free radicals via photodetachment of the corresponding mass-selected negative ion. As an added advantage, the high (5-8 keV) laboratory frame kinetic energy imparted to the radicals during their acceleration prior to detachment allows both the efficient collection and detection of photofragments, since they must simply strike the face of a microchannel plate (MCP) detector.

Two types of photodissociation experiments can be carried out within this general scheme, as alluded to in the previous section. The photofragment flux can be measured as a function of dissociation photon energy, $\mathrm{hv}_{2}$, to map out the dissociative transitions of the free radical. Following this, the dissociation dynamics at a given photon energy can be investigated using a coincidence time- and position-sensitive detector, ${ }^{22,23}$ which allows 
the determination of the photofragment translational energy distribution and energydependent angular distributions.

The version of photofragment translational spectroscopy employed in our experiment differs from Wilson's original method in two major ways, which are ultimately a consequence of the kinemati scheme we use for investigating free radicals. Since free radicals are generated by photodetaching an electron from the negative ion corresponding to the radical of interest, we have the ability to produce the free radicals following acceleration of the precursor negative ions to a high laboratory kinetic energy (resulting in the formation of a 'fast beam'). This fast beam approach is similar to the method pioneered by Los' group, ${ }^{22,24}$ and also used by Helm and Cosby, ${ }^{25}$ for studying dissociative states of small molecules via dissociative charge exchange between a positive ion and metal vapor.

High center-of-mass laboratory kinetic energy allows: (1) efficient $(>50 \%)^{26}$ detection of photofragments through simple collision with the face of a microchannel plate detector, eliminating the need for ionization or other final steps to permit detection, and (2) high collection efficiency, a necessary component of any coincidence detection scheme. If the combined collection and detection probability for a given single fragment is given by the fraction $f$, the probability of a given coincidence event being detected will be only $f^{2}$. However, because in our experiment the kinetic energy release of the fragments is relatively small compared to the large laboratory kinetic energy, the photofragments typically do not scatter far from the beam axis during the flight time prior to detection, and thus both fragments impinge on opposite halves of the same on-axis microchannel r iate detector. In this configuration nearly all of the recoil angle distribution can be accepted at once, which compares favorably with most other methods. When using our time- and position-sensitive detector, we determine the distance between the two fragments and their orientation at the detector face, in addition to the difference in their time-of-flight. This directly yields the magnitude and direction of the center-of-mass velocity vector for a 
single dissociation event in three dimensions. The resolution of our detection scheme is considered more fully in Chapter 2, however, the coincidence aspect of our technique is beneficial in this regard (see $\$$ 2.7.6.5). As a result, our translational energy resolution $\left(\Delta E / E_{T} \approx 0.7 \%\right)^{27}$ also compares favorably with other forms of photofragment translational spectroscopy.

Fast beam photofragment translational spectroscopy is quite a general method for the study of free radical photodissociation. Although photodetachment of a fast, internally cold, mass-selected negative ion beam is a not a highly efficient means to produce free radicals, this has not proven to be a limitation. In particular, the coincidence detection scheme processes only one dissociation event per laser shot; for this reason copious radical production would not benefit us.

Two factors prevent our experiment from being completely universal once the radical beam has been generated. The accuracy of our kinetic energy release measurement requires that the lifetime of the dissociating state not be exceedingly long, since dissociation must occur within a small fraction of the total flight time between the photodissociation region and the detector. For example, $\mathrm{N}_{3}$ at a beam energy of $8 \mathrm{keV}$ has a center-of-mass flight time (using our standard $1 \mathrm{~m}$ flight length) of $\sim 5 \mu \mathrm{s}$. Provided the upper state dissociation lifetime is $<30 \mathrm{~ns}$, no noticeable degradation of resolution will result. These possible lifetime effects can be investigated by performing the experiment with various beam energies or flight lengths. The lifetime 'restriction' is quite reasonable, and has not been an issue thus far.

The second, more strict requirement for any radical system that is to be studied is that dissociation must occur to give products of mass ratio less than approximately four to one. Higher mass ratios would result in unfavorable kinematics: either the light fragment would recoil far enough that it missed the detector, or the heavy fragment would not scatter out of the beam axis enough to avoid the beam block designed to prevent 
undissociated radicals from being detected. This effectively precludes investigation of systems where a hydrogen atom is lost, at least in the current design of the instrument.

In the following section, a general overview of the spectroscopic and dynamical information we gain from our photodissociation experiments is given, and the relation to the potential energy surfaces involved in the dissociation is developed.

\subsection{Photodissociation Spectroscopy and Dynamics}

When embarking on the investigation of a given free radical, we initially wish to determine the locations of the dissociative electronic states. Once an electronic transition has occurred ${ }^{28}$ that places a radical in an excited state at an energy above the lowest dissociation limit, dissociation will occur if a facile pathway exists. This dissociation process may be either direct or involve predissociation; the nature of the dissociation process can be often be discerned through the spectroscopy of the dissociative excited state. This subject is now briefly considered.

\subsubsection{The Spectroscopy of Dissociative Excited States}

By their very nature, conventional spectroscopic studies of free radicals provide the most information about bound ro-vibrational levels within particular electronic states. Knowledge of dissociative states of free radicals is limited to the occasional reported observation of broad absorption continua, broadened absorption lines, or the breaking off of signal in certain regions of emission spectra. From many of these studies, particularly those involving absorption, 29 it is even difficult to accurately determine the onset of dissociation.

As mentioned above, our first goal is to determine the spectroscopy of the dissociative electronic states to be investigated. We thus acquire the total photodissociation cross section spectrum of the radical by measuring the fragment flux as a function of dissociation photon energy. The exact details of our experimental method 
are given in Chapter 2, but these spectra effectively map out the dissociative electronic states of the radical in the photon energy range that we use. When such information already exists, we are guided by previously reported observations of dissociative states. Two types of dissociation processes, resulting in very different photodissociation cross section spectra, can occur.

Direct dissociation involves excitation to a purely repulsive region in one coordinate of an electronic surface. Depending on the presence or absence of active vibrational modes perpendicular to the dissociation coordinate, and the rate of dissociation, direct dissociation may contain some broad structure associated with these bound modes. In any case, the excited molecule immediately begins to form products as it distorts irreversibly along the dissociation coordinate.

Predissociation, an example of which is illustrated by Figure 1.1,30 involves excitation to a particular combination of vibrational levels of an excited electronic state. Depending on the type of predissociation occurring, this system may subsequently couple to a directly repulsive electronic state. Alternately, the system might undergo intramolecular vibrational redistribution (IVR) until enough energy to cause dissociation appears in the vibrational degree of freedom that is the dissociation coordinate (this mechanism often follows internal conversion from the initially excited state to the ground electronic state). The strength of the interaction with the dissociative state, or the rate of IVR, determines the lifetime of the initially prepared states, and correspondingly, the absorption linewidths associated with transitions to these states (the lifetime broadening is, in some sense, a manifestation of the Heisenberg Uncertainty Principle). These absorption lines will be observed as structure in our photodissociation cross section spectra.

Figure 1.1 A diagram showing a predissociative system, and the structure which can appear in the total cross section spectrum of such a system. As with all of the diagrams showing potential energy curves, the radical $A B$ is not restricted to be a diatomic, so fragment $A$ and/or $B$ may represent polyatomic molecules. 


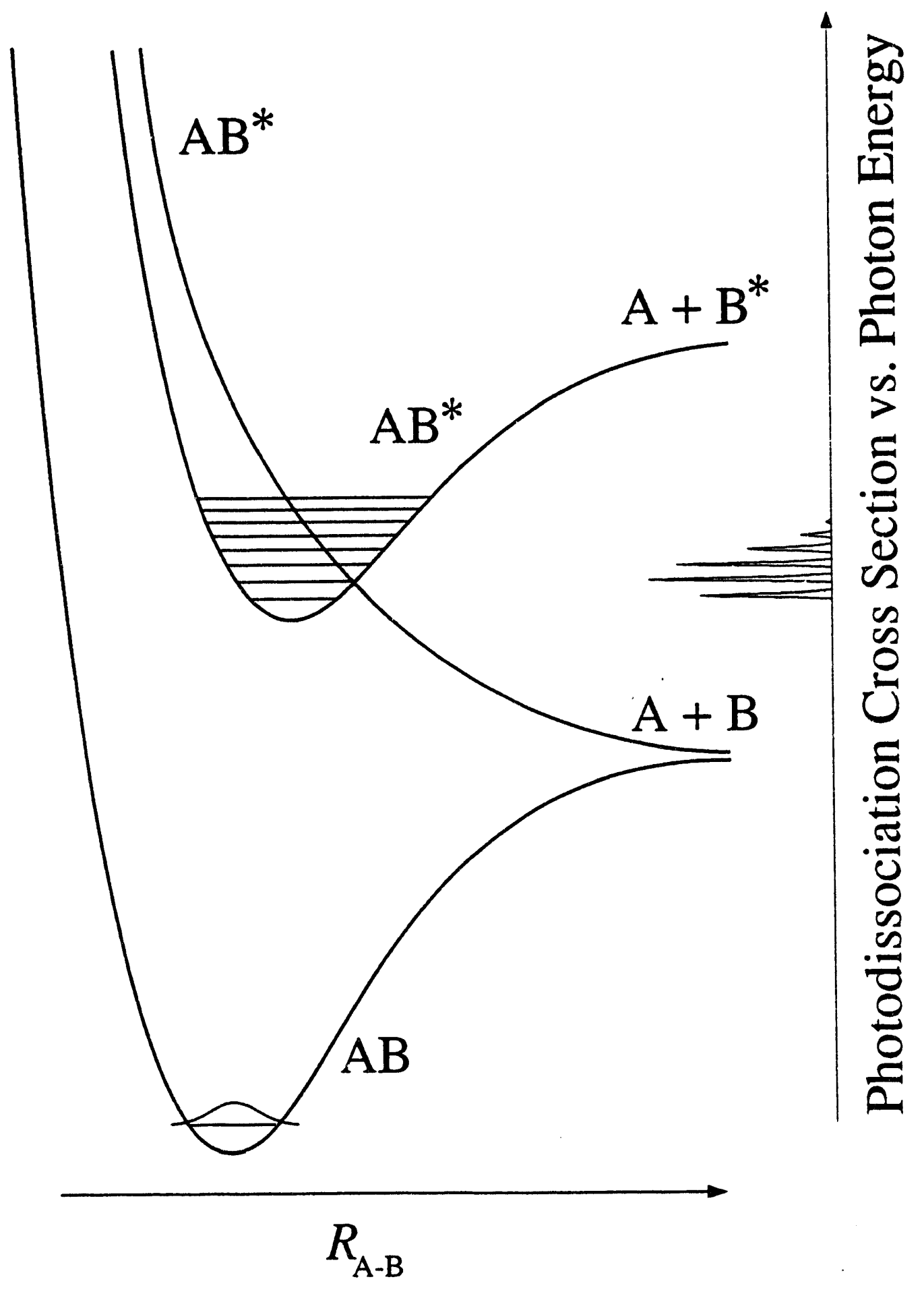

Figure 1.1 
Unstructured photodissociation cross section spectra therefore imply either an extremely fast predissociation process or excitation to a directly repulsive state (discussed above).

It is important to remember that the photodissociation cross section measurements that are obtained in this experiment are what is known as 'action spectra'. Action spectra contain signal that is the result of a particular process (in our case dissociation) which occurs following absorption of a photon. However, quite often the quantum yield for the process that is monitored is not unity, and may vary with wavelength. For example, fluorescence may effectively compete with dissociation at certain photon energies when long-lived predissociative excited states are involved. Therefore, in general, the intensities found in photodissociation cross section spectra must not be equated with the absorption intensity.

Once the spectroscopy of a particular dissociative state has been determined, it is then of interest to determine several key aspects of the dissociation that is occurring, such as: the identity and amount of each product formed; the product translational, electronic, vibrational and rotational (resolution permitting) energies; the angular distribution of the products; and the dissociation lifetimes. A discussion of the extraction of this information from our photodissociation dynamics experiments follows.

\subsubsection{Photodissociation Dynamics}

The quantities of interest in a photodissociation dynamics experiment are the true photofragment center-of-mass translational energy and angular distributions. For a onephoton dissociation the form of the photofragment energy and angular distribution $\rho\left(E_{\mathrm{T}}, \theta\right)$ can be written as

$$
\mathrm{P}\left(E_{\mathrm{T}}, \theta\right)=P\left(E_{\mathrm{T}}\right)\left[1+\beta\left(E_{\mathrm{T}}\right) \mathrm{P}_{2}(\cos \theta)\right]
$$

where $\theta$ is the angle between the photofragment recoil vector and the electric vector of the dissociation laser, $\mathrm{P}_{2}(\cos \theta)$ is the second Legendre polynomial, $\beta\left(E_{\mathrm{T}}\right)$ is the (energy- 
dependent) anisotropy parameter, and $P\left(E_{\mathrm{T}}\right)$ is the angle-integrated kinetic energy distribution. The significance of the anisotropy parameter, which describes the form of the recoil angular distribution, will be discussed below. At this point we will examine the relevance of knowing the translational energy distribution.

With reference to Figure 1.2, it can be seen that Eqn. (1.3) describes the balance of energy in a photodissociation experiment.

$$
E_{I N T, A B}+E_{h v}=E_{I N T, A}+E_{I N T, B}+E_{T}+D_{0}
$$

Here $E_{\mathrm{hv}}$ represents the photon energy, $E_{\mathrm{T}}$ the translational energy (kinetic energy release) and $E_{I N T A B}, E_{I N T A}$, and $E_{I N T, B}$ are the internal energies of the parent radical and the two photofragments, respectively. As will be described, in our experiment great effort is expended to insure $E_{\text {INTAB }} \approx 0$, and for the subsequent discussion it will be assumed to be zero. $D_{0}$ represents the energy required to form the dissociation products in their ground states from ground state radicals at $0 \mathrm{~K}$.

As mentioned earlier, the energetics of free radicals are often somewhat uncertain; for example, large error bars in free radical heats of formation are quite common. However, products from the dissociation of such a radical usually have more well-defined energetics (i.e., the heat of formation of $A B$ might be fairly uncertain, while the heats of formation of $A$ and $B$ could be among the most precisely known.) Because of this, photodissociation studies provide a method with which to investigate the energetics of free radicals. As Eqn. (1.4) shows,

Figure 1.2 The energetics involved in a dissociation process. Note the energy partitioning which occurs between the internal degrees of freedom and the relative translation of the product fragments. On the right is a hypothetical translational energy distribution that might be appropriate for dissociation of a triatomic, with a diatomic and an atom as products. The diatomic is meant to have a long vibrational progression excited, with a rotational contour giving shape to the vibrational peaks. 


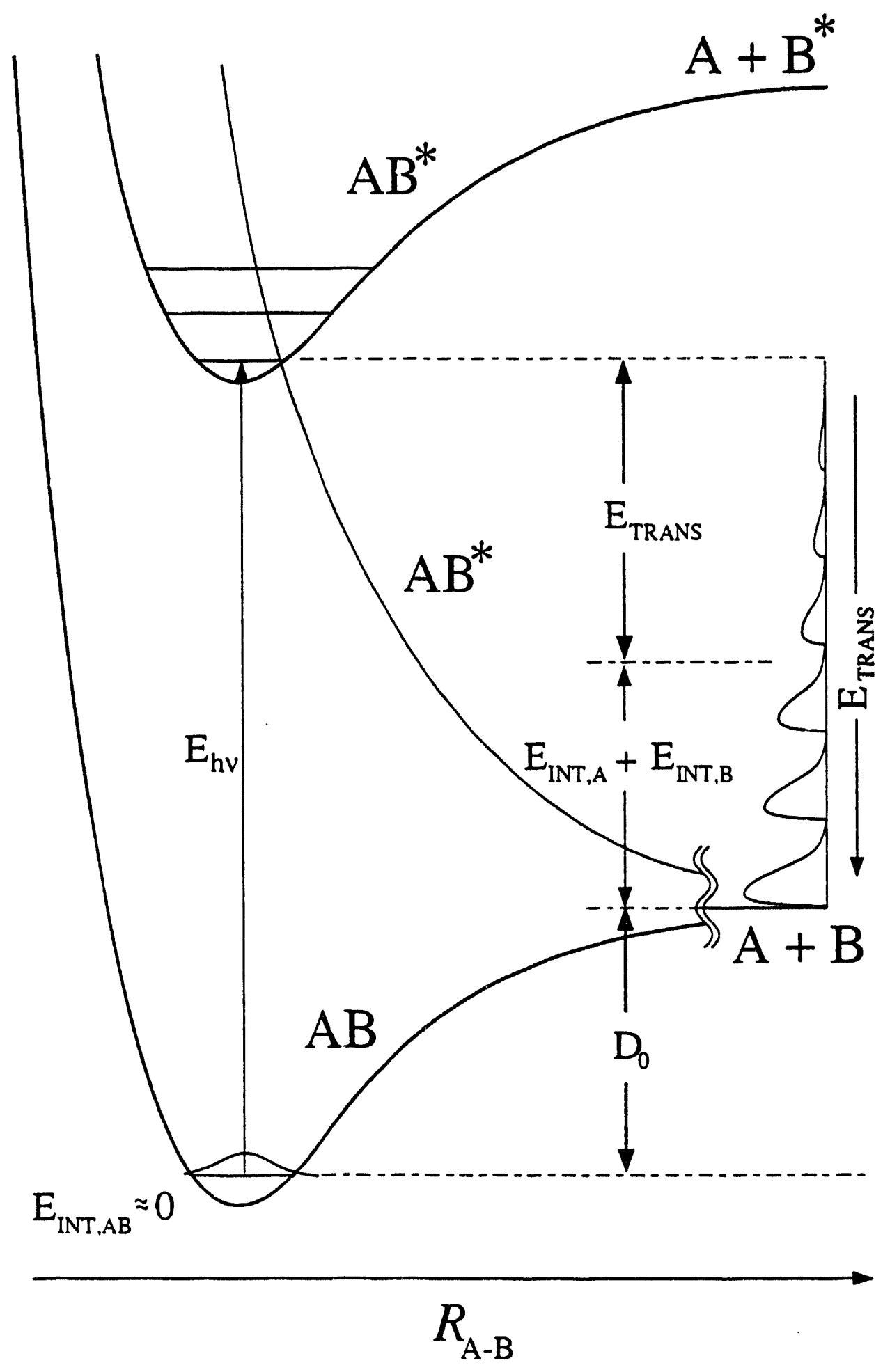

Figure 1.2 


$$
\Delta_{f} H_{0 K}^{0}(\mathrm{AB})=\Delta_{f} H_{0 K}^{0}(\mathrm{~A})+\Delta_{f} H_{0 K}^{0}(\mathrm{~B})-D_{0}(\mathrm{~A}-\mathrm{B})
$$

the quantity linking the heats of formation values is the bond dissociation energy, $D_{0}$, for the bond that is broken.

By measuring the kinetic energy release distribution for a given process, we can discern the maximum kinetic energy that is released. If we assume this kinetic energy release corresponds to the production of photofragments with no internal energy, a upper limit of the bond dissociation energy, $D_{0}$, can be obtained via Eqn. (1.5):

$$
D_{0} \leq E_{h v}-E_{T, M A X}
$$

Then, via Eqn. (1.4), a lower limit of the heat of formation for the radical of interest is found. A variation of this method is used in Chapter 4, where it is determined that the $\mathrm{N}_{3}$ radical is $0.05 \mathrm{kcal} / \mathrm{mol}$ unstable with respect to ground state $\mathrm{N}_{2}+\mathrm{N}$, and in Chapter 5 to determine a value for the heat of formation of $\mathrm{NCO}$, which until our study had been generally overestimated by $>5 \mathrm{kcal} / \mathrm{mol} .^{31,32}$

From the translational energy distributions measured in our experiments one can infer the internal energies of the products. Rearranging Eqn. (1.3) we get

$$
E_{A V A I L}=E_{I N T, A}+E_{I N T, B}+E_{T}=E_{h v}-D_{0},
$$

where we have defined the energy available for distribution amongst the product translational and internal energy degrees of freedom as the photon energy less the bond dissociation energy, $D_{0}$, determined above (or known previously). Therefore, the internal energy distribution of the products is simply the available energy minus the translational energy distribution. We now have detailed knowledge of the energy disposal in the dissociation under investigation. This partitioning of energy into translation and the various internal degrees of freedom reflects the dynamics of the dissociation process. As will be seen in the next section, the observed dynamics relates directly to the nature of the potential energy surface(s) involved in the dissociation. 


\subsubsection{Excited State Potential Energy Surfaces}

The translational energy distributions of the products allow insight into the characteristics of the excited state potential energy surfaces involved in the dissociation process. For example, if excitation occurs to a directly repulsive surface, roughly half of the available energy is channeled in the relative translation of the products. In contrast, a simple bond rupture occurring on the electronic ground state with no exit channel barrier will result in very little kinetic energy release; the translational energy distribution will peak very close to zero (most of the available energy remains in the internal degrees of freedom). This behavior is identical whether the original excitation occurred within the ground electronic state, via infrared multiphoton photodissociation, or to an excited electronic state which undergoes internal conversion to vibrationally excited levels of the ground state. Molecular elimination channels have a large barrier in addition to any exothermicity. This results in a large fraction of the barrier being released into translation as the closed shell product repels the remaining fragment on the way down the exit channel of the potential energy surface.

When the product fragments are small, there is a limited number of internal degrees of freedom that may be populated. In favorable cases (relatively few vibrational modes present or active in the products) our translational energy resolution is sufficient to determine populations of the product vibrational levels, and we can estimate the extent of the rotational excitation by the shape of the ruiaional contour (i.e., we can determine the value of the most probable rotational quantum number, $\mathrm{N}$ ). These considerations allow us to comment in even greater detail on the dissociation dynamics of such a radical. For example, a radical that predissociates following an electronic transition from a linear ground state to a linear excited state, yet produces a highly rotationally excited photofragment, must sample bent geometries as it dissociates.

As can be seen from the above, the translational energy distribution allows a great deal of insight into the nature of the potential energy surfaces involved in the dissociation. 
One last source of dynamical information is available to us, and is discussed in the following section.

\subsubsection{Photofragment Recoil Angular Distributions}

As mentioned at the beginning of this section, in addition to the masses of the photofragments and the kinetic energy released in the dissociation process, our experiment also reveals the angle of the photofragment recoil with respect to the electric field vector $E$ of the linearly polarized laser light (see Figure 1.3). This additional information allows further insight into the nature of the dissociative state under consideration, ${ }^{33}$ since recoil angle distributions contain dynamical information, and can identify the symmetry of an upper state in a dissociative transition.

For a one photon allowed transition the probability is proportional to $|\bar{\mu} \cdot \bar{E}|^{2}$, or $\mu^{2} E^{2} \cdot \cos ^{2} \theta$, where $\bar{\mu}$ is the transition moment vector, $\bar{E}$ is the electric field vector of the polarized light, and $\theta$ is the angle subtended by the two vectors (note that the transition probability is azimuthally symmetric about the electric field vector). In a linear molecule, for example, the transition moment is restricted to lying either along or perpendicular to the molecular axis. Of course, the transition moment has a well-defined set of possible orientations in other symmetry point groups as well.

If dissociation is prompt, that is, recoil occurs before rotation of the molecular frame, the photofragment recoil angular distribution will reflect the initial distribution of the molecular axes on excitation. For example, in the case of a linear molecule ${ }^{34}$ having a transition moment parallel to the molecular axis, a prompt dissociation would result in a $\cos ^{2} \theta$ distribution of photofragment recoil vectors, while if the transition moment were perpendicular to the molecular axis, a prompt dissociation would result in a $\sin ^{2} \theta$ distribution of photofragment recoil vectors.

Figure 1.3 A diagram showing the quantities determined in our experiment. 


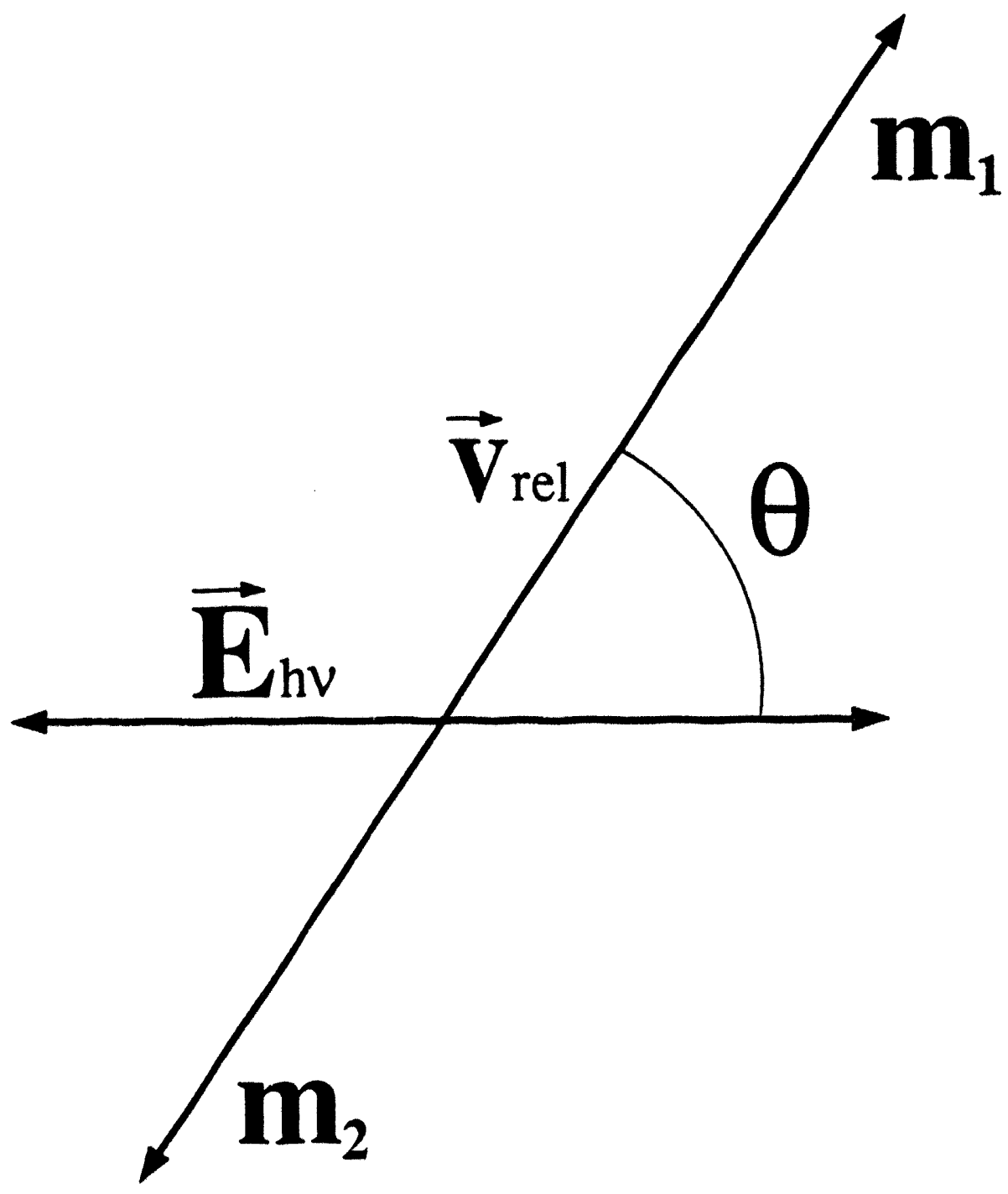

Figure 1.3 
If, however, the excited state has a lifetime on the order of the period of a molecular rotation, the photofragment recoil angular distribution will appear more isotropic because of rotational averaging. Thus, the photofragment angular distribution is sensitive to both the electronic symmetry and the lifetime with respect to dissociation of the upper state.

A standard way of expressing the anisotropy in the recoil angular distribution has been developed by Zare. ${ }^{33}$ This method expresses the probability of recoil at an angle theta with respect to the electric field vector $E$ of the polarized dissociation light as

$$
I(\theta)=\frac{1}{4 \pi}\left[1+\beta \cdot P_{2}(\cos \theta)\right],
$$

where $P_{2}(\cos \theta)$ is the second Legendre polynomial in $\cos \theta$

$$
P_{2}(\cos \theta)=\frac{3 \cos ^{2} \theta-1}{2}
$$

$\beta$ is referred to as the anisotropy parameter, and it ranges from +2 to $-1 . A \cos ^{2} \theta$ distribution corresponds to $\beta$ parameter of $+2, a \sin ^{2} \theta$ distribution corresponds to a $\beta$ parameter of -1 , and an isotropic distribution corresponds to a $\beta$ parameter of 0 .

In our experiment, we have the ability not only to determine a $\beta$ parameter for a given dissociative transition, but to determine $\beta$ as a function of photofragment kinetic energy release from this transition. $\beta\left(E_{T}\right)$ is potentially much more informative. For example, a change in dissociation pathway when forming a given product in a different electronic state may be signaled by a difference in the anisotropy parameter associated with the kinetic energy release values characteristic of each channel.

\subsection{Photodetachment as a Source of Free Radicals}

The main challenge in photodissociation studies of reactive free radicals is preparation of a pure, internally cold sample of sufficiently high number density. 
Traditional sources of free radicals usually cause a stable, closed-shell, precursor molecule to dissociate, forming the reactive free radical as a product. Such sources use techniques such as flash photolysis, in which photodissociation is used to produce the radical of interest, and electric or microwave discharges, which also result in dissociation. These types of sources suffer from two severe difficulties for most applications. First, they are not very selective, producing many by-products, often mixed with unconverted precursor. This mixture will complicate matters when an experiment is subsequently performed: the identity of the signal carrier may be ambiguous. The second difficulty is a consequence of the environment present in these energetic sources. The processes at work in these sources form radicals by adding enough energy to the precursor to break a chemical bond. Unfortunately, energy is generally not deposited into the precursor in a specific manner, and the resulting radicals are formed with a great deal of internal energy. On rare occasions this can be an advantage, i.e., when the spectroscopy of highly vibrationally and rotationally excited levels of the radical is to be investigated. However, in general this is highly undesirable. In particular, if the energetics of a particular radical are to be investigated, one would prefer as small an internal energy distribution as possible in the newly formed radical. This provides more well-defined initial conditions, allowing a more accurate accounting of the energy in the experiment that is subsequently performed.

There have been recent attempts elsewhere to construct free radical sources which typically couple the methods mentioned above to a free jet expansion, thereby obtaining a cold, if often impure, source of free radicals.

Photolytic free radial generation with subsequent free jet expansion is one such method which has undergone development by a number of research groups in the past ten to 15 years. As mentioned in $\S 1.1$, this technique has been used in some of the previous photofragmentation studies of free radicals. The general method is explained in a review by Miller ${ }^{35}$ who has successfully applied it to the spectroscopic study of many radicals under free jet expansion conditions. 
As will be discussed more thoroughly in $\$ 2.3 .5$, pulsed discharges have also been coupled to pulsed free jet expansions in an effort to form free radicals. In particular, more exotic species, with no obvious photolytic precursor, can often be made in this manner.

Another promising alternative radical source has been developed by Chen and coworkers. They have developed a source ${ }^{36}$ that works quite well for selected cases of radicals. ${ }^{37}$ They employ a flash pyrolysis source, having a heated tip on the end of a pulsed free jet expansion. By synthesizing precursors with carefully chosen substituents, nearly quantitative yields of the desired free radical can be formed in the heated section, and internal energy present in these radicals is then removed during the molecular beam expansion. This technique is limited, however, by the ability to identify and then synthesize a precursor which dissociates quantitatively at the appropriate bond on undergoing flash pyrolysis.

Our approach to the difficult problem of preparing reactive free radicals with the goals of highest purity and minimal internal energy is to photodetach the negative ion corresponding to the radical of interest (specifically, the ion having the same chemical structure, but with one extra electron). Since negative ions can be mass selected, an immense benefit of this method is that the radical of interest can be generated exclusively via photodetachment, completely free of impurities (including unconverted precursor). In addition, prior to detachment the ion can be accelerated to high laboratory kinetic energy, permitting uncomplicated detection of the eventual photofragments; the detection efficiency of microchannel plates for neutral particles with $>1 \mathrm{keV}$ kinetic energy is $>50 \% .{ }^{26}$

The possible advantages of this approach make it seem very attractive. However, it is necessary to have the capability of producing sufficient quantities of the negative ion corresponding to the radical of interest. Because of their open-shell nature, free radicals tend to have sizable positive electron affinities, particularly if they contain one or more electronegative atom, and/or can benefit from resonance stabilization of the negative 
charge. This allows negative ion photodetachment to be a very general technique. The method by which we make internally cold negative ions involves crossing an electron beam with the high density region of a pulsed free jet expansion. The details of this source are discussed in $\S 2.3$ and subsections therein. The important consideration for the discussion that follows, however, is that we have the ability to make a sufficient number density of many types of negative ions, typically populating the ground vibrational level and lowest rotational states following expansion.

\subsubsection{Photodetachment Considerations}

The electronic selection rules for photodetachment simply require a one-electron process (i.e., the neutral must be one that can be made by the removal of one electron from the negative ion). For this reason, the ground state of the radical is always an allowed final state in the detachment of an electron from the corresponding negative ion when the anion is closed-shell, and very often an allowed final state otherwise.

The proper photon energy is dictated by two factors. Contrary to the photoionization behavior of neutrals ${ }^{38}$ (where the threshold cross section behaves like a step function), the photodetachment cross-section for negative ions always rises as some function of the energy just above threshold. ${ }^{39,40}$ For this reason, it is beneficial to be as far above threshold as is practical when photodetaching electrons from negative ions.

However, the second factor that is important in choosing the photodetachment photon energy is the extent of any geometry change on going from the negative ion to the neutral. While the full derivation of all considerations involved in photoelectron spectroscopy will not be presented here, one key characteristic of this process must be appreciated. The photodetachment process occurs on a time scale short with respect to vibrational motion. The vibrational population of the radicals formed by photodetachment of a vibrationally cold negative ion is thus determined chiefly by the Franck-Condon overlap between the $v^{\prime \prime}=0$ level of the negative ion and the various energetically accessible 
vibrational levels of the radical. When determining Franck-Condon factors it is common to assume the electronic dipole term is invariant within a given electronic state. The relative intensities of the vibrational levels are therefore computed using the relation

$$
I_{v^{\prime}} \propto\left|\left\langle\Psi_{v^{\prime}}^{0}(R) \mid \Psi_{v^{\prime \prime}=0}^{-}(R)\right\rangle\right|^{2},
$$

where $\Psi_{v^{\prime}=0}(R)$ is the vibrational wavefunction of the ground vibrational level of the negative ion, and $\Psi_{v}^{0}(R)$ are the vibrational wavefunctions of the neutral radical. In pursuing the goal of preparing internally cold radicals via photodetachment of an electron from a negative ion two general cases are possible, as shown schematically in Figure 1.4 and discussed below.

If the change in geometry on photodetachment is minimal, as might be expected for the removal of a nonbonding electron, then the Franck-Condon factors for $\Delta v \neq 0$ transitions will be quite small. In the limit of virtually no geometry change and very small changes in frequency, the Franck Condon factors will be such that the ground vibrational level of the radical will be the only vibrational level populated from the ground vibrational level of the anion. This will hold true even when the detachment photon energy is well above threshold. The first panel in Figure 1.4 illustrates this case, showing how in this situation, it is wise to take advantage of the larger photodetachment cross section expected further above threshold by using as energetic a detachment photon as is practical.

If, on the other hand, the change in geometry on detachment is substantial, the Franck-Condon factors will be large for transitions to excited radical vibrational levels in the one or more modes which most closely resemble the movement of the nuclei. As a result, if the photon energy is far above photodetachment threshold a manifold of radical

Figure 1.4 A diagram showing the two different cases of photodetachment with respect to geometry change between the negative ion and the radical. See text. 

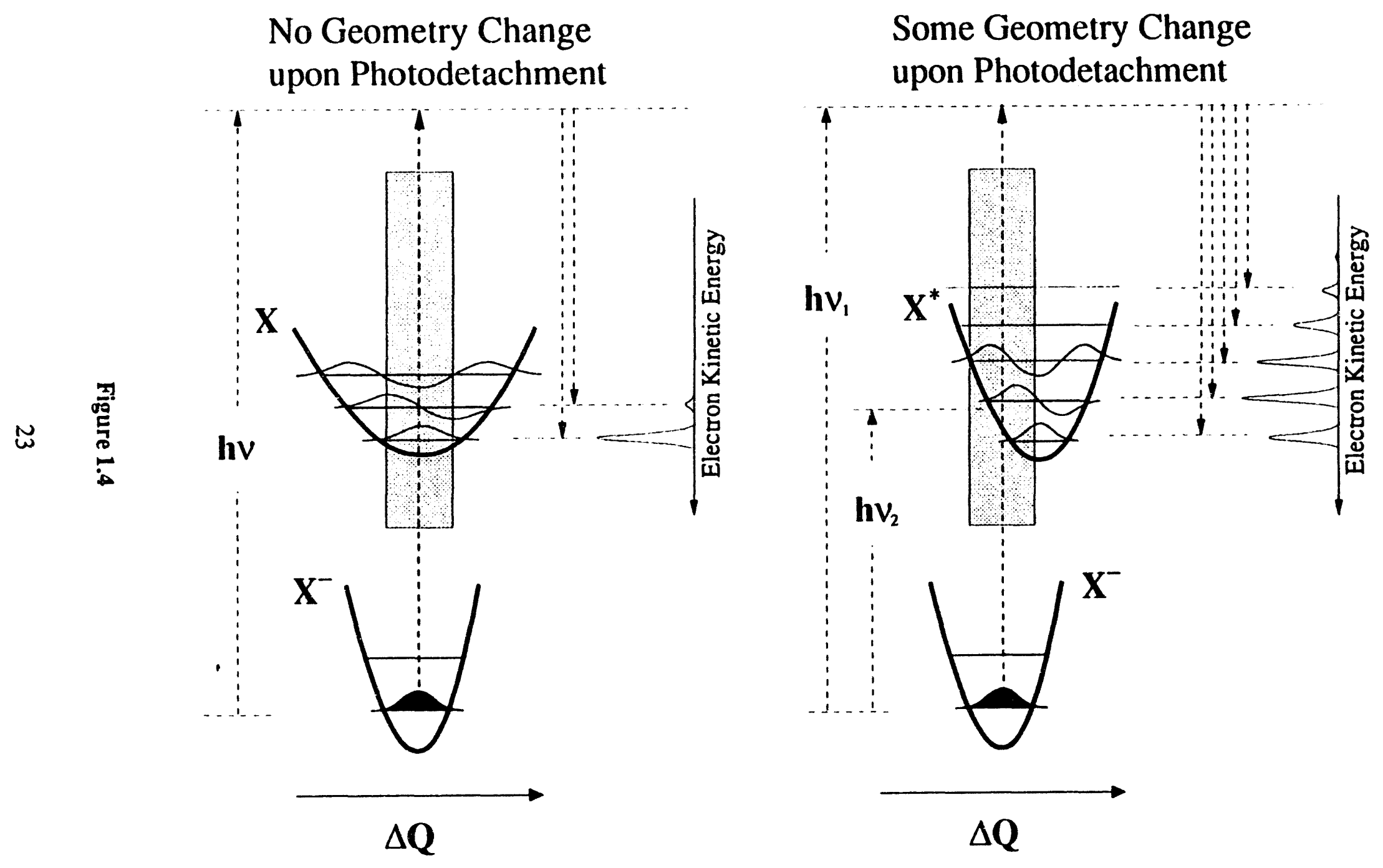
vibrational levels will be populated. As the second panel of Figure 1.4 shows, there is nevertheless a strategy by which radicals can be formed exclusively in the ground vibrational level, even in the case of substantial geometry change. This strategy entails limiting the detachment photon energy to the threshold energy plus an amount just less than the least energetic active vibrational mode (as shown by $h v_{2}$ in Figure 1.4).

In short, judicious choice of photodetachment photon energy will typically allow photodetachment yield to be maximized while resulting in no additional excitation of the neutral radical which is formed.

Occasionally, the Franck-Condon factors for the 0-0 transition between the negative ion and the neutral radical will be unfavorable, and the photoelectron spectrum will show only a broad progression in one or more modes, with no evidence for the origin. In this case, it must be decided if uncertainty in the internal energy of the radicals prior to photodissociation is acceptable. Since there is no shortage of systems that are completely amenable to our photodetachment scheme, this case has yet to be explored. However, the diminished ability to form free radicals in a well-defined initial state is expected to reduce the clarity of subsequent photofragment translational spectroscopy results in a certain number of cases, since the total energy within a given dissociating radical is somewhat unclear. An exception to this expectation are systems that have a structured total photodissociation cross section spectrum (see $\S 4.1$ and $\S 4.2$ ). In this case, the initial energy found in the radical on photoexcitation will still be well-defined, even if a variety of vibrational levels in the ground state of the radical are populated via photodetachment.

\subsubsection{Examples}

Examples of the two photodetachment cases outlined above are now given. The first example illustrates the case involving a negative ion which has very little geometry change upon photodetachment of an electron. $\mathrm{N}_{3}^{-}$is a 'textbook example' of this case because of the non-bonding nature of the electron that is detached to form the ground 
state $\mathrm{N}_{3}$ radical. Rotationally resolv'sd spectra of the $\mathrm{N}_{3}$ radical have been obtained, ${ }^{41}$ and more recently vibration-rotation spectra were obtained for $\mathrm{N}_{3}^{-} \cdot{ }^{42}$ These spectra show that both the radical and negative ion are linear, with a very small geometry change upon detachment.

The photoelectron spectrum of $\mathrm{N}_{3}^{-}$, shown in the top panel of Figure 1.5, was recorded $^{43}$ with a separate fixed-frequency negative ion photoelectron apparatus ${ }^{44}$ using $355 \mathrm{~nm}(3.49 \mathrm{eV})$ photons. The lone feature in the spectrum corresponds mainly to the $000 \leftarrow 000$ (often abbreviated as $0-0$ ) transition between the negative ion and the neutral free radical. The minor contribution of sequence bands involving the relatively low frequency bending mode (i.e., $010 \leftarrow 010,020 \leftarrow 020$, etc.) is suggested by the width of this peak, and eventually confirmed in the total photodissociation cross section spectrum (see $\S 4.3$ and Figure 4.6).

The photoelectron spectrum indicates that virtually no vibrational excitation of the neutral will be occur on photodetachment of the ground vibrational level of the negative ion, even with a photon energy well above the photodetachment threshold. In preparing $\mathrm{N}_{3}$ it was typical to photodetach $\mathrm{N}_{3}^{-}$using the peak of the PTP dye curve, at $343 \mathrm{~nm}$. This corresponds to a photon energy of $3.62 \mathrm{eV}$, well above the measured $2.68 \mathrm{eV}$ electron affinity. ${ }^{43}$ The benefit of detaching this far above threshold is enhanced photodetachment cross section, as described above. Detachment efficiencies of up to $80 \%$

Figure 1.5 Negative ion photoelectron spectra illustrating the two cases with respect to geometry change between the negative ion and the neutral radical. Top panel: The $355 \mathrm{~nm}(3.49 \mathrm{eV})$ photoelectron spectrum of $\mathrm{N}_{3}^{-} .{ }^{43}$ The photon energy used for detachment to form $\mathrm{N}_{3}$ in the present experiment is $\sim 0.94 \mathrm{eV}$ above the detachment threshold.

Bottom panel: The $266 \mathrm{~nm}(4.66 \mathrm{eV})$ photoelectron spectrum of $\mathrm{NCO}^{-} .45$ In contrast to the strategy employed in the case of $\mathrm{N}_{3}^{-}$photodetachment, the photon energy used for detachment to form NCO in the present experiment is only $-0.05 \mathrm{eV}$ above the detachment threshold, as indicated by the arrow. 

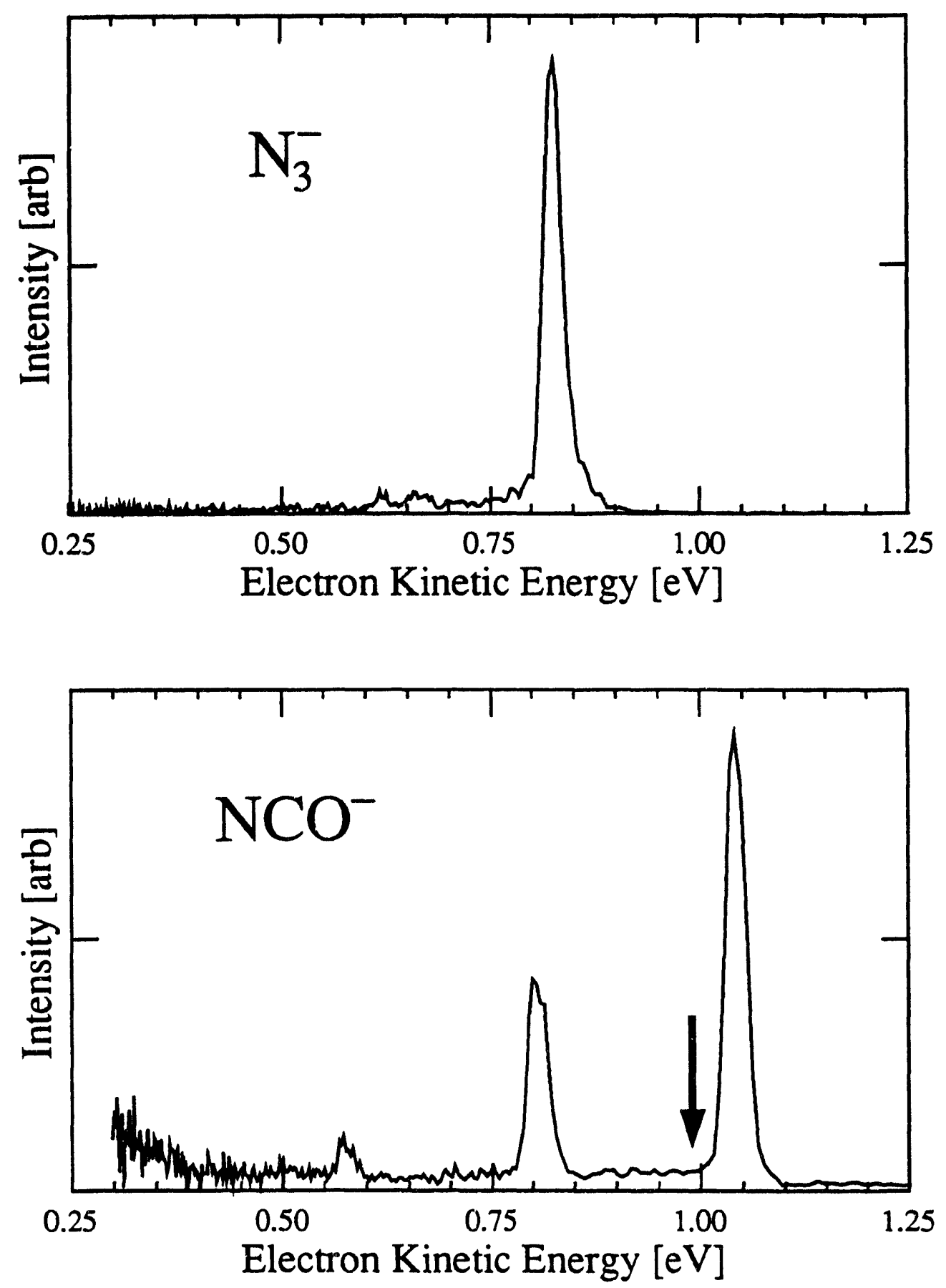

Figure 1.5 
could be observed for $\mathrm{N}_{3}^{-}$with fresh excimer and dye lasers.

An second photodetachment example illustrates the case where geometry change does occur upon photodetachment. The $266 \mathrm{~nm}(4.66 \mathrm{eV})$ photoelectron spectrum of $\mathrm{NCO}^{-}$reported by Bradforth et al., ${ }^{45}$ reproduced in the bottom panel of Figure 1.5, shows a progression in the $v_{3}$ mode. In order to avoid vibrational excitation in the radicals formed via photodetachment, the photon energy must be limited to the electron affinity (determined by Bradforth et al. to be $3.609 \mathrm{eV}$ ) plus an amount just less than the $v_{3}$ fundamental $\left(-1950 \mathrm{~cm}^{-1}\right)$. In our photodissociation studies of NCO, to be described in chapter 5, we therefore use $339 \mathrm{~nm}(3.66 \mathrm{eV})$ photons to detach the electron from $\mathrm{NCO}^{-}$. While slightly more energetic photons could have conceivably been used without exceeding our self-imposed detachment photon energy limit, the maximum for the product of the dye curve and the detachment cross section was found to be at $339 \mathrm{~nm}$. 


\subsection{References}

1 G. Hertzberg, The Spectra and Structure of Simple Free Radicals, (Cornell University Press, Ithica, NY, 1971); J. K. Kochi, Ed. Free Radicals, (Wiley, New York, 1973) Vols. I and II.; J. M. Hay, Reactive Free Radicals, (Academic Press, London, 1974).

2 For a recent review see M. N. R. Ashfold, S. G. Clement, J. D. Howe, and C. M. Western, J. Chem. Soc. Faraday Trans. 89, 1153 (1993).

3 A fairly lengthy article entitled 'Spectra and Structure of Gaseous Organic Free Radicals: A Status Report', S. C. Foster and T. A. Miller, J. Phys. Chem. 93, 5986 (1989) attests to the recent progress made in this area.

4 E. J. Hintsa, X. Zhao, W. M. Jackson, W. B. Miller, A. M. Wodtke, and Y. T. Lee, J. Phys. Chem. 95, 2799 (1991).

5 H. F. Davis and Y. T. Lee, J. Phys. Chem. 96, 5681 (1992).

6 H. F. Davis, B. Kim, H. S. Johnston, and Y. T. Lee, J. Phys. Chem. 97,2172 (1993).

7 A. Weaver, D. W. Arnold, S. E. Bradforth, and D. M. Neumark, J. Chem. Phys. 94, 1740 (1991).

8 A. M. Wodtke and Y. T. Lee, J. Chem. Phys. 89, 4744 (1988).

9 R. E. Continetti, B. A. Balko, and Y. T. Lee, Chem Phys. Lett. 182, 400 (1991).

10 X. Liu and R. D. Coombe, J. Chem. Phys. 91, 7534 (1989).

11 S. H. Kable, J. C. Loison, and P. H. Houston, J. Chem. Phys., 92, 6332 (1990); J. C. Loison, S. H. Kable, P. L. Houston, and I. Burak, J. Chem. Phys. 94, 1796 (1991); S. H. Kable, J. C. Loison, D. W. Neyer, P. H. Houston, I. Burak, and R. N. Dixon, J. Phys. Chem., 95, 8013 (1991); D. W. Neyer, S. H. Kable, J. C. Loison, P. H. Houston, I. Burak, and E. M. Goldfield, J. Chem. Phys., 97, 9036 (1992). D. W. Neyer, X. Luo, P. H. Houston, and I. Burak, J. Chem. Phys., 98, 5095 (1993).

12 R. N. Dixon, Mol. Phys. 54, 333 (1985).

13 For reviews of the Renner-Teller effect, see Ch. Jungen and A. J. Merer in Modern Spectroscopy: Modern Research, K. N. Rao, Ed., (Academic, New York, 1976), Vol. 2, Chapter 2; J. M. Brown and F. Jørgensen, Adv. Chem. Phys. 52, 117 (1983).

14 S. Nourbakhsh, K. Norwood, H.-M. Yin, C.-L. Liao, and C. Y. Ng. J. Chem. Phys. 95,946 (1991); C.-W. Hsu, C.-L. Liao, Z.-X. Ma, P. J. H. Tjossem, and C. Y. Ng, Chem. Phys. Lett. 199, 78 (1992).

15 S. Nourbakhsh, C.-L. Liao, and C. Y. Ng. J. Chem. Phys. 92, 6587 (1990); C.-W. Hsu, C.-L. Liao, Z.X. Ma, P. J. H. Tjossem, and C. Y. Ng, J. Chem. Phys. 97,6283 (1992).

16 J. Chen and P. J. Dagdigian, J. Chem. Phys. 98, 3554 (1993).

17 A. M. Wodtke and Y. T. Lee in Molecular Photodissociation Dynamics, M. N. R. Ashfold, J. E. Baggot, Eds. (Royal Society of Chemistry, London, 1987), p. 31. 
18 For a recent review, see M. N. R. Ashfold, I. R. Lambert, D. H. Mordaunt, G. P. Morley, and C. M. Western, J. Phys. Chem. 96, 2938 (1992).

19 G. E. Busch, J. F. Comelius, R. T. Mahoney, R. I. Morse, D. W. Schlosser, and K. R. Wilson, Rev. Sci. Instrum. 41, 1066, (1970).

20 D. W. Chandler and P. L. Houston, J. Chem. Phys. 87, 1445, (1987); D. W. Chandler, J. M. Thoman, Jr., M. H. M. Janssen, D. H. Parker, Chem. Phys. Lett. 156, 151 (1989); D. W. Chandler, M. H. M. Janssen, S. Stolte, R. N. Strickland, J. W. Thoman, Jr., D. H. Parker, J. Phys. Chem. 94, 4839 (1990); D. P. Baldwin, M. A. Buntine, and D. W. Chandler, J. Chem. Phys. 93, 6578 (1990); M. A. Buntine, D. P. Baldwin, R. N. Zare, and D. W. Chandler, J. Chem. Phys. 94, (1991); M. H. M. Janssen, D. H. Parker, G. O. Sitz, S. Stolte, D. W. Chandler, J. Phys. Chem. 95, 8807 (1991); M. A. Buntine, D. P. Baldwin, and D. W. Chandler, J. Chem. Phys. 96, 5843 (1992); W. P. Hess, D. W. Chandler, and J. W. Thoman Jr., Chem. Phys. 163, 277 (1992); T. Suzuki, V. P. Hradil, S. A. Hewitt, P. L. Houston, and B. J. Whitaker, Chem. Phys. Letl. 187, 257 (1991); A. G. Suits, R. L. Miller, L. S. Bontuyan, and P. L. Houston, J. Chem. Soc. Faraday Trans. 89, 1443, (1993); V. P. Hradil, T. Suzuki, S. A. Hewitt, P. L. Houston, and B. J. Whitaker, J. Chem. Phys. 99, 4455 (1993).

21 J. Biesner, L. Schnieder, G. Ahlers, X. Xie, K. H. Welge, M. N. R. Ashfold, and R. N. Dixon, J. Chem. Phys. 91, 2901 (1989); X. Xie, L. Schnieder, H. Wallmeier, R. Boettner, K. H. Welge, and M. N. R. Ashfold, J. Chem. Phys. 92, 1608 (1990); L. Schnieder, W. Meier, K. H. Welge, M. N. R. Ashfold, and C. M. Westem, J. Chem. Phys. 92, 7027 (1990); G. P. Morley, I. R. Lambert, M. N. R. Ashfold, K. N. Rosser, and C. M. Westem, J. Chem. Phys. 97, 3157 (1992); D. H. Mordaunt, I. R. Lambert, G. P. Morley, M. N. R. Ashfold, R. N. Dixon, C. M. Western, L. Schnieder, and K. H. Welge, J. Chem. Phys. 98, 2054 (1993).

22 D. P. DeBruijn and J. Los, Rev. Sci. Instrum. 53, 1020 (1982).

23 R. E. Continett, D. R. Cyr, D. L. Osborn, D. J. Leahy, and D. M. Neumark, J. Chem. Phys. 99, 2616 (1993).

24 L. D. A. Siebbeles, J. M. Schins. W. J. Van der Zande, J. Los, and M. Glass-Maujean, Phys. Rev. A 44, 343 (1991), and references therein.

25 H. Helm and P. C. Cosby, J. Chem. Phys. 86, 6813 (1987); H. Helm and P. C. Cosby, J. Chem. Phys. 90, 4208 (1989).

26 See Chapter 1 of Microchannel Plate Report, H. Kersten, Ed., (FOM-Instituut voor Atoom- en Molecuulfysica, Amsterdam, 1982), p. 32.

27 D. J. Leahy, D. R. Cyr, D. L. Osborn and D. M. Neumark, Chem. Phys. Lett. (in press).

28 This process is subject to selection rules, which must be satisfied for absorption to occur. These rules determine the spectroscopy associated with a given radical, and derive from the consideration of the quantum states and symmetries of the initial and final states in a given transition. See, for example, the discussion of this subject in reference 29 .

29 G. Herzberg, Molecular Spectra and Molecular Structure, III. Electronic Spectra and Electronic Structure of Polyatomic Molecules, (Van Nostrand, New York, 1966), pp. 457-458.

30 A word of caution must be extended concerning thie potential energy diagrams used to illustrate various processes. The two-dimensional nature of the printed page is insufficient to fully represent the 
multidimensional nature of polyatomic molecular potential energy surfaces. For this reason, the diagrams cannot be taken as any more than two dimensional 'cartoons' of a multidimensional system.

31 M. W. Chase, Jr., C. A. Davies, J. R. Downey, Jr., D. J. Frurip, R. A. McDonald, and A. N. Syverud, JANAF Thermochemical Tables, 3rd. ed. (American Chemical Society and American Institute of Physics, New York, 1986).

32 K.-Y. Du and D. W. Setser, Chem. Phys. Lett. 153, 393 (1988).

33 R. N. Zare, Mol. Photochem. 4, 1 (1972).

34 The theory of the anisotropy of the flux of molecular fragments produced by polarized light is analysed for arbitrary molecules by: S.-C. Yang and R. Bersohn, J. Chem. Phys. 61,4400 (1974).

35 T. A. Miller, Science 223, 545 (1984).

36 D. W. Kohn, H. Clauberg, and P. Chen, Rev. Sci Instrum. 63. 4003 (1992).

37 J. A. Blush, H. Clauberg, D. W. Kohn, D. W. Minsek, Xu Zhang, and P. Chen, Acc. Chem. Res. 25. 385 (1992).

38 J. Berkowitz, Photoabsorption, Photoionization, and Photoelectron Spectroscopy, (Academic Press. New York, 1979), p. 36.

39 E. P. Wigner, Phys. Rev. 73, 1002 (1948).

40 K. J. Reed, A. H. Zimmerman, H. C. Anderson, and J. I. Brauman, J. Chem. Phys. 64, 1368 (1976).

41 A. E. Douglas and W. J. Jones, Can. J. Phys. 43, 2216 (1965).

42 M. Polak, M. Gruebele, and R. J. Saykally, J. Am. Chem. Soc. 109, 2884 (1987).

43 R. E. Continetti, D. R. Cyr, R. B. Metz and D. M. Neumark, Chem. Phys. Lett. 182, 406 (1991).

44 A. Weaver, Ph. D. Thesis, University of California, Berkeley, 1991.

45 S. E. Bradforth, E. H. Kim, D. W. Amold, and D. M. Neumark, J. Chem. Phys. 98, 800 (1993). 


\section{Chapter 2}

\section{The Fast Radical Beam Machine}

\subsection{Introduction}

The Fast Radical Beam Machine (FRBM) is the experimental apparatus constructed in our laboratory to undertake photodissociation studies of free radicals. The apparatus is built to enable the photodissociation of a fast beam of free radicals and subsequent detection of the resulting photofragments. Generation of the fast beam of free radicals is achieved through photodetachment of the corresponding negative ion.

The machine is a fairly complicated entity, as illustrated by a schematic diagram shown in Figure 2.1. To minimize its complexity, it is advantageous to describe the machine in sections. By way of an overview, the apparatus consists of a source region, where negative ions corresponding to the radicals of interest are generated, internally cooled in a supersonic expansion, and accelerated. Next is a region where the negative ions are steered and focused with electrostatic optics and mass selected via time-of-flight to the photodetachment region. Within the photodetachment region, a tunable pulsed laser is timed to photodetach the mass of interest, with the detached electrons collected to allow monitoring of the detachment efficiency. Undetached negative ions are deflected out of the beam axis onto a detector which allows the source performance to be monitored. In the following region the radicals formed by photodetachment are photodissociated and the product fragments detected.

Two types of photodissociation experiments are possible. In a total photodissociation cross section experiment, the total fragment signal is measured as a

Figure 2.1 This diagram shows the overall layout of the Fast Radical Beam Machine. Various components of the machine are labeled, as are the differential pumping regions. This diagram is roughly to scale; the total length of the machine is -13 feet. 


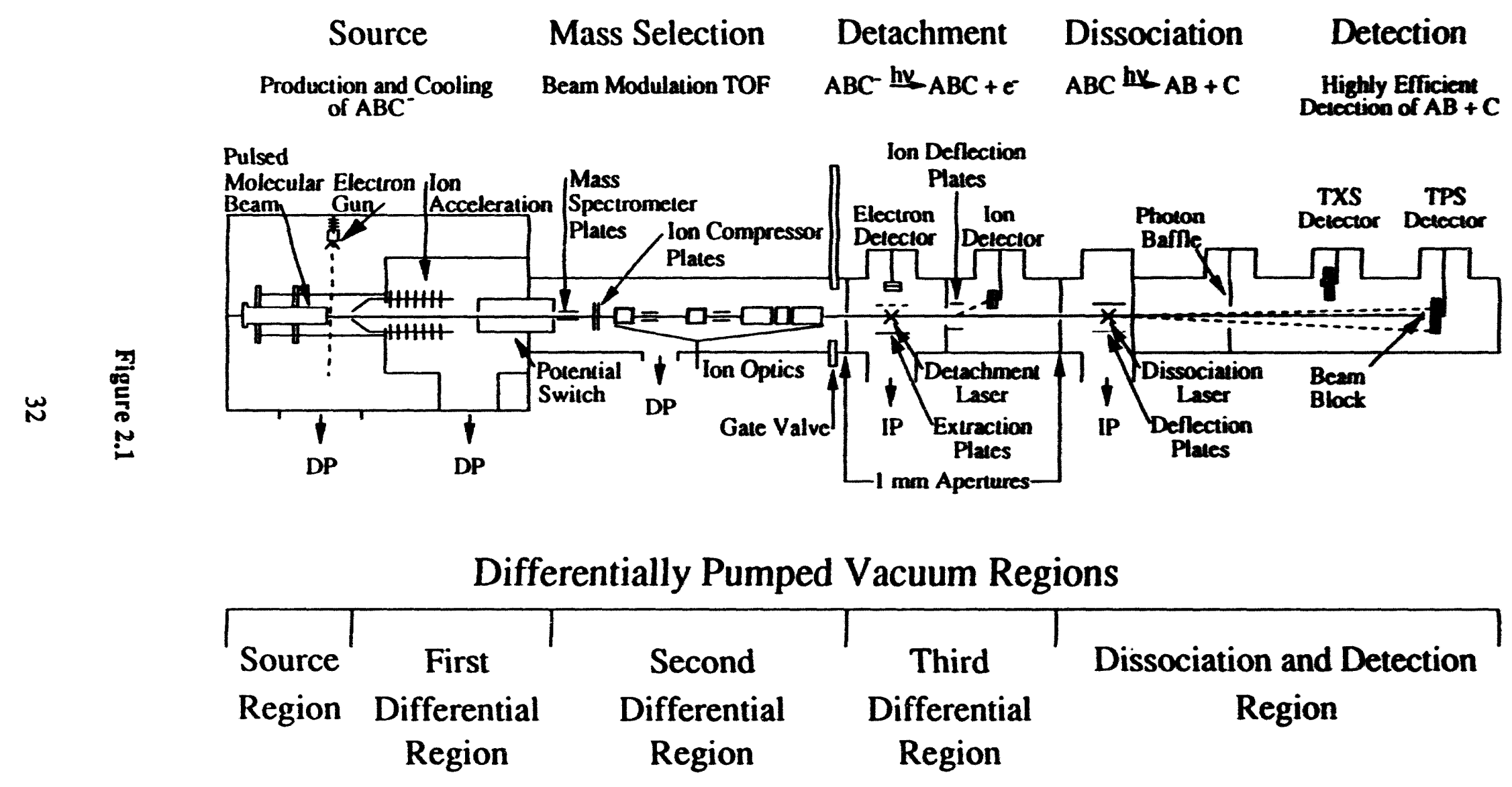


function of dissociation wavelength. To then perform a detailed investigation of the dissociation dynamics at a certain fixed wavelength, the kinetic energy and recoil angle distributions for each product channel are determined using a time- and position-sensitive detector.

In the rest of this chapter the instrument is discussed at length, as this is the first detailed account of its construction.

\subsection{Vacuum Systems}

The machine has five differentially pumped vacuum regions. They are referred to imaginatively as the source, first differential, second differential, third diff rential and detector regions, and are labeled in Figure 2.1. An electropneumatic gate valve delineates the boundary between the first three high vacuum regions (on the source side) and the two ultrahigh vacuum regions downstream (on the detector side).

On the source side of the gate valve, flanges are sealed with viton o-rings and vacuum is provided by diffusion pumps, backed by mechanical pumps. The source region is pumped by a 10", $2300 \mathrm{l} / \mathrm{s}$ diffusion pump (Edwards High Vacuum, Series 250 Diffstak) backed by a $40 \mathrm{l} / \mathrm{s}$ mechanical pump (Edwards E2M40). The first differential region, separated from the source region by the $3 \mathrm{~mm}$ aperture in the skimmer, is pumped by a 6 ", $805 \mathrm{l} / \mathrm{s}$ diffusion pump (Edwards High Vacuum, Series 160 Diffstak) backed by an $18 \mathrm{l} / \mathrm{s}$ mechanical pump (Edwards High Vacuum, E2M18). The second differential region, separated from the first differential region by a $4 \mathrm{~mm}$ aperture, is pumped by a 2", $173 \mathrm{l} / \mathrm{s}$ diffusion pump (Edwards High Vacuum, Series 63 Diffstak) backed by an $81 / s$ mechanical pump (Edwards High Vacuum, E2M8). The diffusion pumps are charged with a polyphenyl ether fluid (Monsanto, Santovac 5(8) because of its superior electrical conducting property. This avoids a problem that can occur when insulating varieties of diffusion pump oil are used - inner surfaces of the chamber, coated with a thin layer of 
non-conducting oil can develop a potential and establish stray fields that affect ion production and/or transmission.

In contrast to the regions prior to the gate valve, the regions following the gate valve employ knife edge/copper gasket seals and relatively cleaner sources of vacuum having lower ultimate pressure. Immediately beyond the electropneumatic gate valve is a $1 \mathrm{~mm}$ aperture which facilitates differential pumping between the second and third differential regions. A second $1 \mathrm{~mm}$ aperture separates the third differential region from the detector region. The third differential and detector regions are pumped by $120 \mathrm{~V} / \mathrm{s}$ and $220 \mathrm{~V} / \mathrm{s}$ ion pumps (Perkin Elmer, Model D-I) respectively. These pumps require no backing, but must not be tumed on when the pressure is above $10^{-4}$ Torr. Longer electrode life is expected if the high vacuum regions are brought to $10^{-5}-10^{-6}$ Torr prior to the ion pumps becoming operational. This is accomplished by temporarily uniting the third differential and detector regions and evacuating them via a $75 \mathrm{~V} / \mathrm{s}$ turbomolecular pump (Varian, Model V-80) backed by an $8 \mathrm{~V} / \mathrm{s}$ mechanical pump (Edwards High Vacuum, E2M8). The ion and turbomolecular pumps can pump in unison in the $10^{-5}$ to $10^{-7}$ Torr range, but once the pressure is below $1 \times 10^{-7}$ Torr the turbomolecular pump has approached its ultimate pressure, and is best isolated from the machine.

Pressures in all five regions are measured by ion gauges, with foreline pressures determined using thermocouple gauges. Typical background pressures for the source, first differential, and second differential region are $1 \times 10^{-7}, 2.5 \times 10^{-7}$, and $2 \times 10^{-7}$ Torr, respectively. After pump down and bake-out of the ultrahigh vacuum region, background and operating pressures are in the low to mid $10^{-9}$ Torr range.

\subsection{Interlock Circuits}

The vacuum system and electronics are protected in case of equipment failure, abnormally high pressure in critical regions, and many types of operator error by an interlock system. The interlock system allows manual control as well as fail-safe 
automatic control of the gate valve, all high voltage electronics and various vacuum equipment. A schematic of the circuit is shown in Figure 2.2 (a) and (b). All relays are shown in their fail-safe positions (i.e., the state that they revert to if the $A C$ power to the interlock box failed). The orientation of the indicator lights was chosen such that green would be visible when conditions are as they should be while running an experiment. This means, for example, that when the electropneumatic gate valve separating the regions on the source side from the regions on the detector side is open, the green light is on. The idea was that if the bell sounds during an experiment, it will be easy to determine the status of the interlocks with a glance; in particular, which interlocks have tripped. For the most part, the symbols in Figure 2.2 can be interpreted with ease. It should be pointed out that the circle containing an altemating current symbol indicates $A / C$ power that is fed into the interlock box directly from a separate circuit breaker on the rack panel. This power then flows out to the designated circuit after passing through a relay controlled by the various interlocks appropriate for the control of that circuit. Some details of the interlock circuits follow.

The interlock allows power to all three diffusion pumps to be turned off manually, while interrupting power automatically if their respective foreline pressures rise above a preset limit, usually $\sim 0.2-0.5$ Torr, or if there is an interruption in cooling water, as determined by a bi-metallic temperature sensor mounted on the diffusion pump cooling coils.

Both the first and third differential region ion gauge controllers have ion gauge high pressure trip points. The first differential region ion gauge controller was chosen to have this capability because it contains the bulk of components that are at the highest voltage in the source region. Of the two ultrahigh vacuum regions, the third differential

Figure 2.2 (a) and (b) The circuit diagram for the FRBM interlock box. See text for details. 


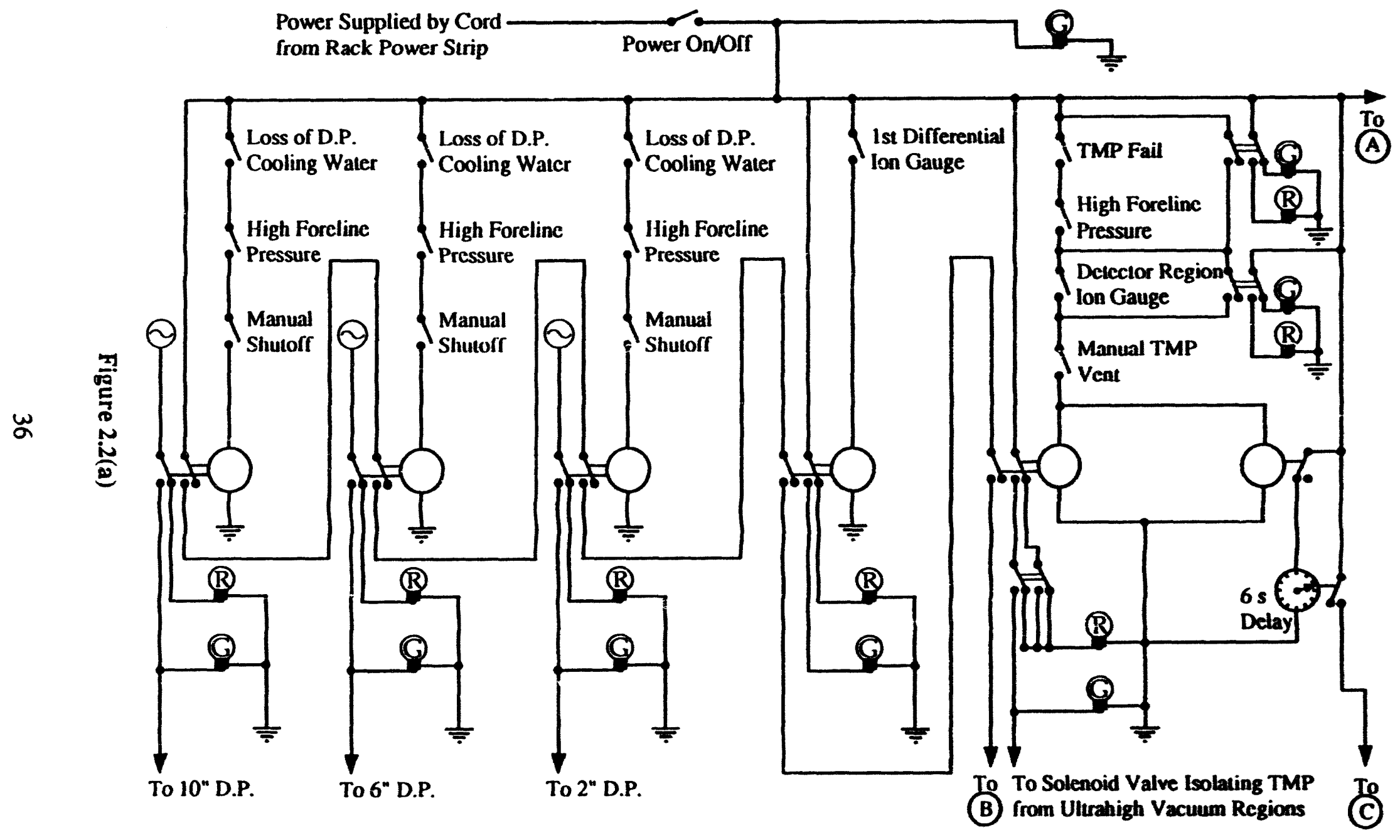




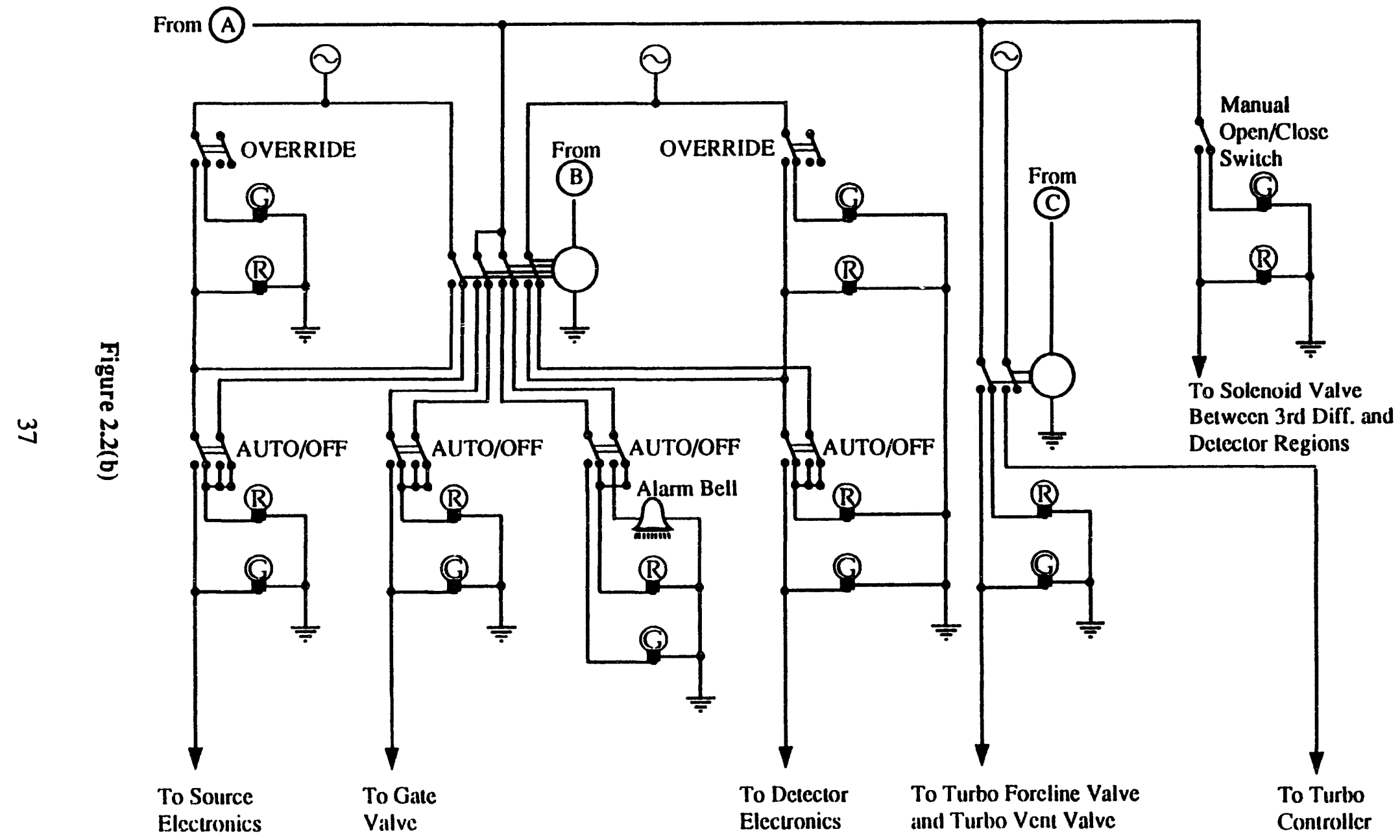


region ion gauge controller was chosen because the detector ion gauge is often turned off while an experiment is in progress.

If any of the diffusion pumps or either of the ion gauges has their interlock trip, a number of things will occur immediately. The gate valve will close, the source and detection electronics plug strips will have their power interrupted, and an ear-piercing, bell will commence ringing. It should be stressed that for safe operation, it is essential that all high voltage power supplies for the source and detection region be plugged in to their interlocked power strips.

Several features exist in the form of front panel AUTO/OFF switches and OVERRIDE switches. As mentioned above, the diffusion pumps have AUTO/OFF switches; the user can definitely turn them off using this switch, but with the switch in the AUTO position all other conditions must be satisfied for the interlock to allow operation. The same is true for the source and detection electronics, and the gate valve. The alarm bell also has an AUTO/OFF switch, although of course the conditions for the operation of the alarm bell are somewhat opposed to most other components, in that it turns on when a problem exists.

OVERRIDE switches will allow the source and detection electronics to receive power despite high pressure or other interlocks being tripped, the sole exception being if the manual AUTO/OFF switch corresponding to the respective OVERRIDE switch is set to OFF. An OVERRIDE switch is also provided to specifically circumvent the effect of a high pressure reading from the detector region ion gauge.

Two other manual switches are present on the front panel. They control electropneumatic valves which can unite the third differential and the detector regions and open the turbomolecular pump (TMP) to these regions, respectively. The color of indicator light associated with these switches is consistent with our original belief that during an experiment the third differential region and the detector region would be isolated from each other (true), but that the TMP would be open to the detector region to 
assist in pumping (false). In fact, as mentioned above, it is found that the ultimate pressure of the TMP is greater than the pressure we can attain in these regions without the assistance of the TMP. Therefore, contrary to the general rule of thumb stated above, under normal operations the indicator corresponding to the switch controlling the solenoid between the TMP and detection region is RED.

The TMP interlock section of this circuit was designed before we acquired the TMP, and has never been fully implemented. There is little incentive to do so because we do not have the TMP open to the machine except during pumpdown. Therefore, in practice the Turbo Fail and High Foreline Pressure sensors are not present in the circuit shown in Figure 2.2, and these 'switches' can be considered to be always closed.

To provide an fail-safe interlock for the TMP a very simple circuit was built and is housed separately. This interlock ensures that if the TMP failed, a valve located on the foreline to the pump would close, isolating the relatively 'dirty' foreline and mechanical pump from the TMP and the ultrahigh vacuum regions of the machine. A switch allows the bypass of this interlock during the initial stages of a pumpdown, where the foreline valve must be open to allow the vacuum chamber to be roughed out by the mechanical pump even when the TMP is not operational.

The ion pumps need not be interlocked, and the ultrahigh vacuum region is not vented in case of loss of power. Ion pumps will turn themselves off in a pressure excursion, and will not create a mess if power is lost. In fact, through 'gettering' action, ${ }^{1}$ these pumps retain pumping ability for some time after being shut off (useful during power outages).

\subsection{Source Region}

Within the source region, we wish to generate negative ions corresponding to the radical of interest. It is usually highly desirable that these ions have very low internal energies, with most of the ions being in the ground vibrational level and the lowest 
rotational states. The advantage of populating relatively few quantum states lies in the resulting narrow distribution of internal energies. This narrow distribution, if transferred to the free radical on photodetachment, will allow for elementary accounting of the total energy in the radical on excitation, and a very precise determination of the amount of energy partitioned into the internal degrees of freedom of the recoiling photofragments, via subtraction of the kinetic energy release from this total energy. As discussed in $\$ 1.4$, if negative ions are formed primarily in the ground vibrational level, photodetachment can usually be undertaken in such a way as to populate only the ground vibrational level of the radical.

The method which is typically used to generate negative ions in this experiment is the electron beam-free jet expansion technique developed by Lineberger and coworkers. ${ }^{2,3}$ In this technique, the continuum flow region of a free jet expansion is crossed at 90 degrees by a $1 \mathrm{keV}$ electron beam. The high energy electrons create lower energy electrons via electron-impact ionization of the molecules in the free jet:

$$
M+e^{-}(\sim 1 \mathrm{keV}) \rightarrow M^{+}+e^{-}(<1 \mathrm{keV})+e^{-}(>0 \mathrm{eV})
$$

The lower energy electrons are rapidly thermalized via inelastic collisions within the expansion and can then participate in the formation of negative ions. Dissociative attachment is the major pathway to negative ion production. ${ }^{3}$ "Dissociative" may mean the breaking of a conventional chemical bond, or the "evaporation" of molecules that had been bound in a van der Waals cluster. Eqns. (2.2) and (2.3) give examples of both of these possibilities, while illustrating our method of producing the azide anion and the oxygen anion, respectively:

$$
\begin{aligned}
& \mathrm{C}_{6} \mathrm{H}_{5} \mathrm{CH}_{2} \mathrm{~N}_{3}+\mathrm{e}^{-} \rightarrow \mathrm{N}_{3}^{-}+\mathrm{C}_{6} \mathrm{H}_{5} \mathrm{CH}_{2} \\
& \mathrm{O}_{2}\left(\mathrm{O}_{2}\right)_{n}+\mathrm{e}^{-} \rightarrow \mathrm{O}_{2}^{-}\left(\mathrm{O}_{2}\right)_{n-m}+\mathrm{m} \mathrm{O}_{2}
\end{aligned}
$$

A second route to negative ions that can be formed via proton abstraction involves formation of $\mathrm{F}^{-}$via dissociative detachment of $\mathrm{NF}_{3}$, followed by the abstraction of a 
(relatively) acidic proton to make the desired negative ion and HF. This process is shown in Eqn. (2.4), illustrating our method of producing the nitromethyl anion:

$$
\begin{aligned}
& \mathrm{NF}_{3}+\mathrm{e}^{-} \rightarrow \mathrm{F}^{-}+\mathrm{NF}_{2} \\
& \mathrm{CH}_{3} \mathrm{NO}_{2}+\mathrm{F}^{-} \rightarrow \mathrm{CH}_{2} \mathrm{NO}_{2}^{-}+\mathrm{HF}
\end{aligned}
$$

At some point in the future, the laser vaporization source $e^{4}$ that has been used with great success on the other two experiments in the Neumark Group will be adapted to our machine, enabling the production and study of various metallic and semiconductor clusters. The pulsed discharge source, discussed further in $\S 2.3 .5$, is also expected to prove very valuable in the future.

\subsubsection{Source Chamber Design}

The source chamber was designed to maintain maximum flexibility without compromising on integrity of construction. The floor of the source chamber, to which the large diffusion pumps are bolted, and the downstream wall, to which the remainder of the machine is attached, are fabricated from 0.75 " stainless steel for high strength. Flexibility in the design is achieved by having the remaining frame made from 3 " wide, 0.5 " thick strips of stainless steel, with all of the pieces inside-welded vacuum-tight along the edges. The chamber walls consist of $1 "$ aluminum plate bolted on to this skeleton, sealed with o-ring gaskets. In this way, the large diffusion pumps can be hung from the floor of the chamber and the interface to the remainder of the machine is robust, while any of the remaining sides or the top can be easily removed for wide-open access to the interior of the source chamber. It is also possible to quickly substitute alternate chamber walls machined for specialized applications, though this option has not been exercised to this point. 


\subsubsection{Pulsed Molecular Beam Valve}

A supersonic free jet expansion is used so that negative ions that are formed will undergo internal cooling. The conversion of internal energy to translational energy of molecules during a series of collisions in a free jet expansion is well established, and documented extensively elsewhere. 5 Pulsed lasers are used in both detaching the negative ions and to cause dissociation of the radical because of their high peak power. It is therefore logical to employ a pulsed free jet expansion. In exchange for the more complex operation of a pulsed versus continuous nozzle, the instantaneous number density in a pulsed expansion is much higher, concurrent with the more modest vacuum pumping speed requirements and low background pressures characteristic of a pulsed molecular beam.

Originally, we experimented with using a standard solenoid-driven pulsed valve (General Valve Corporation, Series 9) to produce our pulsed molecular beam. This arrangement worked relatively well, but was subject to certain problems. Solenoid varieties of pulsed valves draw the poppet back from where it sits covering the faceplate by generating a magnetic field which acts on an armature attached to the poppet. The magnetic field results from the flow of current through a coil surrounding the armature. This flow of current dissipates power and, particularly at high repetition rates, causes excessive heating of the pulsed valve. In addition, the poppet that gave the best results was made of Kel-F and was easily scored, resulting in a leak that could only be repaired by the replacement of the poppet. Even if the poppet did not become badly scored, it often became compressed over time so that it would require adjustment. The adjustments that were necessary to restore proper operation, principally the faceplate tightness, needed to be made outside the machine. This meant venting at least once (but often more that once) in order to adjust the valve. Lastly, the armature of the solenoid appeared to lose its magnetic properties over time, and needed to be replaced periodically. 
The solenoid valve was soon replaced by a Trickl-type ${ }^{6}$ piezoelectric valve at the urging of Dr. Bob Continetti, who had used this design in his past work ${ }^{7}$ in the laboratory of Prof. Y.T. Lee. There was a noticeable improvement in performance using the new pulsed valve, principally in the intensity and full width at half maximum of the gas pulse, as measured using a Fast Ion Gauge (the FIG is discussed in § 2.3.3). In contrast to the General Valve, the piezoelectric valve dissipates very little power while running, and thus does not become warm/hot. Additionally, the piezoelectric valve can achieve 'choke flow', where the flat-topped shape of the gas pulse indicates that the faceplate orifice is fully open and the pulse is limited by the conductance through the orifice.

Another important benefit of the piezoelectric pulsed valve design is the ability to perform dynamic adjustments on the valve while it is in operation in a test configuration. This configuration consists of the valve mounted on the exterior of a flange so that it seals a hole which opens to the interior of the source region. The back of the valve casing is removed so adjustments to the poppet depth and carrier nuts can be made as the valve performance is monitored using the FIG. Once the valve is adjusted optimally, it can be reassembled in its case and the mounted inside the machine. An extremely long period between adjustments or replacement of parts can be expected. Since the beginning of the "Piezoelectric Era" we have not considered a return to the General Valve.

A short description of the construction of the piezoelectric pulsed valve that is currently in use follows. The variations in design that are necessary for using gases that swell or damage flexible o-ring material are also given. Lastly, the driving circuit for the piezoelectric valve is shown and briefly outlined.

A schematic showing the cross section of the piezoelectric pulsed valve is shown in Figure 2.3. This pulsed valve is designed to rapidly and completely open the small $(\sim 375 \mu \mathrm{m})$ hole in the faceplate. A gas at some backing pressure, (typically 10-40 PSIG) rushes into the source chamber through this hole for a short period of time $(\sim 100-200 \mu \mathrm{s})$ before the orifice is sealed again. The size of the faceplate orifice can be varied by the 
substitution of alternate faceplates. The faceplate is normally sealed by a $2-002$ viton o-ring mounted in a groove cut on the end of the aluminum poppet. The poppet is mounted perpendicular to the faceplate through the center of a ceramic disk (Physik Instrumente, U.S. Distributor: Polytec Optronics, Part No. P-910.160) which has piezoelectric properties. ${ }^{8}$ The rapid opening of the faceplate orifice is accomplished by applying high voltage to one side of the disk, which causes it to flex like a drum head. The induced translation is up to 100 microns at $1000 \mathrm{~V}$, drawing the poppet and o-ring back away from the faceplate.

In cases where gases are present that will swell or deform the flexible o-ring material that normally makes the seal with the faceplate, the standard configuration becomes unreliable and a different poppet and faceplate combination is used. The alternate poppet is constructed using a Kel-F ball tip screwed onto and aluminum shaft, which is again mounted onto the piezoelectric crystal. The Kel-F ball tip seals against a conical seat in a modified faceplate. Kel-F is a much stiffer material, which resists swelling much better than viton (minor swelling may still occur). However, the stiffness of the Kel-F ball means that if it is scored or deformed, it will leak. Kel-F tips also require very precise adjustments to achieve a proper seal against the faceplate. To its credit, the Kel-F ball tip allows certain gases to be present in the expansion that could not be used otherwise, but is no match for the convenience and reliability of the viton o-ring design.

The circuit that is used to drive the disk translator was built from circuit diagrams supplied by the group of Hanna Riesler at USC, and is shown in Figure 2.4. The main components of this circuit are two $1 \mathrm{kV}$ MOSFETs which act as switches. They are

Figure 2.3 This schematic is a cross section of the piezoelectric pulsed valve used in the source. (1) Stainless steel body. (2) High voltage connector. (3) Stainless steel faceplate with 0.015 " orifice. (4) Piezoelectric translator disk. (5) Aluminum poppet affixed to disk with lock nut/screw assembly to adjust tension of o-ring seal against faceplate. (6) Carrier o-ring used to damp disk. 


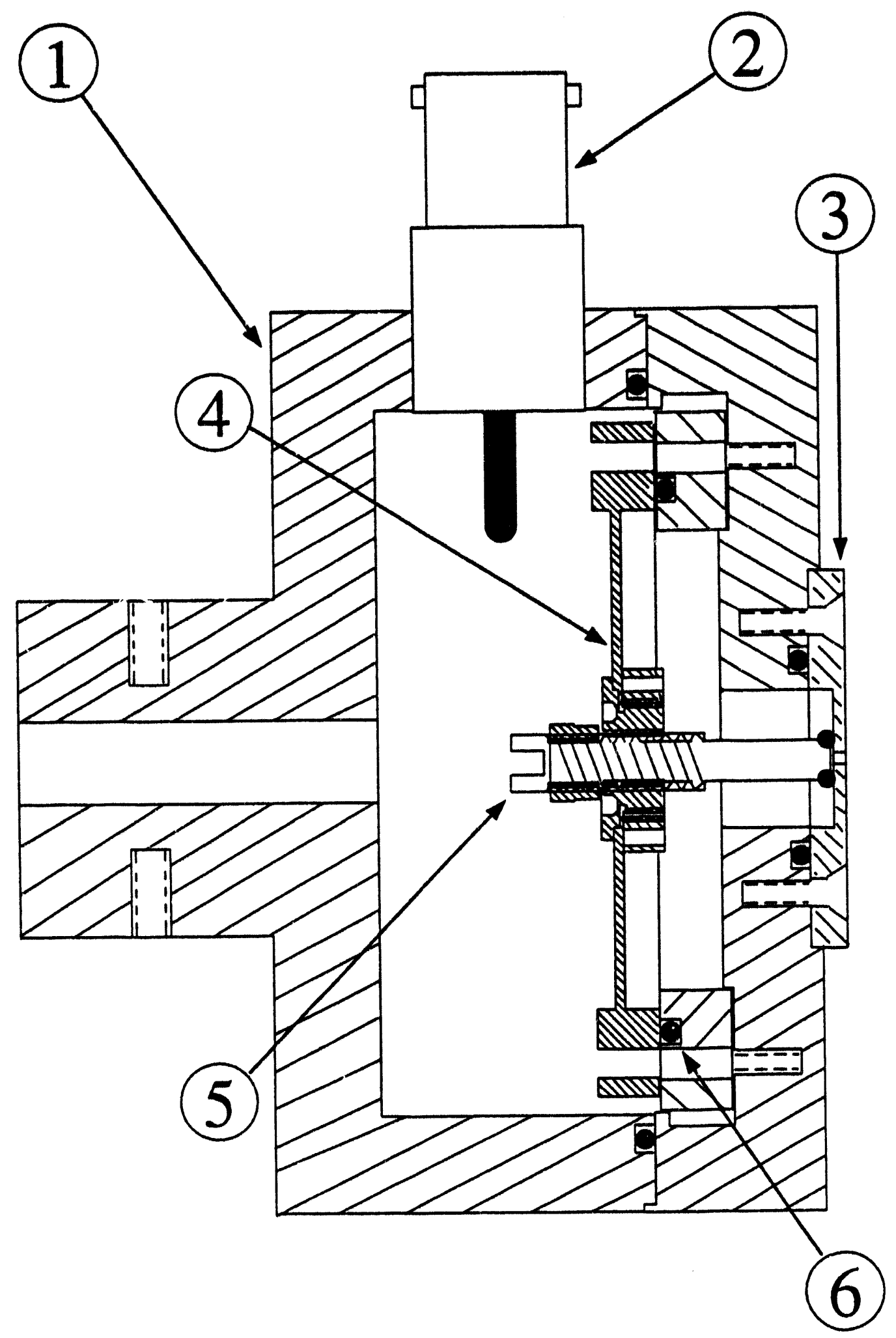

Figure 2.3 
arranged in a push-pull configuration, with one MOSFET switching a connection between negative high voltage and the piezoelectric disk, and the other MOSFET switching a connection between the disk and ground. Normally, the connection to ground is present, but when triggered, the driver opens this switch, isolating the crystal from ground. Simultaneously the other switch closes, allowing the disk to be brought to the negative high voltage. At the end of a user-set delay, both switches revert to their normal state. A critical factor in driving the disk is that the fall-and rise-times of the potential that is applied to the piezoelectric disk must have a minimum RC time constant of $\sim 50 \mu \mathrm{s}$. This results in a pulse shape resembling an invered shark fin. The natural frequency of the disk is $2.5 \mathrm{kHz},{ }^{8 b)}$ and, while this can be safely exceeded to some degree, the disk will crack if a high voltage step function pulse is used.

\subsubsection{Fast Ion Gauge}

A fast ion gauge (FIG) is used to monitor the on-axis temporal profile of the pulsed free jet expansion. By placing the FIG in front of the free jet expansion the local pressure as a function of time can be determined. This allows the performance of the pulsed valve to be monitored, an especially valuable asset while the valve is being adjusted. The FIG thus allows the narrowest, most intense temporal profiles to be obtained in a straightforward manner.

The FIG is home-built in our laboratory by Dr. Robert Continetti, based on a design described by Giese and Gentry ${ }^{9}$ and his previous experience while a graduate student. ${ }^{7}$ It operates on the same principles as an ordinary Bayard-Alpert ion gauge, the difference being a more compact size and the ability for fast time response. Relative pressure is determined by ionizing nearby molecules, collecting the ions and measuring the

Figure 2.4 (a) and (b) The circuit diagram for the piezoelectric pulsed valve driver circuit. 


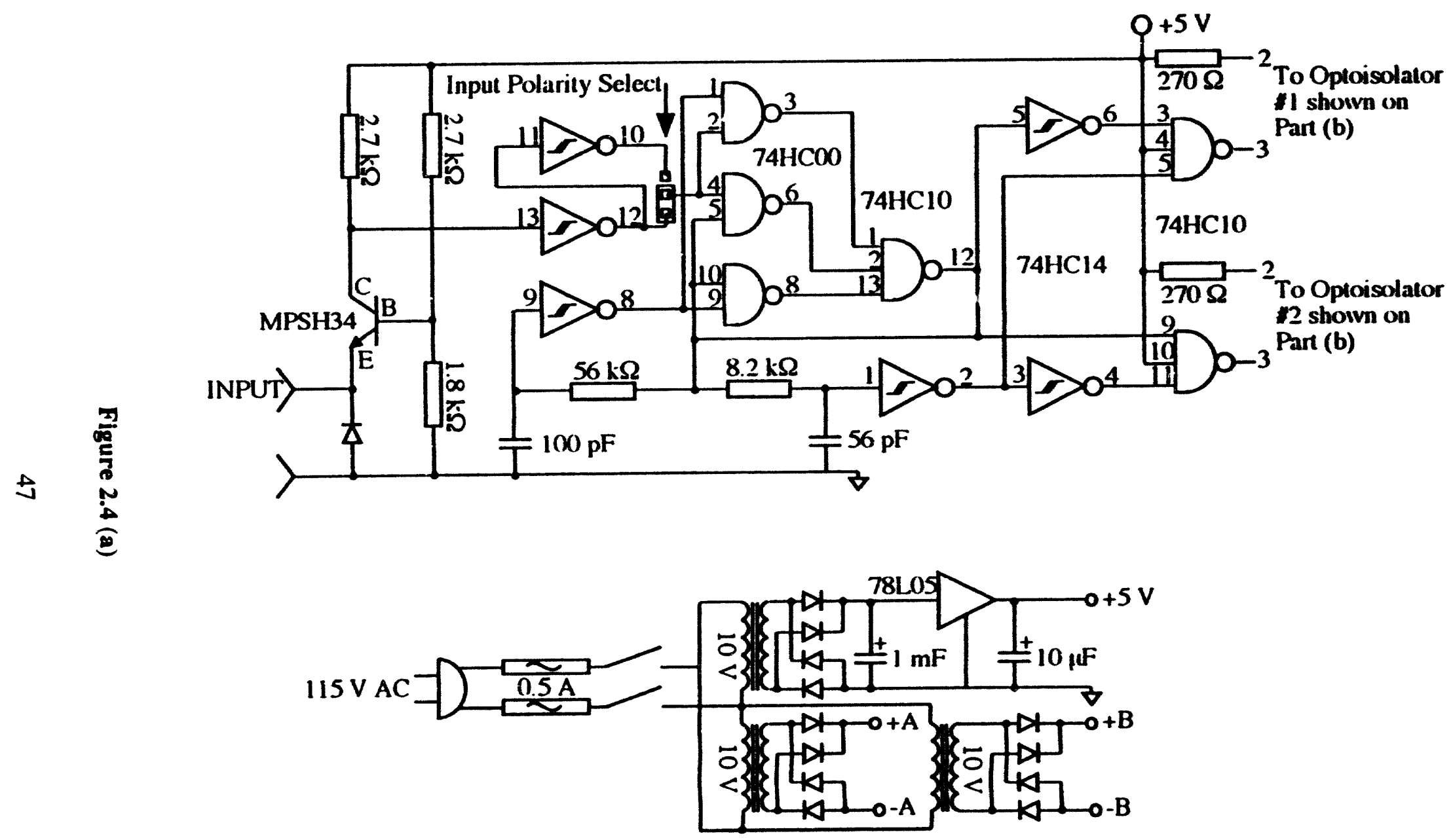

Signal Transformers: ST2-10 


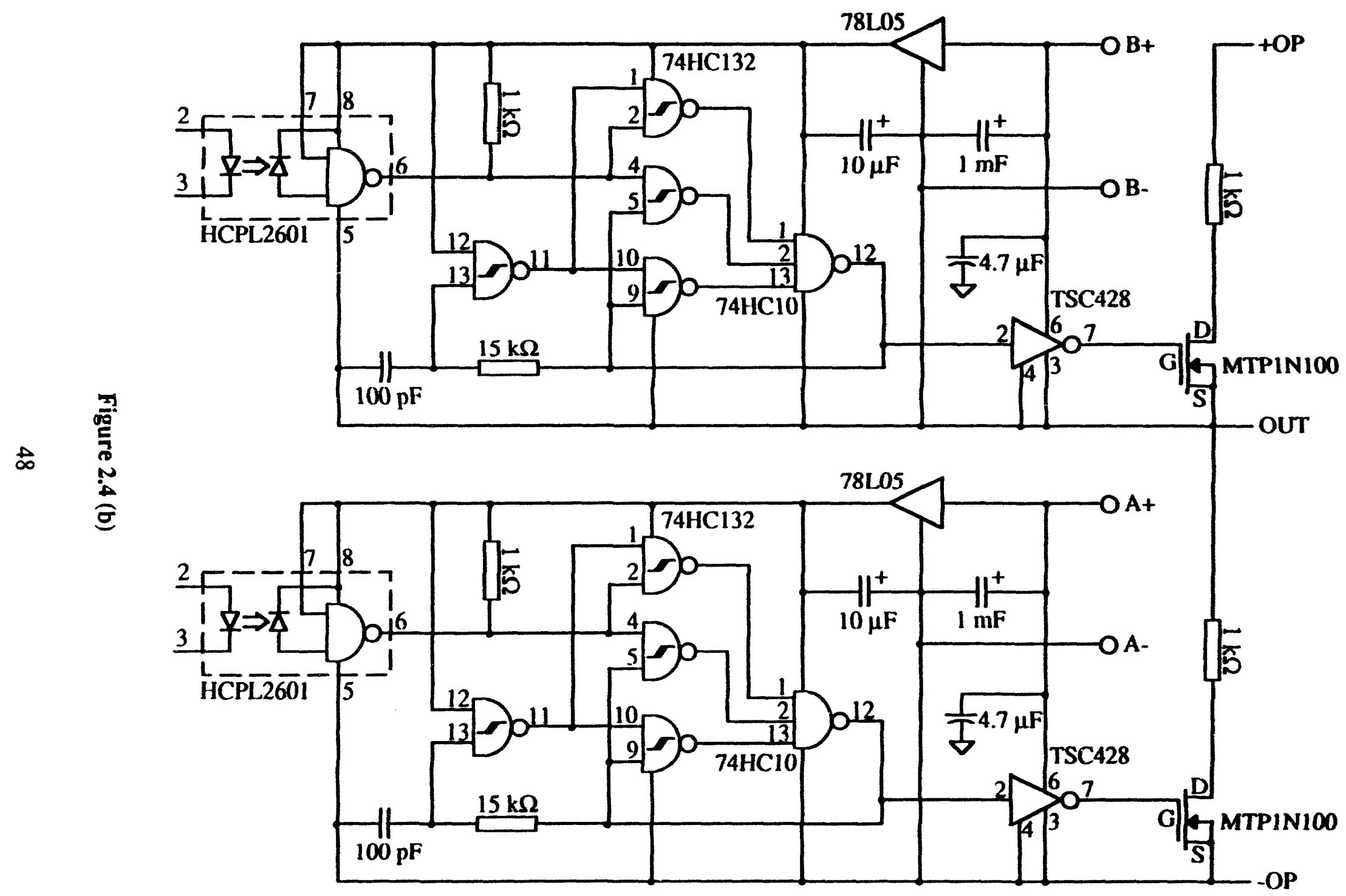


current, which is taken to be proportional to the pressure.

Figure 2.5 shows the geometry of the FIG components. A pair of $2.6 \mathrm{~cm}$ long, $1 \mathrm{~mm}$ diameter tungsten filaments are connected to the leads of a constant voltage/current power supply (Power Designs, Model 3650R). When a $\sim 4 \mathrm{~V}$ potential is across the filaments the resultant current heats the resistive material enough that thermionic emission occurs. This emission of electrons causes the ionization of nearby molecules. The filament power supply is biased at $+10 \mathrm{~V}$ by a second power supply (Acopian, Model A060NT12). The emission current of the filaments corresponds to the current drawn from this float power supply in order to maintain the $+10 \mathrm{~V}$ bias on the filament power supply. An emission current of $10 \mu \mathrm{A}$ is typically used. A grid, made from a spring and thus having a coil shape, is biased at $\sim+200 \mathrm{~V}$ with respect to ground using a third power supply (Acopian, Model U300Y20). This cylindrical coil encircles the length of a thin wire which is nominally a ground potential and acts as a collector of the positive ions. The collector leads to the input of the electrometer circuit that provides a time-resolved voltage reading corresponding to the temporal evolution of the local pressure.

The electrometer circuit that provides time-resolved pressure measurement is also shown in Figure 2.5. The pressure-dependent current on the collector causes a set of two LF 357J operational amplifiers connected in series to produce an inverted signal corresponding to the temporal profile of the local pressure in the pulsed beam. The FIG output typically has $100 \mathrm{mV}$ of high frequency noise superimposed on a $5-10 \mathrm{~V}$ signal. This is an unavoidable consequence of such high amplification by the two operational amplifiers, but is hardly noticeable on the larger scales required to view the full signal.

Care must be taken to keep the filament emission current constant, because ion current at the collector will reflect any fluctuation or long-term drift. Other problems with

Figure 2.5 A diagram of the fast ionization gauge geometry and electrometer circuit. The location in the circuit of the microammeter used to monitor the emission current of the filament is denoted by a circle surrounding an " $\mathrm{A}$ ". 

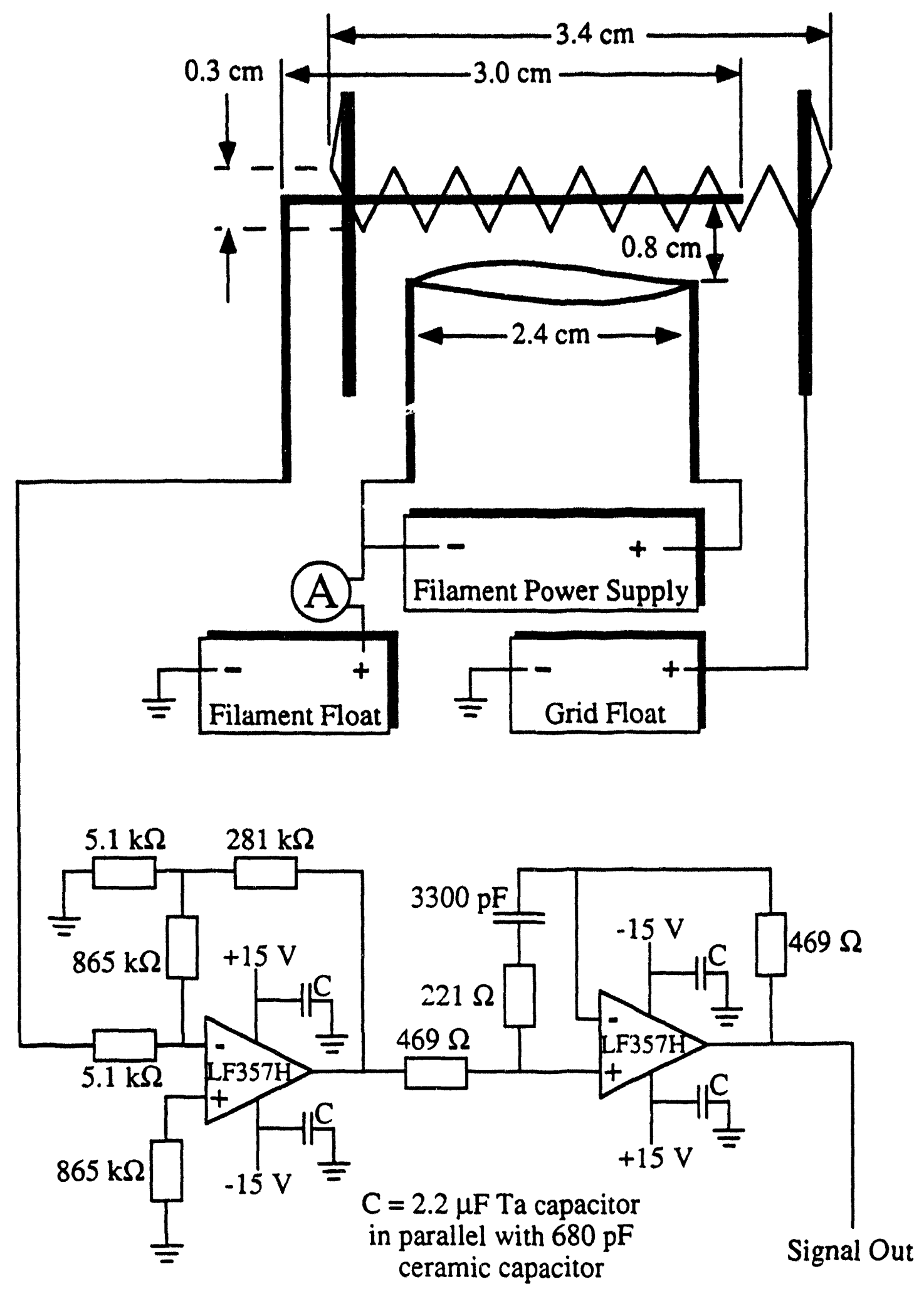

Figure 2.5 
reproducibility and comparability of results stem from the exact location of the FIG with respect to the nozzle orifice. The velocity spread present in the gas pulse serves to broaden the pulse width as it propagates. Therefore, a given pulse will always have a wider temporal profile and lower peak intensity when measured further from the nozzle. Lastly, different gases will have different propensities to ionize under these conditions and therefore give different signal intensities at similar number densities. This also hinders meaningful comparison under different conditions.

\subsubsection{Electron Beam}

The $1000 \mathrm{eV}$ continuous electron beam that crosses the high pressure region of the molecular beam expansion is generated in an identical manner to previous Neumark Group experiments. 4,10 These implementations in tum owe much to Lineberger and coworkers, ${ }^{11}$ who were the originators of the basic design. The electron bram is generated using a Tektronix oscilloscope electron gun assembly. To adapt this electron gun for operation in the relatively high pressure environment of our source region, an iridium filament coated with thorium oxide is used as a thermionic source of electrons. These electrons are then extracted through an anode, accelerated to $\sim 1 \mathrm{keV}$, focused by an einzel lens and steered by a set of deflectors. The electron beam is collected by a Faraday cup, located on the opposite side of the chamber, which is kept at $9 \mathrm{~V}$ relative to ground and is connected to a microammeter. Maximum output is measured to be $>500 \mu \mathrm{A}$, but quite often only a reading of $100 \mu \mathrm{A}$ or less results in optimum conditions for producing negative ions of interest. The microammeter readings are almost certainly underestimation of the true electron beam current during an experiment however, because in normal operating conditions part of the electron beam is intercepted by the faceplate of the pulsed valve and, to a lesser extent, scattered by the molecular beam expansion, and will not be collected on the Faraday cup. 
The electronics associated with the electron gun are displayed in Figure 2.6. The electron energy and einzel focus element power supplies are two 0-2000V (Kepco, Model APH2000M), which are kept at $1000 \mathrm{~V}$ and between $600-950 \mathrm{~V}$ respectively. Only the electron energy supply draws current, the amount of which is equal to the total emission of the filament. The filament power supply is a constant voltage/current supply (Kepco, Model ATE15-15M) which must provide between 4.5 and 8 Amperes at low voltage. The anode and deflector supplies are home-built units containing 150 and 60 VDC supplies (Acopian, Model A0150NT05 and A060NT12, respectively). Because the anode potential and the filament potential need to be close to the electron energy, these power supplies are 'floated' at the electron energy potential. A 1:1 isolation transformer ${ }^{12}$ is used to accomplish this, and the anode potential and filament current power supplies are kept in a Plexiglas enclosure for safety.

\subsubsection{Pulsed Discharge Source}

Feasibility studies have been carried out on a new negative ion source that will serve as an alternative to our usual electron beam-free jet expansion source. This new source involves a pulsed discharge through a mixture of precursor molecules seeded in a carrier gas just prior to undergoing a free jet expansion. It shares certain characteristics of sources used by Bondybey and co-workers, ${ }^{13}$ Ohshima and Endo, ${ }^{14}$ Sharpe and Johnson, ${ }^{15}$ and Bramble and Hamilton. ${ }^{16}$ These workers have generally used this type of source to make and cool transient species, typically radicals or van der Waals complexes involving

free radicals. With the exception of the recent short description of $\mathrm{C}_{2}^{-}$production by Bondybey and co-workers, ${ }^{13}$ very little effort has been expended characterizing the negatively charged species formed with such a source.

The pulsed discharge source is expected to be a powerful tool for making negative

Figure 2.6 A circuit diagram and schematic of the electron gun. 


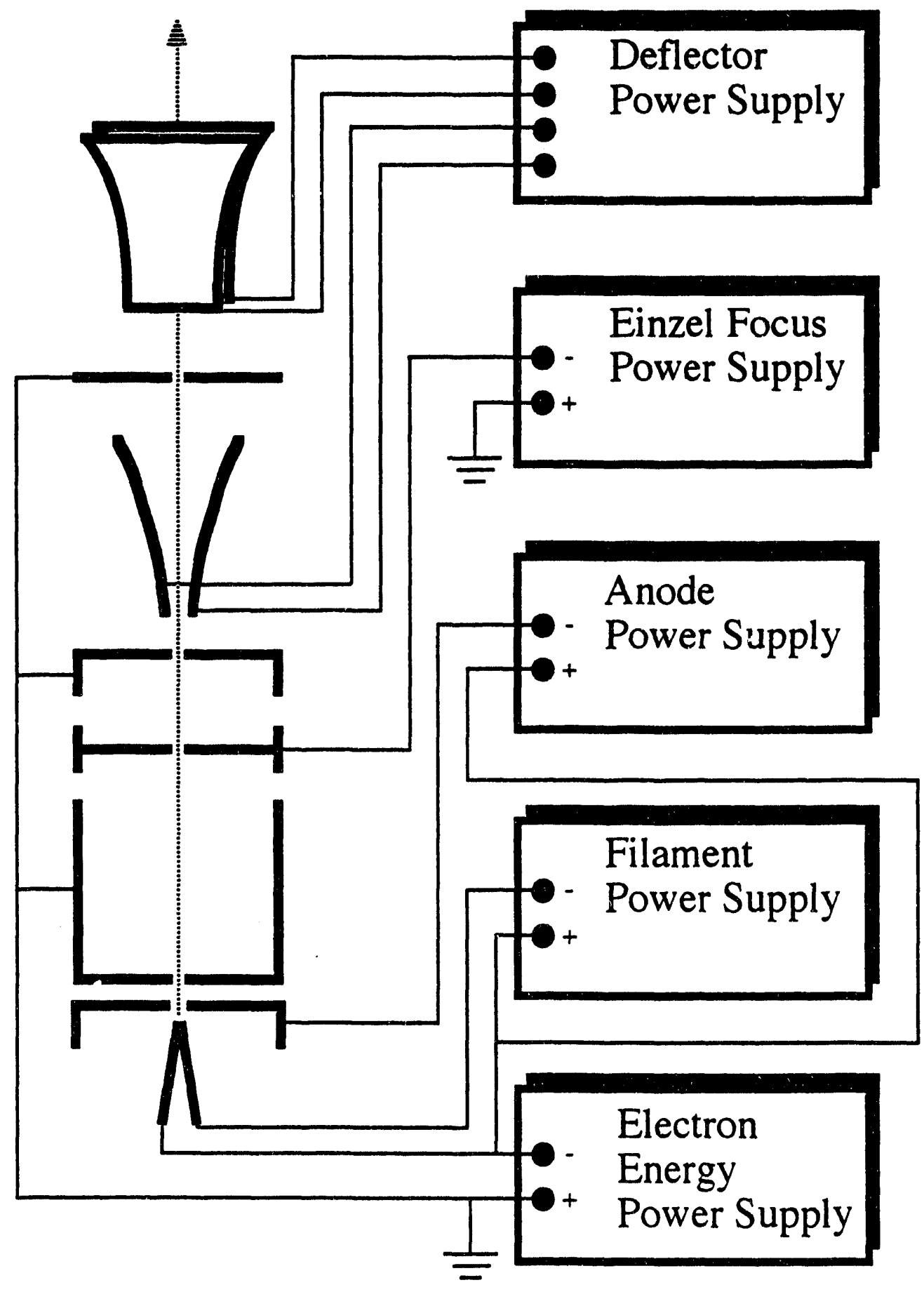

Figure 2.6 
ions. The combination of the presence of large numbers of radicals, typically having positive electron affinities, and the electrons associated with the discharge plasma seems to be an attractive one for negative ion formation. In addition, as researchers have shown in the production of radicals, the negative ions that may be produced do not necessarily have to bear close resemblance to the precursor molecule(s). This is typically a requirement for the electron gun/molecular beam source currently in use, and it is easy to imagine cases where this is restrictive.

We therefore can hope to expand our arsenal of negative ions, forming species through rearrangements and breakdown of larger molecules in the relatively violent environment of the discharge. As an example, it is not clear how to use the current source to make $\mathrm{CCN}^{-}$. Since Bondybey and co-workers were able to generate $\mathrm{CNN}$ using a mixture of $\mathrm{CS}_{2}, \mathrm{~N}_{2}$, and Ar in a pulsed discharge source, ${ }^{13}$ it is hoped to be able to make $\mathrm{CCN}^{-}$simply by using a mixture of $\mathrm{HCCH}, \mathrm{N}_{2}$, and $\mathrm{Ar}$ in such a source.

In certain instances the pulsed discharge source may also be able to produce larger amounts of negative ions that can be made with sources currently in use. Recently, Baker and co-workers ${ }^{17}$ studied $\mathrm{C}_{3}$ by passing neat $\mathrm{CO}$ through a pulsed discharge. Carbon clusters have been studied within the Neumark group ${ }^{18}$ but were formed using laser ablation. While quite effective, this technique does necessitate a Nd:YAG laser, introducing both repetition rate and financial limitations.

The pulsed discharge source also offers the possibility (thus far not pursued) of vaporizing normally-solid material, without the use of a laser.

The design of the pulsed discharge source consists of a pair of electrodes having a channel through them, mounted directly onto the end of the pulsed valve. The version tested thus far, ${ }^{19}$ shown in Figure 2.7, uses a modified pulsed valve faceplate as one electrode. This electrode remains at ground, since the body of the pulsed valve is grounded. The faceplate has a 0.015 " orifice with a flat face on the pulsed valve side to provide a good seal for the o-ring and a beveled face on the discharge side to match the 
0.080 " diameter channel in the insulator. The insulator is $4 \mathrm{~mm}$ thick and consists of a Teflon disk with a 0.25 " inner diameter hole in the center, into which a 0.25 " outer diameter alumina $\left(\mathrm{Al}_{2} \mathrm{O}_{3}\right)$ tube, having an 0.080 " diameter channel through the center, is inserted.

During operation a second stainless steel electrode, fixed to the front of the insulator, is pulsed to a potential above that where breakdown occurs. This electrode is designed to accept inserts that can be used to easily vary the channel length and width, allowing the mixing and expansion conditions to be varied. The inserts are generally fabricated from stainless steel, but can be made from any conductive material. For example, smaller $C_{n}^{-}$clusters, particularly $C_{2}^{-}$, have been observed from this source when the electrode insert was composed of graphite.

By pulsing the electrode potential, two advantages are gained. First, the breakdown can be made to occur in the middle of the expansion, for a relatively brief time, which may assist in cooling the subsequently formed species. Second, the ability to quickly remove the potential from the electrode allows the negative ions that are formed to undergo the subsequent expansion unperturbed by an electric field associated with the

Figure 2.7 A cross section of the pulsed discharge source attachments which affix to the pulsed valve in place of the usual faceplate. The top half of this drawing shows a section that is at $45^{\circ}$ relative to the bottom half. (1) Stainless steel electrode which functions as faceplate to which the poppet makes an o-ring seal. (2) Alumina $\left(\mathrm{Al}_{2} \mathrm{O}_{3}\right)$ cylinder which serves as the inert section separating the two electrodes in which the discharge plasma is created. (3) Teflon spacers. The first helps isolate the two electrodes as well as providing tapped holes for the outer electrode assembly to be fastened. The second, disk shaped spacer covers the steel screws used to fasten the faceplate and first spacer to the pulsed valve body. (4) High voltage electrode insert. This piece, which may be fabricated of any conducting material, has the final orifice through which the free jet expansion occurs. (5) Stainless steel high voltage electrode holder. 


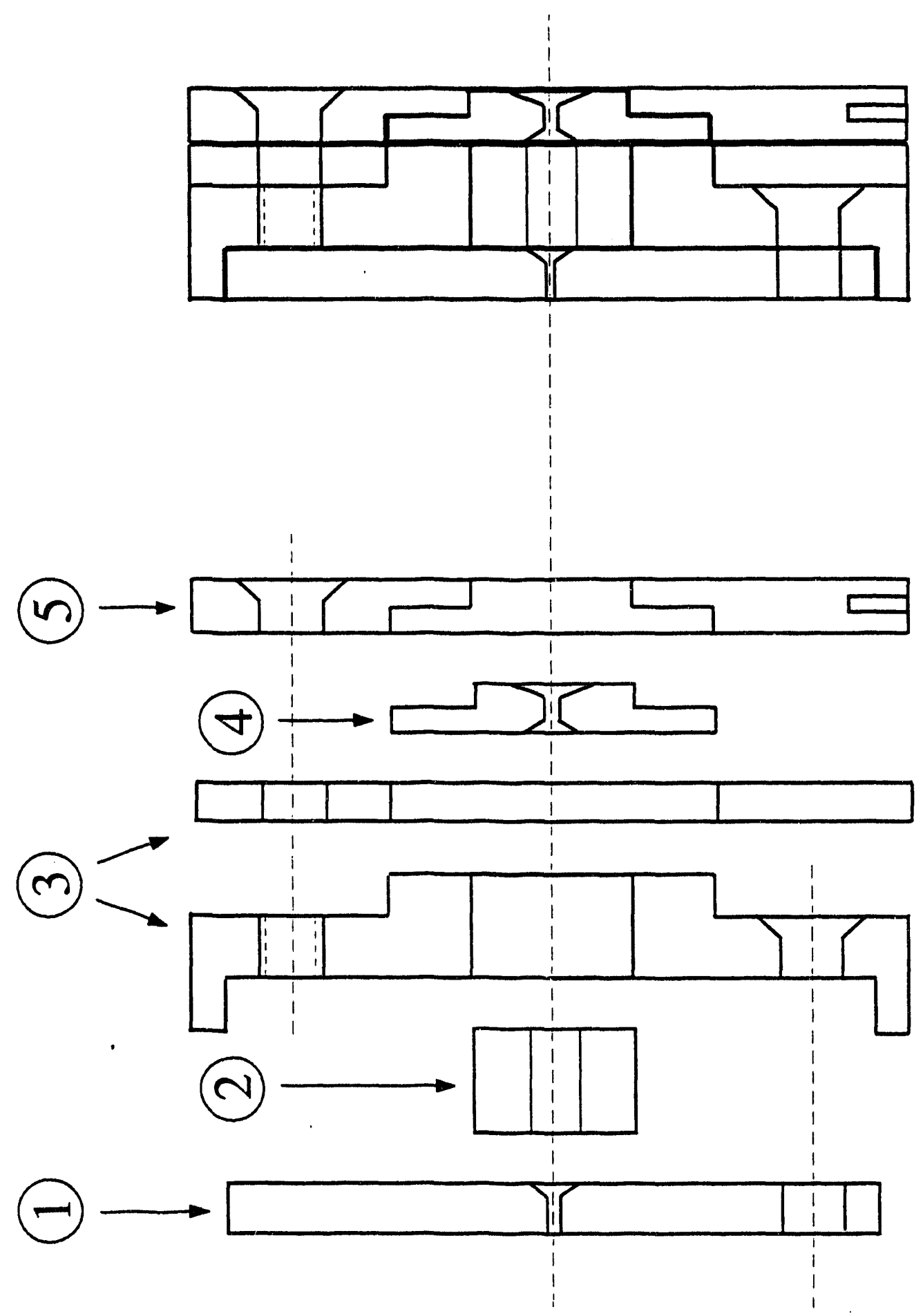

Figure 2.7 
electrode. Negative-going pulses of variable duration (1-100 $\mu \mathrm{s})$ with about a $800 \mathrm{~ns}$ fall time and similar rise time are provided by a pulsed, 'square wave', high voltage power supply (Velonex High Power Pulse Generator, Model 350 with V-1097 direct output plug-in module) connected to a very simple circuit, illustrated in Figure 2.8. This particular power supply requires a $200 \Omega$ load to form properly shaped pulses, this resistance value is approached in the circuit shown in Figure 2.8 if breakdown occurs. Otherwise, the load will be $250 \Omega$, which is completely acceptable. It should be emphasized that non-inductive (denoted NI in Figure 2.8) resistors able to dissipate high power must be used in this circuit. As an illustration, the instantaneous current flowing through $250 \Omega$ when $1000 \mathrm{~V}$ is applied is $4 \mathrm{~A}$. This corresponds to a dissipated power of $4000 \mathrm{~W}$ if the applied potential were continuous. At our typical duty cycle of $(60 \mathrm{~Hz} \times 40$ $\mu \mathrm{s}=0.24 \%$ the power dissipated is still $9.6 \mathrm{~W}$. The theoretical limitation of the circuit actually lies with the single $1500 \Omega$ resistor, rated at $10 \mathrm{~W}$. When breakdown occurs and there is a potential of $1000 \mathrm{~V}$ across this resistor, nearly $5 \mathrm{~W}$ will have to be dissipated under the conditions of the above example. Raising the repetition rate to $100 \mathrm{~Hz}$, or significantly lengthening the discharge time will cause the power dissipated to approach the rating of this resistor. The necessity for these conditions is unlikely, however.

The voltage that is required for a stable and productive discharge depends on many factors, including: instantaneous pressure at the point in the expansion where the discharge is to occur, the specific gases that are present in the expansion, the materials and detailed shapes of each electrode, the inter-electrode spacing etc. In short, because of the wide number of variable:, some amount of trial and error is expected in the production of every new species.

The utility of the pulsed discharge source in making negative ions has been the

Figure 2.8 A schematic of the pulsed discharge source circuit and a typical discharge oscilloscope trace. 


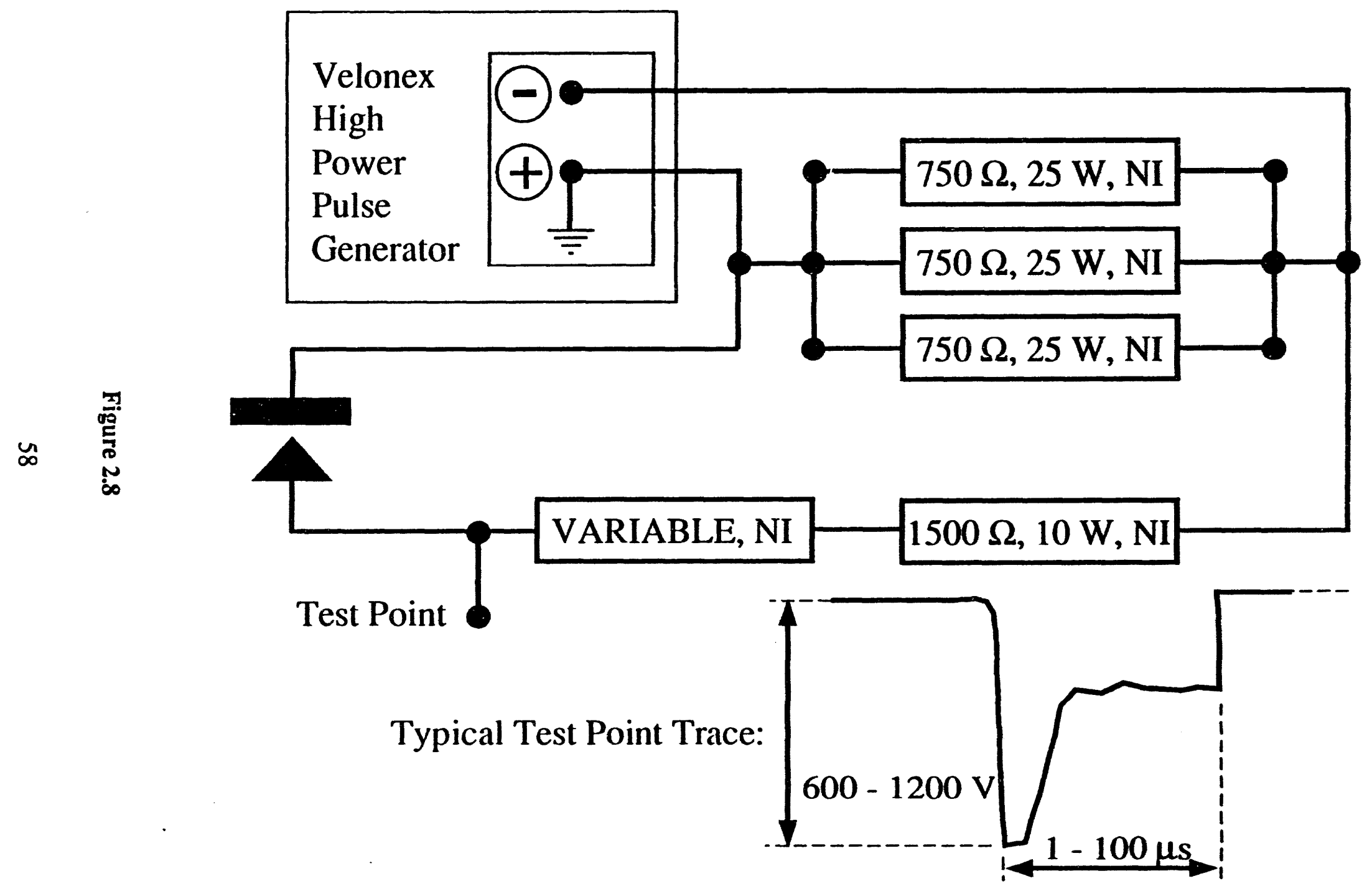


subject of a preliminary investigation using the fast radical beam machine. One critical factor that has been found to be important for satisfactory operation is a stable discharge. A stable discharge can be assured in our case by 'seeding' the discharge; using the electron gun to produce charged species near the front electrode. We have found (quite by accident) that by training a very weak $(>10 \mu \mathrm{A})$ electron beam on the exit of the discharge electrode enhances the stability of the discharge enormously. Positive ions accelerated towards the negative high voltage of the outer electrode are thought to be the key to this effect. In the absence of such seeding, the initiation of a discharge is a random process, and therefore an inherently unstable one. By increasing the potential difference between the electrodes the discharge stability can also be improved, but this action can have other effects and may not be desirable in all cases.

One aspect of the discharge circuit that was not fully investigated was the effect of varying the value of the resistor located in series with the discharge (see Figure 2.8). This resistor has the effect of limiting the current flowing in the discharge. This is a very critical factor, effectively determining the type of discharge that is formed. The high currents ( $~ 0.5 \mathrm{~A}$, instantaneous current) found in the discharges we have been using likely created a very violent arc-type discharge. Evidence that this is so can be found in the mass spectra obtained under these conditions, with very small fragments dominating. In fact, when a Teflon disk was used as the inter-electrode insulating spacer, so that the walls of the insulator channel were composed of Teflon, the most abundant negative ion species present on operation of the pulsed discharge source was $\mathrm{F}^{-}$! This is a reflection both of the electronegativity of fluorine and of the violent conditions during the discharge. While these conditions may be desirable in some instances (perhaps the evaporation of solids might then be possible), more delicate negative ions are not likely to survive. In addition, the ability of the free jet expansion to cool such a tremendously hot distribution of ions may be diminished, resulting in vibrationally and rotationally excited ions being produced. 
In summary, the pulsed discharge source shows great promise as an alternate method for making certain types of negative ion species. It should be investigated further with the goal of optimizing yields of desired negative ions while maintaining low internal energy distributions following free jet expansion.

\subsection{First Differential Region}

Within the first differential region, illustrated in Figure 2.9, two important steps in forming a fast ion beam occur. It is here that acceleration to the appropriate beam energy occurs. Subsequently, the ion beam is rereferenced so that we avoid floating either the source or detection regions.

\subsubsection{Ion Acceleration}

Negative ion acceleration takes place in the first differential region, with the initial effects of the acceleration field felt by the ions as soon as they pass through the skimmer. Ions are accelerated through a potential that is typically 5 to $8 \mathrm{kV}$. The lower and higher limits are usually dictated by kinematics; the center-of-mass velocity of the radical beam with ideally be chosen such that the largest fraction of coincidence photofragmentation events will hit the active area of the detector face. Achieving this goal involves some trial and error, and compromise between the fraction of events where one (or both) of the fragments impinge on the blocking strip and the fraction where one (or both) of the fragments recoils far enough from the center of mass to miss the detector. The absolute lower limit for acceleration potential depends mostly on the decreasing efficiency of detection of neutral particles with a small amount of laboratory frame kinetic energy; the guideline enforced in this experiment is to use an acceleration voltage high enough so that

Figure 2.9 A cross section of the first differential region. Shown are the skimmer, the acceleration plates and the metal cylinder used for rereferencing. 


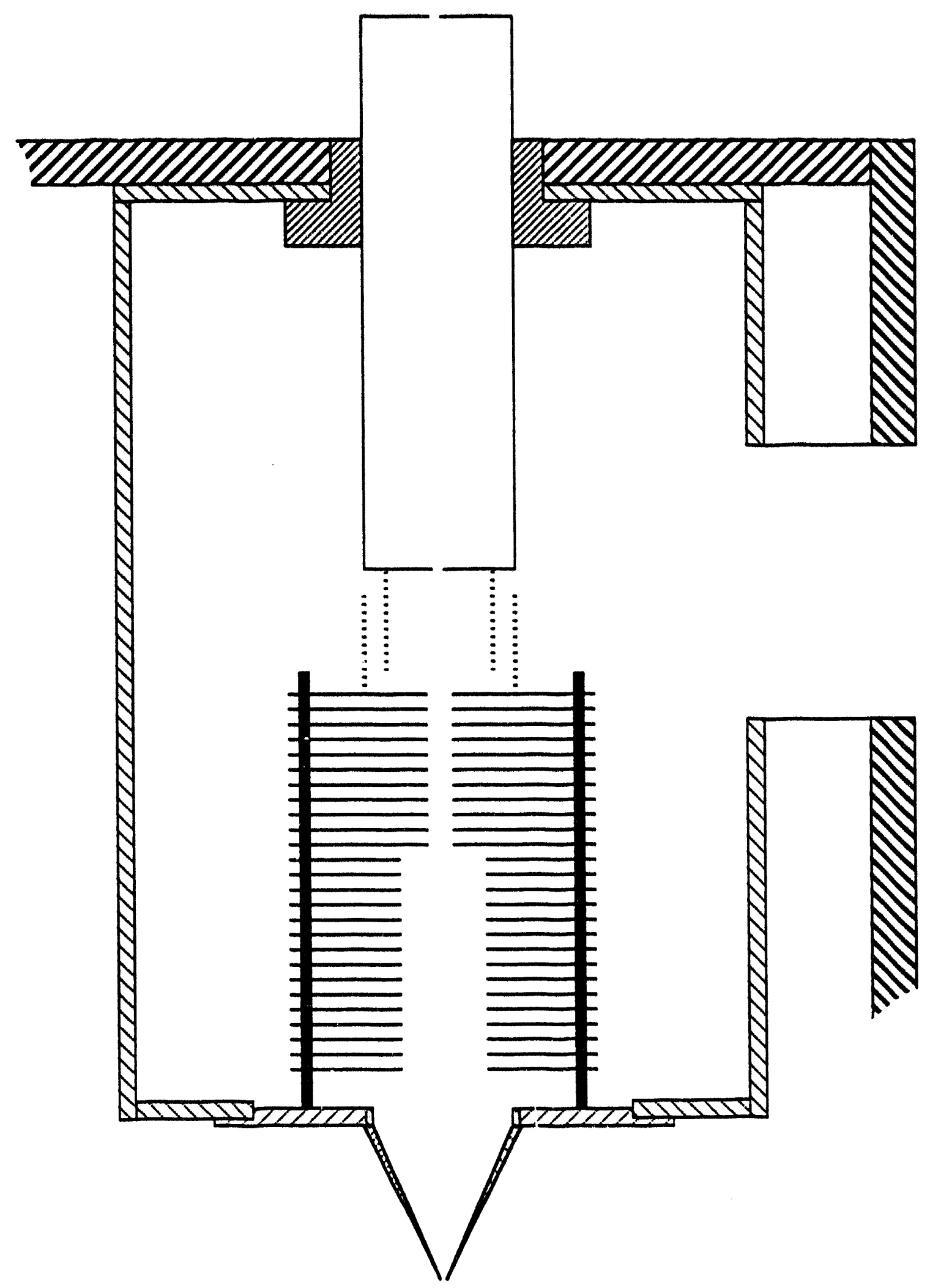

Figure 2.9 
all anticipated and/or observed fragment mass's will possess greater than $1 \mathrm{keV}$ laboratory frame kinetic energy. The absolute upper limit for acceleration potential is determined chiefly by the limits of the acceleration power supply used and the high voltage capacity of our 'potential switch', to be discussed below.

Figure 2.9 shows the arrangement of the acceleration stack within the first differential region, while Figure 2.10 shows the circuit used to apply the proper potentials to the stack, and the location within the circuit of the 'potential switch', which is to be discussed in the following section. A stack of 26 plates, 6 inches in outer diameter are used to accelerate and gently focus the ions. The first 16 plates are joined through $698 \mathrm{k} \Omega$ resistors and have a 2 inch inner diameter. Additional focusing is achieved by having the final ten plates in an decelerating einzel configuration, ${ }^{20}$ with their inner diameter reduced to 0.5 inch. The first three and last four plates of these ten are at the voltage corresponding to the final ion beam energy, while the middle three plates are at some variable, though lesser, voltage. The acceleration region einzel lens allows optimization of the focusing effects due to acceleration, and the voltage on the middle three plates in the einzel lens is adjusted to allow the largest number of ions to be detected downstream.

\subsubsection{Rereferencing the Ion Beam Potential}

After the acceleration process the ions have gained kinetic energy equal to the product of the acceleration potential and the elementary charge on the ion. At this point,

Figure 2.10 A schematic of the acceleration stack, showing the acceleration einzel lens and the location of the 'potential switch' within the overall circuit. Details of the 'potential switch' circuit are given in Figure 2.11. Note that the geometry of these components relative to one another is better depicted in Figure 2.9. 


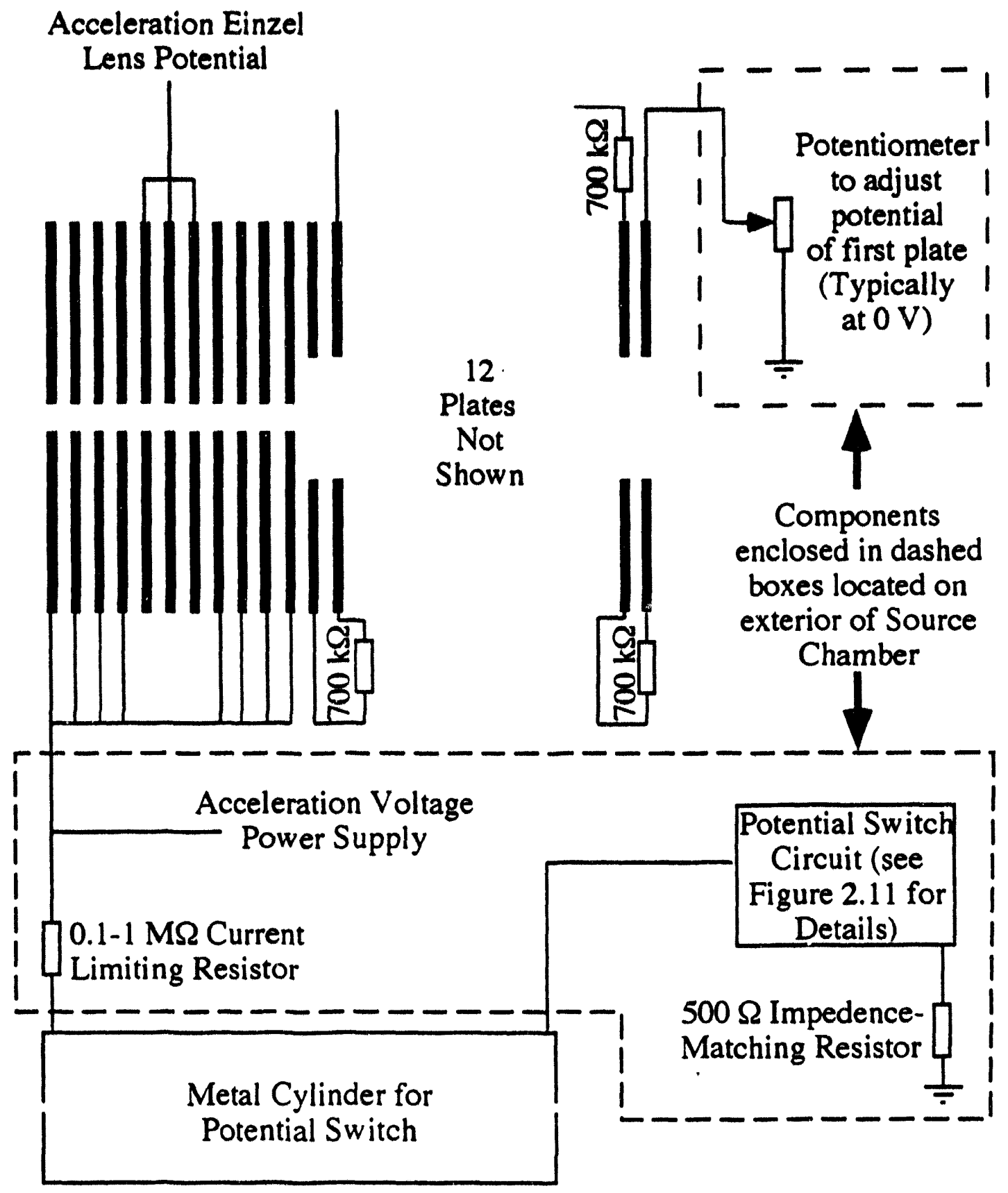

Figure 2.10 
the designers of many ion beam experiments face a dilemma. For effective beam transport, either the source or the detector section of the apparatus has to float, maintaining a potential difference corresponding to the ion beam energy. In our case this situation would result in many obvious inconveniences.

Pulsed ion beam experiments have been shown by Johnson and co-workers ${ }^{21}$ to be amenable to another solution known as rereferencing. However, to our knowledge prior examples where rereferencing has been used were not at the high (4-10 keV) beam energies that are typical for the experiments discussed here.

Rereferencing involves allowing ions to enter an isolated metal cylinder that is initially at the acceleration potential. While the ions are in the cylinder, the voltage is rapidly brought to ground potential. Ions which enter the cylinder before the potential falls, and exit while the potential is at ground, leave the cylinder with virtually the same kinetic energy as they entered, but their kinetic energy has been 'rereferenced'. These ions now possess kinetic energy equal to the beam energy with respect to ground potential, rather than the acceleration potential.

Although perhaps initially non-intuitive, rereferencing works because the ions within the cylinder cross no field lines, and experience no gradient while the potential falls uniformly around them. The fall time of the voltage on the cylinder must be fast so that only a small fraction of the ions inside the cylinder when the potential drop begins will exit prior to the completion of the voltage drop. The specific details of the application of this technique to our apparatus are discussed next, with details of the switching circuit that allows us to satisfy the demanding switching requirements posed by this technique given at the end of this section.

On exiting the acceleration plates, ions enter an isolated $25 \mathrm{~cm}$ long, $8 \mathrm{~cm}$ diameter, hollow, stainless steel cylinder having $3 \mathrm{~mm}$ apertures at the center of each end. This cylinder is normally at the same potential as the final acceleration plate, and is pumped out through mesh that covers portions of the end extending back into the first 
differential region. The aperture on the end of the cylinder extending into the second differential serves as the differential pumping aperture between the first and second differential regions. There is a ground shield surrounding the end of the cylinder that extends towards the detector. This ground shield will confine the potential on the cylinder, preventing any possible perturbations of the detached electrons by electric fields from the cylinder.

At some experimentally optimized delay relative to the triggering of the pulsed valve (ca. 280-380 $\mu$ s, depending on the mass of the desired ions), the potential of this cylinder is taken from the ion beam potential to very close to ground in $\sim 150 \mathrm{~ns}$. When the potential of the cylinder reaches a minimum, all the ions that subsequently emerge from the cylinder will maintain a kinetic energy approximately equal to the full acceleration energy. Only the ions within the cylinder during the rereferencing process will proceed down the machine towards the detector. In this way, the rereferencing process effectively selects a section of ions from a longer stream emanating from the source. ${ }^{22}$

Evidence of this selection can be observed in a time-of-flight spectrum of ions that emerge from the potential switch, even with the mass spectrometer apparatus disabled. Since different masses travel at different speeds if they possess the same kinetic energy, it is possible to obtain a very low resolution mass spectrum by using the potential switch to select an initial bunch of ions, which then separates according to mass. A spike on the short-time side of the broad time-of-flight peak for: ions mass selected in this manner is thought to come from ions that leave the cylinder before the cylinder potential hits the minimum. These ions enjoy a comparative 'head start', but are handicapped by having a lower kinetic energy. They therefore pile up, arriving simultaneously with the earliest ions of proper kinetic energy. Thus, although it is quite tempting to optimize this spike for use in an experiment, it must be avoided. Since the ions in this spike have a large kinetic energy spread, they will diverge as they continue down the machine. 


\subsubsection{Details of the 'Potential Switch' Rereferencing Circuit}

For several months we flirted with an unsatisfactory circuit for quickly dropping the voltage on the metal cylinder from the acceleration voltage to ground, which involved capacitively coupling the output of a pulsed high voltage power supply. It was decided that this method involved too much shot-to-shot jitter in beam energy and a somewhat unreliable power supply; in short, a new scheme was needed.

The new generation of potential switch ${ }^{23}$ was assembled and tested primarily by Dr. Robert Continetti following a suggestion from George Gabor and advice from Oren Milgrome, both of whom are associated with Lawrence Berkeley Laboratory. It involves an inexpensive yet reliable fast-switching metal-oxide semiconductor field-effect transistor (MOSFET) circuit to function as a switch, quickly closing to complete a connection from the cylinder to ground when triggered. Although power MOSFETs capable of rapidly switch up to $1 \mathrm{kV}$ are available, switching higher voltages demands the use of schemes in which MOSFETs are implemented in series. The design utilized in the original MOSFET potential switch to rapidly switch up to $8 \mathrm{kV}$ is based on a series of 10 transformerisolated, varistor-bypassed, $1 \mathrm{kV}$ power MOSFETs. A key to this approach is the use of metal-oxide varistors in parallel with each MOSFET in order to clamp the voltage across a given MOSFET to less than the rated voltage. Metal-oxide varistors display a nonlinear resistance as a function of voltage, having extremely high resistance below their characteristic turn-on voltage, while becoming quite conductive if the turn-on voltage is exceeded. Because of these characteristics they serve as rugged, fast-acting surge suppressers. ${ }^{24}$ Their presence in series with the power MOSFETs allows the requirement for accurate synchronization of the MOSFET triggering to be relaxed, greatly simplifying this aspect of the design.

The current version of this circuit was recently assembled by David Osborn, closely following the original circuit design. However, it contains more recently available components and boasts both an upper voltage limit of up to $\sim 15 \mathrm{kV}$ (using a maximum of 
16 MOSFET stages) and a modular design, with MOSFET stages on plug-in circuit board cards. This affords the ability to use a minimum number of stages for the voltage to be switched to ground, which has a slight minimizing effect on fall time. It is also very convenient for when the rare but inevitable component failure occurs.

As can be seen in Figure 2.11, the circuit consists of up to 16 identical varistorbypassed $1 \mathrm{kV}$ MOSFET stages (Harris, RFP4N100) connected in series, with one end of the chain connected to the cylinder and the other end at ground. The metal oxide varistors used are rated at 920 VDC (Panasonic ZNR 20K102U). The MOSFETs are simultaneously triggered in groups of up to five each through transmission line transformers by high speed FET drivers (Teledyne, TC4422CPA). Each transmission line transformer was made by wrapping 17 turns of RG 316/U coaxial cable around a ferrite toroid (Fair-Rite \#59-77-001601), using the outer conductor of the coaxial cable as the primary and the inner conductor as the secondary of the transformer. ${ }^{25}$ An optoisolator (Hewlett-Packard, HCPL-2601) is used to prevent damage to the external pulse generator supplying the trigger pulse in the event of a failure.

A current-limiting resistor is placed between the acceleration voltage power supply and the cylinder. This is necessary since the ON resistance of the power MOSFETs is quite low ( $3.5 \Omega$ per MOSFET); without this resistor there would effectively be a short circuit from the high voltage power supply to ground. This would not only quickly destroy the MOSFETs but exceed the current rating of the high voltage power supply. The current-limiting resistor value should be large enough to allow the cylinder to closely approach ground. There is effectively a voltage divider determining the potential on the cylinder, with the current limiting resistor dropping most of the potential, but the combined resistance of the MOSFETs accounting for a small potential drop as well. The

Figure 2.11 A schematic of the 'potential switch' circuit used to rereference the ion beam by bringing a metal cylinder from as high as $15 \mathrm{kV}$ down to ground potential in $<150$ ns. 


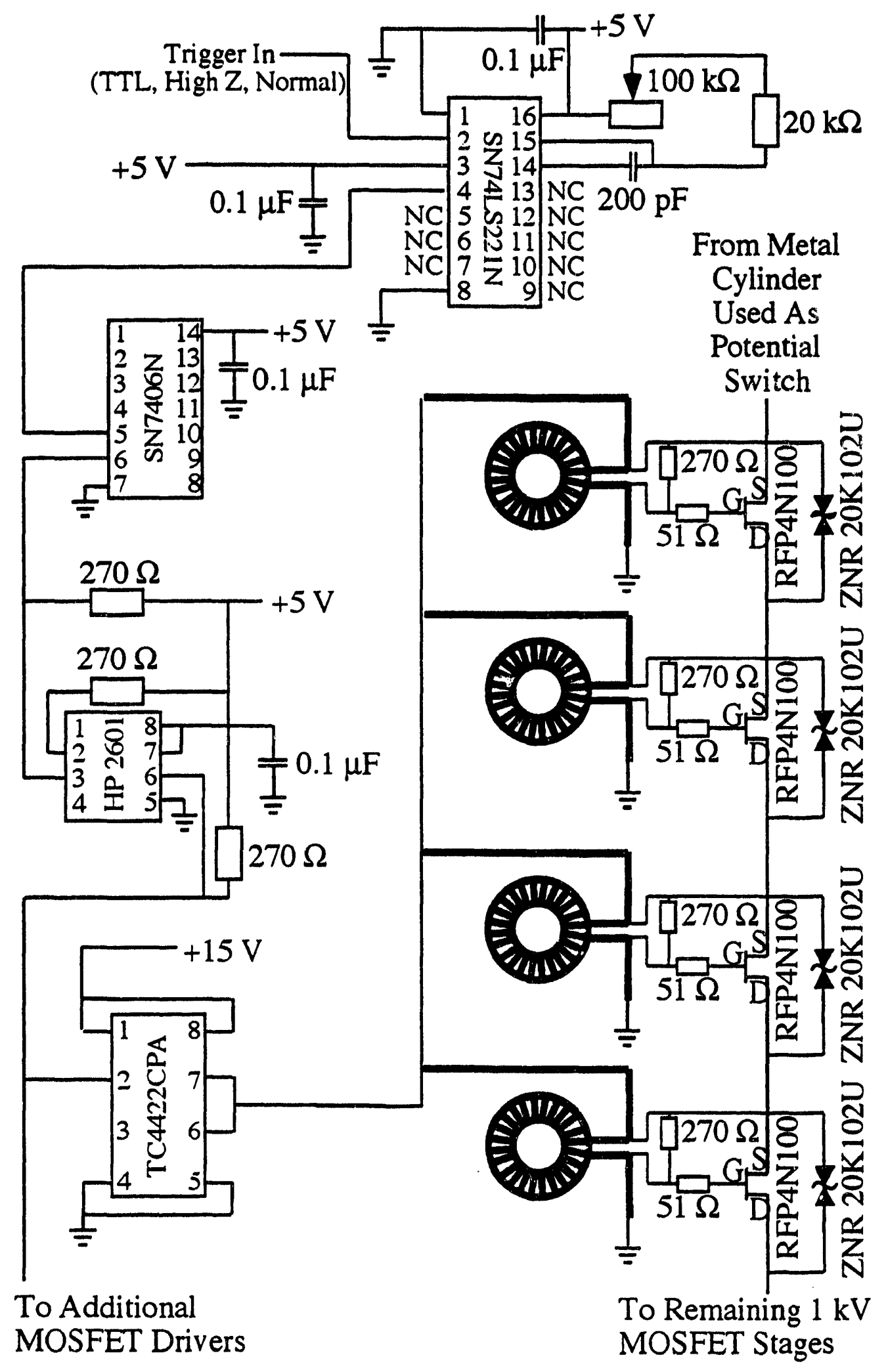

Figure 2.11 
current limiting resistor value cannot be too large, however, because the cylinder must charge back up to the acceleration potential between consecutive iterations. Charging of the cylinder occurs when the MOSFET switch opens and current trickling through the current limiting resistor can no longer go to ground. Instead, this current brings the potential of the metal cylinder up to the acceleration voltage.

A final concern is that the current limiting resistor be of appropriate power rating. At least a 2 Watt rating has been found to be necessary, in agreement with quick calculations (i.e., assuming $10 \mathrm{kV}$ acceleration voltage, $100 \mathrm{k} \Omega$ resistor, and a $60 \mathrm{~Hz} \times 20$ $\mu \mathrm{s}=2 \times 10^{-3}$ duty cycle, one gets 1.2 Watts as the power dissipated).

Since our application requires only that the falling edge be fast, the rising edge is described by the $\mathrm{RC}$ time constant $\mathrm{R}_{\text {lim }} \mathrm{C}_{\mathrm{cyl}}$, or $10 \mu \mathrm{s}$, for a $100 \mathrm{k} \Omega$ current limiting resistor and a $100 \mathrm{pF}$ cylinder. Were it necessary to have two fast edges, it would be possible to use two MOSFET switches in a push-pull version of this circuit. This type of circuit is well suited to fast, high voltage switching applications, and should be considered in all cases where these characteristics are desired.

\subsection{Second Differential Region}

The second differential region contains a beam modulation time-of-flight mass spectrometer, ion beam deflectors and various focusing optics. The geometries of these components and their locations within this region are illustrated in Figure 2.12. These components, discussed below, provide mass selection of a specific ion and the ability to

Figure 2.12 A diagram illustrating the various components present within the second differential region. (1) Metal cylinder used for rereferencing surrounded by a ground shield. (2) Beam modulation deflection plates. (3) Ion compressor plates. (4) Ion beam vertical and horizontal deflection plates. (5) Einzel lens. (6) Gate valve. (7) $1 \mathrm{~mm}$ aperture that serves as a differential pumping aperture in addition to the beam modulation time-of-flight mass spectrometer slit. 


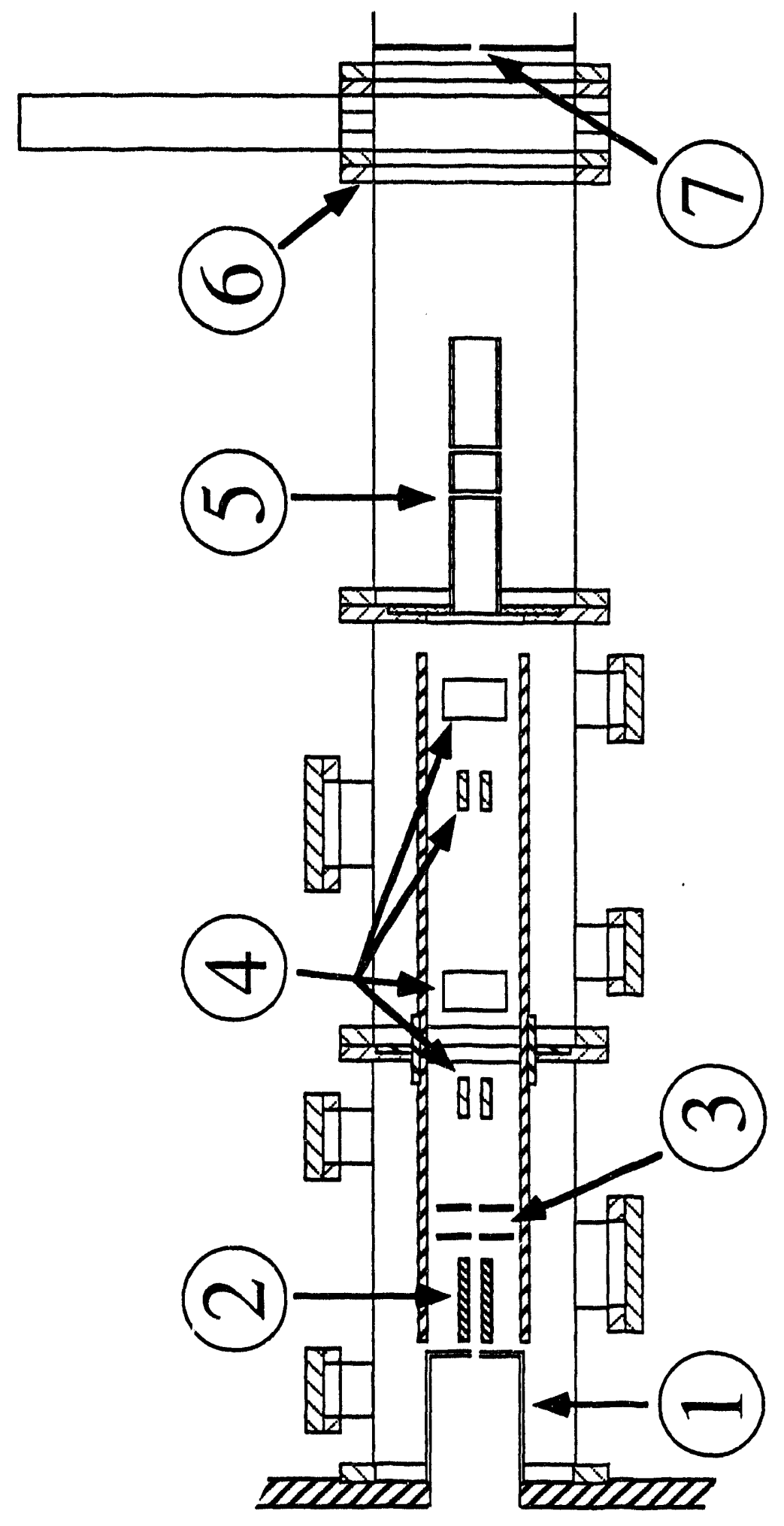

Figure 2.12 
steer and focus the ions as necessary, including the compression of an ion packet along the beam axis.

\subsubsection{Beam Modulation Mass Spectrometer}

The time-of-flight (TOF) mass spectrometer used in this experiment is a collinear beam modulation type, first described by Bakker. ${ }^{26}$ The Bakker-type beam modulation mass spectrometer works by in effect selecting a compact bunch of ions, which will then separate spatially and temporally according to mass because their equal kinetic energies imply different, mass-dependent velocities. The selection process is accomplished simply by rapidly reversing the electric field between a pair of plates whose faces are perpendicular to the ion beam axis. The beam modulation deflection plates are the first components located in the second differential region, [labeled (2) in Figure 2.12]. Only the ions which experience an equal but opposite amount of acceleration from the initial field and the reversed field (i.e. those ions very near the center of the plates when the field is reversed) will continue along parallel to the beam axis and be admitted through the initial $1 \mathrm{~mm}$ defining aperture $0.86 \mathrm{~m}$ from the center of the plates [labeled (7) in Figure 2.12].

Figure 2.13 shows the elect onic circuit that is used to generate the rapidly reversing electric field. This circuit reverses the field between the two mass spectrometer plates by maintaining a voltage $\mathrm{V}_{0}$ on one plate until the arrival of a trigger pulse, at which point the voltage on this plate goes to ground in $<20 \mathrm{~ns}$. The other plate is held at a continuous potential of $\mathrm{V}_{0} / 2$, as determined by a voltage divider formed by the two $10 \mathrm{k} \Omega$ resistors shown in Figure 2.13.

The beam modulation mass spectrometer has the advantage of introducing only a negligible kinetic spread in the ion beam energy. The commonplace Wiley-McClaren ${ }^{27}$

Figure 2.13 A schematic of the beam modulation mass spectrometer circuit. 


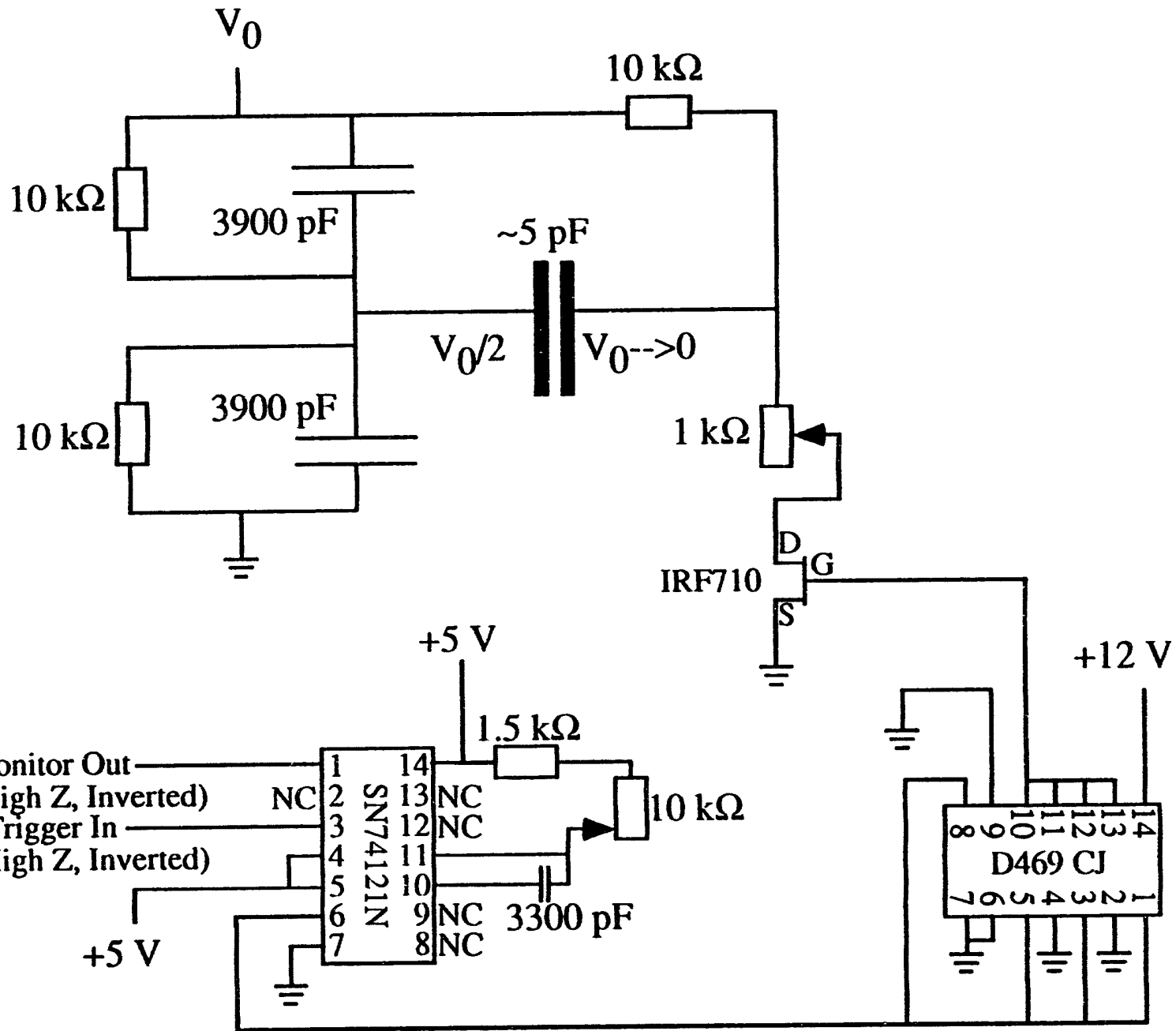


type space-focusing technique was not used in this experiment because it, on the other hand, introduces a significant kinetic energy spread. Maintaining a compact kinetic energy distribution within the negative ions is an important and desirable characteristic for our dynamics experiments. Any increase in the spread of the beam energy, and therefore velocity, results in the formation of a packet of ions that is divergent along the beam axis. If the kinetic energy distribution of the ions in the beam is broad enough, it will be harder to overlap with the detachment and dissociation laser pulses. In addition, a wider kinetic energy distribution in the ions transfers directly to the radicals formed in detachment. For reasons to be addressed in $\S 2.7 .6 .5$, this ultimately results in an increased uncertainty in the measurement of photodissociation kinetic energy release, and to a minor, extent, the recoil vector angle.

Bakker has shown ${ }^{26}$ that the theoretical resolving power is given by:

$$
R=\frac{L^{2} V_{0}}{2 D U(B+S)}
$$

This formula is appropriate when the rise time of the square wave is short compared to the transit time between the plates, which itself is short compared with the field-free drift region, as is the case in our apparatus. Here, $L$ is the length of the field-free drift region, $V_{0}$ the potential difference of the step function applied to the plates, $D$ the separation of the deflection plates, $U$ the ion beam acceleration voltage, $B$ the beam width and $S$ the defining aperture width. The values in Eqn. (2.5) that are fixed in our machine are as follows: $D=1 \mathrm{~cm}, B=0.4 \mathrm{~cm}, S=0.1 \mathrm{~cm}$. In terms of $L$, the initial $1 \mathrm{~mm}$ beam defining aperture is located $86 \mathrm{~cm}$ from the center of the beam modulation plates. However, for the full experiment the ultimate mass spectrometer resolution depends on a second $1 \mathrm{~mm}$ aperture located $153 \mathrm{~cm}$ from the center of the beam modulation plates. This er ,.... vely results in a factor of $(153 / 86)^{2}=3.17$ higher resolution, since Eqn. (2.5) shows that the resolving power is proportional to the square of the drift region length. 
$V_{0}$ and $U$ are variables for any given system, but a typical value for $U$ is $6000 \mathrm{~V}$. In this situation the resolving power past the first $1 \mathrm{~mm}$ aperture is roughly $\sim 1.2 \times V_{0}$. Therefore, if $100 \mathrm{~V}$ is used, the mass resolution will be $\sim 120 \mathrm{amu}$. Typically, however, the minimum voltage is used to resolve a given mass to charge ratio, and these values of $V_{0}$ are closer to $40 \mathrm{~V}$. The values appropriate at the first aperture are given because resolution effects of the mass spectrometer voltage are most conveniently monitored using the ion time-of-flight measured at the ion detector. This detestor is located after the first $1 \mathrm{~mm}$ aperture but before the second.

As can be seen from Eqn. 2.5, resolution is independent of ion mass. It can also be shown ${ }^{26}$ that there is no mass-dependent discrimination, i.e., all masses are transmitted with equal efficiency by the beam modulation mass spectrometer. These last two favorable characteristics round out the list of reasons the beam modulation time-of-flight mass spectrometer is well suited for our experiment.

Some idea of the ion burst duration can be obtained from an additional formula given by Bakker, ${ }^{26}$ again appropriate when the rise time of the square wave is short compared to the transit time between the plates, which itself is short compared with the field-free drift region:

$$
\Delta t=\frac{(B+S) D \sqrt{\frac{2 U m}{e}}}{2 V_{0} L} .
$$

When typical values of $B=0.4 \mathrm{~cm}, S=0.1 \mathrm{~cm}, D=1 \mathrm{~cm} U=6000 \mathrm{~V}, V_{0}=100 \mathrm{~V}$ and $\mathrm{L}=86.6 \mathrm{~cm}$ are used, the ion burst duration for mass $32 \mathrm{amu}$, corresponding to the $\mathrm{O}_{2}^{-}$ anion, will be $\sim 18 \mathrm{~ns}$. This illustrates how temporally compact the ior packet is. Under these conditions the size of the packet along the beam axis will be $\sim 3 \mathrm{~mm}$ if the ion compressor, to be described next, is not used. 


\subsubsection{Ion Compressor}

The ion compressor can be used to focus a group of ions in the dimension parallel to the ion beam axis. The focal point is usually chosen to be midway between the laser interaction regions to improve overlap with the output of both lasers, thus improving overall signal intensity.

Figure 2.12 shows the location of the ion compression components. The ion compressor consists of two $7.5 \mathrm{~cm}$ outer diameter, $0.5 \mathrm{~cm}$ inner diameter plates spaced $2 \mathrm{~cm}$ from each other, concentric with, and perpendicular to the ion beam. These plates are located immediately following the mass spectrometer plates. Ion compression involves the application of a pulsed negative potential to the rear plate with the front plate held at ground, while the negative ions to be focused are completely between the two plates. In this way, ions closest to the back of the packet are accelerated through the largest potential, gain the most kinetic energy, and will therefore eventually overtake negative ions that were closer to the front plate when the potential was switched on.

Figure 2.14 shows the electronic circuit that is used to generate the pulsed electric field used in the operation of the ion compressor. This circuit is extremely similar to the beam modulation mass spectrometer circuit, with the exception that the front plate is held at ground potential, rather than at half the step function voltage of the other plate.

Use of the ion cumpressor has no adverse effects on a total cross section experiment, and is recommended without reservation in that instance. Ion compression will, however, degrade the kinetic energy release resolution in the dynamics experiments for the same reason a Wiley-McClaren design mass spectrometer would, by increasing the spread of the ion beam energy. The increased uncertainty in the quantity will ultimately blur the determination of the photodissociation kinetic energy release distribution.

Figure 2.14 A schematic of the ion compressor circuit. 


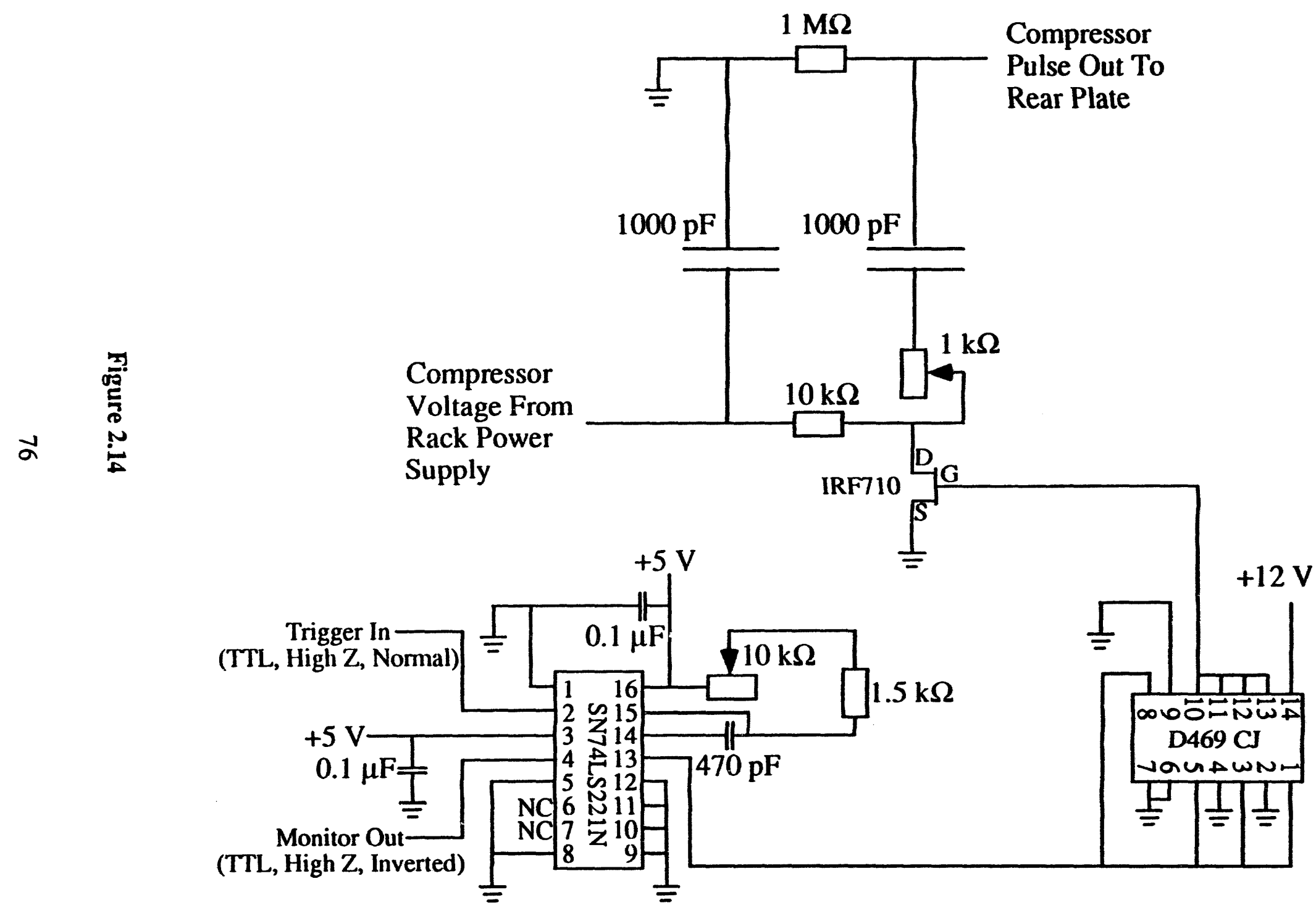




\subsubsection{Ion Optics}

The ion optics, save the acceleration einzel lens, are found in the second differential region. As depicted in Figure 2.12, they follow the mass spectrometer plates and ion compression plates and consist simply of two sets of vertical and horizontal deflector plates and a second einzel lens. The deflectors allow a slight redirection of the ion beam to maximize ion and neutral transmission through the two $1 \mathrm{~mm}$ collimation holes downstream. Each set of deflection plates are 1" long in the direction of the beam axis and have a 1 " separation, symmetric about the beam axis. They are mounted on a rail which allows their position along the beam axis to be easily adjusted when accessed through side flanges. Quite often, however, it is the case that these deflector plates are kept at ground, their influence unnecessary. When they are required to be on at any significant voltage it is usually a clear sign that something else is amiss.

An einzel lens comprised of three cylindrical elements follows these deflectors. It is generally operated in the deceleration mode, with the central element at a negative potential and the first and last elements at ground. The voltage used is mass- and beam energy-dependent, but is typically around $-700 \mathrm{~V}$. Higher voltages can improve throughput of ions by focusing the ion beam more tightly between the two $1 \mathrm{~mm}$ apertures. However, this results in a larger degree of divergence at the fragment detector(s). This can be a problem. For example, the radical beam diameter may grow larger than the $3 \mathrm{~mm}$ radical beam blocking strip on the total photodissociation cross section detector. When this happens, background from undissociated radicals will contaminate the photofragment signal.

\subsection{Third Differential Region}

The third differential region is illustrated in Figure 2.15. Within this region the ion, and then radical, beam undergoes its most rigorous collimation, with $1 \mathrm{~mm}$ apertures forming the entrance and exit to this region. Here the ions having the appropriate mass 
are photodetached, and the photoelectrons generated in this process are collected. In addition, determination of the negative ion species present in the beam can be undertaken simultaneously via time-of-flight mass spectroscopy. This ability assists in determining the intensity and identity of a given negative ion, particularly useful when initially generating a negative ion that had previously not been produced using this apparatus.

\subsubsection{Photodetachment}

Photodetachment is carried out using the output of a tunable excimer-pumped dye laser that produces $20 \mathrm{~ns}, \sim 20-50 \mathrm{~mJ}$ pulses at wavelengths extending from $\sim 335 \mathrm{~nm}$ to $>700 \mathrm{~nm}$ (Lambda Physik LPX 210i and FL3002, respectively). The laser output is gently focused by a $1 \mathrm{~m}$ lens, with the photodetachment volume located about $0.3 \mathrm{~m}$ from the lens. The laser is timed to intercept the negative ions that have a mass corresponding to the radical of interest.

The detachment wavelength is judiciously chosen so as to form radicals in the ground vibrational state, according to the princi jles discussed in $\$ 1.4$. Detachment efficiencies can approach 80\%; with high laser power and optimal timing, this efficiency can be observed in the depletion of the ion nass of interest or, in relative terms, by the size of the detached electron signal.

\subsubsection{Laser Timing Jitter}

Timing jitter is a serious concem in this experiment. The dye laser temporal profiles for both the detachment and dissociation laser systems (as the laser systems are

Figure 2.15 A diagram of the third differential region. (1) Gate valve. (2) Initial $1 \mathrm{~mm}$ aperture. (3) Photodetachment volume straddled by electron extraction plates. (4) Detached electron detector surrounded by grounded mesh. (5) Unde.ached ion deflector. (6) Ion detector, shown in the fully retracted position. (7) Final $1 \mathrm{~mm}$ aperture. 


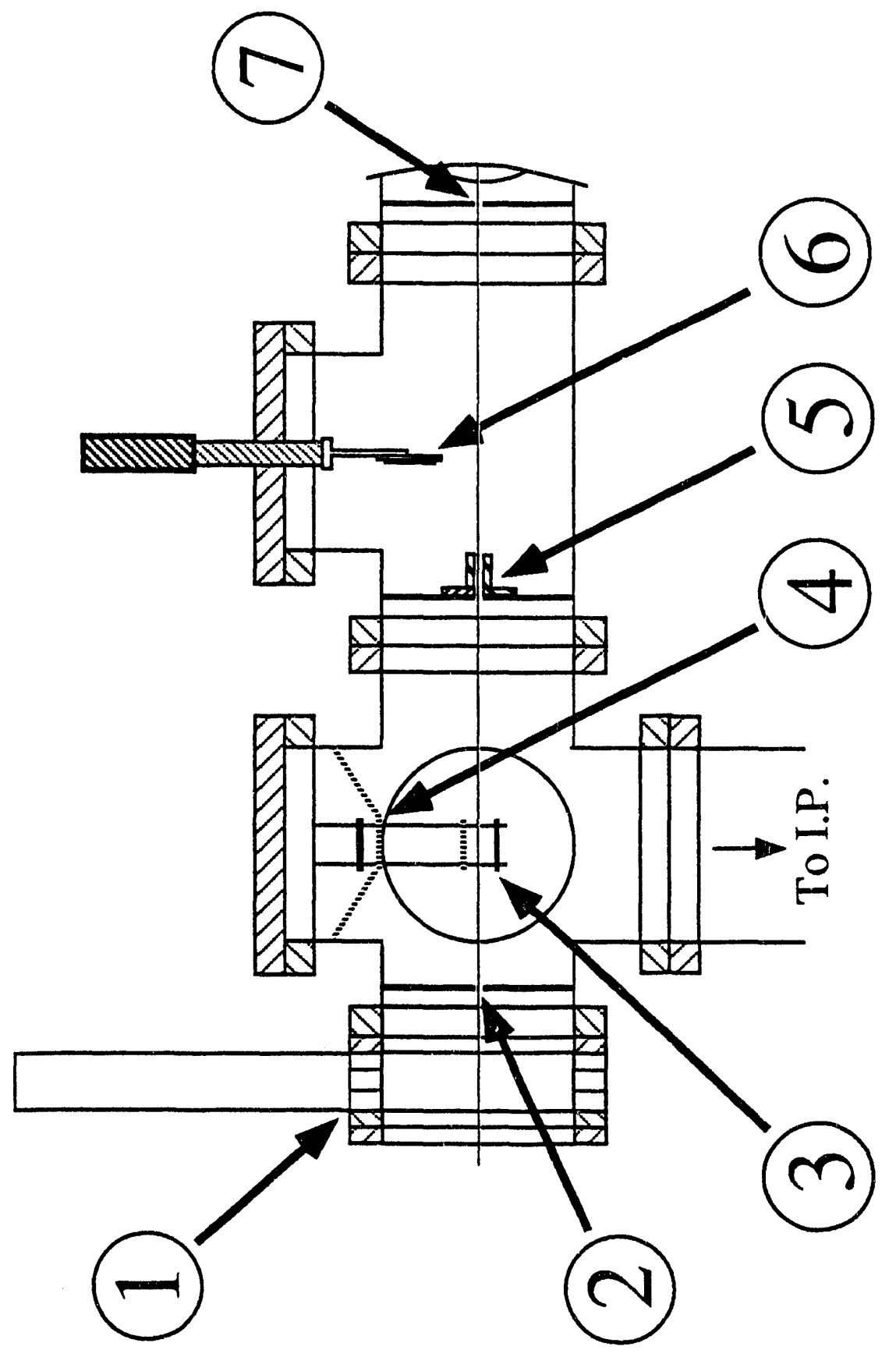

Figure 2.15 
identical) are determined chiefly by that of the excimer pump lasers. The most intense portion of the laser pulse is confined to $\sim 28 \mathrm{~ns}$. The same packet of ions, and, following detachment, radicals, must be intercepted by both detachment and dissociation laser pulses in order for radical photodissociation to occur. Adding to the complexity of this task is the fact that, depending on the ion mass, beam energy and mass spectrometer voltage, the ion burst duration is on the order of $\sim 18 \mathrm{~ns}$, as shown in $\S 2.5 .1$. The excimer laser specification for timing jitter is $4 \mathrm{~ns}$, which is acceptable. However, as the thyratron (the high voltage, high current switch that controls the discharge current) in an excimer laser ages, this specification can be exceeded, sometimes dramatically.

Fast photodiodes are employed to monitor the timing jitter of both the detachment and dissociation lasers. A photodiode circuit is shown in Figure 2.16. The key component of this circuit is a quartzface (for transmission of UV light) photodiode (Motorola, Model MRD510) reverse-biased by a $90 \mathrm{~V}$ potential. A low-intensity backreflection from the output of the dye laser that is to be monitored is made to fall on the face of the photodiode. The response time of the photodiode is approximately $1 \mathrm{~ns}$, sufficient to follow the temporal profile of the dye laser pulse. The output of the photodiodes can be observed using the oscilloscope.

Once the optimal timing for a given laser has been established, monitoring the temporal characteristics of the laser pulses with fast photodiodes can serve two purposes. The photodiode output can be monitored for excessive shot-to-shot timing jitter, as well as long-term drift in laser timing. Adjustment of the thyratron reservoir heater is usually required to correct shot-to-shot timing jitter. Long-term drift is usually the result of thermal variations as the excimer lasers run, except when the gas fill or the thyratron is near the end of its useful life. It is therefore mostly observed as a shortening in the delay between trigger pulse and the excimer laser firing during the initial period of operation,

Figure 2.16 A fast photodiode circuit diagram. 


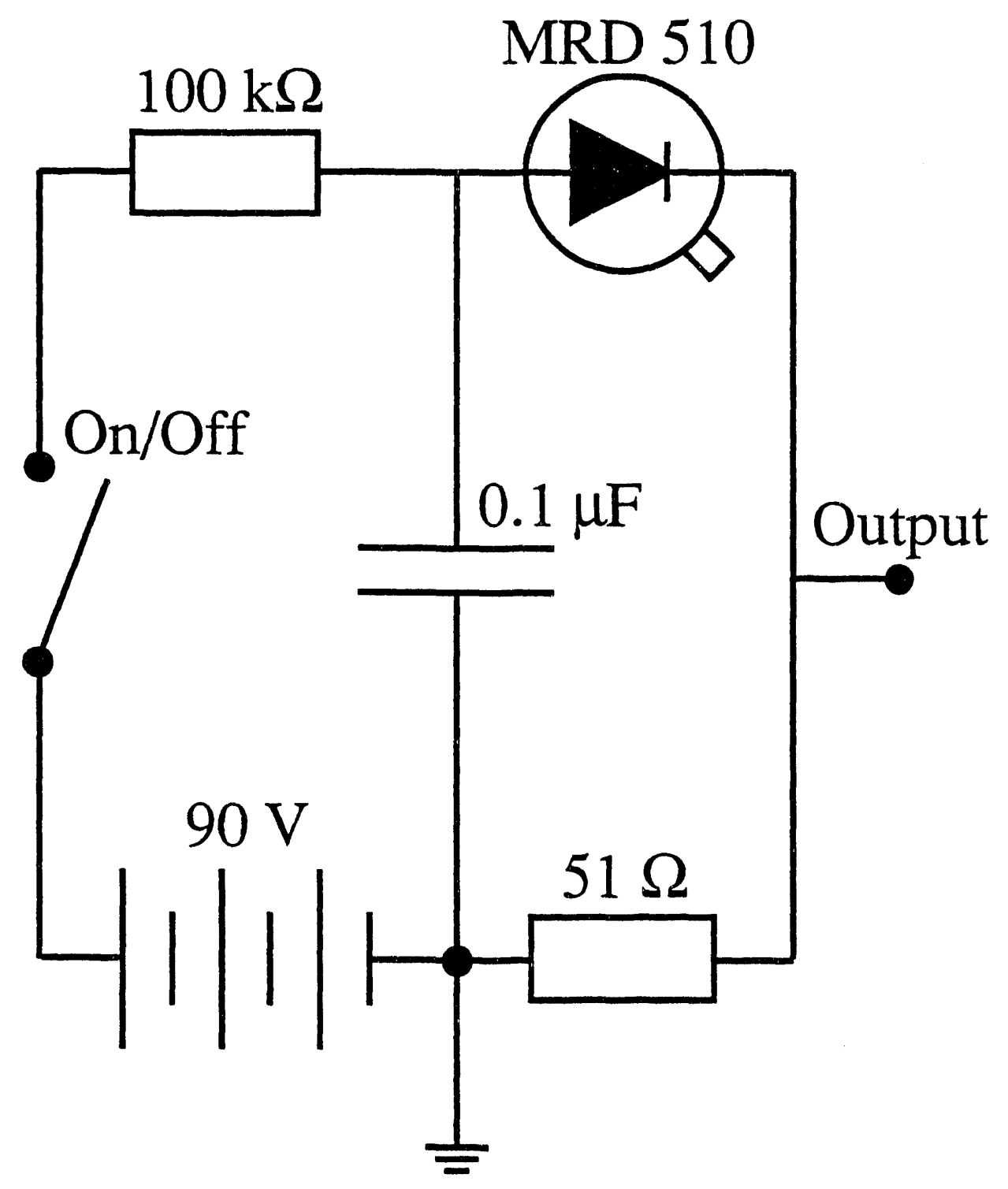

Figure 2.16 
prior to thermal equilibrium within the excimer laser. As the gas fill becomes old, timing drift in the opposite direction is sometimes observed. Small adjustments in trigger pulse timing can be made periodically after consulting the photodiode output to correct for long-term drift.

\subsubsection{Detached Electron Detection}

Detection of detached electrons provides a measure directly proportional to the number of radicals produced in the detachment process. This information is useful for evaluating and adjusting the spatial and temporal overlap of the detachment laser with the negative ion precursor of interest. It is also a critical for proper normalization of total photodissociation cross section spectra.

The gross features of the detached electron detector assembly can be discerned in Figure 2.15. Detached electron detection is accomplished by using a small, pulsed, electric field to accelerate detached electrons perpendicular to the beam axis, up into a final acceleration field which is just prior to the face of a microchannel plate detector assembly.

Microchannel plate detectors serve as large area electron multipliers. The plates themselves are typically thin (1-2 $\mathrm{mm})$ circular disks available in various diameters. All microchannel plate detectors on this machine, with the exception of the time-and positionsensitive detector, use a set of two microchannel plates (Galileo Electro-Optics

Corporation), of diameter $25 \mathrm{~mm}$ or $40 \mathrm{~mm}$. The detached electron detector uses $25 \mathrm{~mm}$ diameter microchannel plates. They are made of a semiconducting glass material and consist of close-packed, regularly spaced channels that are about $10 \mu \mathrm{m}$ in diameter and $12 \mu \mathrm{m}$ from center to center, at an angle of $13^{\circ}$ with respect to the parallel surfaces. The material is highly resistive and a high potential can be placed across them. A photon, neutral particle, ion or electron of sufficient energy will cause electron(s) to be emitted by the microchannel plate at the point of impact. Microchannel plates then function in a 
similar manner to a photomultiplier. The primary electrons are accelerated in the electric field perpendicular to the surface, down into one or more of the channels. Because these channels are at a slight but significant angle away from being perpendicular to the plane of the microchannel plate, the electrons soon collide with the wall of the channel with sufficient energy to cause additional electrons to be released. This cascading effect continues, and gains of up to $10^{3}-10^{4}$ per plate can be achieved. It is estimated that the set of two microchannel plates used in the detached electron detector obtain a gain of $10^{6}$.

The 'chevron mounting' configuration is used in all of the microchannel plate detectors on this machine (save the time- and position-sensitive detector, whose three plates form an analogous 'Z-stack'), in which the angles at which the channels are oriented is reversed between plates. This reduces ion feedback, the process where positive ions are formed through electron bombardment and accelerated back in the opposite direction, eventually causing spurious counts.

The details of the overall detector assembly and its application to the detection of detached electrons will now be given. A set of three threaded rods screwed into the top flange of a six-way cross supports all components of the detector. Extraction plates hang down from the detector, mounted near the end of these threaded rods. The extraction plates are oriented horizontally about 1 " apart, equidistant from, and parallel to the ion beam axis. The upper extraction 'plate' is made using a high ( $90 \%)$ transmittance steel screen spot-welded to a steel ring. A $+6 \mathrm{~V}$ pulse is applied to this upper plate just as detachment occurs. Detached electrons are accelerated through this mesh and on towards the detector by this extraction pulse. The extraction potential is weak compared to the beam potential and pulsed on only just before detachment has occurred (at the earliest) so that the trajectory of the negative ions is not affected.

The detached electron detector is mounted in a perpendicular orientation to the beam axis. A grounded grid made from high transmittance steel mesh spot-welded to a steel ring is supported by the threaded rods, concentric with both the outer diameter of the 
microchannel plates and the extraction plates, and located $\sim 1$ " from the front of the first microchannel plate. An acceleration voltage is set up between this grid and the microchannel plates by biasing the front of the first microchannel plate at $\sim+300 \mathrm{~V}$. This enables the detached electrons (possessing on the order of $3 \mathrm{~V}$ kinetic energy from the extraction pulse) to gain enough kinetic energy to be detected.

When struck by an energetic electron anywhere on the front of the first microchannel plate, $\sim 10^{6}$ electrons will cascade from the back of the final microchannel plate. These electrons are accelerated across a final $100 \mathrm{~V}$ potential difference onto a contiguous steel disk that serves as an anode. This signal is capacitively coupled out to a BNC feedthrough, providing a fast, $5 \mathrm{~ns}$ wide, $10-40 \mathrm{mV}$ negative-going peak.

The circuit which is used in the operation of the electron detector is shown in Figure 2.17. This circuit consists of a resistor network that ensures an equal potential drop across the two MCPs, with a $100 \mathrm{~V}$ Zener diode ensuring a constant drift region potential between the back of the last MCP and the anode. The output is AC coupled from the anode, which is typically at $2150 \mathrm{~V}$, through a $1000 \mathrm{pF}$ capacitor and out through a BNC connector welded in the flange on which the detector is mounted. The detached electron signal is then typically fed directly into a fast preamplifier (EG\&G Ortec, Model 9301). All circuit components (resistor network, zener diode) are located in a small aluminum box mounted on the exterior of a six-conductor feedthrough welded in the flange directly above the detector. This allows easy access should a component fail and avoids component out-gassing concerns which are problematic in the high vacuum environment.

As briefly mentioned above, during total photodissociation cross section scans, the detached electron signal is collected for the purpose of normalizing the observed photofragment signal. In the limit where saturation of dissociative radical transitions is

Figure 2.17 The circuit diagram for the detached electron detector. 


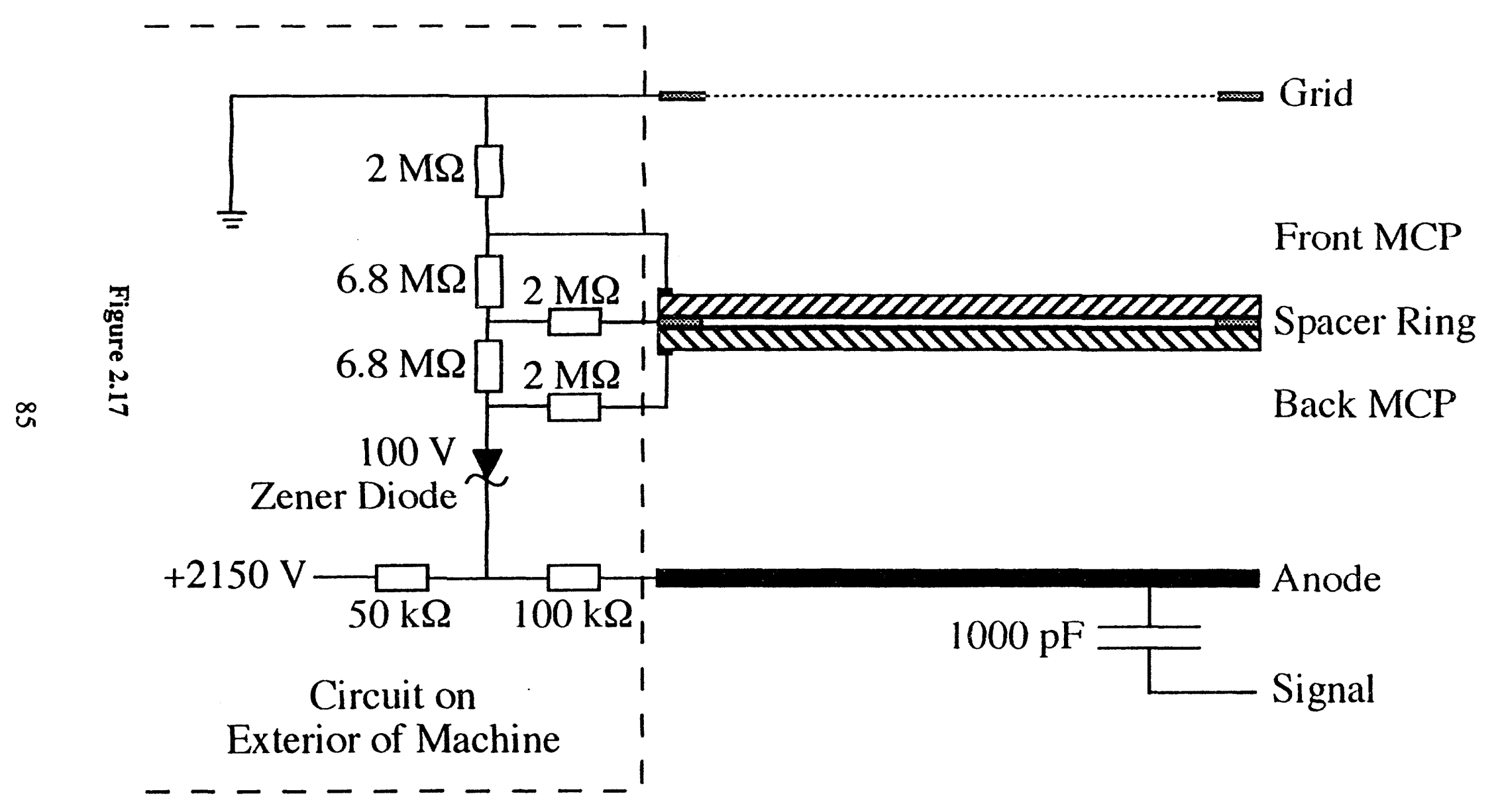


not important, for a given photon energy the number of photofragments should be proportional to the relative number of radicals present in the photodissociation volume. This, in turn, should be proportional to the number of detached electrons.

In contrast, time- and position-sensitive experiments do not need this type of normalization. The detached electron signal is still useful, however, as a gauge of photodetachment efficiency. Any fall-off indicates problems with negative ion production, detachment laser power or detachment laser timing.

\subsubsection{Ion Detection}

It is of interest to be able to detect the negative ions in the beam for a number of reasons. Most importantly, we can carefully monitor the source performance while collecting data, ${ }^{28}$ making adjustments if necessary to maintain a robust ion beam.

Additionally, we can often get a very good idea of the ion depletion due to photodetachment, which at times can be $>75 \%$, by detuning the detachment laser in time and observing the increased intensity of the ion being detached. Lastly, the time-of-flight mass spectrum that is readily obtained with this detector is invaluable during the initial process of investigating the production of a new ion for the study of the corresponding radical.

The ion detector is mounted on a linear motion feedthrough manipulator (PerkinElmer Vacuum Products, Cat. No. 281-6200). Using this manipulator it can easily be raised completely out of the beam or lowered so that it is centered on the beam axis. As illustrated in Figure 2.15 the raised position is typically used since this allows any radicals made by photodetachment to pass; this configuration is necessary during a photodissociation experiment. If the ion detector is in the raised position ion detection is accomplished by deflecting all undetached ions remaining in the beam up out of the beam axis and onto the face of the ion detector. The ion deflection plates are 'hidden' from the photodetachment region so that the static positive potential present on the upper plate 
does not perturb the trajectory of the ions prior to photodetachment. A steel ring. welded onto the inner surface near the entrance of the tee housing the ion detector has been machined to be concentric with the beam axis. On this ring is mounted a 3 " wide thin steel plate, keyed into a groove to insure concentricity for the $\sim 3 \mathrm{~mm}(0.125 ")$ aperture at its center. To minimize the thickness of the hole, the side away from the source is countersunk and expands out to $1 \mathrm{~cm}$. On this same side, two deflection plates are mounted, spaced $1 \mathrm{~cm}$ apart. The steel deflection plate mounted below the aperture is fastened directly on the shielding plate, thereby remaining at ground potential at all times. The upper deflection plate is mounted on Vespel $®$, a machinable plastic with good high vacuum out-gassing properties, which is then fastened to the shielding plate. This deflection plate is then kept at a potential in the vicinity of $1500 \mathrm{~V}$, with the proper value depending primarily on the ion beam energy and the exact vertical position of the detector. Of course, if the detector is in the beam axis, or it is desirable for the ions to be allowed to proceed to subsequent regions of the machine, the deflection plate is grounded.

As Figure 2.18 shows, the components and circuitry for the ion detector are quite similar to that of the detached electron detector. However, it is not necessary to accelerate the ions towards the microchannel plates since they have a kinetic energy determined by the ion beam energy. Therefore the acceleration grid used in the detached electron detector is not present in the ion detector.

The circuit is also has some minor differences. The first relates to the fact that the acceleration grid is not present. Because of this, the total voltage applied to the detector has to be only $1850 \mathrm{~V}$. A second difference is that this detector must be able to translate freely upwards and downwards. It was decided that for this reason all of the voltage dividing resistors should be within the machine, to avjid many long connections that might foul and short circuit upon translation. To minimize outgassing problems, special glass

Figure 2.18 The circuit diagram for the ion detector. 


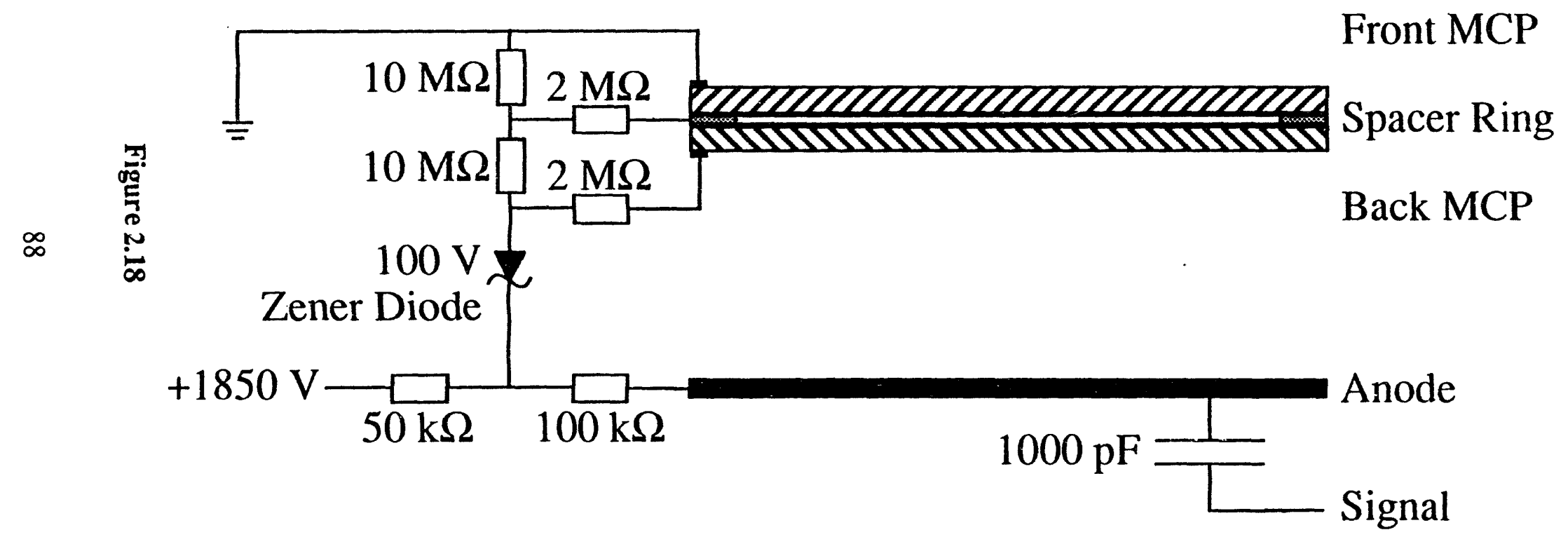


resistors (K \& M Electronics) were used.

\subsubsection{Ion Time-of-Flight Mass Spectroscopy: Monitoring Source Output}

The ion detector is used for investigating the possibility of making new (for this apparatus) negative ion species. The key to determining what negative ions are being produced is the following time-of-flight to mass conversion:

$$
m_{i}=m_{0}+c t_{i}^{2} .
$$

By determining the time-of-flight $t_{i}$ for two ions of known mass $m_{i}$, one can determine the constants $m_{0}$ and $c$ using elementary algebra. The constant $m_{0}$ should be close to zero if the times-of-flight are measured relative to the mass spectrometer trigger pulse, while the constant $c$ will vary with beam energy. For a variety of reasons it is best to have the detector lowered to interseci the beam axis for these measurements. In addition, the ion compressor should be turned off and the mass spectrometer voltage raised to narrow the time-of-flight mass peaks.

While observation of the ion times-of-flight on the oscilloscope can be informative, it is also possible to record mass spectra for posterity by feeding the ion detector signal along with an appropriately timed trigger pulse into a transient recorder (DSP, Model 2001AS). The output of this module is fed into an averaging memory module (DSP, Model 4101), and the summed and averaged signal can be read out to the PC/AT laboratory computer through an interface to the CAMAC crate controller (DSP, Model 6001). Time-of-flight mass spectrometer data acquisition is a main menu option in the FRBM program (found in Appendix I).

\subsection{Photodissociation and Detection Region}

Within this final region, photodissociation of radicals is undertaken followed by detection of the photofragments produced in this process. This region is illustrated in Figure 2.19. 


\subsubsection{Photodissociation}

We customarily frequency-double the output of a second excimer-pumped dye laser system (Lambda Physik LPX-210i and Lambda Physik FL-3002, respectively) to achieve the photon energies necessary for our radical dissociation studies. We use a frequency doubling unit (Interactive Radiation, Inc. Model AT-II), equipped with either $\mathrm{BBO}$ and KDP crystals (as appropriate), to allow the production of doubled light from as low as $\sim 206 \mathrm{~nm}$ up to the lowest wavelength where the fundamental of the dye laser may be used $(-340 \mathrm{~nm})$. Frequency doubling is a nonlinear optical process dependent upon the second order polarizability of the doubling medium, and will not be discussed further here. ${ }^{29}$ The fundamental can be separated from the frequency doubled output, if desired, by the use of an in-line four-prism assembly which spatially separates the two colors and transmits only the frequency doubled light. In addition, the frequency-doubled output is highly polarized in the horizontal plane. This orientation can be preserved, resulting in polarization of the $\bar{E}$ vector of the laser light parallel to the radical beam axis. Alternately, a periscope arrangement of prisms may be used to rotate the polarization to the vertical orientation, perpendicular to the radical beam axis. This ability is useful in cases where a very thorough investigation of the recoil angle distribution is desired.

\subsubsection{Acquiring Photodissociation Laser Timing}

A previously mentioned, the timing of the photodetachment laser can be optimized by inspection of photoelectron signal, or even depletion of ion signal. However, due to the relative paucity of radicals as compared with ions, and the therefore even smaller fragment signal levels, finding the proper timing of the dissociation laser system could be

Figure 2.19 A diagram of the dissociation and detection region. (1) Initial $1 \mathrm{~mm}$ aperture. (2) Pulsed deflection plates. (3) Photon baffle. (4) Total photodissociation cross section detector, shown raised out of the beam axis. (5) Time- and position-sensitive detector. 


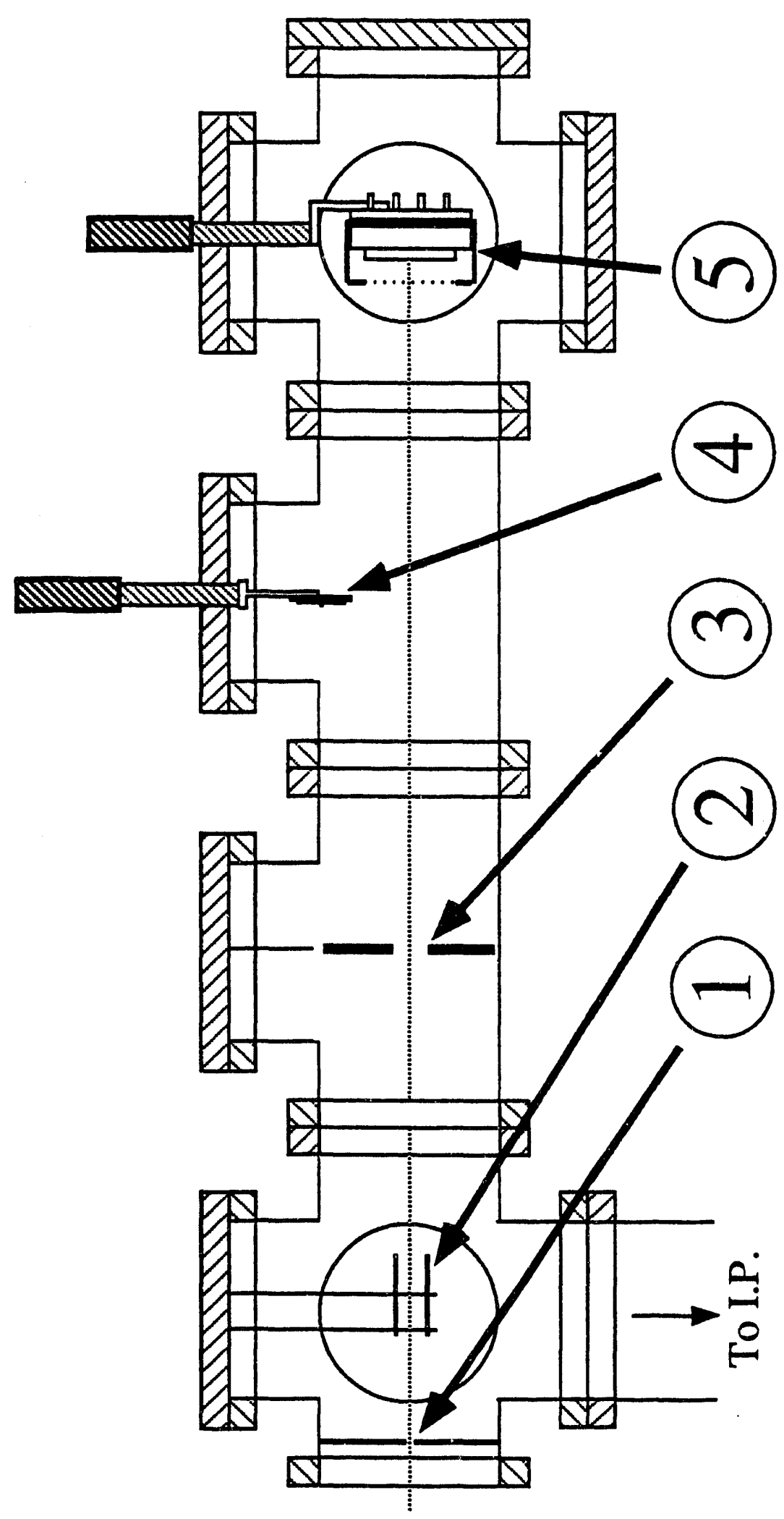

Figure 2.19 
extremely difficult. Timing can be calculated to perhaps better than $0.5 \mu \mathrm{s}$, but the uncertainty in the size of the delay between the excimer laser trigger pulse and the actual firing of the laser is fairly large, as well as being dependent on the operating temperature and discharge voltage of the laser. The required accuracy of timing is on the order of 20 $\mathrm{ns}$, as this is roughly the duration of the laser pulse. The solution to this difficult problem lies in the moderately clever use of a set of deflection plates suspended from the flange above the dissociation volume [see label (2) in Figure 2.19].

The proper dissociation laser timing can be determined by initially allowing ions to propagate through the dissociation volume and impinge on the face of the fragment microchannel plate detector, which has been positioned just off-center to allow the ions to pass by the blocking strip. Of course, this implies that the detachment laser is not in operation, the ion deflector is grounded and the ion detector is grounded and up out of the beam axis. After establishing a respectable signal of ions on the fragment detector, the time of flight of the mass of interest is noted. A pulsed field is then applied to one of the deflection plates straddling the photodissociation volume just early enough to deflect all the ions so that they miss the detector. These plates are extended towards the photofragment detectors by $\sim 5 \mathrm{~cm}$, primarily to increase the amount of time an applied potential can act on the ions, thereby reducing the required strength of the field. However, so that the deflector plates do not perturb the ion beam prior to reaching the photodissociation volume, it is essential that the potential is pulsed on only after the ions have passed this volume. By applying a relatively large potential ions can still be easily made to miss the detector. Invariably the wavelength that is to be used to photodissociate the radicals will be sufficient to detach the ions. Therefore, by not using the detachment laser system and by allowing the ions to continue along undeflected in to the dissociation region, one will be able to detach the ions using the dissociation laser system once the laser timing is correct. The resulting neutrals will not be deflected by the pulsed electric 
field and a clear signal will be present on the neutral detector at the time-of-flight of the corresponding ion.

The usefulness of the preceding method is that it is easier to see the relatively plentiful radicals formed by detaching a large number of negative ions, than the much fewer number of photofragments formed by the dissociation of radicals previously formed by photodetachment. This is particularly true if it is not yet known at which wavelengths the radical in question will dissociate, a scenario quite common with predissociating systems. In this case wavelength scanning concurrent with the laser timing search would be necessary. This method does have the requirement that the electron affinity of the radical must be less than the relevant bond dissociation energy. This situation has always been the case in the experiments thus far and is generally true.

\subsubsection{Monitoring Photodissociation Laser Power}

In order to have the capability to normalize to the intensity of photodissociation laser during total photodissociation cross section measurements, a pyroelectric detector is used to measure laser power. The pyroelectric detector (Molectron, Model P1-61) circuit is shown in Figure 2.20. The active area of this detector is quite small. For this reason, care must be taken that the laser beam does not 'walk' during a laser scan, which would change the relative amount of the beam that falls on the face of the detector, and thus the apparent intensity. To minimize this effect, as well as to attenuate the high laser intensity, ground glass diffusers are mounted in front of the pyroelectric detector.

\subsubsection{Laser Light Baffles}

Scattered photons must be controlled in our experiment, both in the photodetachment region and in the photodissociation region. The detached electron

Figure 2.20 A schematic of the Molectron pyroelectric detector circuit. 


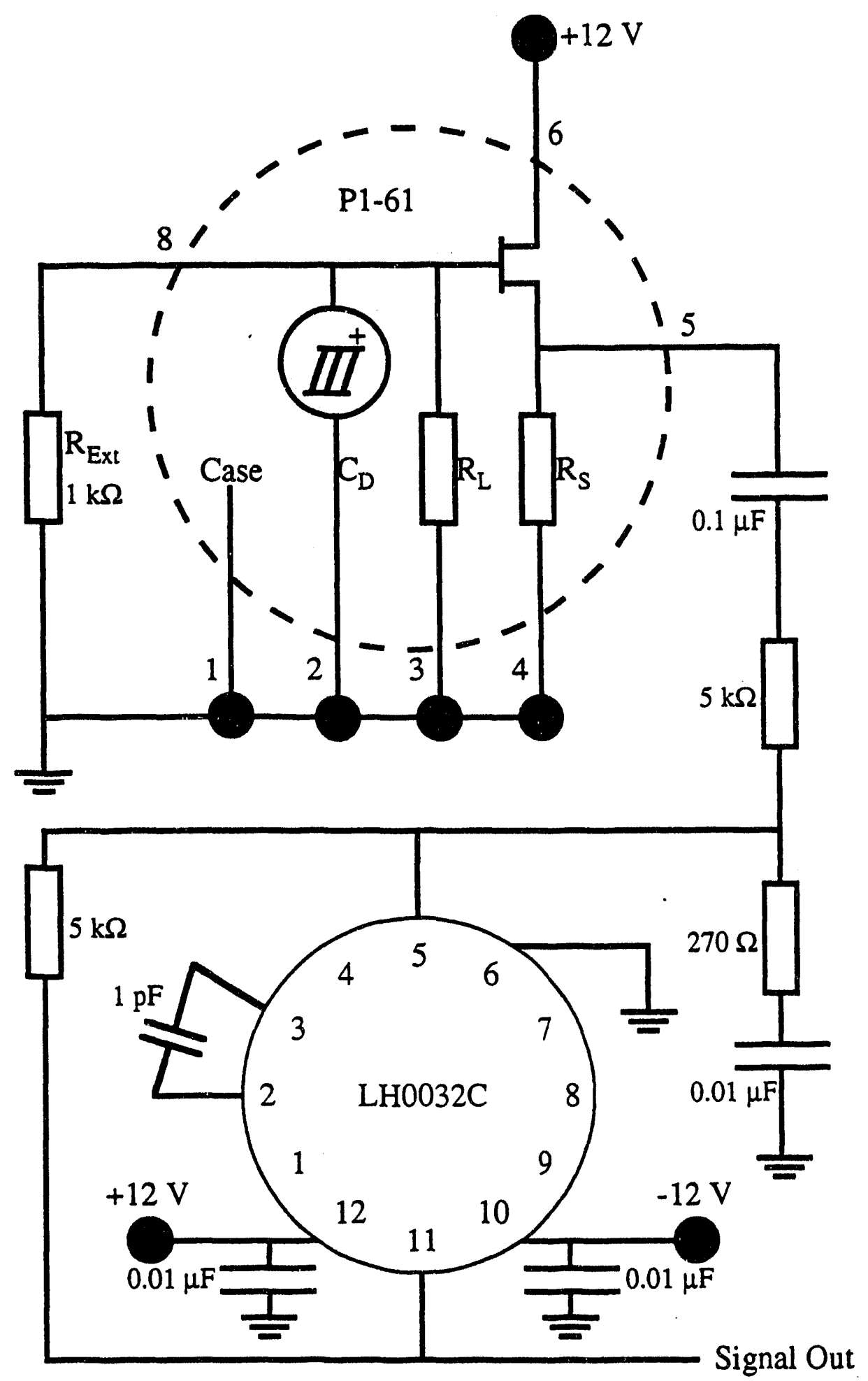

Figure 2.20 
detector can register photons as well as photoelectrons from scattered light hitting the inner surfaces of the machine; both will interfere with the accurate appraisal of detached electron signal. In the photodissociation region stray photons are a particular problem when we acquire time- and position-sensitive data. Photons, which reach the detector a few microseconds prior to the arrival of fragments, can cause the detector electronics to be unable to recover to baseline in time to register the fragments properly.

To address these concerns, we have installed anodized aluminum light baffles on the input and output flanges of both the detachment and dissociation regions. In addition, approximately one third of the way to the time- and position-sensitive detector we have installed an anodized aluminum disk that is very nearly flush with the inner walls of the flight tube [see label (3) in Figure 2.19]. This disk has a hole in the center which subtends a slightly greater solid angle than the detector face, preventing photons from reaching the detector by indirect routes (i.e., by bouncing off the wall). These precautions are quite successful, eliminating up to $90 \%$ of spurious signal caused by scattered photons.

\subsubsection{Total Photodissociation Cross Section Detection}

In order to measure the photofragment yield as a function of photon energy, giving the total (integrated over all product states) photodissociation cross section, fragments are detected using a MCP detector as the photodissociation laser wavelength is varied. This detector is located $0.67 \mathrm{~m}$ from the photodissociation volume and has an active area that is $40 \mathrm{~mm}$ in diameter. The detector assembly has a $3 \mathrm{~mm}$ wide blocking strip that is mounted in front of the active area and extends completely across the center of the face. This blocking strip insures that undissociated radicals will not strike the detector. The same type of linear motion feedthrough manipulator is used to mount this detector assembly as is used to mount the ion detector assembly. Prior to operation, the detector is lowered down from a fully retracted position (as it is shown in Figure 2.19) until the $3 \mathrm{~mm}$ blocking strip is centered on the beam axis. 
The detector construction is identical to that of the ion detector, described previously, save the presence of the $3 \mathrm{~mm}$ blocking strip. In contrast to the time-and position-sensitive detector to be described below, these detectors are characterized by their contiguous anode and thus uncomplicated output. When struck by a fragment with high laboratory-frame kinetic energy anywhere on the active area of the microchannel plate face, the detection efficiency is roughly $50 \% .30$ The resulting cloud of electrons produced by the microchannel plates is collected on the anode, and a fast-rising, $5 \mathrm{~ns}$ wide, $10-40 \mathrm{mV}$ negative polarity peak is produced. This pulse is AC coupled, using a $1000 \mathrm{pF}$ capacitor, from the anode which is at $1850 \mathrm{~V}$. It is then transmitted using a shielded cable through a BNC connector welded in the flange. The signal is then fed directly into an fast preamplifier with a factor of 10 gain (EG\&G Ortec, Model 9301), and from there sent to the gated input of a charge sensitive analog-to-digital converter (ADC, LeCroy, 2249SG). The ADC also receives input from the detached electron detector and the pyroelectric detector (Molectron P1-61, discussed in § 2.7.3) whose output is proportional to the photodissociation laser power. These measurements can be used to normalize the photofragment signal for variations in these quantities. Lastly, there are provisions for the $A D C$ to collect signal from an iodine absorption cell to be used in subsequent wavelength calibration.

When a total (photodissociation) cross section (TXS) scan is taken, a wavelength range and step size is chosen that will result in usually no more than 200 wavelength steps at 100 shots per point. At $60 \mathrm{~Hz}$, this results in a 6 minute scan. The reason for this limited scan length is to guard against the effects of possible instabilities in the source. Counting statistics are improved by repeating these scans until satisfactory signal-to-noise is achieved. In practice, this can mean up to one thousand or more shots for each point. If the ions are unstable, a greater number of quicker scans can be taken by reducing one of the two above mentioned parameters. 
Total cross section data acquisition is controlled by the program FRBM, which can be found in Appendix A.

\subsubsection{Time- and Position-Sensitive Detector}

Detailed dynamics of dissociation events undertaken at a specific dissociation photon energy are obtained using a time- and position-sensitive detector. The detector allows the simultaneous two-dimensional position detection of both fragments from a given photodissociation event, with excellent timing resolution (typically $<0.5 \mathrm{~ns}$ ) in the relative time-of-flight of the two fragments. This in turn provides a direct measurement of the mass ratio, recoil angle and kinetic energy release for each dissociation event.

Time- and position-sensitive detectors have previously found use in a variety of experiments involving the dissociation of metastable ${ }^{31}$ or photoexcited species. ${ }^{32}$ Typically, however, the detector design incorporated one-dimensional (radial) position sensing. Two advantages are gained by determining the two-dimensional positions of each fragment rather than merely the radial displacements. First, it is necessary to measure fragment positions in two dimensions to obtain recoil angular distributions in experiments where the electric field vector of the dissociation laser is perpendicular to the beam axis, since the photofragments will now longer have azimuthal symmetry about the beam axis. A second advantage is realized in discrimination against false coincidences (the detection of fragments from uncorrelated events), since

$$
\frac{x_{2}}{x_{1}}=\frac{y_{2}}{y_{1}}
$$

must hold true due to conservation of momentum if the two photofragments originated from the same photodissociation event. Detectors with two-dimensional position sensitivity have been developed for a variety of electron spectroscopies ${ }^{33}$ and recently, for charge transfer studies of high energy ion beams at surfaces. ${ }^{34}$ 
Because both fragments from a dissociation event are detected in coincidence, important advantages are gained over single particle detection. Single particle detection can be used to yield significant amounts of information, ${ }^{35}$ however, the coincidence technique allows the unambiguous determination of the masses of the fragments and the center-of-mass dynamical quantities for each dissociation event. Resolution is also enhanced by a coincidence measurement, as will be discussed in $\S 2.7 .6 .5$.

Position sensing is accomplished using the method of charge division, mounting two anodes with three conductors each, whose relative areas vary with position, behind a single set of microchannel plates. Other methods were considered but determined to be not ideal for our purpose. Resistive anodes and CCD-based imaging techniques have slower time responses, and are thus unable to determine the relative time-of-flight of the two photofragments to suitable precision. Multi-anode array detection with multi-event capabilities would be ideal, but extremely complex and exorbitant.

The following subsections deal with various aspects of the time- and positionsensitive detector in some detail.

\subsubsection{Detector Body}

Following the design used by the UC Berkeley Space Sciences Laboratory, we have assembled the time- and position-sensitive detector as shown in Figure 2.21. The

Figure 2.21 A schematic of the TPS detector assembly. (1) Kovar/alumina brazed body assembly. (2) $8 \mathrm{~mm}$ wide blocking strip. (3) Spring-loaded ring assembly for holding microchannel plate Z-stack in place. (4) Z-stack of imagequality microchannel plates. (5) Electron drift region with resistive coating on the inner wall. (6) Fused silica anode substrate with photoetched copper anode. (7) Feedthroughs for output signals. For the detection of neutral particles, the front end of the detector assembly is helr at $-4.15 \mathrm{kV}$, giving microchannel plate voltages of $\approx 1300 \mathrm{~V} /$ plate, and a drift region voltage of $230 \mathrm{~V}$ over $8 \mathrm{~mm}$. 


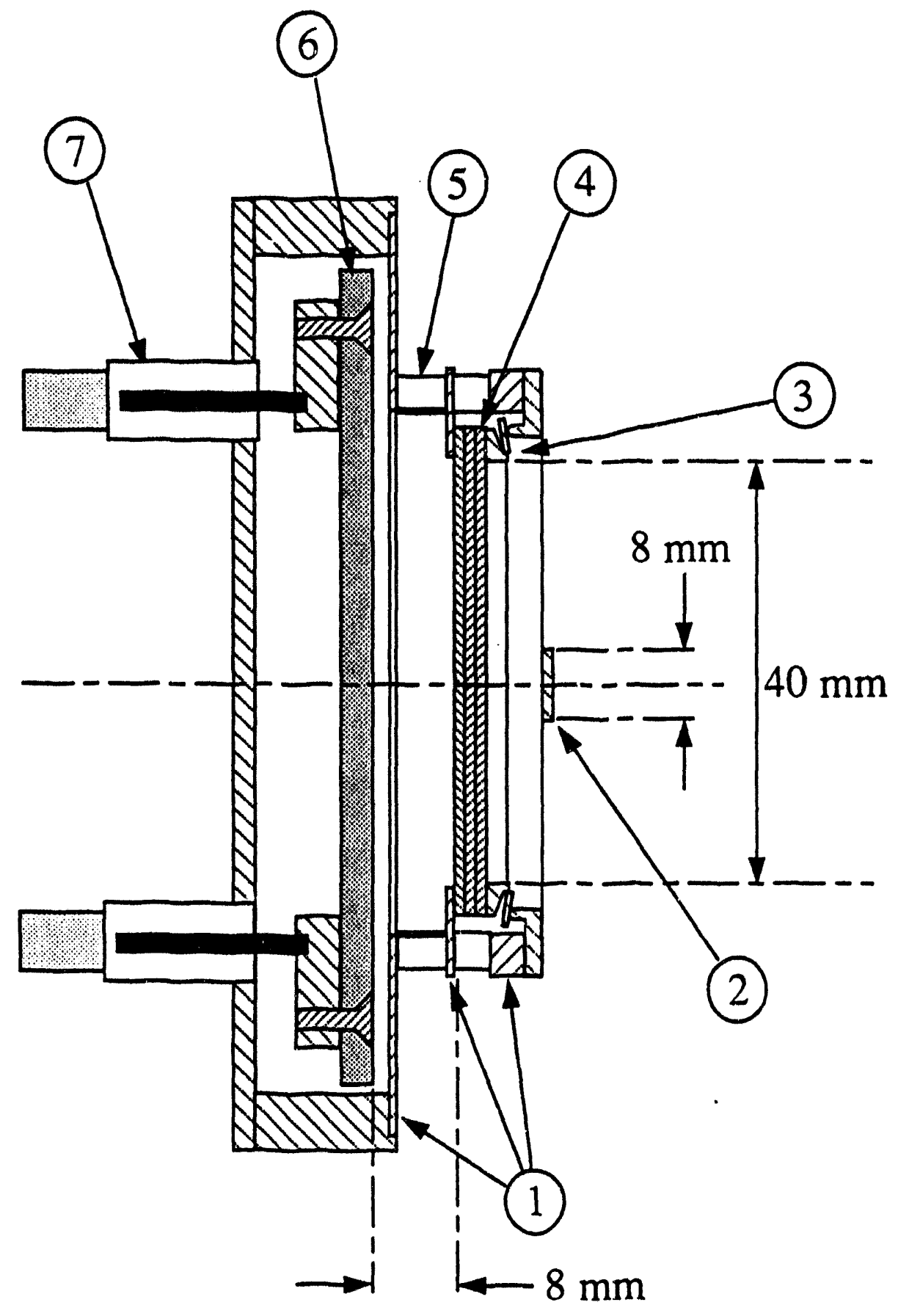

Figure 2.21 
detector body consists of a microchannel plate holder, a resistively coated ceramic (alumina) drift region and an anode mount. The microchannel plate holding section is constructed of Kovar rings brazed on both ends of a ceramic cylinder whose ends have been nickel coated. Both rings have tabs so that leads for the high voltage potentials may be spot-welded on, and the thin inner ring extends inward to form a shelf for the microchannel plates to sit on. The ceramic cylinder provides excellent insulation, isolating the MCPs. The thicker outer ring has threaded holes in it to allow a retaining ring to be screwed in, tightening down on an annular spring which in tum holds the microchannel plates down. The $8 \mathrm{~mm}$ wide blocking strip is also keyed into the retaining ring.

The microchannel plates that are used in this detector are a resistance-matched triplet set of soft edged, double thickness, Z-stacked microchannel plates (Philips, G12/46DT 13-13-13). They have a 12 micron channel diameter, a 13 degree bias angle and a 46 mm plate diameter.

Immediately below the microchannel plate holder is the drift region, an $8 \mathrm{~mm}$ gap between the anode and the back of the MCPs. In order to control the amount of lateral spreading of the electron cloud, and thus the anode area that the electron cloud covers, the potential applied across this region can be varied using the voltage dividing scheme shown in Figure 2.22. The walls of this drift region are made of resistively-coated ceramic (MacroMetallics) so that the field lines will vary smoothly from the top to the bottom of the drift region. The total resistance from top to bottom is $\sim 1 \mathrm{G} \Omega$.

The anode is mounted on seven pads, one for each of the three conductors plus a ground connection. Flat head machine screws are countersunk into the copper-covered fused silica anode, and provide a connection to the pads. These pads are in turn welded to pins that lead to SMA connectors, which extend out the back of the housing. The

Figure 2.22 The voltage divider used to establish the potential across the MCPs and the drift region within the time- and position-sensitive detector. 
High Voltage In

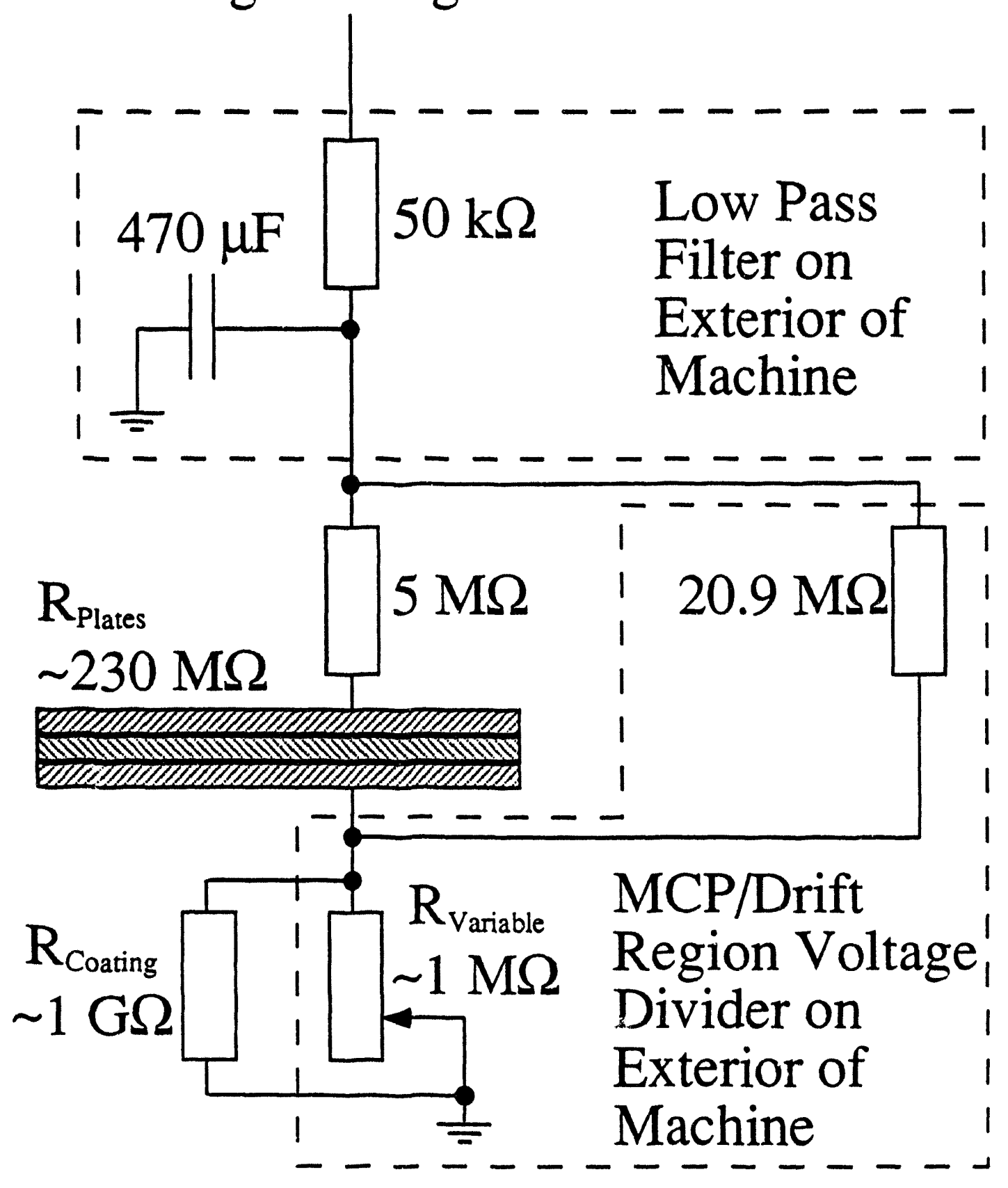

Figure 2.22 
connectors couple the signal to lengths of RG-316/U Teflon-dielectric coaxial cable (chosen chiefly for it's low capacitance) which in turn are connected to BNC feedthroughs on the vacuum chamber nounting flange.

Mounted on the anode assembly and surrounding all of the other sections, save the face of the MCPs themselves, is a magnetic shield made from annealed stainless steel 416 , which guards against the influence of magnetic fields on the electrons produced by the MCPs. Clamped to this magnetic shield is an assembly that supports a $90 \%$ transmittance screen in front of the detector, to reduce the background caused by stray positive ions. In contrast to the other microchannel plate detectors used in this experiment, the anode is held at ground for ease of coupling the signal out to the preamplifiers, while the front of the MCPs are at $\sim-4.15 \mathrm{kV}$. Without this shield, positive ions formed in the ion pumps or the detector region ion gauge will be attracted by this large negative potential and will contribute to spurious background signal. Because the experiment is pulsed, and the detection electronics are gated on for only a few $\mu$ s per laser shot, these background counts would rarely register. Even when they do register, they will be disregarded since they will not meet our coincidence discrimination criteria. If they are too plentiful, however, they will begin to disrupt detection of valid events by causing pile-up. For this reason, we accept a slight diminution of signal which accompanies the installation of the shielding mesh. Additionally, we often turn off the detector region ion gauge during an experiment. These measures cut down drastically on positive ion background.

\subsubsection{Wedge-and-Strip Anode}

Our detector possesses position sensitivity because of a specially designed anode placed behind our stack of microchannel plates. This anode is flat, and is comprised of two separate semicircular sections, each of which has a wedge-and-strip pattern of conductors. A simplified schematic of this pattern is shown in Figure 2.23. It is enlarged and, for the sake of clarity, involves five periods per half rather than 32 , which is the 
number typically used in the anodes installed and used in the detector. It is comprised of interlocking wedges, which taper along their length in the $x$ coordinate, and strips, which vary in width linearly with the $y$ coordinate. A third conductor zigzags between the wedges and strips and fills the remaining area of the anode pattern. Charge division between the three conductors will depend on the area of each conductor, which varies with position. The position of a fragment in the $\mathrm{x}$ and $\mathrm{y}$ directions is nominally given by the following relations

$$
x_{i} \propto \frac{Q_{w i}}{Q_{w i}+Q_{s i}+Q_{z i}} \quad \text { and } \quad y_{i} \propto \frac{Q_{s i}}{Q_{w i}+Q_{s i}+Q_{z i}} .
$$

The pattern which has been used most extensively has a period of $0.8 \mathrm{~mm}$, with minimum and maximum wedge fractions of 0.04 and 0.40 and minimum and maximum strip fractions of 0.06 and 0.40 . Insulating borders $40 \mu \mathrm{m}$ thick separate each conductor.

The substrate on which the anode pattern is fabricated is a custom made fused silica disk ( $\mathrm{S} \& \mathrm{~S}$ Optical), 2.9 inches in diameter and $3 \mathrm{~mm}$ thick. Fused silica is chosen for its low dielectric constant, and consequent small capacitance. Countersunk holes are machined near the outer diameter to allow mounting of the anode and electrical connections to the conductors via the flathead screws which hold the anode in place. This disk then has a $6 \mu \mathrm{m}$ thick layer of copper vapor-deposited onto its surface (Scientific Coatings). The wedge-and-strip pattern is photoetched onto this copper surface.

The pattern is constructed using a modified version of computer code obtained from the UC Berkeley Space Sciences Laboratory. Gerber (plotter format) output from

Figure 2.23 A wedge-and-strip anode pattern, larged and simplified for clarity. The wedges are hatched, the strips have a vertical striped pattern, while the solid line bordering conductors represents a thin insulating gap. W1, S1, Z1 and W2, S2, Z2 measure the charges for the upper and lower halves of the detector, respectively. The actual patterns typically have 32 periods per side, rather than five as shown here, and a diameter of $54 \mathrm{~mm}$. 


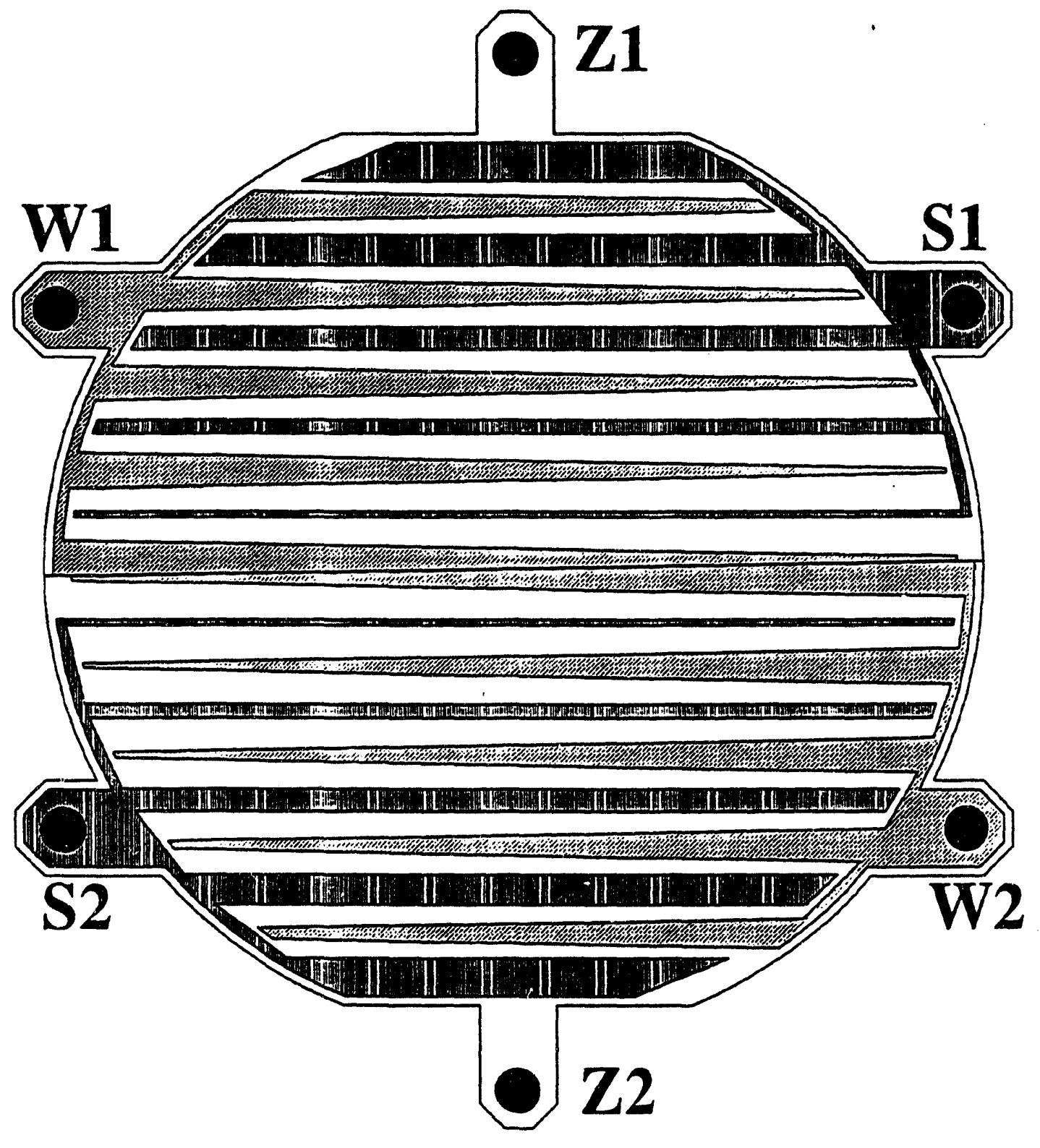

Figure 2.23 
this program is transmitted to a facility (Image Technology Inc.) where a full-scale photomask is made that is seven times larger than the eventual photomask used in the fabrication. Photoreduction of the image is then carried out, with both positives and negatives made of the actual size photomask.

At Space Sciences Laboratory, the wedge-and-strip pattern is then photoetched into the vapor deposited copper using a large mercury lamp to illuminate the treated copper surface through the photomask. Copper is completely and selectively removed where an insulating region is desired, forming conductors separated from each other by an insulating strip of bare fused silica.

\subsubsection{Detection Electronics}

To process the charge fraction and timing information, we must accomplish two separate tasks: determine the charge fractions on each of the three conductors for each half of the anode, and determine the difference in time-of-flight of the two photofragments. A schematic of the data acquisition system that is used for this purpose is given in Figure 2.24.

To accurately determine the charge fractions, the signal from each conductor is fed directly into a charge sensitive preamplifier (Canberra, Model 2004). We have made modifications to the input stages of these units for improved performance in their role in this experiment. Specifically, the input of these preamplifiers is manufactured with the ability to float many kilovolts above the rest of the circuit. This ability is not only unnecessary, certain aspects of the input circuitry were found to be detrimental to our application. Figure 2.25 shows a circuit diagram of the input stage prior to any modifications, followed by the circuit diagram after modifications. Since we do not float our detector, the high voltage input connector is fitted with a grounding plug. The removal of the $100 \mathrm{M} \Omega$ resistor was necessary to allow adequate draining of the accumulated charge on the anode conductors to ground between events, particularly at 
high count rates. The sole purpose of the $1000 \mathrm{pF}$ capacitor to ground originally located between the two resistors on the HV input line was to condition the high voltage power by serving as a low pass filter. It is therefore removed. The last change that was made involves the capacitor that couples charge from the conductor to the charge sensitive amplifier circuit. The value of this capacitor was raised from $1000 \mathrm{pF}$ to $0.01 \mu \mathrm{F}$. The major effects of these modifications are a decrease in inter-conductor cross-talk, thought to occur because the capacitive charge division that occurs between the preamplifier input capacitor on one hand and the neighboring conductors on the other hand is altered to increase charge flowing to the preamplifier, and decrease charge coupling to neighboring conductors. A slight gain in signal is observed because the increased capacitor value allows a lower frequency cut-off than was the case previously.

The output of the charge sensitive preamps is a fast-rising pulse with a relatively long fall-time; the amplitude of the pulse is proportional to the amount of charge detected by the preamplifier. The preamplifier output is sent to a spectroscopy amplifier (EG\&G Ortec, Model 575A) which forms an amplified, shaped peak whose amplitude is

Figure 2.24 Schematic of the data acquisition system. W1,S1,Z1 and W2,S2,Z2, charge signals from the three conductors on both halves of the wedge-and-strip anode; QA, Canberra 2004 charge sensitive preamplifiers; FA, ComLinear CLC 100 fast timing amplifiers, AC coupled to the strip conductor from each half of the anode to provide fast timing pulses; SA, Ortec 575A spectroscopy amplifiers; CFD, Tennelec TC455 constant fraction discriminator, DDG, Precision Instruments 9650 digital delay generator; TAC, Canberra 2145 TAC/SCA time-to-amplitude converter; ADC, Ortec 413A analog-to-digital converter; CAMAC, Kinetic Systems CAMAC crate interfaced with a DSP Model 6001 CAMAC crate controller to a 386based PC/AT clone. Externally supplied gating allows the ADC's and the TAC to accept data only when photofragments are expected.

Figure 2.25 Schematic of the Canberra 2004 input stage before, and after modification to remove the components deemed unnecessary and detrimental to the present application. See text. 


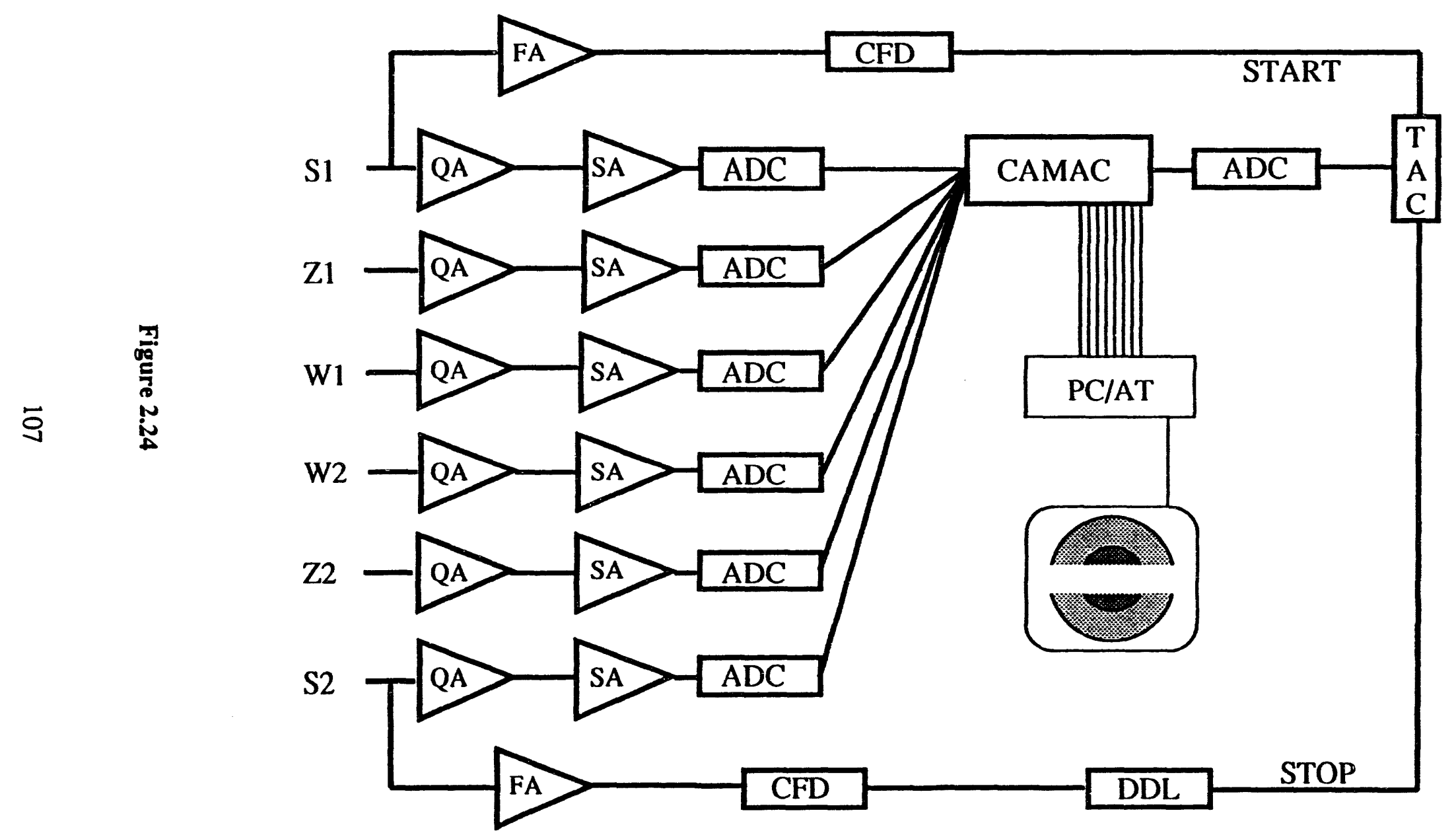




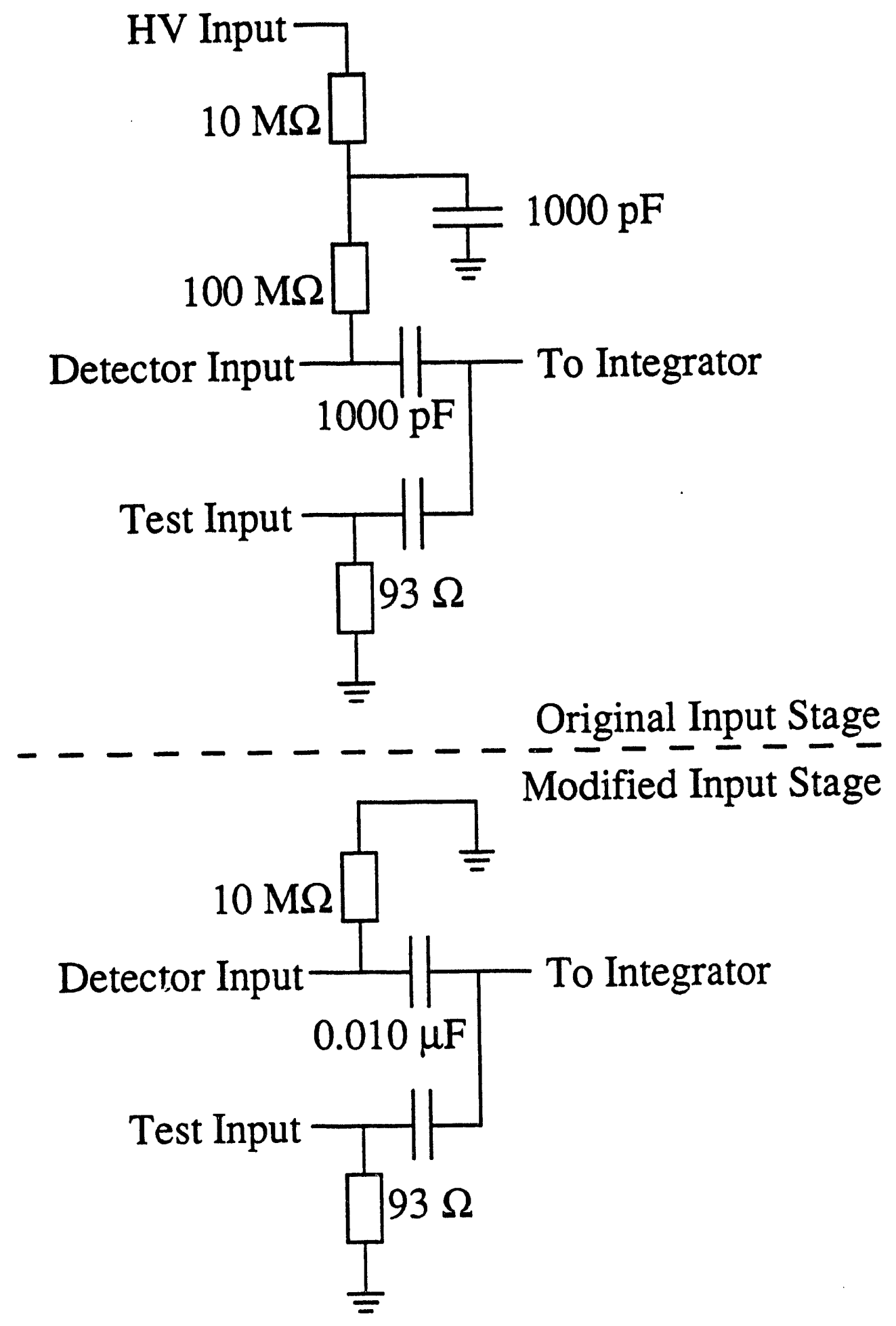

Figure 2.25 
proportional to the amplitude of the rising edge of the preamplifier output. The shaping time constant can be $0.5,1.5$ or $3.0 \mu \mathrm{s}$, but is in our case dictated by two competing factors. A more precise output is obtained when the shaping time constant is longer. However, at the photodissociation photon energies we typically use, scattered photons are occasionally detected at the time- and position-sensitive detector. If scattered photons are being registered, it is best to use the smallest shaping time constant, thus returning the output of the spectroscopy amplifier to baseline promptly (the difference in time of flight for the photons and the fragments is only $4-8 \mu \mathrm{s})$.

The six spectroscopy amplifier outputs are fed into channels 1-3 of two 13-bit quad analog-to-digital converters (ADCs, EG\&G Ortec, Model AD413). These are gated using a home-built gating circuit to accept signal only for a few microseconds during the period when photofragments are expected. The ADCs also have a hardware discriminator that can be set at any value up to $0.5 \mathrm{~V}$, causing pulses of smaller amplitude to be rejected. This prevents line noise or spurious events from causing the ADC channels to be busy.

The difference in time-of-flight of the two fragments is measured by first splitting off, on each anode, a portion of the strip conductor signal through a $2 \mathrm{pF}$ capacitor to the input of a $500 \mathrm{MHz}$ video amplifier (ComLinear Corp., Model CLC100). These negative going signals, having been amplified by 10 times, are then routed into two channels of a quad constant fraction discriminator (CFD, Tennelec, Model TC455). The CFD is typically set up to run in the $50 \%$ mode, with the fixed delay being provided by $0.5 \mathrm{~ns}$ cables plugged into the front panel. A threshold is set for each channel with the knowledge that the output of the CLC100's is biased at $\sim 0.019 \mathrm{~V}$. Thus a threshold setting of $-5 \mathrm{mV}$ actually cuts out all peaks less negative than $\sim-25 \mathrm{mV}$. In the case of a real event, where both the upper and lower anode register signal, the discriminator then feeds the upper timing pulse to the START input of a time-to-amplitude converter (TAC, Canberra, Model 2145 TAC/SCA). The lower timing pulse is sent through a long delay cable or is used as the external trigger for a digital delay generator (DDG, Precision 
Instruments, Model 9650) that is set to provide a delay of greater duration than the greatest difference in time-of-flight possible for the system being studied. This ensures that the lower timing pulse, after being subjected to this fixed delay, always follows the upper timing pulse and can be reliably used as a STOP pulse for the TAC. The TAC is gated on for only a few $\mu$ s per laser shot using a gate delayed to the appropriate time by a gate generator (EG\&G Ortec, Model 416). This gate generator is triggered by the synchout pulse of the dissociation excimer laser.

The experiment is controlled by a PC/AT clone interfaced to the CAMAC crate where the ADC's are housed. The program used for data acquisition is called TPS (an acronym for Time- and Position-Sensitive), which can be found in Appendix B.

\subsubsection{Calibration}

Calibration of the time- and position sensitive detector involves both calibration of the electronics, so that a reliable correlation between signal level and charge fraction (or time) is established, and the calibration of coefficients within the algorithm that converts the measured charge fractions to positions.

The level of amplification of the charge collected on each conductor is determined by the individual amplification settings on the spectroscopy amplifiers. Making this amplification uniform amongst all of the conductors is then a matter of simulating the arrival of a specific amount of charge at each conductor by capaciti ' $y$ coupling the falling edge of the square wave output of a pulse generator (Hewlett-Packard, Model 8012B) through a $1 \mathrm{pF}$ capacitor. This charge is injected just outside the feedthrough associated with each anode conductor in turn while the spectroscopy amplifiers for that conductor are adjusted to give an identical reading into the same ADC channel. Using the same ADC channel accounts for possible differences in ADC sensitivities (to be determined below). The zigzag conductors are adjusted to read half of the value returned by the wedge and strip conductors for the same test pulse. This convention is followed 
because on average, the charge fraction on the zigzag conductor is twice as much as on either the wedge or the strip conductors due to the larger relative area of the zigzag conductor. The $A D C$ value given for the zigzag conductors is multiplied by two immediately after being read by the computer during data collection.

Calibration of the ADC channels used in charge fraction measurement is accomplished by noting the ADC output for a range of spectroscopy amplifier output levels from 1 to $9 \mathrm{~V}$, as measured using the oscilloscope. The dependence of ADC channel output on the height of the input pulse can then be determined using a linear leastsquares fit. This information is stored and used to rescale the raw charge measurements during data analysis.

For timing measurement calibration the TAC output is fed into channel 4 of the $A D C$ which measures the $W 1, S 1$ and $Z 1$ output in channels 1-3. The ADC output is noted while injecting charge onto $S 1$ and $S 2$ at various relative delays. A TAC calibration curve can then be constructed and fit by a linear least-squares routine. When using the digital delay generator (DDG) to establish a variable delay, the intrinsic response time (approximately $40 \mathrm{~ns}$ ) of the $\mathrm{DDG}^{36}$ following external triggering must be added to the front read-out. The total delay includes the sum of contributions from this response time as well as the delay dialed into the delay generator. Alternately, a series of long BNC cables can be used to give a precise, if cumbersome delay line.

From this calibration curve, experimental TAC output is easily converted during data analysis to the apparent difference in time-of-flight for the two fragments (including known delay). Subtraction of the total known, fixed delay that is present to insure the upper fragment is always the START pulse and the lower fragment the STOP pulse can then be done. The signed difference in time of arrival, $\tau$, will then be known to better than $0.02 \%$ of full scale on the TAC. This translates to $0.2 \mathrm{~ns}$ on the $1 \mu \mathrm{s}$ full scale TAC setting. 
The relative amounts of charge on the three conductors of each anode are translated into positions in a slightly more complicated manner. The $x$ and $y$ positions on the detector face are roughly proportional to the fraction of total charge found on the wedge and strip conductors, respectively, as given in \$ 2.7.6.2. However, the full formulae for finding position from charge fractions includes empirical coefficients for these terms that are linear in charge fraction as well as other factors to correct various residual nonlinearities in the detector. The actual formulae used for the $x$ and $y$ coordinates of the upper (denoted "1") position are:

$$
\begin{aligned}
& x_{1}=x_{0}+a_{w 1} \cdot\left[f_{w 1}+\left(c t_{w 1}-1\right) \cdot f_{21}+\left(q_{w 1}-1\right) \cdot f_{w 1}^{2}\right]-b_{w 1} \\
& y_{1}=y_{0}+a_{s 1} \cdot\left[f_{s 1}+\left(c t_{s 1}-1\right) \cdot f_{21}+\left(q_{s 1}-1\right) \cdot f_{s 1}^{2}\right]-b_{s 1} .
\end{aligned}
$$

The formulae for the position on the lower half of the detector are identical except for having "2" substituted everywhere for "1". The assumed position of the center of the radical beam axis on the detector face is denoted $x_{0}, y_{0}$. The fraction of the total charge found on the wedge conductor of the upper half of the detector is given by $f_{w 1}$, i.e.,

$$
f_{w 1}=\frac{Q_{w 1}}{Q_{w 1}+Q_{s 1}+Q_{z 1}}=\frac{Q_{w 1}}{Q_{1}} .
$$

A multiplicative and additive coefficient are given as $a$ and $b$, respectively, with subscripts indicating whether they refer to the wedge or strip (i.e., $a_{w 1}$ or $b_{s 1}$ ). Crosstalk between electrodes is possible given their extended shared borders. However, the wedge and the strip are always separated by the zigzag conductor. Since they are not adjacent at any point, crosstalk between them will be minimal and is not treated. The factors $c t_{w 21}$ and $c t_{s z 1}$ represent an attempt to account for wedge-zigzag and strip-zigzag crosstalk, respectively. Lastly, a coefficient for a term quadratic in the total charge found on the wedge (for $x$ coordinate) or strip (for $y$ coordinate) is included to assist help in modeling residual nonlinearities in the detector position sensing. For example, the problem of 'edge 
effect', where the apparent position of events near the outer edge of the detector face begins to curl inwards, cannot be accounted for satisfactorily in the absence of nonlinear terms. This problem is thought to be a consequence of a number of factors, mostly related to the finite extent of the anode pattem and the slightly inhomogeneous drift field potential near the edge of the detector body.

The coefficients needed in Eqns. (2.10) and (2.11) can be deduced by measuring the apparent position of UV photons from a mercury lamp which pass through a regular $4 \times 2 \mathrm{~mm}$ rectangular grid pattern photoetched into a 0.002 inch thick nickel sheet placed on top of the first microchannel plate. The pattern consists of both 50 and $25 \mu \mathrm{m}$ diameter holes, and a non-linear least squares fitting routine is used to determine the coefficients that result in the best fit between the observed pattern image and the knowledge of the true positions of the holes.

Analysis of the intensity profiles of individual imaged pinholes indicate that the spatial resolution is on the order of $\sim 125 \mu \mathrm{m}$ full-width-at-half-maximum in $x$ and $\sim 75 \mu \mathrm{m}$ in $y$. The difference in $x$ and $y$ arises from the fact that both the wedges and strips have nearly exactly the same maximum and minimum charge fractions, but the dynamic range of the wedge partern is spread over the entire diameter of the anode, while the dynamic range of the strip covers only a radius.

\subsubsection{J Translational Energy Resolution Considerations}

Relatively high translational energy resolution can be achieved in our experiment $\left(\Delta \mathrm{E} / \mathrm{E}_{\mathrm{T}}=0.7 \%\right),{ }^{37,38}$ partially because of the coincidence scheme we employ. The coincidence requirement differs in principle from nearly all other translational photofragment spectroscopy experiments, where only one fragment is detected. In our experiment the distance between the two fragments at the detector face, and their difference in time-of-flight, are determined to a precision of $\sim 140 \mu \mathrm{m}$ and $0.5 \mathrm{~ns}$, respectively. A simpler scheme, where the position of only one of the two fragments is 
measured relative to the center of the detector, and the time-of-flight of this fragment determined relative to the dissociation laser pulse, is indeed possible. However, this method would be subject to uncertainty both in the distance measurement (due to the size of the radical beam at the detector, about $1 \mathrm{~mm}$ in our case), and in the flight time (the dissociation laser pulse is $\sim 30$ ns long), that would serve to decrease the energy (and recoil angle) resolution. The coincidence method is insensitive to these effects.

One factor which could affect the resolution of the coincidence method is the spread in the kinetic eilergy of the radical beam. However, $\Delta E / E_{0}$ is also quite small in our experiment, at $0.1 \%^{38}$ it compares favorably to the typical (1-10\%) energy spread of most neutral molecular beam photodissociation studies.

The only single fragment detection scheme which has better translational energy resolution is the hydrogen atom time-of-flight method developed by Welge and Ashfold, ${ }^{39}$ which provides ur:paralleled energy resolution $\left(\Delta \mathrm{E} / \mathrm{E}_{\mathrm{T}}=0.3 \%\right)$. However, this technique, as it is now formulaied, takes advantage of the relatively light mass and long-lived Rydberg states of hydrogen atoms, and is thus restricted to studying systems which lose a hydrogen atom upon photolysis. On the other hand, the ion-imaging technique developed by Chandler and Houston, ${ }^{40}$ while providing quantum state selection of one of the photofragments, actually has fairly poor translational energy resolution $(0.2-0.5 \mathrm{eV})$ for the reasons, given above, that our resolution would suffer if a coincidence measurement was not made.

As mentioned previously, no ionization is required for detection of the photofragments produced in our experiment. This avoids a final resolution consideration which adversely affects experimentalists utilizing an electron-impact ionization region to facilitate fragment detection. The ultimate resolution achieved in this manner is usually limited by the ratio $\Delta L / L$, where $\Delta L$ is the length of the ionization region and $L$ is the flight length to the detector. 


\subsection{Experimental Timing Sequence}

The experimental timing is accomplished using two digital delay generators (Stanford Research Systems, Model DDG-525). The timing sequence used for both total cross section and dissociation dynamics experiments are nearly identical. The internal clock of the first delay generator is used as the base for our experiments, and the repetition rate we most frequently chose is $60 \mathrm{~Hz}$. The delay values given below are representative of those used for a molecule of mass $\sim 42 \mathrm{amu}$, and beam energy of $8 \mathrm{keV}$ - other conditions will, of course, cause these numbers to vary correspondingly.

- $T_{0}$ triggers the pulsed valve driving circuit

- A triggers the 'potential switch', typical delay : $310 \mu \mathrm{s}$ from $\mathrm{T}_{0}$.

- B triggers the mass spectrometer pulsing circuit, typical delay : $1.1 \mu \mathrm{s}$ from A.

- C triggers the ion compressor pulsing circuit, typical delay : $260 \mathrm{~ns}$ from B.

- D triggers home-built gate generator circuits ${ }^{41}$ which gate the detached electron, laser power, and iodine absorption cell (if used for wavelength calibration) signal collection. In addition, the square wave extraction pulse used in the detection of detached electrons is a product of a gate generator circuit.

The second SRS DG-525 is triggered using the $\overline{\mathrm{AB}}$ output of the first, which has a rising edge at the B (mass spectrometer) delay. Its outputs are as follows:

- $\mathrm{T}_{0}{ }^{\prime}$ triggers the oscilloscope used to monitor the experiment

- $\mathrm{A}^{\prime}$ triggers the detachment laser, typical delay $4.100 \mu \mathrm{s}$ from B.

- B' triggers the dissociation laser, typical delay $7.300 \mu$ s from B.

- $C^{\prime}$ is used infrequently to trigger the pulsed deflection plate involved in the determination of photodissociation laser timing (see $\$ 2.7 .2$ ). Otherwise it is unused.

- $D^{\prime}$ triggers the gate generator circuit which gates the detection electronics on when photofragment signal is expected; in this case a typical delay is $12.000 \mu \mathrm{s}$ from B. 
If the pulsed discharge source is used, delay A then controls the firing of the high power pulse generator that provides the discharge voltage/current. All other delays are then shifted down by one position until $\mathrm{C}^{\prime}$ (which is typically not in use) is reached. 


\subsection{References}

1 Ion Pump Operation, Perkin-Elmer Vacuum Products, (Perkin-Elmer, Eden Prairie, MN, 1984).

2 M. A. Johnson, M. L. Alexander, 1. Hertel, and W. C. Lineberger, Chem. Phys. Lett. 12, 285 (1984).

3 M. A. Johnson and W. C. Lineberger, in Techniques of Chemistry 20, 591 (1988), J. M. Farrar and W.H. Saunders, eds. (Wiley, New York, 1988)

4 T. N. Kitsopolous, Ph. D. Thesis, UC Berkeley, 1991.

5 See D. Miller, p. 14, Atomic and Molecular Beam Methods, Vol. I, G. Scoles, Ed., (Oxford University Press, New York, 1988).

6 R. Proch and T. Trickl, Rev. Sci. Instrum. 60, 713 (1989). See also reference 7.

7 R. E. Continetti, Ph. D. Thesis (University of California, Berkeley, 1989).

8 a)W. G. Cady, Piezoelectricity, (Dover, New York, 1964). b) Physik Instrumente Catalog, Number 109, U.S. Edition.

9 W. R. Gentry and C. F. Giese, Rev. Sci. Instrum. 49, 595 (1978).

10 A. Weaver, Ph. D. Thesis, UC Berkcley, 1991.

11 M. A. Johnson, M. L. Alexander, I Hertel, and W. C. Lineberger, Chem. Phys. Lett. 12, 285 (1984); M. A. Johnson and W. C. Lineberger, in Techniques of Chemistry 20, 591 (1988), J. M. Farrar and W.H. Saunders, Eds. (Wiley, New York, 1988)

12 See Reference 4 , pages 37-38.

13 R. Schlachta, G. M. Lask, S. H. Tsay, and V. E. Bondybey, Chem. Phys. 155, 267 (1991); A. Thoma, B. E. Wurfel, R. Schlachla, G. M. Lask, and V. E. Bondybey. J. Phys. Chem. 96, 7231 (1992); B. E. Wurfel, A. Thoma, R. Schlachta, and V.E. Bondybey, Chem Phys. Lett. 190, 119 (1992); G. Schallmoser, B. E. Wurfel, A. Thoma, N. Caspary, and V. E. Bondybey, Chem. Phys. Lett. 201, 528 (1993).

14 Y. Ohshima and Y. Endo, J. Mol. Spectro. 153, 627 (1992); 159, 458 (1993).

15 S. Sharpe and P. Johnson, Chem. Phys. Lett. 107, 35 (1984).

16 S. K. Bramble and P. A. Hamilton, Chem. Phys. Lett. 170, 107 (1990); Meas. Sci. Technol. 2, 916 (1991)

17 J. Baker, S. K. Bramble, and P. A. Hamilton, Chem. Phys. Lett. 213, 297 (1993).

18 T. N. Kitsopoulos, C. J. Chick, Y. Zhao, and D. M. Neumark, J. Chem. Phys. 95, 5479 (1991); D. W. Amold, S. E. Bradforth, T. N. Kitsopoulos, and D. M. Neumark, J. Chem. Phys. 95, 8753 (1991); C. C. Amold, Y. Zhao, T. N. Kitsopoulos, and D. M. Neumark, J. Chem. Phys. 97, 6121 (1992).

19 As this dissertation is written, additional configurations ase also under investigation. D. L. Osbom and D. J. Leahy, unpublished work. 
20 Strictly speaking, einzel lenses are usually comprised of three cylindrical elements (as our einzel lens in the second differential region is constructed). An attempt to approach this configuration was made by decreasing the inner diameter and using 3 or 4 plates for each element in this arrangement.

21 L. A. Posey, M. J. DeLuca, and M. A. Johnson, Chem. Phys. Lett. 131, 170 (1986).

22 This is particularly true in the case of the electron beam/free jet expansion source, where negative ions are made continuously for most of the of the $-200 \mu \mathrm{s}$ expansion. When very short discharge times are used with the pulsed discharge source, the discharge duration can be less than the transit time of the cylinder for certain mass ions.

23 R. E. Continetti, D. R. Cyr, and D. M. Neumark, Rev. Sci. Instrum. 63, 1840 (1992).

24 P. Horowitz and W. Hill, The Art of Electronics, 2nd ed. (Cambridge University, New York, 1989), p. 53.

25 P. Horowitz and W. Hill, The Art of Electronics, 2nd ed. (Cambridge University, New York, 1989), p. 882.

26 J. M. B. Bakker, J. Phys. E, 6, 785 (1973); 7, 364 (1974)

27 W. C. Wiley and I. H. McClaren, Rev. Sci. Instrum. 26, 1150 (1955).

28 It is probably not a good idea to vary conditions too much during a total photodissociation cross section scan, due to normalization difficulties, but during dynamics experiments using time- and positionsensitive detection adjusting ion production variables is considered completely acceptable.

29 M. Young, Optics and Lasers, 3rd. Rev. Ed. Spinger-Verlag, New York, 1986.

30 See Chapter 1 of Microchannel Plate Report, H. Kersten, Ed., (FOM-Instituut voor Atoom- en Molecuulfysica, Amsterdam, 1982), p. 32.

31 D. P. DeBruijn and J. Los, Rev. Sci. Instrum. 53, 1020 (1982); L. D. A. Siebbeles, J. M. Schins. W. J. Van der Zande, J. Los, and M. Glass-Maujean, Phys. Rev. A 44, 343 (1991), and references therein.

32 H. Helm and P.C. Cosby, J. Chem. Phys. 86, 6813 (1987).

33 L. J. Richter and W. Ho, Rev. Sci. Instrum., 57, 1469 (1986).

34 J. M. Schins, R. Vrijen, W. J. Van der Zande and J. Los, Surf. Sci. 280, 145 (1993).

35 L. D. Gardner, M. M. Graff, and J. L. Kohl, Rev. Sci. Instrum. 57, 177 (1986).

36 This is determined by splitting a trigger signal and, keeping cable lengths the same, have one branch trigger the digital delay generator (while the output channel is set to zero delay) and then out to the oscilloscope. Meanwhile, have the other branch go directly to the oscilloscope. The relative delay can thus be measured directly.

37 D. J. Leahy, D. R. Cyr, D. L. Osbom and D. M. Neumark, Chem. Phys. Lett. (in press). 
38 This ratio can be higher depending on experimental considerations such as beam energy and, in particular, whether the on-axis ion compressor (see § 2.5.2) is employed.

39 J. Biesner, L. Schnieder, G. Ahlers, X. Xie, K. H. Welge, M. N. R. Ashfold, and R. N. Dixon, J. Chem. Phys. 91, 2901 (1989); X. Xie, L. Schnieder, H. Wallmeier, R. Boetuner, K. H. Welge, and M. N. R. Ashfold, J. Chem. Phys. 92, 1608 (1990); L. Schnieder, W. Meier, K. H. Welge, M. N. R. Ashfold, and C. M. Western, J. Chem. Phys. 92, 7027 (1990); G. P. Morley, I. R. Lambert, M. N. R. Ashfold, K. N. Rosser, and C. M. Western, J. Chem. Phys. 97, 3157 (1992); D. H. Mordaunt, I. R. Lambert, G. P. Morley, M. N. R. Ashfold, R. N. Dixon, C. M. Western, L. Schnieder, and K. H. Welge, J. Chem. Phys. 98, 2054 (1993).

40 D. W. Chandler and P. L. Houston, J. Chem. Phys. 87, 1445 (1987); D. W. Chandler J. M. Thoman Jr., M. H. M. Janssen, D. H. Parker, Chem. Phys. Lett. 156, 151 (1989); D. W. Chandler, M. H. M. Janssen, S. Stolte, R. N. Strickland, J. W. Thoman, Jr., D. H. Parker, J. Phys. Chem. 94, 4839 (1990); D. P. Baldwin, M. A. Buntine, and D. W. Chandler, j. Chem. Phys. 93, 6578 (1990); M. A. Buntine, D. P. Baldwin, R. N. Zare, and D. W. Chandler, J. Chem. Phys. 94, (1991); M. H. M. Janssen, D. H. Parker, G. O. Sitz, S. Stolte, D. W. Chandler, J. Phys. Chem. 95, 8807 (1991); M. A. Buntine, D. P. Baldwin, and D. W. Chandler, J. Chem. Phys. 96, 5843 (1992); W. P. Hess, D. W. Chandler, and J. W. Thoman Jr., Chem. Phys. 163, 277 (1992); T. Suzuki, V. P. Hradil, S. A. Hewitt, P. L. Houston, and B. J. Whitaker, Chem. Phys. Lett. 187, 257 (1991); A. G. Suits, R. L. Miller, L. S. Bontuyan, and P. L. Houston, J. Chem. Soc. Faraday Trans. 89, 1443, (1993); V. P. Hradil, T. Suzuki, S. A. Hewitt, P. L. Houston, and B. J. Whitaker, J. Chem. Phys. 99, 4455 (1993).

41 See reference 4, page 55 for gate generator circuits of the same design. 


\section{Chapter 3}

\section{Data Analysis: Principles and Procedures}

Because of improvements in the detection scheme used in the experimental apparatus, we have obtained information concerning the dynamics of free radical dissociation through two different techniques. In our initial studies of the photodissociation dynamics of both $\mathrm{N}_{3}$ and $\mathrm{NCO}$ (the investigation of $\mathrm{NCO}$ is detailed in Chapter 5) a somewhat primitive method of probing dissociation dynamics was applied, namely, measuring the time-of-flight distributions of the photofragments. This approach was considered intermediate between having essentially no way of probing the dynamics of dissociation and the imminent implementation of the time- and position-sensitive detector, then under construction. When the time- and position-sensitive detector was fully developed, it immediately became the tool by which dynamical information concerning the dissociating radical under investigation was gleaned. Because Chapter 5 chronicles experiments which were completed before the new detector was completed, while the experiments in Chapter 6 took place after the new detector was put into service, the principles used to analyze both types of data will be discussed.

\subsection{Time-of-Flight Distributions: Monte Carlo Forward Convolution}

As mentioned above, the time- and position-sensitive detector was developed after the rest of the apparatus. In order to obtain some information about the dynamics of the dissociation processes under study, a much simpler, although admittedly somewhat less informative detection scheme was assembled. By using a $200 \mathrm{MHz}$ transient digitizer to record and sum the time of arrival of all fragments from $1-4 \times 10^{4}$ laser shots, we were able to build up a time-of-flight distribution. This was typically done for both vertical and horizontal laser polarizations, in order to be able to deconvolve the photofragment angular distribution from the data unambiguously. 
It must be acknowledged that this technique is somewhat limited, in that it is only applicable if the fragment masses are known, for reasons which will become apparent. For this reason, while it was quite successful when used to investigate simple systems such as $\mathrm{N}_{3}$ and $\mathrm{NCO}$, it would be inappropriate for a system with multiple and/or undetermined product channels.

The time-of-flight distribution is typically comprised of signal from both fragments of a given dissociation event. However, there is no upper detection limit of one event per laser shot as there is with the more sophisticated time- and position-sensitive detection scheme that is currently employed. There is also no coincidence requirement, that is, if only one fragment of a given dissociation is detected, whether because of geometric discrimination or detector efficiency, it is not rejected for want of a corresponding fragment. The wings of the fragment time-of-flight distribution will always be from the light fragment of dissociation events releasing the most kinetic energy parallel to the beam axis. Thus, assuming the masses of the fragments are known, the maximum kinetic energy release can be determined by consideration of the kinematics and the observed minimum and maximum flight times. The shape of the time-of-flight distribution is the result of a convolution of the kinetic energy release and the recoil angular distribution. This time-offlight distribution can therefore be analyzed to give internal energy distributions of the products at a moderate level of detail, corresponding to the vibrational populations of all accessible product levels (the data typically does not justify determining rotational distributions), as well as an anisotropy parameter for events associated with each $\mathrm{N}$ atom electronic state.

The method of analysis used to obtain the best fit to the data for these parameters is known as Monte Carlo forward convolution. The program used in this procedure was adapted by Dr. Robert Continetti from the LabAvg code used to analyze $\mathrm{D}+\mathrm{H}_{2}$ reactive scattering data. ${ }^{1}$ LabAvg itself was a modified version of importance-sampling Monte Carlo code originally written by $\mathrm{Dr}$. R. B. Walker, which was based on a publication by 
Dr. R. T. Pack ${ }^{2}$ describing a rigorous treatment of the apparatus averaging of the discrete center-of-mass to laboratory transformation. What follows is a brief explanation of the Monte Carlo forward convolution method of analysis and description of how it applies to our experiment.

'Monte Carlo' refers to the use of random sampling of all of the microscopic experimental variables leading up to a dissociation event. Importance sampling, which allows a weighted random sampling for each variable, is achieved by using normalized probability functions to describe the various distributions of interest. For example, it can be shown experimentally that the ion (and thus the radical) beam energy distribution are well described by a Gaussian having a FWHM of $\sim 0.1 \%$. Randomly sampling this weighted distribution will result in a very realistic treatment, in that energies closer to the mean will be sampled more often than those in the wings of the distribution.

This approach is applied to each variable in turn during an iteration, which itself consists of following an event from radical, to fragments, to the detector face, if the event is successful in propagating that far. Examples of quantities having a distribution that must be sampled during an iteration include: lab kinetic energy of the beam (mentioned above), beam divergence, photodissociation volume, dissociation photon energy, etc. These quantities can all be assigned realistic distributions from which the program samples.

The program keeps track of position and velocity information for all particles as each iteration proceeds; determining, for example, whether the radical trajectory will be successful in propagating through the beam-defining apertures, and whether the photofragments hit the active area of the detector face. A predicted photofragment recoil angular distribution and vibrational level population distribution (the latter implies the translational energy distribution through conservation of energy considerations, as seen in $\S 1.3 .2)$ is sampled as part of the simulation for each iteration. The program continues to follow the fragments to the detector face, noting the time-of-flight, so that a simulated 
time-of-flight distribution can be accumulated following approximately $1 \times 10^{4}$ iterations. This simulation can then be compared to the actual time-of-flight distribution from the experiment, and any deficiencies noted. A new recoil energy and angular distribution can then be constructed, and this used in another simulation.

It is this process of predicting, simulating, comparing simulation to data, and modifying the prediction to attempt to correct deficiencies that results in the name 'forward convolution'. By this iterative, often time-consuming process a recoil and angular distribution can eventually be found that fits the time-of-flight distributions from both polarizations and all flight lengths at a given photon energy.

\subsection{Analysis of Time- and Position-Sensitive Detector Data}

Once the time- and position-sensitive detector became operational a direct measurement of the mass ratio (useful in the case of multiple channels), kinetic energy release (KER) and recoil angle with respect to the direction of laser polarization could be made for each dissociation event through methods outlined below, and analysis of time-offlight distributions became obsolete. The specific implementation of our analysis principles for time- and position-sensitive detector data has evolved to incorporate improvements every time we have undertaken the analysis process for a new system. Several improvements and options in the data analysis which have been thought out remain to be implemented due to lack of time and/or necessity (i.e., provision for analysis of data obtained with the laser electric field vector $\bar{E}$ to be pointing perpendicular to the detector face). In this section, a description of the analysis principles will be given along with an rough idea of their application.

As explained in $\$ 2.7 .6 .2$, the raw information retrieved from our detector is a measure of the amount of charge found to impinge on each of the three elements (wedge, strip, and zigzag), for each of the two halves of the detector face, as well as the output of a time-to-amplitude converter, which corresponds to the difference in time-of-flight for the 
two fragments. This data is then converted using the algorithms given in \$2.7.6.4 to a two-dimensional position of each of the two photofragments on the detector face, and their relative time of arrival. In the remainder of this chapter, the conversion of this position and timing information to kinetic energy release (KER), recoil angle theta with respect to electric field vector $E$ of the laser, and mass ratios will be discussed. This is followed by a discussion of the required normalization of the raw translational energy and recoil angle distributions, necessary to account for the presence of the beam block and finite radius of the detector. Lastly, an account is given of the method by which final determinatio. of the translational energy distribution and energy dependent recoil anisotropy is made.

\subsubsection{Derivation of the Kinematic Equations Involved in Determining the}

Center-of-Mass Recoil Angle, Kinetic Energy Release and Mass Fraction for a Dissociation Event

Each of the above title quantities can be determined from the position and relative time-of-flight data afforded by our detector. With reference to Figure 3.1, the equations which allow the these quantities to be found will be derived. Before beginning, a few definitions and identities will be put forth.

\subsubsection{Preliminaries}

The time-of-flight from center of the photodissociation volume to the detector face for the center-of-mass of the dissociating system is

$$
t_{0}=\frac{L}{\left|\vec{v}_{0}\right|}
$$

Figure 3.1 The relationship between the experimental observables $\left(i, M, v_{0}, r_{1}, r_{2}, R, \tau\right)$ and the desired quantities $\left(m_{l}, m_{2}, \theta, v_{\text {rel }}\right)$ is shown by this diagram. 


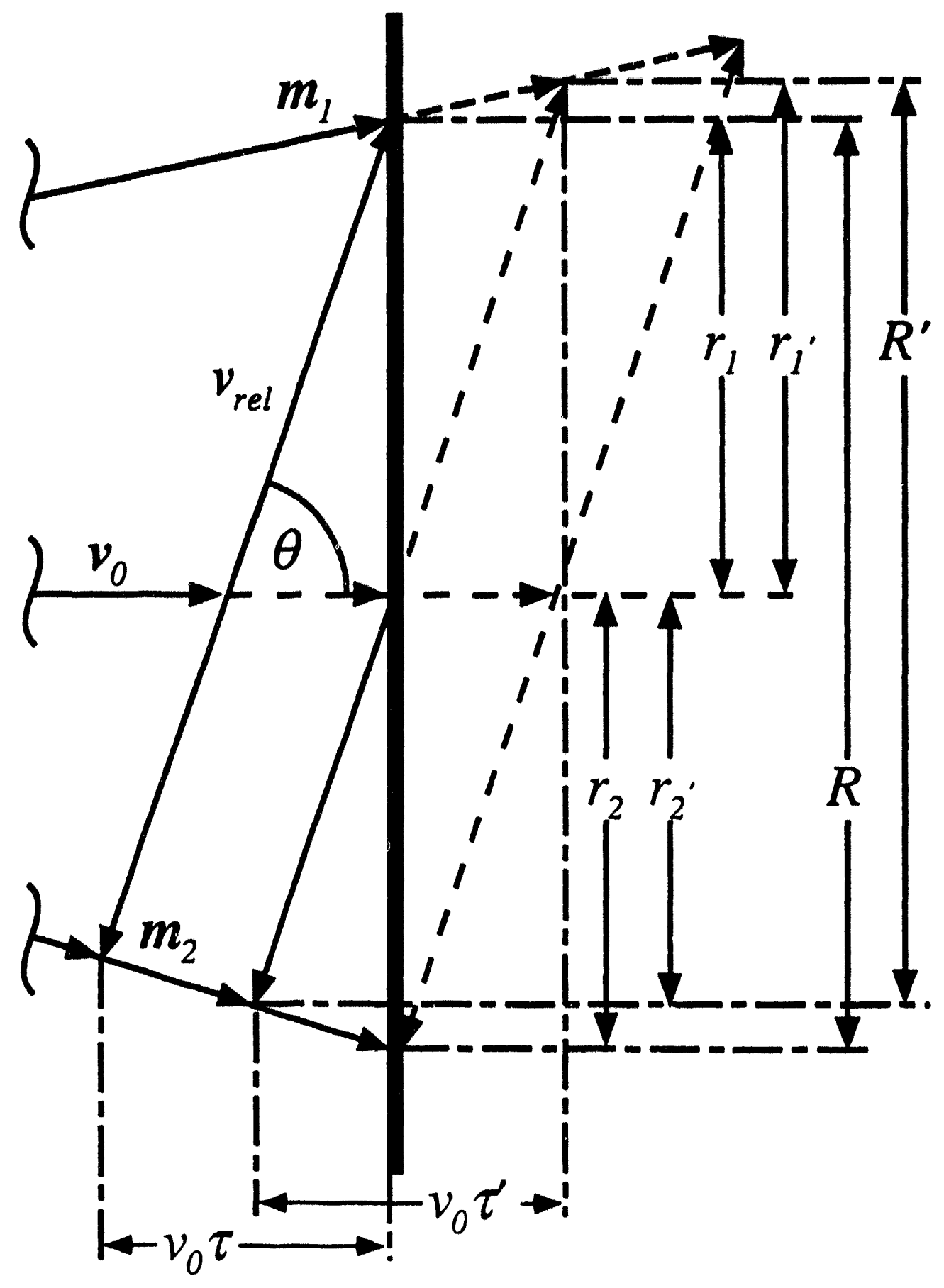

Figure 3.1 
where $\mathrm{L}$ is the flight length from the center of the dissociation volume to the face of the detector, and $\left|v_{0}\right|$ is the velocity of the center-of-mass of the dissociating system, which is determined by the mass of the parent radical $M$ and the ion beam energy $E_{0}$, i.e.,

$$
\left|\nabla_{0}\right|=\sqrt{\frac{2 E_{0}}{M}}
$$

The relative velocity vector between the two fragments can be expressed as

$$
\left|\vec{v}_{\text {red }}\right|=\sqrt{\frac{2 E_{\text {KRR }}}{\mu}} .
$$

where $E_{K E R}$ is the center-of-mass kinetic energy release in the dissociation event and $\mu$ is the reduced mass

$$
\mu=\frac{m_{1} \cdot m_{2}}{m_{1}+m_{2}} \text {. }
$$

The relative velocity vector is defined via the individual center-of-mass velocity vectors of the two fragments in the usual way:

$$
\bar{v}_{\text {rel }}=\bar{v}_{1}-\bar{v}_{2}
$$

We chose particle 1 to be the fragment which is found on the upper half of the detector, as shown in Figure 3.1. Each of $v_{1}$ and $v_{2}$ can be expressed as a sum of parallel and perpendicular components, i.e.,

$$
\bar{v}_{i}=\bar{v}_{i}^{\text {perp }}+\bar{v}_{i}^{\text {par }}
$$

Furthermore, we can express $\bar{v}_{i}^{\text {par }}$ and $\bar{v}_{i}^{\text {parp }}$ in terms of $\left|\bar{v}_{\text {rel }}\right|$ and $\theta . \theta$ is defined relative to the direction of $\bar{E}$ (for the purposes of this derivation the electric field vector $\bar{E}$ of the polarized laser output is assumed to lie parallel to the radical beam axis, therefore $\theta$ is parallel to $\left|\vec{v}_{0}\right|$ and perpendicular to the detector face). $\bar{v}_{i}^{\text {par }}$ and $\vec{v}_{i}^{\text {perp }}$ are thus given by: 


$$
\begin{array}{ll}
\bar{v}_{1}^{\text {par }}=\frac{m_{2}}{M}\left|\vec{v}_{\text {rel }}\right| \cdot \cos \theta & \bar{v}_{1}^{\text {perp }}=\frac{m_{2}}{M}\left|\vec{v}_{\text {rel }}\right| \cdot \sin \theta \\
\vec{v}_{2}^{\text {par }}=-\frac{m_{1}}{M}\left|\vec{v}_{\text {rel }}\right| \cdot \cos \theta & \vec{v}_{2}^{\text {perp }}=-\frac{m_{1}}{M}\left|\vec{v}_{\text {rel }}\right| \cdot \sin \theta
\end{array}
$$

The time-of-flight for each particle can be expressed as

$$
t_{1}=\frac{L}{\left|\bar{v}_{0}\right|+\bar{v}_{1}^{\text {par }}} \quad \text { and } \quad t_{2}=\frac{L}{\left|\bar{V}_{0}\right|+\bar{v}_{2}^{\text {par }}}
$$

If $\tau$ is defined as positive if the upper fragment (particle 1 ) arrives first, then

$$
\tau=t_{2}-t_{1}=\frac{L}{\left|\bar{\nu}_{0}\right|+\bar{\nu}_{2}^{\text {par }}}-\frac{L}{\left|\bar{\nu}_{0}\right|+\bar{\nu}_{1}^{\text {par }}} .
$$

After substitution for the components of $\bar{v}_{1}$ and $\vec{v}_{2}$ which are parallel to the radical beam axis into Eqn. (3.9) an expression for $\left|\bar{v}_{0}\right| \tau$ can be found

$$
\left|\vec{v}_{0}\right| \tau=L\left[\frac{\frac{\left|\vec{v}_{\text {rel }}\right|}{\left|\bar{v}_{0}\right|} \cdot \cos \theta}{1+\frac{m_{2}-m_{1}}{M} \cdot \frac{\left|\vec{v}_{\text {red }}\right|}{\left|\bar{v}_{0}\right|} \cdot \cos \theta-\frac{m_{1} m_{2}}{M}\left(\frac{\left|\vec{v}_{\text {red }}\right|}{\left|\bar{v}_{0}\right|} \cdot \cos \theta\right)^{2}}\right] .
$$

Now we turn our attention to finding an expression for $\vec{R}$, which is defined as the vector connecting the two positions of the fragments from a given dissociation event, from the lower to the upper half of the detector. This is almost (but not quite) equivalent to the two-dimensional projection of the three-dimensional relative velocity vector on the detector face. We begin by expressing $\vec{R}$ in terms of $\bar{r}_{1}$ and $\bar{r}_{2}$ :

$$
\bar{R}=\bar{r}_{1}-\bar{r}_{2}
$$


Although, there is a relatively large uncertainty in the individual measurements of $r_{1}$ and $r_{2}$ (see $\$ 3.2 .1 .4$ ), most of the uncertainty is correlated, and is canceled if we are simply considering $R$.

Now, $r_{1}$ and $r_{2}$ can be expressed as

$$
\nabla_{1}=\nabla_{1}^{\text {parp }} \cdot t_{1} \quad \text { and } \quad r_{2}=\nabla_{2}^{\text {perp }} \cdot t_{2} \text {. }
$$

So

$$
R=\left(\bar{v}_{1}^{\text {parp }} \cdot t_{1}\right)-\left(\bar{v}_{2}^{\text {perp }} \cdot t_{2}\right)
$$

Since we already have expressions for $t_{1}, t_{2}$, and the perpendicular velocity components in terms of $\bar{v}_{\text {red }}$, we substitute to obtain

$$
R=L \cdot\left[\frac{\frac{m_{2}}{M} \cdot \frac{\left|\vec{v}_{\text {rel }}\right|}{\left|\vec{v}_{0}\right|} \cdot \sin \theta}{1+\frac{m_{2}}{M} \cdot \frac{\vec{v}_{\text {rel }} \mid}{\left|\vec{v}_{0}\right|} \cdot \cos \theta}+\frac{\frac{m_{1}}{M} \cdot \frac{\left|\vec{v}_{\text {red }}\right|}{\left|\vec{v}_{0}\right|} \cdot \sin \theta}{1-\frac{m_{1}}{M} \cdot \frac{\left|\vec{v}_{\text {red }}\right|}{\left|\vec{v}_{0}\right|} \cdot \cos \theta}\right],
$$

which can be rearranged to give

$$
R=L \cdot\left[\frac{\frac{\left|\vec{v}_{\text {rel }}\right|}{\left|\vec{v}_{0}\right|} \cdot \sin \theta}{1+\frac{m_{2}-m_{1}}{M} \cdot \frac{\left|\vec{v}_{\text {rel }}\right|}{\left|\vec{v}_{0}\right|} \cdot \cos \theta-\frac{m_{1} \cdot m_{2}}{M^{2}}\left(\frac{\left|\vec{v}_{\text {rel }}\right|}{\left|\vec{v}_{0}\right|} \cdot \cos \theta\right)^{2}}\right] .
$$

\subsubsection{Center-of-Mass Recoil Angle}

If Eqn. (3.15) is compared with Eqn. (3.10) one will note similarities. In particular the denominator and portions of the numerator on both right hand sides are identical. Dividing Eqn. (3.15) by Eqn. (3.10) gives 


$$
\frac{R}{\left|\nabla_{0}\right| \tau}=\frac{\sin \theta}{\cos \theta}
$$

It is now apparent that $\theta$ can be determined from the measured quantities of $R$ and $\tau$, and knowledge of the radical beam velocity, $\left|\vec{v}_{0}\right|$. Rearranging Eqn. (3.16) to solve for $\theta$ gives

$$
\theta=\tan ^{-1}\left(\frac{R}{\left|\vec{v}_{0}\right| \tau}\right)
$$

It should be emphasized that despite its simplicity, Eqn. (3.17) is exact.

\subsubsection{Kinetic Energy Release}

The second quantity that must be extracted from the data is the kinetic energy released in the dissociation or, synonymously, the center-of-mass translational energy of the dissociating system ( $E_{K E R}$ and $E_{r}$ are used interchangeably in our analysis and discussion). This quantity can be determined beginning with the ratio of the beam energy to the kinetic energy released in the dissociation process:

$$
\frac{E_{\text {KER }}}{E_{0}}=\frac{\mu}{M} \cdot \frac{\left|\vec{v}_{\text {rel }}\right|^{2}}{\left|\vec{v}_{0}\right|^{2}}
$$

Within this expression, only $\left|\vec{v}_{\text {rel }}\right|$ (and of course $E_{K E R}$ ) is not known explicitly. However, the ratio of recoil velocity to beam velocity is proportional to the ratio of the recoil distance to the flight length. Unfortunately, unless $\theta=90^{\circ}$, we do not measure the recoil distance of the fragments after propagating for exactly the flight time taken by the centerof-mass of the dissociating system. However, we can determine this quantity from the experimental observables $R$ and $\tau$.

Once again referring to Figure 3.1 , if we call $R^{\prime}$ the separation of the fragments perpendicular to the radical beam axis after time $t_{0}$ (the center-of-mass flight time), and $\left|\vec{v}_{0}\right| \tau^{\prime}$ the separation of the fragments parallel to the radical beam axis after time $t_{0}$, we can write 


$$
E_{K E R}=E_{0} \cdot \frac{m_{1} \cdot m_{2}}{M^{2}} \cdot \frac{\left(R^{\prime}\right)^{2}+\left(v_{0} \tau^{\prime}\right)^{2}}{L^{2}}
$$

Expressions for $R^{\prime}$ and $\left|\vec{\nabla}_{0}\right| \tau^{\prime}$ can be determined to be

$$
\begin{aligned}
& R^{\prime}=t_{0} \cdot \bar{v}_{\text {rel }}^{\text {perp }}=\frac{L}{\left|\bar{v}_{0}\right|} \cdot\left|\bar{v}_{\text {rel }}\right| \cdot \sin \theta \\
& v_{0} \tau^{\prime}=t_{0} \cdot \bar{v}_{\text {ral }}^{\text {par }}=\frac{L}{\left|\bar{v}_{0}\right|} \cdot\left|\bar{v}_{\text {rel }}\right| \cdot \cos \theta
\end{aligned}
$$

Comparing these last Eqns. for $R^{\prime}$ and $\left|\vec{v}_{0}\right| \tau^{\prime}$ to Eqns. (3.10) and (3.15) which give expressions for $R$ and $\left|\vec{v}_{0}\right| \tau$, respectively, it can be seen that

$$
R^{\prime}=R \cdot\left[1+\frac{m_{2}-m_{1}}{M} \cdot \frac{\left|\vec{v}_{\text {rel }}\right|}{\left|\vec{v}_{0}\right|} \cdot \cos \theta-\frac{m_{1} \cdot m_{2}}{M^{2}}\left(\frac{\left|\vec{v}_{\text {rel }}\right|}{\left|\vec{v}_{0}\right|} \cdot \cos \theta\right)^{2}\right]
$$

and similarly

$$
\left|\vec{v}_{0}\right| \tau^{\prime}=\left|\bar{v}_{0}\right| \tau \cdot\left[1+\frac{m_{2}-m_{1}}{M} \cdot \frac{\left|\vec{v}_{\text {rel }}\right|}{\left|\bar{v}_{0}\right|} \cdot \cos \theta-\frac{m_{1} \cdot m_{2}}{M^{2}}\left(\frac{\left|\vec{v}_{\text {rel }}\right|}{\left|\bar{v}_{0}\right|} \cdot \cos \theta\right)^{2}\right]
$$

Substituting for $R^{\prime}$ and $\left|\bar{v}_{0}\right| \tau^{\prime}$ into Eqn. (3.19) gives

$$
E_{K E R}=E_{0} \cdot \frac{m_{1} \cdot m_{2}}{M^{2}} \cdot \frac{\left[\left(\left|\vec{v}_{0}\right| \tau\right)^{2}+R^{2}\right]}{L^{2}} \cdot\left[1+\left(\frac{m_{2}-m_{1}}{M}\right) \cdot \frac{\left|\vec{v}_{r e l}\right|}{\left|\vec{v}_{0}\right|} \cdot \cos \theta-\left(\frac{m_{1} \cdot m_{2}}{M^{2}}\right)\left(\frac{\left|\vec{v}_{\text {rel }}\right|}{\left|\vec{v}_{0}\right|} \cdot \cos \theta\right)^{2}\right]^{2}
$$

We can now expand the final square-bracketed term to be a polynomial in $\frac{\left|\vec{v}_{\text {rell }}\right|}{\left|\bar{v}_{0}\right|} \cdot \cos \theta$. If we recognize that

$$
\frac{\left|\vec{v}_{\text {rel }}\right|}{\left|\bar{v}_{0}\right|}=\sqrt{\frac{E_{K E R}}{E_{0}} \cdot \frac{M}{\mu}},
$$


we can see that $\frac{\left|\vec{v}_{\text {ral }}\right|}{\left|\vec{v}_{0}\right|}$ is determined primarily by the square root of the energies involved.

At a typical beam energy of $6000 \mathrm{eV}$, standard recoil energies in the $0-5 \mathrm{eV}$ range give a $\frac{\left|\vec{v}_{\text {rel }}\right|}{\left|\vec{v}_{0}\right|}$ ratio on the order of 0.01 . Therefore, since $\frac{\left|\vec{v}_{\text {rell }}\right|}{\left|\vec{v}_{0}\right|} \ll 1$, we leave out terms higher than second order in $\frac{\left|\bar{v}_{\text {rell }}\right|}{\left|\bar{v}_{0}\right|} \cdot \cos \theta$ to obtain

$$
E_{K E R}=E_{0} \cdot \frac{m_{1} \cdot m_{2}}{M^{2}} \cdot \frac{\left[\left(\left|\vec{v}_{0}\right| \tau\right)^{2}+R^{2}\right]}{L^{2}} \cdot\left[\begin{array}{l}
1+2\left(\frac{m_{2}-m_{1}}{M}\right) \cdot \frac{\left|\vec{v}_{\text {rel }}\right|}{\left|\vec{v}_{0}\right|} \cdot \cos \theta+ \\
{\left[\frac{\left(m_{2}-m_{1}\right)^{2}-2 m_{1} m_{2}}{M^{2}}\right]\left(\frac{\left|\vec{v}_{\text {rel }}\right|}{\left|\vec{v}_{0}\right|} \cdot \cos \theta\right)^{2}}
\end{array}\right]
$$

At this point we need to eliminate references to $\frac{\left|\vec{v}_{\text {rell }}\right|}{\left|\bar{v}_{0}\right|} \cos \theta$ since we do not know this quantity exactly. We can, however, substitute the equivalent quantity as given by a rearranged Eqn. (3.10), shown here as Eqn. (3.27),

$$
\frac{\left|\bar{v}_{\text {rel }}\right|}{\left|\bar{v}_{0}\right|} \cdot \cos \theta=\frac{\left|\bar{v}_{0}\right| \tau}{L}\left[1+\frac{m_{2}-m_{1}}{M} \cdot \frac{\left|\bar{v}_{\text {rel }}\right|}{\left|\bar{v}_{0}\right|} \cdot \cos \theta-\frac{m_{1} m_{2}}{M}\left(\frac{\left|\bar{v}_{\text {rel }}\right|}{\left|\bar{v}_{0}\right|} \cdot \cos \theta\right)^{2}\right]
$$

Keeping only terms contributing at the second order level or less in $\frac{\left|\bar{v}_{0}\right| \tau}{L}$, which itself is on the order of $\frac{\left|\bar{v}_{\text {rel }}\right|}{\left|\bar{v}_{0}\right|}$, gives the result

$$
E_{K E R}=E_{0} \cdot \frac{m_{1} \cdot m_{2}}{M^{2}} \cdot \frac{\left[\left(\left|\bar{v}_{0}\right| \tau\right)^{2}+R^{2}\right]}{L^{2}} \cdot\left[\begin{array}{l}
1+2\left(\frac{m_{2}-m_{1}}{M}\right) \cdot \frac{\left|\vec{v}_{0}\right| \tau}{L}+ \\
{\left[\frac{3\left(m_{2}-m_{1}\right)^{2}-2 m_{1} \cdot m_{2}}{M^{2}}\right]\left(\frac{\left|\bar{v}_{0}\right| \tau}{L}\right)^{2}}
\end{array}\right],
$$

which in almost all instances does not give a discernibly different result when the quadratic correction is neglected. Therefore the form of the equation we often use in practice is: 


$$
E_{K E R}=E_{0} \cdot \frac{m_{1} \cdot m_{2}}{M^{2}} \cdot \frac{\left[\left(\left|\vec{v}_{0}\right| \tau\right)^{2}+R^{2}\right]}{L^{2}} \cdot\left[1+2\left(\frac{m_{2}-m_{1}}{M}\right) \cdot \frac{\left|\vec{v}_{0}\right| \tau}{L}\right]
$$

\subsubsection{Mass Fractions}

It is sometimes the case that the radical under consideration is sufficiently simple that the identity of the dissociation products is evident. However, as larger and larger radicals are studied it will be critical to be able to identify the product channel(s) that are produced. Analysis of the kinetic energy release, for example, is precluded if the masses of the fragments involved is unknown. For mass ratio measurement, the position of each particle on the detector face relative to the average center of mass position of the radical beam $\left(\mathrm{x}_{0}, \mathrm{y}_{0}\right)$ is required, and is given by $r_{i}=\sqrt{\left(x_{i}-x_{0}\right)^{2}+\left(y_{i}-y_{0}\right)^{2}}$ (where $\left.i=1,2\right)$.

The average center of mass position of the radical beam $\left(x_{0}, y_{0}\right)$ must be determined from the data. This is a trivial procedure for cases where the masses of the products are known, since the center-of-mass coordinates of each event can be calculated in tum, summed and averaged. If the masses of the fragments are unknown, a slightly different procedure is used, which can be explained as follows: Imagine if, for all dissociation events, the positions of both fragments were joined by a straight line. By choosing a circle of diameter $\sim 2 \mathrm{~mm}$, and varying its location in the vicinity of where, experimentally, we know the position of the radical beam should be, we can eventually maximize the number of events whose lines intersect this circle. The position of the center of this circle is then taken to be $\left(x_{0}, y_{0}\right)$, as well as the peak of a two dimensional Gaussian distribution describing the radical beam intensity, having a full width at half maximum of $\sim 1 \mathrm{~mm}$. 
Knowing $r_{1}$ and $r_{2}$, albeit with some uncertainty, will allow the calculation of the mass fraction $\frac{m_{1}}{m_{2}}$ associated with a given event. Remembering that $r_{1}$ and $r_{2}$ can be expressed as

$$
\bar{r}_{1}=\bar{v}_{1}^{\text {perp }} \cdot t_{1} \quad \text { and } \quad \bar{r}_{2}=\bar{v}_{2}^{\text {perp }} \cdot t_{2}
$$

and that we have already developed expressions for $\bar{v}_{1}^{\text {perp }}, \bar{v}_{2}^{\text {perp }}, t_{1}$, and $t_{2}$, we can then set up the ratio

$$
\frac{r_{1}}{r_{2}}=\frac{\frac{m_{2}}{M} \cdot \frac{\left|\bar{v}_{\text {rel }}\right|}{\left|\bar{v}_{0}\right|} \cdot \sin \theta \cdot \frac{L}{1+\frac{m_{2}}{M} \cdot \frac{\left|\bar{v}_{\text {rel }}\right|}{\left|\vec{v}_{0}\right|} \cdot \cos \theta}}{\frac{L}{M} \cdot \frac{\bar{v}_{\text {rel }} \mid}{\left|\bar{v}_{0}\right|} \cdot \sin \theta \cdot \frac{L}{1-\frac{m_{1}}{M} \cdot \frac{\left|\bar{v}_{\text {rel }}\right|}{\left|\vec{v}_{0}\right|} \cdot \cos \theta}}
$$

After canceling terms, this reduces to

$$
\frac{r_{1}}{r_{2}}=\frac{m_{2}}{m_{1}} \cdot \frac{1-\frac{m_{1}}{M} \cdot \frac{\left|\vec{v}_{\text {rel }}\right|}{\left|\vec{v}_{0}\right|} \cdot \cos \theta}{1+\frac{m_{2}}{M} \cdot \frac{\left|\vec{v}_{\text {rel }}\right|}{\left|\vec{v}_{0}\right|} \cdot \cos \theta}
$$

Because the $\frac{\left|\bar{v}_{\text {rei }}\right|}{\left|\bar{v}_{0}\right|}$ ratio is on the order of 0.01 , and $r_{1}$ and $r_{2}$ have a relatively large uncertainty due to the reliance on the average $\left(x_{0}, y_{0}\right)$, it is logical to invoke the approximation $\frac{1}{1+\delta} \approx 1-\delta$, to obtain

$$
\frac{r_{1}}{r_{2}}=\frac{m_{2}}{m_{1}} \cdot\left[1-\frac{\left|\bar{v}_{r e l}\right|}{\left|\bar{v}_{0}\right|} \cdot \cos \theta+\frac{m_{1} m_{2}}{M} \cdot\left(\frac{\left|\bar{v}_{r e l}\right|}{\left|\vec{v}_{0}\right|} \cdot \cos \theta\right)^{2}\right]
$$

We now substitute the expression determined previously in Eqn. (3.27) for $\frac{\left|\vec{v}_{\text {rel }}\right|}{\left|\bar{v}_{0}\right|} \cdot \cos \theta$, keeping terms only to first order in $\frac{\left|\vec{v}_{0}\right| \tau}{L}$, to give 


$$
\frac{r_{1}}{r_{2}}=\frac{m_{2}}{m_{1}} \cdot\left[1-\left(\frac{\left|\vec{v}_{0}\right| \tau}{L}\right)\right] .
$$

Since we are interested in the mass ratio of the photofragments, we rearrange the equation once again to finally obtain

$$
\frac{m_{1}}{m_{2}}=\frac{r_{2}}{r_{1}} \cdot\left[1-\left(\frac{\left|\bar{v}_{0}\right| \tau}{L}\right)\right] .
$$

Since we always know the total mass $M$ of the radical that is being studied, it is a trivial matter to determine the actual masses from the mass fraction information. This expression is intuitively satisfying because if fragment 1 is detected first ( $\tau$ is positive) it does not have as much time to recoil as fragment 2 . Therefore the fraction $\frac{r_{2}}{r_{1}}$ will need a small downward correction, as the factor in the brackets provides. Of course, the situation is reversed when fragment 2 arrives at the detector first, but because $\tau$ is then negative by definition, the formula again provides the proper correction.

If a priori the identities of the fragment channel(s) produced are unknown, we must then examine the photofragment mass spectrum constructed from the data via Eqn. (3.35). Because we know the chemical composition in addition to the mass of the parent radical, there will be a limited number of mass combinations possible, and fewer still that are probable. Generally then, the determination of the identities of the photofragments will be straightforward.

However, mass ratio measurements are relatively imprecise, owing to the abovementioned substitution of $\left(x_{0}, y_{0}\right)$ for the actual location of the center-of-mass of each event; subsequently $\mathrm{m} / \Delta \mathrm{m}$ is on the order of $\sim 20$. Occasionally, therefore, this process will be more challenging. At least two methods of distinguishing between possible mass combinations may be helpfal. Isotopic substitution can be a convenient approach, depending on the structure of the parent radical. The use of $d_{3}$-nitromethane in producing 
the $\mathrm{CD}_{2} \mathrm{NO}_{2}^{-}$ion en route to the photodissociation of $\mathrm{CD}_{2} \mathrm{NO}_{2}$ is illustrated in $\& 6.3$. By examining the photofragment mass spectrum obtained for $\mathrm{CD}_{2} \mathrm{NO}_{2}$ and comparing it to that obtained for $\mathrm{CH}_{2} \mathrm{NO}_{2}$, the identities of two dissociation channels becomes unambiguous. Additionally, molecules sometimes contain an atom that has significant natural abundance at two or more masses (i.e., chlorine, bromine, sulfur, etc.). Since relatively high resolution mass selection of the parent radical occurs during the detachment process, it would be a trivial matter to study molecules containing each isotope in turn, if this would shed light on the identities of the photodissociation products.

\subsubsection{Raw Translational Energy and Recoil Angle Distributions}

As discussed in Chapter 1, the quantities of interest in a photodissociation dynamics experiment are the true photofragment center-of-mass translational energy and angular distributions. Once again, for a one-photon dissociation the form of the photofragment energy and angular distribution $\mathcal{P}\left(E_{T}, \theta\right)$ can be written as

$$
\mathrm{P}\left(E_{\mathrm{T}}, \theta\right)=P\left(E_{\mathrm{T}}\right)\left[1+\beta\left(E_{\mathrm{T}}\right) \mathrm{P}_{2}(\cos \theta)\right]
$$

where $\theta$ is the angle between the photofragment recoil vector and the electric vector of the dissociation laser, $P_{2}(\cos \theta)$ is the second Legendre polynomial, $\beta\left(E_{T}\right)$ is the (energydependent) anisotropy parameter, and $P\left(E_{\mathrm{T}}\right)$ is the angle-integrated kinetic energy distribution.

Eqns. (3.17) and (3.29), originally derived by De Bruijn and Los, ${ }^{3}$ (with the exception of an inconsistent sign convention) are used to obtain the center-of-mass kinetic (translational) energy release ( $E_{\mathrm{KER}}$ or $E_{\mathrm{T}}$ ) and fragment recoil angle $\theta$, measured with respect to the electric field vector $\bar{E}$ of the laser, for each dissociation event. If the timeand position-sensitive detector were able to detect every dissociation event with equal probability, we would at this point be in possession of the actual translational energy and recoil angle distributions. However, due to the blocking strip across the center of the 
detector and the finite radius of the detector face, some (well-defined) geometric discrimination occurs, which must be accounted for.

We begin by constructing the so-called raw translational energy and recoil angle distributions. For each product channel, we process a set of data from the time- and position-sensitive detector, consisting of a total of 50,000 to 100,000 events in most cases, into a two dimensional energy-angle array $\&\left(E_{\mathrm{T}}, \theta\right)$. This is done using energy steps comparable to the instrument resolution, and theta steps of one degree. Each element of this raw data array must then be divided by the relative acceptance of the detector for the energy-angle combination under consideration, in order to allow the final determination of the true translational energy distribution and energy dependent anisotropy parameters.

We now consider the determination of the detector acceptance function in some detail.

\subsubsection{Normalization: The Detector Acceptance Function}

Two geometric factors prevent the detection of all dissociation events. One factor is the presence of a blocking strip across the horizontal axis of the detector. This obstruction will always prevent the accumulation of the lowest KER coincidence events no matter what the recoil angle, and will block even large KER events if the recoil angle with respect to the radical beam axis is small. For example, the minimum kinetic energy release that can be detected for two fragments of masses 14 and 28 amu (which is the case in $\mathrm{N}_{3}$ and $\mathrm{NCO}$ dissociation) is $0.20 \mathrm{eV}\left(\sim 1600 \mathrm{~cm}^{-1}, \sim 4.6 \mathrm{kcal} / \mathrm{mol}\right)$ if $\theta=90^{\circ}$, the detector is in the standard location (centered on the beam axis), the beam energy is $8 \mathrm{keV}$ and the blocking strip is $8 \mathrm{~mm}$ wide. The second factor preventing certain dissociation events from being detected is the finite radius of the detector. This limitation will result in events which have one or both particles miss the active area of the detector face when both the kinetic energy release and/or the recoil angle with respect to the radical beam axis are large. Under the same conditions mentioned in the example above, the onset of field- 
of-view cutoff occurs for events with purely transverse recoil $\left(\theta=90^{\circ}\right)$ at $1.15 \mathrm{eV}$. After this point, more energetic dissociation recoils can still be detected, however the polar angular acceptance decreases as kinetic energy release becomes larger.

These characteristics of our ability to measure coincidence events translates into an energy and angular (both theta and phi) dependent 'acceptance function' for our detector. In order to determine the true photofragment energy and angular distributions from our data we must account for the effects of this detector acceptance function.

Although a Monte Carlo forward convolution approach to this problem is possible, a far more straightforward alternative involves the numerical modeling of the energy and angular dependence of the detector acceptance. This provides unbiased center-of-mass kinetic energy and angular distributions in a very direct manner, as will now be detailed.

We define the detector acceptance function $\mathcal{S}\left(E_{\mathrm{T}}, \theta\right)$ such that:

$$
P\left(E_{\mathrm{T}}, \theta\right)=\delta\left(E_{\mathrm{T}}, \theta\right) / \mathcal{B}\left(E_{\mathrm{T}}, \theta\right)
$$

The function $\mathcal{B}\left(E_{\mathrm{T}}, \theta\right)$ is the probability of observing a coincidence event with values of kinetic energy release and recoil angle of $E_{\mathrm{T}}$ and $\theta$ respectively. While experimentally we can vary the polarization of the laser such that the electric field vector $\vec{E}$ is perpendicular or parallel to the radical beam axis, our default mode of operation is parallel to the radical beam axis, since the frequency doubled output of our dissociation laser is horizontally polarized. The following discussion will assume that this orientation is in use.

As discussed in $\S 1.3 .4$, the photofragment angular distribution is azimuthally symmetric about the electric field vector $\bar{E}$ of the dissociation laser. $\mathscr{S}\left(E_{\mathrm{T}}, \theta\right)$ must be found by determining the range of azimuthal angles over which both fragments strike the active area of the detector, expressed as:

$$
\mathcal{S}\left(E_{\mathrm{T}}, \theta\right)=\frac{1}{2 \pi} \int_{0}^{2 \pi} D\left(E_{T}, \theta, \phi\right) \mathrm{d} \phi .
$$


In Eqn. (3.39), $D\left(E_{\tau}, \theta, \phi\right)$ is the doubly-differential detector acceptance function which gives the probability of detection of a dissociation event with kinetic energy release $E_{T}$, polar recoil angle $\theta$, and azimuthal recoil angle $\phi$. For a point-size radical beam, $D\left(E_{T}, \theta, \phi\right)$ is either 0 or 1 (more realistic beam sizes are discussed below). In practice, the calculation of $\mathcal{S}\left(E_{\mathrm{T}}, \theta\right)$ simply involves stepping through the full range of $\phi$ in steps of degrees, summing the evaluations of $D\left(E_{\mathrm{T}}, \theta, \phi\right)$, and dividing by 360 to obtain the probability of observing dissociation events with a particular kinetic energy release $E_{\tau}$, and polar recoil angle $\theta$. This is done for every $E_{\mathrm{T}}, \theta$ combination, using energy and theta step sizes identical to those used when binning the raw data.

The best agreement with Monte Carlo simulations designed to check the accuracy of these results is obtained when the size and shape of the radical beam is taken into account in the calculation of the doubly differential acceptance function $D\left(E_{\mathrm{T}}, \theta, \phi\right)$. This is accomplished by modeling the beam profile at the detector with a two-dimensional Gaussian with a $1 \mathrm{~mm}$ full width at half-maximum. The relative intensities of this twodimensional Gaussian at each point of a $10 \times 10$ grid are used as coefficients for the overall detector acceptance function found for that point. After all 100 detector acceptance function calculations have been performed, normalized, and summed, a more realistic detector acceptance function is produced. With this modification, $D\left(E_{\top}, \theta, \phi\right)$ takes on values between 0 and 1 , representing what fraction of the radical beam profile gives rise to coincidences for kinetic energy release $E_{\tau}$ and angles $\theta$ and $\phi$.

\subsubsection{Final Determination of the Angle-Integrated Translational Energy Distribution,} $P\left(E_{\mathrm{T}}\right)$, and the Energy Dependent Anisotropy Parameters, $\beta\left(E_{\mathrm{T}}\right)$

Once $\mathcal{A}\left(E_{\mathrm{T}}, \theta\right)$ has been determined, the raw energy and angular distributions can be converted into the (unnormalized) probability distribution $P\left(E_{T}, \theta\right)$, using Eqn. (3.37). We then factor $P\left(E_{\mathrm{T}}, \theta\right)$ into the angle-integrated translational energy distribution, $P\left(E_{\mathrm{T}}\right)$, and 
an enurgy dependent recoil angular distribution, as in Eqn. (3.36). In practice, this entails first determining $\beta\left(E_{\mathrm{T}}\right)$ values followed by the determination of $P\left(E_{\mathrm{T}}\right)$.

Currently, $\beta\left(E_{\mathrm{T}}\right)$ values are obtained for each kinetic energy interval from $E_{i}-\Delta E / 2$ to $E_{i}+\Delta E / 2$ where $\Delta E$ is an arbitrary, constant energy range large enough to provide good statistics for the bulk of the observed range of kinetic energy release. Trese intervals are typically $\sim 10-15$ times the typical kinetic energy bin size. The anisotropy parameter $\beta$ for the kinetic energy release interval centered at the energy $E_{i}$ is then found via summation of the normalized angular distribution data over the angles common to all of the kinetic energy bins in the range from $E_{i}-\Delta E / 2$ to $E_{i}+\Delta E / 2$, followed by a simple least squares fit to Eqn. (3.36).

The final step in this direct inversion analysis is to find the angle-integrated translational energy distribution $P\left(E_{T}\right)$ at the highest energy resolution. The values of $\beta\left(E_{T}\right)$ determined above are used in Eqn. (3.36) while $P\left(E_{\mathrm{T}}\right)$ is found for energy intervals the size of the original bins (i.e., intervals on the order of the instrument resolution).

An improvement to this method is under development, to be implemented in the analysis of the next set of data. It involves using a flexible $\Delta E$ interval to allow the determination of $\beta\left(E_{T}\right)$ to have approximately the same uncertainty across the whole observed kinetic energy range. In addition, the simultaneous fitting of both $P\left(E_{\mathrm{T}}\right)$ and a common $\beta\left(E_{T}\right)$ for the highest resolution energy intervals within the $\Delta E$ interval may result in a better overall determination of these quantities than the two step process we currently employ.

There are two major sources of uncertainty in the determination of $P\left(E_{\uparrow}\right)$. One source is simply associated with the nature of counting experiments, and is well modeled by Poisson statistics. The second arises from the uncertainty in the value we determine for $\beta\left(E_{T}\right)$. This uncertainty, especially when fixed $\Delta E$ intervals are used, can be negligible in some regions and dominate in others. The $\beta\left(E_{T}\right)$ uncertainty is especially large at high $E_{T}$ where the polar acceptance grows small, thereby lowering both the angular range 
contributing to the determination of $\beta$ and the number of dissociation events detected. The uncertainty in $\beta\left(E_{T}\right)$ is locally correlated in the energy release spectrum ( $\beta$ is not expected to randomly jump from one energy bin to another), so the uncertainty in $\beta\left(E_{T}\right)$ is not added in quadrature to the statistical error. Instead, the effect of the uncertainty in $\beta\left(E_{T}\right)$ can be conveyed by using both the upper and lower uncertainty limits of $\beta\left(E_{T}\right)$ in separate determinations of $P\left(E_{\mathrm{T}}\right)$, and comparing the results with the translational energy distribution found using the best-fit values.

Overall, the strengths of coincidence detection and direct inversion of the data are multiple. First, branching ratios between product channels (if applicable) are given by comparison of the integrated area under the angle integrated translational energy distribution for each channel. Second, we obtain the explicit energy dependence of the anisotropy parameter from our experiment, unlike rnost other photodissociation studies. Finally, determination of the angle-integrated translational energy distribution and the energy-dependent anisotropy parameter is through direct inversion of the data, avoiding an iterative and time-consuming forward convolution process. 


\subsection{References}

1 R. E. Continetti, Ph. D. Thesis, University of Califomia, Berkeley, 1989.

2 R. T. Pack, J. Chem. Phys. 81, 1841 (1984).

3 D. P. De Bruijn and J. Los, Rev. Sci. Instrum. 53, 1020 (1982). 


\section{Chapter 4 \\ Photodissociation Studies of $\mathrm{O}_{2}$ and $\mathrm{N}_{3}$ : Examples of FRBM Capabilities}

Our photodissociation studies of the two systems, $\mathrm{O}_{2}$ and $\mathrm{N}_{3}$, are good illustrations of the capabilities of the Fast Radical Beam Machine. Both species have predissociative excited states, resulting in a structured total photodissociation cross section. The simplicity of $\mathrm{O}_{2}$ allows its dissociation to be used as a calibrant and a measure of the ultimate resolution of the detector. On the other hand, $\mathrm{N}_{3}$ is in many ways an ideal polyatomic radical system for investigation using this apparatus, and was chosen as the first radical to be studied using time- and position-sensitive detection. In the following four subsections the total photodissociation cross section experiments followed by the dissociation dynamics experiments for each of $\mathrm{O}_{2}$ and $\mathrm{N}_{3}$ are briefly summarized, with the emphasis on experimental results. A full account of both the $\mathrm{O}_{2}{ }^{1}$ and $\mathrm{N}_{3}{ }^{2}$ work is given elsewhere.

\subsection{Photodissociation Cross Sections in the $B^{3} \Sigma_{u}^{-}\left(v^{\prime}\right) \leftarrow X^{3} \Sigma_{g}^{-}\left(v^{\prime \prime}\right)$}

\section{Schumann-Runge Bands of $\mathrm{O}_{2}$}

Total photodissociation cross sections of $\mathrm{O}_{2}$ were originally obtained in limited spectral regions with the sole expectation of using the predissociation of the SchumannRunge bands as an ideal means to calibrate (and determine the resolution of) the time- and position-sensitive detector, in a complementary manner to the calibration technique discussed in $\$ 2$ 2.6.6.5. (A description of the calibration technique involving $\mathrm{O}_{2}$ dissociation is included in the following section.) Fortunately, a relatively high kinetic energy release resolution was realized in the time- and position-sensitive experimerits, and our important but narrow reasons for studying $\mathrm{O}_{2}$ dissociation became expanded. This development prompted the measurement of the photodissociation cross section for all of the $\mathrm{O}_{2} B^{3} \Sigma_{u}^{-}$state vibrational levels possible using our apparatus, from $v^{\prime}=0$ to $v^{\prime}=11 .^{1}$ 
Here, however, only the photodissociation cross section spectrum of the $B^{3} \Sigma_{u}^{-}\left(v^{\prime}=7\right) \leftarrow$ $X^{3} \Sigma_{z}^{-}\left(\nu^{\prime \prime}=4\right)$ band is discussed as a representative example.

The experimental conditions for $\mathrm{O}_{2}$ photodissociation experiments are now briefly summarized. Neat $\mathrm{O}_{2}$ at a stagnation pressure of 20 PSIG was used to form $\mathrm{O}_{2}^{-}$using the electron beam-pulsed free jet expansion source. By operating the detachment laser at 480 $\mathrm{nm}(2.58 \mathrm{eV})$, we photodetached the electrons well above the $0.45 \mathrm{eV}$ threshold for this process. Vibrationally excited $\mathrm{O}_{2} X^{3} \Sigma_{8}^{-}$as well as $\mathrm{O}_{2} a^{1} \Delta_{8}$ are formed at this detachment wavelength, with the vibrational state distributions governed by the Franck-Condon factors between the $\mathrm{O}_{2}^{-} X^{2} \Pi_{2}$ anion and the respective neutral states. As explained in \$1.4, we normally find it advantageous to avoid vibrational excitation of the radical by detaching near threshold. However, as the potential energy curves sketched in Figure 4.1 show, in studies of the Schumann-Runge $B^{3} \Sigma_{u}^{-}\left(v^{\prime}\right) \leftarrow X^{3} \Sigma_{g}^{-}\left(v^{\prime \prime}\right)$ bands, vibrational excitation in the ground electronic stare of $\mathrm{O}_{2}$ is an advantage as it allows subsequent excitation to the lower vibrational levels of the $B^{3} \Sigma_{u}^{-}$state. In contrast, these lower vibrational levels of the $B^{3} \Sigma_{u}^{-}$state have vanishing Franck-Condon factors from the ground vibrational level of the $X^{3} \Sigma_{g}^{-}$state owing to the significant extension of equilibrium interatomic distance in the excited state.

Originally, $\mathrm{O}_{2}$ total photodissociation cross section scans were undertaken using the detector primarily designated for that purpose, described in $\S 2.7 .5$. The total photodissociation cross section spectrum presented in Figure 4.2, however, was obtained using the TPS detector, after linking together the wedge, strip and zigzag output, and measuring only total photofragment flux. Figure 4.2 shows a total cross section spectrum

Figure 4.1 Schematic of the $\mathrm{O}_{2}$ potential energy curves relevant to the study of predissociation following excitation of the Schumann-Runge $B^{3} \Sigma_{u}^{-}\left(v^{\prime}\right) \leftarrow X^{3} \Sigma_{g}^{-}\left(v^{\prime \prime}\right)$ bands.

Figure 4.2 The total photodissociation cross section of $\mathrm{O}_{2}(0.0025 \mathrm{~nm}$ steps, corrected to vacuum wavelengths) in the region of the $v^{\prime}=7 \leftarrow v^{\prime \prime}=4$ band. 


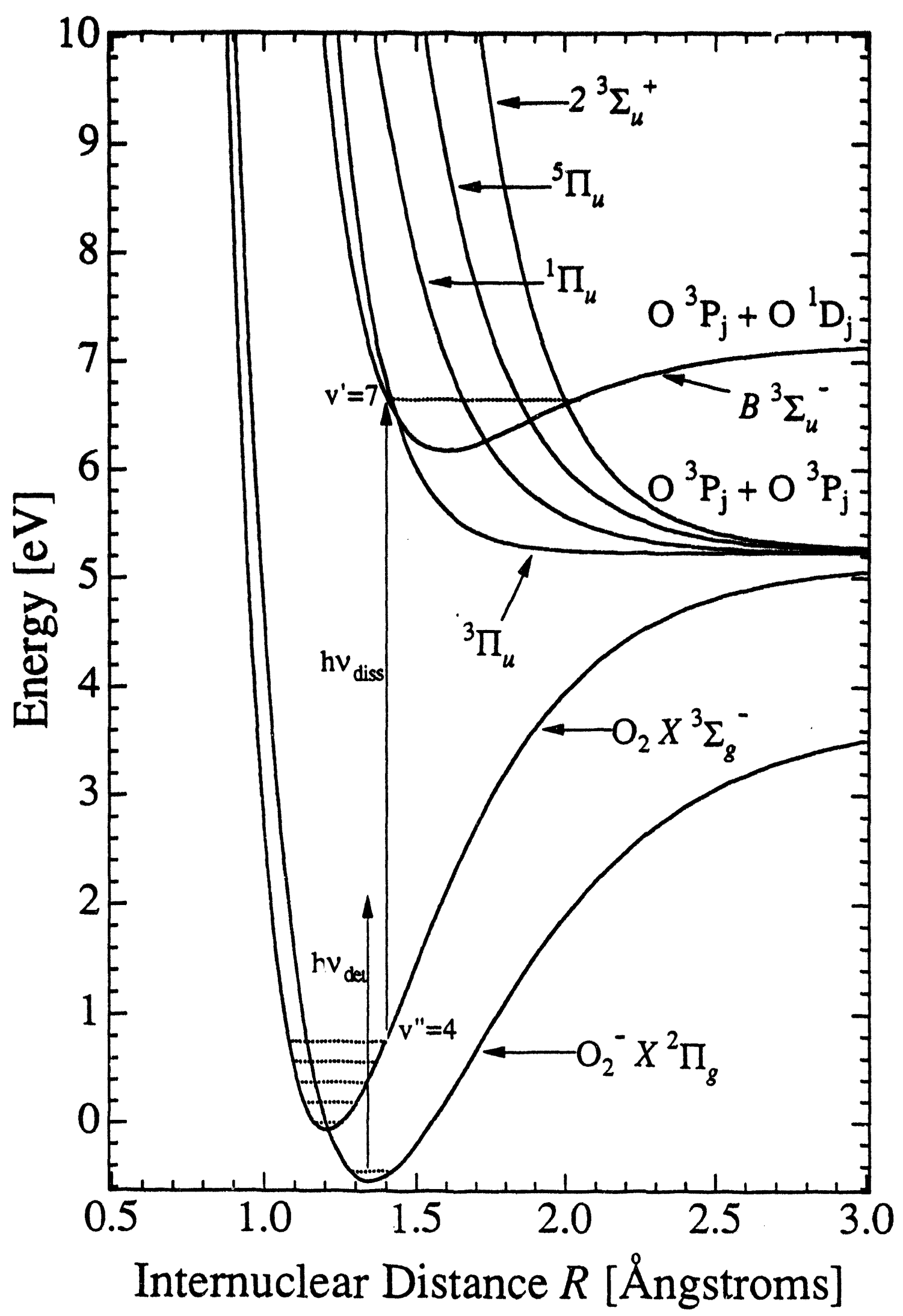

Figure 4.1 


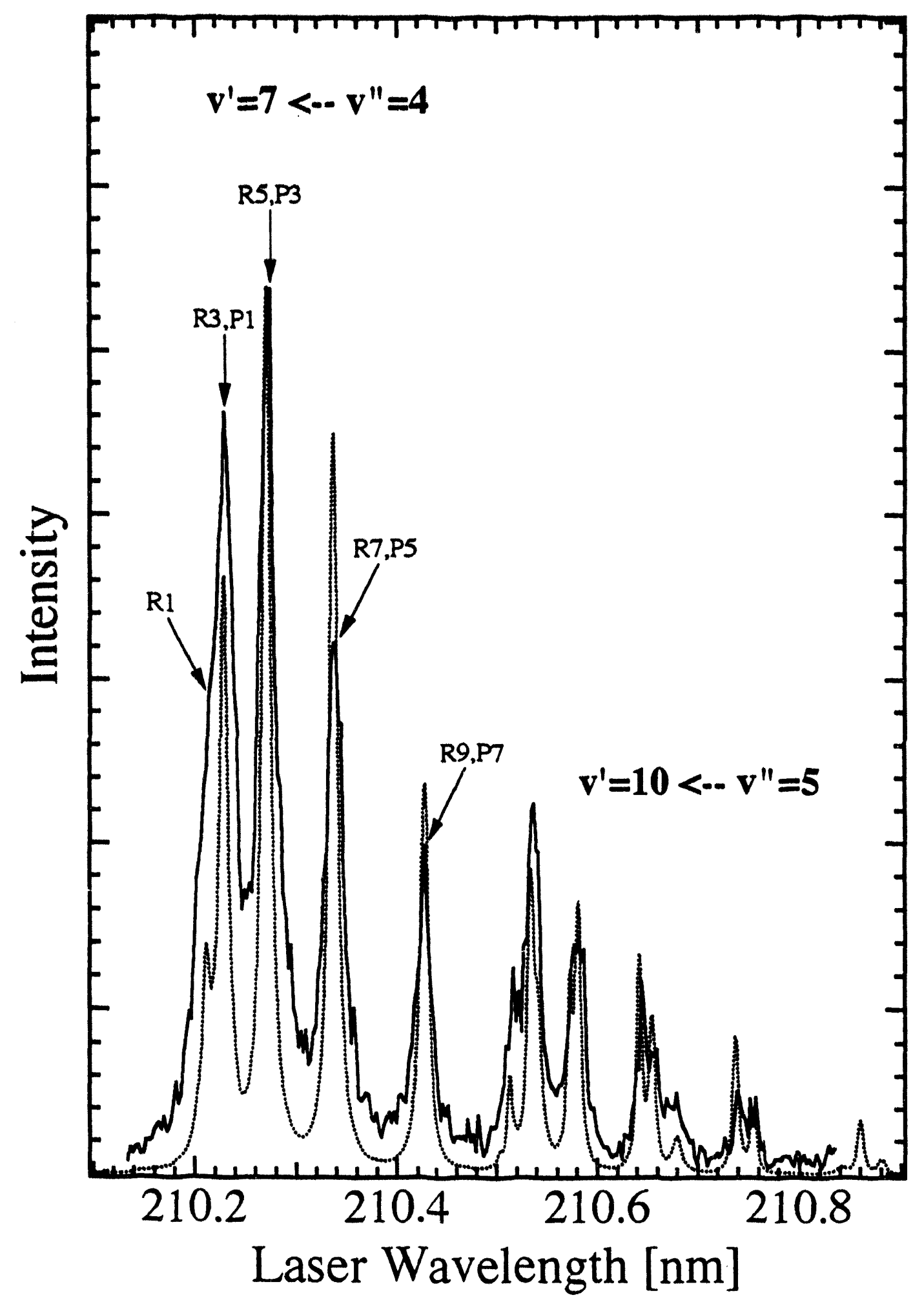

Figure 4.2 
in the region of the $v^{\prime}=7 \leftarrow v^{\prime \prime}=4$ band along with a simulation that assumes a Boltzmann temperature of $50 \mathrm{~K}$ (offset for clarity). The photofragment signal intensity is normalized by detached electron signal and dissociation laser power. This spectrum contains the sum of signal from only 200 laser shots per point. Note that while (partial) rotational resolution is achieved, the natural linewidth is quite large for many of these transitions, obscuring spin-rotation fine structure. The vibrational bands within the $B^{3} \Sigma_{u}^{-} \leftarrow X^{3} \Sigma_{g}^{-}$ electronic transition are degraded to the red quite strongly, with the band-head occurring at the origin in most cases, and the $v^{\prime}=7 \leftarrow v^{\prime \prime}=4$ transition is no exception. The rotational transitions are labeled above the appropriate peaks.

Although there is essentially no new spectroscopic information in this spectrum as the ground and excited levels involved have been thoroughly characterized in the past, ${ }^{3}$ these total photodissociation cross section studies were still important for a number of

reasons. As a test system for the experimental apparatus $\mathrm{O}_{2}$ is quite convenient, both because of the ease of producing $\mathrm{O}_{2}^{-}$and also because the Schumann-Runge bands predissociate, resulting in a structured total photodissociation cross section. However, the major purpose of investigating the total photodissociation cross section of $\mathrm{O}_{2}$ became the mapping out of transitions to vibrational levels of the excited $B^{3} \Sigma_{u}^{-}$state from $v^{\prime}=0$ to $v^{\prime}=11$. Once this was completed, we could calibrate our time- and position-sensitive detector and then use it to further study the dissociation dynamics of the Schumann-Runge bands.

\subsection{TPS Detection of $\mathrm{O}_{2}$ Dissociation:}

\section{Resolving Correlated Spin-Orbit States of the $0{ }^{3} P_{j_{1}}+O{ }^{3} P_{j_{2}}$ Products}

Because the products of $\mathrm{O}_{2}$ dissociation are atoms they can possess no internal energy in the form of vibrational or rotational motion. Initially neglecting the relatively small spin-orbit splitting in the ${ }^{3} P_{\mathrm{j}}$ ground state of the oxygen atoms $\left({ }^{3} P_{0}\right.$ is $28 \mathrm{meV}$, while ${ }^{3} P_{1}$ is $19 \mathrm{meV}$ above the ground state ${ }^{3} P_{2}$ ), the kinetic energy release should resemble a 
delta function. With reference to Figure 4.1, the recoil energy will be approximately $1.5 \mathrm{eV}$, equal to the difference in energy between the $B^{3} \Sigma_{u}^{-}$state ro-vibrational level and the $\mathrm{O}\left({ }^{3} P\right)+\mathrm{O}\left({ }^{3} P\right)$ product asymptote. The utility of this system as a calibrant stems from the fact that if many $\mathrm{O}_{2}$ dissociation events are detected, the distribution of recoil angles will ensure that all regions of the detector face will receive coincidence fragments having recoil vectors with identical magnitudes. If the coefficients in the position algorithm are accurate across the whole detector face, and the electronics are calibrated correctly, then the translational energy distribution calculated from the measured positions and differences in time of flight will be the expected narrow delta function, centered at the proper recoil energy. In this way, the width of the observed translational energy distribution becomes a measure of the calibration of the equipment and algorithms involved in time- and position-sensing and, ultimately, a measure of the resolution.

This approach allows 'js to collect a large body of $\mathrm{O}_{2}$ dissociation data and use it to find the best fitting empirical factors in the position algorithm via a least-squares routine. A compromise exists between using a sufficient number of parameters in the fitting expression and having a tractable fitting problem. The least-squares fit is typically prevented from achieving below $\sim 30 \mathrm{meV}$ resolution over the entire detector face due to the necessity of neglecting higher order empirical corrections.

However, with $\mathrm{O}_{2}$ dissociation data the fit is locally quite good. If the detector face is divided up into $1 \mathrm{~mm} \times 1 \mathrm{~mm}$ squares, a resolution of $<10 \mathrm{meV}$ is found for most squares with sufficient signal for good counting statistics. In the central portions of the upper and lower halves of the detector areas as large as $4 \mathrm{~mm}$ (in the y dimension) $\times 8 \mathrm{~mm}$ (in the $\mathrm{x}$ dimension) can exhibit this high resolution using one set of factors.

The use of this piece-wise high resolution can allow us to obtain $10 \mathrm{meV}$ resolution over most of the detector face, in the following way. Best-fit factors are found for the position equations using all but the edges of the detector face, where we find the nonlinearities are too severe. The detector face is then divided up into $1 \mathrm{~mm} \times 1 \mathrm{~mm}$ 
squares. When the translational energy distributions are determined for each individual area, a correction can be applied to shift the mean kinetic energy release for each area to match the mean found with the best-fit factors applied to all regions. The translational energy distributions for all of the smaller regions can then be summed and the resulting resolution will be $\sim 10 \mathrm{meV}$ over the bulk of the detector face.

It should be mentioned, however, that this particular method of improving the overall resolution was most effective prior to recent modifications in the detector body design. Although our uncorrected resolution is now higher than before these modifications, this method presently results in less of an improvement in overall resolution. It is thought that perhaps the reason for the larger prior success was that it . corrected primarily the same distortions ameliorated by the detector body modifications. The principal improvements in the detector body concerned reducing "edge effects", which are generally ascribed to inhomogeneities in the electric field near the edge of the detector body. These imperfections cause the acceleration of the electron cloud towards the anode to be skewed. This reason for this recent loss in efficiency will have to be investigated and confirmed, however, because it may indicate a change in importance among various resolution-degrading mechanisms, which should be addressed.

When examining $\mathrm{O}_{2}$ dissociation data, and when the number of coincidence events permits, the highest resolution is obtained by selecting the region of the detector face where the position algorithm gives the best results; typically in the center of each half.

Once resolution on the order of $10 \mathrm{meV}$ is achieved, however, the spin-orbit interaction that slightly splits the ground electronic state of the $O$ atoms can no longer be neglected. Therefore we might expect to observe slightly different kinetic energy releases depending on the specific spin-orbit states formed by the two $O$ atom products. As one example, careful measurement of the kinetic energy release distribution of the correlated $O$ atoms following excitation to $v^{\prime}=7$ in the $B^{3} \Sigma_{u}^{-}$upper state allows the kinetic energy release corresponding to the correlated spin-orbit product states to be resolved, as shown 
in Figure 4.3. Subsequent to this achievement, the correlated spin-orbit product state distributions of all of the $B^{3} \Sigma_{u}^{-}$upper state vibrational levels from $v^{\prime}=0$ to $v^{\prime}=11$ have been determined. ${ }^{1(b)}$

Predissociation from the Schumann-Runge $B^{3} \Sigma_{u}^{-}\left(v^{\prime}\right) \leftarrow X^{3} \Sigma_{g}^{-}\left(v^{\prime \prime}\right)$ bands involves interaction of the $B^{3} \Sigma_{u}^{-}$state with one of the four dissociative excited states of allowed symmetry known to intersect it, as shown in Figure 4.1. The pair of $O$ atoms will correlate to one of the five symmetry-and parity-allowed correlated $(j, j)$ spin-orbit energy asymptotes (the sixth possible combination of atomic limits, the $(0,0)$ limit, is rigorously forbidden as it must correlate to a gerade molecular state). Relative populations of these correlated spin orbit states have never been determined before. ${ }^{4}$ The systematic investigation of these distributions over the range of vibrational levels in the $B^{3} \Sigma_{u}^{-}$upper state will help unravel the nature of the dissociative state(s) primarily responsible for the dissociation (i.e. which dissociative state(s) initially couple most strongly to the $B^{3} \Sigma_{u}^{-}$ state) as well as the subsequent interactions between various dissociative states as the interatomic distance grows.

Figure 4.4 shows the angular distribution of recoil vectors found in this experiment. Analysis of this angular distribution gives an anisotropy parameter $\beta$ of 0.76 \pm 0.03 . While it is possible that this value is affected by residual saturation or power broadening, it is quite reproducible. An anisotropy parameter of 0.76 is also consistent

Figure 4.3 The translational energy release distribution $P\left(E_{\mathcal{T}}\right)$ resulting from the predissociation of $\mathrm{O}_{2} B^{3} \Sigma_{u}^{-}\left(v^{\prime}=7\right)$. The circles represent experimental data, and the solid line represents the results of a fit to these data. Every fourth point has an error bar, representing the $1 \sigma$ uncertainty. The sticks denote the positions and intensities of the individual $\left(j_{1}, j_{2}\right)$ limits as deduced from the fit.

Figure 4.4 The photofragment angular distribution $P(\theta)$. Also shown is the least squares fit to the experimental data, which yielded an anisotropy parameter of $\beta=0.76 \pm 0.03$. 


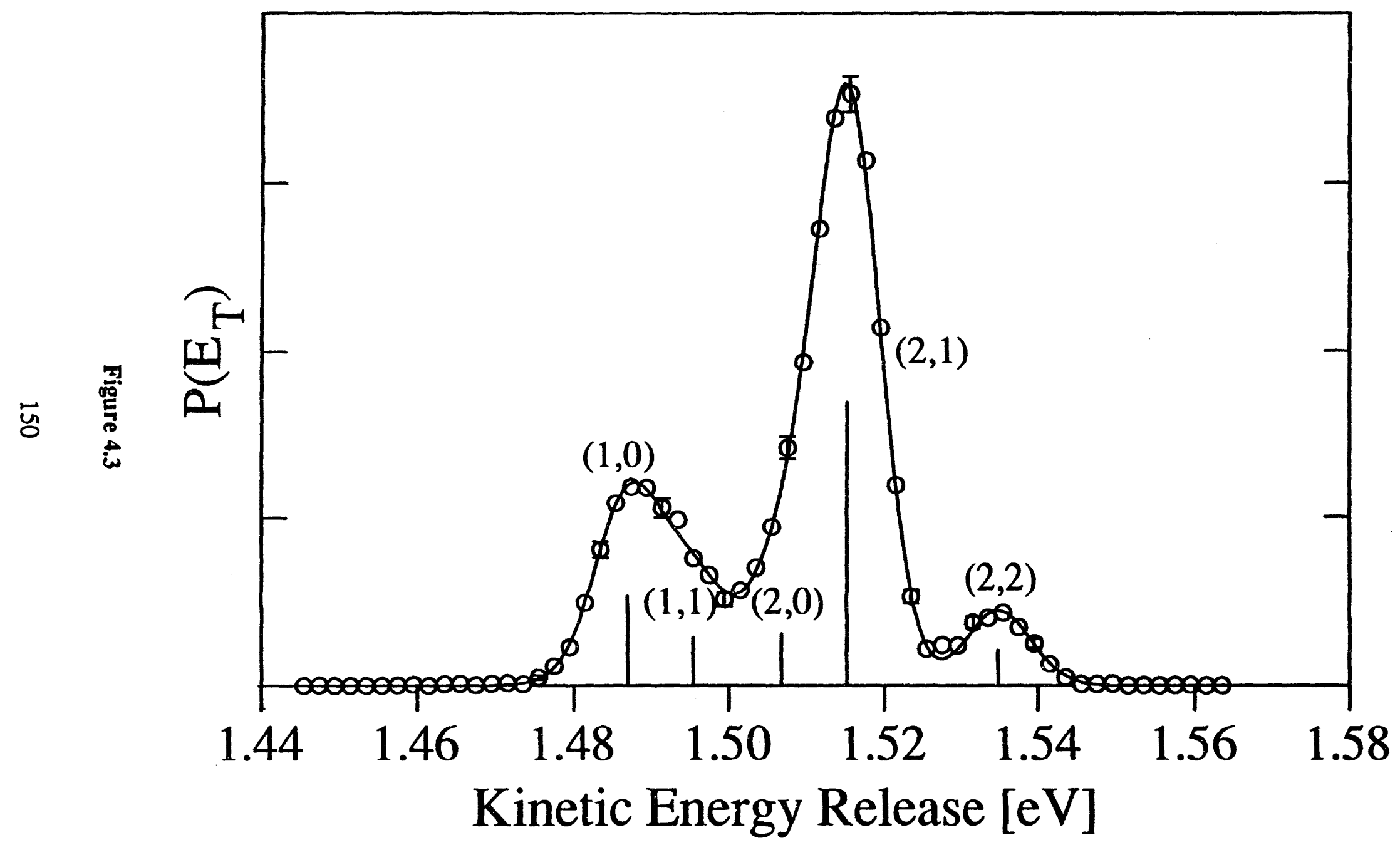




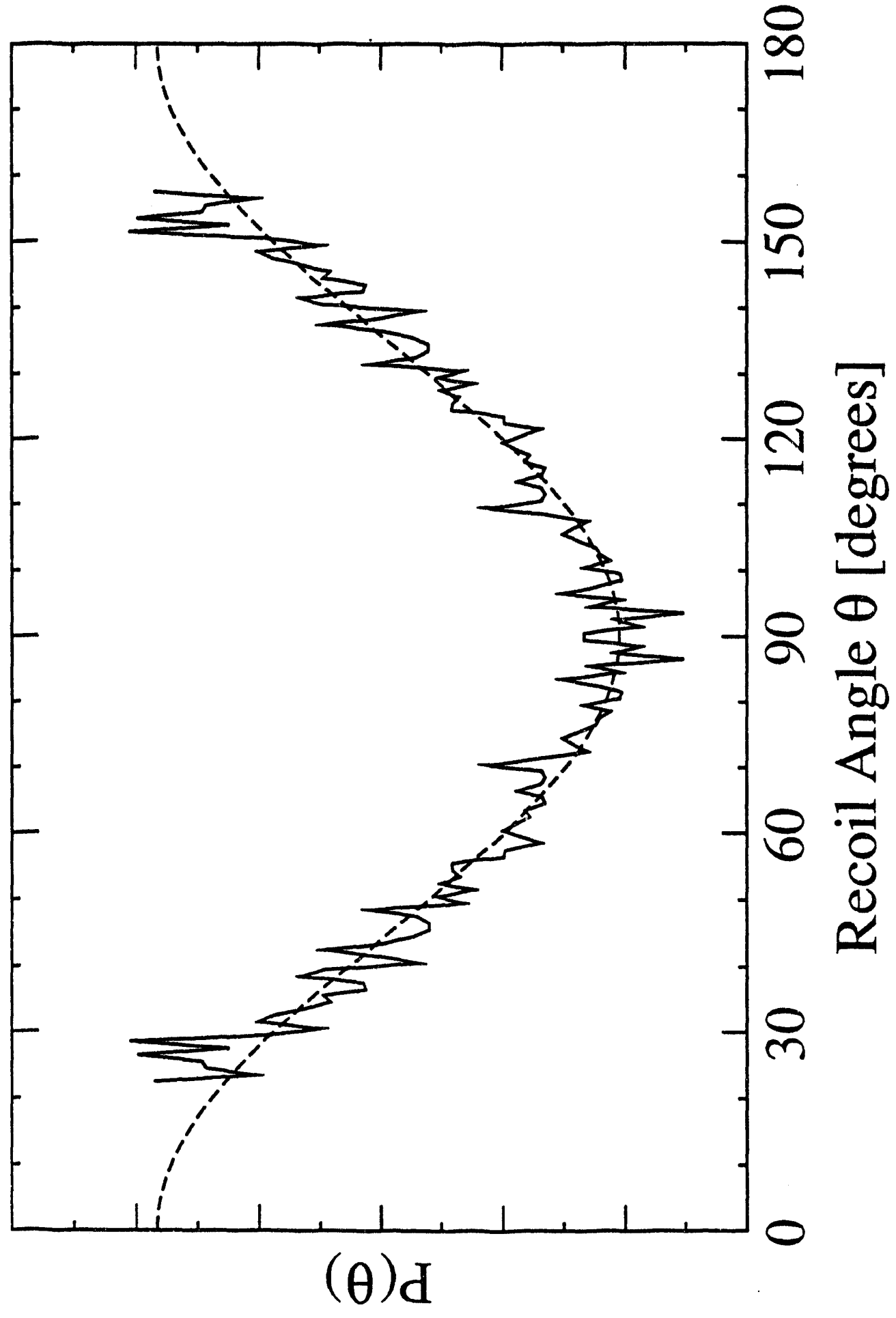

Figure 4.4 
with the observed $2 \mathrm{~cm}^{-1}$ natural linewidth for the $v^{\prime}=7$ level of the $B^{3} \Sigma_{u}^{-}$state, since both measures imply a $\sim 3$ ps lifetime for the excited state. The rotation which occurs in this time (prior to dissociation) results in a reduction of the anisotropy parameter from the limiting value of +2 for a parallel transition (recall $\S 1.3 .4$ ).

\subsection{Total Photodissociation Cross Sections of the $\mathbf{N}_{\mathbf{3}}$ Free Radical}

The $\mathrm{N}_{3}$ radical is, for our experiment, the prototypical polyatomic radical. The precursor anion, $\mathrm{N}_{3}^{-}$, is easily made via dissociative attachment to benzyl azide $\left(\mathrm{C}_{6} \mathrm{H}_{5} \mathrm{CH}_{2} \mathrm{~N}_{3}\right)$. The detachment of this ion is discussed in $\S 1.4 .2$ as an example of a system where little geometry change occurs, resulting in $\Delta v=0$ transitions from the ion to the neutral. This in turn relaxes our detachment photon energy restrictions; we can thus detach using any convenient wavelength above threshold, which maximizes our detachment efficiency.

A diagram showing the energetics of the various $\mathrm{N}_{3}$ electronic states in addition to dissociation product energy levels is shown in Figure 4.5. The $\mathrm{N}_{3}$ radical was first studied spectroscopically in a series of flash photolysis absorption experiments. 5,6 These rotationally-resolved absorption studies identified the main absorption features at $\sim 272 \mathrm{~nm}$ as arising from the $\tilde{B}^{2} \Sigma_{u}^{+} \leftarrow \tilde{X}^{2} \Pi_{g}$ transition. They also showed that $N_{3}$ is a linear centrosymmetric molecule with little change in bond length on undergoing the $\tilde{B} \leftarrow \tilde{X}$ transition. Because the ground electronic state is degenerate and the geometry is linear, transitions originating from $\mathrm{N}_{3} \bar{X}\left(v_{2}=1\right)$ exhibit the expected Renner-Teller effect. ${ }^{7,8}$ For our experiment, the relevant observation fron these previous studies was the presence of diffuse bands reported at slightly shorter wavelength than the main $\tilde{B} \leftarrow \tilde{X}$ transitions. This strongly suggests predissociation is occurring from the upper states involved in these diffuse transitions. These bands were tentatively assigned to a ${ }^{2} \Pi \leftarrow^{2} \Pi$ electronic

Figure 4.5 The energetics of the $\mathrm{N}_{3}$ system. 

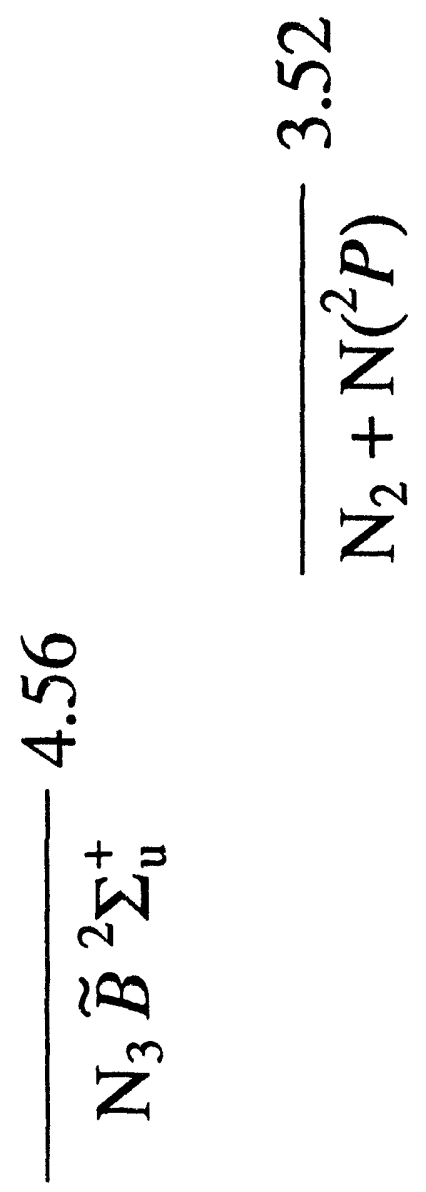

m

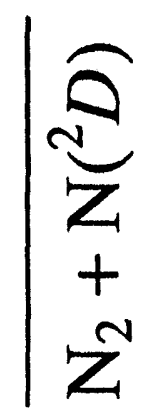

$\theta^{n}$

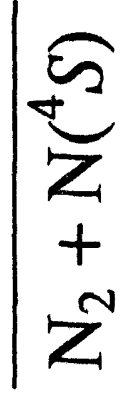

0

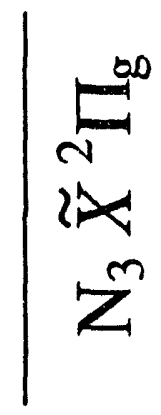

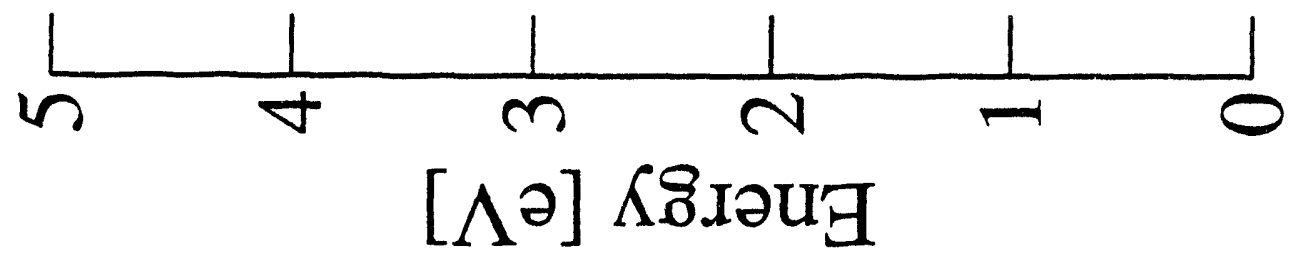

Figure 4.5 
transition expected to be in this region.

The major motivation for obtaining the total photodissociation cross section of the $\mathrm{N}_{3}$ radical was to once again map out and determine the extent of predissociation in these excited state(s). As will be shown below, the $\mathrm{N}_{3}$ radical predissociates throughout the absorption bands due to the main $\tilde{B} \leftarrow \tilde{X}$ transitions in addition to the bands found at slightly higher energy.

$\mathrm{N}_{2}$ or He at a pressure of 10 PSIG was bubbled through room temperature benzyl azide (Alfa) and this mixture was used to form $\mathrm{N}_{3}^{-}$in the electron beam/pulsed free jet expansion source. A repetition rate of $50 \mathrm{~Hz}$ and a beam energy of $8 \mathrm{kV}$ was used. The detachment excimer-pumped dye laser was operated at either $343 \mathrm{~nm}(3.62 \mathrm{eV})$ or $386 \mathrm{~nm}$ $(3.21 \mathrm{eV}$ ), the peak of the dye curve intensity of PTP and BBQ (Exciton) respectively. This allowed the photodetachment of $\mathrm{N}_{3}^{-}$well above the $2.68 \mathrm{eV}$ threshold for this process. The frequency-doubled dissociation laser light had a 0.4 or $0.08 \mathrm{~cm}^{-1}$ bandwidth depending on whether an intracavity etalon was used, and was attenuated from $\sim 2$ $\mathrm{mJ} /$ pulse to $20-50 \mu \mathrm{J} /$ pulse to minimize power broadening in the rotationally resolved high-resolution spectra. Typically 300-500 laser shots per point were acquired and summed for total photodissociation cross section spectra. All other details are as described in $\S 2.7 .5$.

The low resolution survey scan of the $N_{3}$ predissociation spectrum is shown in Figure 4.6. The peaks are labeled according to the vibronic symmetries of the states involved. The two main peaks originate from the spin-orbit split $\bar{X}^{2} \Pi_{g}$ ground state to the excited $\tilde{B}^{2} \Sigma_{u}^{+}$state. Also labeled are sequence bands involving one quantum of bend excitation (and thus vibrational angular momentum) in both the ground and excited states. At first glance the vibrational distribution does appears not to be as cold as might be

Figure 4.6 Low resolution survey scan $(0.05 \mathrm{~nm}$ steps, corrected to vacuum wavelengths) of $\mathrm{N}_{3}$ photodissociation cross section. Transitions are labeled according to the vibronic symmetry of the states involved. 


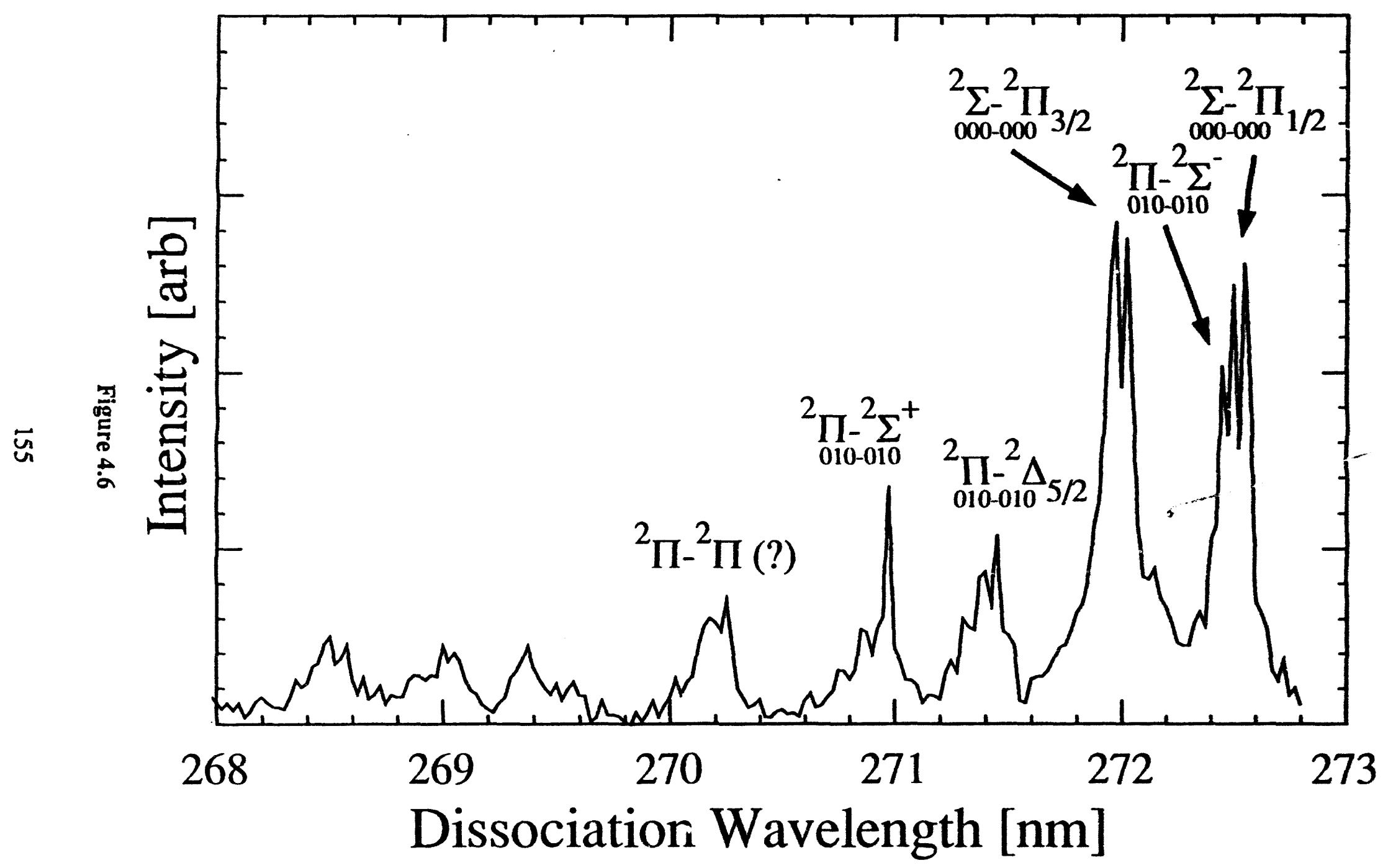


expected. However, as will become apparent below, dynamical effects probably result in a greater quantum yield for dissociation from bend-excited upper state levels. In addition, no correction to the spectra for a possible variation in the branching ratios to the different $\mathrm{N}$ atom electronic states was made. A much larger fraction of those events which proceed to form the ground state (but spin-forbidden) $\mathrm{N}\left({ }^{4} S\right.$ ) atom channel will release enough kinetic energy so that the $\mathrm{N}$ atom recoils out past the detector than will the lowest energy spin-allowed $N(2 D)$ atom channel. This will have an effect on the apparent photodissociation cross section intensity if the branching ratios to the different product channels vary with excited state vibrational level.

The exact nature of the bands below $270.5 \mathrm{~nm}$ remains unidentified. Whereas in the past there was some uncertainty due to the presence of other possible signal carriers, the mass selection feature of this experiment means they are now at least firmly associated with the $\mathrm{N}_{3}$ radical.

The high resolution spectrum of the $\tilde{B}^{2} \Sigma_{u}^{+} \leftarrow \tilde{X}^{2} \Pi_{3 / 2}$ sub-band is shown in Figure 4.7, accompanied by a simulation of the spectrum. The simulation was constructed using the ${ }^{2} \Sigma \leftarrow^{2} \Pi$ transition strengths for a diatomic molecule ${ }^{910}$ and the ground and excited state constants of Douglas and Jones. ${ }^{6}$ The peak intensities show the characteristic 2:1 nuclear spin statistics expected for $\mathrm{N}_{3}$. The spectrum is well-characterized by a Boltzmann temperature of $50 \mathrm{~K}$, confirming that rotational cooling is achieved in our source. The slightly diminished intensity of the middle portion of the spectrum relative to the simulation is likely the signature of residual saturation, although dynamical effects cannot be ruled out. Linewidths are instrument limited to $0.11 \mathrm{~cm}^{-1}$, with the laser

Figure 4.7 Upper panel: High resolution etalon scan $(0.0002 \mathrm{~nm}$ steps, conected to vacuum wavelengths) of the ${ }^{2} \Sigma_{u}^{+} \leftarrow{ }^{2} \Pi_{3 / 2}$ sub-band origin. Rotational branches are labeled using combs. Lower panel: Simulation of the spectrum using an assumed rotational temperature of $50 \mathrm{~K}$. 


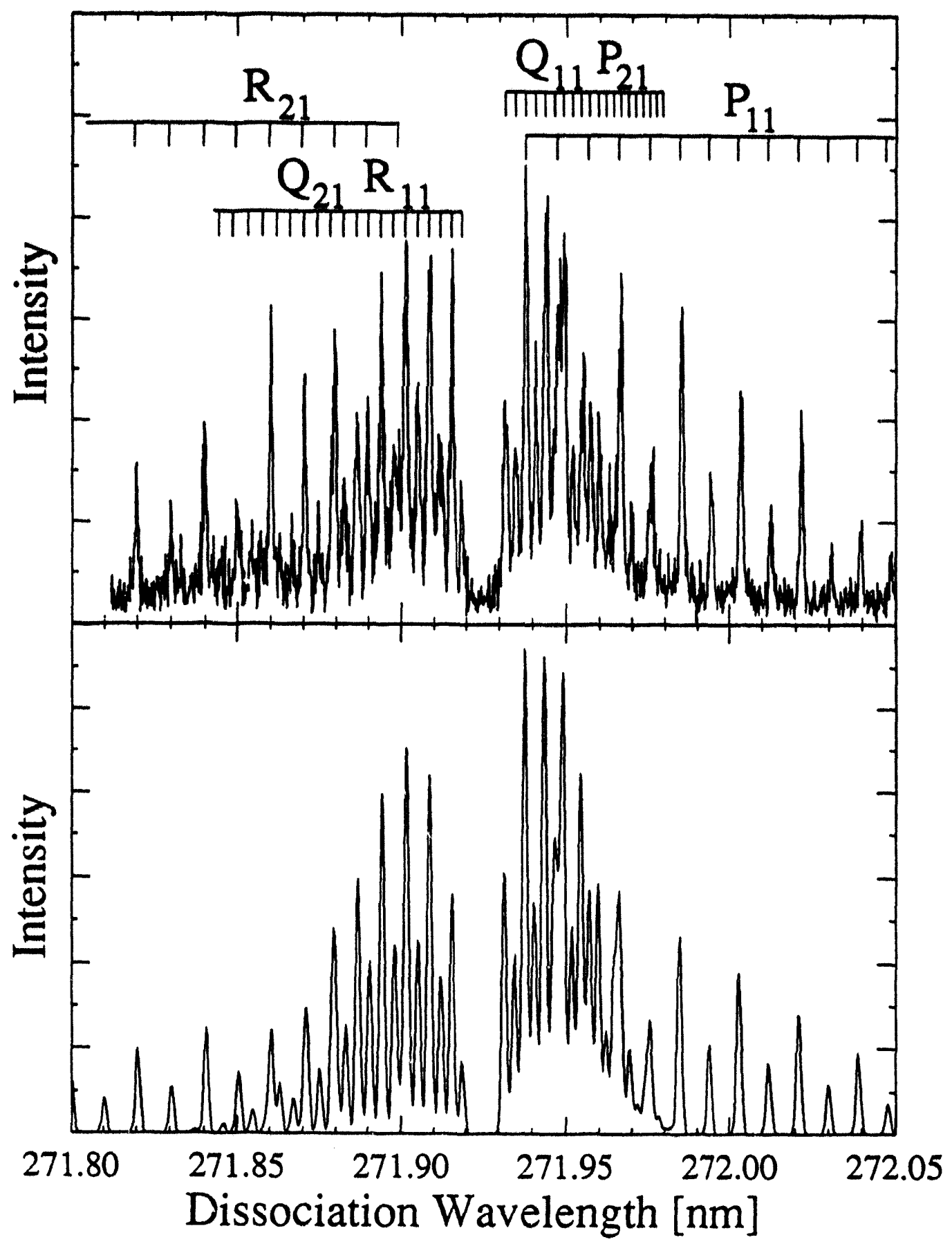

Figure 4.7 
bandwidth and Doppler broadening making the largest contributions to this value.

Once again, the primary result of the total photodissociati .. cross section studies of $\mathrm{N}_{3}$ is not the spectroscopy, which was known at least as well from absorption studies, but rather the fact that predissociation occurs throughout the first electronic absorption band. This system is also a good test of the ultimate resolution of the apparatus for total photodissociation cross section experiments, because of the relatively narrow rotational linewidths. Armed with knowledge of the total photodissociation cross sections, it is possible to perform a detailed study of the translational energy and recoil angle distributions to determine the product electronic and vibrational branching ratios as a function of $\tilde{B}^{2} \Sigma_{u}^{+}$state vibrational excitation. This is the subject of the following section.

\subsection{TPS Detection of $\mathrm{N}_{3}$ Radical Dissociation:}

'Mode Specific' Effects in the N Atom Electronic State Branching Ratio The dissociation dynamics of the $\tilde{B}^{2} \Sigma_{u}^{+}$state of $\mathrm{N}_{3}$ were then investigated using fast radical beam photodissociation coupled with the time- and position-sensitive detection technique described in previous chapters. Detailed photofragment kinetic energy and angular distributions were obtained after excitation to both the $\left(v_{1}, v_{2}, v_{3}\right)=(000)$ and (010) vibrational levels of the $\tilde{B}$ state. The branching ratio to the various $N$ atom product channels varied quite strongly depending if one quantum of bend excitation was present in the upper state.

As was shown in the previous section, the $\mathrm{N}_{3}$ radical undergoes p1 zdissociation following excitation to all accessible levels of the $\widetilde{B}^{2} \Sigma_{u}^{+}$state. At the excitation energies required to access the $\tilde{B}^{2} \Sigma_{u}^{+}$state three different $N$ atom electronic states can be produced in conjunction with ground electronic state $\mathrm{N}_{2}$ (the first electronic excited state of $\mathrm{N}_{2}$ is inaccessible at these energies). Motivation for this detailed study of the disposal of energy in the photodissociation products of the $\mathrm{N}_{3}$ radical was threefold. First, it is of interest to determine the importance of the lowest energy, but spin-forbidden, $N\left({ }^{4} S\right)+\mathrm{N}_{2}$ 
channel. Second, a comparison of dissociation product channel branching ratios depending on whether one quantum of vibration is present in the upper state may illuminate any dynamical effects as dissociation progresses. Finally, as explained in \$1.3.2, this technique results in a very direct measure of the bond dissociation energy from which an accurate heat of formation for the $\mathrm{N}_{3}$ radical can be calculated.

Figure 4.8 presents the translational energy distribution of the photofragments formed upon excitation to the (000) level of the $\tilde{B}$ state, while Figure 4.9 presents the translational energy distribution of the photofragments formed upon excitation to the (010) level of the $\tilde{B}$ state. Both figures are labeled according to the best fit for the origins of the various electronic and vibrational levels. Both distributions share certain characteristics. The translational energy distributions extend out to much higher kinetic energy release than possible for the spin-allowed channels, indicating the presence of spinforbidden products. The most intense portion of both distributions is within the lower vibrational levels of the first spin-allowed $\mathrm{N}\left({ }^{2} D\right)+\mathrm{N}_{2}$ channel. Both distributions indicate inverted and/or long vibrational distributions and peak rotational intensities well away from the vibrational origins. The most striking difference between the two translational energy distributions is the drastic change in the $N$ atom $\left({ }^{2} D\right):\left({ }^{4} S\right)$ branching ratio.

The anisotropy parameter that describes the photofragment recoil angular distributions following excitation to each of the (000) and (010) vibrational levels in the $\tilde{B}$

Figure 4.8 Center-of-mass translational energy distribution $P\left(E_{T}\right)$ obtained following excitation to the (000) level of the $\tilde{B}^{2} \Sigma_{u}^{+}$state.

Figure 4.9 Center-of-mass translational energy distribution $P\left(E_{T}\right)$ obtained following excitation to the (010) level of the $\tilde{B}^{2} \Sigma_{u}^{+}$state.

Figure 4.10 The anisotropy parameter $\beta\left(E_{\mathrm{T}}\right)$ for $\mathrm{N}_{3}$. The solid line with the diamonds shows the results for the $(000)$ band while the dashed line with circles shows the $(010)$ results. Error bars are $1 \sigma$. Vertical dashed arrows indicate the energetic thresholds of the channels involving ${ }^{2} D$ and ${ }^{2} P \mathrm{~N}$ atoms from the 000 level of the $\tilde{B}^{2} \Sigma_{u}^{+}$state. 


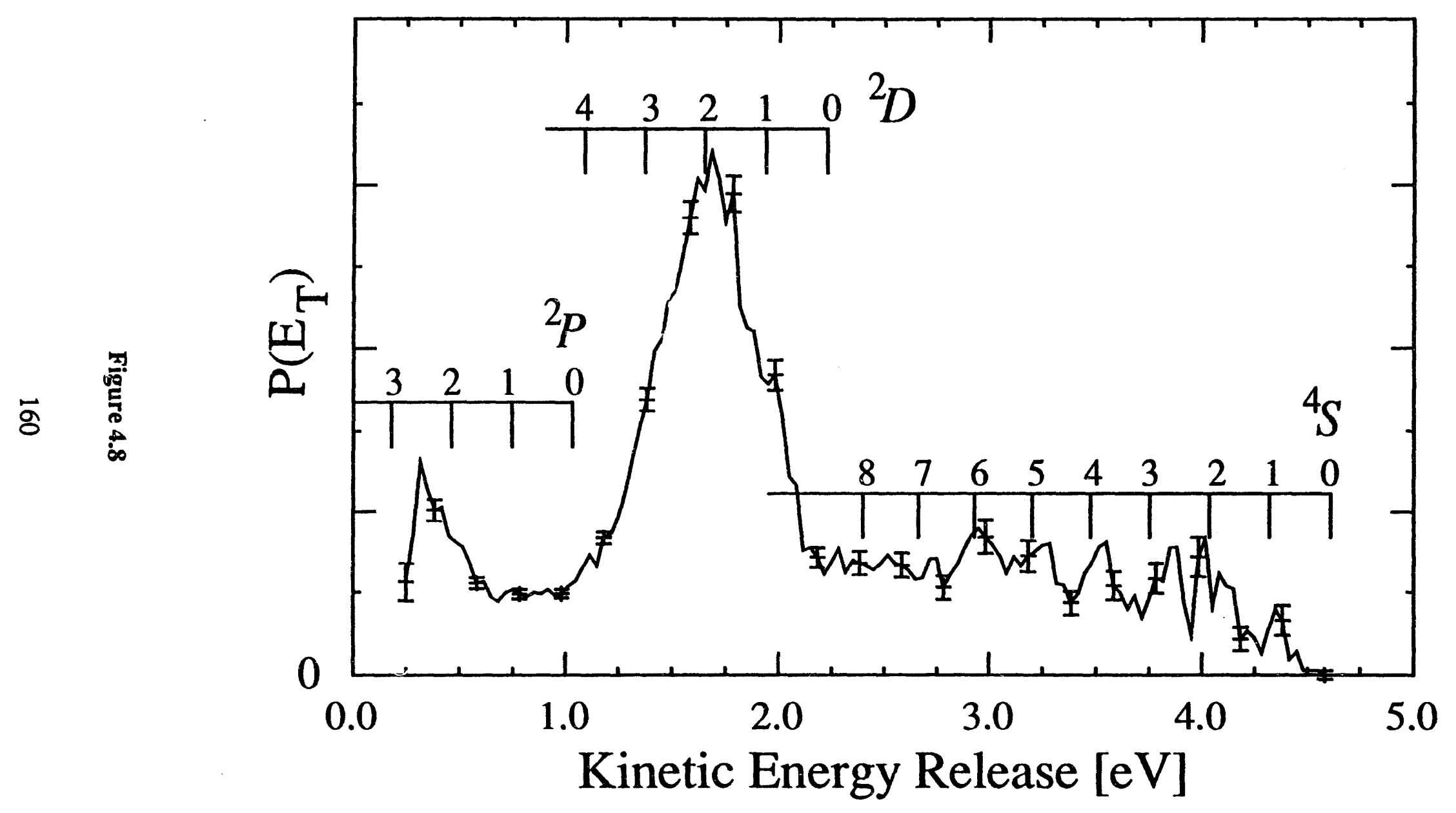




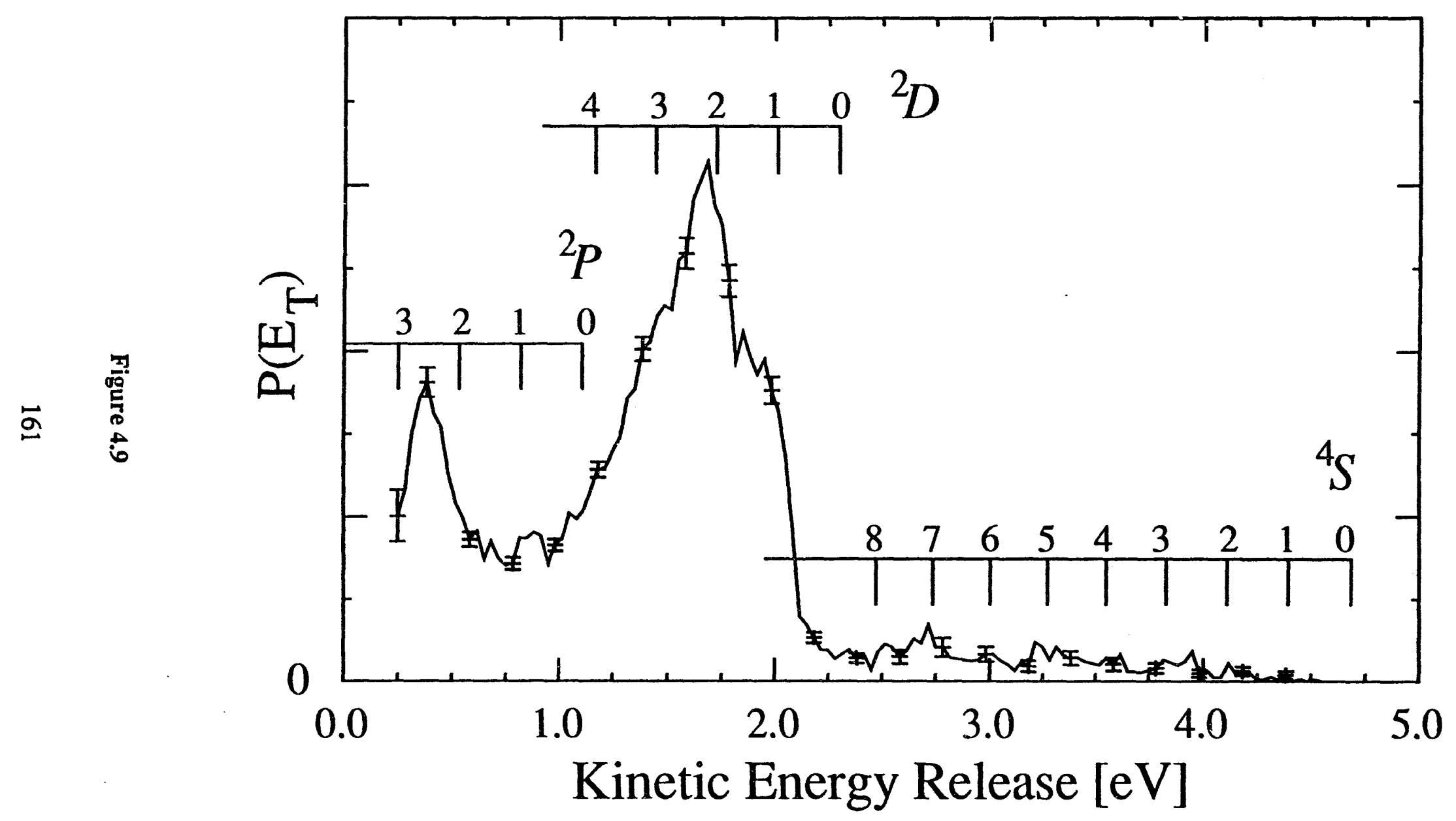




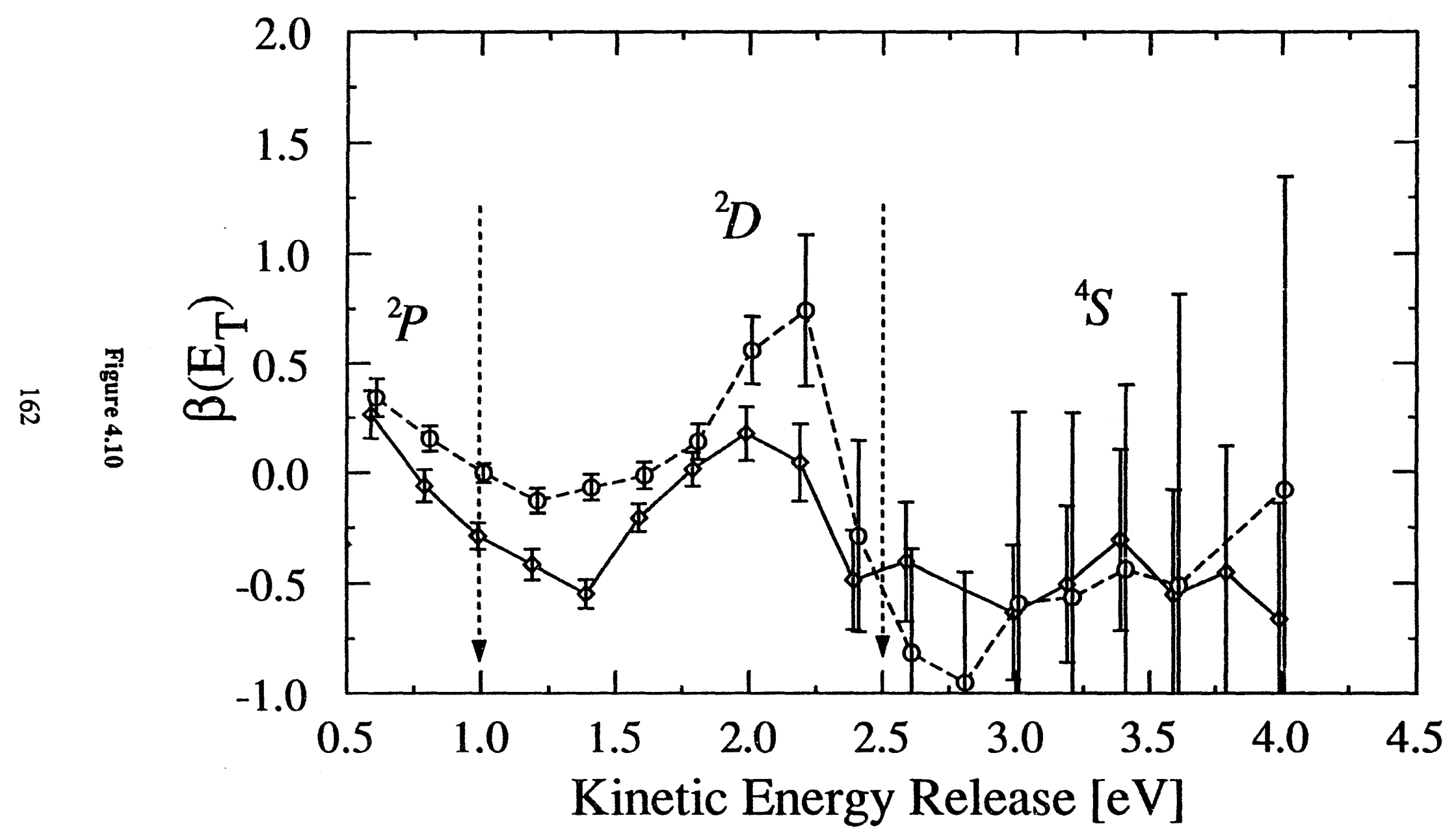


state is plotted as a function of kinetic energy release in Figure 4.10. A change in the value of $\beta$ can be observed if a comparison is made of the regions where each $\mathrm{N}$ atom electronic state dominates the translational energy distribution.

The conclusions which can be drawn about the dissociation of $\mathrm{N}_{3}$ from these results are many. Briefly, it is immediately apparent from Figures 4.8 and 4.9 that $\mathrm{N}_{3}$ dissociates to both spin-allowed and spin-forbidden products, despite the fact that at all times two spin-allowed channels are energetically accessible. As will be seen in Chapter 5, this is in contrast to the behavior of the NCO radical which dissociates predominantly to spin-allowed products once above the energetic threshold for their formation. There is, however, a dramatic change in the $\mathrm{N}$ atom $\left({ }^{2} D\right):\left({ }^{4} S\right)$ branching ratio with the addition of one quantum of bending excitation in the upper state. This, coupled with the high rotational excitation of the $\mathrm{N}_{2}$ products ( $\mathrm{J}_{\text {peak }} \approx 32$ ) required to simulate the observed translational energy distributions, is strong evidence for the participation of bent geometries in the dissociation from the $\tilde{B}$ state. The values of the energy dependent anisotropy parameter are also largely consistent with a bent geometry during dissociation, particularly in the channel producing $N\left({ }^{2} D\right)$ atoms where $\beta$ becomes positive. For a perpendicular transition such as the $\tilde{B}^{2} \Sigma^{+} \leftarrow \tilde{X}^{2} \Pi$ excitation studied here, a purely axial recoil would result in a negative anisotropy parameter.

The thermochemistry of $\mathrm{N}_{3}$ is determined in a very direct way by these experiments, independently confirming that the radical is $0.05 \mathrm{kcal} / \mathrm{mol}$ endoergic with respect to $\mathrm{N}\left({ }^{4} S\right)+\mathrm{N}_{2}$, as reflected by the labels on Figure 4.5. This value is in good agreement with that determined by Brauman and co-workers after performing proton affinity studies ${ }^{11}$ of $\mathrm{N}_{3}^{-}$and electron affinity studies ${ }^{12,13}$ of $\mathrm{N}_{3}$. 


\subsection{References}

1 (a) D. J. Leahy, D. R. Cyr, D. L. Osbom and D. M. Neumark, Chem. Phys. Lett. (in press).

(b) Full details of all $\mathrm{O}_{2}$ dissociation work will forthcoming in a full publication by D. J. Leahy, D. R. Cyr, D. L. Osborn and D. M. Neumark.

2 R. E. Continetti, D. R. Cyr, R. B. Metz, and D. M. Neumark, Chem. Phys. Lett. 182, 406 (1991); R. E. Continetti, D. R. Cyr, D. L. Osborn, D. J. Leahy, and D. M. Neumark, J. Chem. Phys. 99, 2616 (1993).

3 B. R. Lewis, J. H. Carver, T. I. Hobbs, D. G. McCoy, and H. P. F. Gies, J. Quant. Spectrosc. Rad. Trans. 20, 191 (1978); 22, 213 (1973); H. P F. Gies, S. T. Gibson, D. G. McCoy, A. J. Blake, and B. R. Lewis, ibid, 26, 469 (1981); B. R. Lewis, L. Berzins, J. H. Carver, and S. T. Gibson, J. Quant. Spectrosc. Rad. Trans. 36, 187 (1986); A. S-C. Cheung, K. Yoshino, D. E. Freeman, R. S. Friedman, A. Dalgarno, and W. H. Parkinson, J. Mol. Spectrosc. 134, 362 (1989); A. S-C. Cheung, K. Yoshino, J. R. Esmond, S. S-L. Chiu, D. E. Freeman, and W. H. Parkinson, J. Chem. Phys. 92,842 (1990); S. S-L. Chiu, A. S-C. Cheung, K. Yoshino, J. R. Esmond, D. E. Freeman, and W. H. Parkinson, J. Chem. Phys. 93, 5539 (1990).

4 Indeed, only one other recent experiment has measured correlated fine-structure state distributions without a much larger spin-orbit interaction. This was done in the photodissociation of $\mathrm{NO}_{2}$, by U. Robra, H. Zacharias, and K. H. Welge, Z. Phys. D 16, 175 (1990).

5 B. A. Thrush, Proc. R. Soc. London, Ser. A 235, 143 (1956).

6 A. E. Douglas and W. J. Jones, Can. J. Phys. 43, 2216 (1965).

7 J. A. Pople, Mol. Phys. 3, 16 (1960).

8 J. T. Hougen, J. Chem. Phys. 36, 549 (1962).

9 L. T. Earis, Phys. Rev. 48, 423 (1935).

10 I. Kovacs, Rotational structure in the spectra of diatomic molecules, (Elsevier, Amsterdam, 1969) pp. 125-131.

11 M. J. Pellerite, R. L. Jackson, and J. I. Brauman, J. Phys. Chem. 85, 1624 (1981).

12 R. L. Jackson, M. J. Pellerite, and J. I. Brauman, J. Am. Chem. Soc. 103, 1802 (1981).

13 E. Illenberger, P. B. Comita, J. I. Brauman, H.-P. Fenzlaff, M. Heni, N. Heinrich, W. Koch, and G. Frunking, Ber. Bunsenges. Phys. Chem. 89, 1026 (1985). 


\section{Chapter 5}

\section{Photodissociation of the NCO Radical}

\subsection{Introduction}

During the past ten to 15 years, a wide variety of state-selected methods have been developed to study the photodissociation of stable, closed-shell molecules. ${ }^{1}$ Ideally, these experiments enable one to map out the dissociative electronic states in the molecule, find the energy needed to break the dissociating chemical bond, and determine in detail the interactions between the departing photofragments. It is clearly of interest to extend the methods of photodissociation to the study of reactive open-shell radicals. At present, however, even the bond dissociation energies, let alone the detailed interactions among the excited electronic states, are poorly characterized in many of these species. Thus far, successful photodissociation experiments have been performed on only a handful of reactive free radicals, ${ }^{2,3,4}$ largely because of the experimental difficulties involved in preparing these species free of contaminants and in reasonably well-defined quantum states. Recently, we have demonstrated that these problems can be overcome by preparing the radical of interest via photodetachment of a mass-selected negative ion beam. ${ }^{5}$ This paper describes the application of this method to the photodissociation of the NCO radical.

The NCO radical has attracted considerable interest over the years both for its role in combustion chemistry and because of its spectroscopic complexity. NCO is believed to be an intermediate in the combustion of nitrogen containing compounds. ${ }^{6}$ It has been observed in $\mathrm{CH}_{4} / \mathrm{N}_{2} \mathrm{O}$ flames ${ }^{7}$ and is know'n to be the primary product of the $\mathrm{CN}+\mathrm{O}_{2}$ reaction. ${ }^{8}$ The spectroscopy of the ground and excited states of NCO is also of interest. The electronic transitions from the ground $\tilde{X}^{2} \Pi$ state to the low-lying $\tilde{A}^{2} \Sigma^{+}$and $\tilde{B}^{2} \Pi$ excited states were first observed in $1958^{\circ}$ and analyzed in detail by Dixon ${ }^{10,11}$ in 1960 ; 
the $\tilde{A}^{2} \Sigma^{+} \leftarrow \tilde{X}^{2} \Pi$ and $\tilde{B}^{2} \Pi \leftarrow \tilde{X}^{2} \Pi$ origins are at $440 \mathrm{~nm}$ and $315 \mathrm{~nm}$, respectively. Dixon's work on the $\tilde{A}^{2} \Sigma^{+} \leftarrow \tilde{X}^{2} \Pi$ system was followed by several optical absorption ${ }^{12.13}$ and laser-induced fluorescence ${ }^{7 a, 7 c, 14,15,16,17}$ studies. The electronic ground state has been investigated using infrared, ${ }^{12}$ electron paramagnetic resonance, ${ }^{18}$ microwave ${ }^{19}$ and far infrared laser magnetic resonance ${ }^{20}$ spectroscopy. The detailed characterization of the NCO ground state and the $\tilde{A}^{2} \Sigma^{+} \leftarrow \tilde{X}^{2} \Pi$ transition has in turn led to a better understanding of the $\mathrm{CN}+\mathrm{O}_{2}$ reaction; several groups have measured vibrational, rotational, and fine structure distributions of $\mathrm{NCO}$ produced by this reaction using laserinduced fluorescence of the $\tilde{A}^{2} \Sigma^{+} \leftarrow \tilde{X}^{2} \Pi$ transition. ${ }^{21}$

The results presented here provide new insight into the $\tilde{B}^{2} \Pi \leftarrow \tilde{X}^{2} \Pi$ system of NCO which, in contrast to the $\tilde{A}^{2} \Sigma^{+} \leftarrow \tilde{X}^{2} \Pi$ system, remains largely unassigned. This is due to Renner-Teller and Fermi resonance interactions within the $\tilde{B}{ }^{2} \Pi$ state, as well as extensive perturbations of this state by the nearby lower-lying $\tilde{A}^{2} \Sigma^{+}$ state and predissociation to $\mathrm{N}+\mathrm{CO}$. In fact, one of the key unresolved issues with respect to the $\tilde{B}$ 2 $\Pi$ state, and certainly the one which motivated the current study, is the identification of the onset of predissociation from the $\tilde{B}^{2} \Pi$ state and the characterization of the dissociation products. Dixon ${ }^{11}$ originally observed spectral broadening beginning at the transition to the $v_{1}=2, v_{2}=0, v_{3}=0$, or $(200)$, level $r t 33,700 \mathrm{~cm}^{-1}$. This broadening was attributed to predissociation resulting in the spin-allowed $\mathrm{N}\left({ }^{2} D\right)+\mathrm{CO}$ products, thereby giving an upper bound of $40 \mathrm{kcal} / \mathrm{mol}$ for $\mathrm{D}_{0}(\mathrm{~N}-\mathrm{CO})$, the bond dissociation energy of $\mathrm{NCO}$ to form ground state (but spin-forbidden) $\mathrm{N}\left({ }^{4} S\right)+\mathrm{CO}$ products. The corresponding heat of formation is $\Delta_{f} H_{298 \mathrm{~K}}^{0}(\mathrm{NCO})=45 \mathrm{kcal} / \mathrm{mol} .\left[\mathrm{D}_{0}(\mathrm{~N}-\mathrm{CO})=85.3 \mathrm{kcal} / \mathrm{mol}\right.$ $\left[=\Delta_{f} H_{298 \mathrm{~K}}^{0}(\mathrm{~N})+\Delta_{f} H_{298 \mathrm{~K}}^{0}(\mathrm{CO})\right]-\Delta_{f} H_{298 \mathrm{~K}}^{0}(\mathrm{NCO})$, see Discussion.] In a subsequent vacuum ultraviolet photolysis study of $\mathrm{HNCO}$ in which the threshold for production of the NCO Ã $2 \Sigma^{+}$state was measured, Okabe 22 determined $\Delta_{f} H_{298 \mathrm{~K}}^{0}(\mathrm{NCO})$ to be $37 \mathrm{kcal} / \mathrm{mol}$. This implies that predissuciation to $\mathrm{N}\left({ }^{2} D\right)+C O$ can occur only above the $(400)$ level of 
the $\tilde{B}$ ' $\Pi$ state. Okabe's value for $\Delta_{f} H_{298 \mathrm{~K}}^{0}(\mathrm{NCO})$ is consistent with that obtained by Coombe $^{2}$ in a recent study of NCO photodissociation at $193 \mathrm{~nm}$. Crosley ${ }^{23}$ found that the fluorescence lifetime of the $\tilde{B}^{2} \Pi$ state was dramatically shorter for the (100) vibrational level ( $\leq 10 \mathrm{~ns})$ than for the $(000)$ level ( $63 \mathrm{~ns})$. This was interpreted to mean that the spinallowed $N\left({ }^{2} D\right)+C O$ threshold occurs between the (000) and (100) levels of the $\tilde{B}^{2} \Pi$ state, yielding $D_{0}(\mathrm{~N}-\mathrm{CO}) \leq 39 \mathrm{kcal} / \mathrm{mol}$ and $\Delta_{f} H_{298 \mathrm{~K}}^{0}(\mathrm{NCO}) \geq 48 \mathrm{kcal} / \mathrm{mol}$.

The observation that the $\mathrm{CN}+\mathrm{O}_{2} \rightarrow \mathrm{NCO}+\mathrm{O}$ reaction occurs with no appreciable activation barrier ${ }^{24}$ means that $\Delta_{f} H_{298 \mathrm{~K}}^{0}(\mathrm{NCO}) \leq 44 \mathrm{kcal} / \mathrm{mol}$, and this, in conjunction with the considerable $\mathrm{NCO}$ bending excitation produced by the $\mathrm{CN}+\mathrm{O}_{2}$ reaction ${ }^{21 b . c}$ lends support to Okabe's value for $\Delta_{f} H_{298}^{0} \mathrm{~K}(\mathrm{NCO})$. However, the extraction of $\Delta_{f} H_{298}^{0} \mathrm{~K}(\mathrm{NCO})$ from his measurement uses the heat of formation of $\mathrm{HNCO}$, and this in turn requires an accurate value for $\Delta_{f} H_{298 \mathrm{~K}}^{0}(\mathrm{NH})$, for which the spread in recently reported values is several $\mathrm{kcal} / \mathrm{mol}$. The most recent determination of $\Delta_{f} H_{298}^{0} \mathrm{~K}(\mathrm{HNCO})$ by Chandler, ${ }^{25}$ when combined with Okabe's work, yields a revised value of $36.1 \mathrm{kcal} / \mathrm{mol}$ for $\Delta_{f} H_{298 \mathrm{~K}}^{0}(\mathrm{NCO})$; this is the currently accepted literature value. ${ }^{26}$ However, an independent determination of $\Delta_{f} H_{298}^{0} \mathrm{~K}(\mathrm{NCO})$ from a direct measurement of the $\mathrm{N}-\mathrm{CO}$ bond dissociation energy is desirable; an accurate value is vital in modeling reaction schemes involving the NCO radical. In addition, one would like to understand the origin of the widely disparate values discussed above.

The NCO photodissociation study reported here addresses all of these issues. We have investigated the $\tilde{B}^{2} \Pi \leftarrow \bar{X}^{2} \Pi$ electronic transition between 316 and $234 \mathrm{~nm}$. The salient results are as follows. We show unambiguously that all of the vibrational levels of the NCO $\tilde{B} 2 \Pi$ state dissociate. In addition, translational energy measurements on the photofragments show that the spin-allowed $\mathrm{N}\left({ }^{2} D\right)+\mathrm{CO}$ channel is accessible only for vibrational levels higher than the (600) level in the $\tilde{B}^{2} \Pi$ state (greater than $20.3 \mathrm{kcal} / \mathrm{mol}$ above the electronic origin), while the lower vibrational levels undergo predissociation to 
the spin-forbidden $\mathrm{N}\left({ }^{4} S\right)+\mathrm{CO}$ channel. We believe that it is this spin-forbidden dissociation which led to the high values of $\Delta_{f} H_{298 \mathrm{~K}}^{0}(\mathrm{NCO})$ inferred by Dixon ${ }^{11}$ and Crosley. ${ }^{23}$ Finally, our photofragment kinetic energy release measurements near the $\mathrm{N}\left({ }^{2} D\right)+\mathrm{CO}$ threshold yield a value of $30.5 \pm 1 \mathrm{kcal} / \mathrm{mol}$ for $\Delta_{f} H_{298 \mathrm{~K}}^{0}(\mathrm{NCO})$.

\subsection{Experimental Approach}

Our experiments are based on the idea that, since free radicals typically have positive electron affinities, one can generate a very clean source of radicals by laser photodetachment of a mass-selected beam of the precursor negative ion. The resulting radical beam is then dissociated with a second laser. Since the initial kinetic energy of the ion beam is $8 \mathrm{keV}$, the fragments resulting from photodissociation of the radical have high kinetic energies in the laboratory frame of reference and can be directly detected with high $(\sim 50 \%)$ efficiency using a microchannel plate detector. The overall experiment in the case of NCO photodissociation is

$$
\mathrm{NCO}^{-} \stackrel{h v_{1}}{\longrightarrow} \mathrm{NCO}+e^{-} \stackrel{h v_{2}}{\longrightarrow} \mathrm{N}+\mathrm{CO} \text {. }
$$

The higher energy $\mathrm{O}+\mathrm{CN}$ channel is not accessible at the dissociation wavelengths used in this study.

Two types of experiments can be performed. In photodissociation cross section measurements, we determine the total photofragment signal as a function of $\mathrm{hv}_{2}$ and thereby map out the dissociative electronic transitions of the NCO radical. In the case of the $\tilde{B}^{2} \Pi \leftarrow \tilde{X}^{2} \Pi$ transition in NCO, one expects a highly structured spectrum since the $\tilde{B}^{2} \Pi$ state undergoes predissociation rather than direct dissociation. Aiternatively, at fixed $\mathrm{h} v_{2}$, we can measure the photofragment time-of-flight (TOF) distribution at the 
detector and determine the maximum kinetic energy release and (more approximately) the translational energy distribution of the fragments.

The fast radical beam photodissociation spectrometer used in this work is shown in Figure 2.1. The operation of this instrument is described in detail in Chapter 2, so consequently only the most pertinent features will be reviewed here as the details unique to the study of NCO are presented.

Negative ions are generated using the pulsed molecular beam/electron gun source developed by Lineberger and coworkers ${ }^{27}$ and used in several of our other investigations. ${ }^{28}$ To make $\mathrm{NCO}^{-}$, neon (10 psig) is bubbled through benzyl isocyanate [Pfaltz \& Bauer] at room temperature and the resulting gas mixture is expanded through a piezoelectric pulsed molecular beam valve ${ }^{29}$ operating at a $50 \mathrm{~Hz}$ repetition rate. A $1 \mathrm{keV}$ electron beam intersects the free jet expansion downstream of the valve orifice, forming NCO- by dissociative attachment of an electron to the benzyl isocyanate. (A similar scheme was first used by Illenberger et al. to generate $\mathrm{N}_{3}^{-}$from benzyl azide. ${ }^{30}$ ) Since the anions are formed in the continuum flow region of the free jet expansion, they experience significant vibrational and rotational cooling.

The ions pass through a skimmer and are accelerated to $8 \mathrm{keV}$. They then pass through a pulsed high voltage switch ${ }^{31}$ which enables us to run an $8 \mathrm{keV}$ ion beam through the instrument while maintaining the source and detector regions at ground potential. The ions are then mass-selected using a coaxial, beam-modulation TOF mass spectrometer; ${ }^{32}$ this design induces a negligible kinetic energy spread in the ion beam, an important and desirable feature for the photofragment TOF measurements described below.

The ion beam passes through a $1 \mathrm{~mm}$ diameter aperture into the photodetachment region. Here, the ions are crossed by an excimer-pumped dye laser beam (Lambda Physik LPX 210i and FL3002, PTP dye from Exciton) timed to intercept only the ion mass of interest and operating at $339 \mathrm{~nm}$ (nearly the peak of the PTP dye curve). The 
photodetachment wavelength is chosen to allow production of only the ground vibrational state of the neutral free radical, as discussed in $\S 1.4 .2$. The photoelectron spectrum ${ }^{33}$ of $\mathrm{NCO}^{-}$indicates that at $339 \mathrm{~nm}$, no excited vibrational states of the neutral free radical will be formed from the ground vibrational state of the ion, so the vibrational distribution attained during the supersonic expansion is preserved. Photodetached electrons are collected and detected using a microchannel plate (MCP) detector, the resulting signal is stored for subsequent normalization of the photodissociation spectrum. Any undetached anions are deflected out of the beam, leaving a mass-selected, fast beam of neutral free radicals. These pass through another $1 \mathrm{~mm}$ collimating aperture and enter the photodissociation and detection region.

In this region, the radicals are crossed with the laser beam from a second excimerpumped dye laser system, the output of which is doubled using the appropriate doubling crystal. The dyes used to cover the broad range of dissociation wavelengths (from 632 to $468 \mathrm{~nm}$ in the fundamental wavelength) were R640, R6G, R610, R590, C540A, C503 and C480 (Exciton). Laser pulse energies were typically $1-2 \mathrm{~mJ}$ after frequency-doubling, and the linewidth of the doubled light was $0.4 \mathrm{~cm}^{-1}$. This is what determines the resolution of our spectra; the Doppler broadening in our experiment is only $0.02 \mathrm{~cm}^{-1}$. Depending on the arrangement of right angle prisms used to direct the laser beam into the spectrometer, the laser polarization direction could be made either parallel or perpendicular to the radical beam axis.

The resulting photofragments are detected by a $40 \mathrm{~mm}$ diameter MCP which can be placed either 67.8 or $101.1 \mathrm{~cm}$ downstream of the photodissociation region. Any neutrals that strike this detector have sufficient laboratory kinetic energy to be detected with high efficiency. The center of this detector is shielded by a $3.0 \mathrm{~mm}$ wide beam block to prevent undissociated NCO radicals from impinging on the detector face, while the 
$\mathrm{N}+\mathrm{CO}$ fragments typically have enough center-of-mass translational energy to miss the beam block but not so much energy that they miss the detector entirely.

In photodissociation cross section measurements, the photofragment yield is determined as a function of the dissociation dye laser wavelength. At each wavelength, the MCP signal is measured with an analog-to-digital converter interfaced to a microcomputer. A step size of $0.02 \mathrm{~nm}$, with 500-1000 shots per point is typically used to gather a survey scan, which provides sufficient resolution for the observation of rotational contours of NCO bands. Where strong photodissociation signal is observed, scans were taken with a step size of $0.001 \mathrm{~nm}$ and a laser pulse energy of $100 \mu \mathrm{J}$ to minimize power broadening.

In photofragment TOF experiments, the photodissociation laser wavelength is fixed while the photofragment MCP signal is collected and summed over $1-4 \times 10^{4}$ laser shots by a $200 \mathrm{MHz}$ LeCroy transient digitizer interfaced to a microcomputer. TOF spectra were taken with both parallel and perpendicular laser polarization orientations at several wavelengths in order to determine the photofragment energy and angular distribution. For comparison, TOF spectra of the undissociated parent radical were obtained by blocking the dissociation laser and translating the MCP detector so that the radical beam missed the beam block and hit the detector.

\subsection{Results}

Figure 5.1 shows the compilation of our photodissociation cross section measurements ( $0.02 \mathrm{~nm}$ step size) on the $\tilde{B}{ }^{2} \Pi \leftarrow \tilde{X}^{2} \Pi$ system over a total range of $82 \mathrm{~nm}$, from 316 to $234 \mathrm{~nm}$. These measurements cover a wider wavelength range than

Figure 5.1 Compilation of survey scans $\left(0.02 \mathrm{~nm}\right.$ step size) of the NCO $\tilde{B}{ }^{2} \Pi \leftarrow \tilde{X}^{2} \Pi$ photodissociation cross section. Progressions in the $v_{1}$ vibration and the $v_{3}$ vibration are indicated. The spectrum shows that the lowest levels of the $\tilde{B}{ }^{2} \Pi$ state undergo predissociation. Asterisks (*) illustrate dissociation wavelengths where TOF data presented below were obtained. 


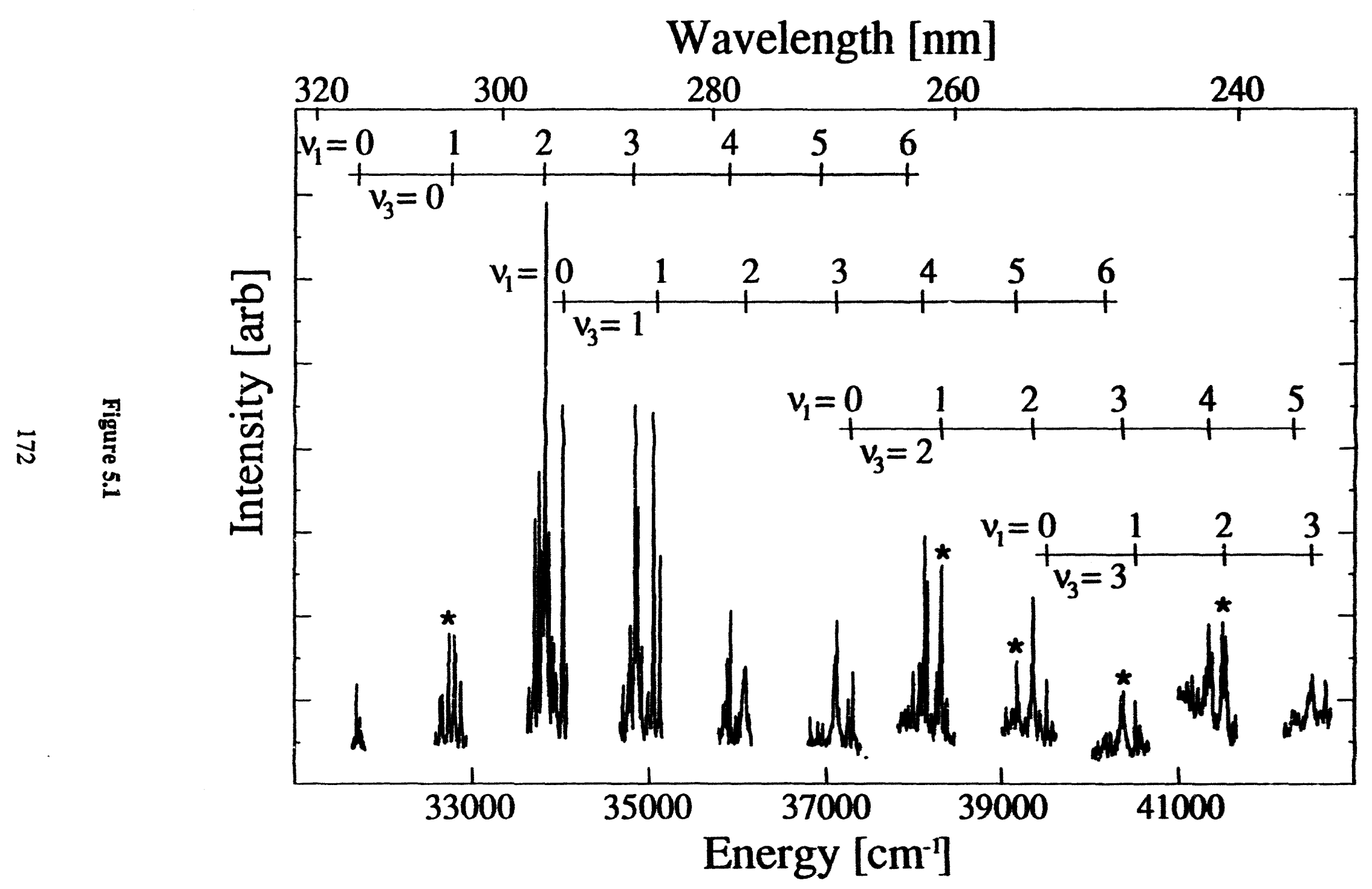


Dixon's original work ${ }^{11}$ and are at higher resolution than the matrix isolation spectra of Milligan and Jacox. ${ }^{12}$ Preliminary scans, at a lower resolution than Figure 5.1, across the entire wavelength range enabled us to concentrate further scans in the regions where predissociation signal was observed. Therefore, the apparent gaps in our survey spectrum are regions that were found not to contain peaks of significant intensity. The intensity of the signal presented here is not normalized to laser power, since at the laser fluence customarily used for these survey scans $\left(100-200 \mathrm{~mJ} / \mathrm{cm}^{2}\right)$ the transitions could have been saturated.

To the extent that the spectrum in Figure 5.1 can be assigned, it appears to consist of progressions in the $v_{1}$ and $v_{3}$ vibrational modes of the $\bar{B} 2 \Pi$ state with fundamental frequencies of approximately $1047 \mathrm{~cm}^{-1}$ and $2303 \mathrm{~cm}^{-1}$, respectively. (These values were obtained in Dixon's study. $\left.{ }^{11}\right)$ The assignments for the location of the various $\left(v_{1} O v_{3}\right)$ vibrational levels of the $\tilde{B}{ }^{2} \Pi$ state are indicated in Figure 5.1. An enlargement of the spectral region in Figure 5.1 containing the $(100) \leftarrow(000)$ band is shown in Figure 5.2. Figure 5.3 shows a higher resolution scan (laser step size $=0.001 \mathrm{~nm}$ ) of the sub-bands labeled (d) and (e) in Figure 5.2; the more intense sub-band (d) is tie main ${ }^{2} \Pi_{3 R^{-}}{ }^{2} \Pi_{3 / 2}$ transition, which was one of the two sub-bands analyzed by Dixon in his original work on this system. Line widths in Figure 5.3 are instrument limited to $\cong 0.4 \mathrm{~cm}^{-1} \mathrm{FWHM}$, corresponding to the laser resolution.

Figure 5.2 Survey scan in the region of the $\tilde{B}^{2} \Pi(100) \leftarrow \tilde{X}^{2} \Pi(000)$ band. Labels a) through $f$ ) indicate individual sub-bands; $b$ ) and d) are the previously assigned (ref. 11) ${ }^{2} \Pi_{1 / 2} \leftarrow^{2} \Pi_{1 / 2}$ and ${ }^{2} \Pi_{3 / 2} \leftarrow^{2} \Pi_{3 / 2}$ sub-bands, while the other peaks have been tentatively assigned here as $(020) \leftarrow(000)$ sub-bands: a) $\mu-2 \Pi_{1 / 2} \leftarrow 2 \Pi_{1 / 2}$, c) $\mu-2 \Pi_{3 R} \leftarrow 2 \Pi_{3 / 2}$, e) $k^{2} \Pi_{1 / 2} \leftarrow^{2} \Pi_{1 / 2}$, and f) $\kappa^{2} \Pi_{3 / 2} \leftarrow^{2} \Pi_{3 / 2}$. These transitions are shown schematically in Figure 5.6.

Figure 5.3 Finer scan ( $0.001 \mathrm{~nm}$ step size) of sub-bands d) and e) in Figure 5.2. See Figure 5.6 for assignments. The $P, Q$ and $R$ branches in the two sub-bands are labeled. 


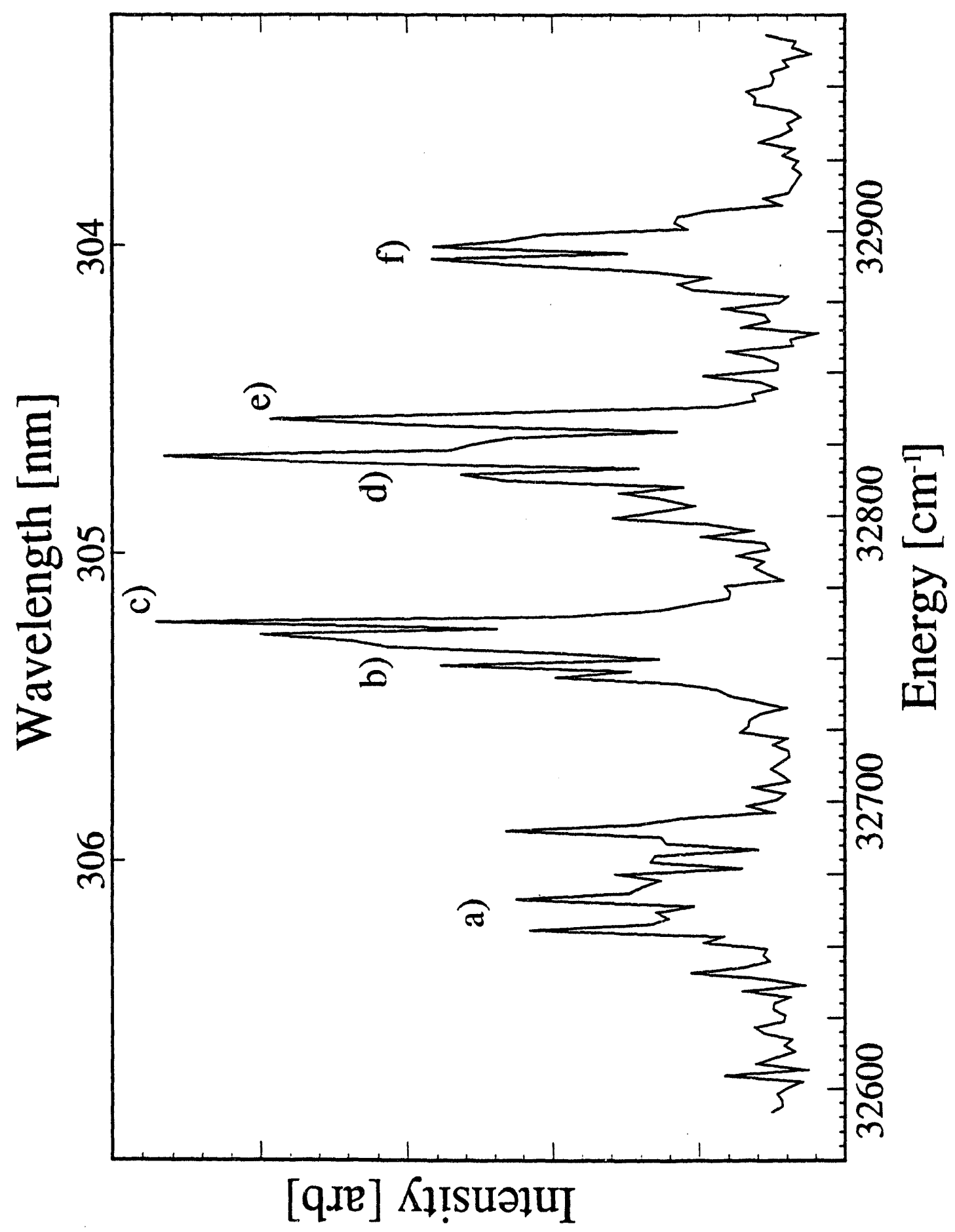

Figure 5.2 


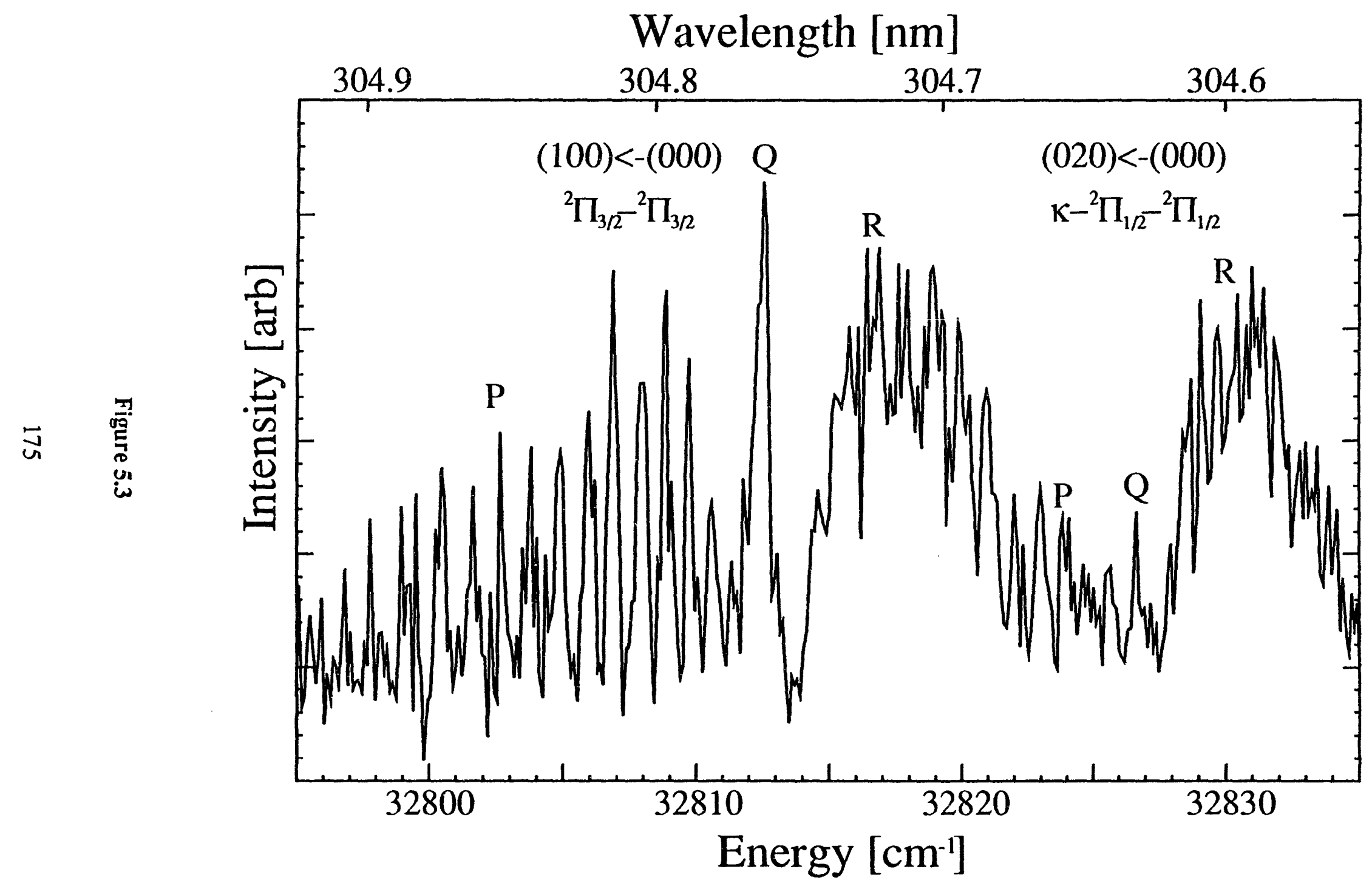


Since we observe signal only when NCO dissociates during the flight time between the photodissociation volume and the photofragment detector (about $5.27 \mu \mathrm{s}$ for a $101.1 \mathrm{~cm}$ flight length), the spectrum in Figure 5.1 shows that the $\tilde{B}^{2} \Pi$ state predissociates over the entire $\tilde{B}^{2} \Pi \leftarrow \tilde{X}^{2} \Pi$ system. Predissociation is observed even from the $\tilde{B}$ state $(000)$ level, although the intensity of the photofragment signal was considerably less than for the bands further to the blue. This differs from the $\tilde{B} 2 \Pi \leftarrow$ $\bar{X}^{2} \Pi$ absorption spectrum, in which transitions to the $(000)$ level appear to be at least as intense as the transitions to higher levels. The implications of these observations for the NCO dissociation dynamics and energetics are discussed below.

Photofragment TOF spectra were taken at dissociation wavelengths of $305.23 \mathrm{~nm}$, $260.69 \mathrm{~nm}, 255.20 \mathrm{~nm}, 247.58 \mathrm{~nm}$, and $240.83 \mathrm{~nm}$ with the detector $101.1 \mathrm{~cm}$ from the photodissociation volume and the laser polarization such that the electric field vector $\bar{E}$ was parallel to the direction of the radical beam propagation. These wavelengths correspond to the peaks marked with an $\left(^{*}\right)$ in Figure 5.1. The five experimental spectra are displayed in Figure 5.4. The narrow peak shown in Figure 5.4 b) is a typical undissociated radical beam TOF profile, which was obtained to allow evaluation of the energy spread in the undissociated radical beam for incorporation into simulations of the dissociation spectra. In addition, the TOF spectra at $260.69 \mathrm{~nm}, 255.20 \mathrm{~nm}$, and $247.58 \mathrm{~nm}$ were taken with the detector $67.8 \mathrm{~cm}$ from the photodissociation volume with the laser polarization parallel and perpendicular to the radical beam axis. These are shown in Figure 5.5. The spectra obtained with the two orthogonal laser polarizations are very

Figure 5.4 TOF data (circles) obtained with a flight length of $101.1 \mathrm{~cm}$ at five photodissociation wavelengths as indicated. Various simulations (solid, dashed lines) are discussed in the text.

Figure 5.5 TOF data (circles) obtained with a flight length of $67.8 \mathrm{~cm}$ at three wavelengths, with the dissociation laser output polarized both parallel and perpendicular to the ion beam axis as indicated. Various simulations (solid, dashed lines) are discussed in the text. 


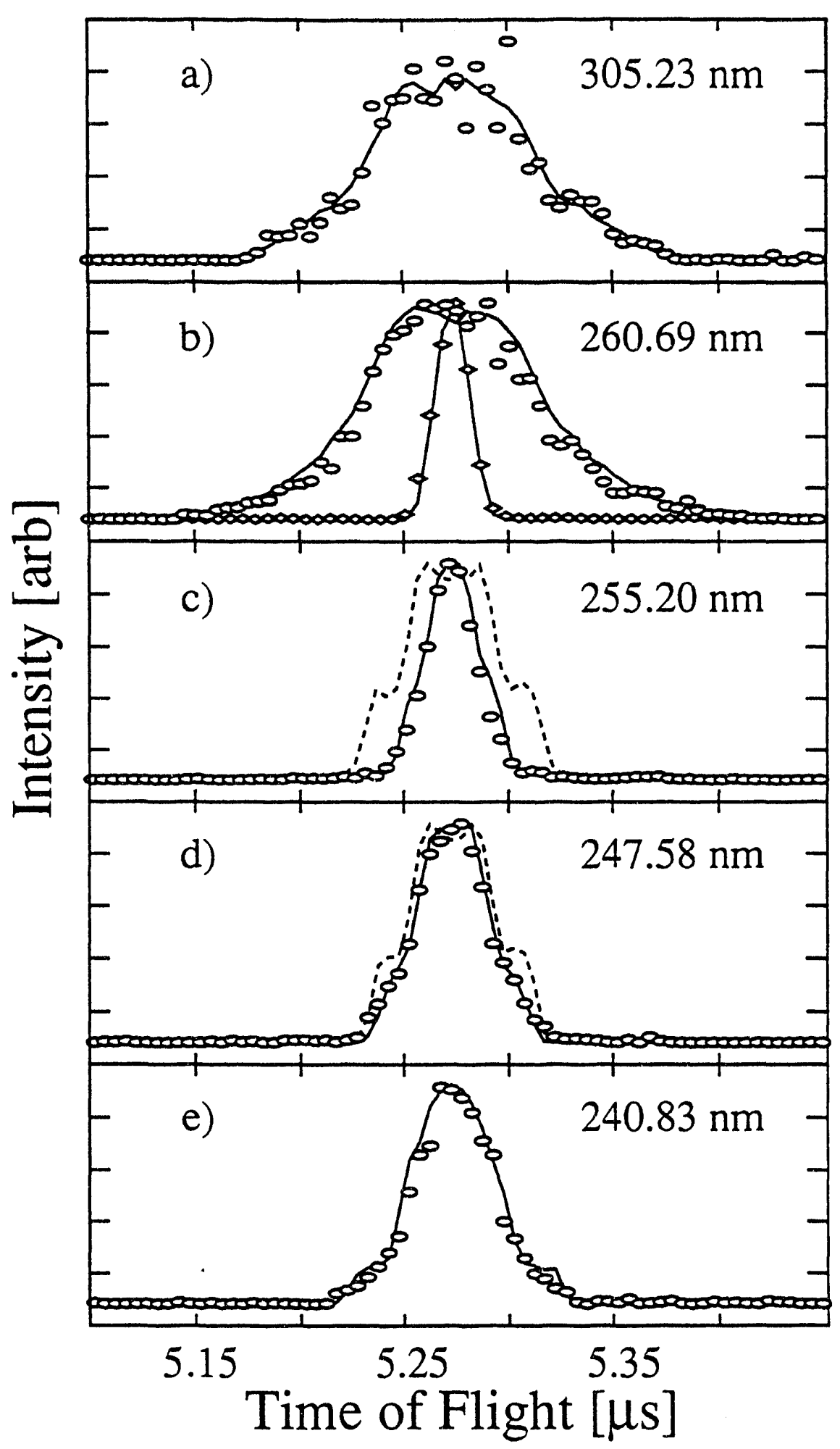

Figure 5.4 


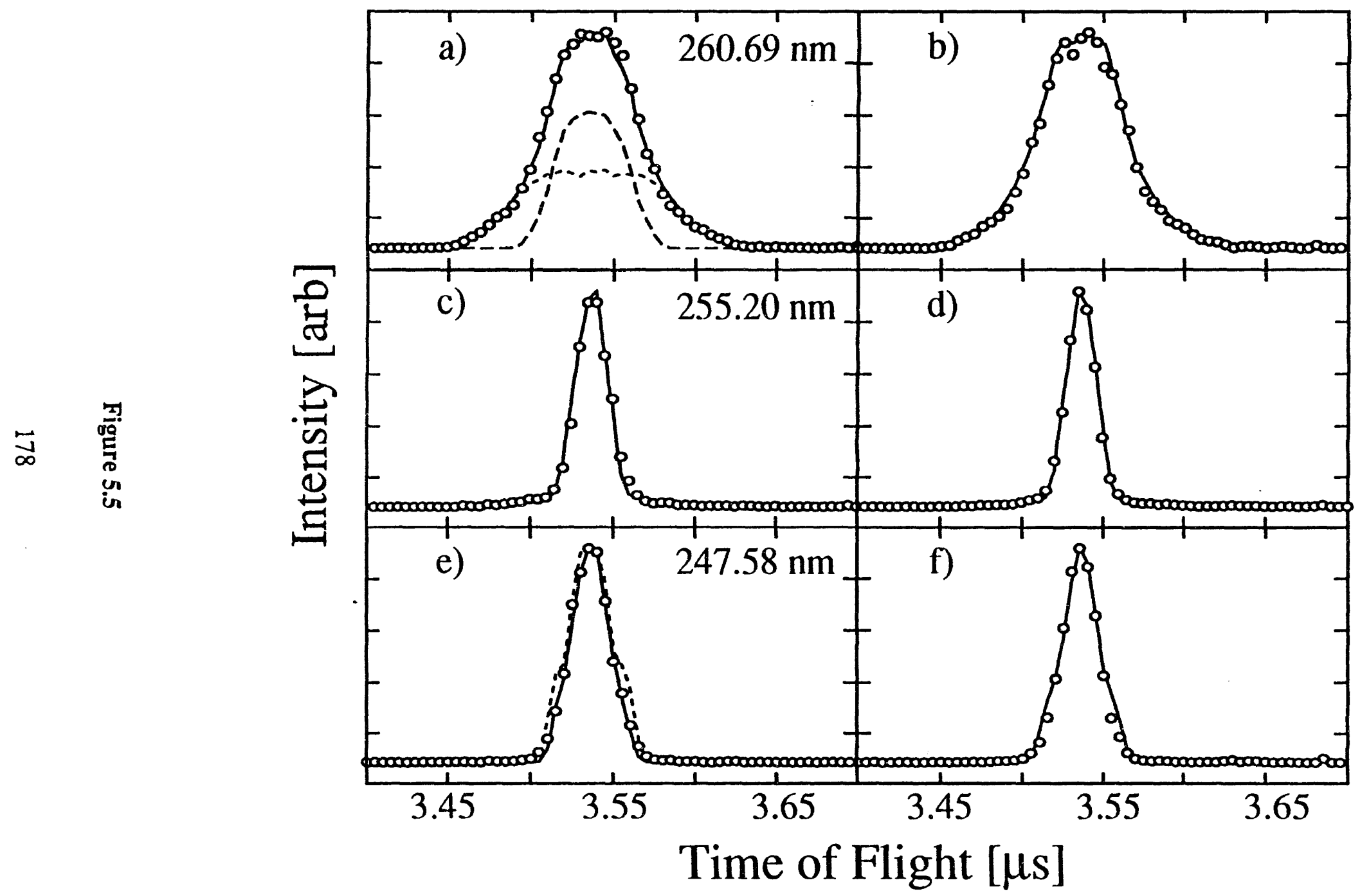


similar, implying a nearly isotropic photofragment angular distribution. The most striking result seen in both figures is the abrupt narrowing of the TOF distribution between 260.69 and $255.20 \mathrm{~nm}$, which suggests that a new dissociation channel opens in that wavelength interval.

\subsection{Analysis}

\subsubsection{NCO Spectroscopy}

The $\tilde{B}^{2} \Pi \leftarrow \tilde{X}^{2} \Pi$ system in NCO has proven notoriously difficult to assign. The $\tilde{X}^{2} \Pi$ state vibrational energy level structure is complicated by Renner-Teller coupling and Fermi resonances between the $v_{1}$ and $v_{2}$ modes. These effects are also expected in the $\tilde{B}^{2} \Pi$ state along with perturbations from the $\tilde{A}^{2} \Sigma^{+}$state. In Dixon's original spectrum, ${ }^{11}$ only the $(100) \leftarrow(000)$ band had enough extended rotational structure and was sufficiently unperturbed to permit any rotational analysis; this led to an identification of the main ${ }^{2} \Pi_{1 / 2}$ $\leftarrow^{2} \Pi_{1 / 2}$ and ${ }^{2} \Pi_{3 / 2} \leftarrow 2 \Pi_{3 / 2}$ sub-bands. A rotational analysis of the $(000) \leftarrow(000)$ band was obtained only recently in a double resonance experiment. ${ }^{34}$ Dixon found that the bands to the blue of the $(100) \leftarrow(000)$ band were broadened, presumably by predissociation, preventing any rotational analysis. Although these higher energy bands can be tentatively assigned to progressions in the $v_{1}$ and $v_{3}$ modes of the $\tilde{B}$ 2 $\Pi$ state, the detailed vibrational structure within each band seen in Figure 5.1 and in Reference 11 remains largely unexplained. In this section we consider what additional information can be gleaned about the spectroscopy of the $\tilde{B}^{2} \Pi \leftarrow \tilde{X}^{2} \Pi$ transition from our photodissociation cross section measurements.

The rotationally-resolved spectrum in Figure 5.3 is a section of the $(100) \leftarrow(000)$ band. The main $P, Q$ and $R$ branches noted are from the previously assigned ${ }^{2} \Pi_{3 / 2} \leftarrow 2 \Pi_{3 / 2}$ sub-band. A second, less intense transition is also shown just to the blue of this main subband, and the $\mathrm{P}$ branch associated with this transition extends into the $\mathrm{R}$ branch head of 
the main sub-band. In comparison to Dixon's spectrum, the intensity distribution of the more intense band in Figure 5.3 reflects a substantially lower rotational temperature for the NCO radicals. The $\mathrm{R}$ and $\mathrm{P}$ branches in Figure 5.3 fall off in intensity at considerably lower $\mathrm{J}$ values. In addition, we observe a strong $\mathrm{Q}$ branch, while none was seen in his spectrum; in a $\Pi \leftarrow \Pi$ ransition the intensity of the $Q$ branch falls off rapidly with increasing $\mathrm{J}$. The lower rotational temperature in our experiment is not too surprising, because our radicals are generated by photodetachment of a jet-cooled anion beam as opposed to flash photolysis. Simulations of our spectrum indicate a rotational temperature of about $50 \mathrm{~K}$.

In the absence of perturbations or sequence bands, the $(100) \leftarrow(000)$ band should consist of only two sub-bands (the aforementioned $2 \Pi_{1 / 2} \leftarrow 2 \Pi_{1 / 2}$ and ${ }^{2} \Pi_{3 / 2} \leftarrow 2 \Pi_{3 / 2}$ transitions) whose origins are separated approximately by $\left|A^{\prime}-A^{\prime \prime}\right|=18.9 \mathrm{~cm}^{-1}$, where $\mathrm{A}^{\prime}=-76.6 \mathrm{~cm}^{-1}$ and $\mathrm{A}^{\prime \prime}=-95.5 \mathrm{~cm}^{-1}$ are the spin-orbit coupling constants in the $\tilde{B}{ }^{2} \Pi$ and $\bar{X}^{2} \Pi$ states of $\mathrm{NCO}$, respectively. ${ }^{13.34}$ Instead, Figure 5.2 shows four well-separated sub-bands labeled (a), (b), (d), and (f), with approximate origins at $32,665 \mathrm{~cm}^{-1}$, $32,750 \mathrm{~cm}^{-1}, 32,814 \mathrm{~cm}^{-1}$, and $32,890 \mathrm{~cm}^{-1}$, respectively. In addition, as mentioned above, there is an additional sub-band just to the blue of the main ${ }^{2} \Pi_{3 / 2} \leftarrow 2 \Pi_{3 / 2}$ band at $32,829 \mathrm{~cm}^{-1}$ (labeled (e)), and a higher resolution scan of the main ${ }^{2} \Pi_{1 / 2} \leftarrow^{2} \Pi_{1 / 2}$ sub-band shows a similar sub-band at $32,760 \mathrm{~cm}^{-1}$ (labeled (c) in Figure 5.2). Thus, the (100) (000) band consists of at least six sub-bands; only two of these have been assigned: (b) to the main ${ }^{2} \Pi_{3 / 2} \leftarrow 2 \Pi_{3 / 2}$ sub-band and (d) to the $2 \Pi_{1 / 2} \leftarrow 2 \Pi_{1 / 2}$ sub-band. However, the spliting between the origins of sub-bands (b) and (d) is $64 \mathrm{~cm}^{-1}$, rather than $19 \mathrm{~cm}^{-1}$ as expected from the difference of spin-orbit coupling constants. Thus, even this relatively well-understood band in the $\tilde{B}{ }^{2} \Pi \leftarrow \tilde{X}^{2} \Pi$ ransition is quite complex, and we wish to consider possible explanations for its appearance. 
Previous studies of the electronic spectroscopy of $\mathrm{BO}_{2}$ and $\mathrm{CO}_{2}^{+}$provide a wealth of information which can be used to interpret the spectrum of the isoelectronic NCO radical. The $\tilde{A}^{2} \Pi_{\mathrm{u}} \leftarrow \tilde{X}^{2} \Pi_{\mathrm{g}}$ absorption system in $\mathrm{BO}_{2}$ was first seen at high resolution by Johns, ${ }^{35}$ while the emission spectroscopy of the $\tilde{A}^{2} \Pi_{u} \rightarrow \tilde{X}^{2} \Pi_{\mathrm{g}}$ system in $\mathrm{CO}_{2}^{+}$has been extensively studied by Rostas and co-workers. ${ }^{36}$ Both the $(100) \leftarrow(000)$ band in $\mathrm{BO}_{2}$ and the $(000) \rightarrow(100)$ band in $\mathrm{CO}_{2}^{+}$exhibit several vibrational features in addition to the two expected sub-bands. In both cases, there are sub-bands due to Fermi resonances between the (100) and (020) levels present; $v_{1} \approx 2 v_{2}$ in the $\tilde{A}^{2} \Pi$ state of $\mathrm{BO}_{2}$ and the $\tilde{X}^{2} \Pi$ state of $\mathrm{CO}_{2}^{+}$. The (020) levels are further split by the combination of Renner-Teller and spinorbit interactions. The theoretical treatment for this combination of effects has been worked out in a series of papers by Pople ${ }^{37}$ and Hougen. ${ }^{38}$

While a (000)-(020) band in a transition between two ${ }^{2} \Pi$ electronic states is normally very weak, the (020) levels of $\Pi$ vibronic symmetry borrow intensity from the nearby (100) levels (which also have $\Pi$ vibronic symmetry). As discussed by Johns ${ }^{35}$ and Larcher et al., ${ }^{36 \mathrm{~b}}$ this interaction leads to as many as six sub-bands in what is nominally a $(100) \leftarrow(000)$ band, since the $\mu$ and $\kappa^{2} \Pi_{P}(P=1 / 2,3 / 2$, where $P=\mid \pm \Omega+l l)$ states associated with the (020) level each borrow intensity from the ${ }^{2} \Pi_{\Omega}$ state associated with the (100) level (see Figure 5.6). In addition, because of energy level shifts associated with the Fermi resonance, the splitting between the (100) ${ }^{2} \Pi_{1 / 2}$ and ${ }^{2} \Pi_{3 / 2}$ levels can be quite different from the value expected based on spin-orbit coupling alone.

In NCO, where $v_{1} \approx 2 v_{2}$ in the $\tilde{X}^{2} \Pi$ and $\tilde{A}^{2} \Sigma^{+}$electronic states, effects from Fermi resonances have been observed in both states. ${ }^{14,20 b}$ Although $v_{2}$ has not been measured for the $\tilde{B}^{2} \Pi$ state, a recent theoretical study by Alexander and Werner ${ }^{39}$ predicts harmonic frequencies of $1080 \mathrm{~cm}^{-1}$ and $522 \mathrm{~cm}^{-1}$ for these stretching and bending modes, so one might expect Fermi resonances in this state as well. The observation of at 
least six sub-bands in Figure 5.2 is consistent with this expectation, as is the deviation of the splitting between bands (b) and (d) from $\left|A^{\prime}-A^{\prime \prime}\right|$.

Figure 5.6, adapted from Johns' paper, ${ }^{35}$ shows an energy level diagram consistent with the structure in the $(100) \leftarrow(000)$ band. The $(100)$ and $(020) \tilde{B}$ state levels with $\Pi$ vibronic symmetry are shown, from left to right, with no interactions, spin-orbit and Renner-Teller coupling, and finally, the additional effect of Fermi resonances. On the left, the unperturbed (100) level lies above the (020) level in accordance with Alexander's calculation. In the middle set of levels, Dixon's value for the $\tilde{B}$ state spin-orbit coupling constant $\left(A^{\prime}=-76.6 \mathrm{~cm}^{-1}\right)$ is used for the $(100)$ and $(020)$ manifolds, along with a RennerTeller parameter sufficiently large so that the (100) levels lie within the (020) levels with $\Pi$ symmetry (the two (020) levels with $\Phi$ symmetry are not shown). The rightmost set of levels shows the qualitative effect of the Fermi resonance between the (100) and (020) manifolds. Since nearby levels with the same $\Omega$ value repel one another, the (100) ${ }^{2} \Pi_{1 / 2}$ and ${ }^{2} \Pi_{3 / 2}$ levels are forced closer together. This results in a splitting between the main ${ }^{2} \Pi_{1 / 2} \leftarrow^{2} \Pi_{1 / 2}$ and ${ }^{2} \Pi_{3 / 2} \leftarrow 2 \Pi_{3 / 2}$ transitions which is larger than $\left|\mathrm{A}^{\prime}-\mathrm{A}^{\prime}\right|$, as is seen in the experimental spectrum. Carrying this one step further, the six sub-bands identified in Figure 5.2 can be assigned to the transitions labeled in Figure 5.6, assuming the energy level spacings indicated in the caption of Figure 5.6.

While Figure 5.6 offers an explanation for the appearance of the $(100) \leftarrow(000)$ band, other possible sources for the multiple sub-bands must also be considered. For example, perturbations from the lower-lying $\tilde{A}{ }^{2} \Sigma^{+}$state are prominent in the $\tilde{B}^{2} \Pi(000)$ $\leftarrow \tilde{X}^{2} \Pi(000)$ band, ${ }^{34}$ and may well be contributing to the $(100) \leftarrow(000)$ band. At higher

Figure 5.6 Schematic showing our tentative assignment for the sub-bands observed in the $(100) \leftarrow(000)$ transition region. The observed transitions yield the following spacings for the 100 and 020 energy levels in the $\tilde{B}{ }^{2} \Pi$ electronic state, relative to the lowest $\mu-2 \Pi_{3 / 2}$ level: $\mu-2 \Pi_{1 / 2}, 0.6 \mathrm{~cm}^{-1},{ }^{2} \Pi_{32}, 54 \mathrm{~cm}^{-1}$, ${ }^{2} \Pi_{12}, 85 \mathrm{~cm}^{-1}, \mathrm{\kappa}^{-2} \Pi_{32}, 130 \mathrm{~cm}^{-1}, \mathrm{k}^{-2} \Pi_{12}, 164.6 \mathrm{~cm}^{-1}$. 


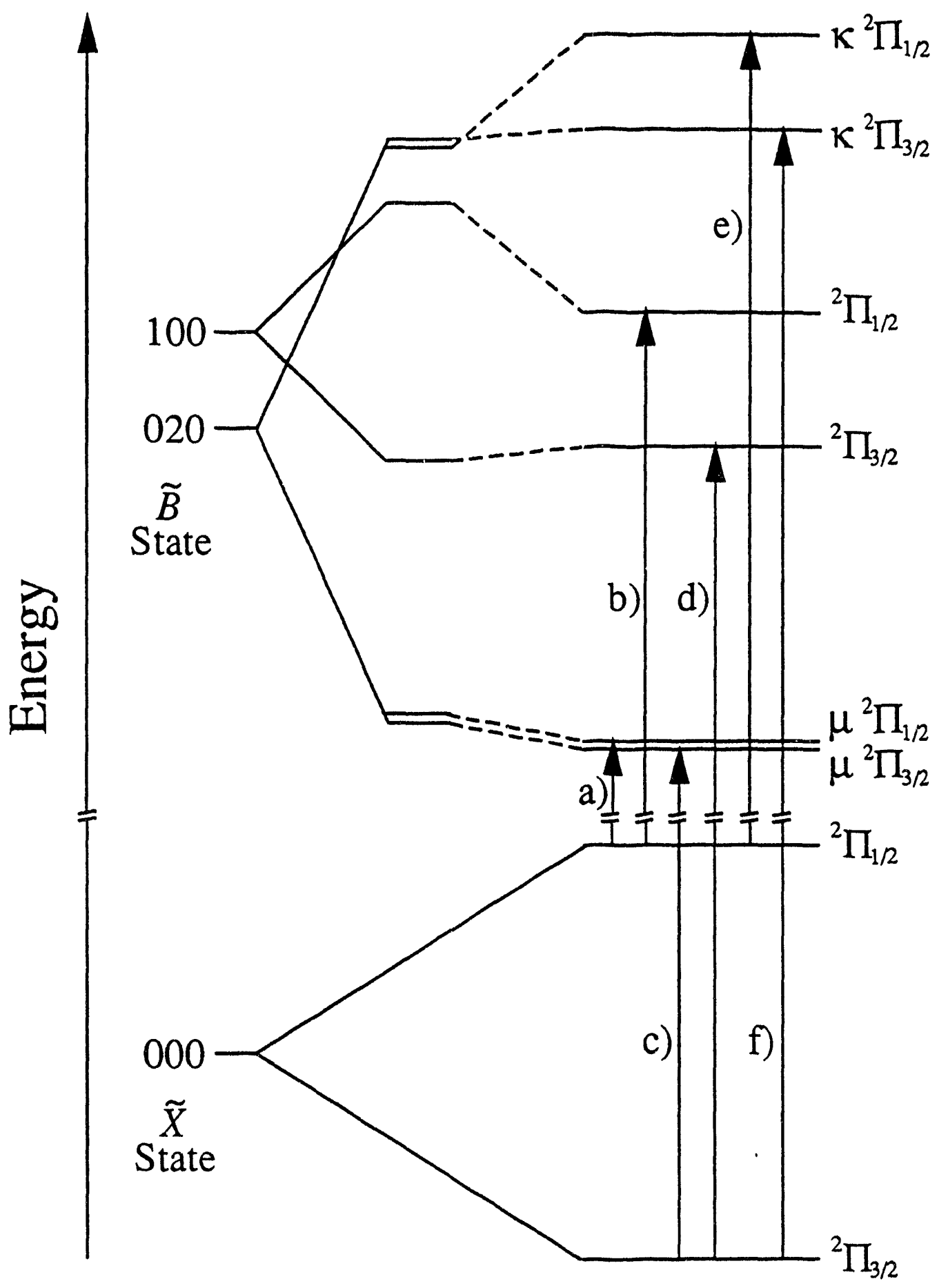

Figure 5.6 
resolution sub-band (a), in particular, appears to actually consist of two distinct features, one centered at $32665 \mathrm{~cm}^{-1}$ (the value used in the above discussion) and the other at $32689 \mathrm{~cm}^{-1}$, possibly resulting from extensive $\tilde{A}$ state perturbations. Similar perturbations have been identified in a double resonance study of $\mathrm{CO}_{2}^{+} .{ }^{40}$ In addition, the $(100) \leftarrow(000)$ transition in NCO may be overlapped by sequence bands in the NCO bend (the $(110) \leftarrow$ (010) band, for example). Any bend excitation in the $\mathrm{NCO}^{-}$is transferred to $\mathrm{NCO}$ when the radical is produced by photodetachment, because bend sequence bands in the $\mathrm{NCO}^{-}$ photoelectron spectrum fall on top of the $\mathrm{NCO}(000) \leftarrow \mathrm{NCO}^{-}(000)$ transition. We note that bend sequence bands were observed in our photodissociation study of $\mathrm{N}_{3}{ }^{5}$

With higher resolution data over a wider frequency range than in the rotationallyresolved spectrum in Figure 5.3, we might be able to definitively sort out these possibilities. As it is, the qualitative similarities with the $\mathrm{CO}_{2}^{+}$and $\mathrm{BO}_{2}$ spectra support the notion that most of the detailed structure in the $(100) \leftarrow(000)$ band in NCO is from Fermi resonances within the $\tilde{B} 2 \Pi$ state. These effects will become even more complex for higher energy transitions in the $\tilde{B}^{2} \Pi \leftarrow \tilde{X}^{2} \Pi$ system.

\subsubsection{Dissociation Dynamics}

In this section, the photofragment time-of-flight (TOF) results are analyzed in order to learn about the dissociation dynamics of the NCO $\tilde{B} 2 \Pi$ state. This data can be considered at two levels of detail. We obtain the approximate maximum photofragment kinetic energy release at each wavelength from the edges of the TOF distribution. We also obtain more precise and detailed photofragment energy and angular distributions by comparing experimental and simulated TOF spectra at various laser wavelengths and polarizations.

We first consider the five TOF spectra shown in Figure 5.4. In the center-of-mass frame of reference, the fastest $\mathrm{N}$ atoms scattered parallel and anti-parallel to the ion beam 


\begin{tabular}{|c|c|c|c|c|}
\hline $\begin{array}{c}\text { Wavelength } \\
{[\mathrm{nm}]}\end{array}$ & $\begin{array}{c}h v \\
{[\mathrm{eV}]}\end{array}$ & $\begin{array}{c}\Delta \tau \\
{[\mathrm{ns}]}\end{array}$ & $\begin{array}{c}\text { KER } \\
{[\mathrm{eV}]}\end{array}$ & $\begin{array}{c}\mathrm{hv}-\mathrm{KER} \\
{[\mathrm{eV}]}\end{array}$ \\
\hline 305.23 & 4.06 & 220 & 1.74 & 2.32 \\
\hline 260.69 & 4.76 & 265 & 2.52 & 2.24 \\
\hline 255.20 & 4.86 & 75 & 0.20 & 4.66 \\
\hline 247.58 & 5.01 & 100 & 0.36 & 4.65 \\
\hline 240.83 & 5.15 & 120 & 0.52 & 4.63 \\
\hline
\end{tabular}

Table 5.1 Summary of results obtained directly from TOF spectra shown in Figure 5.4. Kinetic energy release (KER) values are determined using Eqn. (5.1). See text.

direction correspond to the minimum $\left(t_{\min }\right)$ and maximum $\left(t_{\max }\right)$ flight times at which photofragment signal is observed. If effects due to the energy spread of the radical beam and the extent of the beam block are ignored (these are considered explicitly below), then $\tau=t_{\max }-t_{\min }$ is related to the maximum center-of-mass kinetic energy release, $K E R$, by

$$
K E R=\frac{v_{0}^{4}}{8 L^{2}} \cdot\left(\frac{m_{N} \cdot m_{N C O}}{m_{C O}}\right) \cdot \tau^{2},
$$

where $L$ is the distance the fragments travel to the detector ( $101.1 \mathrm{~cm}$ for these spectra) and $v_{0}$ is the beam velocity $\left(1.91 \times 10^{7} \mathrm{~cm} / \mathrm{sec}\right)$. The results for the spectra in Figure 5.4 are shown in Table 5.1.

Within Table 5.1, the quantity (hv - KER) corresponds to the bond dissociation energy if ground state products are formed. Any excitation present in the products reduces the KER and makes this quantity larger. The more detailed simulations discussed below show that the values for the KER in Table 5.1 are only approximate upper bounds to the maximum photofragment kinetic energy release, and therefore, the values of 
(hv - KER) are only approximate lower bounds for the bond dissociation energy plus product internal energy.

Nonetheless, several features in this table are noteworthy. First, consider the value for the KER at $\lambda=305 \mathrm{~nm}$, which corresponds to excitation of the $(100) \leftarrow(000)$ transition. If the upper state were dissociating to the lowest energy spin-allowed dissociation channel, $\mathrm{N}\left({ }^{2} D\right)+\mathrm{CO}$, then $\mathrm{KER}=1.78 \mathrm{eV}$ yields $\Delta_{f} H_{298 \mathrm{~K}}^{0}(\mathrm{~N}-\mathrm{CO})=$ $88 \mathrm{kcal} / \mathrm{mol}$, more than double the accepted value. This value for $\Delta_{f} H_{298 \mathrm{~K}}^{0}(\mathrm{~N}-\mathrm{CO})$ would mean that the $\mathrm{CN}+\mathrm{O}_{2} \rightarrow \mathrm{NCO}+\mathrm{O}$ reaction is endothermic by $45 \mathrm{kcal} / \mathrm{mol}$, a physically unreasonable result because this reaction is rapid at room temperature. The $305 \mathrm{~nm}$ and $260 \mathrm{~nm}$ TOF results therefore indicate that the upper states for both transitions are dissociating to the energetically allowed but spin-forbidden $\mathrm{N}\left({ }^{4} S\right)+\mathrm{CO}$ channel, which lies $2.38 \mathrm{eV}(54.9 \mathrm{kcal} / \mathrm{mol})$ below the $\mathrm{N}\left({ }^{2} D\right)+C O$ channel. However, at $\lambda=255 \mathrm{~nm}$, the TOF distribution narrows abruptly. This suggests an energy level diagram for NCO and its photofragments as given in Figure 5.7; at $\lambda=255 \mathrm{~nm}$, the spin-allowed channel $\mathrm{N}\left({ }^{2} D\right)+$ CO channel is energetically accessible, whereas it is not at $\lambda=260 \mathrm{~nm}$.

The results in Table 5.1 therefore imply that the $\mathrm{N}\left({ }^{2} D\right)+\mathrm{CO}$ channel becomes accessible between 4.76 and $4.86 \mathrm{eV}$ above the $\mathrm{NCO}$ ground state and allows us to bracket $\Delta_{f} H_{298 \mathrm{~K}}^{0}(\mathrm{NCO})$ between 31.5 and $29.0 \mathrm{kcal} / \mathrm{mol}$. This bracketing is validated by the additional observation that the KER is small at the longest wavelength at which $\mathrm{N}\left({ }^{2} D\right)+\mathrm{CO}$ is formed, implying no substantial barrier to dissociation. Since this range of $\Delta_{f} H_{298 \mathrm{~K}}^{0}(\mathrm{NCO})$ values lies below the accepted literature26 value of $36.1 \mathrm{kcal} / \mathrm{mol}$, we have performed more detailed analyses of photofragment TOF spectra which will now be discussed.

We wish to determine the N-CO bond dissociation energy and the photofragment

Figure 5.7 Energy level diagram showing the position of the NCO electronic energy levels relative to the asymptotic energies of the fragment channels as determined by this work. 


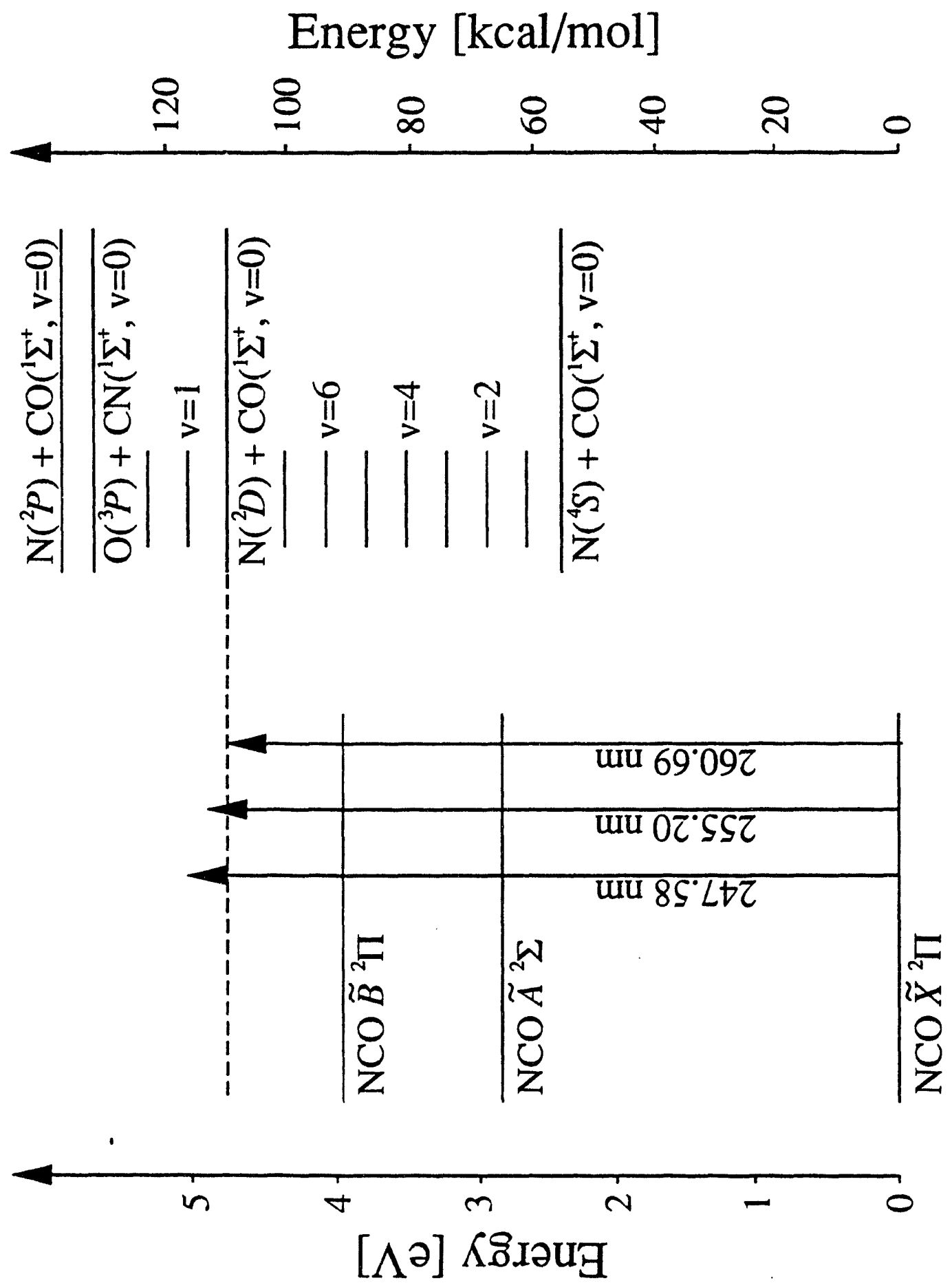

Figure 5.7 
energy and angular distributions consistent with the TOF spectra in Figures 5.4 and 5.5. This is done using a Monte Carlo computer simulation program, ${ }^{41}$ which generates a TOF spectrum for given dissociation wavelength, laser polarization and radical beam characteristics. The simulation program also accounts for the finite size of the photofragment detector and the beam block. The radical beam characteristics are checked by comparing simulated and experimental TOF spectra of the undissociated radical beam; the agreement, shown in Figure 5.4(b), is excellent. The simulation assumes a spread of $8 \mathrm{eV}(0.1 \%)$ in the ion beam energy. This value is consistent with the small energy spread expected from the beam-modulation TOF mass spectrometer. ${ }^{42}$

To carry out a photofragment TOF simulation, we specify a trial photofragment angular distribution and bond dissociation energy. The simulation program accounts for contributions to the TOF spectra by both $\mathrm{N}$ and $\mathrm{CO}$ fragments, since the experimental spectrum has contributions from both. Figure 5.5(a) shows the individual contributions of the $\mathrm{N}$ (dotted lines) and $\mathrm{CO}$ (dashed lines) fragments as determined from the simulation program together with their sum that fits the experimental TOF spectrum. The fact that only $\mathrm{N}$ atoms contribute to the TOF signal close to $t_{\min }$ and $\mathrm{t}_{\max }$ is illustrated clearly by this figure.

The photofragment center-of-mass kinetic energy and angular distribution $P\left(E_{\mathrm{T}}, \theta\right)$ is taken to be

$$
P\left(E_{T}, \theta\right)=\sum_{i=S, D} n_{i} P_{i}\left(E_{T}\right) \cdot\left(1+\beta_{i} P_{2}(\cos \theta)\right) .
$$

The index $i$ specifies the $N$ atom electronic state $\left({ }^{4} S\right.$ or $\left.{ }^{2} D\right)$. Here $n_{i}$ refers to the electronic state populations, $P_{\mathrm{i}}\left(E_{\mathrm{T}}\right)$ is the photofragment kinetic energy distribution in the $i$ th electronic state, $\theta$ is the angle between the fragment recoil and laser polarization vector, and $\beta_{\mathrm{i}}$ is the anisotropy parameter ${ }^{-4}$ for the $i$ th electronic state. $P_{\mathrm{i}}\left(E_{\mathrm{T}}\right)$ is 


\begin{tabular}{|c|c|c|c|c|}
\hline $\begin{array}{c}\lambda \\
{[\mathrm{nm}]}\end{array}$ & $\begin{array}{c}{ }^{4} S:{ }^{2} D \\
\text { Ratio }\end{array}$ & $\beta$ (for ${ }^{2} D$ channel) & $\beta$ (for ${ }^{4} S$ channel) & $\begin{array}{c}D_{0}(\mathrm{~N}-\mathrm{CO}) \\
{[\mathrm{kcal} / \mathrm{mol}]}\end{array}$ \\
\hline 260.69 & $100: 0$ & N/A & +0.1 & 54.9 \\
\hline 255.20 & $6: 94$ & +0.2 & +0.1 & 54.9 \\
\hline 247.58 & $2: 98$ & +0.2 & +0.1 & 54.9 \\
\hline
\end{tabular}

Table 5.2 Simulation parameters used to fit all data in Figure 5.5 (both polarizations) and the common wavelengths in Figure 5.4. The value shown for $\mathrm{D}_{0}(\mathrm{~N}-\mathrm{CO})$ was determined by obtaining the best fit for all data in both Figures 5.4 and 5.5 .

\begin{tabular}{|c|c|c|c|c|c|c|c|c|c|}
\hline $\begin{array}{c}\lambda \\
\text { [nm] }\end{array}$ & $\begin{array}{c}\mathrm{v}=0 \\
\text { pop. }\end{array}$ & $\begin{array}{c}\mathrm{v}=1 \\
\text { pop. }\end{array}$ & $\begin{array}{c}\mathrm{v}=2 \\
\text { pop. }\end{array}$ & $\begin{array}{c}\mathrm{v}=3 \\
\text { pop. }\end{array}$ & $\begin{array}{c}\mathrm{v}=4 \\
\text { pop. }\end{array}$ & $\begin{array}{c}\mathrm{v}=5 \\
\text { pop. }\end{array}$ & $\begin{array}{c}\mathrm{v}=6 \\
\text { pop. }\end{array}$ & $\begin{array}{c}\mathrm{v}=7 \\
\text { pop. }\end{array}$ & $\begin{array}{c}\mathrm{v}=8 \\
\text { pop. }\end{array}$ \\
\hline 260.69 & 1.0 & 1.0 & 1.0 & 1.0 & 1.5 & 2.5 & 3.0 & 2.0 & 1.0 \\
\hline
\end{tabular}

Table 5.3 CO fragment vibrational distribution used in the simulations of the three TOF spectra at $260.69 \mathrm{~nm}$ in Figures 5.4 and 5.5. The simulations assumed no rotational excitation of the $\mathrm{CO}$.

determined by specifying the bond dissociation energy and the $\mathrm{CO}$ vibration-rotation distribution associated with the $i$ th $\mathrm{N}$ atom electronic state.

We first consider the TOF spectra at the three wavelengths for which the photofragment flight length was $67.8 \mathrm{~cm}$ (Figure 5.5), as these were taken with both laser polarization directions. The broad TOF spectrum at $\lambda=260 \mathrm{~nm}$ indicates dissociation to $\mathrm{N}\left({ }^{4} S\right)+\mathrm{CO}$, while the two narrower spectra at $\lambda=255$ and $247 \mathrm{~nm}$ are from dissociation primarily to $\mathrm{N}\left({ }^{2} D\right)+C O$. However, the narrow peak in the $255 \mathrm{~nm}$ TOF spectrum sits atop a small, broad pedestal due to a small amount of $\mathrm{N}\left({ }^{4} S\right)+\mathrm{CO}$ production. At all three wavelengths, the spectra taken with the laser horizontally polarized (parallel to the radical 
beam axis) are slightly broader than the spectra taken with vertical laser polarization. This indicates that the anisotropy parameter $\beta$ is positive for both $N$ atom product electronic states; we find $\beta=+0.1$ for the $N\left({ }^{4} S\right)$ channel and $\beta=+0.2$ for the $N\left({ }^{2} D\right)$ channel. The complete set of parameters used to arrive at the solid line fits for the three sets of TOF spectra are listed in Table 5.2 and Table 5.3.

The narrow peak in the $255 \mathrm{~nm}$ TOF spectrum can be fit successfully assuning a single value for the photofragment kinetic energy of $0.10 \mathrm{eV}$. If this corresponds to the translational energy of $\mathrm{N}\left({ }^{2} D\right)+\mathrm{CO}(\mathrm{v}=0, \mathrm{~J}=0)$ fragments, then the $\mathrm{N}\left({ }^{2} D\right)+\mathrm{CO}$ dissociation channel lies $4.76 \mathrm{eV}(109.8 \mathrm{kcal} / \mathrm{mol})$ above the ground state of $\mathrm{NCO}$, yielding an N-CO bond dissociation energy (to form $\mathrm{N}\left({ }^{4} S\right)+\mathrm{CO}$ ) of $2.38 \mathrm{eV}$ $(54.9 \mathrm{kcal} / \mathrm{mol})$. This value of the bond dissociation energy is the one used to determine the relative energies of $\mathrm{NCO}$ and the dissociation product channels in Figure 5.7.

Our interpretation of the $255 \mathrm{~nm}$ spectrum is supported by the $247 \mathrm{~nm}$ spectrum using the same bond dissociation energy in which the edges of the distribution are fit accurately assuming they are also from $\mathrm{N}\left({ }^{2} D\right)+\mathrm{CO}(\mathrm{v}=0, \mathrm{~J}=0)$ fragments. However, closer to the center of the distribution, the simulated TOF spectrum is too broad (Figure 5.5(e), dashed line) if all the $\mathrm{CO}$ is assumed to be in its ( $(\mathrm{v}=0, \mathrm{~J}=0)$ state, and the best simulation (solid line) includes rotational excitation in the $\mathrm{CO}$ fragment $[\mathrm{CO}(\mathrm{v}=1)$ is not accessible at $247 \mathrm{~nm}$ ]. The rotational excitation was incorporated using the following empirical functional form for the translational energy distribution:

$$
P\left(E_{T}\right)=1-\frac{E_{T}}{E_{T O T}}
$$

Here $E_{\mathrm{T}}$ is translational energy while $E_{\mathrm{TOT}}$ is hv - $\left[\mathrm{D}_{0}(\mathrm{~N}-\mathrm{CO})+\Delta \mathrm{E}\left({ }^{2} D-4 S\right)\right]$, with $\Delta \mathrm{E}\left({ }^{2} D-{ }^{4} S\right)$ equal to the difference in energy between the ${ }^{2} D$ and ${ }^{4} S \mathrm{~N}$ atom electronic states. 


\begin{tabular}{|c|c|c|c|c|c|c|}
\hline $\begin{array}{c}\lambda \\
{[\mathrm{nm}]}\end{array}$ & $\begin{array}{c}\mathrm{v}=0 \\
\text { pop. }\end{array}$ & $\begin{array}{c}\mathrm{v}=1 \\
\text { pop. }\end{array}$ & $\begin{array}{c}\mathrm{v}=2 \\
\text { pop. }\end{array}$ & $\begin{array}{c}\mathrm{v}=3 \\
\text { pop. }\end{array}$ & $\begin{array}{l}\mathrm{v}=4 \\
\text { pop. }\end{array}$ & $\begin{array}{c}\mathrm{v}=5 \\
\text { pop. }\end{array}$ \\
\hline 305.23 & 1.0 & 1.5 & 2.5 & 3.0 & 3.0 & 1.0 \\
\hline 240.83 & 1.0 & 1.0 & $\ldots$ & $\ldots$ & $\ldots$ & $\ldots$ \\
\hline
\end{tabular}

Table 5.4 CO fragment vibrational distribution (neglecting rotational excitation) used in the simulations in of the TOF spectra Figure 5.4 with TOF spectra at 305.23 (for $\mathrm{N}\left({ }^{4} S\right)+\mathrm{CO}$ products) and $240.83 \mathrm{~nm}$ (for $\mathrm{N}\left({ }^{2} D\right)+\mathrm{CO}$ products).

In the simulated $260 \mathrm{~nm}$ TOF spectrum, the photofragment kinetic energy distribution was determined using the bond dissociation energy, determined above, of $2.38 \mathrm{eV}$. The $\mathrm{CO}(\mathrm{v}, \mathrm{J}=9)$ distribution used is given in Table 5.3. A more detailed distribution involving $\mathrm{CO}$ vibrational and rotational excitation is not justified by the data. However, one can safely say that, unless the $\mathrm{CO}$ is extremely rotationally excited, the $\mathrm{CO}$ vibrational distributios. is highly inverted. More significantly, from the perspective of the NCO energetics, $t_{\min }$ and $t_{\max }$ in the simulated TOF distribution, which are due to $\mathrm{N}\left({ }^{4} S\right)+\mathrm{CO}(\mathrm{v}=0)$, match the experimental spectrum very well, further supporting the bond dissociation erergy obtained from the $255 \mathrm{~nm}$ spectrum. As mentioned above, the $255 \mathrm{~nm}$ and, to an even lesser extent, the $247 \mathrm{~nm}$ spectra have a small contribution from $\mathrm{N}\left({ }^{4} S\right)+\mathrm{CO}$ product. The electronic branching ratios in Table 5.2 were determined assuming the same $\mathrm{CO}$ vibrational distribution for the $\mathrm{N}\left({ }^{4} S\right)+\mathrm{CO}$ product that was determined for the $260 \mathrm{~nm}$ spectrum.

As a further check on the values determined in these simulations, the parameters in Table 5.2 and Table 5.3 were used to fit the three TOF spectra in Figure 5.4 at the same three wavelengths but where the photofragment flight length is $101.1 \mathrm{~cm}$. Excellent agreement is found between the experimental and simulated TOF spectra. Finally, the TOF spectra in Figure 5.4 at both $305 \mathrm{~nm}$ and $240 \mathrm{~nm}$ were fit assuming the respective $\beta$ 
values obtained above for the two $\mathrm{N}$ atom electronic channels. The fragment state populations that gave the best-fitting simulations (solid lines, Figure 5.4) are listed in Table 5.4. The $\mathrm{CO}$ vibrational distribution at $305 \mathrm{~nm}$ appears to be highly inverted, just as at $260 \mathrm{~nm}$, while the excellent fit at the extrema of the simulation to the data further confirms our choice of $D_{0}(\mathrm{~N}-\mathrm{CO})$. At $240 \mathrm{~nm}$, again using $\mathrm{D}_{0}(\mathrm{~N}-\mathrm{CO})=2.38 \mathrm{eV}$ in our simulation, the $\mathrm{N}(2 D)+\mathrm{CO}(\mathrm{v}=1)$ channel should be energetically accessible. Indeed, the shape of the TOF spectrum is noticeably different from the $247 \mathrm{~nm}$ spectrum, with broader wings under a central peak, and we find by varying only the relative vibrational level population of $v=0$ and $v=1$ (neglecting rotational excitation) that an adequate fit is obtained if $50 \%$ of the $\mathrm{CO}$ is in the $\mathrm{v}=1$ level.

The value of $2.38 \mathrm{eV}$ for $\mathrm{D}_{0}(\mathrm{~N}-\mathrm{CO})$ was obtained from our analysis assuming the $\mathrm{NCO}$ is in its rotational ground state. Including the correction for the average rotational energy of the $\mathrm{NCO}(0.1 \mathrm{kcal} / \mathrm{mol}$ at $50 \mathrm{~K})$, we obtain $\Delta_{f} H^{0}=30.4 \mathrm{kcal} / \mathrm{mol}$ at $0 \mathrm{~K}$. $\Delta_{f} H^{0}(\mathrm{NCO})$ at $298 \mathrm{~K}$ is only slightly higher, $30.5 \mathrm{kcal} / \mathrm{mol} .^{44}$ This value is significantly lower than the currently accepted literature value ${ }^{26}$ of $36.1 \pm 1 \mathrm{kcal} / \mathrm{mol}\left(\mathrm{D}_{0}(\mathrm{~N}-\mathrm{CO})=\right.$ $2.13 \mathrm{eV}$ ). As a measure of the sensitivity of our TOF spectra to the assumed value of $\mathrm{D}_{0}(\mathrm{~N}-\mathrm{CO})$, Figure $5.4(\mathrm{c})$ shows the results of a simulation (dotted line) at $255 \mathrm{~nm}$ with $\mathrm{D}_{0}(\mathrm{~N}-\mathrm{CO})=2.12 \mathrm{eV}$ but with all the other parameters the same as in Table 5.2. This is clearly inferior to the simulation with $\mathrm{D}_{0}(\mathrm{~N}-\mathrm{CO})=2.38 \mathrm{eV}$. Based on trial simulations at various dissociation wavelengths using different bond dissociation energies, we estimate the error in our determination of $\Delta_{f} H_{298}^{0} \mathrm{~K}(\mathrm{NCO})$ to be $\pm 1 \mathrm{kcal} / \mathrm{mol}$.

\subsection{Discussion}

The results presented here not only settle the question of the onset of predissociation in the NCO $\tilde{B}^{2} \Pi$ state, but also explain the observations in previous experiments. In Dixon's original study of the NCO $\tilde{B}{ }^{2} \Pi \leftarrow \tilde{X}^{2} \Pi$ system, he attributed the 
spectral broadening of the $(200) \leftarrow(000)$ transition to the onset of predissociation forming $\mathrm{N}\left({ }^{2} D\right)+\mathrm{CO}$ products, whereas Crosley ${ }^{23}$ interpreted the lower fluorescence lifetime of the $\tilde{B} 2 \Pi$ state (100) level relative to the (000) level to mean that the (100) level could dissociate to $\mathrm{N}\left({ }^{2} D\right)+C O$. Our photodissociation cross section measurements clearly show that all vibrational levels of the NCO $\tilde{B}^{2} \Pi$ state predissociate. The explanation of these seemingly discordant results is given by the photofragment TOF spectra. These show that, in Dixon's and Crosley's experiments, predissociation of the $\tilde{B}^{2} \Pi$ state was indeed occurring, but it was spin-forbidden dissociation to $\mathrm{N}\left({ }^{4} S\right)+\mathrm{CO}$ products. Their results can therefore be explained if the dissociation rate to these products increases with the level of $\tilde{B}^{2} \Pi$ state vibrational excitation, causing a noticeable decrease in the fluorescence lifetime for the (100) level and observable broadening in the absorption spectrum for the (200) and higher levels. As mentioned in the Results section, the intensity of the photofragment signal from the $(000)$ level relative to the signal from the higher levels appears to be smaller than in the $\tilde{B}{ }^{2} \Pi \leftarrow \tilde{X}^{2} \Pi$ absorption spectrum. This suggests that the dissociation rate from the $(000)$ level is sufficiently slow that the photofragment signal is depleted by spontaneous emission.

The next important issue is the discrepancy between our value for $\Delta_{f} H_{298}^{0}(\mathrm{NCO})$ and the literature value. Our experiment is, in principle, a direct measurement of the $\mathrm{N}-\mathrm{CO}$ bond dissociation energy, but there are several assumptions in our analysis which must be discussed. The $\tilde{B}^{2} \Pi \leftarrow \tilde{X}^{2} \Pi$ transitions in the range of the $N\left({ }^{2} D\right)+C O$ threshold have not been definitively assigned. In using the photofragment TOF spectra to determine this threshold, we have assumed all the spectroscopic transitions originate from the (000) level of the NCO ground electronic state. This may not be correct, as some of these transitions may be sequence bands originating from vibrationally excited NCO. As discussed in the Analysis section, vibrationally excited NCO can result from photodetachment of vibrationally excited $\mathrm{NCO}^{-}$. However, in the unlikely event that this 
were somehow the case for all the transitions in Figure 5.4, then the true bond dissociation energy would be higher than $2.38 \mathrm{eV}$ and the true $\Delta_{f} H_{298 \mathrm{~K}}^{0}(\mathrm{NCO})$ would be lower than $30.5 \mathrm{kcal} / \mathrm{mol}$, a shift in the opposite direction from the literature value. We also assume in our simulations that dissociation occurs in a time which is less than $1 \%$ of the total flight time $(-3.5 \mu \mathrm{s})$. This is strongly supported by the observation that the TOF spectra which were taken at both photofragment flight lengths, $l=67.8 \mathrm{~cm}$ and $101.1 \mathrm{~cm}$, could be fit with a single fragment translational energy distribution.

Another source of possible error is that our analysis assumes that $t_{\min }$ and $t_{\max }$ in the TOF distributions are from $\mathrm{N}+\mathrm{CO}(\mathrm{v}=0)$ fragments, and, in particular, that the TOF spectra at $255 \mathrm{~nm}$ and $247 \mathrm{~nm}$ are due entirely to $C O(v=0)$. Suppose, however, that no $\mathrm{CO}(\mathrm{v}=0)$ is produced at those wavelengths, and that the TOF spectra are from $\mathrm{N}\left({ }^{2} D\right)+\mathrm{CO}(\mathrm{v}=1)$. If this were the case, $\mathrm{D}_{0}(\mathrm{~N}-\mathrm{CO})$ would have to be lowered by $5 \mathrm{kcal} / \mathrm{mol}$ (corresponding to the $\mathrm{CO}$ vibrational frequency) from our value and $\Delta_{f} H_{298 \mathrm{~K}}^{0}(\mathrm{NCO})$ would be raised by the same amount, which would bring it in close agreement with the literature value. However, this would imply that the $\mathrm{CO}$ distribution at $241 \mathrm{~nm}$ [Figure 5.4(e)] is approximately $50 \% \mathrm{v}=2,50 \% \mathrm{v}=1$, and no $\mathrm{v}=0$, a most unusual distribution. In addition, at $260 \mathrm{~nm}$ and $305 \mathrm{~nm}$, where only $\mathrm{N}\left({ }^{4} S\right)+\mathrm{CO}$ is formed, the edges of the TOF spectra are reproduced in our simulations using $\mathrm{D}_{0}(\mathrm{~N}-\mathrm{CO})$ $=2.38 \mathrm{eV}$. In order to fit these spectra with a $5 \mathrm{kcal} / \mathrm{mol}$ lower bond dissociation energy, one would have to assume no $\mathrm{CO}(\mathrm{v}=0)$ was produced at these wavelengths, as well. This also seems unlikely, considering the substantial range of $\mathrm{CO}$ vibrational states populated at both of these wavelengths, and the different mechanism producing the spin-forbidden product channel. We therefore believe that the bond dissociation energy obtained from our TOF spectra is correct, and that the literature value of $\Delta_{f} H_{298 \mathrm{~K}}^{0}(\mathrm{NCO})$ is too large by several $\mathrm{kcal} / \mathrm{mol}$. 
The literature value 26 of $\Delta_{f} H_{298 \mathrm{~K}}^{0}(\mathrm{NCO})$ is obtained from three quantities which contain possible sources of error: (1) DA (H-NCO), the threshold for formation of $\mathrm{NCO}$ $\tilde{A}^{2} \Sigma^{+}$from the photolysis of $\mathrm{HNCO}$, (2) $\mathrm{D}_{0}(\mathrm{HN}-\mathrm{CO})$, the dissociation energy of HNCO to form $\mathrm{NH}+\mathrm{CO}$, and (3) $\Delta_{f} H_{298 \mathrm{~K}}^{0}(\mathrm{NH})$. The $\mathrm{H}-\mathrm{NCO}$ bond dissociation energy, $\mathrm{D}_{0}(\mathrm{H}-\mathrm{NCO})$, is found from $\mathrm{D}^{\mathrm{A}}(\mathrm{H}-\mathrm{NCO})$ by subtracting $2.82 \mathrm{eV}$, the electronic energy of the NCO $\tilde{A}^{2} \Sigma^{+}$state. We then have

$$
\begin{aligned}
\Delta_{f} H_{0 \mathrm{~K}}^{0}(\mathrm{NCO}) & =D_{0}(\mathrm{H}-\mathrm{NCO})-D_{0}(\mathrm{HN}-\mathrm{CO}) \\
& +\Delta_{f} H_{0 \mathrm{~K}}^{0}(\mathrm{NH})+\Delta_{f} H_{0 \mathrm{~K}}^{0}(\mathrm{CO})-\Delta_{f} H_{0 \mathrm{~K}}^{0}(\mathrm{H}) .
\end{aligned}
$$

The standard heats of formation of $\mathrm{CO}$ and $\mathrm{H}$ are well known. ${ }^{44}$ The literature value of $\Delta_{f} H_{298 \mathrm{~K}}^{0}(\mathrm{NCO})$ is based on Okabe's value 22 of $7.73 \mathrm{eV}$ for DA(H-NCO), Chandler's value $^{25}$ of $\mathrm{D}(\mathrm{HN}-\mathrm{CO})=82.9(+2.8,-0.7) \mathrm{kcal} / \mathrm{mol}$, which was obtained from the threshold for $\mathrm{NH}\left(\mathrm{a}^{1} \Delta\right)$ production from $\mathrm{HNCO}$ photodissociation, and Berkowitz's value ${ }^{45}$ of $\Delta_{f} H_{298 \mathrm{~K}}^{0}(\mathrm{NH})=85.2 \mathrm{kcal} / \mathrm{mol}$, which was obtained from $\mathrm{NH}_{2}$ ionization and dissociative ionization potentials. Two more recent independent measurements ${ }^{46}$ of $\Delta_{f} H_{298}^{0} \mathrm{~K}(\mathrm{NH})$ are in excellent agreement with Berkowitz's value. However, a more recent measurement of $\mathrm{D}^{\mathrm{A}}(\mathrm{H}-\mathrm{NCO})$ by Shobatake ${ }^{47}$ yields a slightly lower value, $7.65 \mathrm{eV}$, lowering $\Delta_{f} H_{298 \mathrm{~K}}^{0}(\mathrm{NCO})$ by $1.8 \mathrm{kcal} / \mathrm{mol}$. This brings $\Delta_{f} H_{298}^{0} \mathrm{~K}(\mathrm{NCO})$ down from 36.1 to $34.3 \mathrm{kcal} / \mathrm{mol}$. In addition, using the upper bound on $\mathrm{D}_{0}(\mathrm{HN}-\mathrm{CO})$ of $85.7 \mathrm{kcal} / \mathrm{mol}$ from Chandler's study lowers $\Delta_{f} H_{298}^{0} \mathrm{~K}(\mathrm{NCO})$ still more, to $31.5 \mathrm{kcal} / \mathrm{mol}$, bringing it within the error bars of our measurement. Our value for $\Delta_{f} H_{298 \mathrm{~K}}^{0}(\mathrm{NCO})$ therefore is not as inconsistent with previous work as it might first appear, and a further lowering of $\mathrm{D}^{\mathrm{A}}(\mathrm{H}-\mathrm{NCO})$ would yield even better agreement.

We also note that Coombe ${ }^{2}$ obtained a lower bound to $\Delta_{f} H_{298 \mathrm{~K}}^{0}(\mathrm{NCO})$ of $37 \mathrm{kcal} / \mathrm{mol}$ in a photodissociation study of $\mathrm{NCO}$ at $193 \mathrm{~nm}$, based on the highest internal energy seen in the $\mathrm{CN}$ fragment. However, his value assumes no internal excitation in the 
$\mathrm{NCO}$, which is generated by the $\mathrm{F}+\mathrm{HNCO}$ reaction. The $\mathrm{CO}$ bond distances in $\mathrm{HNCO}$ and NCO are $1.167 \AA^{48}$ and $1.21 \AA^{49}$ respectively. Based on a simplistic Franck-Condon view of the F + HNCO reaction, this geometry change would lead one to expect the NCO product to have significant stretching excitation which could, in principle, appear as CN internal excitation when the $\mathrm{NCO}$ is photodissociated.

Finally, we consider the mechanism for the various NCO dissociation processes. Until very recently, little was known about the detailed structure and interaction of the excited state potential energy surfaces of NCO. Past calculations have been completed at the INDO-CI ${ }^{50}$ and $\mathrm{MINDO} / 3^{51}$ level; predictions of electronic state levels varied widely between these two calculations, with neither being close to the experimentally determined values for the $\tilde{A}^{2} \Sigma^{+}$and $\tilde{B}^{2} \Pi$ states. However, a theoretical study just completed by Alexander and Werner ${ }^{39}$ addresses in detail the nature of the potential energy surfaces and the mechanism of the spin-forbidden decomposition of the NCO $\tilde{B}^{2} \Pi$ state using $a b$ initio methods. They propose that the dissociation pathway for this process first passes through a conical intersection between the $\tilde{B}^{2} \Pi$ and $\tilde{A}^{2} \Sigma^{+}$states, both of which have components of $\mathrm{A}^{\prime}$ symmetry when the molecule is bent. Dissociation then occurs through the interaction, via spin-orbit coupling, of the $\tilde{A}^{2} \Sigma^{+}$state with the repulsive ${ }^{4} \Sigma^{-}$state which correlates with $\mathrm{N}\left({ }^{4} S\right)+\mathrm{CO}$ products. An attractive feature of their calculation is that, for collinear geometries, the minimum in the crossing seam between the $\tilde{B}^{2} \Pi$ and $\tilde{A}^{2} \Sigma^{+}$ states occurs slightly above the $\tilde{B}{ }^{2} \Pi$ state minimum. This is consistent with the apparent rapid increase in dissociation rate as the vibrational energy of the $\tilde{B} 2 \Pi$ state increases.

This calculation also predicts that the lowest energy crossing between the $\tilde{A}$ state and the repulsive ${ }^{4} \Sigma$ - state occurs at a bond angle of $145.5^{\circ}$ and $\mathrm{CO}$ bond length of $1.21 \AA$, which is considerably longer than the bond length of $1.13 \AA$ in diatomic $C O$. One therefore expects considerable rotational and vibrational excitation of the $\mathrm{CO}$ produced by spin-forbidden dissociation of NCO. While we cannot distinguish rotational from 
vibrational excitation in our TOF spectra of the spin-forbidden channel, the spectra are certainly consistent with extensive excitation of the $\mathrm{CO}$ product. Planned experiments with a two-particle position and time sensing detector similar to that used by Los and coworkers ${ }^{52}$ should yield more complete product energy distributions, enabling a detailed comparison with theory.

Finally, although Alexander and Werner did not explicitly discuss photodissociation to the spin-allowed products, one can speculate on the possible mechanism based on their results. Neither the $\tilde{A}^{2} \Sigma^{+}$nor $\tilde{B}{ }^{2} \Pi$ states correlate to $\mathrm{N}\left({ }^{2} D\right)+\mathrm{CO}$ products for linear $\mathrm{NCO}$ geometries, but both states correlate to these products for bent geometries. The excited stretching levels of the $\tilde{B}^{2} \Pi$ state that are accessed in our experiment should have some bending character due to Fermi resonances, so dissociation to spin-allowed products should be reasonably facile once this channel becomes energetically accessible. This is consistent with the experimental observation that the spin-allowed channel dominates immediately above the $\mathrm{N}\left({ }^{2} D\right)+\mathrm{CO}$ threshold.

\subsection{Conclusions}

We have used photodissociation spectroscopy to study the $\tilde{B}^{2} \Pi \leftarrow \tilde{X}^{2} \Pi$ electronic transition in NCO. Photodissociation cross section measurements show that predissociation occurs throughout the $\tilde{B}^{2} \Pi$ state, including the ground vibrational level. Time-of-flight spectroscopy of the $\mathrm{N}+\mathrm{CO}$ photofragments reveal that the first several vibrational levels of the NCO $\tilde{B} 2 \Pi$ state undergo spin-forbidden dissociation to $\mathrm{N}\left({ }^{4} S\right)+\mathrm{CO}$ products, but $20.3 \mathrm{kcal} / \mathrm{mol}$ above the $\tilde{B} 2 \Pi$ state origin, the spin-allowed $\mathrm{N}\left({ }^{2} D\right)+\mathrm{CO}$ channel becomes energetically accessible and immediately dominates the photofragment distribution. From our determination of this threshold, we obtain a $\Delta_{f} H_{298 \mathrm{~K}}^{0}$ of $30.5 \mathrm{kcal} / \mathrm{mol}$ for the NCO free radical, which is significantly lower than the current literature value of $36.1 \mathrm{kcal} / \mathrm{mol}$. We also obtain approximate $\mathrm{CO}$ internal energy 
distributions from the time-of-flight spectrum. The dynamics of NCO photodissociation are briefly discussed in terms of recent theoretical results by Alexander and Werner which indicate that spin-forbidden dissociation from the $\tilde{B} 2 \Pi$ state occurs via a conical intesection with the $\tilde{A}^{2} \Sigma^{+}$state followed by a non-adiabatic transition to a repulsive quartet state which correlates to $\mathrm{N}\left({ }^{4} S\right)+\mathrm{CO}$ products. 


\subsection{References}

1 Molecular Photodissociation Dynamics, edited by M. N. R. Ashfold and J. E. Baggott (Royal Society of Chemistry, London, 1987).

2 X. Liu and R. D. Coombe, J. Chem. Phys. 91, 7543 (1989).

3 J. C. Loison, S. H. Kable, P. L. Houston, and I. Burak, J. Chem. Phys. 94,1796 (1991).

4 E. J. Hintsa, X. S. Zhao, W. M. Jackson, W. B. Miller, A, M. Wodtke, and Y. T. Lee, J. Phys. Chem. 95, 2799 (1991).

5 R. E. Continetti, D. R. Cyr, R. B. Metz, and D. M. Neumark, Chem. Phys. Lett., 182, 406 (1991).

6 B. S. Haynes, Combust. Flame 28, 113 (1977); J. A. Miller, M. C. Branch, W. J. McLean, D. W. Chandler, M. D. Smooke, and R. J. Kee, Symp. (Int.) Combust. [Proc]., 12th, 673 (1984).

7 a) K. N. Wong, W. R. Anderson, A. J. Kollar, and J. A. Vanderhoff, J. Chem. Phys. 81, 2970 (1984). b) W. R. Anderson, J. A. Vanderhoff, A. J. Kotlar, M. A. Dewilde, and R. A. Beyer, J. Chem. Phys. 77, 1677 (1982).

c) R. A. Copeland and D. R. Crosley, Can J. Phys. 62, 1488 (1984).

8 K. Schmatjko and J. Wolfram, Ber. Bunsenges. Physik. Chem. 82, 419 (1978).

9 R. Holland, D. W. G. Style, R. N. Dixon, and D. A. Ramsay, Nature 182, 337 (1958).

10 R. N. Dixon, Phil. Trans. Roy. Soc. (London), A252, 165 (1960).

11 R. N. Dixon, Can. J. Phys., 38, 10, (1960).

12 D. E. Milligan and M. E. Jacox, J. Chem. Phys. 47, 5157 (1967).

13 P. S. H. Bolman, J. M. Brown, A. Carrington, I. Kopp, and D. A. Ramsay, Proc. R. Soc. Lond. A. 343, 17 (1975).

14 V. E. Bondybey and J. H. English, J. Chem. Phys. 67, 2868 (1977).

15 T. R. Chariton, T. Okamura and B. A. Thrush, Chem. Phys. Lett. 89, 98 (1982).

16 D. R. Woodward, D. A. Fletcher, and J. M. Brown, Mol. Phys. 62, 517 (1987).

17 D. Patel-Misra, D. G. Sauder, and P. J. Dagdigian, J. Chem. Phys. 93, 5448 (1990).

18 A. Carrington, A. R. Fabris, B. J. Howard, and N. J. D. Lucas, Mol. Phys., 20, 961 (1971).

19 a) K. Kawaguchi, S. Saito, and E. Hirota, Mol. Phys. 49, 663 (1983).

b) K. Kawaguchi, S. Saito, and E. Hirota, Mol. Phys. 55, 341 (1985).

20 a) C. E. Barnes, J. M. Brown, A. D. Fackerell, and T. J. Sears, J. Mol. Spectrosc. 92,485 (1982). b) J. Werner, W. Seebass, M. Koch, R. F. Curl, W. Urban, and J. M. Brown, Mol. Phys. 65, 453 (1985). 
c) P. B. Davies and I. H. Davis, Mol. Phys. 69, 175 (1990).

21 a) D. M. Sonnenfroh, R. G. MacDonald, and K. Liu, J. Chem. Phys. 93, 1478 (1990).

b) D. G. Sauder, D. Patel-Misra, and P. J. Dagdigian, J. Chem. Phys. 95, 1696 (1991).

c) L. F. Phillips, I. A. W. Smith, R. P. Tuckett, and C. J. Whitham, Chem. Phys. Lett. 183, 254 (1991).

22 H. Okabe, J. Chem. Phys. 53, 5307 (1970).

${ }^{23}$ B. J. Sullivan, G. P. Smith and D. R. Crosley, Chem. Phys. Lett. 96, 307 (1983).

24 R. J. Balla and K. H. Casleton, J. Phys. Chem. 95, 2344 (1991), and references therein.

25 a) T. A. Spiglanin, R. A. Perry, and D. W. Chandler, J. Phys. Chem. 90, 6184 (1986).

b) T. A. Spiglanin and D. W. Chandler, Chem. Phys. Lett. 141, 428 (1987).

${ }^{26}$ K. - Y. Du and D. W. Setser, Chem. Phys. Lett. 153, 393 (1988).

27 M. A. Johnson, M. L. Alexander, and W. C. Lincberger, Chem. Phys. Lett. 112, 285 (1984).

${ }^{28}$ R. B. Metz, S. E. Bradforth, and D. M. Neumark, Adv. Chem. Phys. 81, 1 (1992).

29 D. Proch and T. Trickl, Rev. Sci. Instrum. 60, 713 (1989).

30 E. Illenberger, P. B. Comita, J. I. Brauman, H. -P. Fenzlaff, M. Heni, N. Heinrich, W. Koch, and G. Frenking, Ber. Bunsenges Physik. Chem. 89, 1026 (1985).

31 R. E. Continetti, D. R. Cyr, and D. M. Neumark, Rev. Sci. Instrum. 63, 1840 (1992).

32 J. M. B. Bakker, J. Phys. E 6, 785 (1973); 7, 364 (1974).

33 S. E. Bradforth, E. H. Kim, D. W. Amold, and D. M. Neumark, J. Chem. Phys. 98, 800 (1993).

34 R. N. Dixon, M. J. Trenouth, and C. M. Westem, Mol. Phys. 60, 779 (1987).

35 J. W. C. Johns, Can. J. Phys. 39, 1738 (1961).

36 a) D. Gauyacq, C. Larcher, and J. Rostas, Can. J. Phys. 57, 1634 (1979).

b) C. Larcher, D. Gauyacq and J. Rostas, J. de Chim. Phys. 77, 655 (1980).

37 J. A. Pople, Mol. Phys. 3, 16 (1960).

38 J. T. Hougen, J. Chem. Phys. 36, 519 (1962); J. Chem. Phys. 37, 403 (1962).

39 M. H. Alexander and H.-J. Werner, submitted to J. Chem. Phys.

40 a) M. A. Johnson, J. Rostas, and R. N. Zare, Chem. Phys. Lett. 92, 225 (1982).

b) M. A. Johnson, R. N. Zare, J. Rostas and S. Leach, J. Chem. Phys. 80, 2407 (1984).

41 R. E. Continetti, Ph. D. Thesis, University of California, Berkeley, 1989. 
42 T. N. Kitsopoulos, Ph. D. Thesis, University of Califomia, Berkeley, 1991.

43 R. N. Zare, Mol. Photochem. 4, 1 (1972).

44 M. W. Chase, Jr., C. A. Davies, J. R. Downey, Jr., D. J. Frurip, R. A. McDonald, and A. N. Syverud, JANAF Thermochemcial Tables, 3rd Edition (American Chemical Society and American Institute of Physics, New York, 1986).

45 S. T. Gibson, J. P. Greene, and J. Berkowitz, J. Chem. Phys. 83, 4319 (1985).

46 K. M. Ervin and P. B. Armentrout, J. Chem. Phys. 86, 2659 (1987); J. W. Sutherland and J. V. Michael, J. Chem. Phys. 88, 830 (1988).

47 K. Uno, T. Hikida, A. Hiraya, and K. Shobatake, Chem. Phys. Lett. 166, 475 (1990).

48 K. Yamada, J. Mol. Spectrosc. 79, 323 (1980).

49 P. Misra, C. W. Mathews, and D. A. Ramsay, J. Mol. Spectrosc. 130, 419 (1988).

50 P. Carskey, J. Kuhn, and R. Zahradnik, J. Mol. Spectrosc. 55, 120 (1975).

51 B. F. Minaev, N. M. Ivanova, and Z. M. Muldahmetov, Spectrosc. Lett. 22, 901 (1989).

52 D. P. de Brujin and J. Los, Rev. Sci. Instrum. 53, 1020 (1982). 


\section{Chapter 6 \\ Photodissociation of the $\mathrm{CH}_{2} \mathrm{NO}_{2}$ Radical}

\subsection{Introduction}

The study of molecular photodissociation processes has developed into one of the most productive and valuable areas of chemical physics in recent years. ${ }^{1}$ This body of work has led to a detailed understanding of both the spectroscopy and dynamics associated with the dissociative electronic states in a wide variety of molecules. Moreover, photodissociation experiments provide one of the most direct means of determining bond dissociation energies. However, the vast body of these investigations have been undertaken on stable molecules; only a handful of photodissociation experiments on reactive free radicals have been performed to date. For most radicals, the excited states in general and the dissociative states in particular are poorly characterized, and the bond dissociation energies are not known very accurately. Hence, these species present an inviting target for photodissociation experiments.

This situation provided the motivation for the development in our laboratory of a photodissociation experiment especially well-suited for studies of free radicals, based on a novel form of photofragment translational spectroscopy. In the standard version of photofragment translational spectroscopy, ${ }^{2}$ a molecular beam is photolyzed, and one measures the translational energy and angular distributions of the resulting fragments by a variety of techniques. ${ }^{3}$ Thus far, the primary challenge met in extending this method to reactive free radicals ${ }^{4,5}$ has been the difficulties involved in generating a clean, wellcharacterized molecular beam of these species. Our experiment uses a different approach in order to eliminate this problem. We produce mass selected, neutral free radicals from photodetachment of a fast $(6-8 \mathrm{keV})$ beam of the corresponding negative ions. The resulting radicals are then photodissociated, and the photofragments are detected with high efficiency. We can map out the dissociative excited states of a given radical by 
determining how the photodissociation cross section varies with dissociation photon energy. Subsequently, we perform dynamics experiments at selected wavelengths, using a coincidence detection scheme to determine the masses, kinetic energy release (KER), and recoil angle with respect to the laser polarization for each pair of photofragments formed. From this, we obtain photofragment translational energy and angular distributions as well as the yield for each fragmentation channel. Previously, we have applied this method to diatomic and triatomic species. ${ }^{67,8,9}$ In this paper, we report our first results for a polyatomic molecule, the nitromethyl radical $\mathrm{CH}_{2} \mathrm{NO}_{2}$.

The nitromethyl radical is thought to be a primary intermediate in the combustion of nitromethane, $\mathrm{CH}_{3} \mathrm{NO}_{2}$, formed by simple hydrogen abstraction. ${ }^{10,11}$ Past experimental work on $\mathrm{CH}_{2} \mathrm{NO}_{2}$ is fairly limited. Chachaty and coworkers ${ }^{12,13}$ obtained electron spin resonance (ESR) spectra of the nitromethyl radical following $\gamma$ irradiation of nitromethane at 77 and $195 \mathrm{~K}$. Matrix isolation work using isotopic substitution performed by Jacox ${ }^{11,14}$ resulted in the identification of all but one of the twelve ground state vibrational frequencies by infrared absorption, and the subsequent determination of the planar, $C_{2 v}$ geometry of the ground state. Jacox also ascertained that the threshold for photodissociation lies between 280 and $300 \mathrm{~nm}$. The photoproducts resulting from dissociation at $280 \mathrm{~nm}$ were determined to be $\mathrm{H}_{2} \mathrm{CO}$ and NO. It was noted that these could be either the nascent fragments or the products from caged recombination of $\mathrm{CH}_{2}+\mathrm{NO}_{2}$ formed by $\mathrm{C}-\mathrm{N}$ fission. More recently, Metz et al. ${ }^{15}$ recorded the photoelectron spectrum of the nitromethyl anion $\left(\mathrm{CH}_{2} \mathrm{NO}_{2}^{-}\right)$. They determined the electron affinity of $\mathrm{CH}_{2} \mathrm{NO}_{2}$ to be $2.475 \pm 0.010 \mathrm{eV}$, and located an excited electronic state (the $1{ }^{2} \mathrm{~A}_{2}$ state) $1.591 \mathrm{eV}$ above the $\tilde{X}{ }^{2} \mathrm{~B}_{1}$ ground state. In addition, ab initio calculations were performed to assist in identifying the one remaining unknown groundstate vibrational frequency (for the torsional mode) from the experimental data. Other theoretical work on the nitromethyl radical includes two previous $a b$ initio calculations 
completed at the single and multiconfigurational self-consistent field (MCSCF) levels by McKee, ${ }^{16,17}$ which attempted to identify and characterize the lowest electronic states.

In contrast to the relatively few studies of $\mathrm{CH}_{2} \mathrm{NO}_{2}$, the photodissociation of the analogous closed shell species, nitromethane, has been thoroughly studied. Depending on the method of excitation, three major pathways can contribute to dissociation:

$$
\begin{aligned}
\mathrm{CH}_{3} \mathrm{NO}_{2} & \rightarrow \mathrm{CH}_{3} \mathrm{NO}+\mathrm{O} \\
& \rightarrow \mathrm{CH}_{3} \mathrm{O}+\mathrm{NO} \\
& \rightarrow \mathrm{CH}_{3}+\mathrm{NO}_{2} .
\end{aligned}
$$

The ultraviolet photodissociation of $\mathrm{CH}_{3} \mathrm{NO}_{2}$ in the wavelength range from 190 to $220 \mathrm{~nm}$ has been investigated in the gas phase ${ }^{18,19,20,21}$ and also in solution. 22 This is a $\pi^{*} \leftarrow \pi$ excitation and leads exclusively to $\mathrm{C}-\mathrm{N}$ bond fission, Eqn. (6.3). Butler ${ }^{20}$ has proposed two pathways to these products. The primary pathway is adiabatic, involving crossing onto a $\sigma^{*} \leftarrow n$ surface of the same symmetry as the initially excited state and leading to the production of $\mathrm{NO}_{2}$ in the $1{ }^{2} \mathrm{~B}_{2}$ electronic state. At the highest energies in this range a diabatic dissociation pathway becomes energetically allowed, producing $\mathrm{NO}_{2}$ in the $2{ }^{2} \mathrm{~B}_{2}$ electronic state. Schoen et al..$^{23}$ have reported picosecond photodissociation studies of nitromethane at wavelengths between $238 \mathrm{~nm}$ and $337 \mathrm{~nm}$, again finding only C-N bond fission in this region. The amount of electronically excited $\mathrm{NO}_{2}$ was shown to increase with increasing photon energy, as determined by the ratio of fluorescence yield to laserinduced fluorescence yield measurements on the photoproducts. In contrast, infrared multiphoton dissociation (IRMPD) studies by Wodtke et al..24.25 determined that when dissociation occurs on the ground state surface, there is a competition between the $\mathrm{C}-\mathrm{N}$ bond fission process and rearrangement to form methyl nitrite, followed by nitric oxide elimination, as illustrated by Eqn. (6.2). Beijersbergen et al. ${ }^{26}$ have recently published a study in which the dissociative charge transfer neutralization of the nitromethane radical cation is described. This technique results in the formation of neutrals with excitation energies centered at $5.9 \mathrm{eV}$ ( $\mathrm{Na}$ charge exchange) or $7.2 \mathrm{eV}$ (Cs charge exchange). In 
their study, they determined the major channels to be $\mathrm{N}-\mathrm{O}$ and $\mathrm{C}-\mathrm{N}$ fission [Eqns. (6.1) and (6.3)], while nitric oxide elimination [Eqn. (6.2)] was observed as a minor channel.

The work on the nitromethyl radical reported here was largely motivated by our interest in learning how its photodissociation dynamics might differ from nitromethane. The matrix results obtained by Jacox ${ }^{11}$ are particularly intriguing in this regard. If the $\mathrm{H}_{2} \mathrm{CO}+\mathrm{NO}$ photoproducts observed by Jacox are indeed nascent fragments, this would indicate that the primary $\mathrm{UV}$ photochemistry of $\mathrm{CH}_{2} \mathrm{NO}_{2}$ involves $\mathrm{NO}$ elimination rather than $\mathrm{C}-\mathrm{N}$ bond fission as in $\mathrm{CH}_{3} \mathrm{NO}_{2}$. The collision-free environment in our experiment makes it possible to clearly identify the primary photofragments.

In the following section details of the experiment are discussed, while results and analysis of the photodissociation of the nitromethyl radical are presented in $\S 6.3$. We unambiguously identify and characterize the nascent photoproducts of the ultraviolet photodissociation of $\mathrm{CH}_{2} \mathrm{NO}_{2}$. In doing so, we identify the following two dissociation channels: (I) $\mathrm{CH}_{2} \mathrm{NO}_{2} \rightarrow \mathrm{CH}_{2} \mathrm{NO}+\mathrm{O}$ and (II) $\mathrm{CH}_{2} \mathrm{NO}_{2} \rightarrow \mathrm{H}_{2} \mathrm{CO}+\mathrm{NO}$. The dissociation process that involves simple $\mathrm{C}-\mathrm{N}$ bond fission, (III) $\mathrm{CH}_{2} \mathrm{NO}_{2} \rightarrow \mathrm{CH}_{2}+\mathrm{NO}_{2}$, is not observed. For the two observed channels, the branching ratio, expressed as the quotient (I)/(II), changes from 1.19 to 1.76 on varying the photodissociation wavelength from $270 \mathrm{~nm}$ to $240 \mathrm{~nm}$. In addition, the translational energy distribution, $P\left(E_{\mathrm{T}}\right)$, and the recoii anisotropy parameter as a function of translational energy release, $\beta\left(E_{\mathrm{T}}\right)$, are determined for each channel. These data imply fundamental differences in $t^{\prime}:$ dissociation dynamics for the two observed channels, providing insight into the dissociative mechanism involved in each channel. In $\S 6.4$, we discuss the implications of these observations on the dissociation mechanisms for the various channels. It is noted that a preliminary account of this work has been published elsewhere. ${ }^{27}$ 


\subsection{Experimental Approach}

Due to their open shell nature, free radicals typically have positive electron affinities. Our experiment utilizes this property in order to generate a pure, well-characterized packet of free radicals via photodetachment of the corresponding mass-selected negative ion. As an added advantage, the high laboratory frame kinetic energy imparted to the radicals during their acceleration prior to detachment allows the efficient collection and detection of photofragments simply by striking the face of a microchannel plate (MCP) detector.

A schematic of the fast radical beam photodissociation spectrometer used in this work is shown in Figure 2.1. Details concerning the design of the fast radical beam spectrometer and the photofragment detection scheme are given in Chapter 2, but there are several aspects of the experiment particularly germane to the present study which will be described here.

The basic experiment is as follows:

$$
\mathrm{CH}_{2} \mathrm{NO}_{2}^{-} \stackrel{h v_{1}}{\longrightarrow} \mathrm{CH}_{2} \mathrm{NO}_{2}+\mathrm{e}^{-} \stackrel{h v_{2}}{\longrightarrow} \text { fragments. }
$$

The nitromethyl anion, $\mathrm{CH}_{2} \mathrm{NO}_{2}^{-}$, is prepared following the procedure of Metz et al., ${ }^{15}$ using a pulsed molecular beam ion source similar to that developed by Lineberger and coworkers. ${ }^{28}$ We first bubble a mixture of $10 \% \mathrm{NF}_{3}$ in $\mathrm{N}_{2}$ through neat nitromethane (Eastman) at room temperature and 18 PSIG total pressure. A pulsed molecular beam valve operating at $60 \mathrm{~Hz}$ introduces this mixture to the source vacuum chamber. Immediately outside the nozzle orifice, the supersonic expansion is crossed with a continuous $1 \mathrm{keV}$ electron beam. Low energy secondary electrons formed by electron impact ionization result in the production of $\mathrm{F}^{-}$via dissociative attachment to $\mathrm{NF}_{3}$. This fluorine anion abstracts a proton from nitromethane, forming $\mathrm{CH}_{2} \mathrm{NO}_{2}^{-}$, which undergoes cooling during the remainder of the expansion. Ions pass through a $3 \mathrm{~mm}$ diameter 
skimmer and are accelerated to $6 \mathrm{keV}$. They are then rereferenced to ground potential by the use of a potential 'switch', 29 and mass selected via a Bakker-type time-of-flight mass spectrometer. ${ }^{30}$ After passing through a $1-\mathrm{mm}$ beam-defining aperture, the anions are then photodetached using a tunable, pulsed, excimer-pumped-dye laser system, timed to intersect the mass of interest. Detachment laser energies are $30-50 \mathrm{~mJ} / \mathrm{pulse}$ and the laser is loosely focused with a $1 \mathrm{~m}$ focal length lens, $50 \mathrm{~cm}$ prior to intersecting the ion packet. Attenuation of the ion beam by the photodetachment light pulse can be as high as $50 \%$.

The anions are vibrationally cold, and the detachment wavelength is chosen to avoid the production of vibrationally excited radicals, as is discussed in $\$ 1.4 .2$. This is done with the aid of Figure 6.1, which shows the fixed frequency photoelectron spectrum of $\mathrm{CH}_{2} \mathrm{NO}_{2}^{-}$obtained previously using a separate apparatus in our laboratory. This photoelectron spectrum was taken using a photon energy of $3.49 \mathrm{eV}\left(\lambda_{\text {det }}=355 \mathrm{~nm}\right)$, and revealed the electron affinity of $\mathrm{CH}_{2} \mathrm{NO}_{2}$ to be $2.475 \mathrm{eV} .{ }^{15}$ In this spectrum, the measured kinetic energy of the electrons represents the photon energy minus the difference in energy between the ground vibrational state of the anion and vibrational levels in the neutral. Hence, in Figure 6.1 the peak at highest electron kinetic energy corresponds to the ground vibrational level in the neutral. The spectrum consists of a strong vibrational progression in the $v_{3}$ symmetric stretch mode. We therefore photodetach the anions at a photon energy of $2.59 \mathrm{eV}\left(\lambda_{\text {det }}=479 \mathrm{~nm}\right)$, just above the electron affinity of $\mathrm{CH}_{2} \mathrm{NO}_{2}$. As illustrated by the location of the arrow in Figure 6.1, this is not energetic enough to excite the active $v_{3}$ mode of the neutral.

Following detachment, all remaining negative ions are deflected out of the beam. The radicals must pass through a second $1 \mathrm{~mm}$ collimating aperture and are then

Figure 6.1 Photoelectron spectrum of nitromethyl anion obtained using a photon energy of $3.49 \mathrm{eV}\left(\lambda_{\text {det }}=355 \mathrm{~nm}\right)$. The prominent $v_{3}$ progression is labeled, and the position of the arrow relative to the origin peak indicates the energy available upon photodetachment using $2.59 \mathrm{eV}$ photons $\left(\lambda_{\text {det }}=479 \mathrm{~nm}\right)$. 


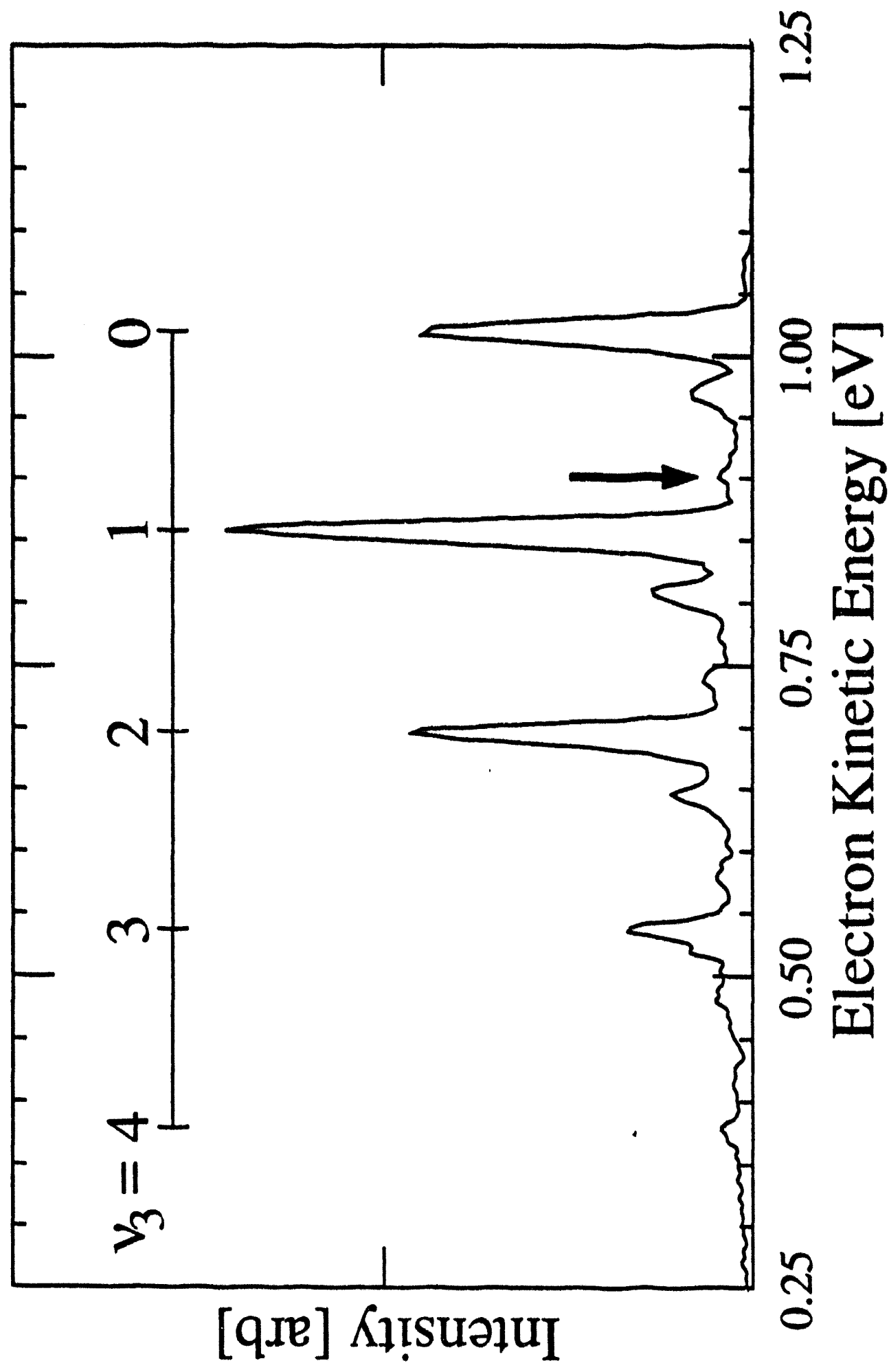

Figure 6.1 
intersected in the photodissociation region by the horizontally polarized, frequencydoubled output of a second pulsed excimer-pumped-dye laser, loosely focused by a $2 \mathrm{~m}$ lens. The photofragments are detected with one of two microchannel plate detectors, lying 0.67 and $1.0 \mathrm{~m}$ downstream of the photodissociation region respectively; the closer detertor is movable and is removed from the beam path when the more distant detector is in use. Photofragments typically have enough recoil kinetic energy to scatter out from the beam axis, thereby missing the blocking strips placed across the center of the detectors ( 3 or $8 \mathrm{~mm}$ wide, respectively) to prevent undissociated radicals from impinging on the active area. The laboratory kinetic energies of the photofragments are generally $>1 \mathrm{keV}$, ensuring a high ( $-50 \%)$ detection efficiency for those that strike the active area. The detector at $0.67 \mathrm{~m}$ is used for photodissociation cross section measurements; the total photofragment yield is monitored as the dissociation laser wavelength is varied. In general, this yields an approximate mapping of the dissociative electronic states of a given radical.

The photodissociation dynamics experiments are done using a more complex photofragment coincidence detection scheme. The laser is tuned to a wavelength at which photodissociation occurs, and the photofragments are detected with the second detector, $1 \mathrm{~m}$ from the photodissociation region. This detector is a two-particle time- and positionsensitive detector, similar in principle to the detector first developed by de Bruijn and Los, ${ }^{31}$ although we use a coincidence wedge-and strip anode (C-WSA) ${ }^{8,32,33}$ rather than capacitive charge division for the position sensing. As illustrated in Figure 6.2, for each photodissociation event we measure the position of both fragments at the detector, denoted $\left(x_{1}, y_{1}\right)$ and $\left(x_{2}, y_{2}\right)$, as well as the time delay between their arrival, $\tau$. This enables us to determine the photofragment masses, kinetic energy release, and scattering

Figure 6.2 Schematic of a typical dissociation event, with the measured quantities necessary for calculation of fragment mass ratio, KER and recoil angle with respect to laser polarization labeled. 


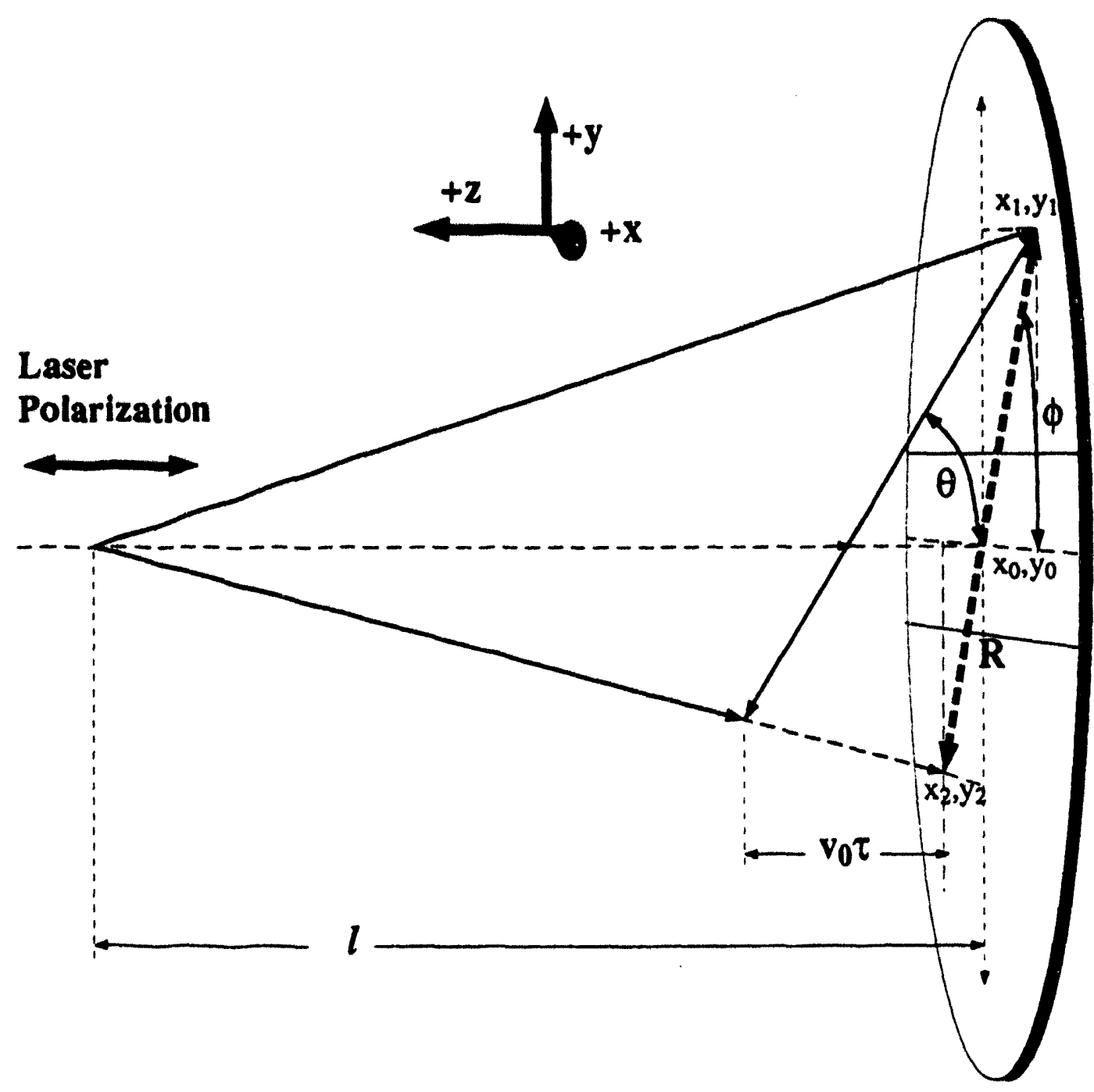

Figure 6.2 
angle with respect to the dissociation laser polarization direction for each event, as fully discussed in $\$ 3.2$. Because this is a coincidence measurement, we can process at most one event per laser pulse; ion densities in this experiment are sufficiently low so that this was not a problem. By summing over large numbers of dissociation events $\left(2-8 \times 10^{4}\right)$, we have obtained branching ratios as well as translational energy and angular distributions for each channel observed in this experiment.

The mass ratio of the two fragments is found by first determining $r_{i}=\sqrt{\left(x_{i}-x_{0}\right)^{2}+\left(y_{i}-y_{0}\right)^{2}}$, the displacement of each fragment from the location of the radical beam center $\left(x_{0}, y_{0}\right)$ on the detector face. The mass ratio of the two fragments is then given approximately by the inverse of the ratio of these displacements:

$$
\frac{m_{1}}{m_{2}}=\frac{r_{2}}{r_{1}} \cdot\left[1-\left(\frac{\mathrm{v}_{0} \tau}{L}\right)\right] \equiv \frac{r_{2}}{r_{1}}
$$

Once the fragment masses are determined, Eqns. (6.6) and (6.7), originally derived by De Bruijn and Los, ${ }^{31}$ are used to obtain the center-of-mass kinetic energy release (KER) and fragment recoil angle $\theta$, measured with respect to the polarization vector of the laser, for each dissociation event.

$$
\begin{aligned}
& \theta=\tan ^{-1}\left(\frac{\mathrm{R}}{\mathrm{v}_{0} \tau}\right) \\
& K E R=E_{0} \cdot\left(\frac{m_{1} \cdot m_{2}}{M^{2}}\right) \cdot \frac{\left[\left(\mathrm{v}_{0} \tau\right)^{2}+\mathrm{R}^{2}\right]}{L^{2}} \cdot\left[1+2\left(\frac{m_{2}-m_{1}}{M}\right) \cdot \frac{\mathrm{v}_{0} \tau}{L}\right]
\end{aligned}
$$

Here $R=\sqrt{\left(x_{2}-x_{1}\right)^{2}+\left(y_{2}-y_{1}\right)^{2}}$ is the relative separation of the two fragments, $M$ is the parent radical mass, $m_{1}$ and $m_{2}$ the fragment masses striking the upper and lower half of the detector, respectively, $L$ the flight length, and $E_{0}$ and $v_{0}$ the primary beam 
energy and velocity, respectively. $\tau$ is taken to be positive if the upper fragment strikes the detector first.

In Eqn. (6.5) the chief source of uncertainty is the dependence of $r_{1}$ and $r_{2}$ on an explicit knowledge of $\left(x_{0}, y_{0}\right)$, the detector coordinates where the center of mass of the dissociating system would be found (i.e. where the parent radical, if it had not dissociated, would strike the detector). The radical beam is about $1 \mathrm{~mm}$ FWHM in diameter, resulting in a fragment mass resolution $(\mathrm{m} / \Delta \mathrm{m})$ of about 15 for the results reported here. In contrast, Eqns. (6.6) and (6.7) do not require an explicit knowledge of $\left(x_{0}, y_{0}\right)$, and therefore the center-of-mass uncertainty does not contribute to the energy and scattering angle, assuming the product masses can be identified unambiguously. The measured spatial resolution is on the order of $75 \mu \mathrm{m}$ full-width-at-half-maximum in $y$ and $130 \mu \mathrm{m}$ in $x$, while timing resolution is about $0.5 \mathrm{~ns}$. Energy resolution as high as $10 \mathrm{meV}$ has been demonstrated during calibrations using the predissociation of $\mathrm{O}_{2}$ through the SchumannRunge band, ${ }^{9}$ but is approximately $30 \mathrm{meV}\left(0.69 \mathrm{kcal} / \mathrm{mol}, 240 \mathrm{~cm}^{-1}\right)$ in these studies.

As discussed in Chapter 3, upon the completion of a photodissociation dynamics experiment, we first separate the data into different mass channels following Eqn. (6.5). For each channel we then construct a two-dimensional array in KER and theta into which each coincident dissociation event is binned. The channel specific energy-angle arrays, denoted here as $\delta\left(E_{T}, \theta\right)$, are determined using Eqns. (6.6) and (6.7). From this, we obtain the true joint energy and angular distribution $P\left(E_{T}, \theta\right)$ for each product channel by dividing by the "detector acceptance function" $D\left(E_{\mathrm{T}}, \theta\right)$ :

$$
\mathscr{P}\left(E_{T}, \theta\right)=\$\left(E_{T}, \theta\right) / \mathscr{A}\left(E_{T}, \theta\right) .
$$

The detector acceptance function accounts for the finite acceptance of the detector as a function of $E_{\mathrm{T}}$ and theta. For example, fragments with lower recoil energies and/or values of theta close to 0 or 180 degrees may not strike the detector because of the beam block, while some high energy fragments at values of theta close to 90 degrees miss the detector 
because of its finite size. The straightforward numerical determination of $\mathcal{A}\left(E_{\mathrm{T}}, \theta\right)$ is discussed in § 3.2.3.

As mentioned above, the dual-wedge-and-strip anode requires an $8 \mathrm{~mm}$ wide horizontal blocking strip which shields the microchannel plates over a small region above the interface of the two anodes to prevent the undissociated radical beam from impinging on the detector face. However, it also prevents photofragments with very low center-ofmass kinetic energy from being detected. This effect is magnified in the case of fragments with unequal masses. The heavy fragment scatters out of the beam more slowly than the light fragment and is more likely to be blocked, but both fragments must be detected to be recorded as a coincidence event. In order to improve coincidence detection of low energy, unequal mass fragments, the detector can be positioned slightly off-center vertically with respect to the radical beam axis. In the experiments reported here, such a product channel was identified early on, and the data were then collected with the center of the detector $1.9 \mathrm{~mm}$ lower than the radical beam axis. This enables heavy fragments that would have otherwise struck the blocking strip to clear the top edge. Because the correlated light fragments have a greater recoil velocity due to momentum conservation, they are able to travel the correspondingly larger distance necessary to clear the bottom of the blocking strip. The effect of this offset on the detector acceptance function is included in the analysis of the data.

\subsection{Results and Analysis}

Photodissociation cross section measurements of the $\mathrm{CH}_{2} \mathrm{NO}_{2}$ radical were taken across the wavelength range from 240 to $270 \mathrm{~nm}$. The photodissociation cross section scans appeared to be structureless, with the cross section peaking near $240 \mathrm{~nm}$ and decreasing monotonically by about a factor of five at $270 \mathrm{~nm}$. Most of the photodissociation dynamics data were taken at $240 \mathrm{~nm}$, corresponding to a photon energy of $119.1 \mathrm{kcal} / \mathrm{mol}$, where the dissociation signal was most intense. Supplementary data 
were recorded using longer wavelengths of $255 \mathrm{~nm}$ and $270 \mathrm{~nm}$, corresponding to photon energies of 112.1 and $105.9 \mathrm{kcal} / \mathrm{mol}$, respectively.

\subsubsection{Identification of Photodissociation Channels}

Prior to any further analysis, it is necessary to determine which fragmentation channels occur in the photodissociation of $\mathrm{CH}_{2} \mathrm{NO}_{2}$. Many channels are energetically allowed in the present case of $\mathrm{CH}_{2} \mathrm{NO}_{2}$, a six atom radical to which $\sim 5 \mathrm{eV}$ excitation energy is imparted. Even if only one dissociation channel is active, the analysis of time and position data to obtain the translational energy distribution for that channel depends on first identifying the masses of the photofragments produced. The presence of two or more active dissociation channels demands the ability to determine which channel each dissociation event corresponds to.

The photofragment mass spectrum for $\mathrm{CH}_{2} \mathrm{NO}_{2}$ at $240 \mathrm{~nm}$ is shown in Figure 6.3. Note that this plot is constructed from the data by determining the photofragment mass ratio in each event, and thus the fraction of the total mass $M(=60 \mathrm{amu})$ of the parent radical for each fragment, resulting in a mirror image about $M / 2$. The mass spectrum indicates the presence of two dissociation channels. Although the mass resolution is limited, it appears that in one channel, the fragment masses are 16 and $44 \mathrm{amu}$, while in the other, both fragments have mass $30 \mathrm{amu}$. This would indicate that the two dissociation channels are (I) $\mathrm{CH}_{2} \mathrm{NO}+\mathrm{O}$ and (II) $\mathrm{H}_{2} \mathrm{CO}+\mathrm{NO}$.

In order to verify these assignments, the photofragment mass spectrum of $\mathrm{CD}_{2} \mathrm{NO}_{2}$ was also obtained at $2 \mu 0 \mathrm{~nm}$, and the results are superimposed on the $\mathrm{CH}_{2} \mathrm{NO}_{2}$ mass

Figure 6.3 Photofragment mass spectrum from the $240 \mathrm{~nm}$ dissociation of the nitromethyl radical $\left(\mathrm{CH}_{2} \mathrm{NO}_{2}\right.$; solid line) and the $\mathrm{d}_{2}$-nitromethyl radical $\left(\mathrm{CD}_{2} \mathrm{NO}_{2}\right.$; dashed line). Areas under the mass peaks do not accurately reflect the mass channel branching ratio because they are uncorrected for detector acceptance (see text). 


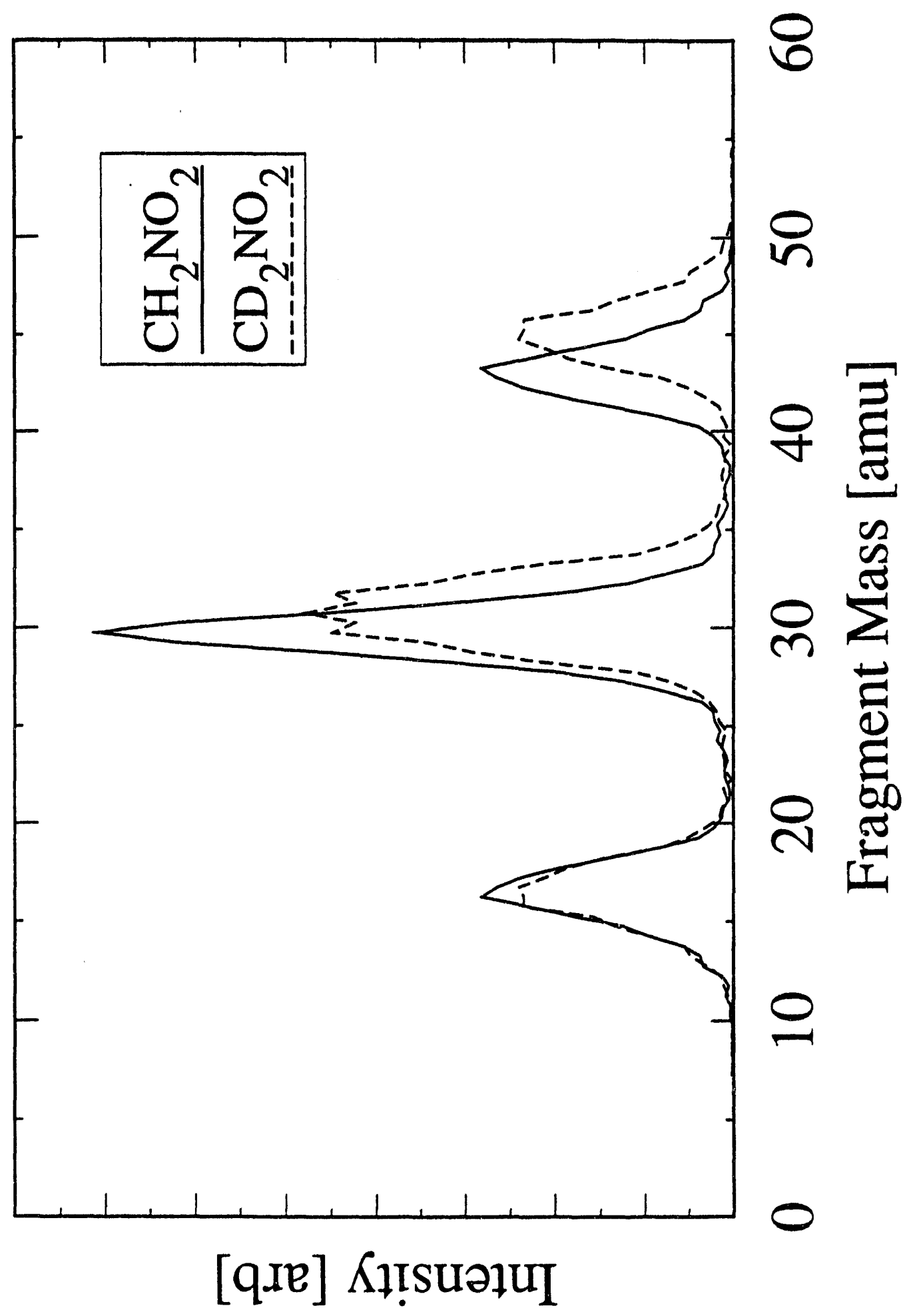

Figure 6.3 
spectrum in Figure 6.3. The differences in the two spectra upon deuteration are the following: the mass peak centered at 16 amu remains fixed while the mass peak at $44 \mathrm{amu}$ shifts to $46 \mathrm{amu}$, and the mass peak centered at $30 \mathrm{amu}$ broadens towards higher mass and appears to be centered at $31 \mathrm{amu}$. The first difference shows that the photofragments with mass ratio close to 3:1 indeed corresponds to (I) $\mathrm{CH}_{2} \mathrm{NO}_{2} \rightarrow \mathrm{CH}_{2} \mathrm{NO}+\mathrm{O}$, since there can be no H/D atoms in the lighter mass fragment. Although the mass fragments from channel (III), $\mathrm{CH}_{2} \mathrm{NO}_{2} \rightarrow \mathrm{CH}_{2}+\mathrm{NO}_{2}$, (14 and 46) are similar to those of channel (I), upon isotopic substitution the lower mass peak would shift from 14 to $16 \mathrm{amu}$, while the heavier mass fragment would remained fixed at $46 \mathrm{amu}$. We therefore estimate an upper limit for the presence of channel (III) products of $4 \%$. The second observed difference confirms the presence of channel (II), $\mathrm{CH}_{2} \mathrm{NO}_{2} \rightarrow \mathrm{H}_{2} \mathrm{CO}+\mathrm{NO}$, since the dissociation products from $\mathrm{CH}_{2} \mathrm{NO}_{2}$ should both have a mass of $30 \mathrm{amu}$ while the deuterated analogue, $\mathrm{CD}_{2} \mathrm{NO}_{2}$, should have dissociation products with masses of 32 and $30 \mathrm{amu}$ respectively. The deuterated mass spectrum eliminates another energetically feasible channel with comparable masses corresponding to $\mathrm{O}_{2}$ elimination, $\mathrm{CH}_{2} \mathrm{NO}_{2} \rightarrow \mathrm{H}_{2} \mathrm{CN}+\mathrm{O}_{2}$. The fragment masses of this excluded channel would change from 28:32 to 30:32 upon deuteration resulting in an expected narrowing of the central peak of the mass spectrum, contrary to our observation. This channel is assigned a similar estimated upper limit of $4 \%$.

While the mass spectra we have obtained do not show evidence for the presence of any dissociation channels other that the two identified above, we are unable to comment on channels involving extreme mass ratios because of the unfavorable kinematics involved. When two fragments have very disparate masses, as in the case of hydrogen bond fission, either the light fragment will recoil outside the maximum radius of the detector, or the heavy fragment will fail to clear the blocking strip, or both, depending on the recoil energy and angle of the event. Therefore, a conceivable channel that would go undetected in our experiment involves loss of a hydrogen atom, forming $\mathrm{HCNO}_{2}+\mathrm{H}$. Although there is no 
literanure value for the $\mathrm{C}-\mathrm{H}$ bond energy of nitromethyl radical, we expect it to be close to the $100 \mathrm{kcal} / \mathrm{mol}$ value found for the $\mathrm{C}-\mathrm{H}$ bond in $\mathrm{CH}_{3} \mathrm{NO}_{2},{ }^{15,34}$ largely unaffected by the open shell nature of the radical. This is quite high compared to the available energy in these experiments and the energies of the channels which are known to be occurring (see below), so it is doubtul that this channel is active.

\subsubsection{Translational Energy and Angular Distributions}

Once the photodissociation channels are identified, we can determine the centerof-mass translational energy and angular distributions for each channel. The energy and angular distribution, $\mathcal{P}\left(E_{T}, \theta\right)$, for a one-photon dissociation can be expressed as

$$
P\left(E_{T}, \theta\right)=P\left(E_{T}\right)\left[1+\beta\left(E_{T}\right) \mathrm{P}_{2}(\cos \theta)\right],
$$

where $\theta$ is the angle between the photofragment recoil vector and the electric vector of the dissociation laser, $P_{2}(\cos \theta)$ is the second Legendre polynomial, $\beta\left(E_{\mathrm{T}}\right)$ is the anisotropy parameter as a function of translational (or kinetic) energy, and $P\left(E_{\mathrm{T}}\right)$ is the angleintegrated translational energy distribution. The anisotropy parameter $\beta$ can range from $\beta=+2$ to $\beta=-1$, corresponding to $a \cos ^{2} \theta$ distribution and a $\sin ^{2} \theta$ distribution of recoil vectors, respectively. ${ }^{35}$

$P\left(E_{\mathrm{T}}, \theta\right)$ is derived from the data as described in $\S 6.2$, and, in greater detail,

\$ 3.2.4. The photofragment anisotropy parameter $\beta\left(E_{\mathrm{T}}\right)_{i}$ is found for each kinetic energy interval $\left(E_{i}-\Delta E / 2, E_{i}+\Delta E / 2\right)$ by performing a least squares fit to Eqn. (6.9). The $\Delta E$ intervals chosen for this procedure were $4.6 \mathrm{kcal} / \mathrm{mol}$ for channel (I) and $9.2 \mathrm{kcal} / \mathrm{mol}$ for channel (II). Only when the data becomes very sparse (i.e. in the 'tail' of the KER distribution near the energetic limit for a given channel) do we find that this method of analysis breaks down. In these restricted regions, containing very little data, we cannot determine $\beta\left(E_{T}\right)$, and it is assumed to be zero (implying an isotropic recoil angular distribution). The final step in this direct inversion analysis is to find the angle-integrated 
translational energy distribution $P\left(E_{\mathrm{T}}\right)$ at the highest energy resolution. Using the appropriate $\beta\left(E_{T}\right)$ as determined above, we determine $P\left(E_{T}\right)$ for energy intervals of $0.575 \mathrm{kcal} / \mathrm{mol}$ in channel (I) and $1.150 \mathrm{kcal} / \mathrm{mol}$ in channel (II). These intervals are comparable to the instrumental resolution.

Table 6.1 shows the energies relative to the nitromethyl radical of the two observed product channels in addition to the absent $\mathrm{C}-\mathrm{N}$ bond fission channel. The heat of formation of $\mathrm{CH}_{2} \mathrm{NO}_{2}$ is taken to be $30.4 \pm 3 \mathrm{kcal} / \mathrm{mol},{ }^{15}$ while the heat of formation for $\mathrm{CH}_{2} \mathrm{NO}$ has been calculated by Melius ${ }^{36}$ to be $43 \pm 6 \mathrm{kcal} / \mathrm{mol}$. All other thermochemical data are relatively well known and are obtained from Reference 34.

Figure 6.4 shows the $P\left(E_{\uparrow}\right)$ distribution of channel (I), $\mathrm{CH}_{2} \mathrm{NO}_{2} \rightarrow \mathrm{CH}_{2} \mathrm{NO}+\mathrm{O}$, at the three wavelengths used in this study, 240, 255 and $270 \mathrm{~nm}$. All three of these distributions are peaked close to zero KER, with the $240 \mathrm{~nm}$ distribution peaking furthest away at $5-8 \mathrm{kcal} / \mathrm{mol}$. The $P\left(E_{\mathrm{T}}\right)$ distributions obtained at the other wavelengths peak closer to zero, but larger statistical uncertainties make the exact determination of their maxima difficult. For this channel the lowest observable KER, because of the presence of the blocker, is $2.0 \mathrm{kcal} / \mathrm{mol}$. The observed mean KERs are $11.4,11.0$, and $10.8 \mathrm{kcal} / \mathrm{mol}$ for 240,255 and $270 \mathrm{~nm}$ photodissociation, respectively. These values are upper bounds for the true mean KER, because of the unobserved fragments with a KER of less than $2.0 \mathrm{kcal} / \mathrm{mol}$. Barring the presence of an excursion in the $P\left(E_{\mathrm{T}}\right)$ distribution below $2.0 \mathrm{kcal} / \mathrm{mol}$, the overestimate in the mean KERs listed above is less than $10 \%$. The maximum kinetic energy release in the $240 \mathrm{~nm}$ data, for which the statistical error bars are smallest, is $44 \pm 1 \mathrm{kcal} / \mathrm{mol}$. This observation, combined with the well known $\Delta_{\mathrm{f}} \mathrm{H}_{(0 \mathrm{~K})}^{0}$ of an oxygen atom $(59.0 \mathrm{kcal} / \mathrm{mol})^{34}$ and the $\Delta_{\mathrm{f}} \mathrm{H}_{(0 \mathrm{~K})}^{0}$ for $\mathrm{CH}_{2} \mathrm{NO}_{2}$ given by Metz et al., 15 places an upper bound on the $\Delta_{\mathrm{f}} \mathrm{H}_{(0 \mathrm{~K})}^{0}$ for $\mathrm{CH}_{2} \mathrm{NO}$ of $47 \pm 3 \mathrm{kcal} / \mathrm{mol}$. This upper bound is

Figure 6.4 $P\left(E_{\tau}\right)$ distribution plots for the $\mathrm{CH}_{2} \mathrm{NO}+\mathrm{O}$ photodissociation channel taken at the three labeled wavelengths. Error bars represent $1 \sigma$ uncertainty. 


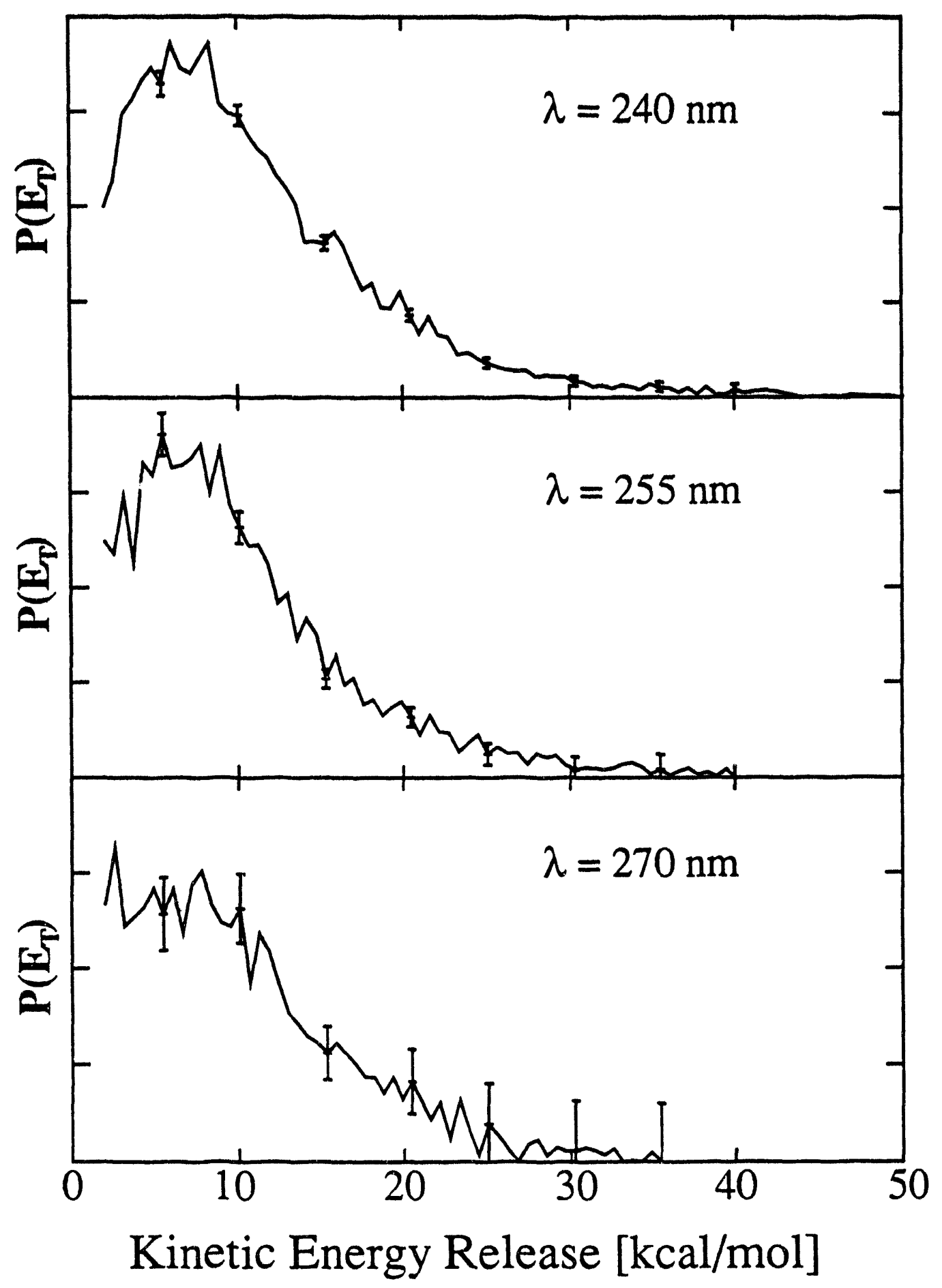

Figure 6.4 


\begin{tabular}{|c|c|c|c|c|c|}
\hline $\begin{array}{c}\text { Product } \\
\text { Channel }\end{array}$ & $\begin{array}{c}\text { Masses } \\
\text { [amu] }\end{array}$ & $\begin{array}{c}D_{0} \\
{[\mathrm{kcal} / \mathrm{mol}]}\end{array}$ & \multicolumn{3}{|c|}{$h \mathrm{hv}-\mathrm{D}_{0}[\mathrm{kcal} / \mathrm{mol}]$} \\
\hline (I) $\mathrm{CH}_{2} \mathrm{NO}+\mathrm{O}$ & $44+16$ & $72 \pm 6$ & 47 & 40 & 34 \\
\hline (II) $\mathrm{H}_{2} \mathrm{CO}+\mathrm{NO}$ & $30+30$ & $-33.7 \pm 3$ & 152.8 & 145.8 & 139.6 \\
\hline (III) $\mathrm{CH}_{2}+\mathrm{NO}_{2}$ & $14+46$ & $71.2 \pm 3$ & 47.9 & 40.9 & 34.7 \\
\hline
\end{tabular}

Table 6.1 Some possible product channels for the ultraviolet photodissociation of the nitromethyl radical. On the right, the energy available to the products following dissociation at the three wavelengths used in this work are listed. Heats of formation for $\mathrm{CH}_{2} \mathrm{NO}_{2}$ and $\mathrm{CH}_{2} \mathrm{NO}$ are taken from Metz et al. ${ }^{15}$ and Melius, ${ }^{36}$ respectively, while all other thermochemical information is obtained from Ref. 34. Channels (I) and (II) were observed as major channels in these experiments, while channel (II), corresponding to $\mathrm{C}-\mathrm{N}$ bond fission, was found to be absent.

very close to the $\Delta_{\mathrm{f}} \mathrm{H}_{(0 \mathrm{~K})}^{0}$ for $\mathrm{CH}_{2} \mathrm{NO}$ of $43 \pm 6 \mathrm{kcal} / \mathrm{mol}$ given by Melius. ${ }^{36}$ It is well below the upper bound of $\Delta_{\mathrm{f}} \mathrm{H}_{(298 \mathrm{~K})}^{0}\left(\mathrm{CH}_{2} \mathrm{NO}\right)=99 \mathrm{kcal} / \mathrm{mol}$ given by Beijersbergen $e t$ $a l .2^{26}$ however, in their calculations they use the most probable kinetic energy release rather than the maximum observed kinetic energy release. This, coupled with their assumption that all excess energy is measured as kinetic energy, results in a very conservative upper bound.

Figure 6.5 shows the $P\left(E_{\mathrm{T}}\right)$ of channel (II), $\mathrm{CH}_{2} \mathrm{NO}_{2} \rightarrow \mathrm{H}_{2} \mathrm{CO}+\mathrm{NO}$, for 240,255 and $270 \mathrm{~nm}$ photolysis. All three energy distributions have a broad peak near $55 \mathrm{kcal} / \mathrm{mol}$, with a mean KER of $58 \mathrm{kcal} / \mathrm{mol}$. High-energy tails in the $P\left(E_{\mathrm{T}}\right)$ plots extend up to about $20 \mathrm{kcal} / \mathrm{mol}$ below the energetic limit of this channel, which is $153 \mathrm{kcal} / \mathrm{mol}$ at $240 \mathrm{~nm}$. Upon changing photon energies, the mean KER changes very little; the majnr effect

Figure 6.5 $P\left(E_{\uparrow}\right)$ distribution plots for the $\mathrm{H}_{2} \mathrm{CO}+\mathrm{NO}$ photodissociation channel taken at the three labeled wavelengths. Error bars represent $1 \sigma$ uncertainty. 


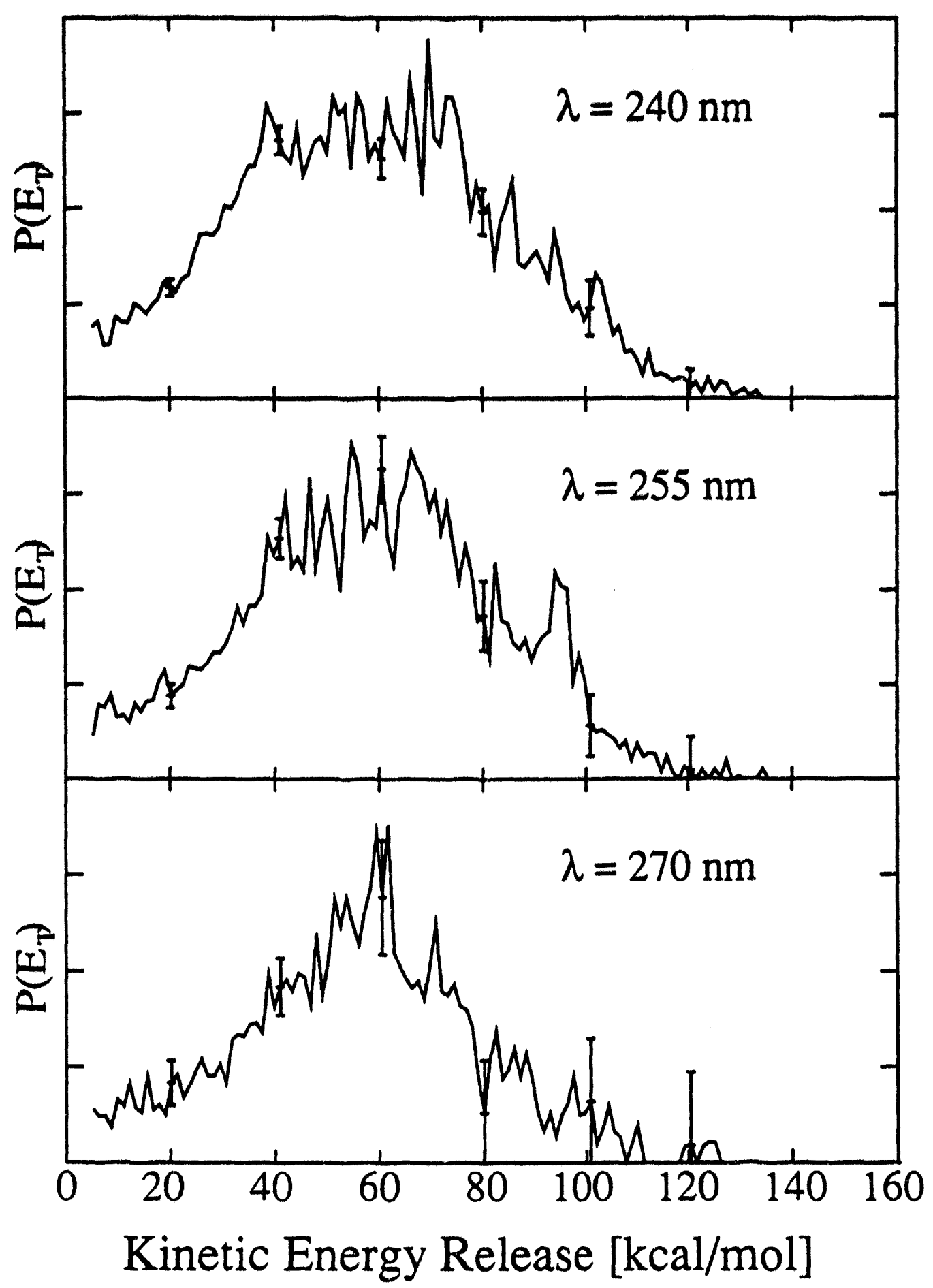

Figure 6.5 
instead is that the higher wavelength $P\left(E_{T}\right)$ distributions are narrower, being more strongly peaked near the mean KER.

Figure 6.6 is a plot of the fragment recoil anisotropy parameter, $\beta$, as a function of recoil energy, for channels (I) and (II) as determined from the $240 \mathrm{~nm}$ data. $\beta$ for channel (I) appears to be essentially zero until the upper limit of observed KER's, where the uncertainty associated with the measured values of $\beta$ includes zero in all but one case. $\beta$ for channel (II) is also found to be quite close to zero at low values of KER. Both of these plots are representative of the dependence of $\beta$ on KER at all three wavelengths for the respective channels; within the experimental uncertainty, the $\beta(E)$ plots did not appear to depend on the photon energy. We note that nearly isotropic angular distributions were also seen in the $193 \mathrm{~nm}$ photodissociation of nitromethane. 18

Channel (II) may also involve the secondary dissociation of the most highly intemally excited fortion of the $\mathrm{H}_{2} \mathrm{CO}$ formed. The exothermicity of channel (II) is $34 \mathrm{kcal} / \mathrm{mol}$, so that at $240 \mathrm{r.m}(119.1 \mathrm{kcal} / \mathrm{mol})$, fully $153 \mathrm{kcal} / \mathrm{mol}$ of energy is available for distribution among the products' degrees of freedom. This energy is well above the $80 \mathrm{kcal} / \mathrm{mol}$ barrier to dissociation to $\mathrm{H}_{2}+\mathrm{CO}$. Of course, Figure 6.5 shows that much of the available energy appears as product translation. In addition, the NO bond order changes from 1.5 to 2.5 during elimination, which is likely to result in vibrationally excited $\mathrm{NO}$, further decreasing the energy available for partitioning into $\mathrm{H}_{2} \mathrm{CO}$ internal degrees of freedom. Nonetheless, some fraction of $\mathrm{H}_{2} \mathrm{CO}$ might be expected to possess sufficient internal energy to undergo secondary dissociation on the nanosecond time scale. A second dissociation pathway for $\mathrm{H}_{2} \mathrm{CO}$, forming $\mathrm{H}\left({ }^{2} \mathrm{~S}\right)+\mathrm{HCO} \tilde{X}$ with very little KER, is expected to dominate the decomposition of $\mathrm{H}_{2} \mathrm{CO}$ much above its threshold of $96 \mathrm{kcal} / \mathrm{mol}^{37}$ In both cases, however, the kinematics are such that the velocity of the

Figure 6.6 Recoil anisotropy factor $\beta$, determined from the data as a function of recoil energy $E_{\mathrm{T}}$, for both channel (I), $\mathrm{CH}_{2} \mathrm{NO}_{2} \rightarrow \mathrm{CH}_{2} \mathrm{NO}+\mathrm{O}$, and channel (II), $\mathrm{CH}_{2} \mathrm{NO}_{2} \rightarrow \mathrm{H}_{2} \mathrm{CO}+\mathrm{NO}$, at $240 \mathrm{~nm}$. 

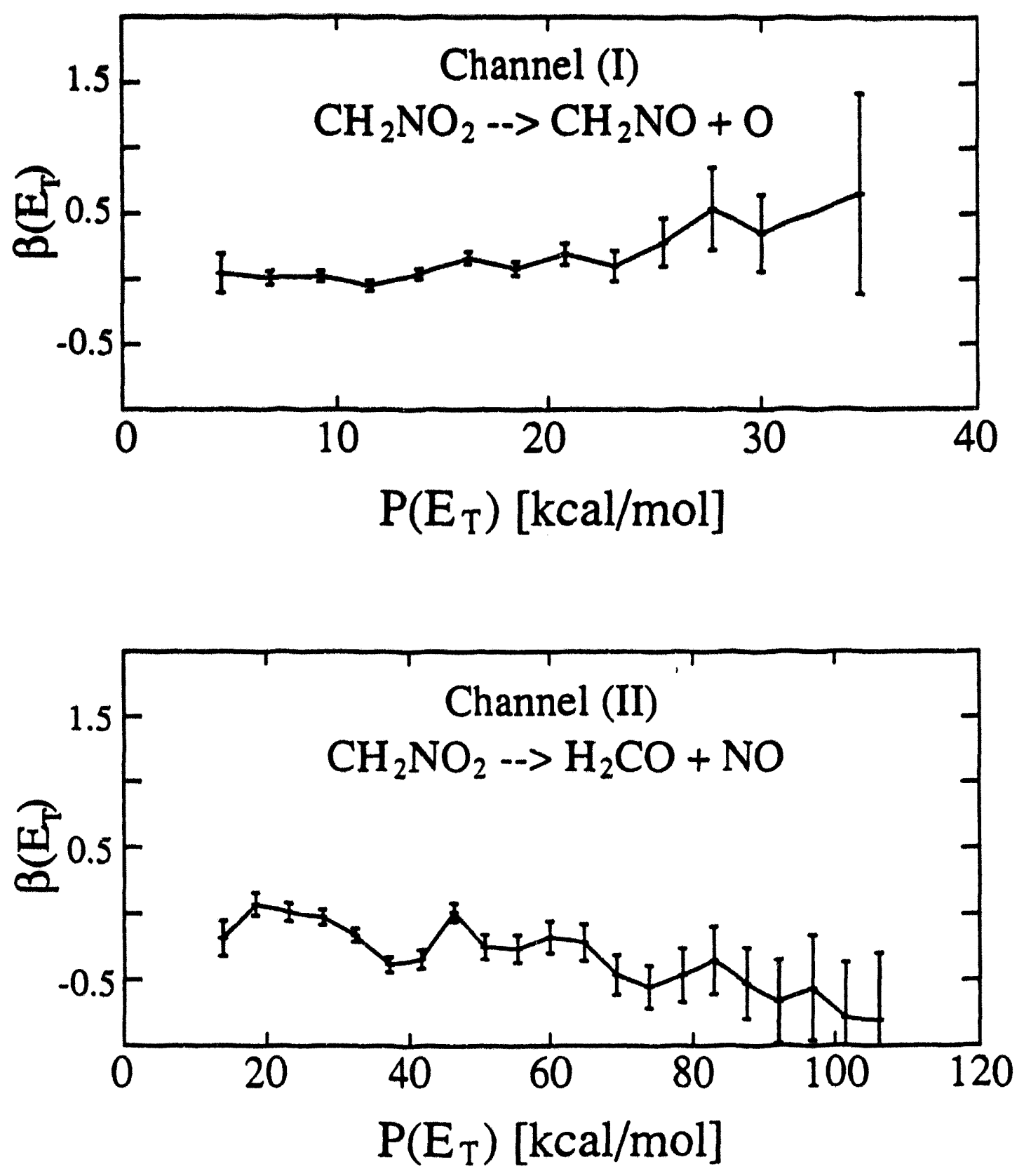

Figure 6.6 


\begin{tabular}{|c|c|c|c|c|}
\cline { 3 - 5 } \multicolumn{1}{c|}{} & Channel (I) & Channel (II) & . \\
\hline Wavelength & $\begin{array}{c}\text { Photon } \\
\text { Energy }\end{array}$ & $\begin{array}{c}\mathrm{CH}_{2} \mathrm{NO}+\mathrm{O} \\
\langle\mathrm{KEKK}\rangle\end{array}$ & $\begin{array}{c}\mathrm{H}_{2} \mathrm{CO}+\mathrm{NO} \\
\langle\mathrm{KER}\rangle\end{array}$ & Branching Ratio \\
\hline$[\mathrm{nm}]$ & {$[\mathrm{kcal} / \mathrm{mol}]$} & {$[\mathrm{kcal} / \mathrm{mol}]$} & {$[\mathrm{kcal} / \mathrm{mol}]$} & {$[$ Channel(I)/Channel(ID)] } \\
\hline 270 & 105.9 & 11.4 & 59 & $1.19(+0.37,-0.21)$ \\
\hline 255 & 112.1 & 11.0 & 59 & $1.29(+0.25,-0.16)$ \\
\hline 240 & 119.1 & 10.8 & 56 & $1.76(+0.27,-0.16)$ \\
\hline
\end{tabular}

Table 6.2 This table is a compilation of the observed data for both channels at the three wavelengths (photon energies) used in our experiments. The average kinetic energy release for each channel and the branching ratio measurements [expressed as the quotient channel (I)/channel (II)] are given.

heavy fragment ( $\mathrm{CO}$ or $\mathrm{HCO}$ ) will be very close to that of the original $\mathrm{H}_{2} \mathrm{CO}$ photofragment, whereas the light fragment will fly out of the beam and miss the detector. The net result, at worst, will be a slight blurring of the translational energy distribution for channel (II). Even in the absence of secondary dissociation, one would not expect to resolve any structure in the translational energy distribution for channel (II) since two molecular fragments are produced. Hence, the effects of secondary dissociation on the overall appearance of the distribution should be very small.

\subsubsection{Branching Ratios}

Branching ratios are easily determined from the foregoing KER analysis by summing the area under the KER curves to obtain the total population of each channel at each wavelength. As can be seen from Table 6.2, the branching ratio is near unity at the lowest photon energy, but definitely favors channel (I), involving an oxygen atom loss, as 
the photon energy is increased. The uncertainties given with these numbers derive chiefly from two sources: statistical error, and uncertainly in $\beta\left(E_{\tau}\right)$. Statistical error in each channel is well modeled by counting statistics. The second source of uncertainty is due primarily to the absence of knowledge of $\beta\left(E_{\mathrm{T}}\right)$ for the small amount of data found at energies where the $P\left(E_{T}\right)$ is very low. By assuming values of $\beta\left(E_{T}\right)$ that represent the possible extremes in these regions, and the limits of uncertainty in the regions where $\beta\left(E_{\mathrm{T}}\right)$ is well determined, we estimate the uncertainty in branching ratio from this source.

\subsection{Discussion}

In the following discussion, we compare our results for the photodissociation of the nitromethyl radical to the known photodissociation behavior of nitromethane. We then identify the initial electronic transition used in the excitation of $\mathrm{CH}_{2} \mathrm{NO}_{2}$ in the present experiment. Subsequently, we develop the argument that the observed product branching ratios (particularly the absence of $\mathrm{C}-\mathrm{N}$ bond fission), combined with the translational energy distribution for channel (II), imply that dissociation is not occurring via a statistical process on the ground state $\mathrm{CH}_{2} \mathrm{NO}_{2}$ surface. Rather, $\mathrm{NO}$ elimination most likely results from nuclear dynamics on an excited state surface, aided by the coordinative unsaturation of the carbon atom in the nitromethyl radical. We will also argue that the other observed channel, involving $\mathrm{N}-\mathrm{O}$ bond fission, must similarly be occurring along an excited pathway, and we identify a plausible mechanism.

\subsubsection{Comparison to Nitromethane}

The identities of the product channels we observe in the ultraviolet photodissociation of the nitromethyl radical represent a significant departure from what one might expect based on the known UV photofragmentation behavior of the closest closed-shell analogue, nitromethane. Nitromethane exclusively undergoes $\mathrm{C}-\mathrm{N}$ bond fission following UV absorption, $18,19,20,21,23$ whereas the analogous channel [channel (III)] 
is absent in $\mathrm{CH}_{2} \mathrm{NO}_{2}$ photodissociation. Instead, we have identified two major channels: channel (I), N-O bond fission, and channel (II), the elimination of NO. Primary N-O bond fission has not been observed in any collision-free nitromethane photodissociation experiments, although it has been observed in the nitromethane radical cation dissociative charge neutralization experiments of Beijersbergen et al. ${ }^{26}$ They also reported a minor channel identified as $\mathrm{H}_{2} \mathrm{CO}+\mathrm{NOH}$ (or $\mathrm{HNO}$ ). Only in infrared multiphoton dissociation (IRMPD) studies 24,25 has the analogous $\mathrm{CH}_{3} \mathrm{O}+\mathrm{NO}$ photodissociation product channel been seen for nitromethane.

Some of the differences between $\mathrm{CH}_{2} \mathrm{NO}_{2}$ and $\mathrm{CH}_{3} \mathrm{NO}_{2}$ can be understood in terms of the relevant bond dissociation energies. As shown in Table 6.1, the $\mathrm{N}-\mathrm{O}$ and $\mathrm{C}-\mathrm{N}$ bond strengths are roughly comparable in $\mathrm{CH}_{2} \mathrm{NO}_{2}(=71 \mathrm{kcal} / \mathrm{mol})$. In contrast, the bond dissociation energies for the $\mathrm{N}-\mathrm{O}$ and $\mathrm{C}-\mathrm{N}$ bonds in $\mathrm{CH}_{3} \mathrm{NO}_{2}$ are 95 and $60.6 \mathrm{kcal} / \mathrm{mol}$, respectively. ${ }^{34}$ The slightly stronger $\mathrm{C}-\mathrm{N}$ bond in $\mathrm{CH}_{2} \mathrm{NO}_{2}$ presumably arises from the $\mathrm{C}-\mathrm{N} \pi$ bonding interaction via the half-filled $2 \mathrm{~b}_{1}$ molecular orbital. The $\mathrm{N}-\mathrm{O}$ bond is considerably stronger in $\mathrm{CH}_{3} \mathrm{NO}_{2}$ than in $\mathrm{CH}_{2} \mathrm{NO}_{2}$ or other related radicals: e.g., the $\mathrm{N}-\mathrm{O}$ bond dissociation energies of $\mathrm{NO}_{2}$ and $\mathrm{NO}_{3}$ are $72.7^{34}$ and $49.2 \mathrm{kcal} / \mathrm{mol},{ }^{5}$ respectively. While the absence of $\mathrm{N}-\mathrm{O}$ fission in the UV photodissociation of nitromethane is perhaps not so surprising in light of the bond strengths, the preference of $\mathrm{N}-\mathrm{O}$ over $\mathrm{C}-\mathrm{N}$ bond fission in $\mathrm{CH}_{2} \mathrm{NO}_{2}$ photodissociation is an intriguing bond selective effect worthy of further consideration.

The observation of $\mathrm{NO}$ elimination in the $\mathrm{UV}$ photodissociation of $\mathrm{CH}_{2} \mathrm{NO}_{2}$ but not $\mathrm{CH}_{3} \mathrm{NO}_{2}$ is also of interest. As mentioned above, $\mathrm{CH}_{3} \mathrm{O}+\mathrm{NO}$ products have been seen in the infrared multiphoton dissociation of nitromethane; 25 these are produced in competition with the products of $\mathrm{C}-\mathrm{N}$ bond fission with a branching ratio of $\left(\mathrm{CH}_{3} \mathrm{O}+\mathrm{NO}\right) /\left(\mathrm{CH}_{3}+\mathrm{NO}_{2}\right) \approx 0.6$. In these studies, the mechanism proposed for $\mathrm{CH}_{3} \mathrm{O}+\mathrm{NO}$ formation was isomerization to methyl nitrite $\left(\mathrm{CH}_{3} \mathrm{ONO}\right)$ over a barrier involving a three-center $\overline{\mathrm{C}-\mathrm{N}-\mathrm{O}}$ transition state followed by $\mathrm{CH}_{3} \mathrm{O}-\mathrm{NO}$ bond fission, all 
on the ground state electronic surface of the system. Using a statistical model for the competition between isomerization and $\mathrm{C}-\mathrm{N}$ bond fission, the isomerization barrier height was estimated to be $55.5 \mathrm{kcal} / \mathrm{mol}$. Saxon and Yoshimine ${ }^{38}$ later performed calculations to more fully characterize this nitro-nitrite isomerization transition state and determined that the $\mathrm{C}-\mathrm{N}$ and $\mathrm{C}-\mathrm{O}$ bond lengths are $3.4 \AA$ and $3.65 \AA$ respectively. These bond lengths are very large compared with the $\mathrm{CH}_{3} \mathrm{NO}_{2}$ ground state $\mathrm{C}-\mathrm{N}$ equilibrium bond distance of $1.489 \AA,{ }^{39}$ resulting in a transition state whose energy is calculated to be $56.7 \mathrm{kcal} / \mathrm{mol}$, only $0.4 \mathrm{kcal} / \mathrm{mol}$ less than their calculated $\mathrm{C}-\mathrm{N}$ bond dissociation energy. The very unfavorable geometry of the rearrangement transition state and accompanying high barrier is a consequence of the inability of the fully saturated carbon atom in nitromethane to form a new bond without first breaking an existing bond. In the case of NO elimination from $\mathrm{CH}_{2} \mathrm{NO}_{2}$, one also expects to pass through some type of $\overline{\mathrm{C}-\mathrm{N}-\mathrm{O}}$ transition state, but the formation of such a structure should be facilitated because the carbon atom in $\mathrm{CH}_{2} \mathrm{NO}_{2}$ is coordinatively unsaturated. This effect should not be limited to the ground state surface.

The dynamics involved in the NO elimination channel that we observe are also quite different from the above-mentioned IRMPD study of $\mathrm{CH}_{3} \mathrm{NO}_{2}$. In the IRMPD experiment, the translational energy distribution for NO elimination peaked at zero kinetic energy. This is the characteristic distribution for a statistical bond fission in the absence of a barrier in the reverse direction, and indicates that the $\mathrm{CH}_{3} \mathrm{ONO}$ well is sufficiently deep for "re-randomization" of the available energy to occur after crossing the isomerization transition state. Indeed, the heat of formation of methylnitrite is virtually identical to that of nitromethane (only $\sim 2 \mathrm{kcal} / \mathrm{mol}$ higher), resulting in methylnitrite being $\sim 40 \mathrm{kcal} / \mathrm{mol}$ stable with respect to $\mathrm{CH}_{3} \mathrm{O}+\mathrm{NO}$ products. ${ }^{34}$ The situation in our experiment is quite different; the translational energy distribution for NO elimination peaks at high kinetic energy (around $60 \mathrm{kcal} / \mathrm{mol}$ ). This implies that, regardless of the details, little or no rerandomization occurs prior to dissociation to $\mathrm{H}_{2} \mathrm{CO}+\mathrm{NO}$ products. 


\subsubsection{Nature of the Initially Excited Electronic State}

In order to have any detailed understanding of the dissociation mechanisms operative in $\mathrm{CH}_{2} \mathrm{NO}_{2}$, we need to know which electronic transition is responsible for the absorption band probed in our experiment. $\mathrm{CH}_{2} \mathrm{NO}_{2}$ has a ${ }^{2} \mathrm{~B}_{1}$ ground state with molecular orbital configuration $\left[\ldots\left(8 a_{1}\right)^{2}\left(5 b_{2}\right)^{2}\left(1 a_{2}\right)^{2}\left(2 b_{1}\right)\right]$. Figure 6.7 gives a qualitative picture of the $1 a_{2}$ and $2 b_{1}$ molecular orbitals as well as the unoccupied $3 b_{1}$ molecular orbital, obtained by undertaking a minimal basis set [Slater-type orbital (STO-3G)] geometry optimization calculation using the GAUSSIAN 88 package ${ }^{40}$ on $\mathrm{CH}_{2} \mathrm{NO}_{2}^{-}$. A low-lying excited state of $\mathrm{CH}_{2} \mathrm{NO}_{2}$, the $I^{2} \mathrm{~A}_{2}$ state, results from the $2 \mathrm{~b}_{1} \leftarrow 1 \mathrm{a}_{2}$ transition. This was the excited state observed in the $\mathrm{CH}_{2} \mathrm{NO}_{2}^{-}$photoelectron spectrum, ${ }^{15}$ formed by the removal of an electron from the $1 \mathrm{a}_{2}$ molecular orbital of the anion, and found to lie only $36.7 \mathrm{kcal} / \mathrm{mol}$ above the ground state. Figure 6.7 shows that the $2 b_{1} \leftarrow 1 a_{2}$ transition involves excitation from a non-bonding $\mathrm{N}-\mathrm{O} \pi$ orbital to a $\pi$ orbital which is $\mathrm{N}-\mathrm{O}$ antibonding and $\mathrm{C}-\mathrm{N}$ bonding. However, because the $1{ }^{2} \mathrm{~A}_{2}$ state lies so far below the photon energies used in our experiment ( $>105 \mathrm{kcal} / \mathrm{mol})$ it is an unlikely candidate for the initial excited state. The $2 b_{1} \leftarrow 5 b_{2}$ transition yielding a ${ }^{2} B_{2}$ excited state is not optically allowed in $C_{2 v}$ symmetry. Two other excited states resulting from $\pi^{*} \leftarrow \pi$ transitions are more attractive options: the $1{ }^{2} \mathrm{~B}_{1}$ state, from the $3 b_{1} \leftarrow 2 b_{1}$ transition, and the $2{ }^{2} \mathrm{~A}_{2}$ state from the $3 b_{1} \leftarrow 1 a_{2}$ transition. The $3 b_{1} \leftarrow 2 b_{1}$ transition is analogous to the transitions seen around $230 \mathrm{~nm}$ for several alkanenitronate $\left(\mathrm{R}^{\prime} \mathrm{RC}=\mathrm{NO}_{2}^{-}\right)$anions in solution. ${ }^{41}$ The $3 \mathrm{~b}_{1} \leftarrow$ $1 a_{2}$ transition is more localized on the $\mathrm{NO}_{2}$ group than the other two transitions, and strongly resembles the $\pi^{*} \leftarrow \pi$ transition in nitromethane between $190-220 \mathrm{~nm}$. This is the transition accessed in most nitromethane UV photodissociation experiments. 18,19,20,21

The lowest energy transitions in solvated alkanenitronate anion spectra result in

Figure 6.7 Form of the $1 a_{2}, 2 b_{1}$, and $3 b_{1}$ molecular orbitals determined from ab initio calculations performed on $\mathrm{CH}_{2} \mathrm{NO}_{2}^{-}$. 

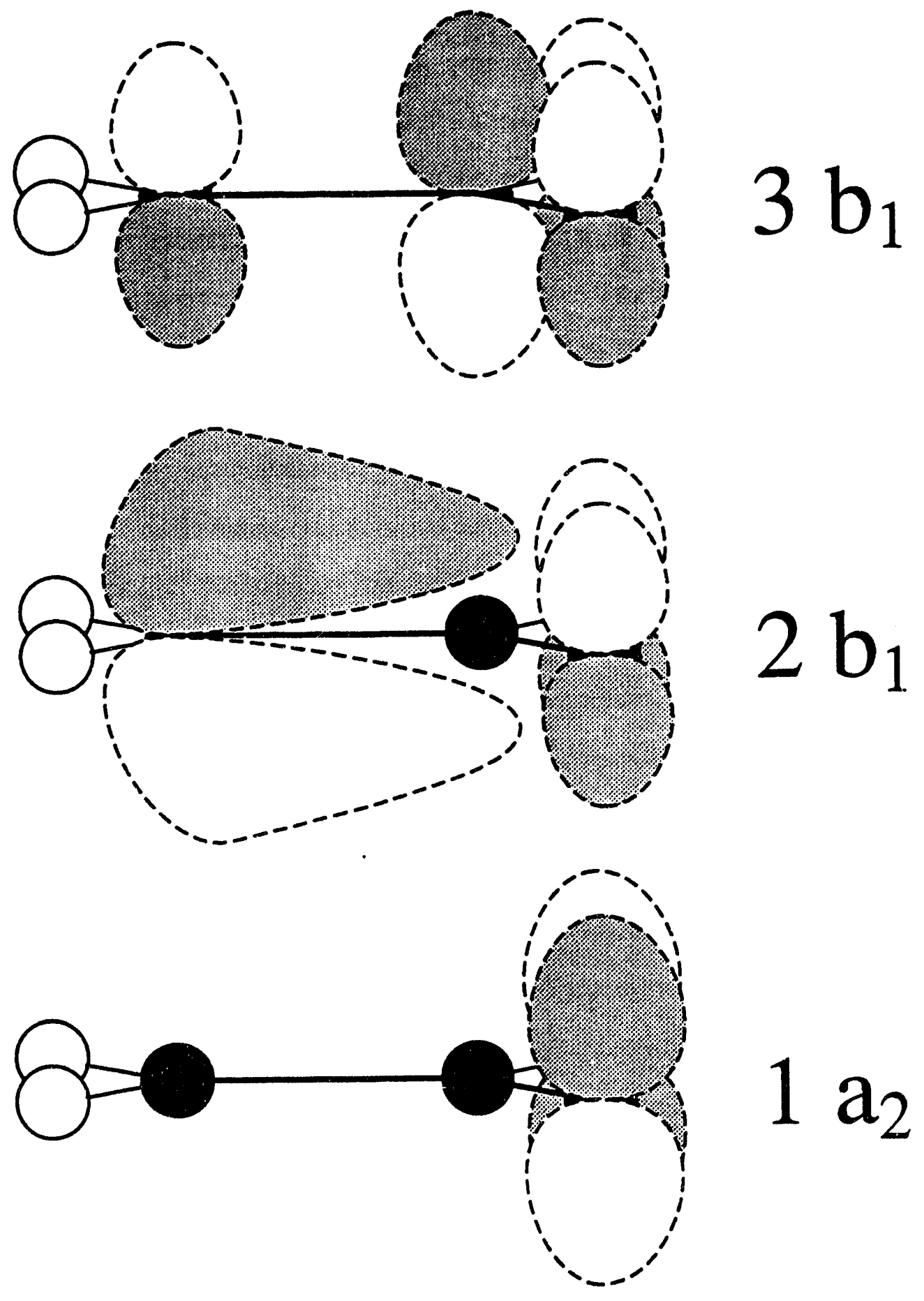

Figure 6.7 
bands that peak near $230 \mathrm{~nm}$ and extend up to $250-260 \mathrm{~nm} .{ }^{41}$ This corresponds approximately with our photodissociation cross section measurements, although the band in the radical appears somewhat red-shifted. The $\mathrm{C}-\mathrm{N}$ bond distance in the anion ground state is considerably shorter than in the neutral (1.347 $\AA$ vs. $1.430 \AA$, according to our previous.$b$ initio calculations ${ }^{15}$ ), and the $\pi^{*}$ orbital populated by this transition is C-N antibonding. One would expect the vertical transition energy to be lower in the radical than in the corresponding anion, so the observed red shift appears reasonable. Conversely, one might expect the $3 b_{1} \leftarrow 1 a_{2}$ transition to occur at a higher energy in $\mathrm{CH}_{2} \mathrm{NO}_{2}$ than the $\pi^{*} \leftarrow \pi$ transition in nitromethane because the $\mathrm{C}-\mathrm{N} \pi^{*}$ antibonding interaction in $\mathrm{CH}_{2} \mathrm{NO}_{2}$ is absent in nitromethane. Any blue shifting of this transition would place it well beyond the range of photodissociation wavelengths used in our experiments. Based on these considerations, we assign the $1{ }^{2} B_{1}$ state resulting from the $3 b_{1} \leftarrow 2 b_{1}$ transition as the initially prepared excited electronic state.

Figure 6.8 shows the energetics of the $\mathrm{CH}_{2} \mathrm{NO}_{2}$ electronic states relative to the dissociation channels (I), (II) and (III). The location of the $1{ }^{2} \mathrm{~B}_{1}$ state on this diagram is intended to indicate the lowest energy at which transitions from the ground state to the $1{ }^{2} \mathrm{~B}_{1}$ state occur, and is estimated according to the lowest energy at which electronic excitation of $\mathrm{CH}_{2} \mathrm{NO}_{2}$ leads to dissociation. According to the matrix isolation study of Jacox, ${ }^{11}$ the dissociation threshold is known to be between the wavelengths of $300 \mathrm{~nm}$ $(4.13 \mathrm{eV})$ and $280 \mathrm{~nm}(4.42 \mathrm{eV})$. We therefore place the $l^{2} \mathrm{~B}_{1}$ state (somewhat arbitrarily) at $4.25 \mathrm{eV}$. The energetics of low lying electronic states of channels (I) and (III) are also shown; the excited states of $\mathrm{CH}_{2}$ and $\mathrm{NO}_{2}$ are reasonably well-characterized

Figure 6.8 Energetics of the nitromethyl radical and selected energetically allowed product channels (not all products shown are observed). States whose energies are known relatively well are represented by solid lines located at their origin, while a dashed line is used to denote the approximate location of the $I^{2} \mathrm{~B}_{1}$ state (see text). 


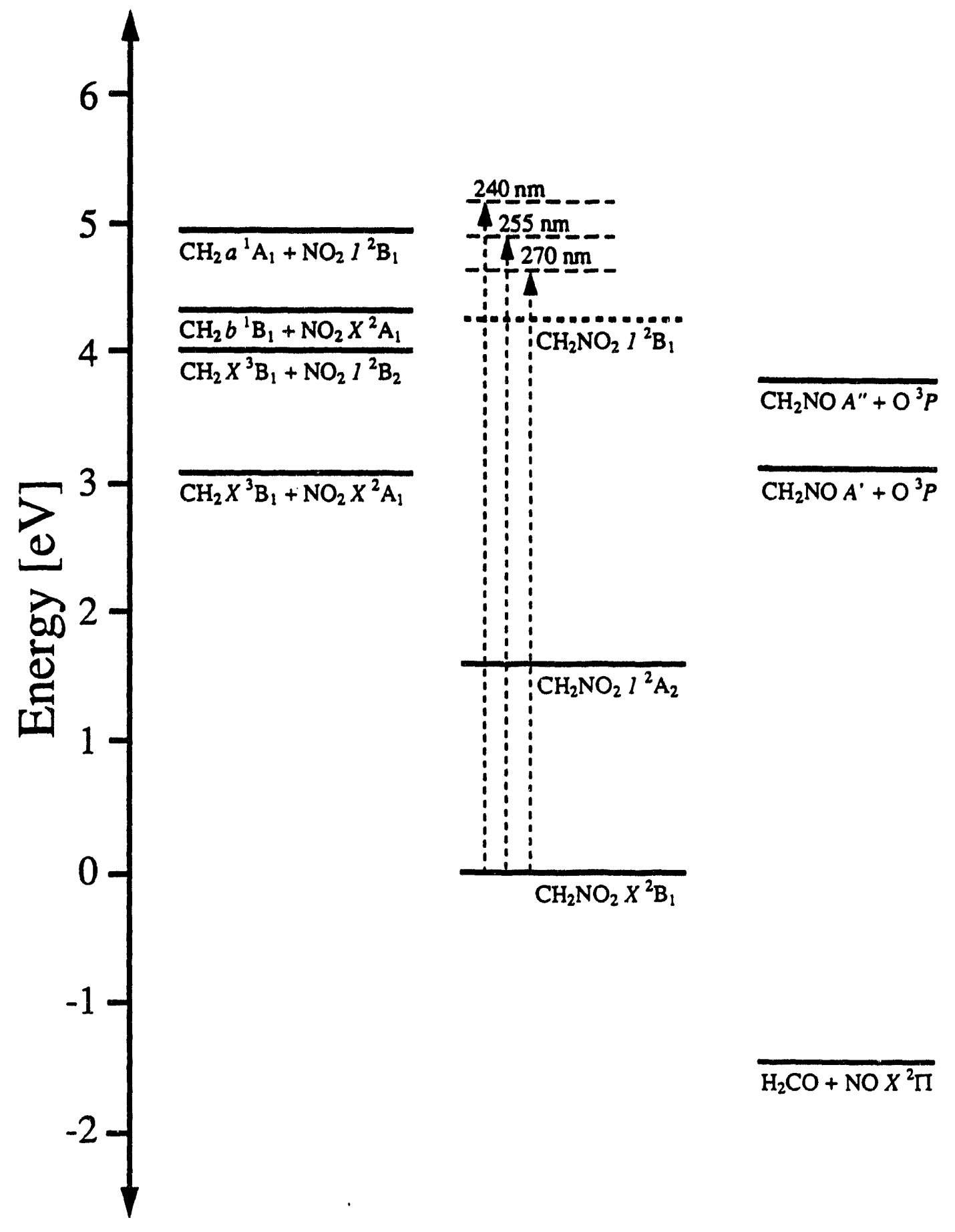

Figure 6.8 
experimentally, while the locations of the ground and first excited states of $\mathrm{CH}_{2} \mathrm{NO}$ are from an MP4-BAC $a b$ initio calculation by Melius. ${ }^{36}$

\subsubsection{Dissociation Mechanisms}

Our assignment of the electronic transition accessed in these experiments offers an immediate if somewhat unspectacular reason for the different product channels seen in the $\mathrm{CH}_{2} \mathrm{NO}_{2}$ and $\mathrm{CH}_{3} \mathrm{NO}_{2}$ photodissociation experiments. In nitromethane dissociation, the $\pi^{*}$ state accessed near $200 \mathrm{~nm}$, which correlates diabatically to $\mathrm{CH}_{3}+\mathrm{NO}_{2}\left(2{ }^{2} \mathrm{~B}_{2}\right)$ products, is believed to be predissociated by a $\sigma^{*}$ repulsive state of the same symmetry that correlates to lower energy $\mathrm{CH}_{3}+\mathrm{NO}_{2}\left({ }^{2}{ }^{2} \mathrm{~B}_{2}\right)$ products. Since we believe we are exciting a qualitatively different transition in $\mathrm{CH}_{2} \mathrm{NO}_{2}$, involving a different excited state surface, this mechanism would not be operative. It remains to be understood how $\mathrm{CH}_{2} \mathrm{NO}_{2}$ does dissociate, and this will now be considered in detail.

\subsubsection{Possible Role of Ground State Statistical Dissociation}

To explain product branching ratios and translational energy distributions in a given photodissociation experiment, a simple model to consider is rapid internal conversion to the ground state potential energy surface. One should then be able to predict the product branching ratios by a statistical model such as Rice-RamspergerKassel-Marcus (RRKM) theory. ${ }^{42,43}$ Such a statistical treatment was successfully used by Wodtke et al. to interpret the IRMPD of nitromethane. ${ }^{25}$ Figure 6.9 shows a schematic of the potential energy along the reaction coordinates for channels (I)-(III) on the ground state surface of $\mathrm{CH}_{2} \mathrm{NO}_{2}$. We would expect "loose" transition states for the simple bond fission channels (I) and (III), and a "tight" transition state, presumably involving a three-

Figure 6.9 Schematic of the adiabatic minimum energy pathways to all three product channels on the ground state surface of $\mathrm{CH}_{2} \mathrm{NO}_{2}$. 


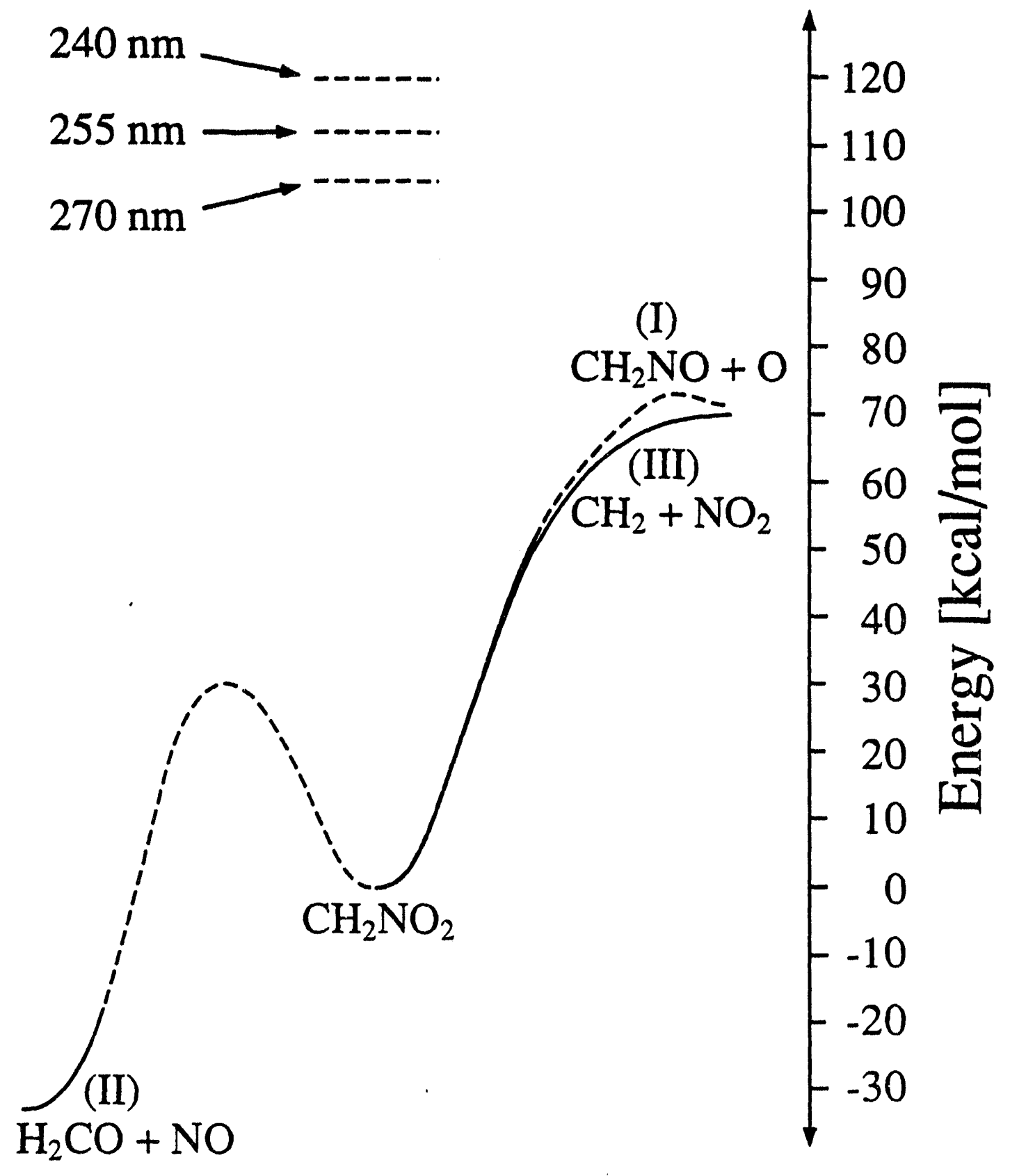

Figure 6.9 
membered $\overline{\mathrm{C}-\mathrm{N}}-\mathrm{O}$ ring, for NO elimination channel (II). ${ }^{44}$ The high kinetic energy release associated with channel (II) is consistent with passage over a barrier associated with the tight transition state, while the translational energy distribution for channel (I) peaks near zero, the expected "statistical distribution" for simple bond fission (perhaps over a small barrier in the reverse direction, consistent with the $P\left(E_{\mathrm{T}}\right)$ distribution maximum at several kilocalories per mole.) However, ground state $\mathrm{CH}_{2}+\mathrm{NO}_{2}$ correlate to the ground state of $\mathrm{CH}_{2} \mathrm{NO}_{2}$ both diabatically and adiabatically. This means there should be no barrier to the $\mathrm{CH}_{2}+\mathrm{NO}_{2}$ recombination reaction, so the loose transition state associated with C-N bond fission will lie about $71 \mathrm{kcal} / \mathrm{mol}$ (i.e. the bond dissociation energy) above the $\mathrm{CH}_{2} \mathrm{NO}_{2}$ well. Since the $\mathrm{N}-\mathrm{O}$ and $\mathrm{C}-\mathrm{N}$ bond dissociation energies are very close, the transition state for channel (I) must also lie at least as high; it will be higher if there is a barrier along the reaction coordinate for channel (I). In addition, the preexponential $\mathrm{A}$ factor in the Arrhenius rate equation for the $\mathrm{C}-\mathrm{N}$ bond fission channel will be larger since channel (III) yields two polyatomics, as opposed to a polyatomic plus an atom, and will thus have a greater gain in entropy on going from the reactant to the activated complex. ${ }^{43}$ Thus, even with no barrier to $\mathrm{N}-\mathrm{O}$ bond fission above the bond dissociation energy, we expect a statistical model to yield a greater rate of $\mathrm{C}-\mathrm{N}$ bond fission than $\mathrm{N}-\mathrm{O}$ bond fission. This is incompatible with the absence of $\mathrm{C}-\mathrm{N}$ bond fission products observed in the present experiment and immediately indicates that the $\mathrm{N}-\mathrm{O}$ bond fission channel cannot be explained by statistical dissociation on the ground state surface. We shall return to this channel subsequently.

While it appears certain that a statistical dissociation on the ground state surface cannot explain all of the observed dissociation dynamics, it may be possible that $\mathrm{N}-\mathrm{O}$ bond fission [channel (I)] occurs on an excited state surface, while rearrangement and elimination of NO [channel (II)] is statistically favored on the ground state surface and dominates $\mathrm{C}-\mathrm{N}$ bond fission [channel (III)] once on that surface. This requires that the RRKM rate constant for NO elimination at a reasonable barrier height be substantially 
larger than that for $\mathrm{C}-\mathrm{N}$ bond fission. We have performed RRKM calculations ${ }^{45}$ for the two channels to test if this is plausible.

RRKM theory is the most widely accepted and generally employed method of describing and modeling unimolecular decomposition processes. It is based on two assumptions: that vibrational energy is randomized rapidly on the time scale of a reaction throughout all vibrational degrees of freedom; and that all trajectories of a system which cross a judiciously chosen surface separating reactants from products are considered to have irreversibly reacted. RRKM theory therefore allows the rate of reaction at a given energy to be expressed as:

$$
k(E)=\frac{W(E)}{h \rho(E)}
$$

Here, $W^{\dagger}(E)$ is the sum of internal states of the dissociating complex at the transition state, and $\rho(E)$ is the density of states of the reactant. Because any recrossing of the dividing surface is neglected by the second assumption given above, a slight overestimation of reaction rate can result. Therefore, this expression is strictly speaking an upper bound.

The parameters required to undertake the sum- and density-of-states calculations which are necessary to evaluate Eqn (6.10) are the vibrational frequencies and parameters that describe any internal rotational degrees of freedom present in either the reactant or the transition state. While these quantities are usually well known for the reactant, transition states are often not as well characterized. Fortunately, it is generally acknowledged that the calculated RRKM unimolecular decomposition rate is insensitive to the exact values given the vibrational frequencies in the critical configuration, so long as they are consistent with the correct high-pressure preexponential A-factor.

The high-pressure preexponential A-factor, denoted $A_{\infty}$ in Eqn (6.11), ${ }^{46}$

$$
k_{u n i}^{\infty}=A_{\infty} \cdot \exp \left\{-\frac{E_{\infty}}{k_{B} T}\right\}
$$


can be recognized as the preexponential term in the familiar Arrhenius rate equation. It is at high pressures that collisionally activated unimolecular decomposition truly follows first-order kinetics, since the rate limiting step will not be the second order (bimolecular) activation process. Values of $A_{\infty}$ and $E_{\infty}$ are determined experimentally by examining the temperature dependence of reaction rates under high pressure conditions.

The $A_{\infty}$ factor is related to the change in entropy on going from the reactant to the activated complex in the following way:

$$
A_{-}=\frac{e k_{B} T}{h} \cdot \exp \left\{\frac{\Delta S}{k_{B} T}\right\}
$$

For this reason 'loose' unimolecular decomposition transition states involving a long separation of incipient fragments, conversion of vibrational modes to hindered rotors and/or a net lowering of remaining vibrational mode frequencies relative to the reactant molecule have large $A_{\infty}$ factors.

Since we know all the reactant vibrational frequencies from the works of Jacox and Metz et al., we then calculated vibrational frequencies for the critical configurations that are consistent with the expected A-factors for the individual processes under consideration. Vibrational frequencies for the tight three-center transition state in NO elimination were chosen to yield a preexponential $\log _{10} \mathrm{~A}$-factor in the Arrhenius rate expression of 13 , based on comparisons to previously determined values for comparable types of unimolecular decompositions. ${ }^{43,47}$ The vibrational frequencies in the transition state for $\mathrm{C}-\mathrm{N}$ bond fission were chosen to be consistent with a $\log _{10} \mathrm{~A}$-factor of 15.6 , determined by Benson and O'Neal from the analysis of kinetics data. ${ }^{48}$ The program AFAC $^{49}$ is used, which calculates the A-factor associated with changing from the known reactant frequencies to a proposed set of frequencies for the critical configuration. By iteratively varying the proposed critical configuration vibrational frequencies, one can find 
a set of frequencies that is realistic and which results in the desired A-factor. These vibrational frequencies were then used in RRKM rate calculations.

In our RRKM rate calculations, all vibrations were assumed to be separable harmonic oscillators. The rotational temperature was estimated to be $50 \mathrm{~K}$, which is consistent with previous experiments using this apparatus.6,7.8.9 The overall rotations were treated adiabatically, so that the change in moments of inertial from reactant to critical configuration served only to modify slightly the energy available above the barrier to a particular decomposition pathway.

The moments of inertia for the reactant, $\mathrm{CH}_{2} \mathrm{NO}_{2}$, were obtained from the

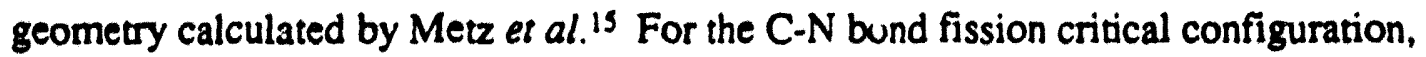
moments of inertia were calculated assuming a $\mathrm{C}-\mathrm{N}$ bond distance of $2.3 \AA$, s0 with both of the nascent $\mathrm{CH}_{2}$ and $\mathrm{NO}_{2}$ fragments having the same bond lengths and angles as the respective free molecules. The critical configuration for the isomerization-elimination channel was taken to be an equilateral $\overline{\mathrm{C}-\mathrm{N}-\mathrm{O}}$ ring, with the second oxygen extending out from the nitrogen, bent out of the plane of the ring by $60^{\circ}$. The change in available energy due to change in moments of ineria, and thus changes in rotational energy, on transforming from reactant to transition state geometries was very small compared to the overall excess energy in all cases.

The most informative results from our RRKM calculations are shown in Table 6.3. First, the statistical rates of $\mathrm{C}-\mathrm{N}$ bond fission at the three different wavelengths used in our study are reported. Then, the rate of the rearrangement-elimination channel is examined by assuming several values for the height of the barrier to a possible ground state rearrangement. We find that even for a barrier as low as $10 \mathrm{kcal} / \mathrm{mol}$ with respect to ground state $\mathrm{CH}_{2} \mathrm{NO}_{2}$, $\mathrm{NO}$ elimination is only four times as fast as $\mathrm{C}-\mathrm{N}$ bond fission at $\lambda_{\text {diss }}=240 \mathrm{~nm}$. This is the dissociation wavelength where our signal-to-noise ratio is best, and we would definitely observe $\mathrm{C}-\mathrm{N}$ fission were it occurring to this extent. Moreover, a $10 \mathrm{kcal} / \mathrm{mol}$ barrier is not consistent with the translational energy distribution for the NO 


\begin{tabular}{|c|c|c|c|c|c|}
\hline Channel & $\log _{10} \mathrm{~A}$-factor & $\begin{array}{c}E^{*} \\
{[\mathrm{kcal} / \mathrm{mol}]}\end{array}$ & $\begin{array}{c}E_{0} \\
{[\mathrm{kcal} / \mathrm{mol}]}\end{array}$ & $\begin{array}{c}E^{\dagger} \\
{[\mathrm{kcal} / \mathrm{mol}]}\end{array}$ & $\begin{array}{c}\text { Harmonic } \mathrm{k}(E) \\
{\left[10^{12} \mathrm{~s}^{-1}\right]}\end{array}$ \\
\hline $\mathrm{CH}_{2}+\mathrm{NO}_{2}$ & 15.6 & 105.9 & 71.1 & 34.8 & 0.223 \\
\hline & 15.6 & 112.5 & 71.1 & 41.4 & 0.487 \\
\hline & 15.6 & 119.1 & 71.1 & 48.0 & 0.931 \\
\hline $\mathrm{H}_{2} \mathrm{CO}+\mathrm{NO}$ & 13 & 105.9 & 10 & 95.9 & 3.62 \\
\hline & 13 & 112.5 & 10 & 102.5 & 3.81 \\
\hline & 13 & 119.1 & 10 & 109.1 & 3.99 \\
\hline $\mathrm{H}_{2} \mathrm{CO}+\mathrm{NO}$ & 13 & 105.9 & 30 & 75.9 & 0.444 \\
\hline & 13 & 112.5 & 30 & 82.5 & 0.531 \\
\hline & 13 & 119.1 & 30 & 89.1 & 0.621 \\
\hline
\end{tabular}

Table 6.3 The results of several RRKM calculations which were performed to investigate the possibility that the experimentally observed products could be a result of statistical unimolecular decomposition on the ground electronic state of $\mathrm{CH}_{2} \mathrm{NO}_{2}$. Specifically, the statistical rate for channel (III), $\mathrm{C}-\mathrm{N}$ bond fission, is calculated and compared to that of channel (II), the rearrangement-elimination channel, at two different values of the main unknown quantity, the barrier to channel (II). The labels $E^{*}$, $E_{0}$, and $E^{\dagger}$ refer to the photon energy, the energy below which classical reaction cannot occur, and the difference between the two, respectively. See text.

elimination channel. This distribution peaks around $60 \mathrm{kcal} / \mathrm{mol}$ (see Figure 6.4), indicating that the barrier with respect to the products is at least this high. Since NO elimination from $\mathrm{CH}_{2} \mathrm{NO}_{2}$ is exothermic by $33 \mathrm{kcal} / \mathrm{mol}$, the barrier height with respect to $\mathrm{CH}_{2} \mathrm{NO}_{2}$ must be at least $30 \mathrm{kcal} / \mathrm{mol}$. For this barrier height, statistical $\mathrm{C}-\mathrm{N}$ bond fission on the ground state surface is faster than NO elimination at $240 \mathrm{~nm}$. Judging by the 
observed $P\left(E_{T}\right)$ distribution for this channel, the true barrier height is probably larger than this. A barrier of $30 \mathrm{kcal} / \mathrm{mol}$ with respect to $\mathrm{CH}_{2} \mathrm{NO}_{2}$ implies that the potential energy at the barrier must be very efficiently channeled into product kinetic energy, and this is unlikely considering how strained the transition state must be with respect to the products. Our calculations therefore imply that in the statistical limit, elimination of NO cannot be the dominant dissociation channel on the ground state. While there is considerable leeway in some of the assumptions made in our analysis, this conclusion seems quite secure.

One final scenario that deserves attention is the possibility that statistical C-N bond fission does occur from the ground state but that the statistical kinetic energy release distribution peaks so sharply near zero that detection of the products is overwhelmingly diminished by the blocking strip at the center of the detector.

For molecules the size of $\mathrm{CH}_{2} \mathrm{NO}_{2}$, a 'prior distribution' model ${ }^{\mathrm{s1}}$ can be used to give a reasonable approximation of the statistical product translational energy distribution. By comparing this distribution to our known low kinetic energy cut-off, we can determine if the products from statistical C-N fission will be undetected.

As given by Levine, ${ }^{51}$ the prior distribution has the form:

$$
P\left(E \mid f_{T}\right) \propto f_{T}^{1 / 2} \cdot\left(1-f_{T}\right)^{\alpha},
$$

where for a given total energy $E, f_{T}$ is the fraction of energy found in translation (i.e., $f_{T}=E_{T}(E)$. The exponent $\alpha$ is found using the formula $\alpha=s-1+r / 2, s$ being the number of vibrational modes and $r$ the number of rotational degrees of freedom in the products. For channel (III), $\mathrm{CH}_{2} \mathrm{NO}_{2} \rightarrow \mathrm{CH}_{2}+\mathrm{NO}_{2}, s=6$ and $r=6$ so $\alpha$ has the value of 8. At a dissociation wavelength of $240 \mathrm{~nm}$ this results in the predicted translational energy distribution shown in Figure 6.10. Since the mass ratio is very similar between channel (I)

Figure 6.10 The translational energy distribution predicted by a prior distribution calculation for the channel $\mathrm{CH}_{2} \mathrm{NO}_{2} \rightarrow \mathrm{CH}_{2}+\mathrm{NO}_{2}$ at $240 \mathrm{~nm}$. 


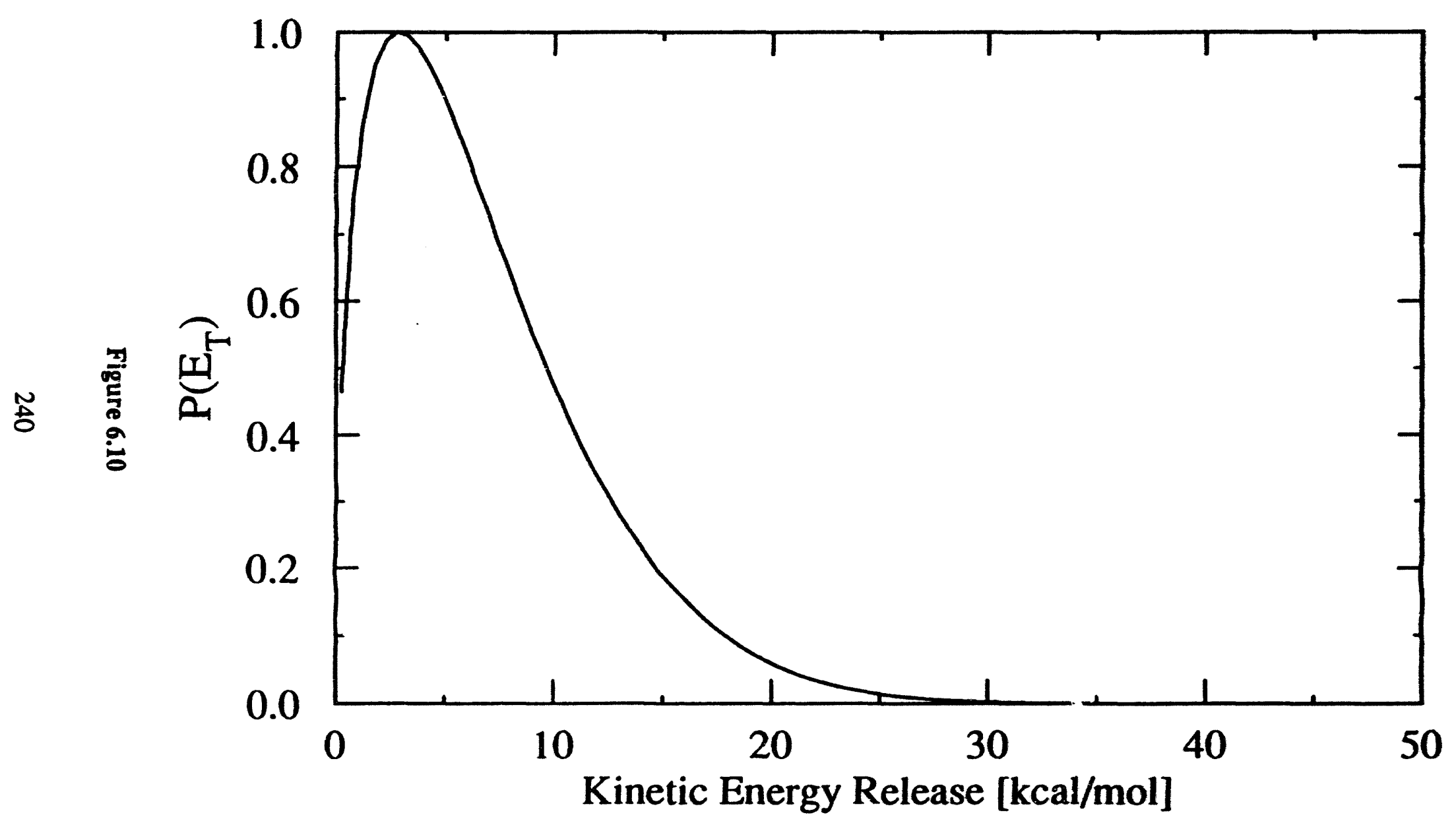


and channel (III) products, the blocking-strip-induced low kinetic energy release cut-off for the $\mathrm{CH}_{2}+\mathrm{NO}_{2}$ products will be virtually the same as in channel (I), shown to be $\sim 2 \mathrm{kcal} / \mathrm{mol}$ in Figure 6.4. Figure 6.10 clearly shows that a large fraction of the translation energy distribution extends out well past $2 \mathrm{kcal} / \mathrm{mol}$. We are thus very confident that $\mathrm{C}-\mathrm{N}$ bond fission is not occurring to any significant extent.

\subsubsection{2 $\mathrm{CH}_{2} \mathrm{NO}_{2} \rightarrow \mathrm{H}_{2} \mathrm{CO}+\mathrm{NO}$ Dissociation Mechanism}

We are left with two options regarding channel (II). Either it proceeds on ground state surface in a highly non-statistical (i.e., mode-specific) fashion, or it results from the nuclear dynamics on one or more of the excited state surfaces. Given the considerable molecular rearrangement necessary to produce the observed channel (II) products, in contrast to the very direct $\mathrm{C}-\mathrm{N}$ simple bond fission channel, the first option seems (quite unlikely. This suggests the existence of a mechanism on excited state surfaces that would facilitate dissociation to channel (II) products. After presenting and discussing such a mechanism, we then consider the mechanism for dissociation channel (I), which, based on the above discussion, also most likely occurs on an excited state surface.

Figure 6.11 depicts the likely nuclear dynamics for the rearrangement-elimination process of channel (II), irrespective of the details of the potential energy surfaces involved. This channel presumably involves (a) rotation of the $\mathrm{CH}_{2}$ plane perpendicular to the $\mathrm{NO}_{2}$ plane, (b) oxygen atom transfer though a $\overline{\mathrm{C}-\mathrm{N}-\mathrm{O}}$ three-membered-ring, and (c) elimination of $\mathrm{H}_{2} \mathrm{CO}$ from $\mathrm{NO}$. We note that steps (a) and (b) are the first steps in the mechanism proposed by Yamada and co-workers ${ }^{52,53}$ for the photochemical rearrangement of $\mathrm{R}^{\prime} \mathrm{RC}=\mathrm{NO}_{2}^{-}$anions in solution as a synthetic route to the corresponding hydroxamic acids [i.e. $\mathrm{RC}(\mathrm{O})-\mathrm{N}(\mathrm{OH}) \mathrm{R}$ ]. In their mechanism, as applied to $\mathrm{CH}_{2} \mathrm{NO}_{2}^{-}$, the anion first

Figure 6.11 Nuclear dynamics presumably involved in channel (II) rearrangementelimination mechanism resulting in the reaction $\mathrm{CH}_{2} \mathrm{NO}_{2} \rightarrow \mathrm{H}_{2} \mathrm{CO}+\mathrm{NO}$. 

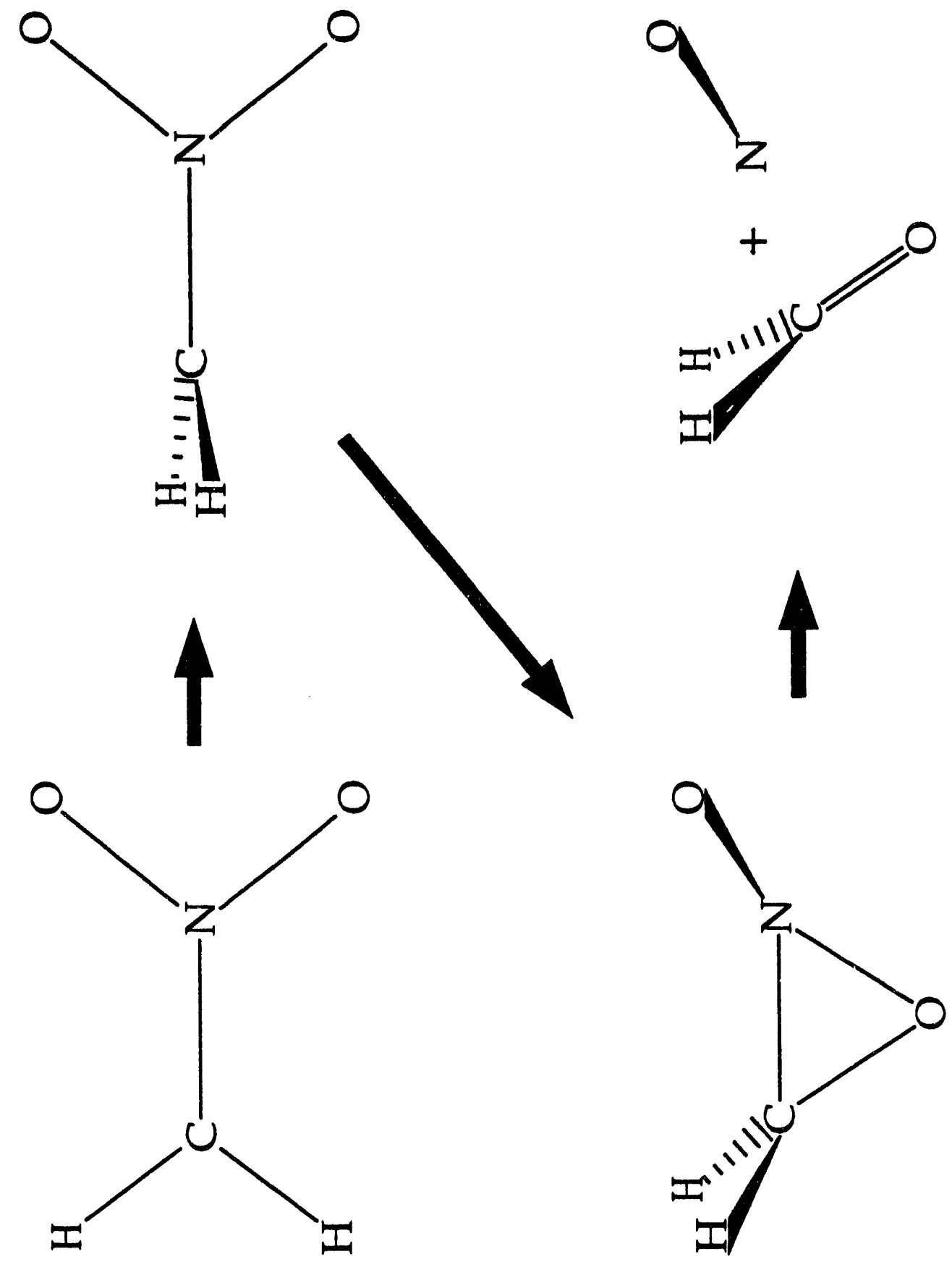

Figure 6.11 
undergoes $\pi^{*} \leftarrow \pi$ excitation corresponding to one electron being transferred from the $2 b_{1}$ HOMO to the $3 b_{1}$ molecular orbital (see Figure 6.7). The subsequent dynamics involve $\mathrm{CH}_{2}$ moiety rotation, which minimizes the $\mathrm{C}-\mathrm{N}$ antibonding interaction, formation of a three-membered oxaziridine ring as an oxygen atom is transferred from nitrogen to carbon atoms, and finally hydrogen atom migration resulting in the observed net isomerization from $\mathrm{CH}_{2} \mathrm{NO}_{2}^{-}$to $\mathrm{HC}(\mathrm{O}) \mathrm{N}(\mathrm{O}) \mathrm{H}^{-}$. The similarities between the observed rearrangementelimination products in the neutral $\mathrm{CH}_{2} \mathrm{NO}_{2}$ radical and the postulated $\mathrm{CH}_{2} \mathrm{NO}_{2}^{-}$anion rearrangement dynamics are compelling. Not only are the nuclear motions in the initial two stages of the anion rearrangement mechanism very similar to what must occur in the rearrangement-elimination channel observed with nitromethyl radical, but the initial electronic transition is proposed to be the same in each case (as discussed in § 6.4.2).

Although the anion has an additional $2 b_{1}$ electron in both the ground and excited states, one expects the $I^{2} \mathrm{~B}_{1}$ state in the radical to be significantly stabilized by a $90^{\circ}$ intermal rotation about the $\mathrm{C}-\mathrm{N}$ bond for the same reason as in the anion: reduction of the $\mathrm{C}-\mathrm{N}$ antibonding interaction as a result of the half-filled $3 \mathrm{~b}_{1}$ orbital. The resulting stabilization may be greater in the radical because there is no half-filled $\mathrm{C}-\mathrm{N} \pi$-bonding orbital as there is in the anion excited state, or it might be smaller due to the larger C-N bond distance in the radical. In any case, let us consider what effect the $90^{\circ}$ internal rotation might have on the relevant molecular orbitals in the excited state. Figure 6.12 (a) shows the $3 b_{1}$ molecular orbital after the internal rotation has occurred. While this orbital has some amplitude on the carbon atom in the planar geometry, it becomes an orbital localized on the $\mathrm{NO}_{2}$ group in the $90^{\circ}$ geometry, leaving the p-orbital on the carbon atom perpendicular to the $\mathrm{CH}_{2}$ plane empty. This geometry places an excess positive charge on

Figure 6.12 (a) The form of the $3 b_{1}$ molecular orbital following a $90^{\circ}$ internal rotation.

(b) Top view of $\mathrm{CH}_{2} \mathrm{NO}_{2}$ subsequent to internal rotation, showing how a $\mathrm{C}-\mathrm{O}$ bond may form from the interaction of the $5 \mathrm{~b}_{2}$ oxygen atom lone pair molecular orbital with the empty p orbital on the carbon atom. 

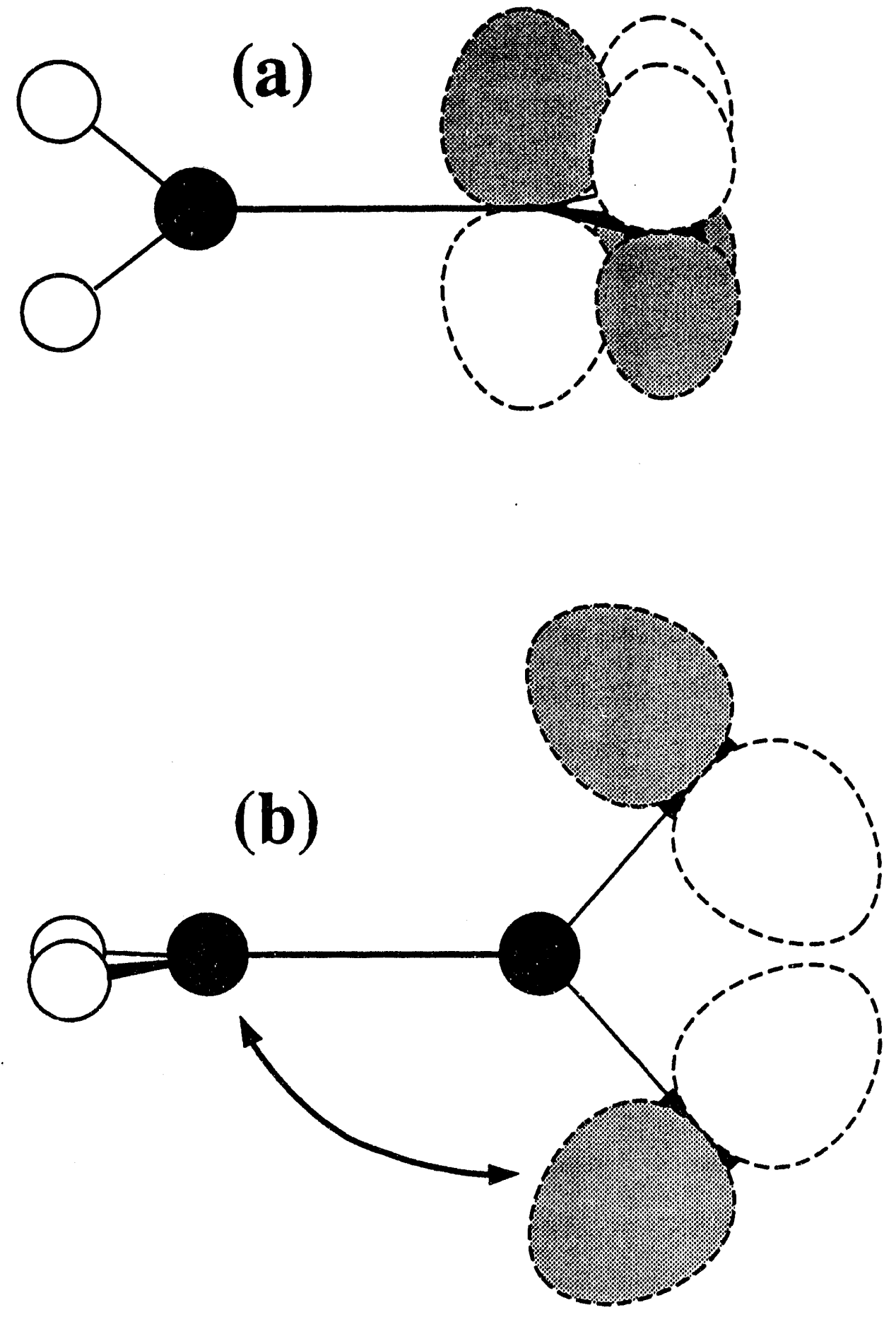

Figure 6.12 
the carbon atom and corresponding excess negative charge on the $\mathrm{NO}_{2}$ moiety. This should facilitate formation of the three-member $\overline{\mathrm{C}-\mathrm{N}-\mathrm{O}}$ ring intermediate, since the lone pair on either of the oxygen atoms in the $\mathrm{NO}_{2}$ plane [see Figure 6.12 (b)] will be attracted to the empty orbital on the electron-poor carbon atom, forming an $\mathrm{O}-\mathrm{C}$ bond. Figure 6.12 may be somewhat of a simplification in that rehybridization of the nitrogen atom may occur subsequent to internal rotation, resulting in an $\mathrm{sp}^{3}$ hybridization with a lone pair of electrons localized on the nitrogen, but this does not affect the overall argument.

As far as the energetics along the rather complex reaction coordinate associated with the nuclear dynamics depicted in Figure 6.11 are concerned, we expect the highest energy geometry to occur either en route to formation of the three-member ring, or at the three-member ring itself. While one might wonder if the three-member oxaziridine ring represents a local minimum, prior theoretical studies ${ }^{53}$ suggest that the structurally identical intermediate postulated for $\mathrm{CH}_{2} \mathrm{NO}_{2}^{-}$rearrangement is unstable. In addition, only oxaziridines having aryl-, alkyl-, H-, or acyl-substituted nitrogen atoms have been isolated; no stable oxaziridines with $O$-substituted nitrogen atoms (i.e. the current case) are known. ${ }^{54}$ In any case, regardless of the exact location of the barrier along the reaction coordinate, we expect it to lie well above ground state $\mathrm{H}_{2} \mathrm{CO}+\mathrm{NO}$ products, consistent with the high translational energy seen for this channel. On the other hand, the geometry found at the barrier is only beginning to resemble the products, and as a result there should be extensive internal excitation found in the products. This implies that the actual barrier height with respect to the products is significantly higher than the minimum value of $\sim 60 \mathrm{kcal} / \mathrm{mol}$ implied by the translational energy distribution.

The central element in the above mechanism is that the coordinatively unsaturated carbon atom provides a relatively facile pathway for NO elimination. Such a pathway does not exist in $\mathrm{CH}_{3} \mathrm{NO}_{2}$, consistent with the absence of $\mathrm{NO}$ production as a result of electronic excitation. Moreover, other examples of dissociation channels analogous to 
channel (II) in our study have been observed in which the carbon atom in the parent molecule is unsaturated. For example, in the experiments of Beijersbergen et al.,26 three structural isomers of nitromethane radical cation and their deuterated analogs were neutralized by resonant charge exchange. This process forms the neutral with a significant amount of excitation ( $6.9 \mathrm{eV}$ for $\mathrm{Na}$ and $7.2 \mathrm{eV}$ for $\mathrm{Cs}$ charge exchange). Below are the structures of two of the isomers studied, nitromethane 1 and its tautomer, acinitromethane 2:

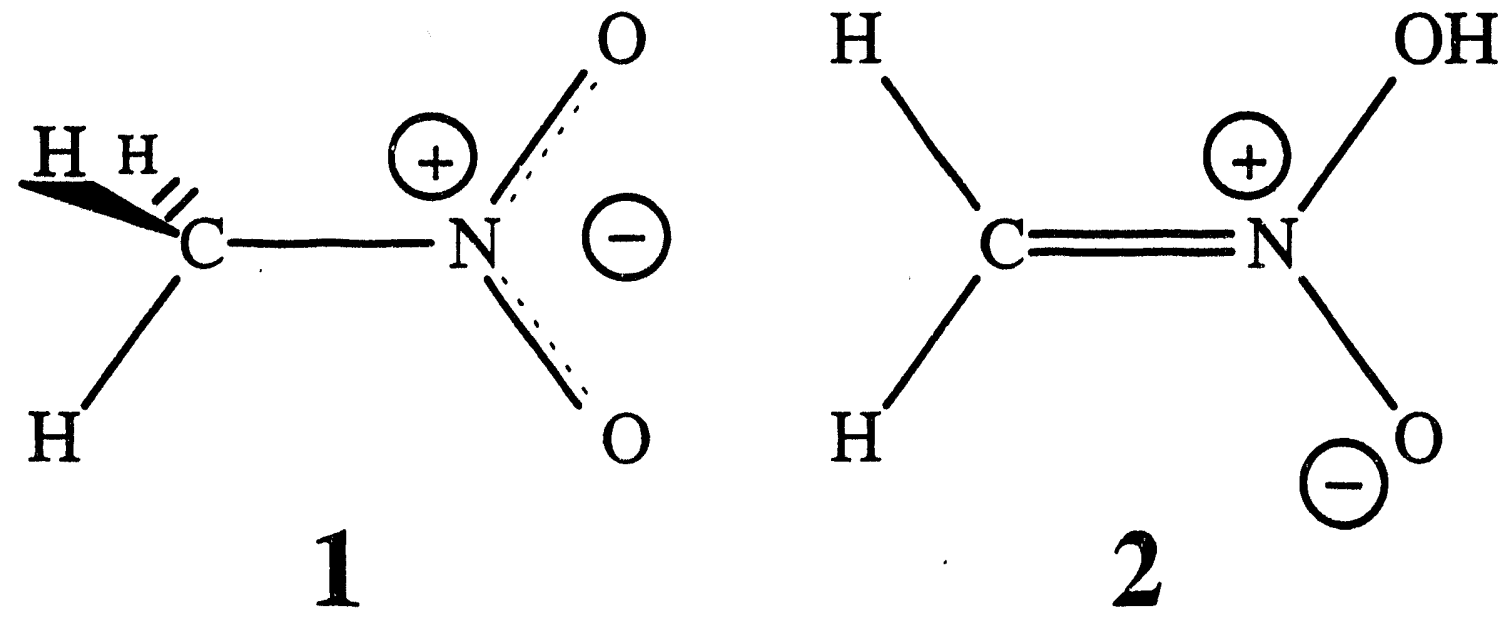

Rearrangement followed by elimination of $\mathrm{NOH}$ (or $\mathrm{HNO}$ ) was found to be the major pathway only upon charge exchange neutralization yielding the aci form of nitromethane, which contains a $\mathrm{CH}_{2}$ group rather than a $\mathrm{CH}_{3}$ group bonded to the nitrogen atom. Also, in the recent study by Galloway et al. 55 of the UV photodissociation of nitrobenzene, NO elimination was observed, along with both $\mathrm{C}-\mathrm{N}$ and $\mathrm{N}-\mathrm{O}$ bond fission. While nitrobenzene is a closed shell molecule, the carbon atom bound to the $\mathrm{NO}_{2}$ group is coordinatively unsaturated, and this may enhance the NO elimination channel by the mechanism discussed above. 


\subsubsection{3 $\mathrm{CH}_{2} \mathrm{NO}_{2} \rightarrow \mathrm{CH}_{2} \mathrm{NO}+\mathrm{O}$ Dissociation Mechanism}

Having proposed a possible mechanism by which channel (II) proceeds to products, the next step is to determine whether the absence of $\mathrm{C}-\mathrm{N}$ bond fission and the presence of $\mathrm{N}-\mathrm{O}$ fission can also be explained in terms excited state dynamics. We first consider why $\mathrm{C}-\mathrm{N}$ fission might not readily occur from the initially accessed $1^{2} \mathrm{~B}_{1}$ state. As shown in Figure 6.8, the three lowest lying $\mathrm{C}-\mathrm{N}$ bond fission channels which could correlate to this state in $\mathrm{C}_{2 v}$ symmetry are (IIIa) $\mathrm{CH}_{2}\left(\tilde{X}^{3} \mathrm{~B}_{1}\right)+\mathrm{NO}_{2}\left(\tilde{X}^{2} \mathrm{~A}_{1}\right)$, (IIIb) $\mathrm{CH}_{2}\left(\tilde{b}^{1} \mathrm{~B}_{1}\right)+\mathrm{NO}_{2}\left(\tilde{X}^{2} \mathrm{~A}_{1}\right)$, and (IIIc) $\mathrm{CH}_{2}\left(\tilde{a}^{1} \mathrm{~A}_{1}\right)+\mathrm{NO}_{2}\left({ }^{2}{ }^{2} \mathrm{~B}_{1}\right)$. The observed absence of $\mathrm{C}-\mathrm{N}$ bond fission in our results requires that there is no low energy pathway for dissociation of this state to the ground state fragments [channel (IIIa)]. If we examine how the molecular orbitals of the parent molecule correlate with those of the fragments, we indeed find that the ground state fragments correlate diabatically (and adiabatically) with the $\tilde{X}{ }^{2} \mathrm{~B}_{1}$ state of $\mathrm{CH}_{2} \mathrm{NO}_{2}$ and not with the $I^{2} \mathrm{~B}_{1}$ state. Hence, channel (IIIc) is the lowest $\mathrm{C}-\mathrm{N}$ bond fission channel to which the $I^{2} \mathrm{~B}_{1}$ state can correlate diabatically. This channel lies $45 \mathrm{kcal} / \mathrm{mol}$ above the lowest $\mathrm{CH}_{2} \mathrm{NO}+\mathrm{O}$ channel. $\mathrm{C}-\mathrm{N}$ bond fission is therefore less favorable than might be expected at first glance, and any additional barrier along the reaction coordinate further reduces the expected contribution from this channel.

To complete the overall picture, we need to identify a facile dissociation pathway to $\mathrm{N}-\mathrm{O}$ bond fission consistent with the observed translational energy distribution. This distribution, which at $240 \mathrm{~nm}$ peaks at less than $15 \%$ of the available kinetic energy, indicates that the surface on which dissociation occurs is not strongly repulsive along the $\mathrm{N}-\mathrm{O}$ coordinate. We first consider which electronic states of $\mathrm{CH}_{2} \mathrm{NO}_{2}$ might lead to $\mathrm{CH}_{2} \mathrm{NO}+\mathrm{O}$ production. The geometry of the ground state of $\mathrm{CH}_{2} \mathrm{NO}$ calculated by Balakina et al. ${ }^{56}$ is shown in Figure 6.13. This radical has $\mathrm{C}=\mathrm{N}$ double bond character,

Figure 6.13 Geometry of ground state $\mathrm{CH}_{2} \mathrm{NO}$, as determined in Ref. 56. Bond lengths are in Ångstroms, bond angles are in degrees. 


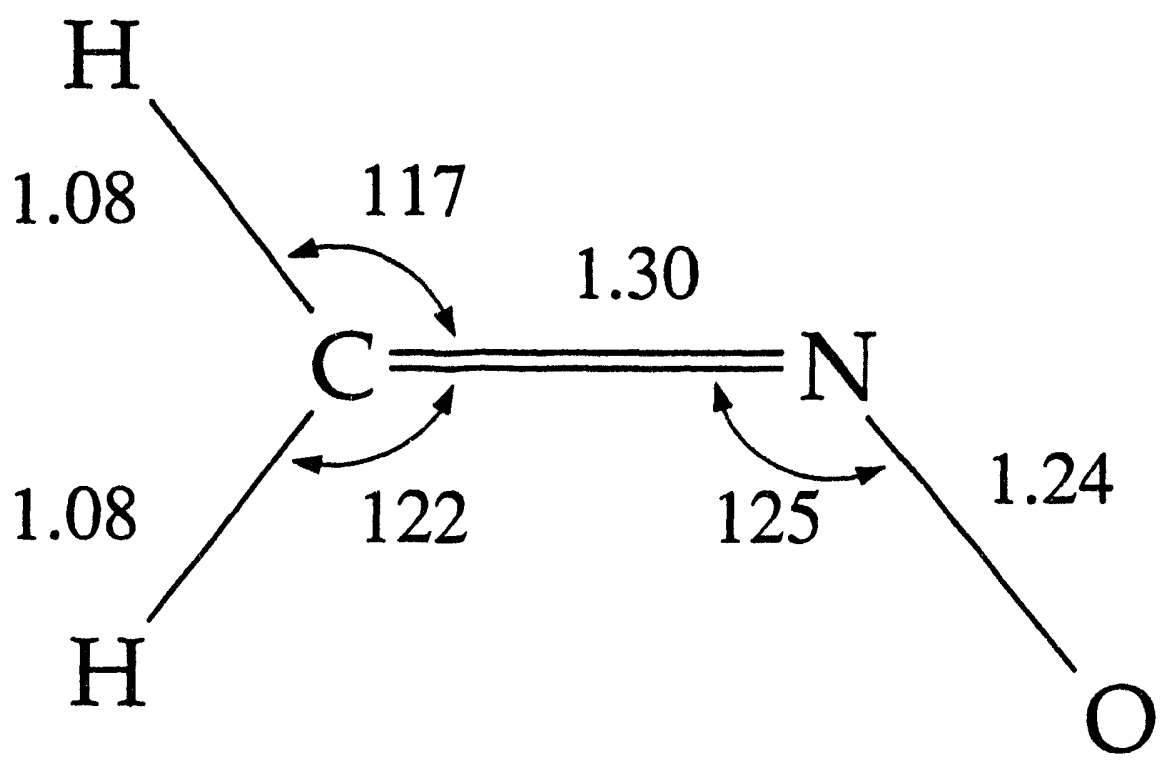

Figure 6.13 
while the HOMO of the $1{ }^{2} \mathrm{~B}_{1}$ state of $\mathrm{CH}_{2} \mathrm{NO}_{2}$ (the $3 \mathrm{~b}_{1}$ molecular orbital) is $\mathrm{C}-\mathrm{N} \pi$ antibonding. Hence, the $1{ }^{2} \mathrm{~B}_{1}$ state should not diabatically correlate to the ground state of $\mathrm{CH}_{2} \mathrm{NO}$. On the other hand, the I ${ }^{2} \mathrm{~A}_{2}$ state has a $\mathrm{C}-\mathrm{N}$ double bond and lies well below the $\mathrm{CH}_{2} \mathrm{NO}+\mathrm{O}$ asymptote. Moreover, the $\mathrm{C}-\mathrm{N}$ double bond makes dissociation to $\mathrm{CH}_{2}+\mathrm{NO}_{2}$ unlikely. Dissociation to $\mathrm{CH}_{2} \mathrm{NO}+\mathrm{O}$ from the $1^{2} \mathrm{~A}_{2}$ state therefore presents an attractive possibility.

The next issue to address is the mechanism which allows the radical to transfer onto the $1{ }^{2} \mathrm{~A}_{2}$ surface after initial excitation to the $1{ }^{2} \mathrm{~B}_{1}$ state. The molecular orbital occupancies for these states in the planar geometry, ...(1 $\left.a_{2}\right)\left(2 b_{1}\right)^{2}$ for the former and ...( $\left(a_{2}\right)^{2}\left(3 b_{1}\right)$ for the latter, differ by two electrons, and are therefore coupled only by the configuration interacticn term in the electronic Hamiltonian. This coupling should be strong only in the vicinity of an intersection between the two surfaces; such an intersection can be brought about by internal rotation about the $\mathrm{C}-\mathrm{N}$ bond. As discussed above, this torsional motion is expected to stabilize the initially excited $l^{2} \mathrm{~B}_{1}$ state. In contrast, it should strongly destabilize the $1{ }^{2} \mathrm{~A}_{2}$ state as it disrupts the $\mathrm{C}-\mathrm{N}$ double bond in the planar geometry. One can therefore imagine a crossing between the two surfaces at an internal rotation angle between $0^{\circ}$ and $90^{\circ}$, as shown in Figure 6.14. The radical has no symmetry at the intermediate angles, so these would actually be avoided crossings, with the adiabatic surfaces repelling each other near where the intersections would occur in the absence of configuration interaction.

However, it is perhaps easier to think about the overall dynamics in terms of diabatic surfaces. If, at the crossing points, the radical remains on the initial diabatic surface, it will continue to undergo internal rotation until it reaches the $90^{\circ}$ geometry, at which point NO elimination is most favorable. On the other hand, it can undergo a

Figure 6.14 Schematic of the potentials for the $1{ }^{2} \mathrm{~B}_{1}$ state and the $1{ }^{2} \mathrm{~A}_{2}$ state along the torsion coordinate. 


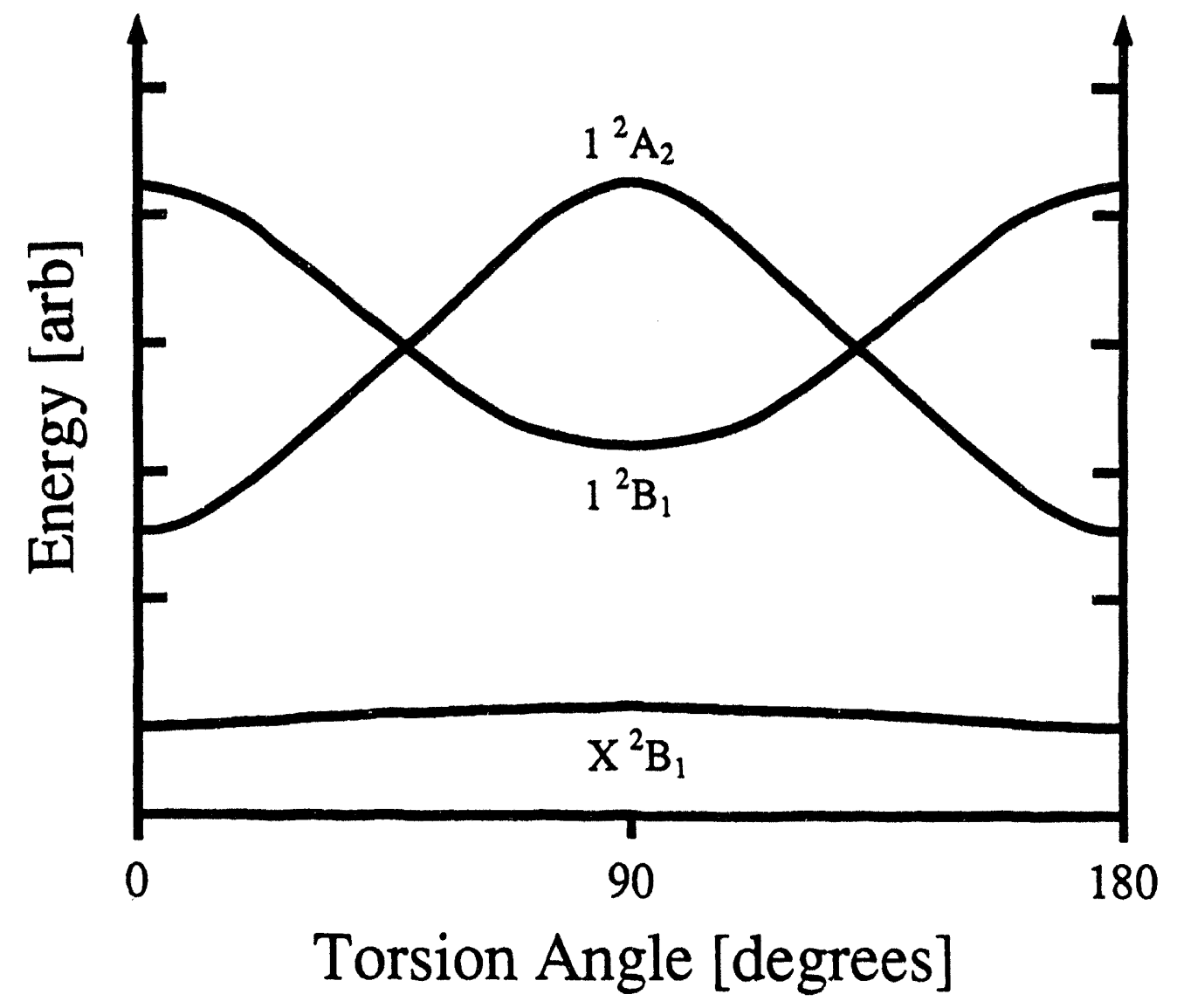

Figure 6.14 
transition to the diabatic surface which correlates to the $1{ }^{2} \mathrm{~A}_{2}$ state in the planar geometry. Such a diabatic transition would most likely result in a highly vibrationally excited molecule. If we apply the concepts developed for statistical dissociation on ground state surfaces to this situation, we would expect that dissociation to fragments with high kinetic energy is unlikely because the $I^{2} \mathrm{~A}_{2}$ state is strongly bound with respect to $\mathrm{CH}_{2} \mathrm{NO}+\mathrm{O}$. This is consistent with our observed distribution. However, a translational energy distribution described by phase space theory would peak very close to zero, while ours actually peaks at $5-8 \mathrm{kcal} / \mathrm{mole}$. This may indicate a small barrier to $\mathrm{N}-\mathrm{O}$ bond fission. Alternatively, peaking away from zero may indicate that the dissociation rate is sufficiently rapid as to render the statistical perspective somewhat inappropriate.

Overall, the picture we are proposing to explain the photodissociation of $\mathrm{CH}_{2} \mathrm{NO}_{2}$ is that the important nuclear dynamics are occurring on excited state potential energy surfaces. In particular, torsional motion on the initially excited surface plays a major role in the two observed dissociation channels, and the outcome depends on whether a diabatic transition occurs while the radical is undergoing internal rotation. We acknowledge that aspects of these mechanisms are somewhat speculative. Nonetheless, as the overail scheme is consistent with the experimental results, it should at least provide a starting point for more detailed experimental and theoretical studies of this species. We believe that $a b$ initio investigations of the excited states of $\mathrm{CH}_{2} \mathrm{NO}_{2}$ would be particularly helpful in assessing some of the ideas put forward here.

\subsection{Conclusions}

We have studied the photodissociation dynamics of the $\mathrm{CH}_{2} \mathrm{NO}_{2}$ radical at three wavelengths: 240,255 , and $270 \mathrm{~nm}$. We have measured branching ratios for the various dissociation channels, as well as translational energy and angular distributions for each major channel. We observe only two channels and find these have comparable yields: (I) $\mathrm{CH}_{2} \mathrm{NO}_{2} \rightarrow \mathrm{CH}_{2} \mathrm{NO}_{2}+\mathrm{O}$ and (II) $\mathrm{CH}_{2} \mathrm{NO}_{2} \rightarrow \mathrm{H}_{2} \mathrm{CO}+\mathrm{NO}$. No C-N bond fission is 
observed. These results are in sharp contrast to the ultraviolet photodissociation of nitromethane, which undergoes exclusive $\mathrm{C}-\mathrm{N}$ bond fission near $200 \mathrm{~nm}$. We have assigned the electronic transition in these experiments to the $I^{2} \mathrm{~B}_{1} \leftarrow \bar{X}{ }^{2} \mathrm{~B}_{1}$ transition, in which the $2 b_{1} C-N \pi$-bonding electron is promoted to the $3 b_{1} \pi^{*}$ molecular orbital. An analysis of the observed branching ratios and translational energy distributions indicates that statistical dissociation on the ground state surface is most likely not occurring, and that both of the observed channels are occurring on excited state surfaces. Mechanisms for both channels are proposed in which the initial nuclear dynamics involves intemal rotation about the $\mathrm{C}-\mathrm{N}$ bond in the initially excited state. A $90^{\circ}$ internal rotation facilitates formation of the three-center $\overline{\mathrm{C}-\mathrm{N}-\mathrm{O}}$ transition state needed for NO elimination, while internal rotation also provides a reasonable coupling mechanism between the $l{ }^{2} \mathrm{~B}_{1}$ and lower-lying $\mathrm{I}^{2} \mathrm{~A}_{2}$ surface, with $\mathrm{N}-\mathrm{C}$ bond fission occurring on the latter surface. 


\subsection{References}

1 Molecular Photodissociation Dynamics, edited by M. N. R. Ashfold and J. E. Baggr" (Royal Society of Chemistry, London, 1987).

2 G. E. Busch, J. F. Comelius, R. T. Mahoney, R. 1. Morse, D. W. Schlosser, and K. R. Wilson, Rev. Sci. Instrum. 41, 1066 (1970); G. E. Busch and K. R. Wilson, J. Chem Phys. 56, 3626 (1972).

3 For a recent review, see M. N. R. Ashfold, I. R. Lambert, D. H. Mordaunt, G. P. Morley and C. M. Western, J. Phys. Chem. 96, 2938 (1992).

4 E. J. Hincsa, X. Zhao, W. M. Jackson, W. B. Miller, A. M. Woduke, and Y. T. Lee. J. Phys. Chem. 95 , 2799 (1991).

5 H. F. Davis, B. Kim, H. S. Johnston, and Y. T. Lee, J. Phys. Chem. 97,2172 (1993).

6 R. E. Continetti, D. R. Cyr, R. B. Mctz, and D. M. Neumark, Chem. Phys. Lett. 182, 406 (1991).

7 D. R. Cyr, R. E. Continetti, R. B. Mc1z, D. L. Osborn, and D. M. Neumark, J. Chem. Phys. 97,4937 (1992).

8 R. E. Continetti, D. R. Cyr, D. L. Osborn, D. J. Leahy and D. M. Neumark, J. Chem. Phys. 99, 2616 (1993).

9 D. J. Leahy, D. R. Cyr, D. L. Osborn, and D. M.Neumark, Chem. Phys. Lett. (in press).

10 L. F. Salter and B. A. Thrush, J. Chem. Soc., Furaday Trans. I 37, 2025 (1977).

11 M. E. Jacox, J. Phys. Chem. 87, 3126 (1983).

12 C. Chachaty, J. Chim. Phys. 62,728 (1965).

13 C. Chachaty, C. Rosilio, J. Chim. Phys. 64,777 (1967).

14 M. E. Jacox, J. Phys. Chem. 91,5038 (1987).

15 R. B. Mctz, D. R. Cyr, and D. M. Neumark, J. Phys. Chem. 95, 2900 (1991).

16 M. L. McKee, J. Am. Chem. Soc. 107, 1900 (1985).

17 M. L. McKee, J. Chem. Phys. 81, 3580 (1984).

18 L. J. Butler, D. Krajnovich, Y. T. Lec, G. Ondrey, and R. Bersohn, J. Chem. Phys. 79, 1708 (1983).

19 N. C. Blais, J. Chem. Phys. 79, 1723 (1983).

20 K. Q. Lao, E. Jensen, P. W. Kash, and L. J. Butler, J. Chem. Phys. 93, 3958 (1990).

21 D. B. Moss, K. A. Trentelman, and P. L. Houston, J. Chem. Phys. 96, 237 (1991).

22 D. L. Phillips and A. B. Myers, J. Phys. Chem. 95, 7164 (1991).

23 P. E. Schoen, M. J. Marrone, J. M. Schnur, and L. S. Goldberg, Chem. Phys. Lett. 90, 272 (1982). 
24 A. M. Wodtke, E. J. Hintsa, and Y. T. Lee, J. Chem. Phys. 84, 1044 (1986).

25 A. M. Wodtke, E. J. Hinsta, and Y. T. Lee, J. Phys. Chem. 90,3549 (1986).

26 J. H. M. Beijersbergen, W. J. van der Zande, P. O. Kistemaker, J. Los, T. Drewello, and N. M. M. Nibbering, J. Phys. Chem. 96,9288 (1992).

27 D. J. Leahy, D. R. Cyr, D. L. Osbom, and D. M. Neumark, SPIE Prcseedings 1858, 49 (1993).

28 M. A. Johnson, M. L. Alexander, and W. C. Lincberger, Chem. Phys. Lett. 112, 285 (1984).

29 R. E. Continetti, D. R. Cyr, and D. M. Neumark, Rev. Sci. Instrum. 63, 1840 (1992).

30 J. M. B. Bakker, J. Phys. E 6, 785 (1973); 7, 364 (1974).

31 D. P. de Bruijn and J. Los, Rev. Sci. Instrum. 53, 1020 (1982).

32 C. Martin, P. Jelinsky, M. Lampton, R. F. Malina, and H. O. Anger, Rev. Sci. Instrum. 52, 1067 (1981).

33 D. F. Ogletree, G. S. Blackman, R. Q. Hwang, U. Stark, G. A. Somorjai and J. E. Katz, Rev. Sci. Instrum. 63, 104 (1992).

34 S. G. Lias, J. E. Barmess, J. F. Liebman, J. L. Holmes, R. D. Levin, and W. G. Mallard, J. Phys. Chem. Ref. Data, 17, Supplement 1 (1988).

35 R. N. Zare, Mol. Photochem. 4, 1 (1972).

36 C. F. Melius, private communication.

37 P. Ho, D. J. Bamford. R. J. Buss, Y. T. Lee, and C. B. Moore, J. Chem. Phys. 76, 3630 (1982).

38 R. P. Saxon and M. Yoshimine, Can. J. Chcm. 70, 572 (1992).

39 A. P. Cox and S. Waring, J. Chem. Soc. Faraday Trans. 2, 1060 (1972).

40 M. J. Frisch, M. Head-Gordon, H. B. Schlegel, K. Raghavachari, J. S. Binkley, C. Gonzalez, D. J. DeFrees, D. J. Fox, R. A. Whiteside, R. Seeger, C. F. Mclius, J. Baker, R. L. Martin, L. R. Kahn, J. J. P. Stewart, E. M. Fluder, S. Topiol, and J. A. Pople, Gaussian Inc., Pittsburgh, PA.

41 F. T. Williams, Jr., P. W. K. Flanagan, W. J. Taylor, and H. Schechter, J. Org. Chem. 30, 2674 (1965).

42 For general reviews of RRKM theory, see W. Forst, J. Chim. Phys. 87, 715 (1990); Theory of Unimolecular Reactions, (Academic, New York, 1973); P. J. Robinson and K. A. Holbrook, Unimolecular Reactions, (Wiley-Interscience, Now York, 1972).

43 R. G. Gilbert and S. C. Smith, Theory of Unimolecular and Recombination Reactions, (Blackwell, Oxford, 1990). 
44 'Loose' and 'tight' relate to the relative gain in entropy on going from the equilibrium geometry to the activated complex, with 'loose' implying a larger gain and vise-versa.

45 D. L. Bunker and W. L. Hase, Quantum Chemistry Program Exchange catalog number QCPE-234, Department of Chemistry, Indiana University.

46 D. J. Krajnovich, Ph. D. Thesis, UC Berkeley, 1983, p. 85.

47 S. W. Benson, Thermochemical Kinetics, 2nd ed., (Wiley, New York, 1976).

48 S. W. Benson and H. E. O'Neal, Natl. Stand. Ref. Data Ser., Natl. Bur. of Stand. 21, 473 (1970).

49 AFAC was obtained from the group of Prof. Y. T. Lee. It calculates the expected $A_{\infty}$ factor based on Eqn. 6.12 , by first calculating $\Delta S$ given vibrational frequencies for both the reactant and transition state.

$50 \mathrm{Dr}$. Sean Smith determined this $\mathrm{C}-\mathrm{N}$ bond distance value, using a variational calculation, to have the minimum sum of states in the dissociating complex at the energy available when a $240 \mathrm{~nm}$ photon is used in our experiment.

51 R. D. Levine in Theory of Chemical Reaction Dynamics, Vol. IV, M. Baer, Ed., (CRC Press, Boca Raton, FL, 1984), p. 31.

52 K. Yamada, T. Kanekiyo, S. Tanaka, K. Naruchi, and M. Yamamoto, J. Am. Chem. Soc. 103, 7003 (1981).

53 O. Kikuchi, T. Kanekiyo, S. Tanaka, K. Naruchi, and K. Yamada, Bull. Chem. Soc. Jpn. 55, 1509 (1982).

54 E. Schmitz in Comprehensive Heterocyclic Chemistry, W. Lwowski, Ed., (Pergamon Press, Oxford, 1984).

55 D. B. Galloway, J. A. Bartz, L. G. Huey, and F. F. Crim, J. Chem. Phys. 98, 2107 (1993).

56 M. Y. Balakina, M. B. Zuev, and I. D. Morozova, J. Mol. Struct. (Theochem) 183, 291 (1989). 


\section{Appendix A}

\section{FRBM - The Ion Mass Spectrum and Total Photodissociation Cross Section Experiment Control and Data Acquisition Program}

This Appendix presents FRBM the data acquisition program used both to collect ion mass spectra (see § 2.6.4.1) as well as radical total photodissociation cross section spectra (see § 2.7.5). Both options are invoked as selections from the main menu. Other main menu items include provision for 'manual' control of the lasers, setting experimental parameters, plotting and file manipulation. Sub menus are found within these options. In addition to the source files listed below, several units supplied by Turbo Pascal are compiled into the program, such as crt, dos and graph. A third-party unit, graphadd, allows extra printing capabilities. A file containing routines for interface to the CAMAC crate, namely camturbo.v4, must be compiled as an include file. Far calls must be disabled via compiler directive in the sections where this file is included. See the Turbo Pascal manuals for details and ramifications.

\section{Description of Files}

frbm.pas Contains the list of units to be built into the program; calls initialization routines, followed by the main menu.

init_ovr.pas Initializes the Turbo Pascal overlay manager to automatically overlay all units in frbm.pas marked with the compiler directive \$O+.

initial.pas Initializes all parameters necessary for the program to run properly, checks with the user that the system knows the proper date and time for the. $\log$ files that will be made, insures subdirectories for data exist (or 
creates them), installs external graphing units and initializes GPIB controller and the dye lasers.

mainmenu.pas Presents the user with an array of menu items, calling the routine corresponding to the selection made by the user.

globals.pas Declares all variables, constants, data types, strings, etc. that need to be shared among units.

utility.pas This is a catch-all unit which includes somewhat common functions and procedures that can be called from other units. The general headings that are included here are: math, user input, saving and reading data files, and 'miscellaneous'

insgrdrv.pas Installs the third-party graphics driver that allows us to print to the laserwriter from within the program

grafutil.pas A pared-down version of graphlib.tgt (written by William Polik) modified to include routines from the Turbo Pascal graph unit where possible.

txsplot.pas Handles plotting for total photodissociation cross section data, including raw photodissociation signal, detached electron signal, laser power signal and normalized signal.

tofplot.pas Provides plots of ion signal vs. time-of-flight.

plotutil.pas Contains routines allowing plots to be sent to the laserwriter from within the program.

parameters.pas Searches for and reads in a setup file containing initial parameters for experiments. If this file is not found it prompts the user for this information and allows such a setup file to be written.

dyelaser.pas Allows full control of the detachment (grating) and dissociation (grating and etalon) dye lasers via GPIB interface.

twkplot.pas Provides graphical representation of the status of the various measured quantities (photodetached electrons, laser power, dissociated radical 
signal) measured via the charge sensitive ADC's. Useful for efficient

'tweaking' of various parameters to improve output of these signals.

txs_exp.pas Controls the total cross section scanning and data acquisition and related routines such as the signal tweaking mode.

tof_exp.pas Controls the data acquisition during ion time-of-flight experiments.

gpibutil.pas Contains utilities for use with the National Instruments GPIB controller, which is used primarily to interface with the dye lasers.

tpdecl.pas Contains declarations necessary for GPIB operation.

\section{Source Code Listing}

\section{1 frbm.pas}

( frbm.pas

)

( This program is a result of major modifications, additions, deletions and revisions to the ACROPOLIS program originally written by J. G. Loeser to control an experiment with similar components. It has been upgraded to version 5.5 of Turbo Pascal, and the include files converted to separate units. Overlays are used to enable the program to be quite large. Graphics routines from TP are now used, with the small number of useful routines from the old graphlib unit now found in grafutil. Graphics drivers from Graf/Drive Plus have been utilized to allow graphing directly to our PostScript LaserWriter from within the program.

Questions that may arise concerning various aspects of this code can be addressed by the appropriate section of either the Turbo Pascal 5.5 Reference Manual or Users Guide supplied by Borland, which are altogether quite helpful.

( main program FRBM

program FRBM (input, output);

$\{\mathrm{SF}+\}$

\{\$M 16384,200000,300000\}

uses overlay,init_ovr,initial,mainmenu;

(SO initial)

[\$O globals] 
[\$O utility)

(\$O insgrdrv]

(\$O grafutil)

(\$O oxsplot)

\{\$O tofplot\}

[\$O plotutil\}

[\$O parameters]

[\$O dyelaser]

[SO twkplot]

[SO txs_exp]

[\$O tofms]

(\$O mainmenu]

$\{* * * * * * * * * * * * * * * * * * * * * * * * * * * * * * * * * * * * * * * * * * * * * * * * * * * * * * * * * * * * * * * * * * * * * * * * * * * *)\}$

begin ( main program FRBM )

initialize_ovr;

initialize;

main_menu;

end. (main program FRBM \}

\section{2 init_ovr.pas}

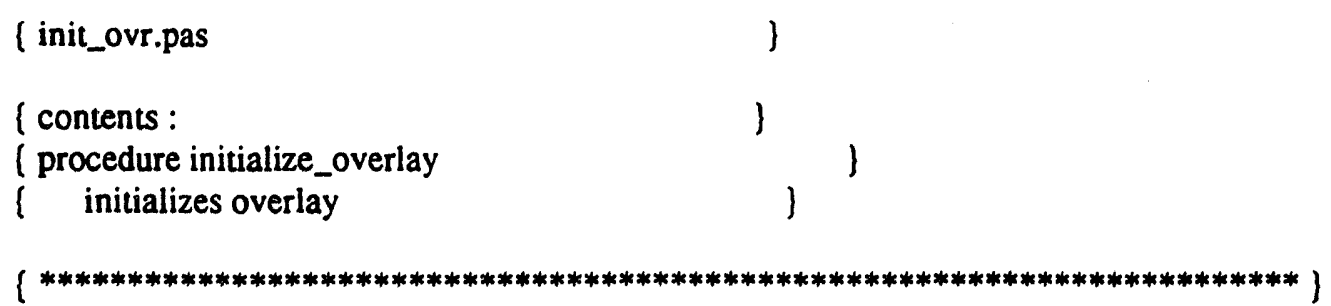

unit init_ovr;

$\{\mathrm{SF}+\}$

interface

uses overlay;

procedure initialize_overlay;

$\{* * * * * * * * * * * * * * * * * * * * * * * * * * * * * * * * * * * * * * * * * * * * * * * * * * * * * * * * * * * * * * * * * * * * * * * * * * * * *)\}$

implementation

procedure initialize_overlay;

const

OvrMaxSize $=40000$;

var 


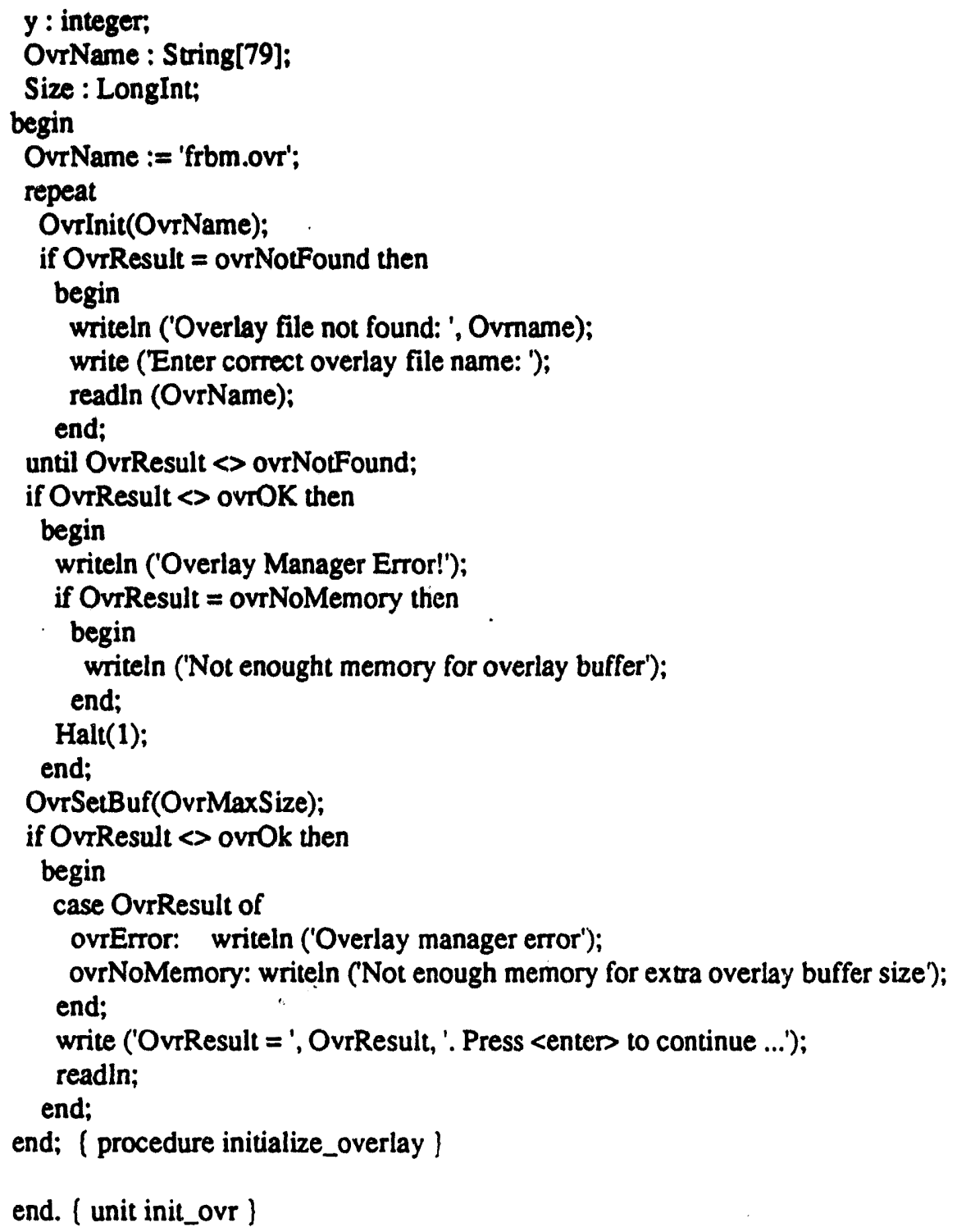

\section{3 initial.pas}

( initial.pas

)

( contents :

1

( procedure initialize

i initializes everything (except for overlay manager)

unit initial;

[SO+]

$[\mathrm{SF}+]$ 


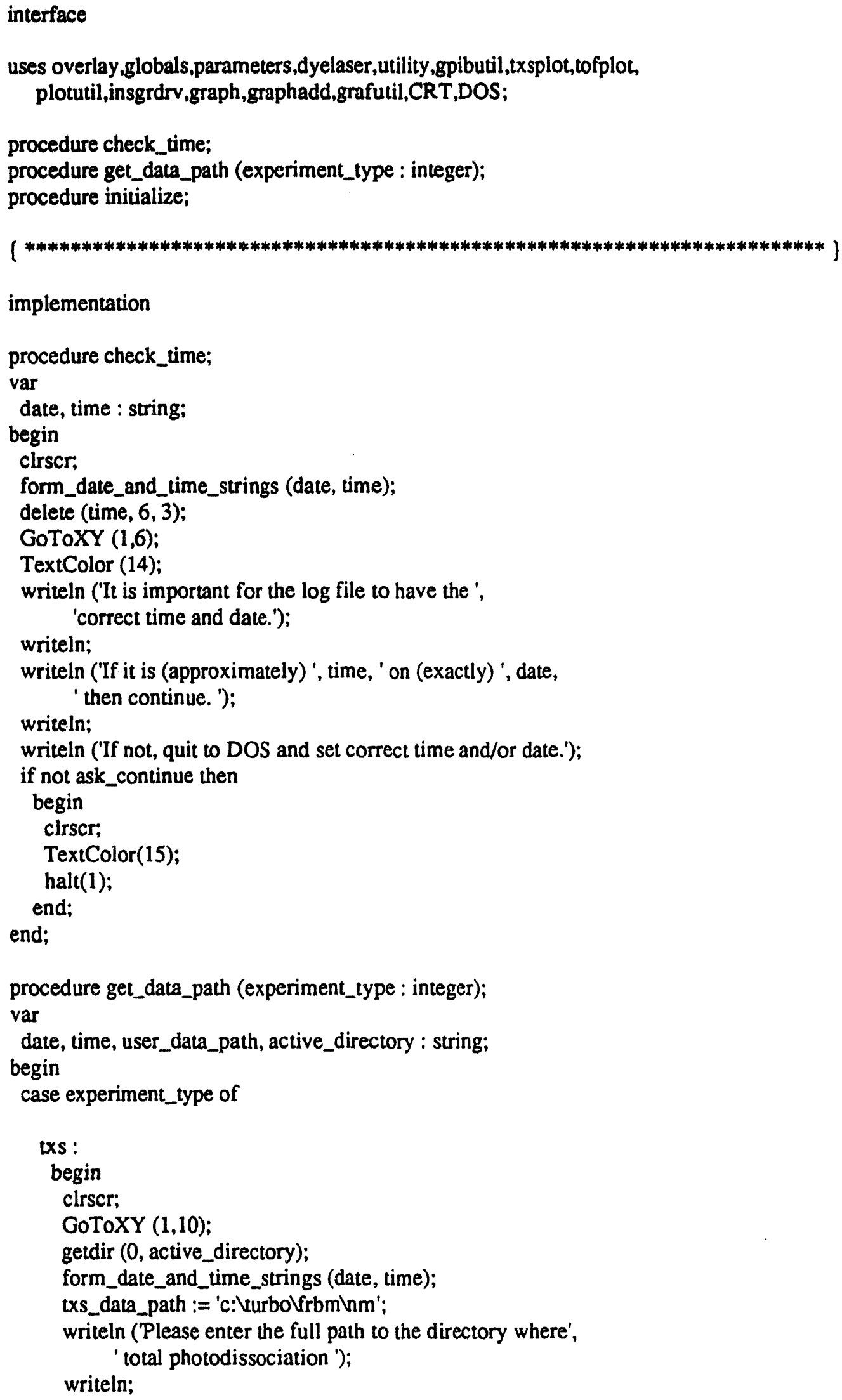




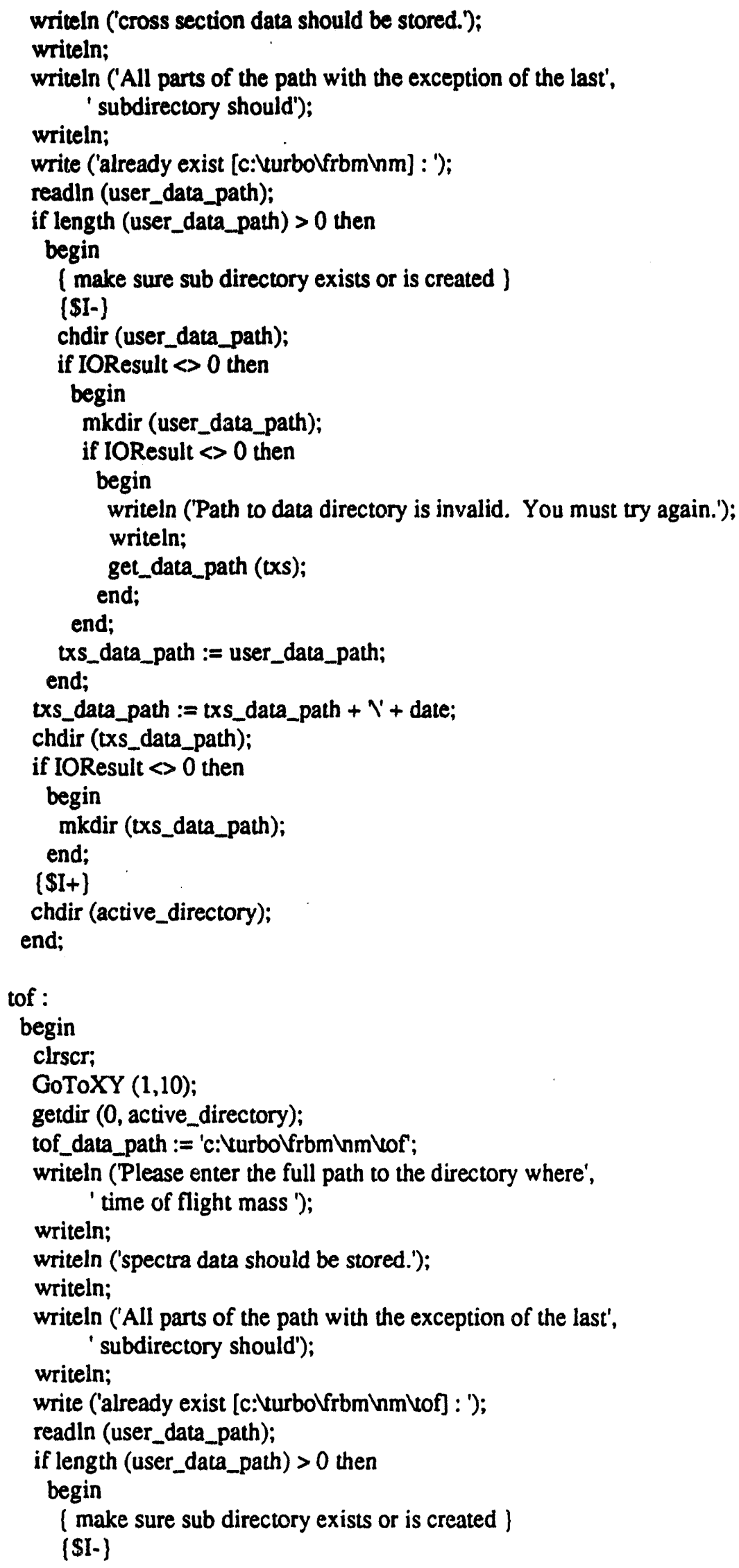




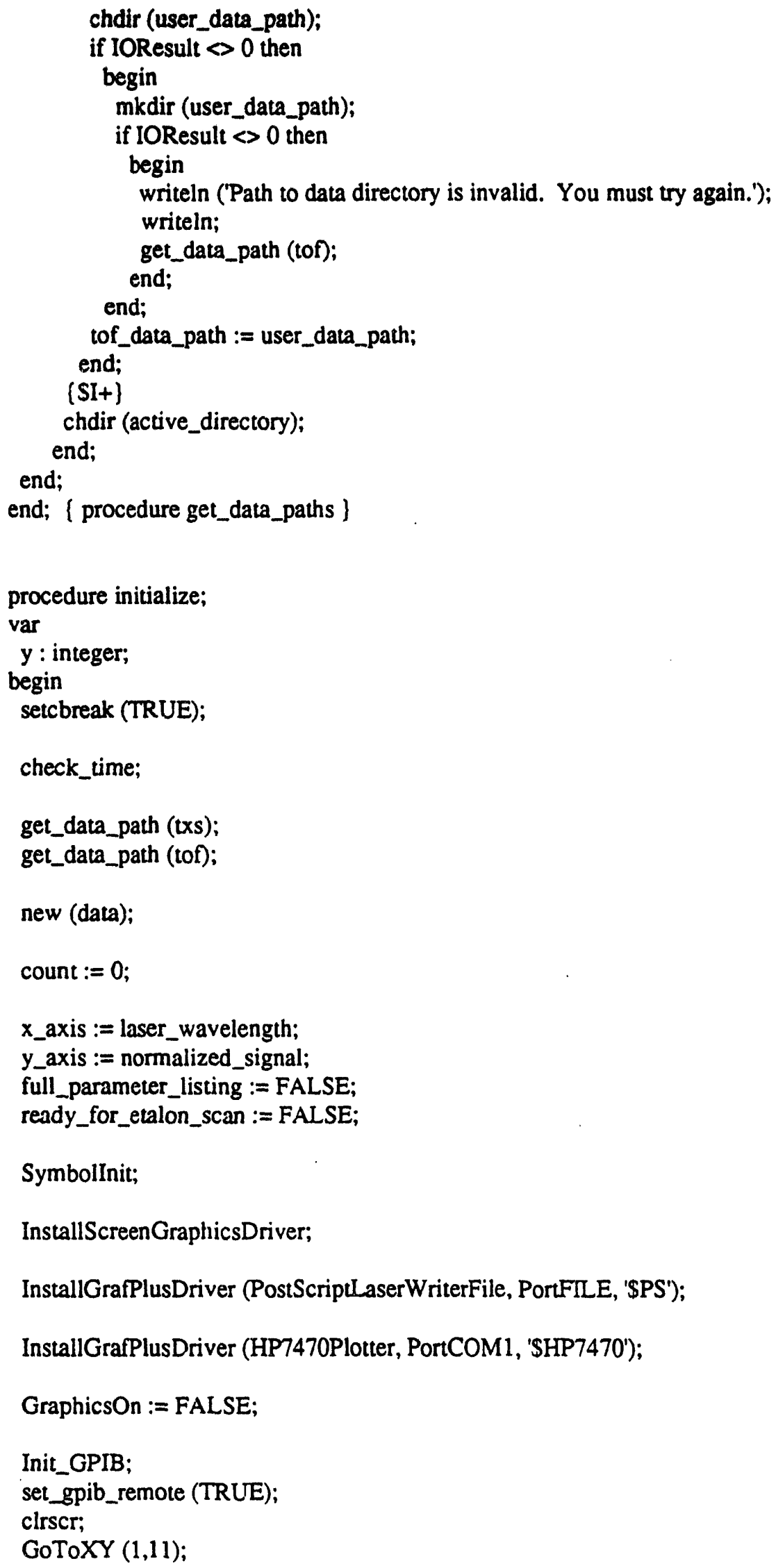




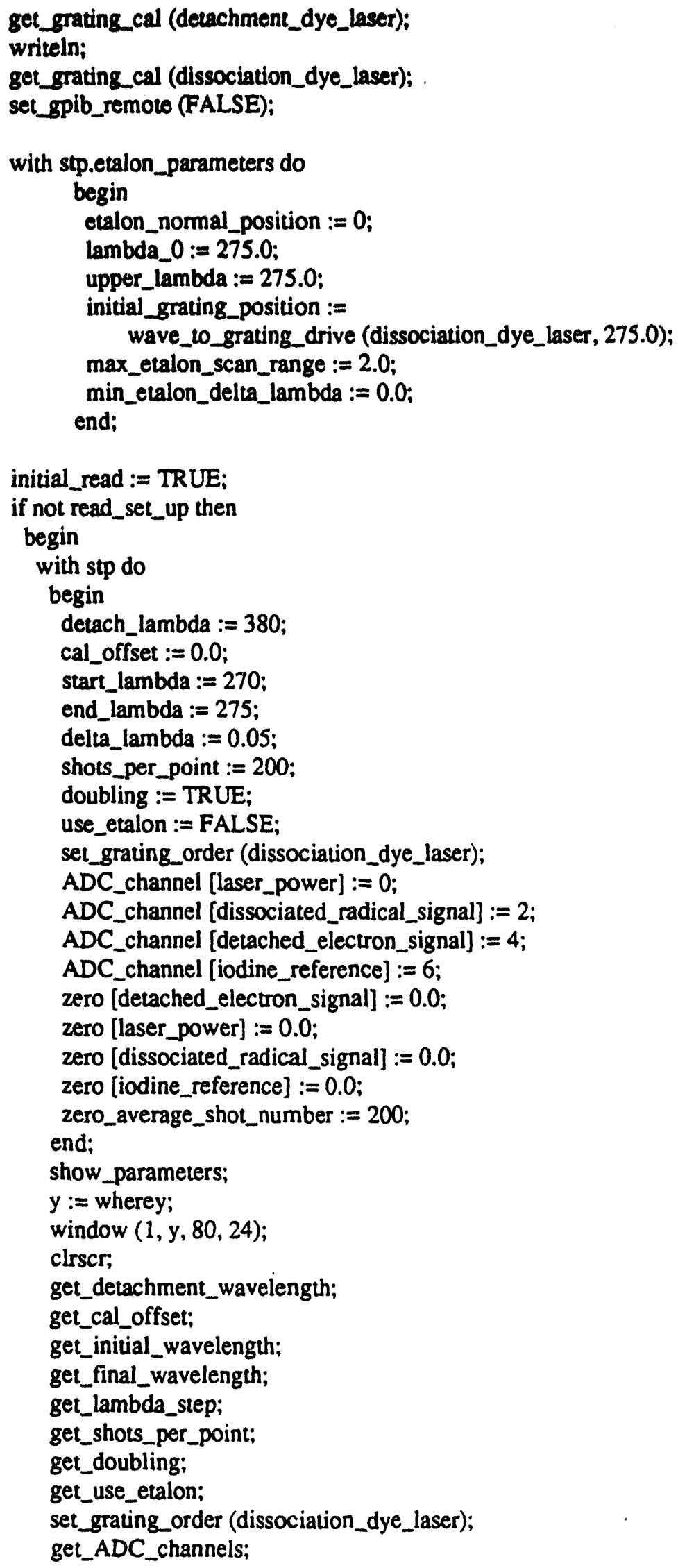




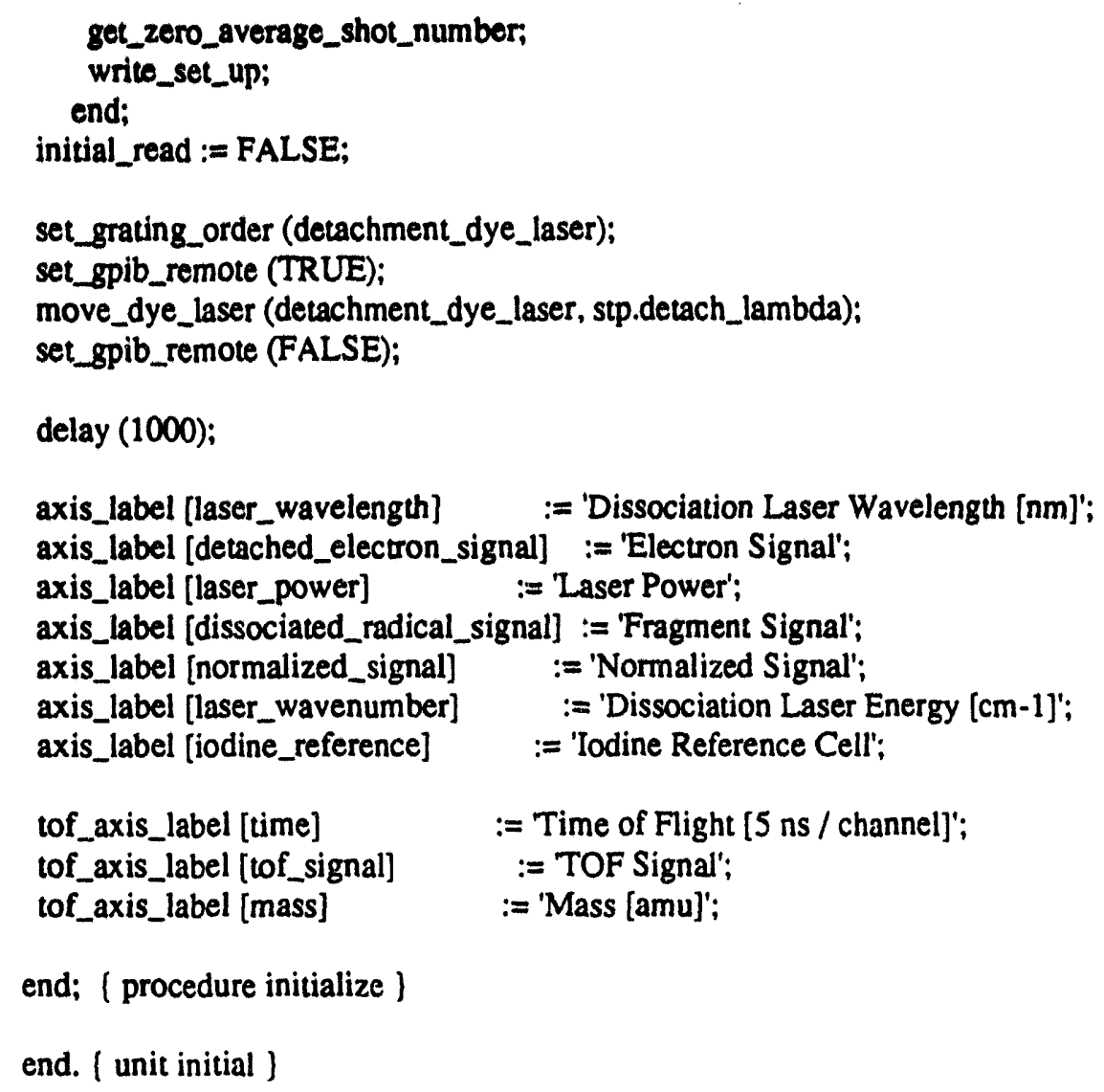

end; ( procedure initialize ]

end. ( unit initial )

\section{4 mainmenu.pas}

( mainmenu.pas

I contents :

( procedure main_menu

menu of things to do before and after experiment
了

了

)

$\{* * * * * * * * * * * * * * * * * * * * * * * * * * * * * * * * * * * * * * * * * * * * * * * * * * * * * * * * * * * * * * * * * * * * * * * * * * * * * * * *)\}$

unit mainmenu;

$\{\mathrm{SO}+\}$

$\{\mathrm{SF}+\}$

interface

uses globals,initial,parameters,utility,txs_exp,tofms,txsplot,CRT;

procedure main_menu;

$\{* * * * * * * * * * * * * * * * * * * * * * * * * * * * * * * * * * * * * * * * * * * * * * * * * * * * * * * * * * * * * * * * * * * * * * * * * *)\}$ 


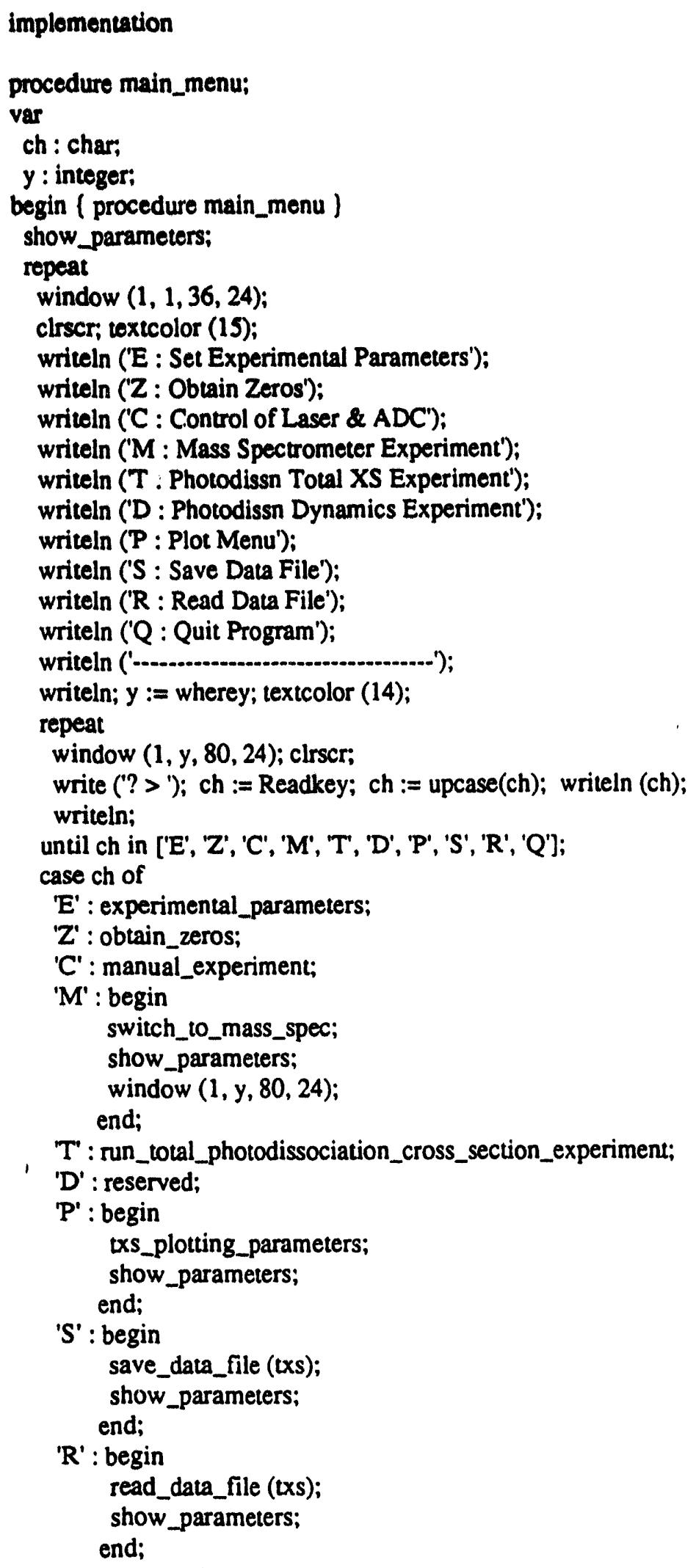




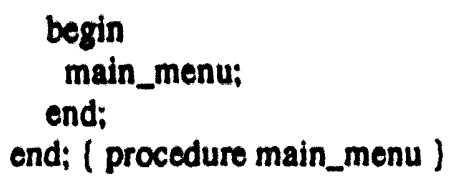

end. \{ unit mainmenu \}

\section{5 globals.pas}

\section{I globals.pas}

this unit contains all global variables neccessary for frbm.pas ]

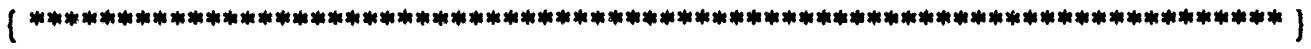

unit globals;

$(\mathrm{SO}+]$

$(\$ \mathrm{~F}+\}$

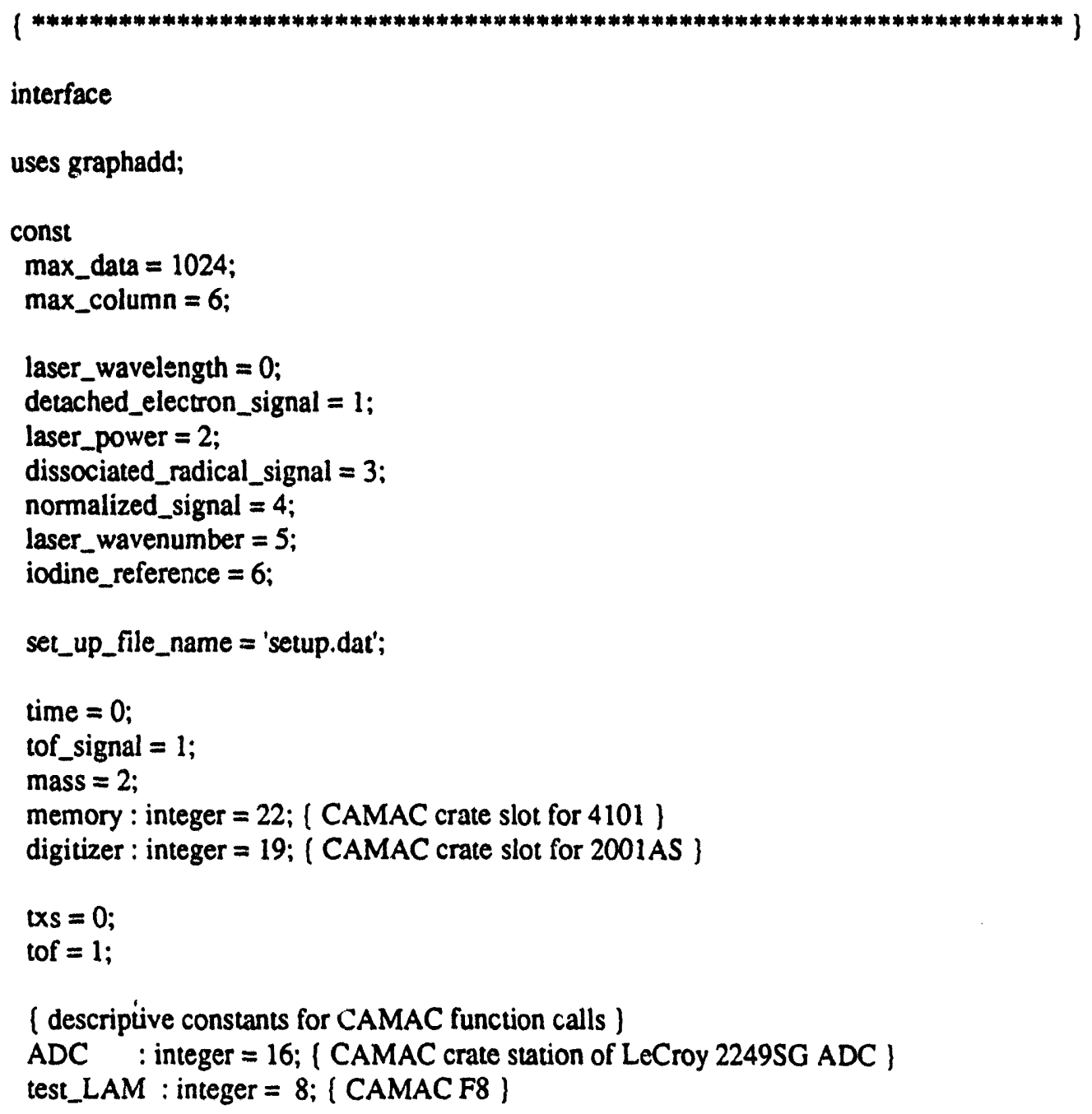

(descriptive constants for CAMAC function calls )

ADC : integer = 16; ( CAMAC crate station of LeCroy 2249SG ADC ) test_LAM : integer $=8$; $\{$ CAMAC F8 $\}$ 
read_channel : integer $=0 ;($ CAMAC FO $)$

dataway_z: integer $=1 ;$ ( CAMAC Initialize )

dacaway_c $:$ integer $=2 ;$ ( CAMAC Clear)

dataway_ $i:$ integer $=4 ;$ ( CAMAC Inhibit )

CRIACL : integer $=64 ;$ ( Clear Request Inhibit \& ACL detection registers )

max_tweak_limit $=200$;

lines : boolean = TRUE;

plot_lines : boolean = TRUE:

plot_from_zero : boolean = TRUE;

info : DrvINFO = (DrvMem:36000; DrwWrkdrive:'e';

DrvOutfile : 'e: Plot.out';

escchk : TRUE; nohead : FALSE );

PathToGraphics : string = 'c: Vurbolgraphics';

GraphComm : word = Baud9600 or ParNone or Data8 or Stopl;

$\mathrm{XON}$ : boolean = FALSE;

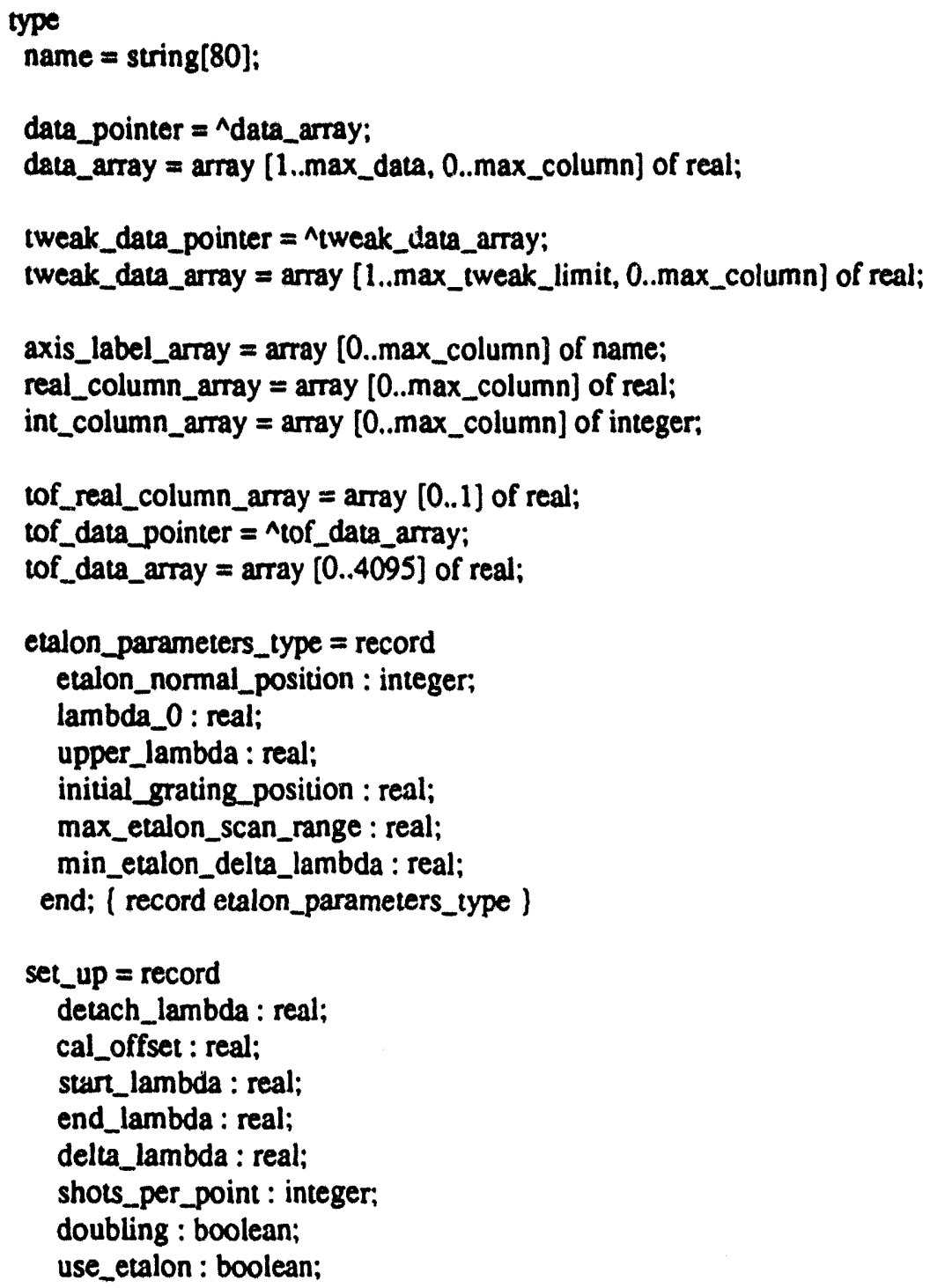




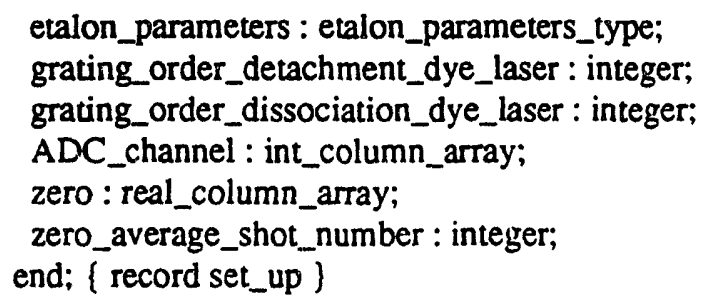


full_parameter_listing : boolean;

ready_for_etalon_scan : boolean;

new_zeros_obtained : boolean;

sweeps_to_average : integer;

digitizer_control : integer;

memory_control : integer;

data_count : integer;

tof_data : tof_data_pointer;

tof_data_max, tof_data_min : real;

mass_spec_delay : real;

tof_plot_min, tof_plot_max : tof_real_column_array;

df_data_count : integer;

df_digitizer_control : integer,

df_memory_control : integer;

df_sweeps_to_average : integer;

df_mass_spec_delay : real;

GraphDriver : integer; ( The Graphics device driver )

GraphMode : integer; [ The Graphics mode value \}

ErrorCode : integer; (Reports any graphics errors )

MaxColor : word; [ The maximum color value available ]

OldExitProc : Pointer; [ Saves exit procedure address ]

GraphicsOn : Boolean; ( Saves status of CRT mode (graphics or text))

HP7470Plotter : integer;

PostScriptLaserWriterFile : integer;

status : word;

magic : ^byte;

wordptr : ^ word;

hold : byte;

implementation

end. [ unit globals ]

\section{6 utility.pas}

( utility.pas

( contents :

\{i. math

( function int_power (base : real;

power : integer) : real

\{ii. user input

[ii.a. yes/no

\{ function ask_for_boolean (prompt : name) : boolean

( user must answer yes/no question, 'prompt'
)

\}

1

\}

\}

了 
(ii.b. continue/quit

( function ask_continup ' 'oolean

f user must hit <enter> to continue or $Q$ to quit

]

\{ ii.c. numbers

)

( these functions convert strings to numbers and ignore inappropriate ]

( characters -- beware of misinterpretation of invalid input

( true if user enters 'response' between ' $m$ in' and ' $m a x$ ' or '<' which sets 'response' to 'min' or

'>' which sets 'response' to 'max'

( false if user just presses 'enter'; 'response' is unchanged

( 'prompt' is repeated if user enters something else

[ function ask_for_integer (var response : integer;

( prompt : name;

$\min , \max :$ integer $):$ boolean

\{ function ask_for_real (var response : real;

l prompt : name;

( $\min , \max :$ integer) : boolean

\}

\{ ii.d. wavelength to wavenumber conversion and vise versa

( function wl2wn (wl : real) : real;

( function wn2wl (wn : real) : real;

\{ ii.e. file names

了

( function exist (file_name : name) : boolean

\{ true if file called 'file_name' is present on disk

\{ function legal_name (file_name : name) : boolean

( true if file name 'file_name' is a legal one

\{ procedure get_out_file_name (var file_name : name;

\{

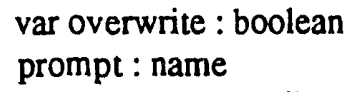

true if user enters a new 'file_name' or wants to overwrite an

( existing file; if overwrite, sets overwrite to TRUE

\{ function get_in_file_name (var file_name : name;

l prompt : name) : boolean

true if user entered 'file_name' of an existing file

\{ iii. save and read data

( procedure save_data (experiment_type : integer);

( save data to individual'.dat' file, append scan parameter info

I to one 'date.log' file per day 
\{ iv. miscellaneous

\}

( procedure reserved;

\section{)}

$\{$ displays message : 'reserved for future development'

\section{\{}

unit utility;

$\{\mathrm{SO}+\}$

$\{\$ \mathrm{~F}+\}$

interface

uses globals,DOS,CRT;

function int_power (base : real; power : integer) : real;

function ask_for_boolean (prompt : name) : boolean;

function ask_continue : boolean;

function ask_for_integer (var response : integer; prompt : name;

$\min , \max :$ integer) : boolean;

function ask_for_real (var response : real; prompt : name;

min, max : real) : boolean;

function wl2wn (wl : real) : real;

function wn2wl (wn : real) : real;

function I2S (Val, Digit : Integer) : String;

function R2S (Val : real; Digit, Decimal : Integer) : String;

function exist (file_name : name) : boolean;

function legal_name (file_name : name) : boolean;

procedure get_out_file_name (var file_name : name; var overwrite : boolean;

experiment_type : integer; prompt : name;

vargot_good_out_file_name : boolean);

function get_in_file_name (var file_name : name; experiment_type : integer;

prompt : name) : boolean;

procedure form_date_and_time_strings (var date_string : name;

var time_string: name);

procedure save_data_file (experiment_type : integer);

procedure read_data_file (experiment_type : integer);

procedure reserved;

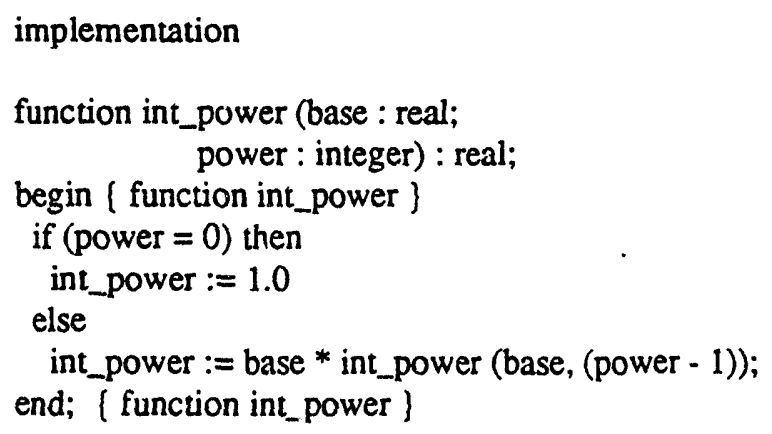




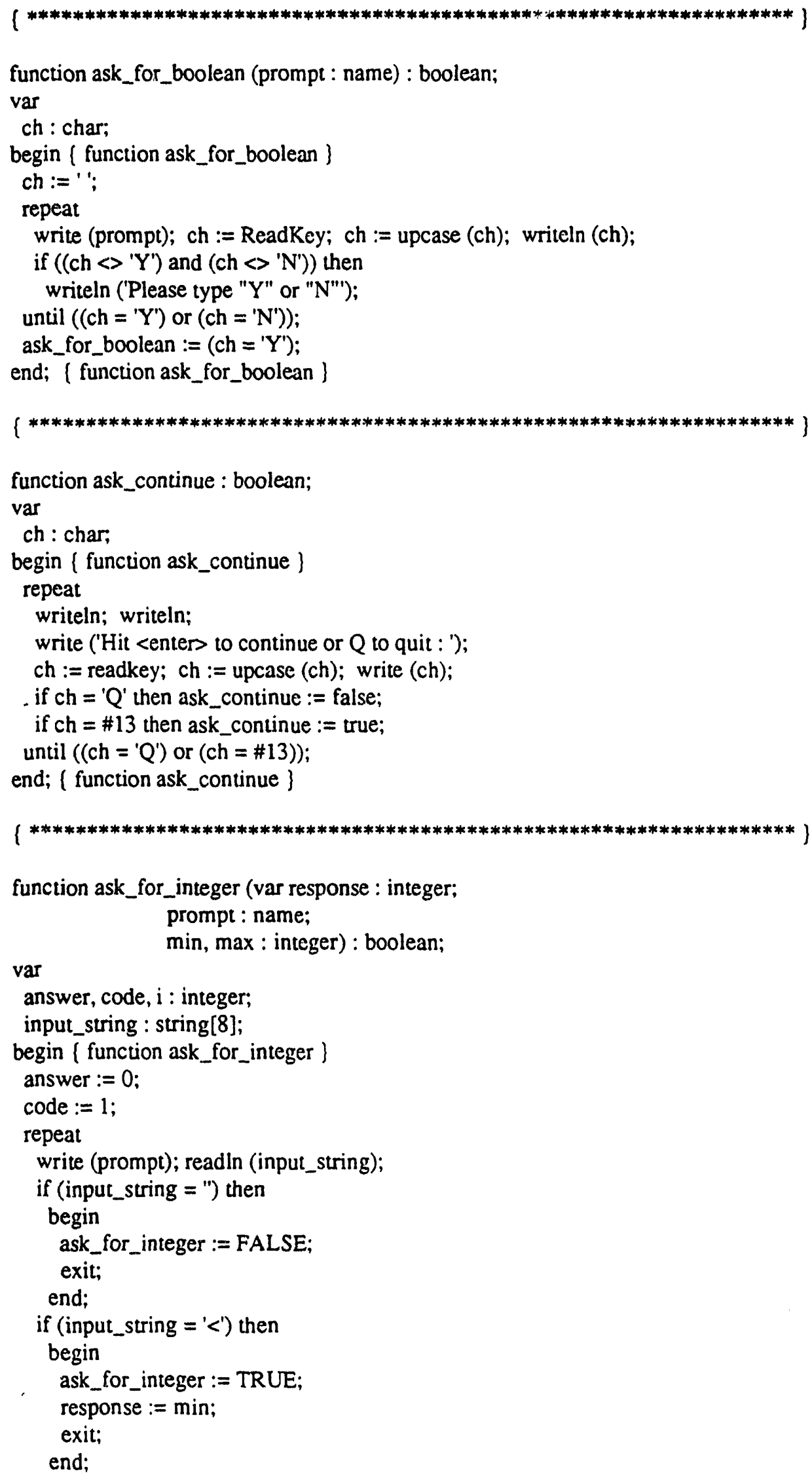




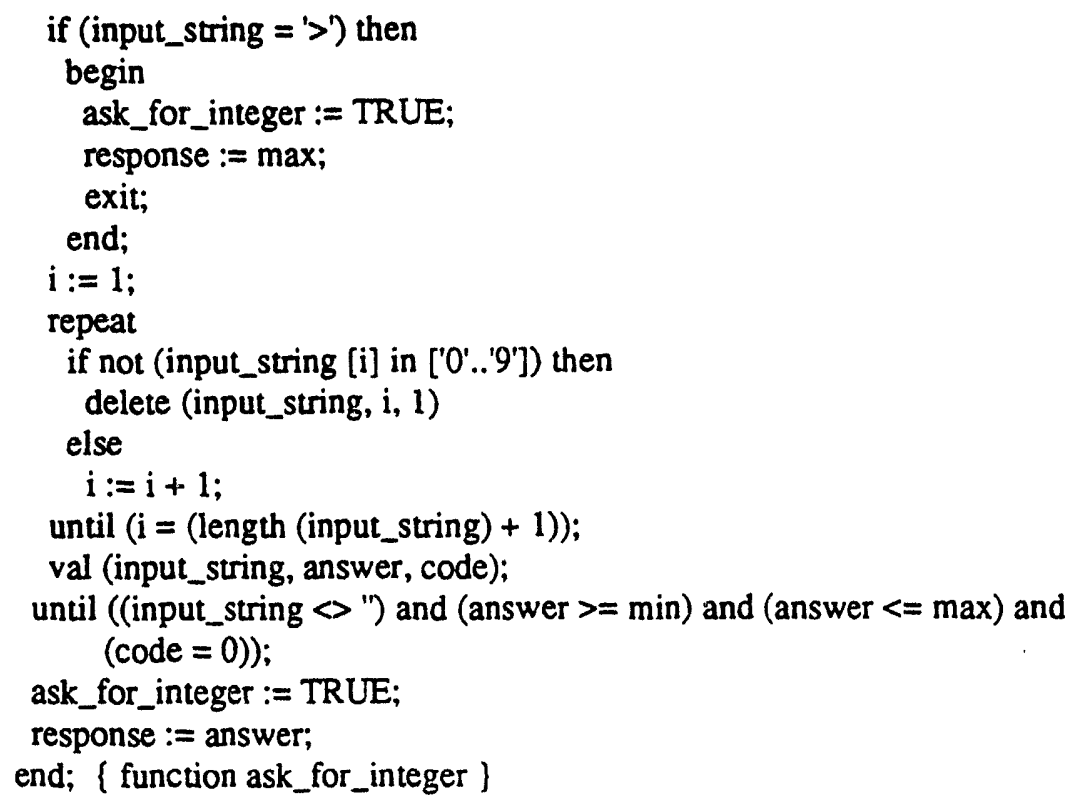




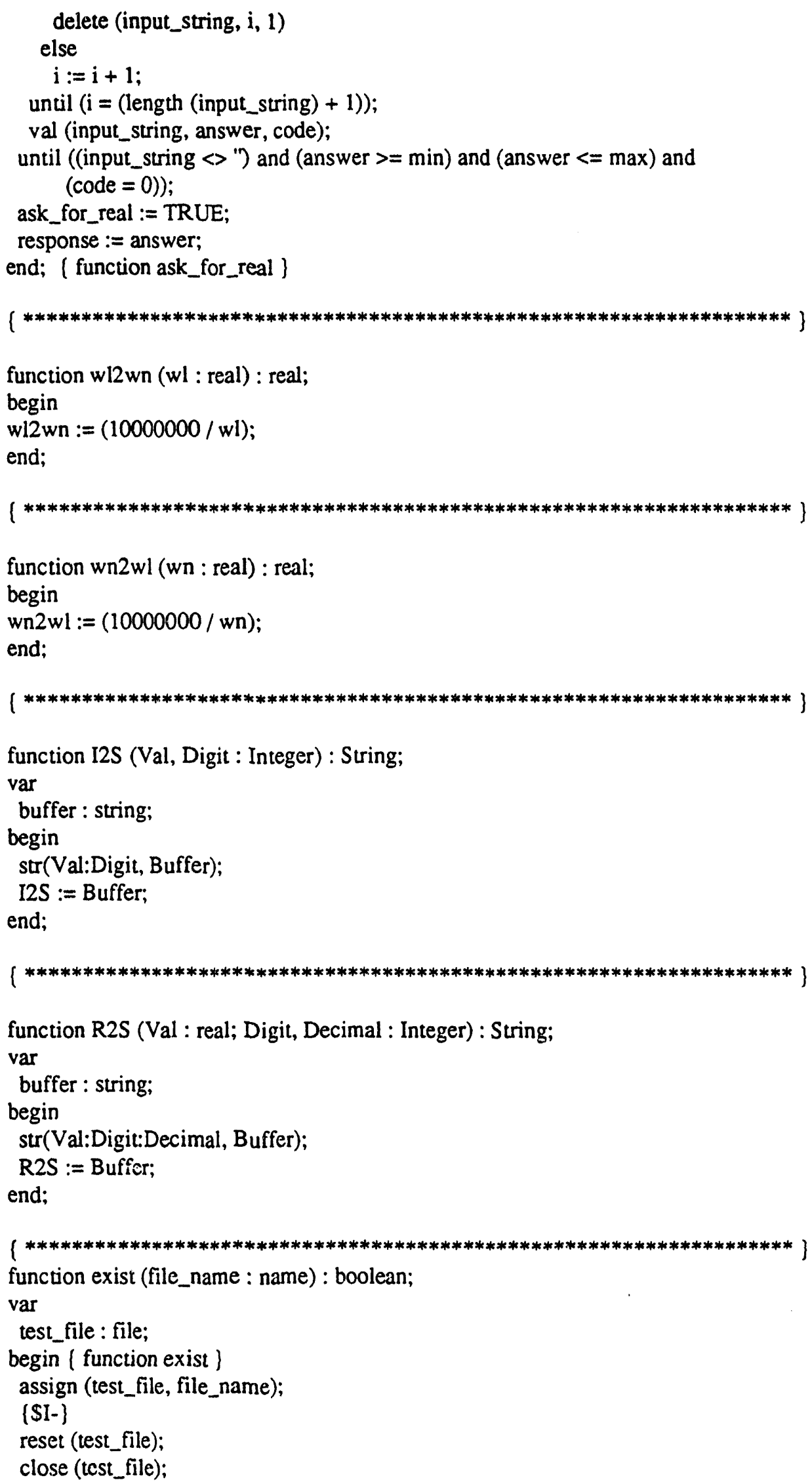


$\{\$ I+\}$

exist : $=($ IOresult $=0)$;

end; ( function exist \}

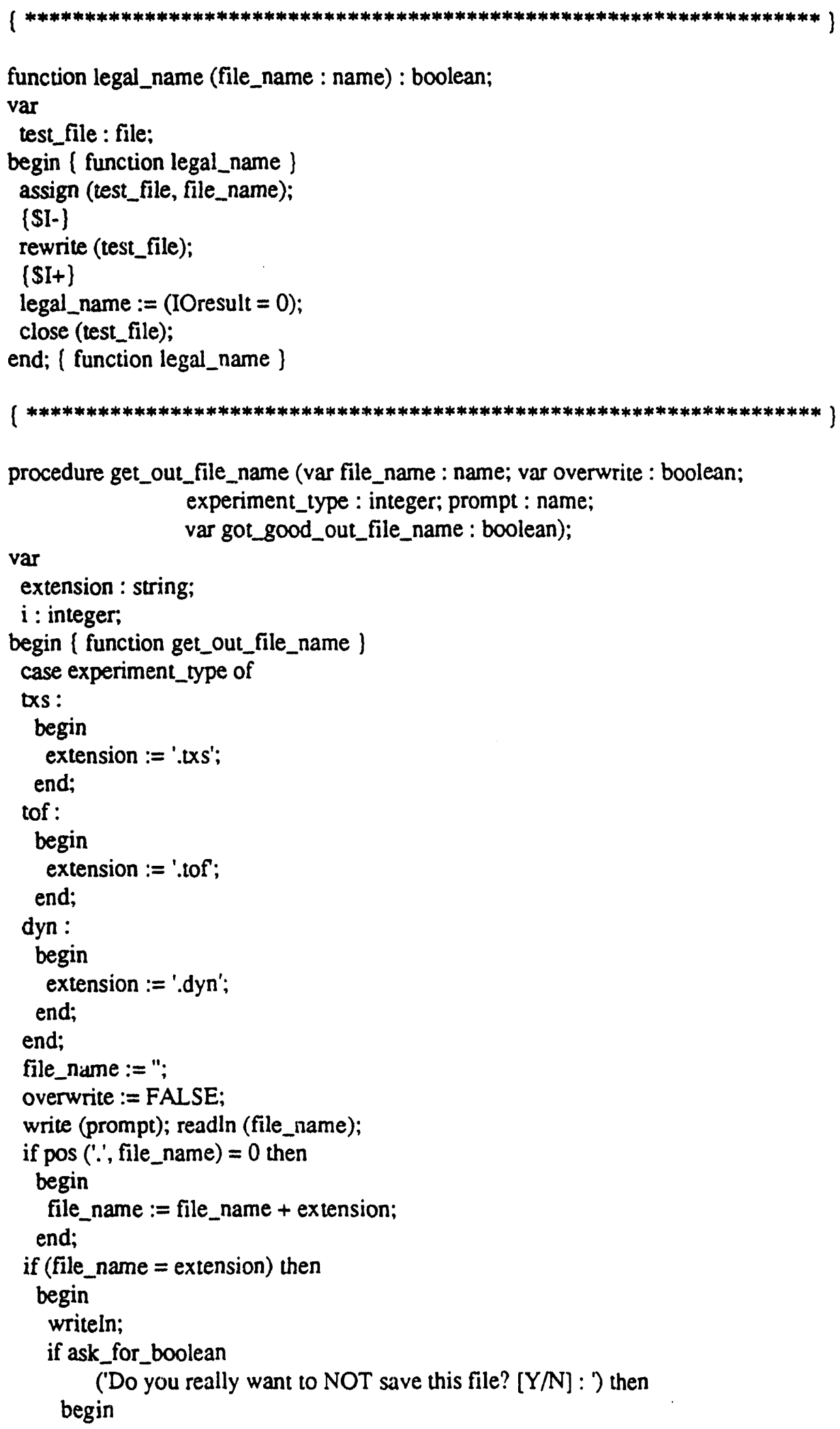




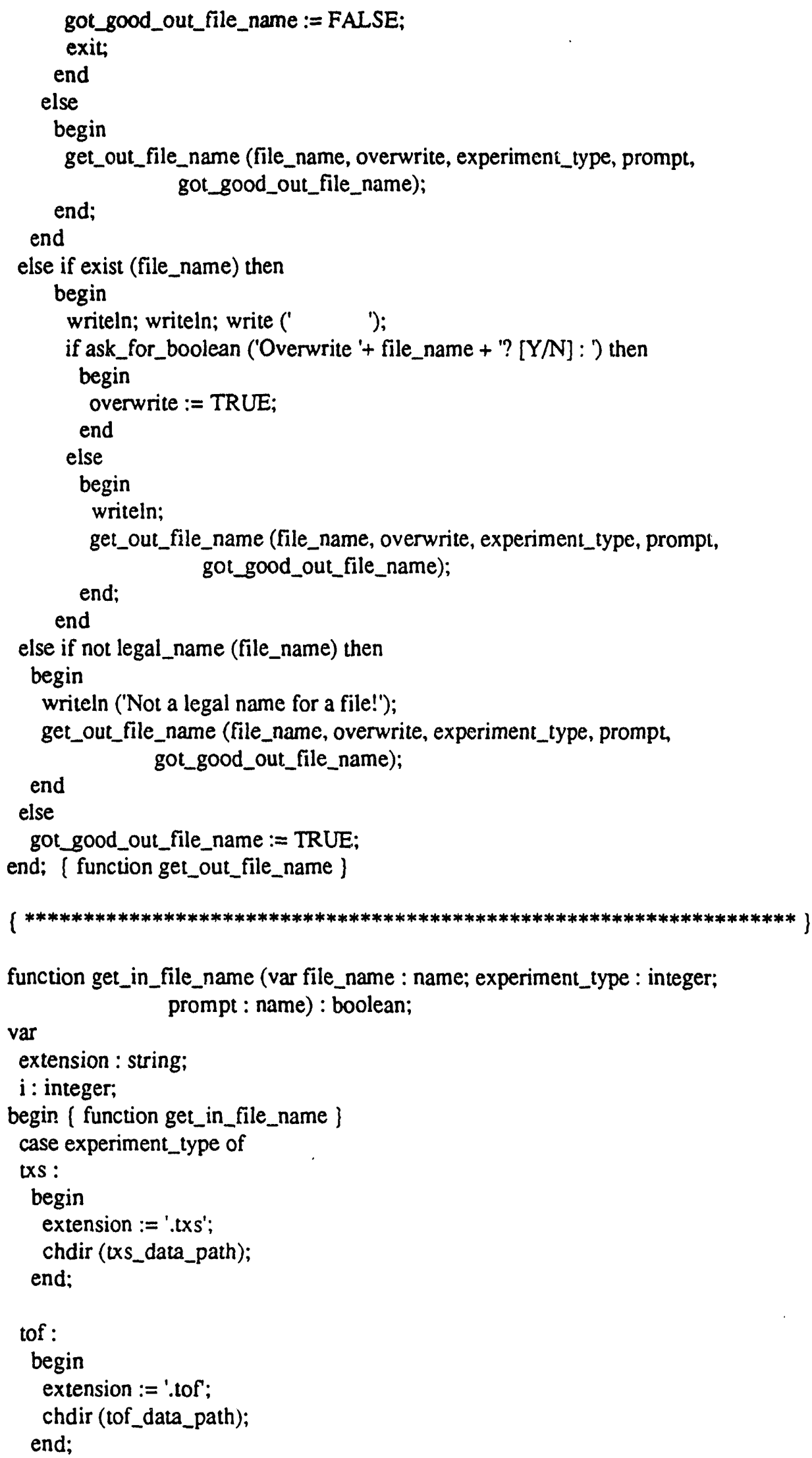




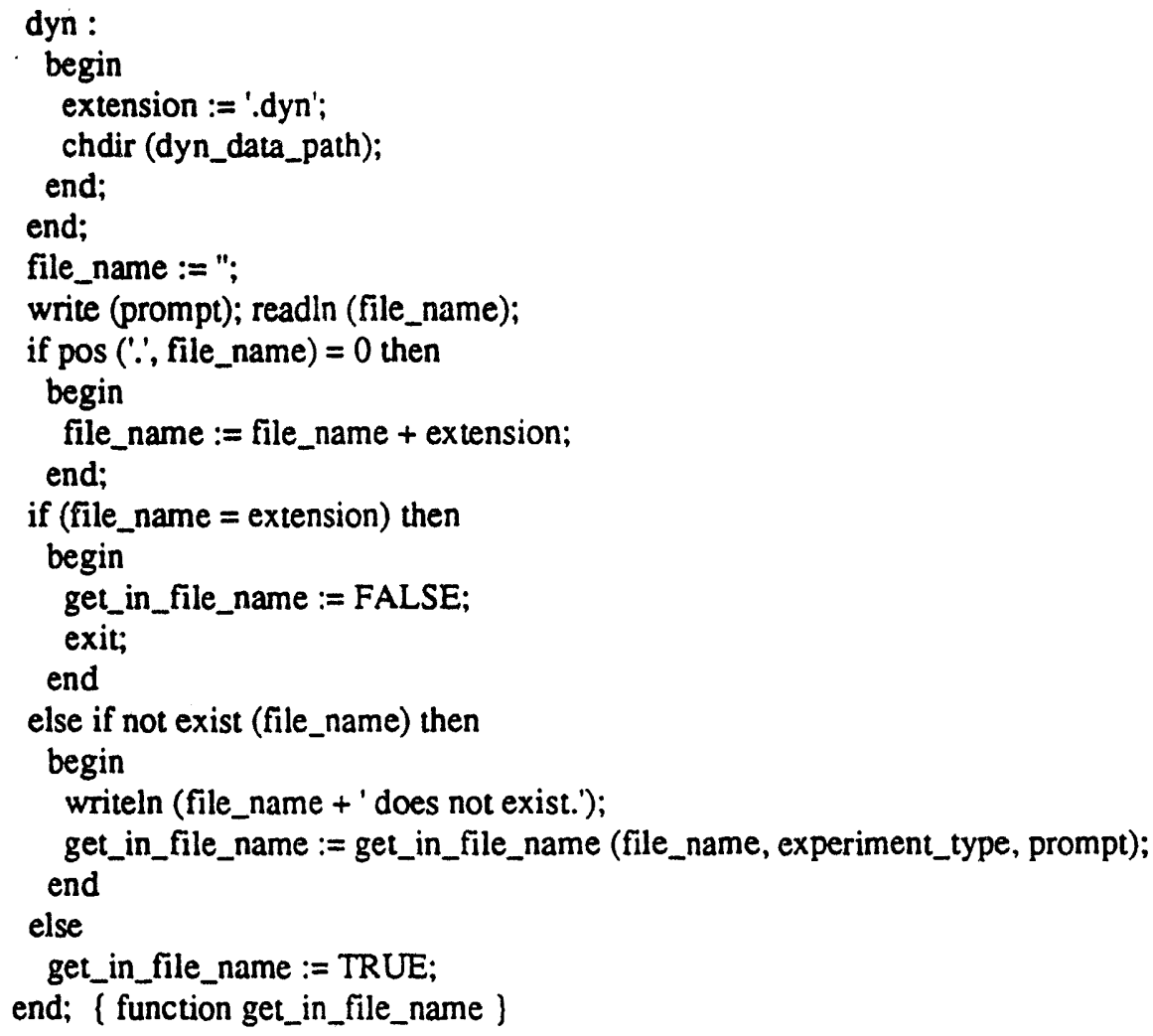




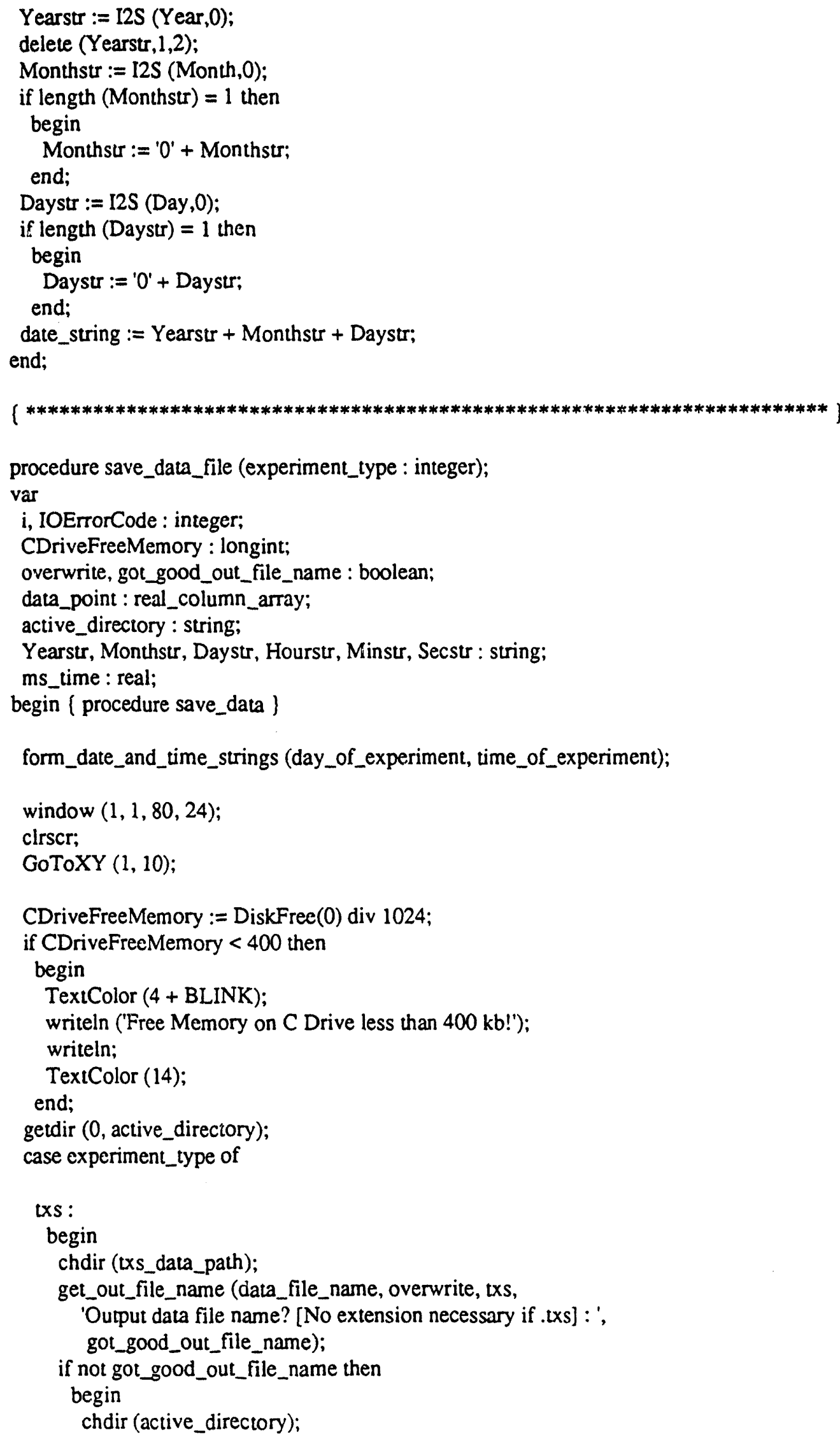




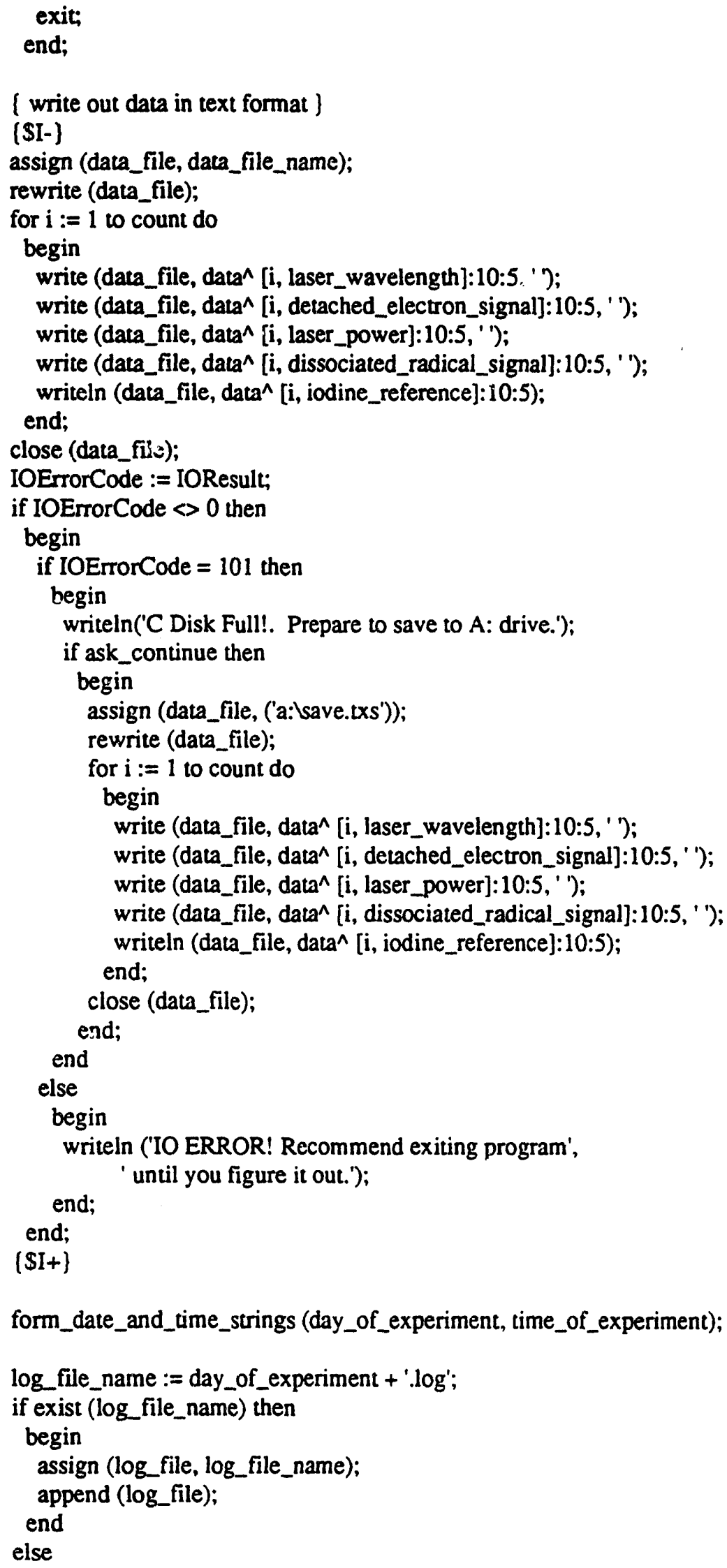




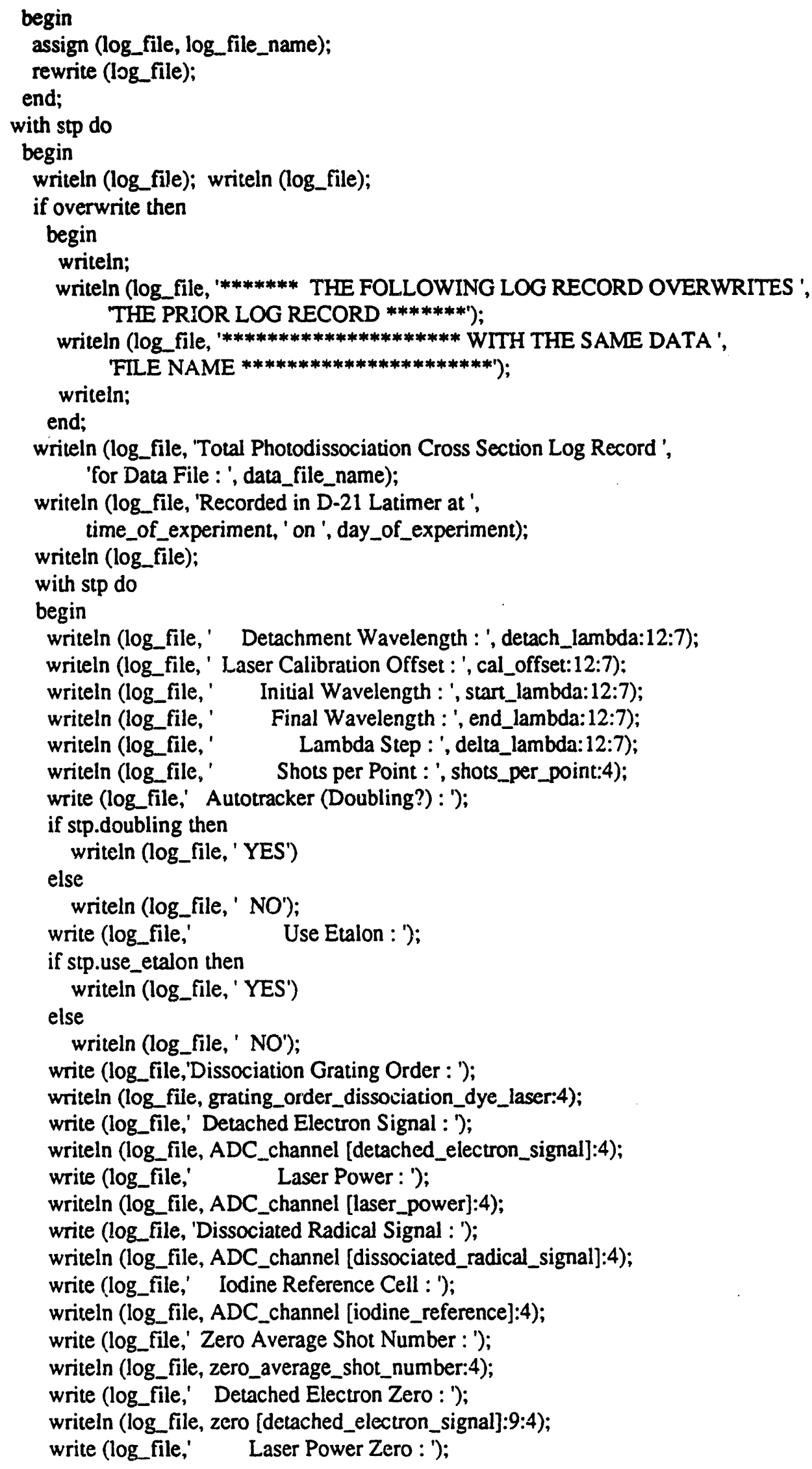




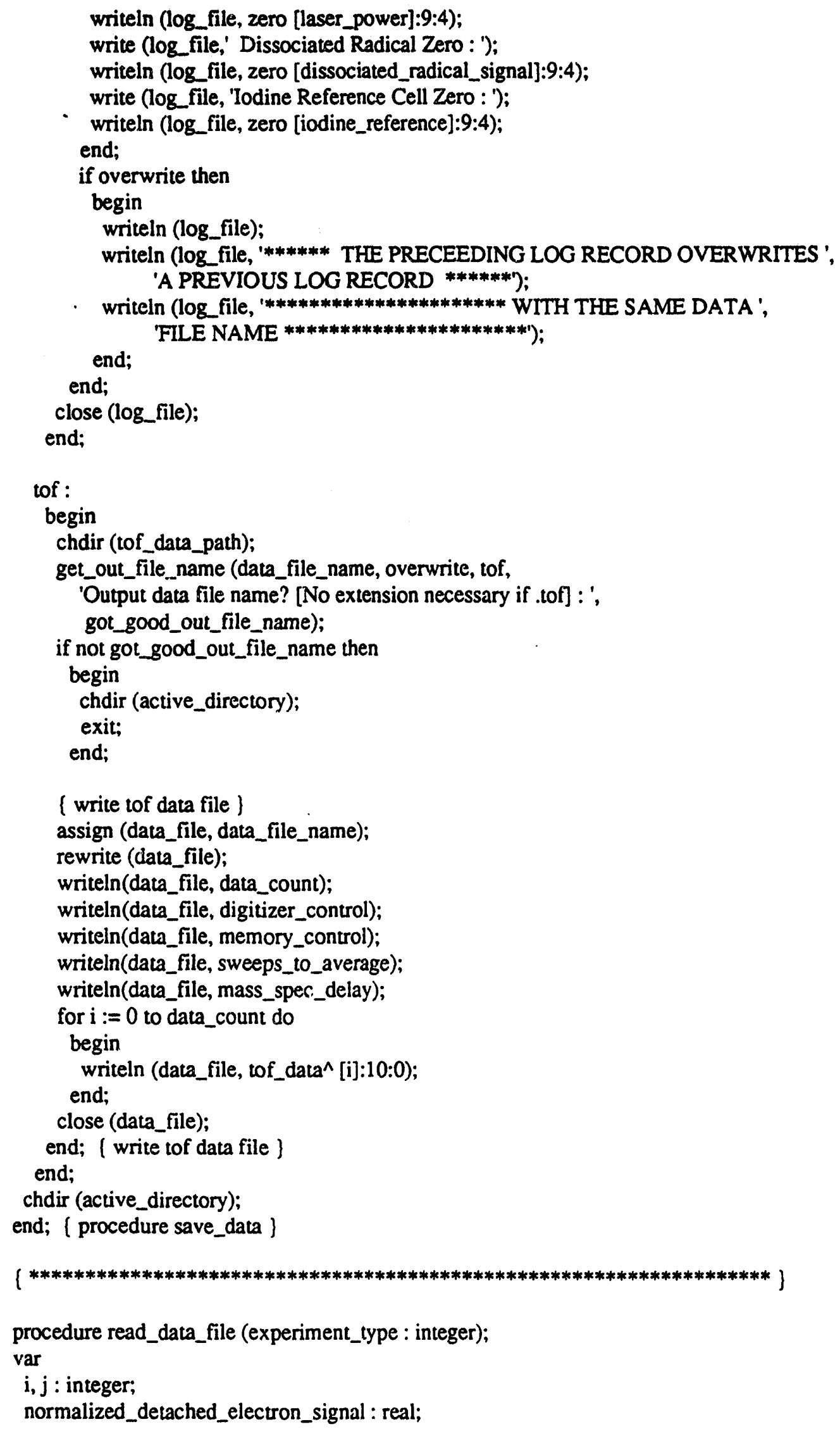




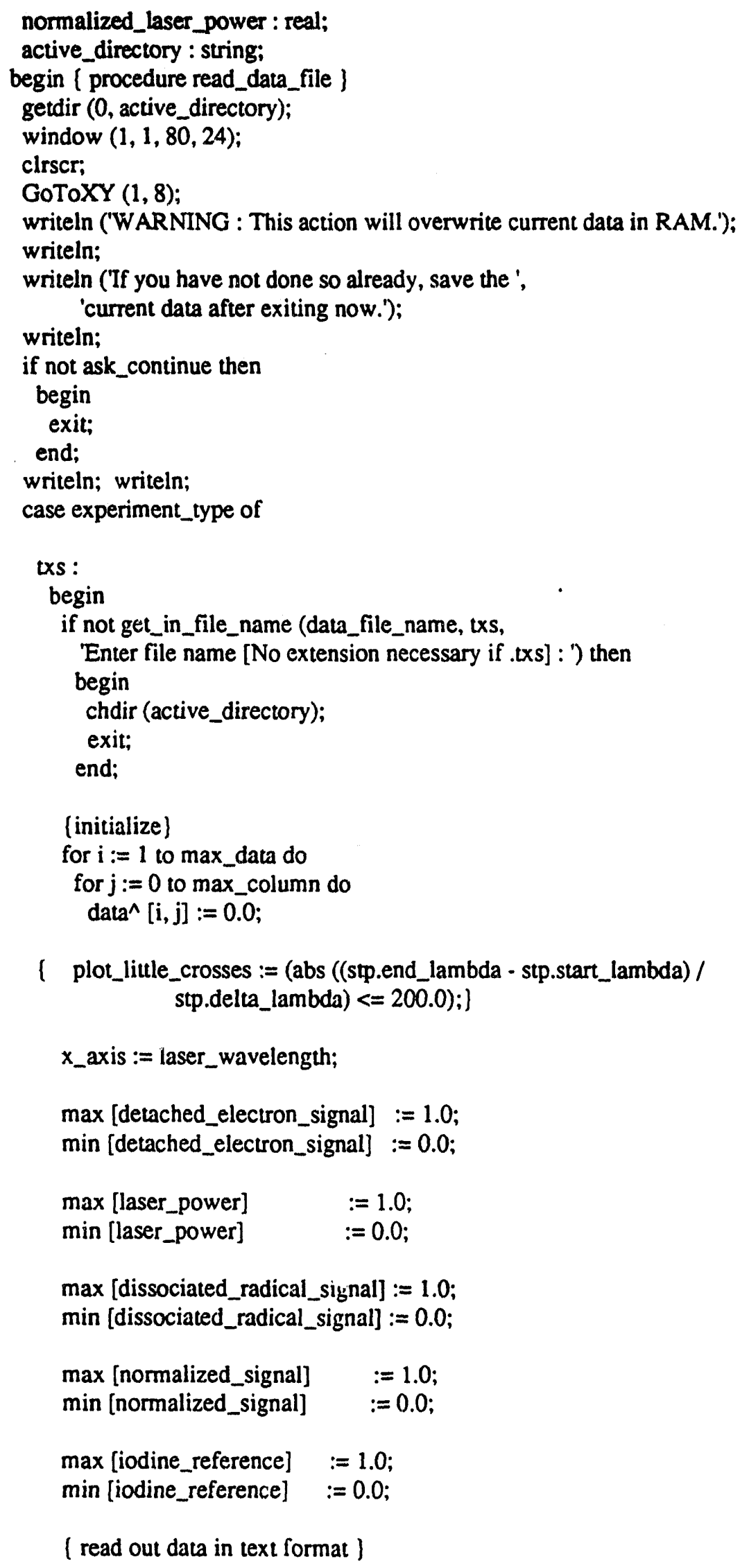




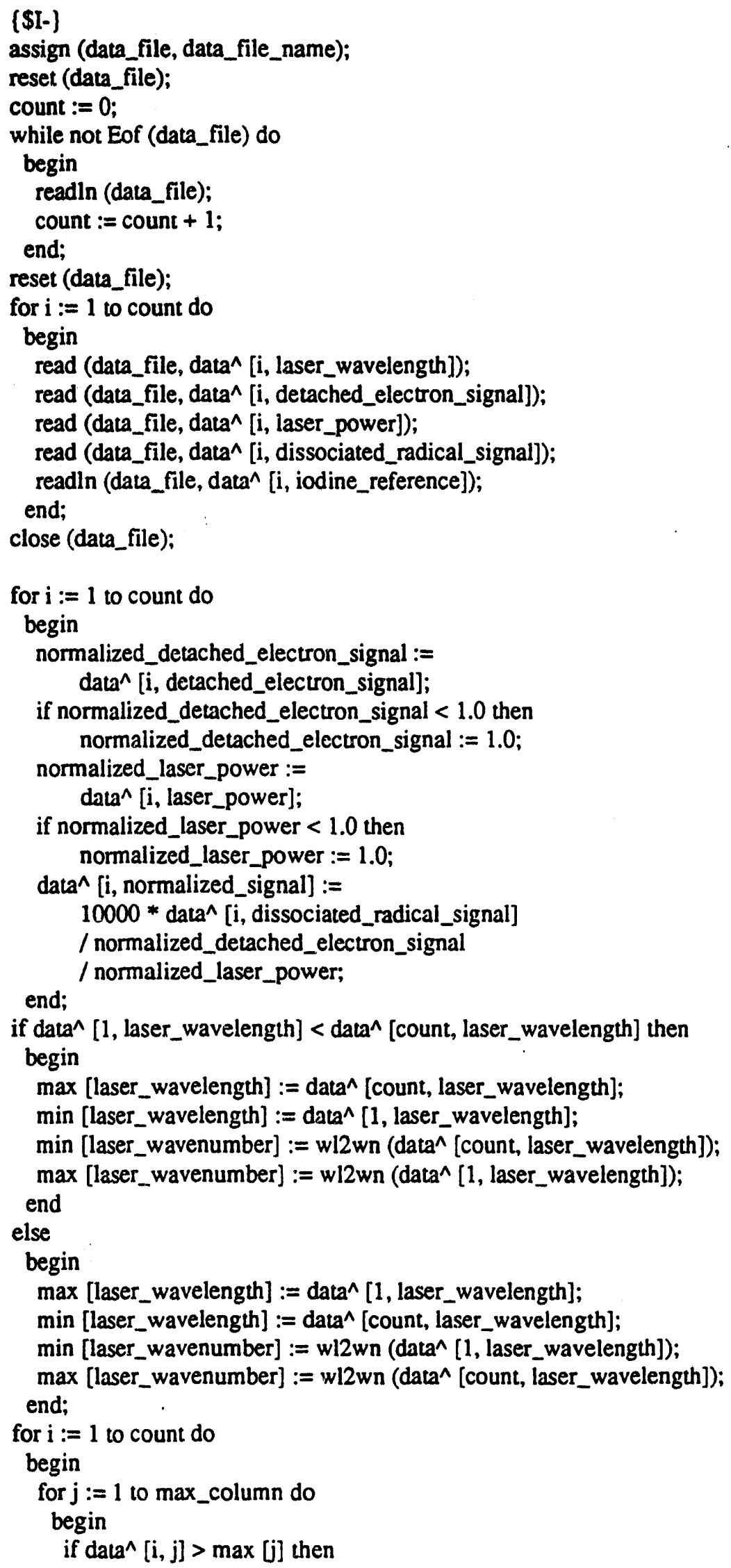




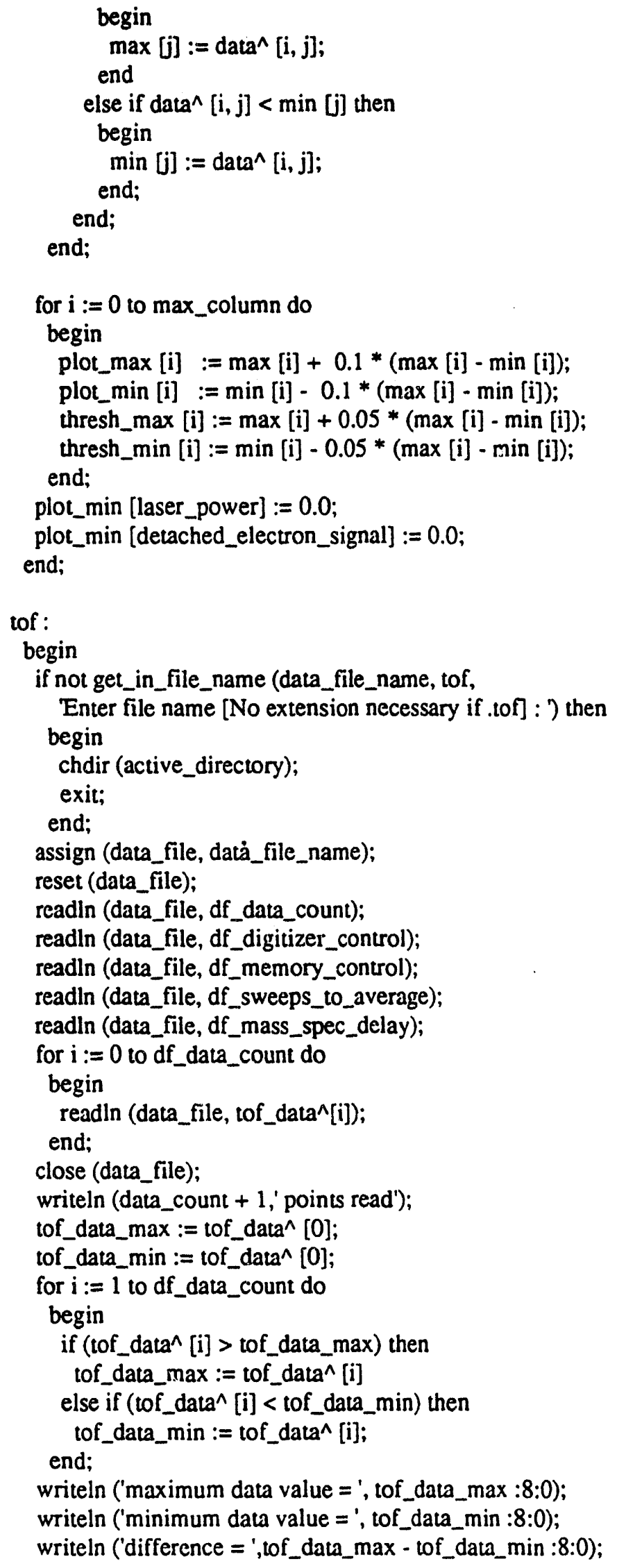




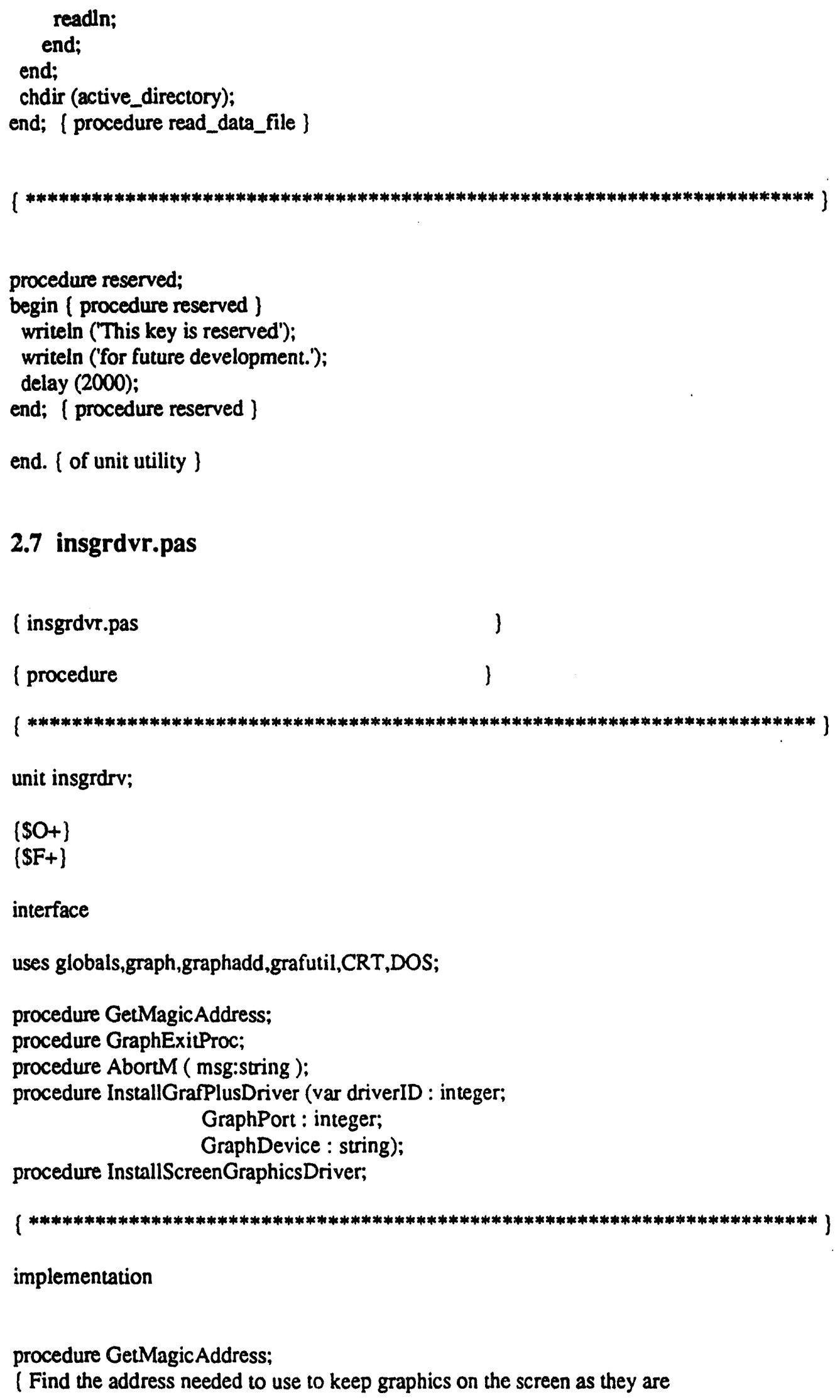


plotted out on the HP plotter or the PostScript printer

begin

wordpt := ptr(seg(closegraph),8+ofs(closegraph));

magic := ptr(DSeg,2+wordptr $)$;

end: (procedure GetMagicAddress )

procedure GraphExitProc;

begin

ExitProc := OldExitProc; \{ Restore exit procedure address \}

bCloseGraph; [Shut down the graphics system ]

end; ( GraphExitProc )

procedure AbortM ( msg:string );

begin

WriteLn(msg);

Halt(1);

end;

procedure InstallGrafPlusDriver (var driverID : integer; GraphPort : integer, GraphDevice : string);

begin (procedure InstallGrafPlusDriver ) (Install the graphics driver specified by GraphDevice ]

driverID := InstallUserDriver(GraphDevice,nil) + 5;

ErrorCode := GraphResult;

if ErrorCode $<>0$ then

begin

AbortM (GraphErrorMsg(ErrorCode));

end;

end; ( procedure InstallGraphPlusDriver \}

procedure InstallScreenGraphicsDriver;

begin (procedure InstallScreenGraphicsDriver )

[ Initialize graphics and report any errors that may occur )

(when using Crt and graphics, turn off Crt's memory-mapped writes ]

[DirectVideo := False; ]

OldExitProc := ExitProc;

ExitProc :=@GraphExitProc;

[save previous exit proc \}

GraphDriver := Detect;

[ insert custom exit proc in chain ]

repeat

bInitGraph(GraphDriver, GraphMode, PathToGraphics, nil);

ErrorCode := GraphResult; [ preserve error return )

if ErrorCode $<>$ grOK then (error?

begin

Writeln('Graphics error: ', GraphErrorMsg(ErrorCode));

if ErrorCode $=$ grFileNotFound then ( Can't find driver file \}

begin

Write ('Enter full path to BGI driver or type $<$ Ctrl-Break> to quit : ');

Readin(PathToGraphics);

Writeln;

end 


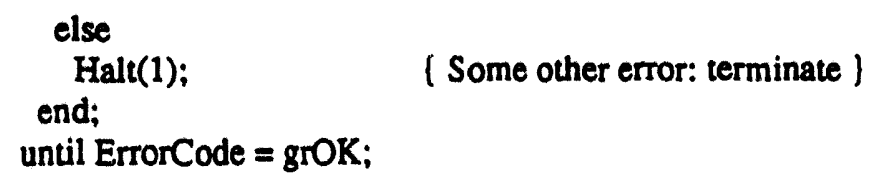

Plotinit;

RestoreCRTMode;

end; ( procedure InstallScreenGraphicsDriver )

end. ( unit insgrdvr )

\section{8 grafutil.pas}

( This unit uses a few routines from Turbo Graphics Tools 1.1 in order to ensure compatibility between our current programs and the new Turbo 5.5 Graph unit. Where ever possible, however, the TP unit has been used to minimize duplication. \}

unit grafutil;

$\{\mathrm{SO}+\}$

[SF+]

interface

uses graph,graphadd,DOS,CRT;

const

MaxSymbol $=9 ; \quad$ \{0..any positive integer allowable

ULXScreen $=0 ; \quad$ (screen coordinate system defined here;)

ULYScreen $=0 ; \quad$ (may be changed by the user)

LRXScreen $=1000$;

LRYScreen $=1000$;

type

SymbolMask $=$ Array $[0.6]$ of Byte;

var

xdpi, ydpi : word; $\quad$ (Dots per inch of current graphics output]

DrawColor : Integer;

Symbol : Array[0..MaxSymbol] of SymbolMask; [Symbol patterns]

ErrorCode : Integer;

XabsGbl,YabsGbl : integer; $\quad$ [Current graphics cursor location]

ULXABS,ULYABS,

LRXABS,LRYABS : integer; [Screen comers in pixels]

$\operatorname{Max} X, \operatorname{Max} Y$ : integer; [Saves the maximum graphics coordinates]

ULXWIN,ULYWIN,

LRXWIN,LRYWIN : integer; [Current window comers in pixels]

ULXSCR,ULYSCR, 


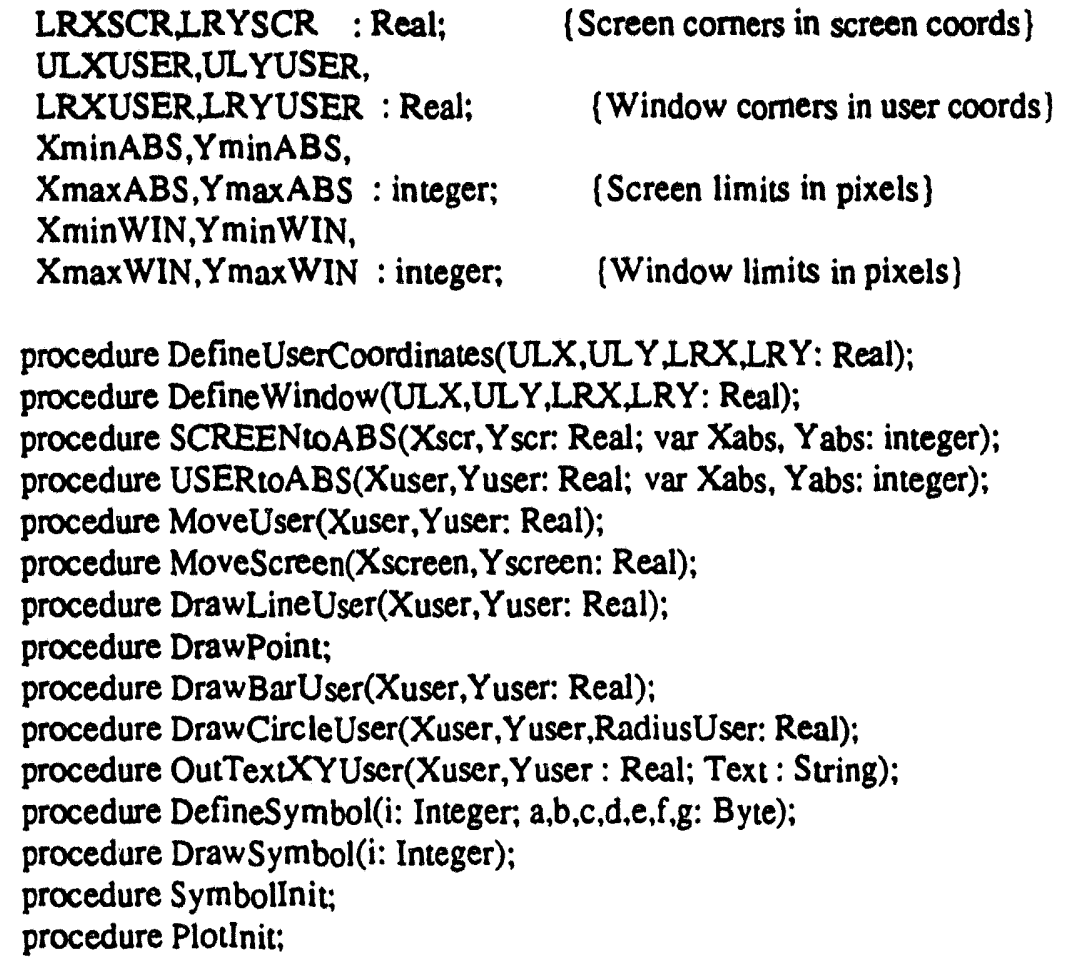

procedure DefineWindow(ULX,ULY,LRX,LRY: Real);

(Input parameters are given in screen coordinates; absolute coordinates of new window are calculated and global variables for clipping are set)

var

XScaleFactor, YScaleFactor : Real;

begin (of procedure DefineWindow)

XScalcFactor := (LRXABS-ULXABS)/(LRXSCR-ULXSCR); (abs coords of window)

YScaleFactor: $=($ LRYABS-UL YABS)/(LRYSCR-ULYSCR);

ULXWIN := Round((ULX - ULXSCR)*XScaleFactor + ULXABS);

ULYWIN := Round((ULY - ULYSCR)*YScalcFactor + ULYABS); 


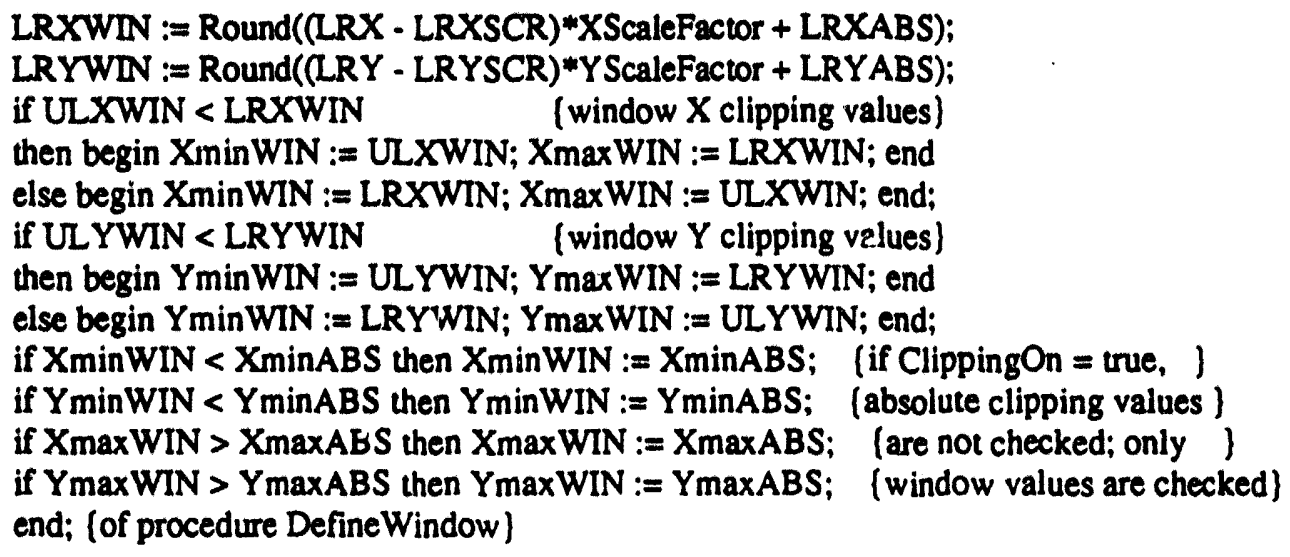

procedure SCREENtoABS(Xscr,Yscr: Real; var Xabs, Yabs: integer);

[Converts screen coordinates into absolute coordinates]

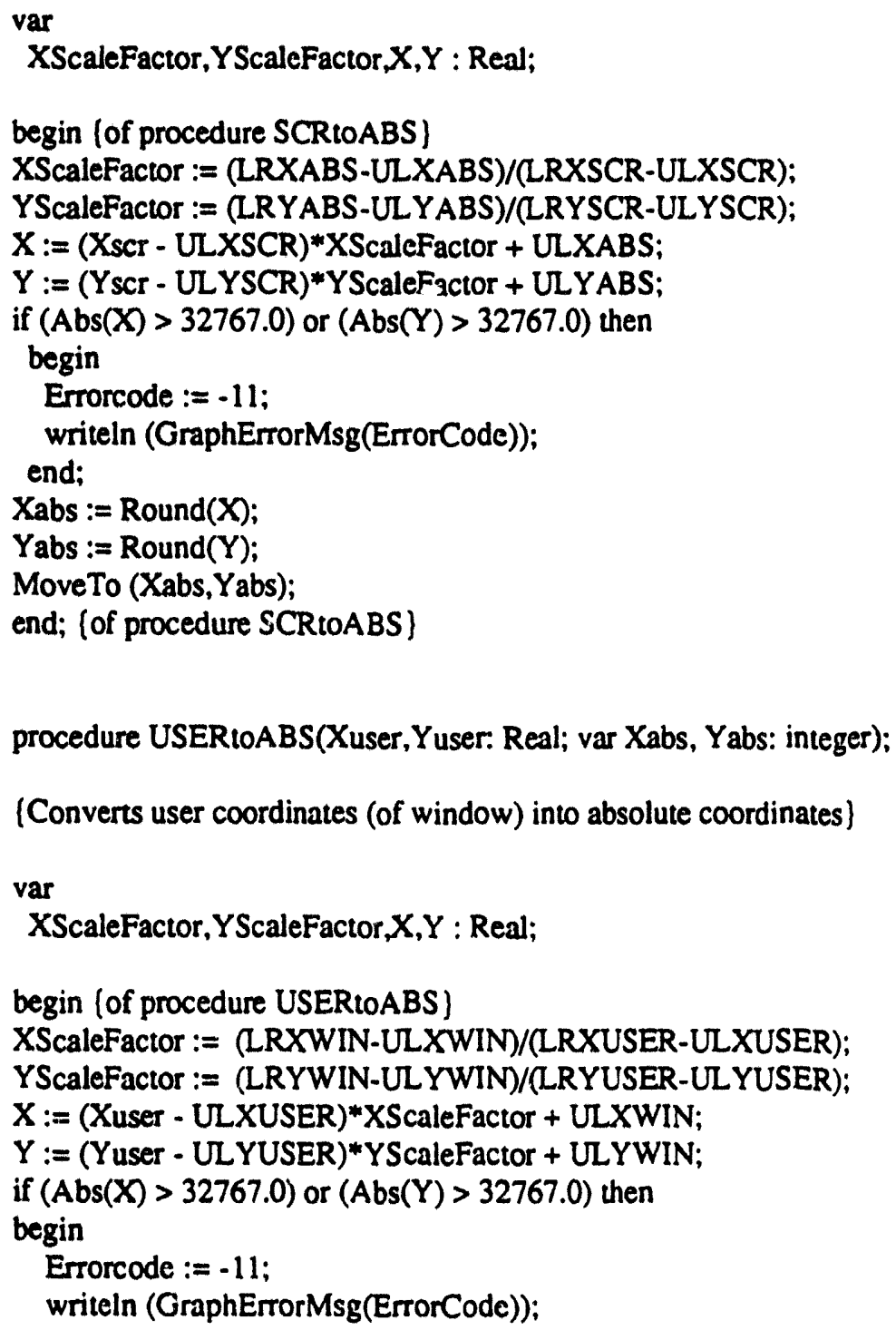

procedure USERtoABS(Xuser,Yuser: Real; var Xabs, Yabs: integer);

[Converts user coordinates (of window) into absolute coordinates] 
end;

Xabs := Round $(X)$;

Yabs := Round $(Y)$;

MoveTo (Xabs,Yabs);

end; (of procedure USERtoABS)

procedure MoveUser(Xuser,Yuser: Real);

[Moves invisible graphics cursor to (Xuser,Yuser) without drawing line]

begin (of procedure Move)

USER toABS(Xuser,Yuser,XabsGbl,YabsGbl);

end; (of procedure Move)

procedure MoveScreen(Xscreen, Yscreen: Real);

(Moves invisible graphics cursor to (Xscreen,Yscreen) without drawing line)

begin \{of procedure MoveScr)

SCREENioABS (Xscreen, Yscreen,XabsGbl,YabsGbl);

end; (of procedure MoveScr)

procedure DrawLineUser(Xuser,Yuser: Real);

(Draws a line from current invisible graphics cursor to (Xuser,Yuser); leaves invisible graphics cursor at (Xuser,Yuser) after drawing line)

var

X2abs, Y2abs : integer;

begin (of procedure DrawLineUser)

USERtoABS(Xuser,Yuser,X2abs,Y2abs);

Line(XabsGbl,YabsGbl,X2abs, Y2abs);

$\mathrm{XabsGbl}:=\mathrm{X} 2 \mathrm{abs}$;

YabsGbl := Y2abs;

end; \{of procedure DrawLineUser\}

procedure DrawPoint;

[Draws a point at current invisible graphics cursor position]

begin (of procedure DrawPoint)

PutPixel(XabsGbl,YabsGbl,DrawColor);

end; (of procedure DrawPoint)

procedure DrawBarUser(Xuser, Yuser: Real);

(Draws a bar with current invisible graphics cursor as one corner and the comer diagonal to it given by (Xuser,Yuser); leaves invisible 
graphics cursor at (Xuser, Yuser) after drawing line\}

$\operatorname{var}$

X2abs,Y2abs : integer;

begin (of procedure DrawBarUser)

USERtoABS (Xuser,Y Yser,X2abs,Y2abs);

Bar(XabsGbl,YabsGbl,X2abs, Y2abs);

XabsGbl := X2abs;

YabsGbl := Y2abs;

end; ( procedure DrawBarUser )

procedure DrawCircleUser(Xuser,Yuser,RadiusUser: Real);

(Draws a circle with center given by first two parameters and radius given by the third

var

Xabs,Yabs,origin, intersection,RadiusAbs : integer;

RadiusXuser : real;

pixels : string;

begin (of procedure DrawCircleUser)

USERtoABS(Xuser,Yuser,Xabs,Yabs);

origin := Xabs;

RadiusXuser := Xuser + RadiusUser;

USERtoABS(RadiusXuser,Yuser,Xabs, Yabs);

intersection := Xabs;

RadiusAbs := abs(intersection - origin);

(str (RadiusAbs:1, pixels);

OutTextXY (MaxX div 2, (MaxY div 2+50), pixels);

MoveUser $(8000,8000)$;

Bar (XabsGbl,YabsGbl,XabsGbl + 3,YabsGbl + 3); \}

USERtoABS(Xuser,Yuser,Xabs, Yabs);

Circle(Xabs, Yabs,RadiusAbs);

end; ( procedure DrawCircleUser ]

procedure OutTextXYUser(Xuser,Yuser : Real; Text : String);

var

Xabs,Yabs : integer;

begin

USERtoABS(Xuser,Yuser,Xabs, Yabs);

OutTex XYY(Xabs, Yabs,Text);

end;

procedure DrawSymbol(i: Integer);

[Draws $7 \times 7$ symbol[i] centered at invisible graphics cursor position] 


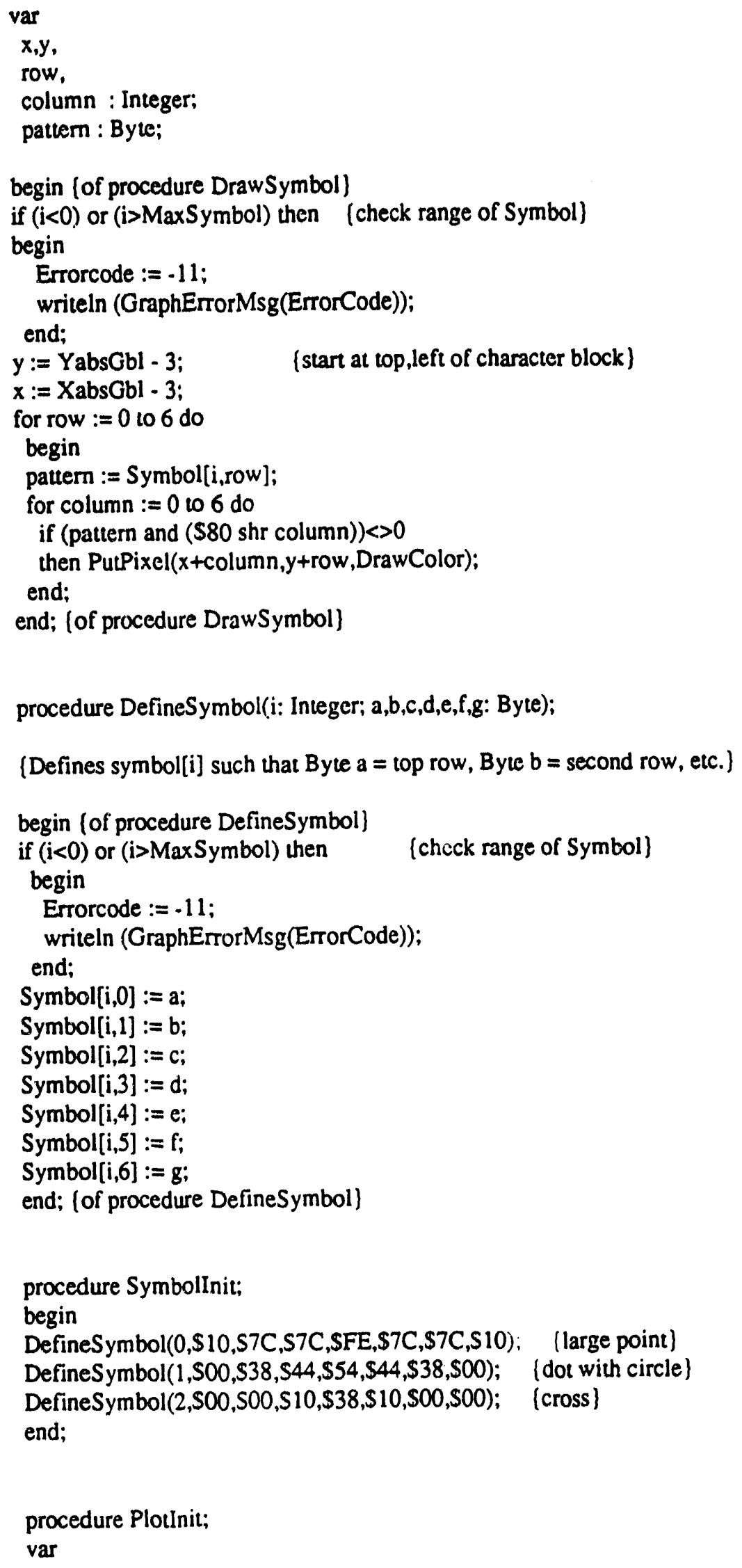




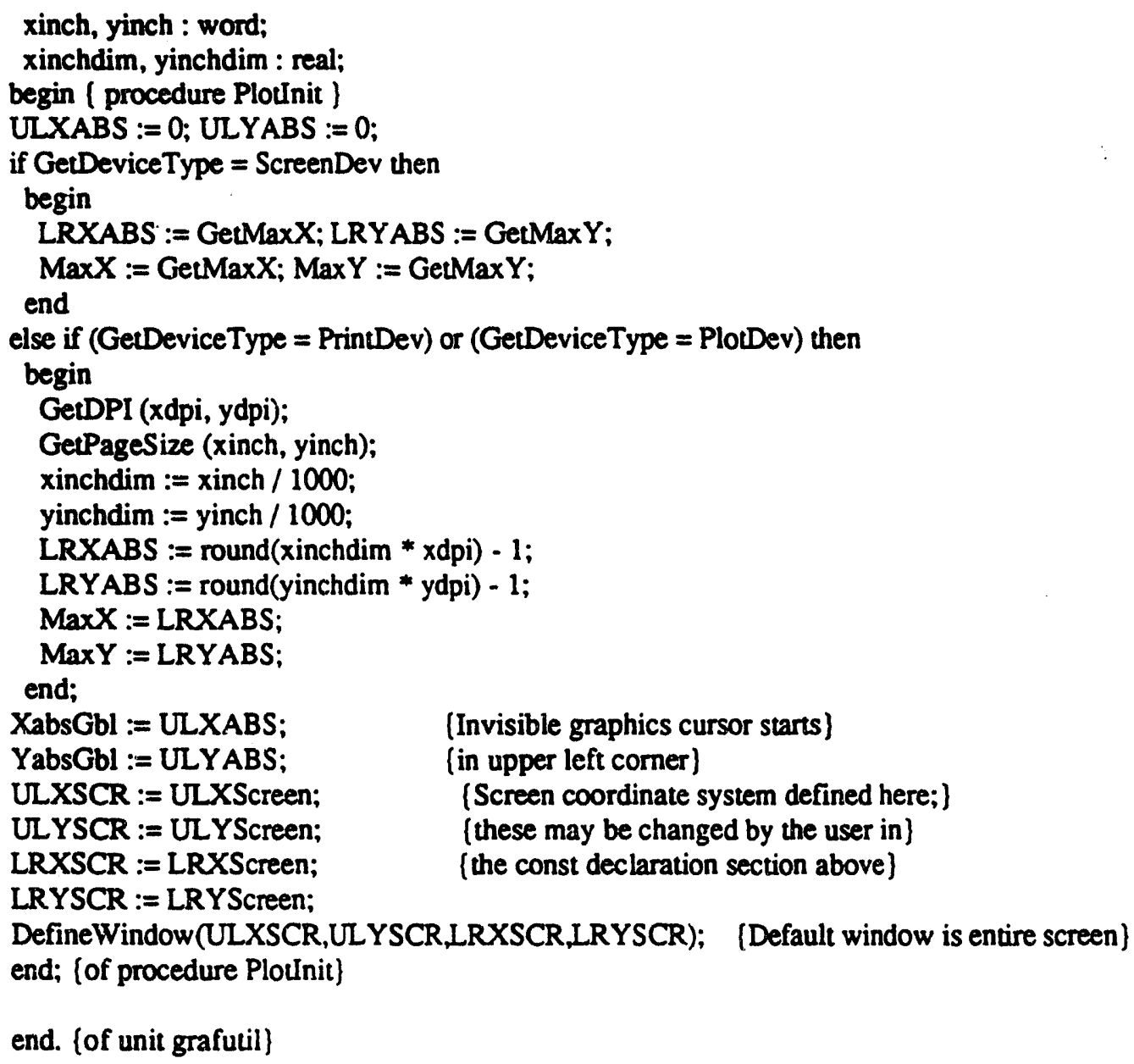

\section{9 txsplot.pas}

( txsplot.pas ) ]

[ contents : ]

\{i. useful (repetitive) \}

$\left\{\begin{array}{ll}\text { \{ procedure uxs_draw_ticks (tick_axis : integer, } \\ \text { constant_limit : real; } \\ \text { code : integer; } \\ \text { label_ticks : boolean) }\end{array}\right\}$

[ii. while experiment is running ]

( procedure ixs_label_axes (axis : integer) ]

( procedure lxs_draw_axes )

( procedure txs_x_y_ticks (y_data : integer) ] 
\{ procedure txs_replot (y_data, point_count : integer; visible : boolean) \}

( procedure txs_plot_point (count, y_data : integer) \}

\{ procedure set_done_flag ]

[iii. after experiment \}

( procedure p_txs_draw_axes (x_axis, y_axis : integer) \}

\{ procedure p_txs_plot_points (x_axis, y_axis : integer) \}

(procedure j_hard_copy (x_axis, y_axis : integer) \}

\{ function set_txs_axis (var axis : integer) : boolean \}

( procedure dump_experiment_screen )

\{ procedure show_txs_plot_parameters \}

[ procedure plot_menu ]

$\{* * * * * * * * * * * * * * * * * * * * * * * * * * * * * * * * * * * * * * * * * * * * * * * * * * * * * * * * * * * * * * * * * * * * * * * * *\}$

unit txsplot;

$\{\mathrm{SO}+\}$

$\{\mathrm{SF}+\}$

interface

uses globals, utility,insgrdrv,plotutil,graph,graphadd,grafutil,CRT,DOS;

const

Font $:$ Integer $=$ DefaultFont;

procedure txs_draw_ticks (tick_axis : integer; constant_limit : real; code : integer; label_ticks : boolean);

procedure txs_label_axes (axis : integer);

procedure txs_draw_axes;

procedure txs_x_y_ticks (y_data : integer);

procedure txs_replot (y_data, point_count : integer; visible : boolean);

procedure txs_plot_point (count, y_data : integer);

procedure set_done_flag;

procedure p_txs_draw_axes (x_axis, $y$ _axis : integer);

procedure $p_{-} t x x_{-}$plot_points (x_axis, $y_{-}$axis : integer);

function set_txs_axis (var axis : integer) : boolean;

procedure dump_txs_experiment_screen;

procedure show_txs_plot_parameters;

procedure txs_plotting_parameters; 


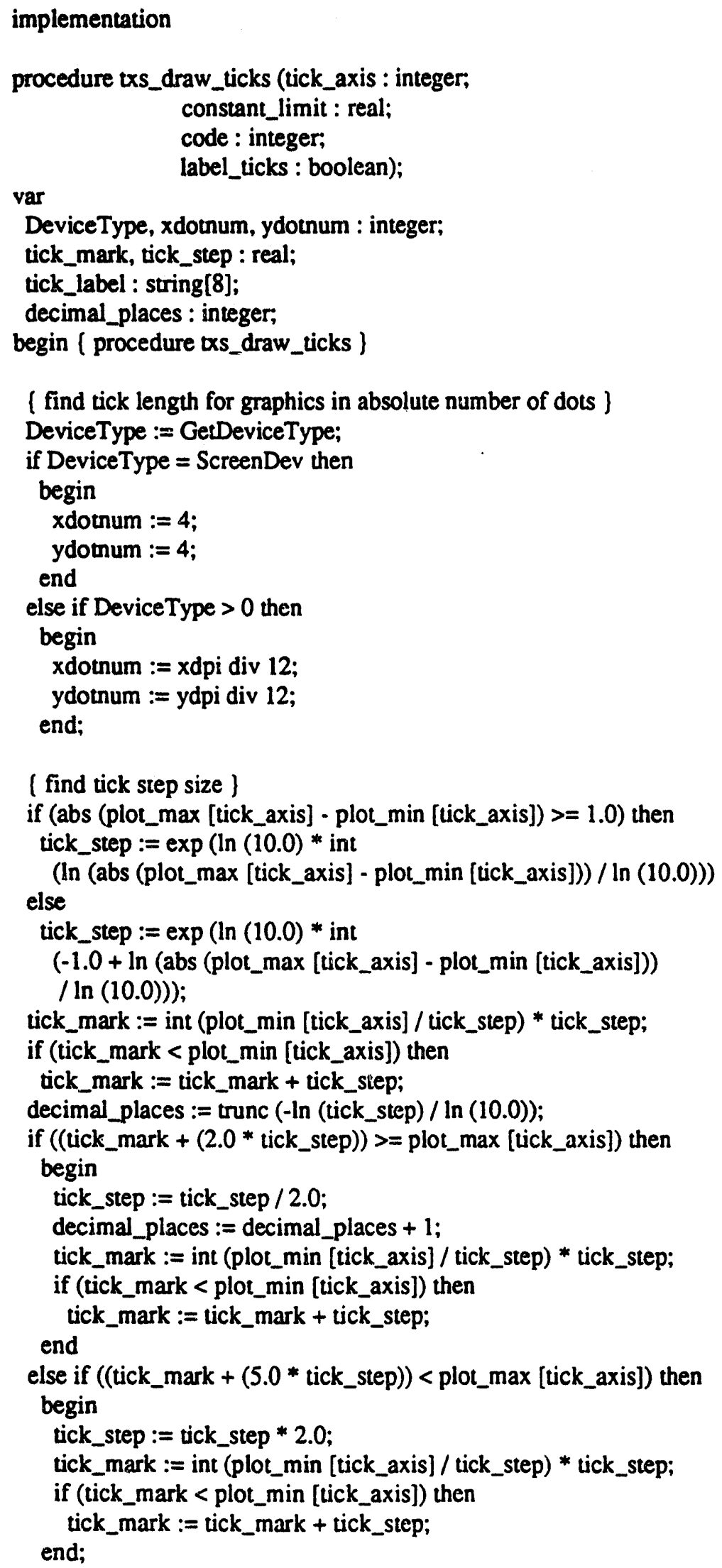




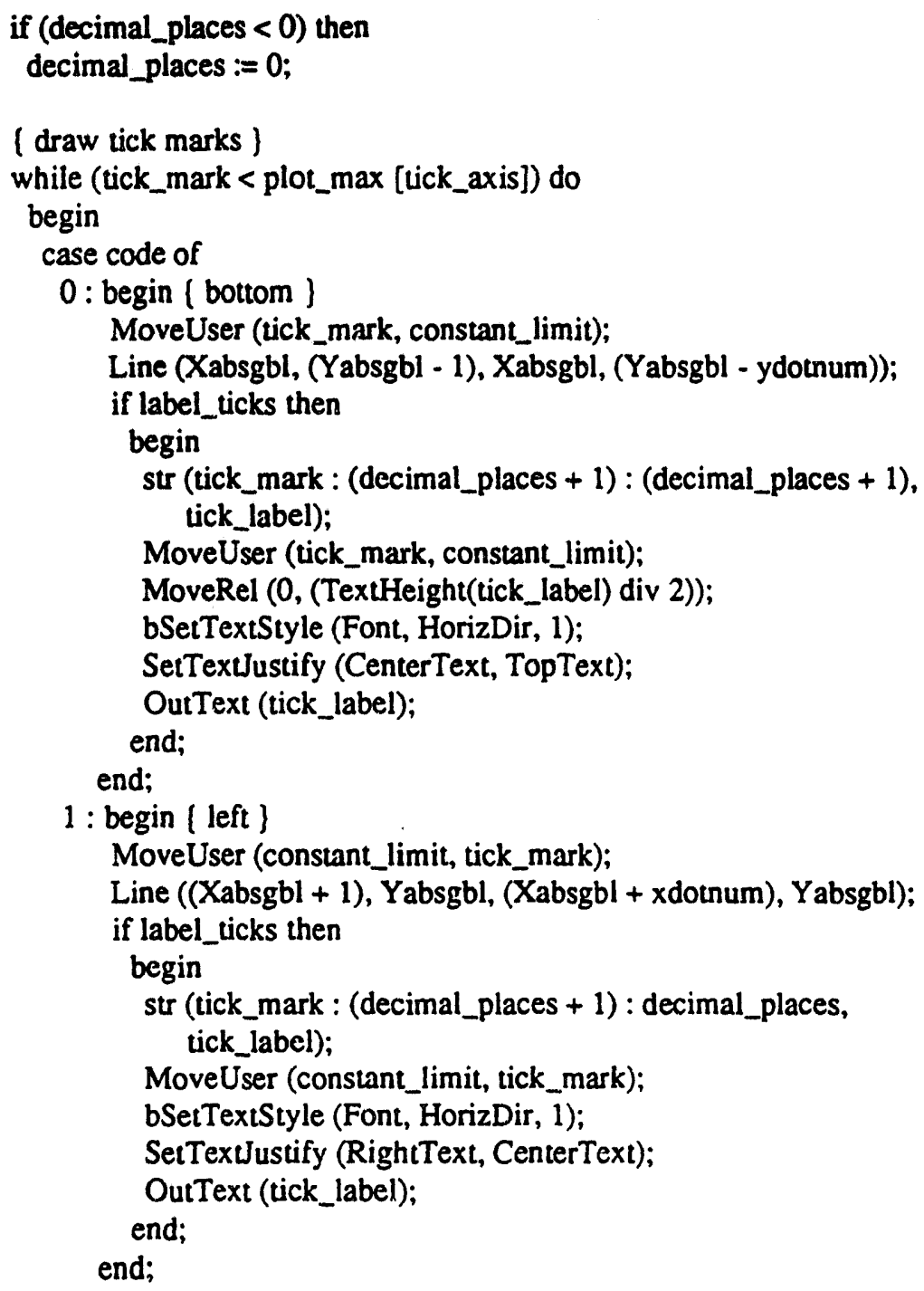




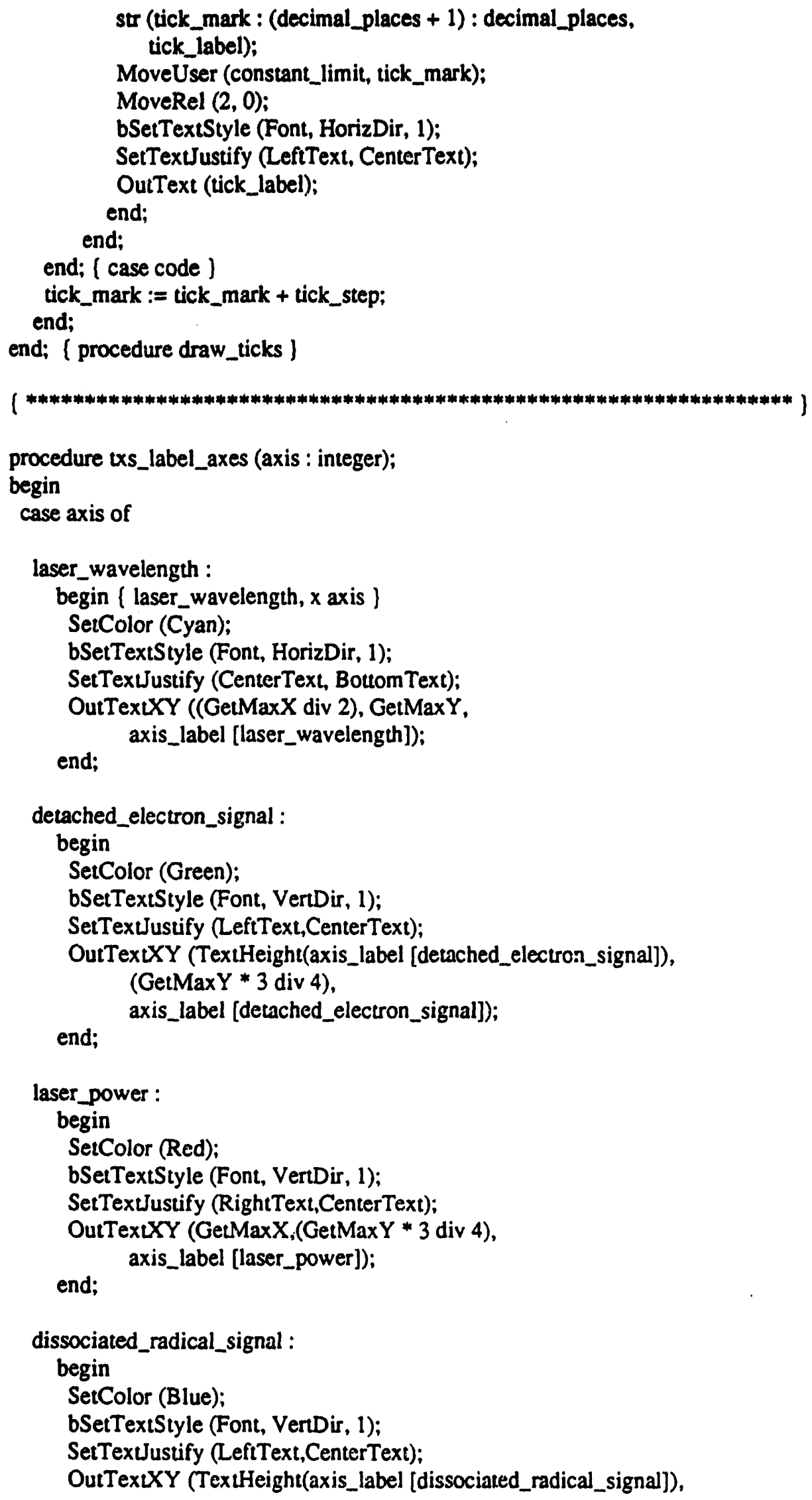


(GetMaxY div 4),

end;

axis_label [dissociated_radical_signal]);

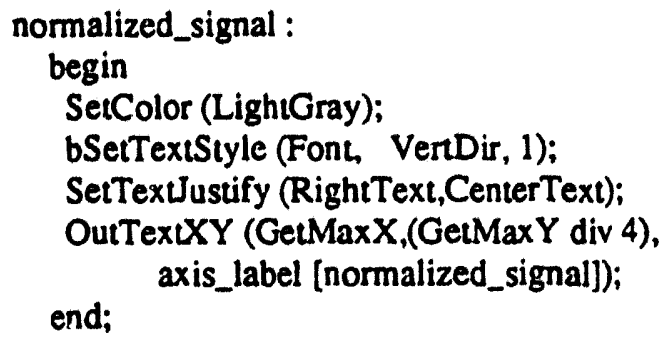

end; \{ case axis \}

end; (procedure txs_label_axes )

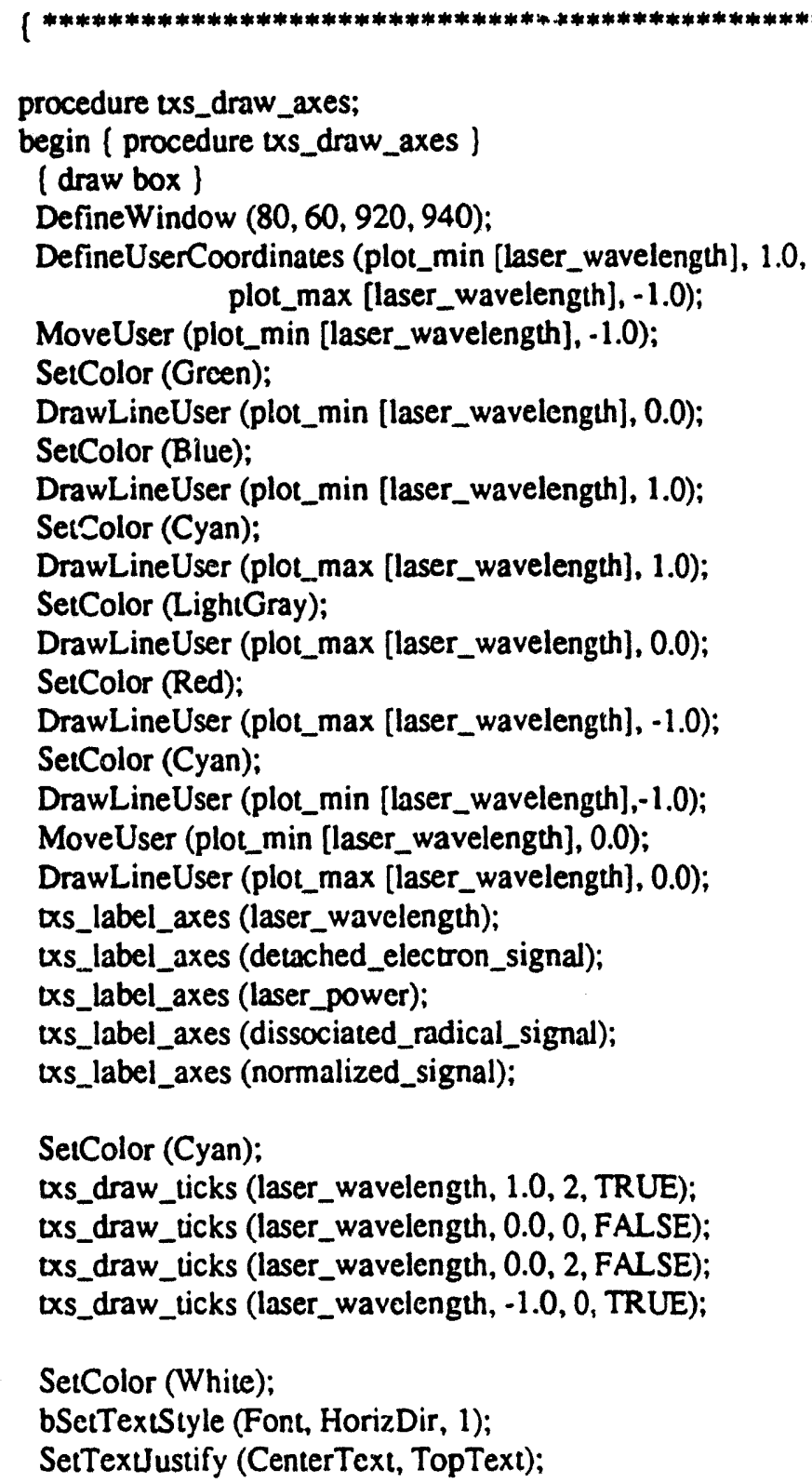


OutTexLXY (GetMaxX div 2, 0 ,

Total Photodissociation Cross Section Experiment');

end: (procedure txs_draw_axes )

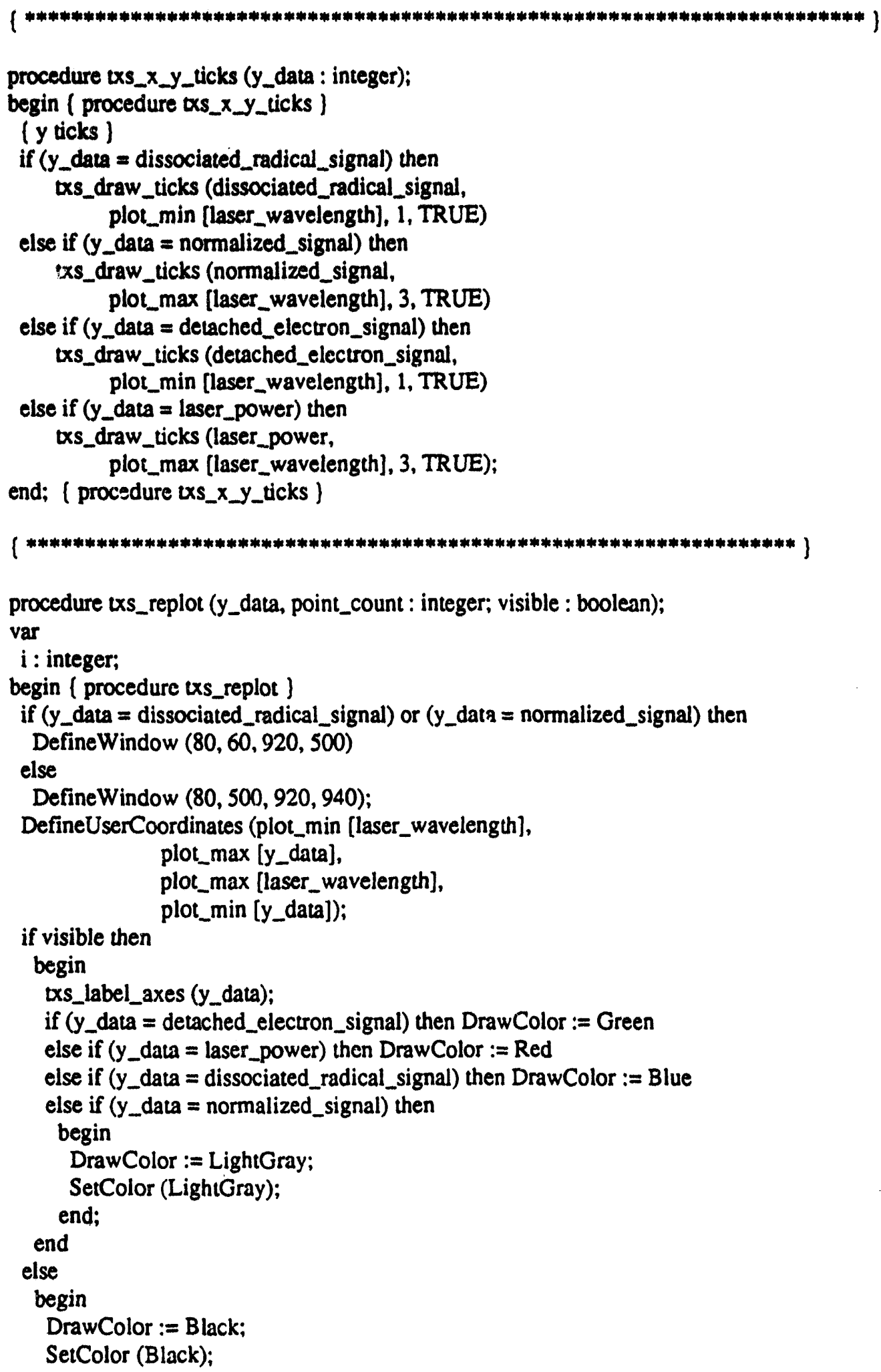




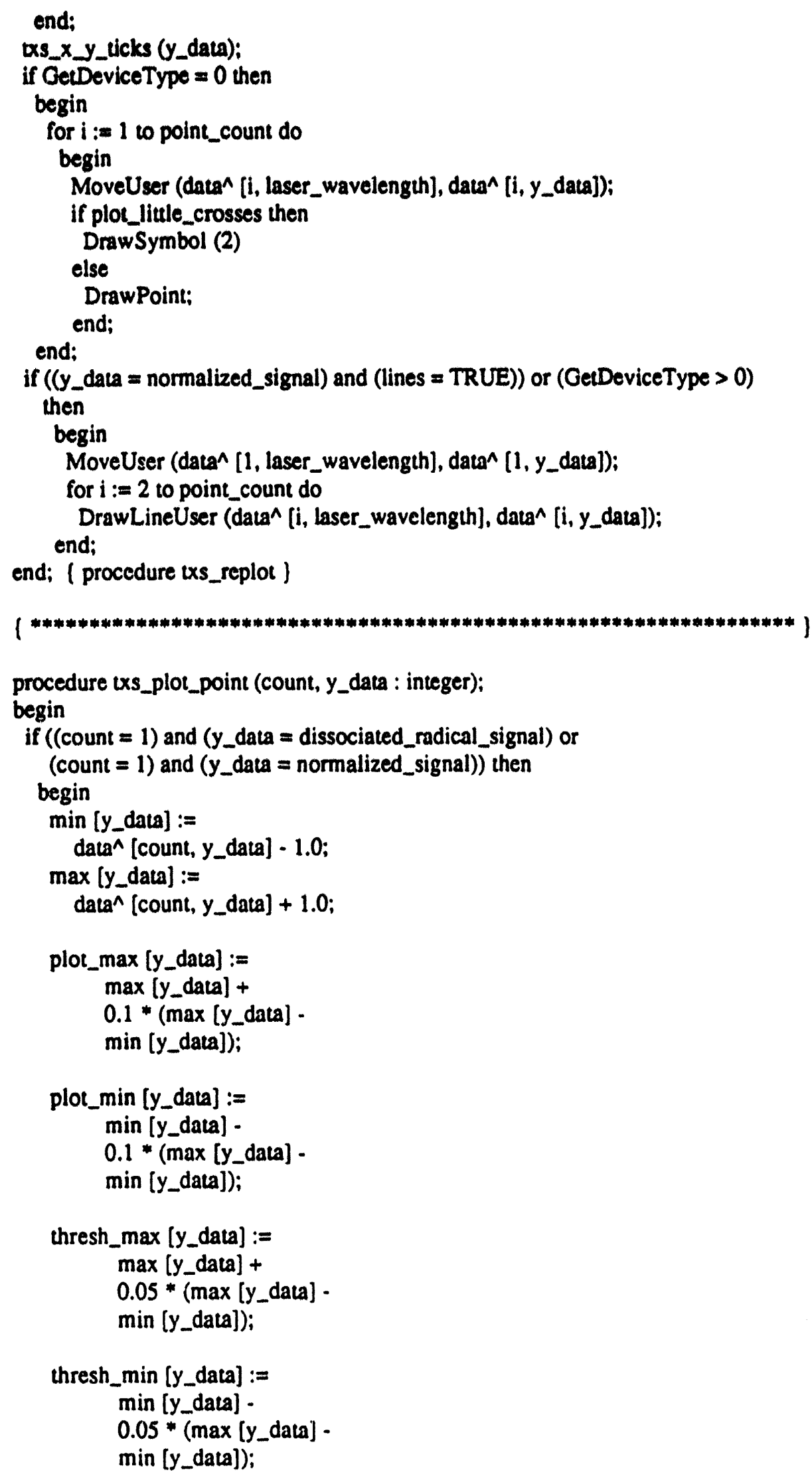


DefineWindow $(80,60,920,500)$;

DefineUserCoordinates (plot_min [laser_wavelength], plot_max [y_data],

plot_max [laser_wavelength], plot_min [y_data]);

if $y_{\text {_data }}=$ dissociated_radical_signal then

begin

SetColor (Blue);

DrawColor := Blue;

end

else

begin

SecColor (Lightoray);

DrawColor : = LightGray;

end;

uxs_x_y_ticks (y_data);

MoveUser (data^ [count, laser_wavelength], data^ [count, y_data]):

if plot_little_crosses then

DrawSymbol (2)

else

DrawPoint:

end;

if (data^ [count, $y_{-}$data] $>$thresh_max [y_data]) then

begin

txs_replot (y_data, (count - 1), FALSE);

$\max \left[y_{-}\right.$data] $:=$data $^{\wedge}$ [count, y_data];

plot_max [y_data] := $\max \left[y_{-}\right.$data $]+$

$\left(0.1 *\right.$ (max [y_data] - $\min \left[y_{-}\right.$data]));

thresh_max $\left[y_{\text {_data }}\right]:=\max \left[y_{-}\right.$data $]+$

$\left(0.05 *\right.$ (max [y_data] - $\min \left[y_{-}\right.$data $\left.\left.]\right)\right)$;

txs_replot (y_data, count, TRUE);

end

else if (data^ [count, y_data] < thresh_min [y_data]) then begin

txs_replot (y_data, (count - 1), FALSE);

$\min \left[y_{-}\right.$data] $:=$data^ [count, $y_{-}$data];

plot_min [y_data] $:=\min \left[y_{\text {_data }}\right.$ -

$\left(0.1 *\right.$ (max [y_data] - $\min \left[y_{-}\right.$data]));

thresh_min [y_data] $:=\min \left[y_{-}\right.$data] -

$\left(0.05 *\left(\max\right.\right.$ [y_data] - $\min \left[y_{-}\right.$data]));

txs_replot (y_data, count, TRUE);

end

else

begin

if ( $y$ _data $=$ dissociated_radical_signal)

or (y_data = normalized_signal) then

DefineWindow $(80,60,920,500)$

else

DefineWindow $(80,500,920,940)$;

DefineUserCoordinates (plot_min [laser_wavelength), plot_max [y_daca],

plot_max [laser_wavelength],

plot_min [y_data]); 
begin

DefineWindow $(100,100,1000,900)$;

DefineUserCoordinates (plot_min [x_axis], plot_max [y_axis],

plot_max [x_axis], plot_min [y_exis]):

SetColor (Whice);

(draw box)

MoveUser (plot_min [x_axis], plot_min [y_axis]);

DrawLineUser (plot_min [x_axis], plot_max [y_axis]);

DrawLineUser (plot_max [x_axis], plot_max [y_axis]):

DrawLineUser (plot_max [x_axis], plot_min [y_axis]);

DrawLineUser (plot_min [x_axis], plot_min [y_axis]);

[ label y axis ]

bSetTextStyle (Font, VertDir, 1):

SerTexiJusufy (LeftText, CenterText);

OutTextXY (Textheight(axis_label [y_axis]), (GetMaxY div 2), axis_label [y_axis]);

( label $x$ axis )

bSetTextStyle (Font, HorizDir, 1);

SetTextJustify (CenterText, BottomText);

OutTexLXY ((GetMaxX div 2 + GetMaxX div 20), GetMaxY, axis_label [X_axis]);

SetColor (White);

txs_draw_ticks (x_axis, plot_min [y_axis], 0, TRUE);

txs_draw_ticks (x_axis, plot_max [y_axis], 2, FALSE);

ixs_draw_ticks (y_axis, plot_min [x_axis], 1, TRUE);

us_draw_ticks (y_axis, plot_max [x_axis], 3, FALSE);

(label title)

bSetTextStyle (Font, HorizDir, 1);

SetTextJustify (CenterText, TopText);

OutTexLXY (GetMaxX div $2+$ GetMaxX div 20, 0 , Total Photodissociation Cross Section Experiment');

OutTexLXY (GetMaxX div $2+$ GetMaxX div 20, (Textheight ( $T$ ') *3), (axis_label [y_axis] + 'vs. ' + axis_label [x_axis]));

[ label file name ]

SetColor (Red);

bSetTextStyle (Font, HorizDir, 1);

SetTextJustify (LeftText, Bottom Text);

OutTexLXY $(0$, GetMaxY, data_file_name);

end: (procedure p_lxs_draw_axes )

(

procedure p_txs_plot_points (x_axis, y_axis : integer);

var

plot_litule_crosses : boolean;

i : integer,

begin ( procedure p_lxs_plot_points ) 


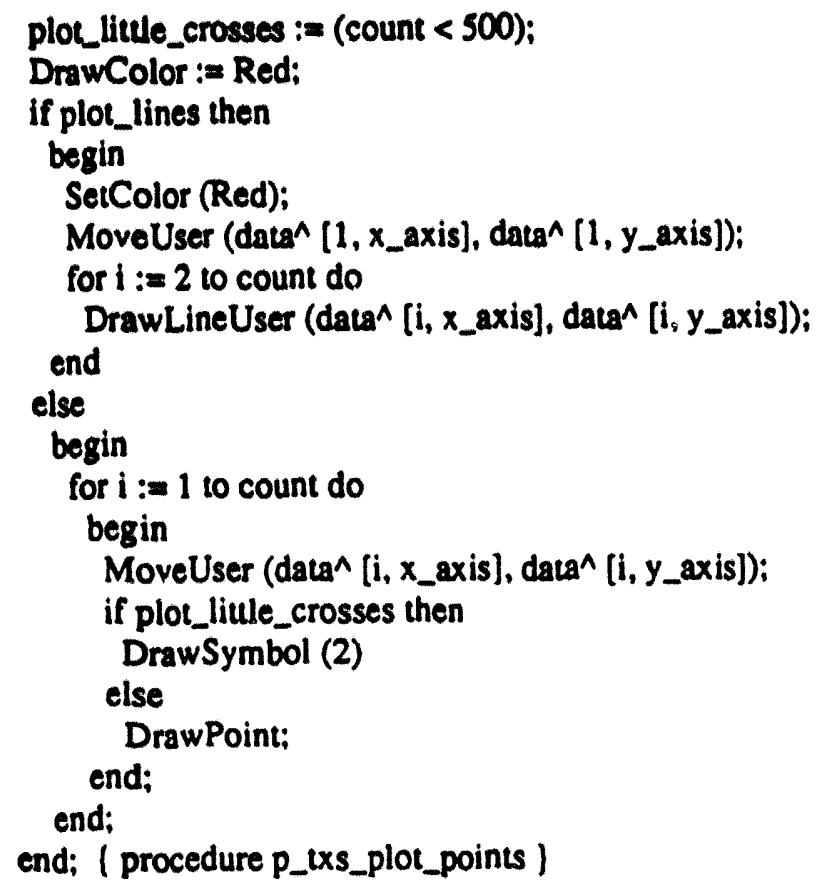


txs_draw_axes;

( plot data)

SeuLineStyle $(3,0,1)$;

oxs_replot (laser_power, counh, TRUE);

SetzineStyle $(2,0,1)$;

txs_replot (dissociated_radical_signal, count, TRUE);

SeLLineStyle $(1,0,1)$;

txs_replot (detached_electron_signal, count, TRUE);

SeuLineStyle $(0,0,1)$;

txs_replot (normalized_signal, count, TRUE);

(label file name )

(SetColor (Red);]

bSetTextStyle (Font, HorizDir, 1);

SetTextJustify (LeftText, BottomText);

OutTextXY $(0$, GetMaxY, data_file_name);

end;

procedure dump_txs_experiment_screen;

begin

(show_screen_dump_parameters;

screen_dump_menu;)

(Put on screen what will go to output device \}

SetGraphMode(GraphMode);

GraphicsOn := TRUE;

draw_txs_dump_screen;

(Now output to PS LaserWriter )

prevent_clear_screen;

erase_old_porfile;

enter_laserwriter_graphics_mode;

(Draw Plot of Data)

draw_lxs_dump_screen;

plot_graph_and_exit:

Ipr_plot_to_laserwriter;

InstallScreenGraphicsDriver;

TextColor (14);

end;

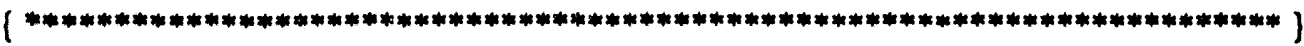

procedure put_plot_to_screen;

(called just before putting plot to graphics device ) 


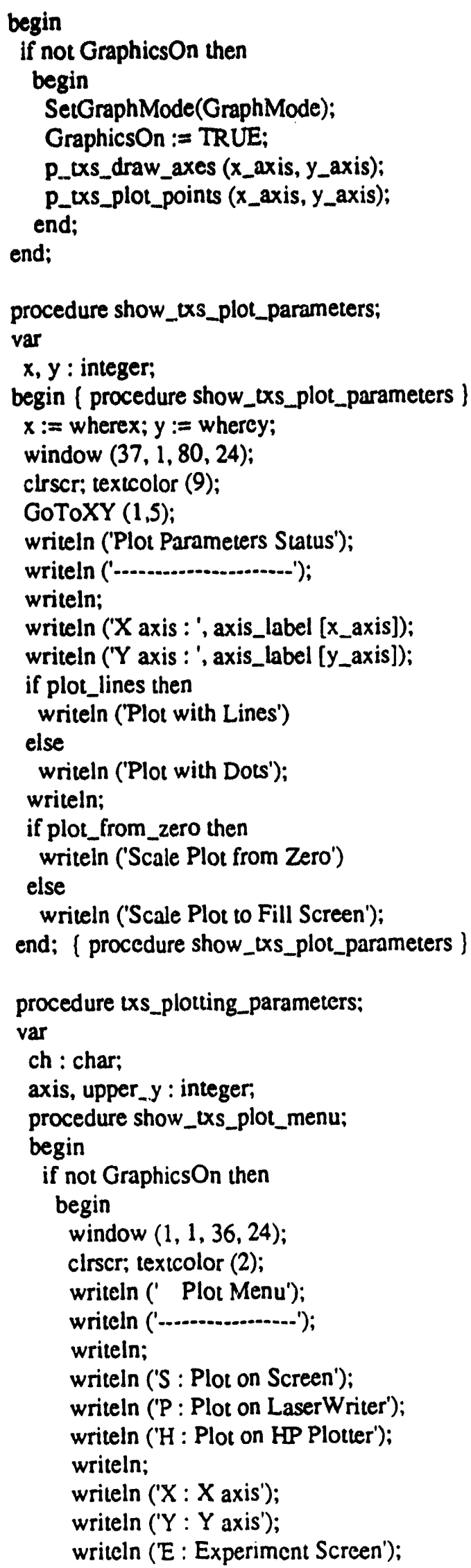




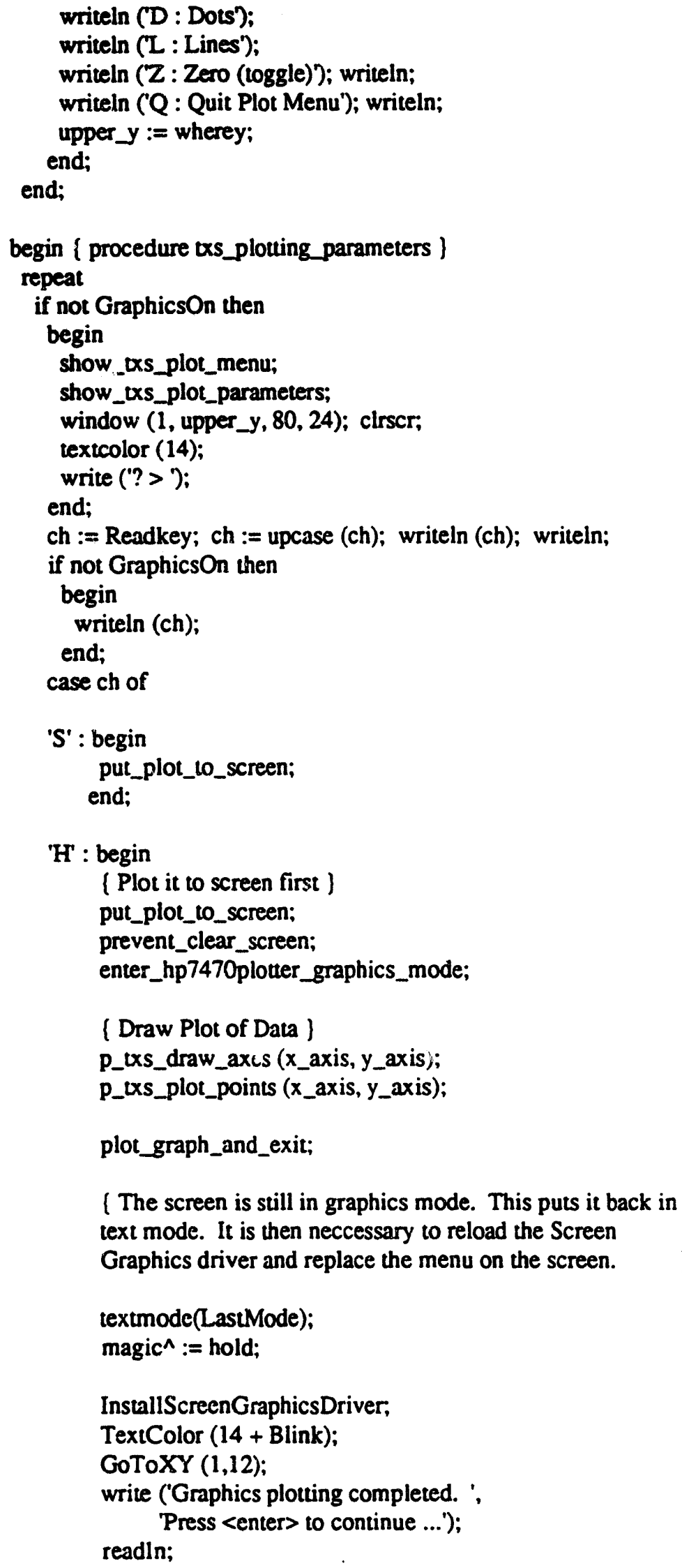

( The screen is still in graphics mode. This puts it back in text mode. It is then neccessary to reload the Screen Graphics driver and replace the menu on the screen.

textmode(LastMode);

$\operatorname{magic}^{\wedge}:=$ hold;

InstallScreenGraphicsDriver,

TextColor (14 + Blink);

GoToXY $(1,12)$;

write ('Graphics plotting completed. ',

'Press <enter> to continue ...'); readln; 
end;

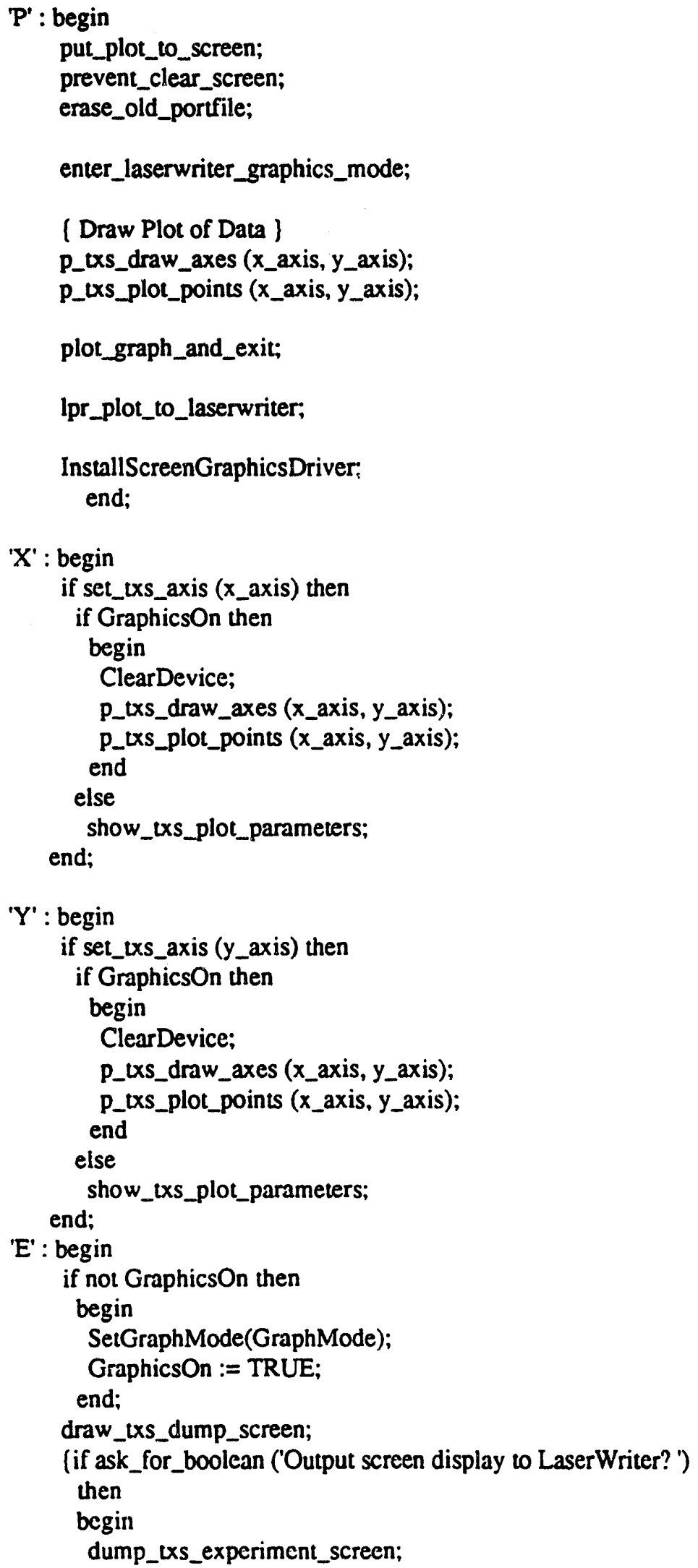




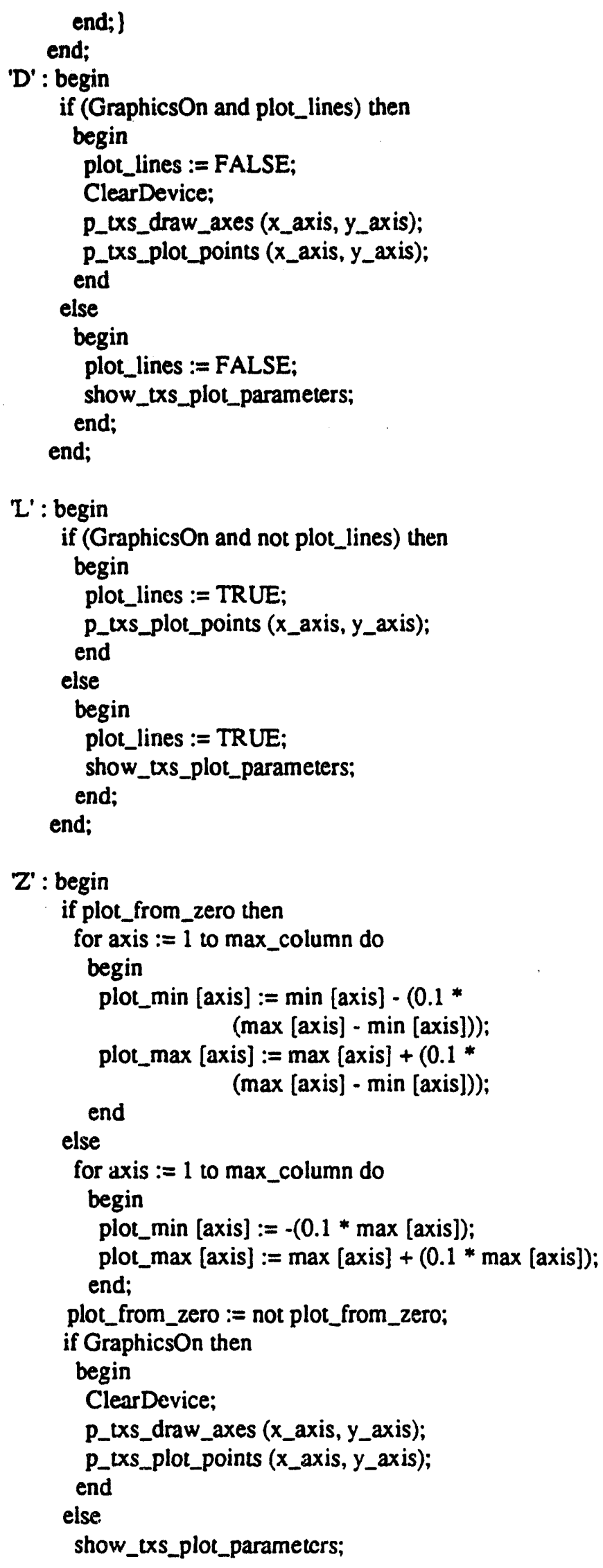


end;

\#13 : begin

if GraphicsOn then

begin

RestoreCRTMode;

GraphicsOn := FALSE;

end;

show_txs_plot_parameters;

end;

'Q' : begin

if GraphicsOn then

begin

RestoreCRTMode;

GraphicsOn := FALSE; end:

end;

end; ( case ch )

until (ch = ' $Q$ ');

end; ( procedure txs_plotting_parameters )

end. ( unit txsplot )

\subsection{0 tofplot.pas}

\section{( tofplot.pas}

\{ contents :

(i. useful (repetitive)

( procedure draw_ticks (tick_axis : integer;

1 constant_limit : real;

code : integer;

label_ticks : boolean)

\{ ii. while experiment is running

( procedure label_axes (axis : integer)

I procedure x_draw_axes

( procedure $x_{-} y_{-}$ticks (y_data : integer)

( procedure $x \_r c p l o t$ (y_data, point_count : integer, visible : boolcan) ]

( procedure x_plot_point (count, y_data : integer)

\{ procedure set_done_flag

\section{\}}

1

]

\}

)

I

\}

) 
[ iii. after experiment

( procedure p_draw_axes (x_axis, y_axis : integer) )

( procedure p_plot_points (x_axis, y_axis : integer) ]

(procedure j_hard_copy (x_axis, y_axis : integer) \}

( function set_axis (var axis : integer) : boolean )

\{ procedure dump_experiment_screen \}

( procedure show_plot_parameters ]

( procedure plot_menu ) ]

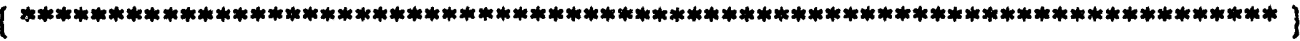

unit tofplot;

$\{\mathrm{SO}+\}$

$\{\mathrm{SF}+\}$

interface

uses globals,utility,insgrdrv,graph,plotutil,graphadd,grafutil,CRT,DOS;

const

Font $:$ Integer $=$ DefaultFont;

procedure tof_draw_ticks (tick_axis : integer;

constant_limit : real;

code : integer;

label_ticks : boolean);

procedure tof_draw_axes (x_axis, $y \_a x i s$ : integer);

procedure dump_tof_experiment_screen (x_axis, $y \_a x i s$ : integer;

data_count : integer);

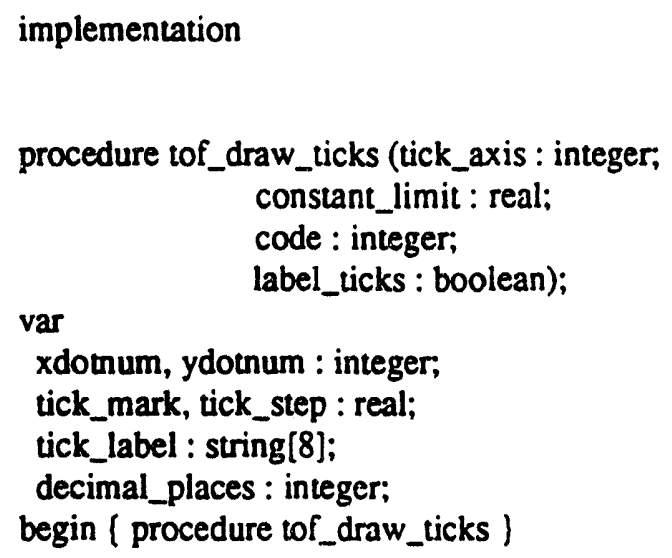




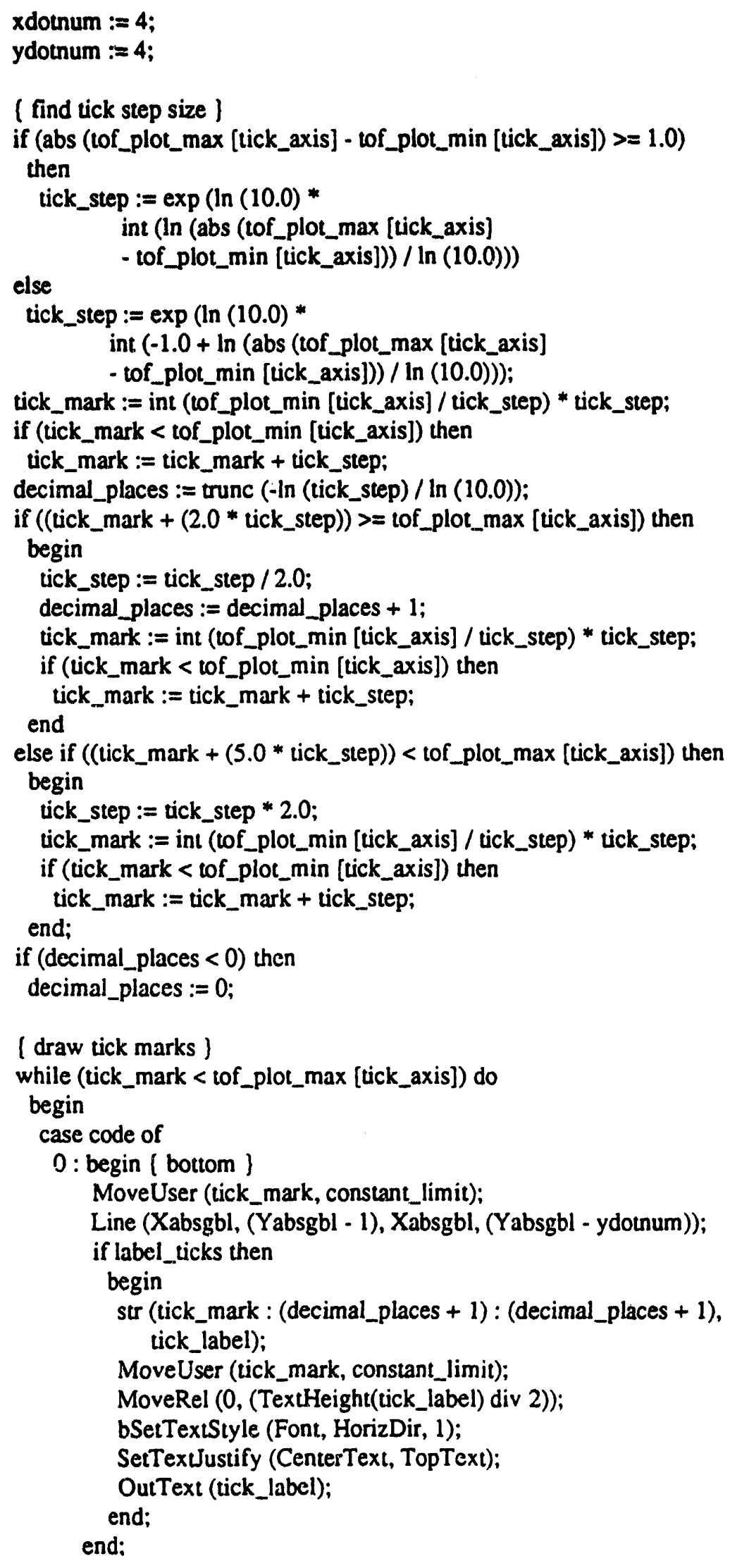




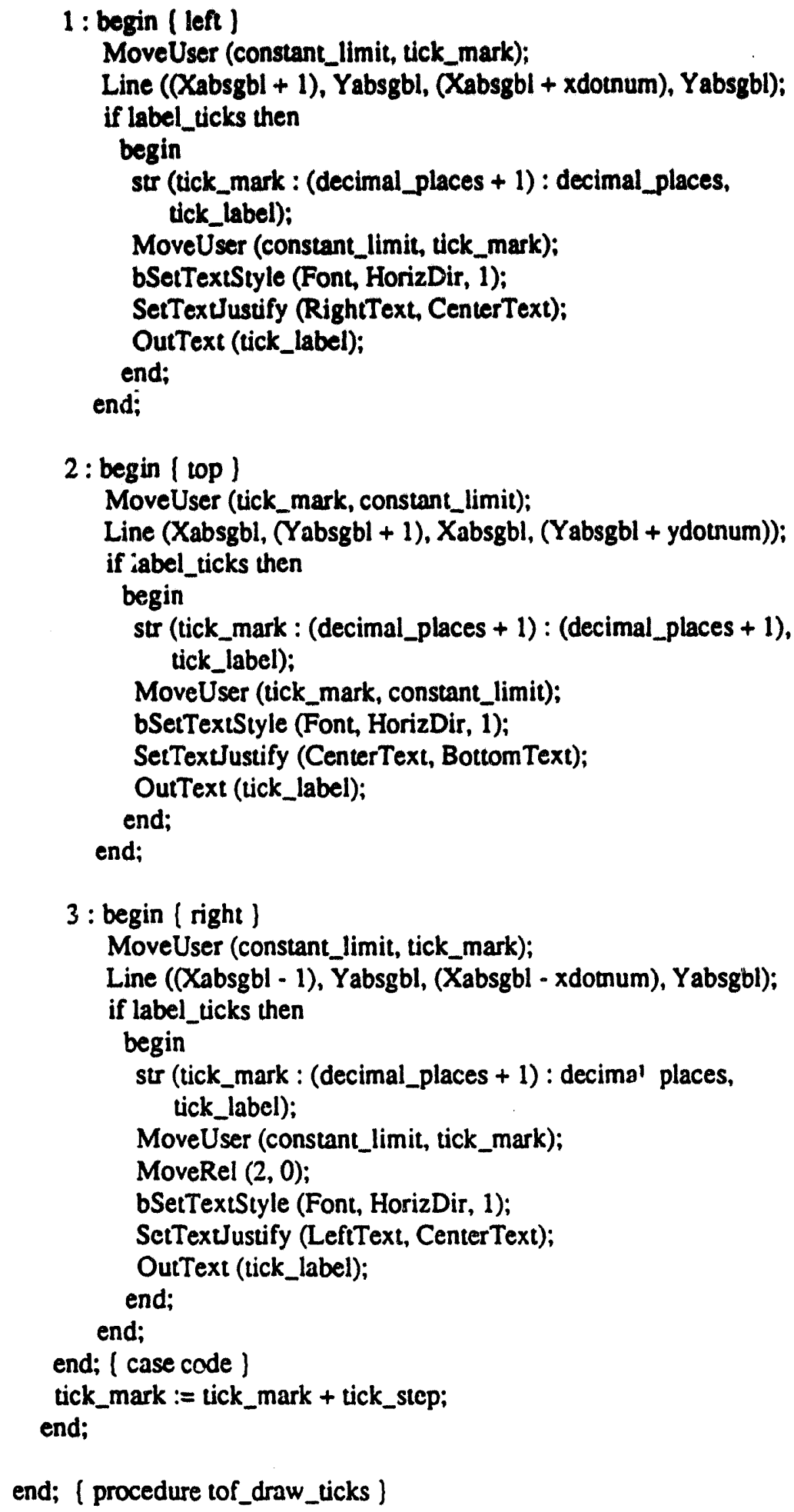


DefineWindow $(100,100,1000,900)$;

DefineUserCoordinates (tof_plot_min [tume], tof_plot_max [tof_signal], tof_plot_max [time], tof_plot_min [tof_signal]);

SetColor (Lightoray);

MoveUser (tof_plot_min [time], tof_plot_min [tof_signal]);

DrawLineUser (tof_plot_min [time], tof_plot_max [tof_signal]);

DrawLineUser (tof_plot_max [time], tof_plot_max [tof_signal]);

DrawLineUser (tof_plot_max [time], tof_plot_min [tof_signal]);

DrawLineUser (tof_plot_min [time], tof_plot_min [tof_signal]);

[ label y axis ]

bSetTextStyle (Font, VeriDir, 1);

SetTextJustify (LeftText, CenterText);

OutTexLXY (TextHeight(tof_axis_label [y_axis]), (GetMaxY div 2), tof_axis_label [y_axis]);

(label $x$ axis )

bSetTextStyle (Font, HorizDir, 1);

SetTextJustify (CenterText, BottomText);

OutTexLXY ((GetMaxX div 2 + GetMaxX div 20), GetMaxY, tof_axis_label [x_axis]);

tof_draw_ticks (uime, tof_plot_min [tof_signal], 0, TRUE);

tof_draw_ticks (time, tof_plot_max [tof_signal], 2, FALSE);

tof_draw_ticks (tof_signal, tof_plot_max [time], 3, FALSE);

tof_draw_ticks (tof_signal, tof_plot_min [time], 1, TRUE);

(label title)

bSetTextStyle (Font, HorizDir, 1);

SetTextustify (CenterText, TopText);

OutTextXY (GetMaxX div $2+$ GetMaxX div 20, 0 , 'Time of Flight Mass Spectrum');

OutTexLXY (GetMaxX div 2+ GetMaxX div 20, (TextHeight (T) *3), (tof_axis_label [y_axis] + ' vs. ' + tof_axis_label [x_axis]));

(label file name )

SetColor (Red);

bSetTextStyle (Font, HorizDir, 1);

SetTextJustify (LeftText, Bottom Text);

OutTexuXY (0, GetMaxY, data_file_name);

end; ( procedure tof_draw_axes )

(

procedure draw_tof_dump_screen (x_axis, $y_{\text {_axis }}$ : integer;

data_count : integer);

var

i : integer;

begin

tof_draw_axes (x_axis, y_axis); 


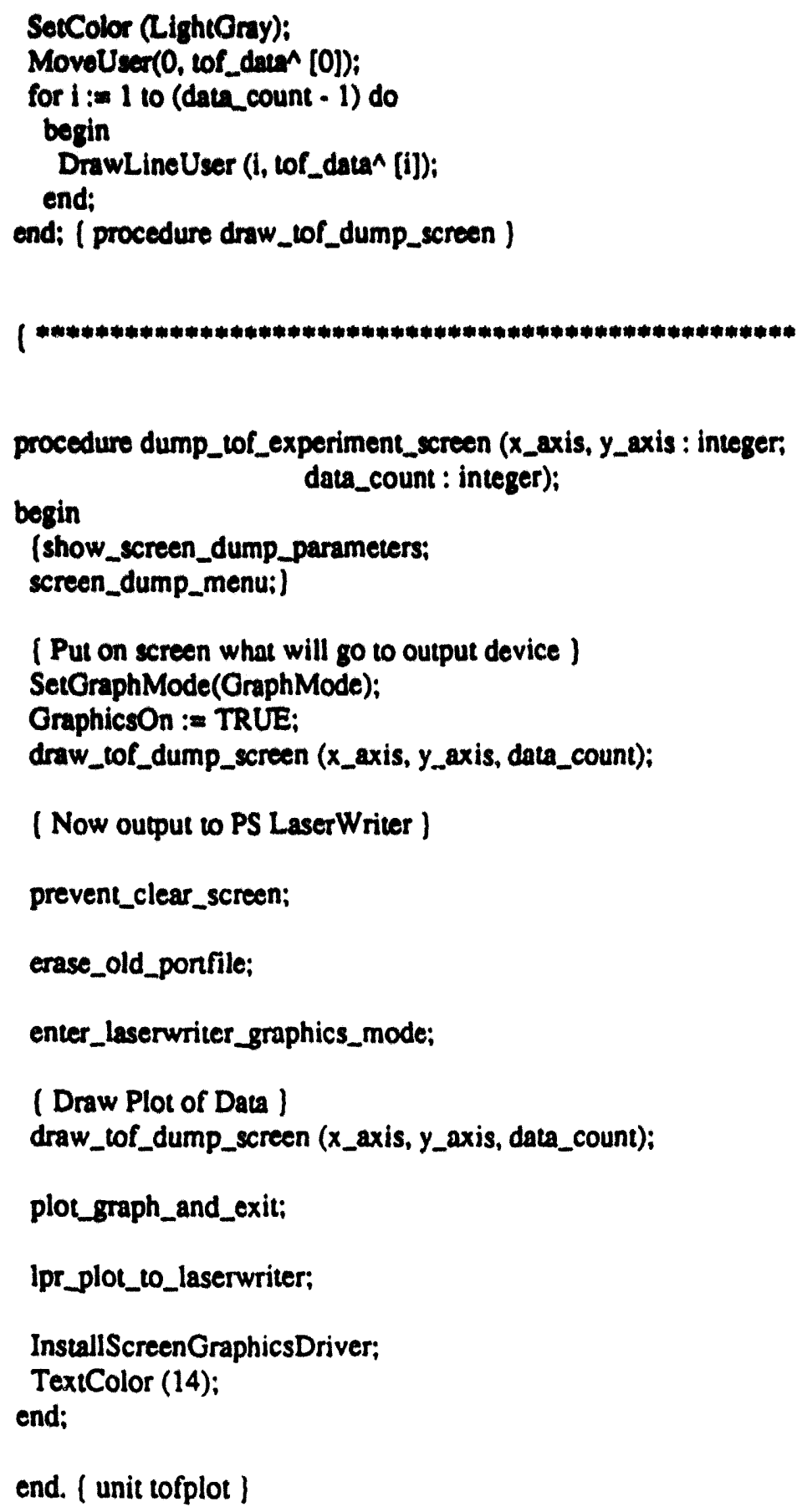

\subsection{1 plotutil.pas}

( plotutil.pas

unit plotutil;

$[\mathrm{SO}+]$ 
interface

uses globals, utility,inggrdrv,graph,graphadd,grafutil,CRT,DOS;

const

Font : Integer $=$ DefaultFont:

procedure set_done_flag;

procedure prevent_clear_screen:

procedure plot_graph_and_exit;

procedure ipr_plot_to_laserwriter;

procedure erase_old_porfile:

procedure enter_laserwriter_graphics_mode;

procedure enter_hp7470plotter_graphics_mode;

(

implementation

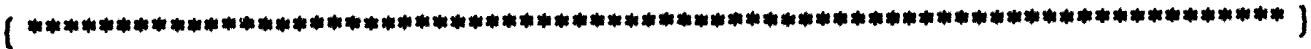

procedure set_done_flag;

var

i : intsger;

begin ( procedure set_done_flag )

bSetTexistyle (Font, HorizDir, 1);

SetTexUustify (?enterText,TopText);

SetColor (Yellow);

OutTexLXY ((MaxX div 2), (MaxY*4 div 5),

DONE! Hit any key to continue ...);

For $i:=1$ to 8 do

begin

Sound (1000);

Delay (100);

NoSound:

Delay (100);

end:

Repeat

SetFillStyle (EmptyFill, EmptyFill);

Bar ((MaxX div 2 - (TextWidth ('DONE! Hit any key to continue ...') div 2)),

(MaxY* 4 div 5),

(MaxX div $2+$ (TextWidth ('DONE! Hit any key to continue ...') div 2)),

(MaxY * $4 \operatorname{div} 5+$ TextHeight('D')));

Delay (200);

SetColor (Yellow);

OutTexLXY ((MaxX div 2), (MaxY * 4 div 5),

'DONE! Hit any key to continue ...');

Delay (200);

until KeyPressed;

end: (procedure set_done_flag ) 
procedure prevent_clear_ecreen;

begin

( After puting a graph on the screen, save the magic address

contents, then set it to SaS before calling closegraph to

prevent clearing the screen.

hold := magic^; magic^ := Sas;

belosegraph;

end: ( procedure prevent_clear_screen )

procedure plot_graph_and_exit;

begin

\section{[ Plot the graph ]}

\section{ClearDevice:}

status := GraphStatus:

if (status and $\$ 8000$ ) $<>0$ then begin

bCloseGraph;

AbortM (GraphStatusMsg(status));

end:

[ Exit Graphics ]

bClose-Graph;

end: ( procedure plot_graph_and_exit )

procedure erase_old_portile;

var

f : file;

begin

( Erase any existing old PortFILE plots so we don't print them as well )

if exist('e: (plot.out') then

begin

assign (f, 'e: plot.out');

erase (f);

end:

end: ( procedure erase_old_portfile )

procedure Ipr_plot_to_laserwriter;

begin

Swap Vectors:

Exec('c: lcommand.com','/c lpr e: (plot.out');

SwapVectors;

if DosError $<0$ then

- begin

TextMode(LastMode);

magic^ := hold:

TextColor (14 + Blink);

GraphicsOn := FALSE;

GoToXY $(10,12)$;

writeln ('Dos Error \# ', DosError); 


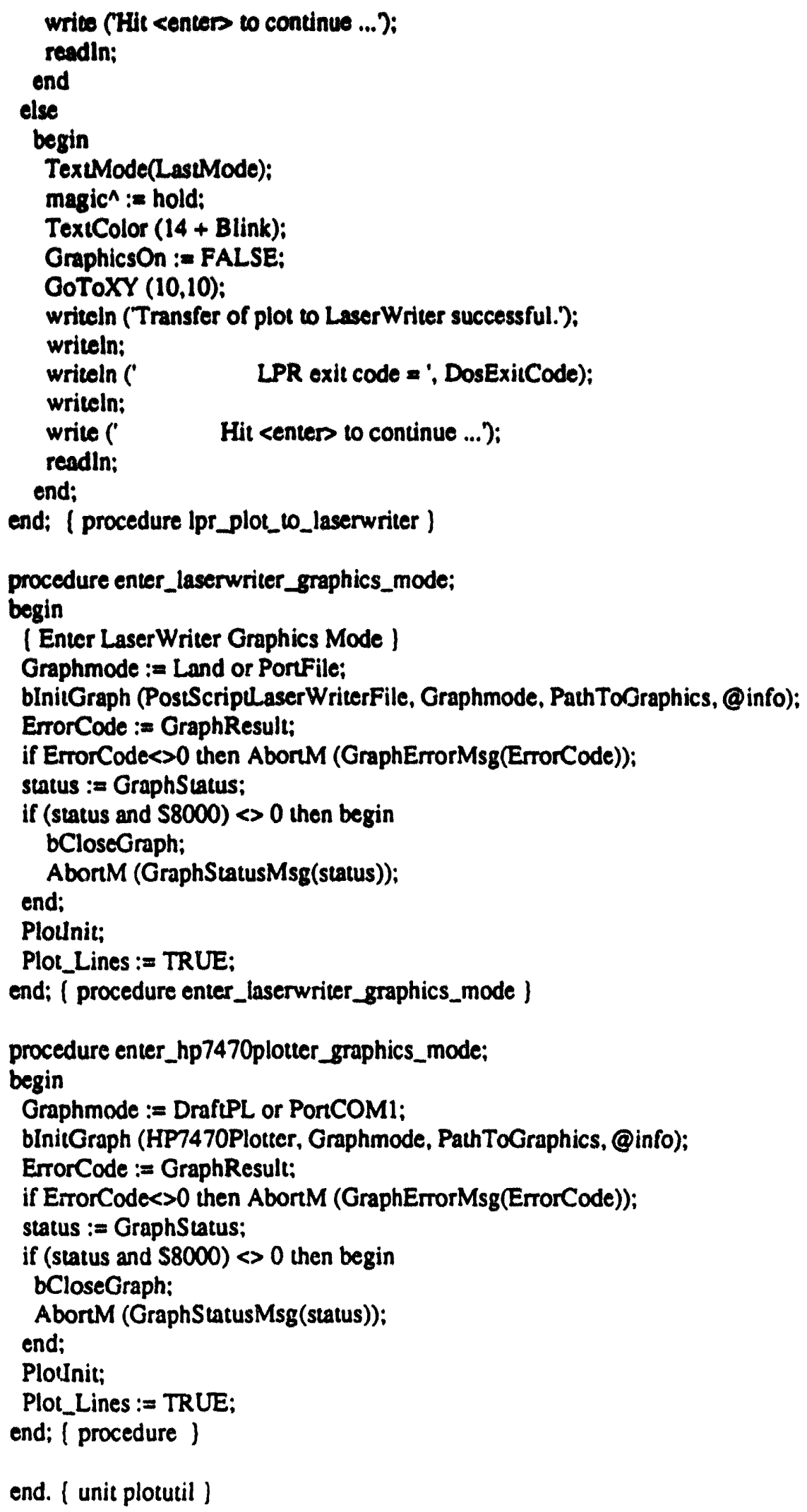




\subsection{2 parameter.pas}

( parameters.pas

J

I contents :

( procedure experimental_parameters

i menu for changing experimental set_up parameters

( procedure get_detachment_wavelength

( procedure get_cal_offset

\section{1}

I procedure check_range_vs_step

( procedure get_initial_wavelength

( procedure get_final_wavelength

I procedure get_lambda_step

I procedure get_shots_per_point

1

I procedure get_doubling

I procedure get_use_etalon

\section{)}

I procedure get_grating_orders

I

( procedure get_ADC_channels

I procedure get_zero_average_shot_number

I function read_set_up : boolcan

1 initialize experimental set-up parameters from a file

I procedure write_set_up

i write experimental set_up parameters to a file

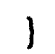

write experimental set_up parameters to a file

( procedure show_parameters

( display experimental set_up parameters on right hand side of screen ]

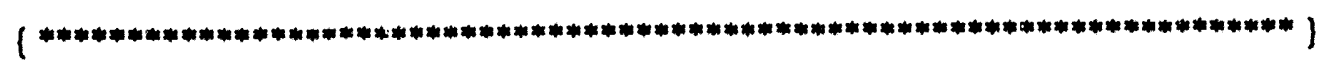

unit parameters;

[SO+]

(SF+)

interface

uses globals,utility,gpibutil,dyelaser,DOS,CRT; 


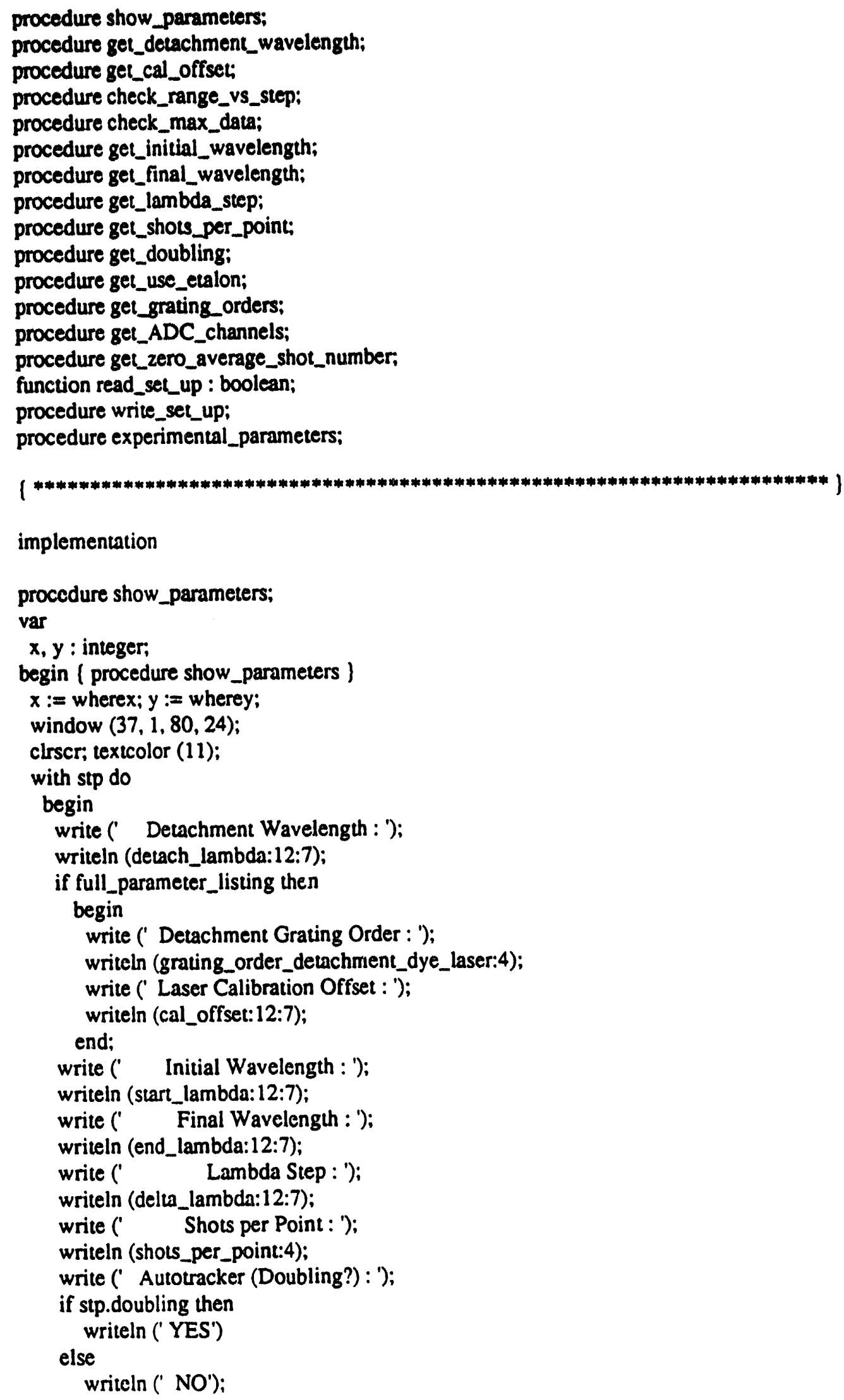




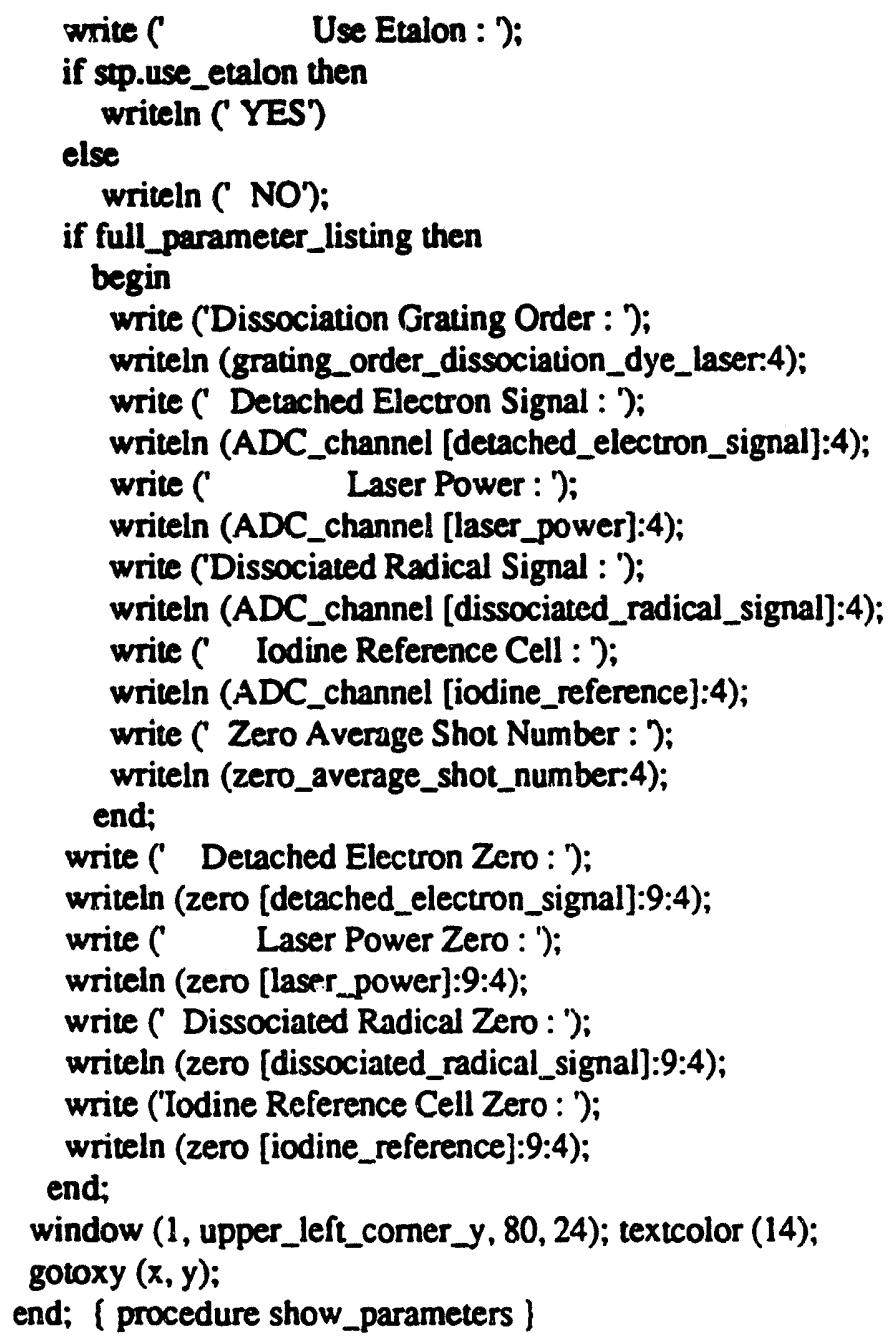


end: [ procedure cal_offset ]

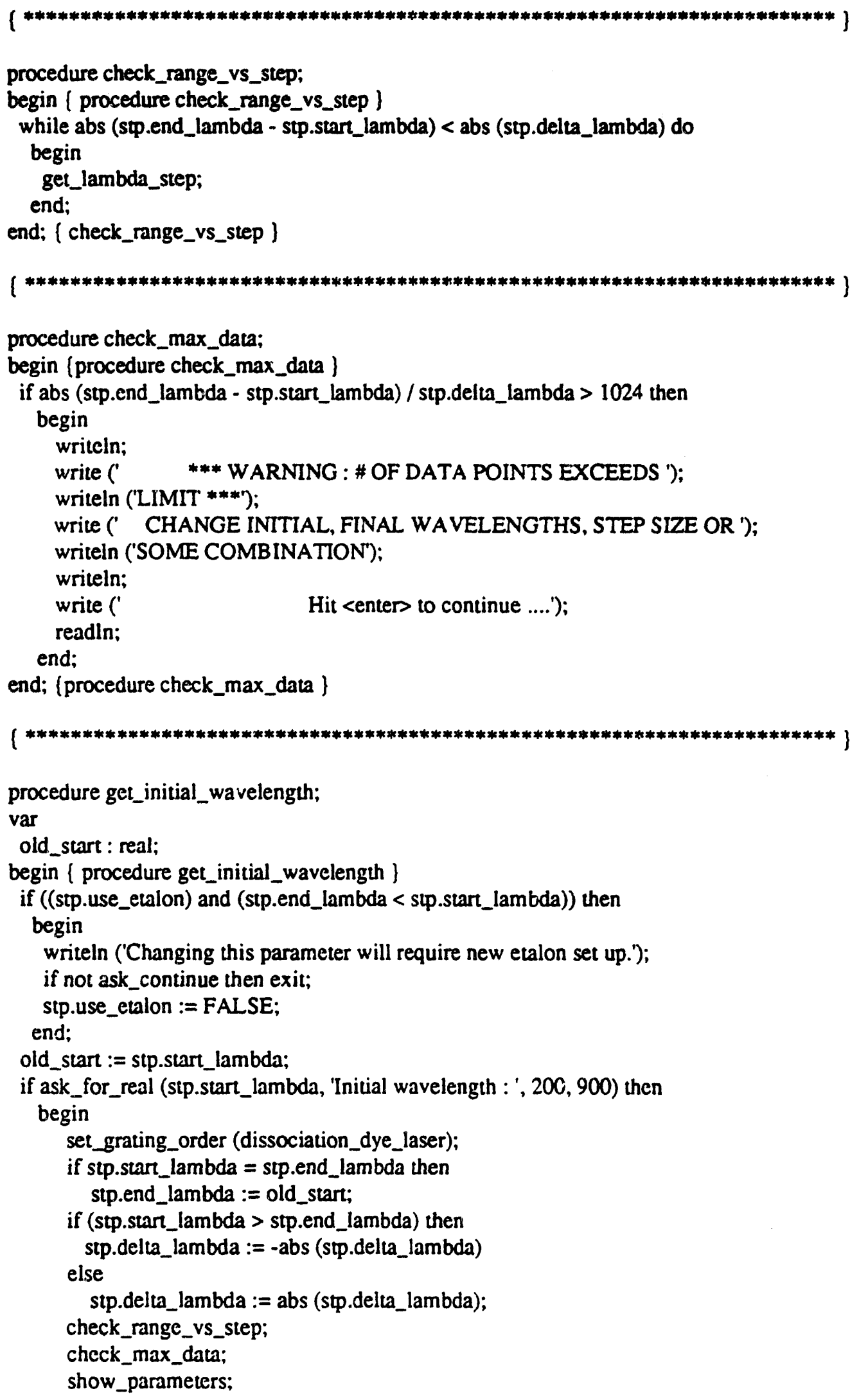


end;

end: (procedure get_initial_wavelength)

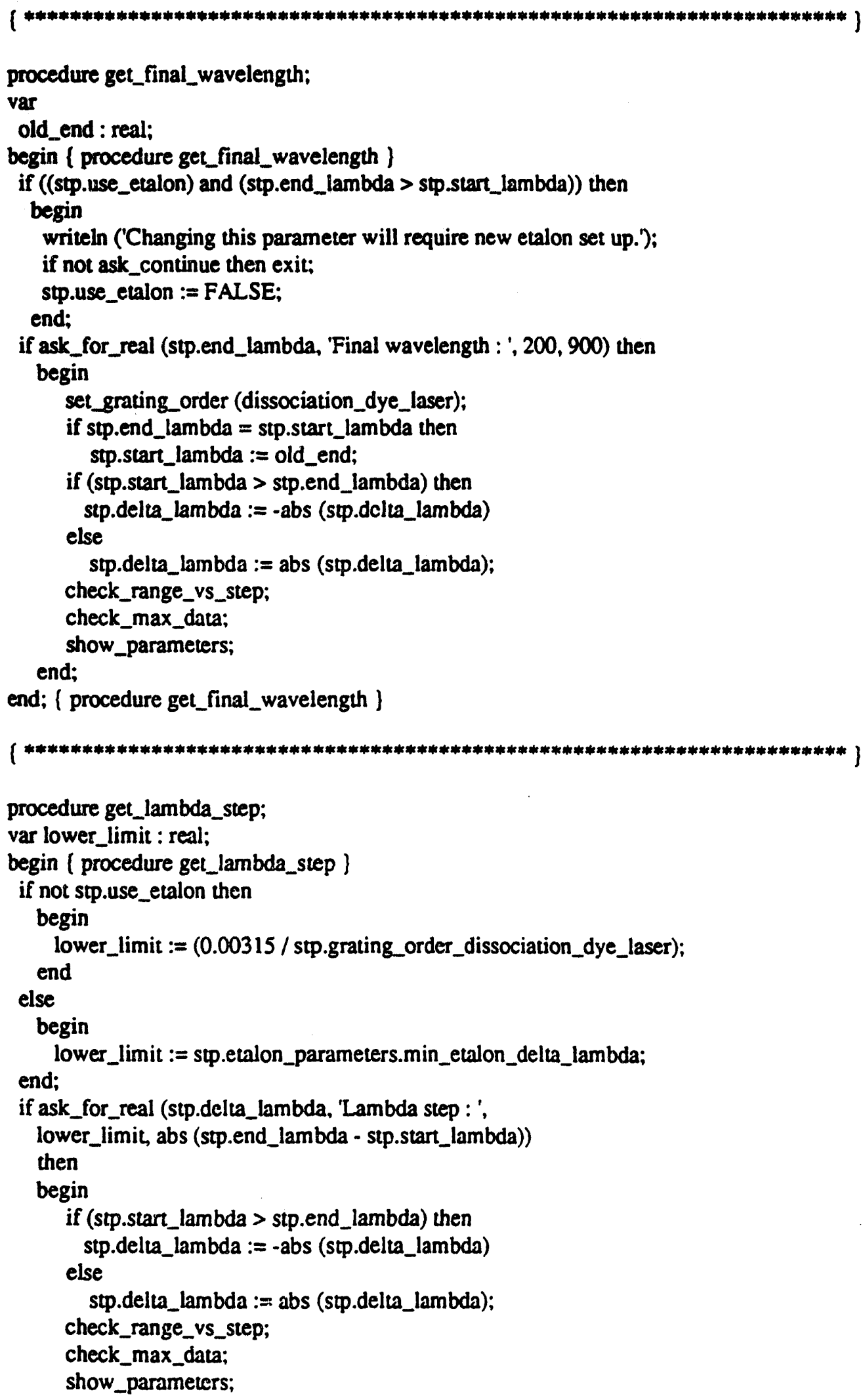


end:

end ( procedure get_lambda_step \};

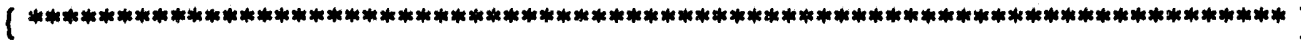

procedure get_shots_per_point;

begin ( procedure shots_per_point )

if ask_for_integer (stp.shots_per_point, 'Shots per Point : ',

$1,32767)$ then

show_parameters;

end ( procedure get_shots_per_point \};

1

procedure get_doubling;

begin ( procedure get_doubling \}

stp.doubling := ask_for_boolean ('Use Autotracker? (Double?));

set_grating_order (dissociation_dye_laser);

show_parameters;

end (procedure get_doubling );

( $* * * * * * * * * * * * * * * * * * * * * * * * * * * * * * * * * * * * * * * * * * * * * * * * * * * * * * * * * * * * * * * * * * * * * * * * * * *)$ )

procedure get_use_etalon;

var

lambda, old_lambda : real;

normal_position_set : boolean;

end_step : integer;

begin (procedure get_use_etalon \}

stp.use_etalon := ask_for_boolean ('Use Etalon? : ');

if stp.use_etalon then

begin

with stp do

begin

clrscr; get_cal_offset;

use_etalon := FALSE;

get_initial_wavelength;

get_final_wavelength;

use_etalon := TRUE;

get_lambda_step; clrscr; writeln;

writeln ('Be patient, etalon is proceeding to set up position!');

if start_lambda $<$ end_lambda then

begin

lambda := end_lambda;

end

else

begin

lambda := start_lambda;

end;

etalon_parameters.upper_lambda := lambda;

etalon_parameters.lambda_0 :=

use_etalon := FALSE;

lambda / $(1-\operatorname{sqr}(800 * 3.125 \mathrm{e}-6) / 2)$;

set_gpib_remote (TRUE); 


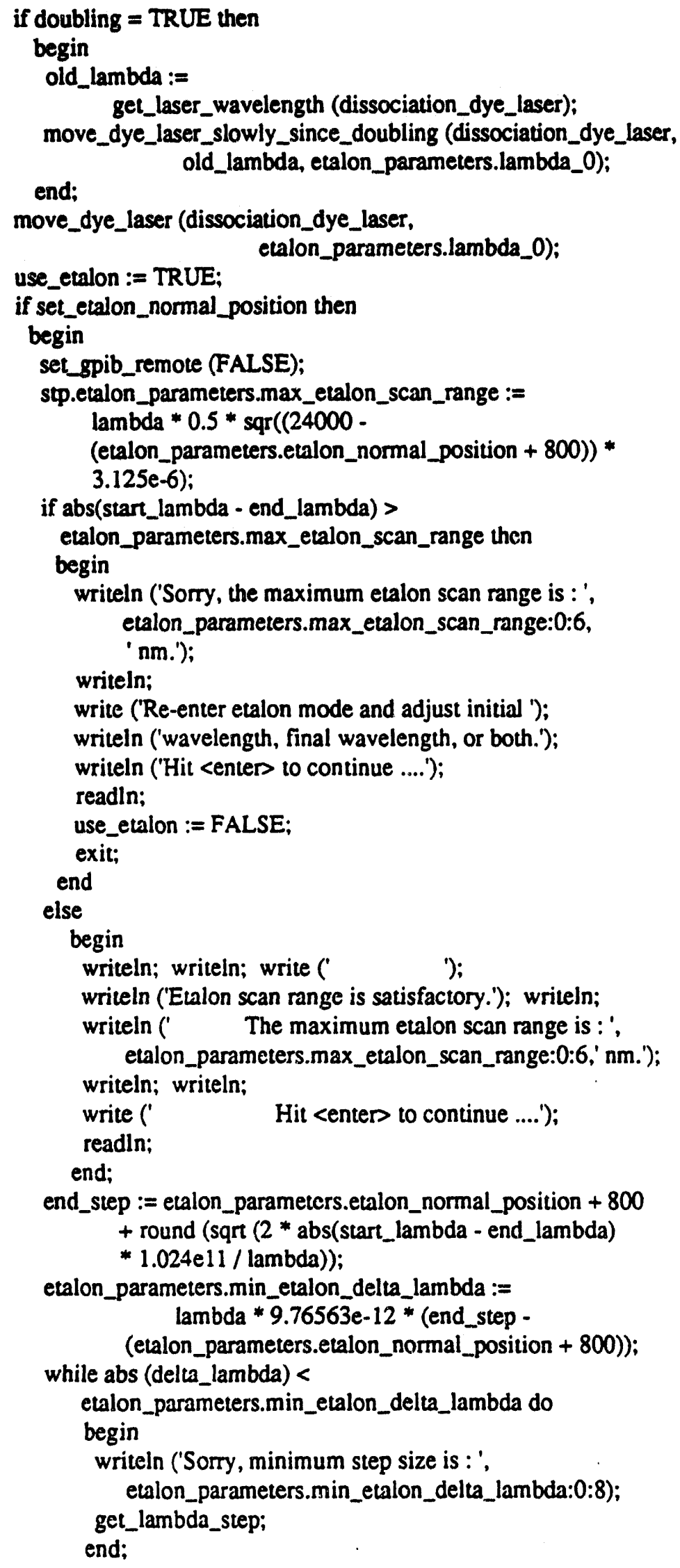




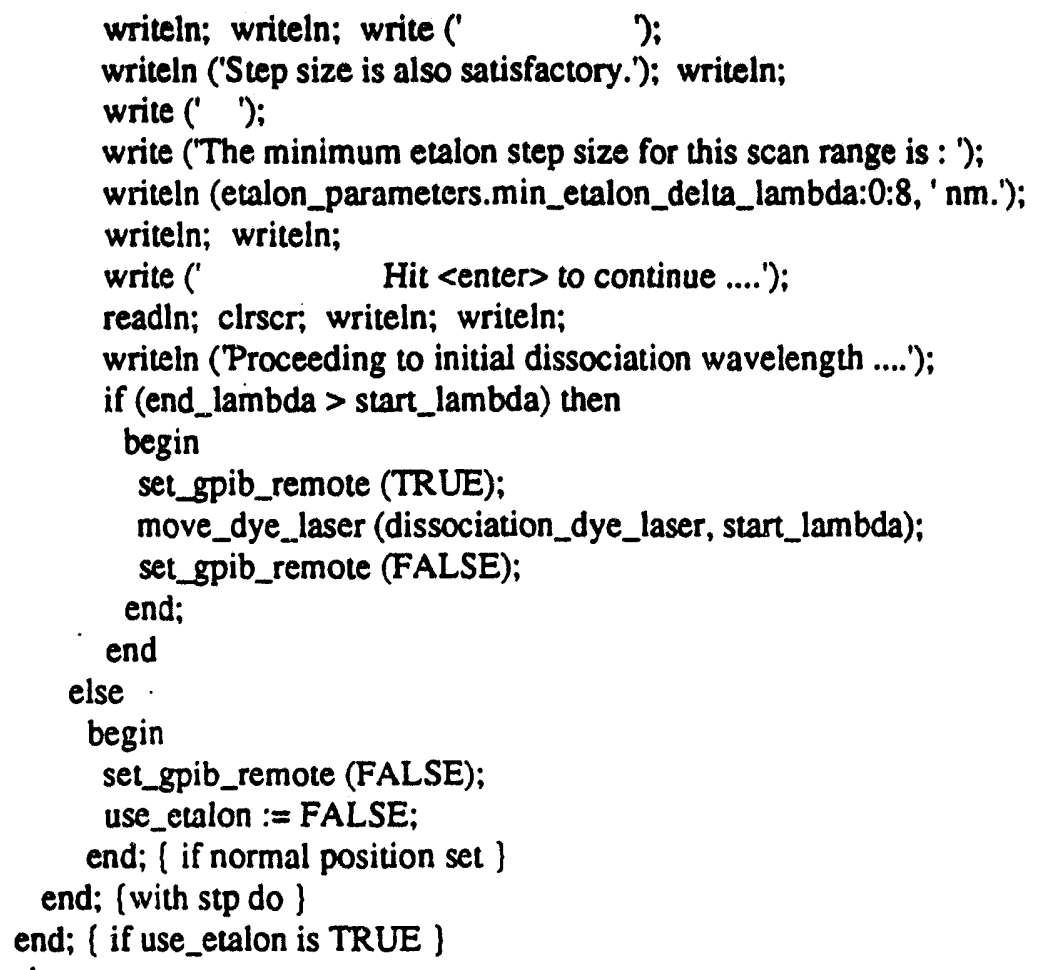




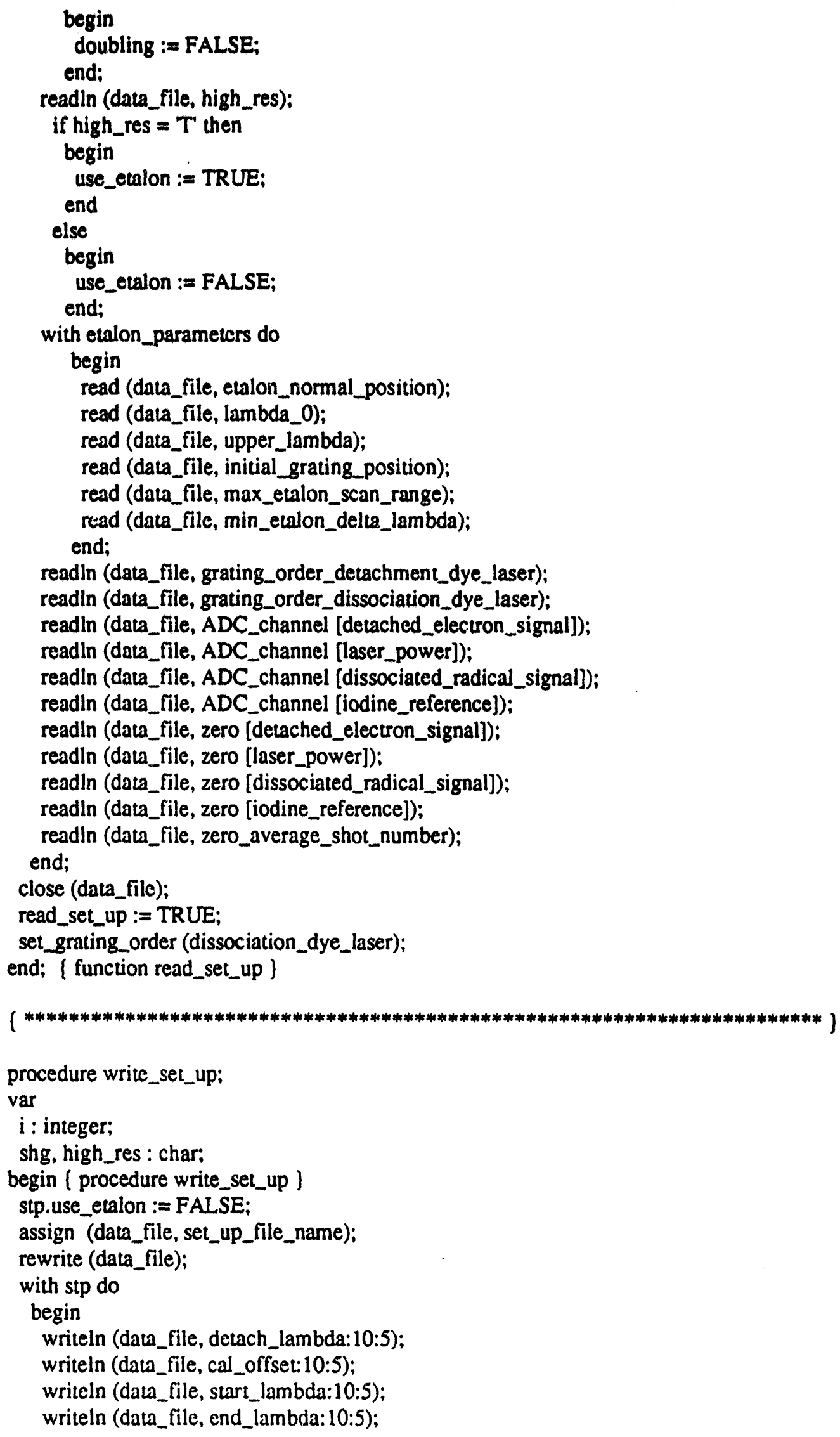




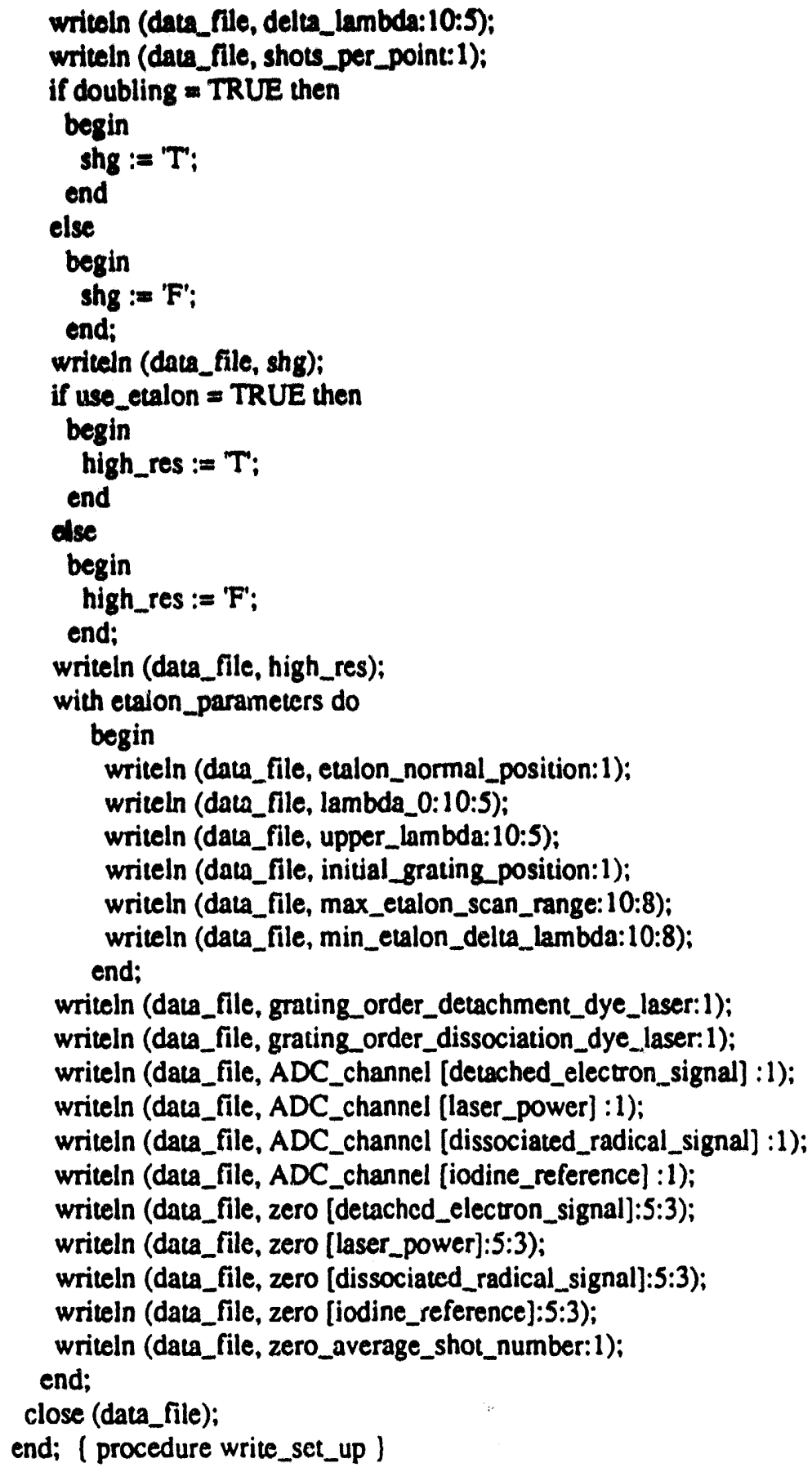




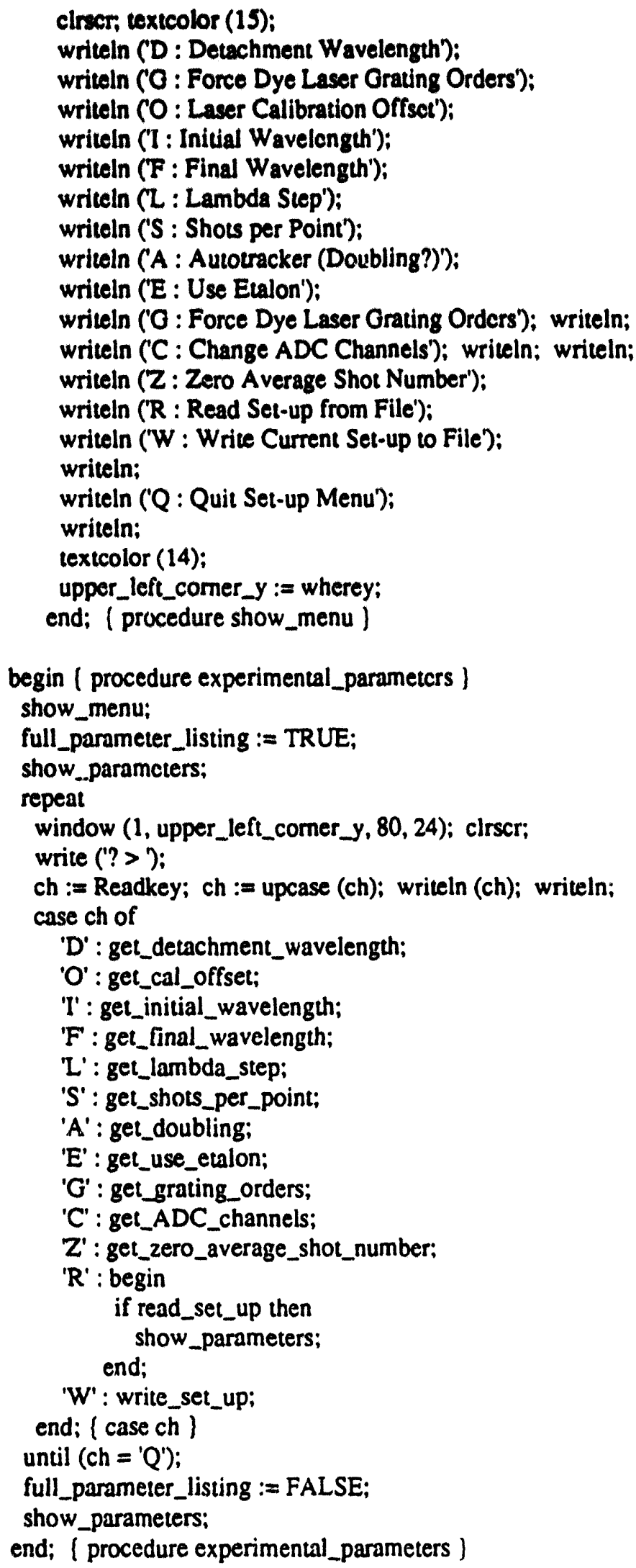


end. ( unit parameters )

\subsection{3 dyelaser.pas}

\section{I dyelaser.pas \\ procedures used for GPIB and dye laser control}

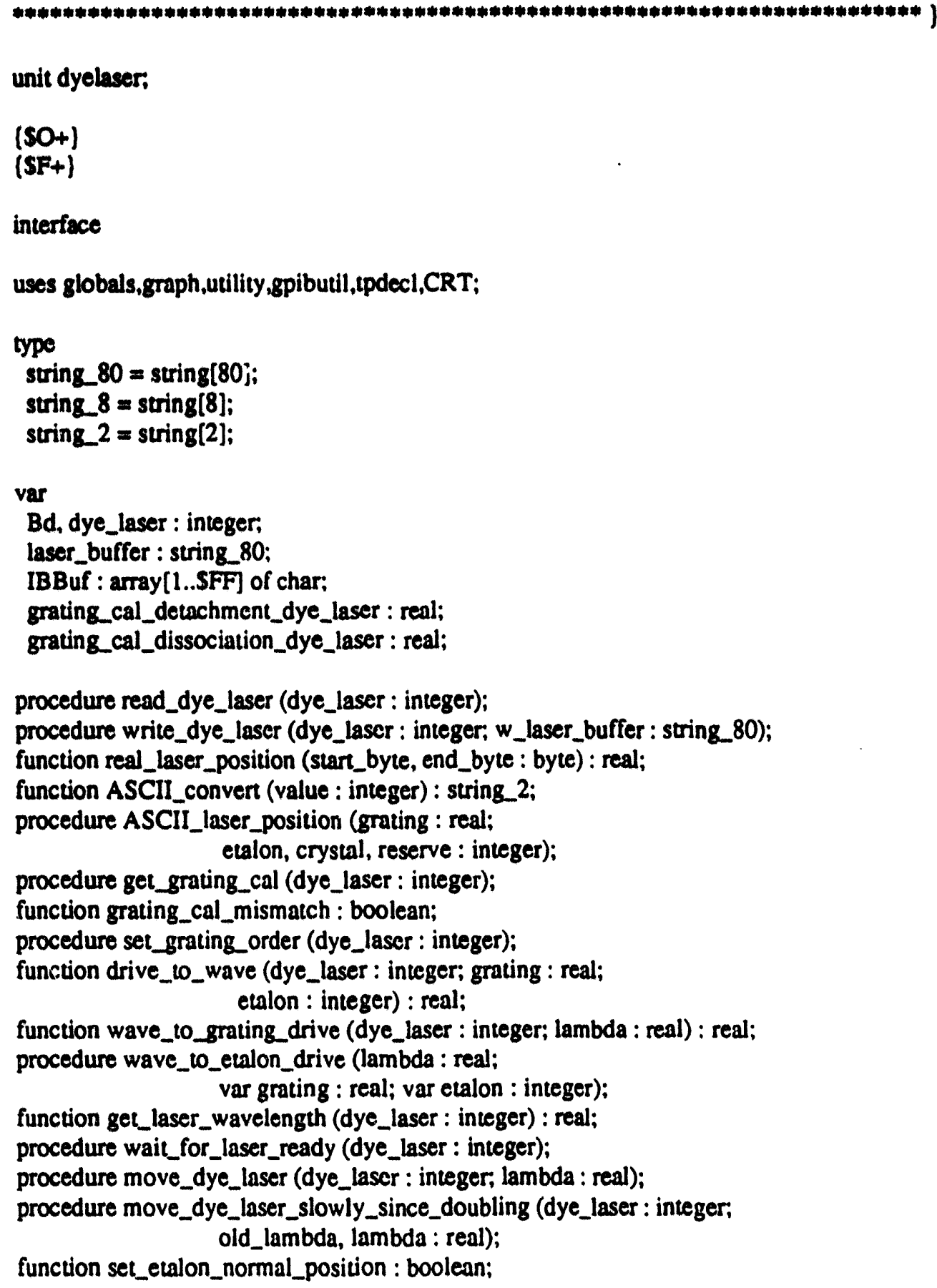


implementation

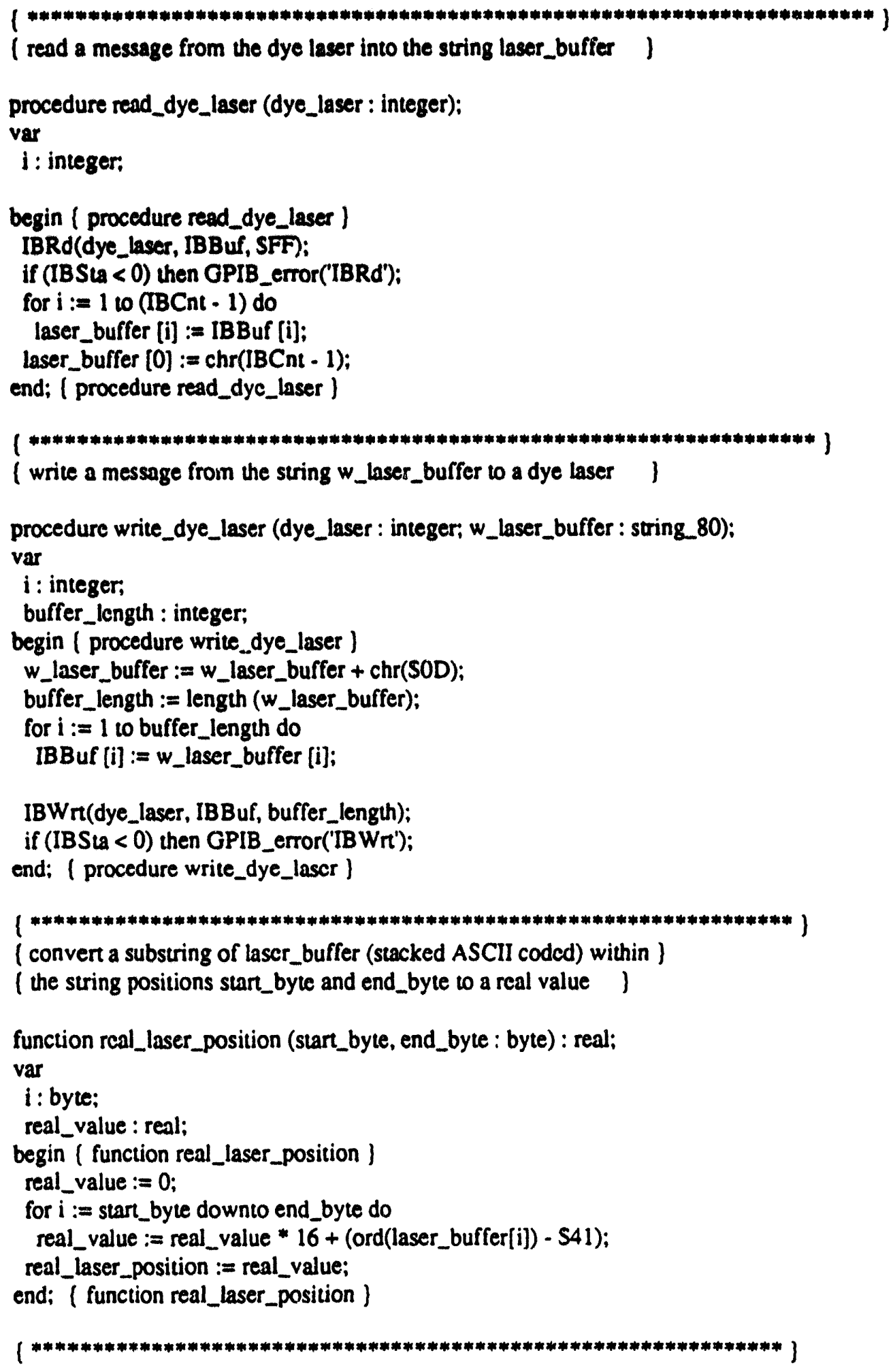


end: ( procedure get_grating_cal )

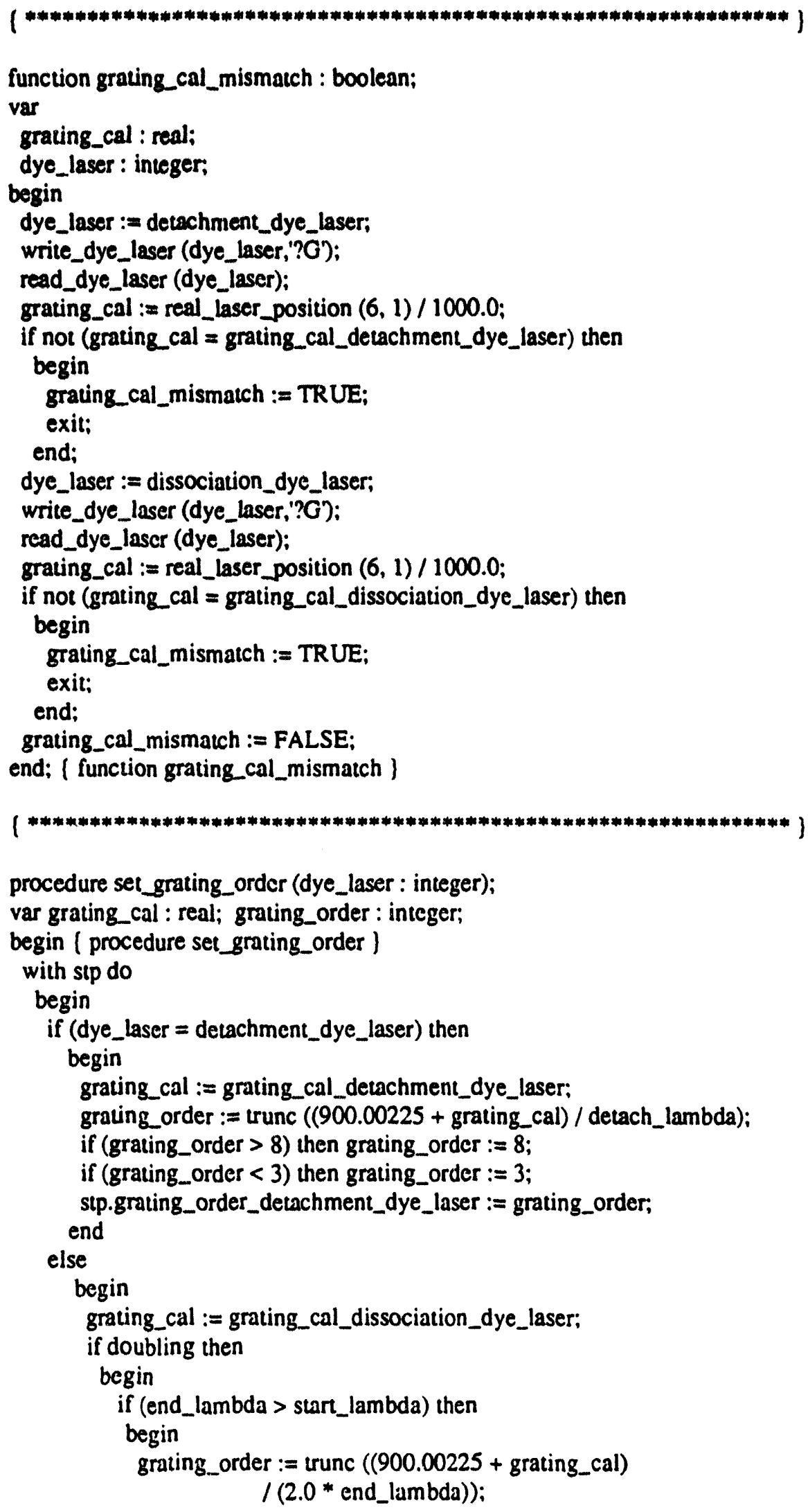




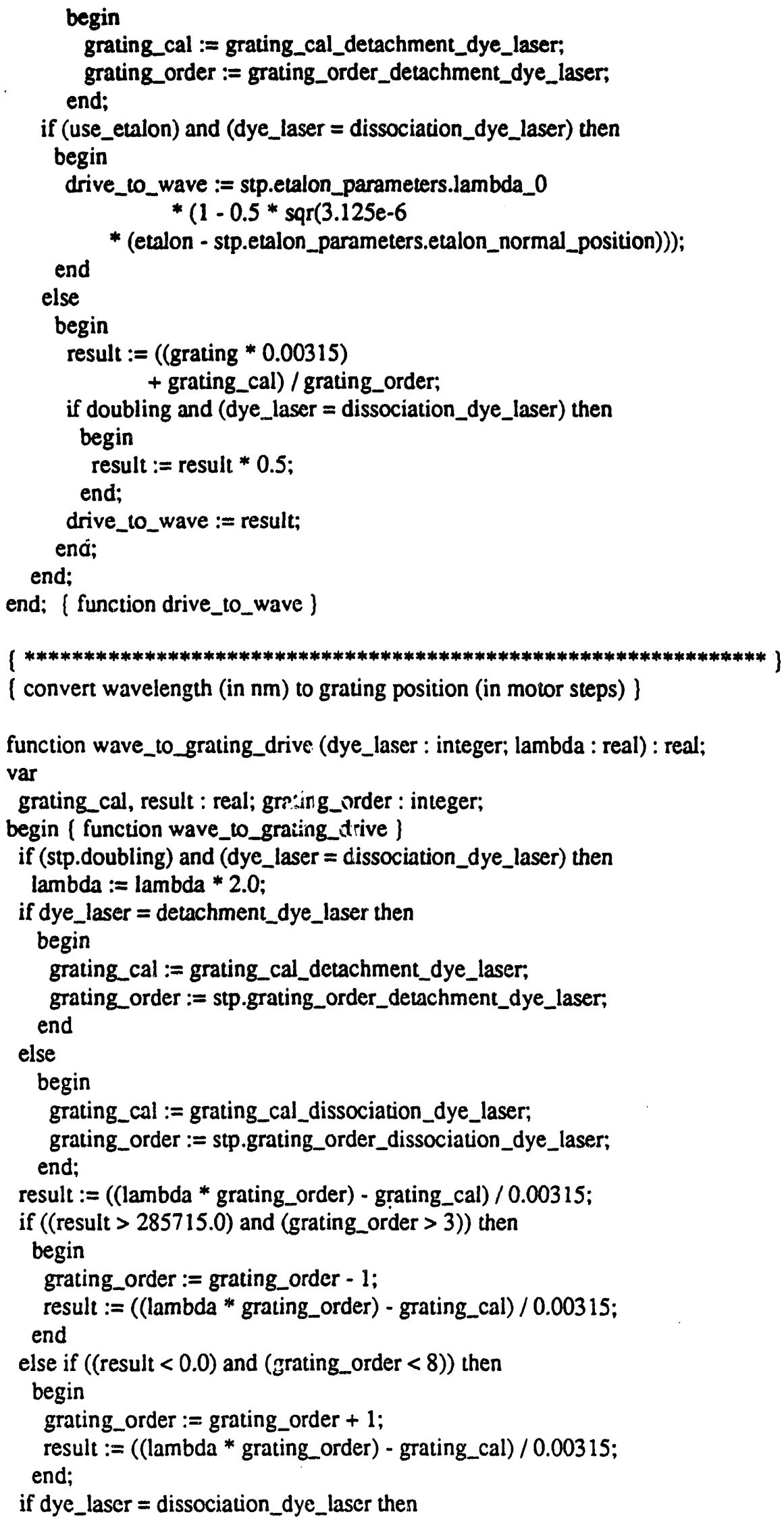


stp.grating_order_dissociation_dye_laser := grating_order

else

- stp.grating_order_detachment_dye_laser := grating_order;

wave_to_grating_drive := result:

end; ( function wave_to_drive )

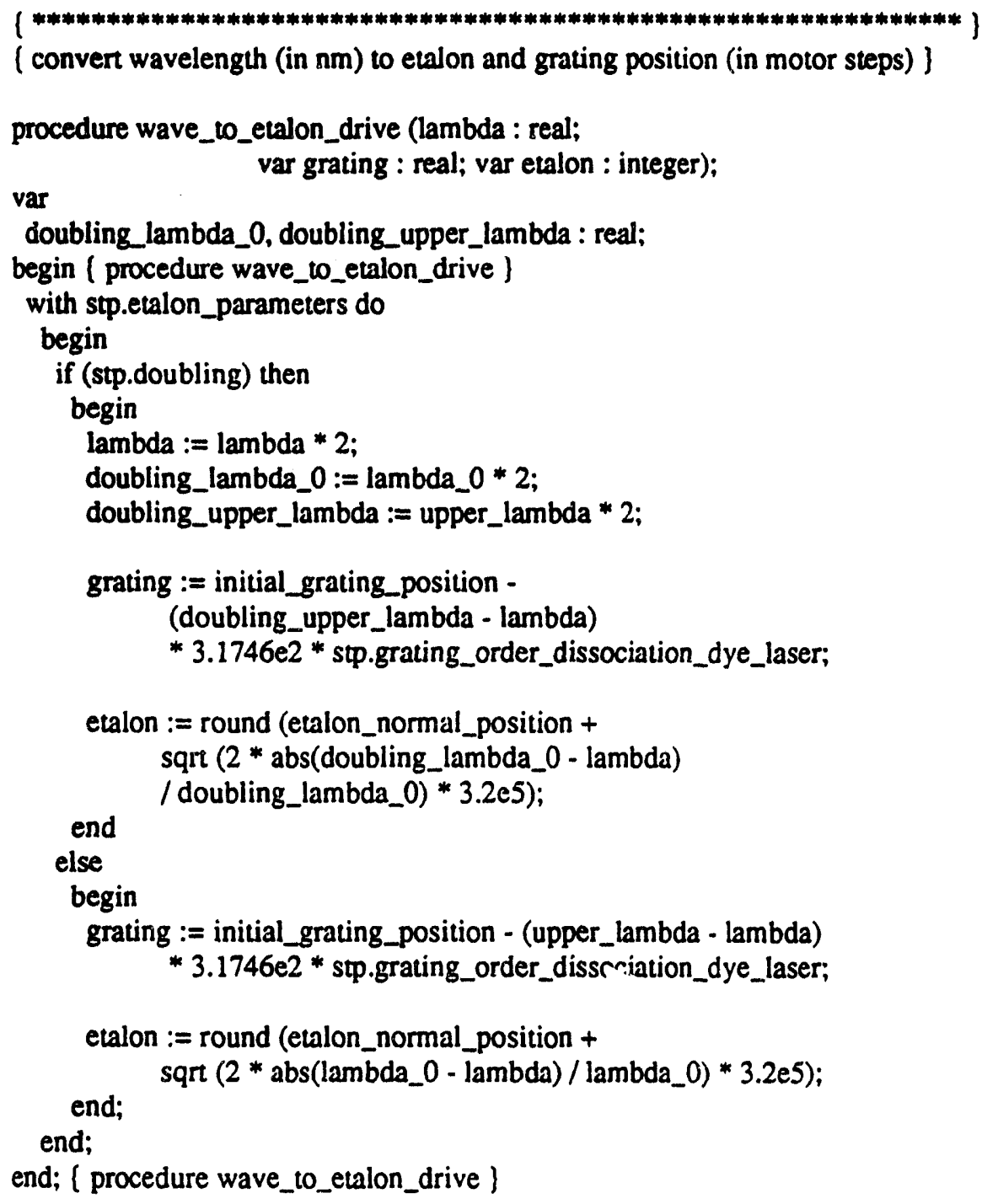




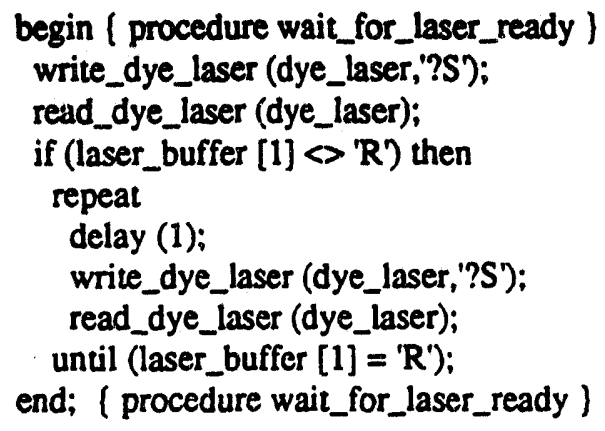




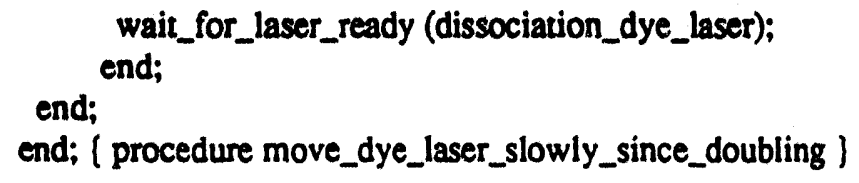




\subsection{4 twkplot.pas}

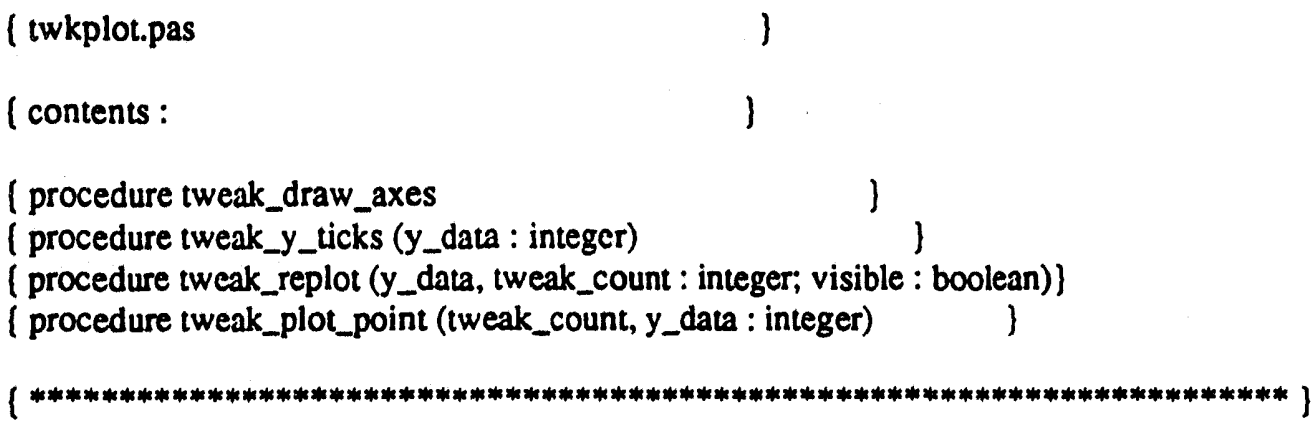

unit twkplot;

$\{\mathrm{SO}+\}$

$\{\mathrm{SF}+\}$

interface

uses globals, Lxsplot,plotutil,graph,graphadd,grafutil,CRT;

procedure tweak_draw_ticks (tick_axis : integer;

constant_limit : real:

code : integer;

label_ticks : boolean);

procedure tweak_draw_axes;

procedure tweak_y_ticks (y_data : integer);

procedure tweak_replot (y_data, tweak_count : integer; visible : boolean);

procedure tweak_replot_all (tweak_count : integer);

procedure tweak_plot_point (tweak_count, y_data : integer);

$\{* * * * * * * * * * * * * * * * * * * * * * * * * * * * * * * * * * * * * * * * * * * * * * * * * * * * * * * * * * * * * * * * * * * * * * * * *)\}$

implementation

procedure tweak_draw_ticks (tick_axis : integer;

constant_limit : real;

code : integer;

label_ticks : boolean);

var

xdotnum, ydotnum : integer;

tick_mark, tick_step : real;

tick_label : string[8];

decimal_places : integer;

begin \{ procedure tweak_draw_ticks \}

xdotnum :=4;

ydotnum :=4;

[ find tick step size ]

if (abs (tweak_plot_max [tick_axis] - tweak_plot_min [tick_axis]) $>=1.0$ ) 


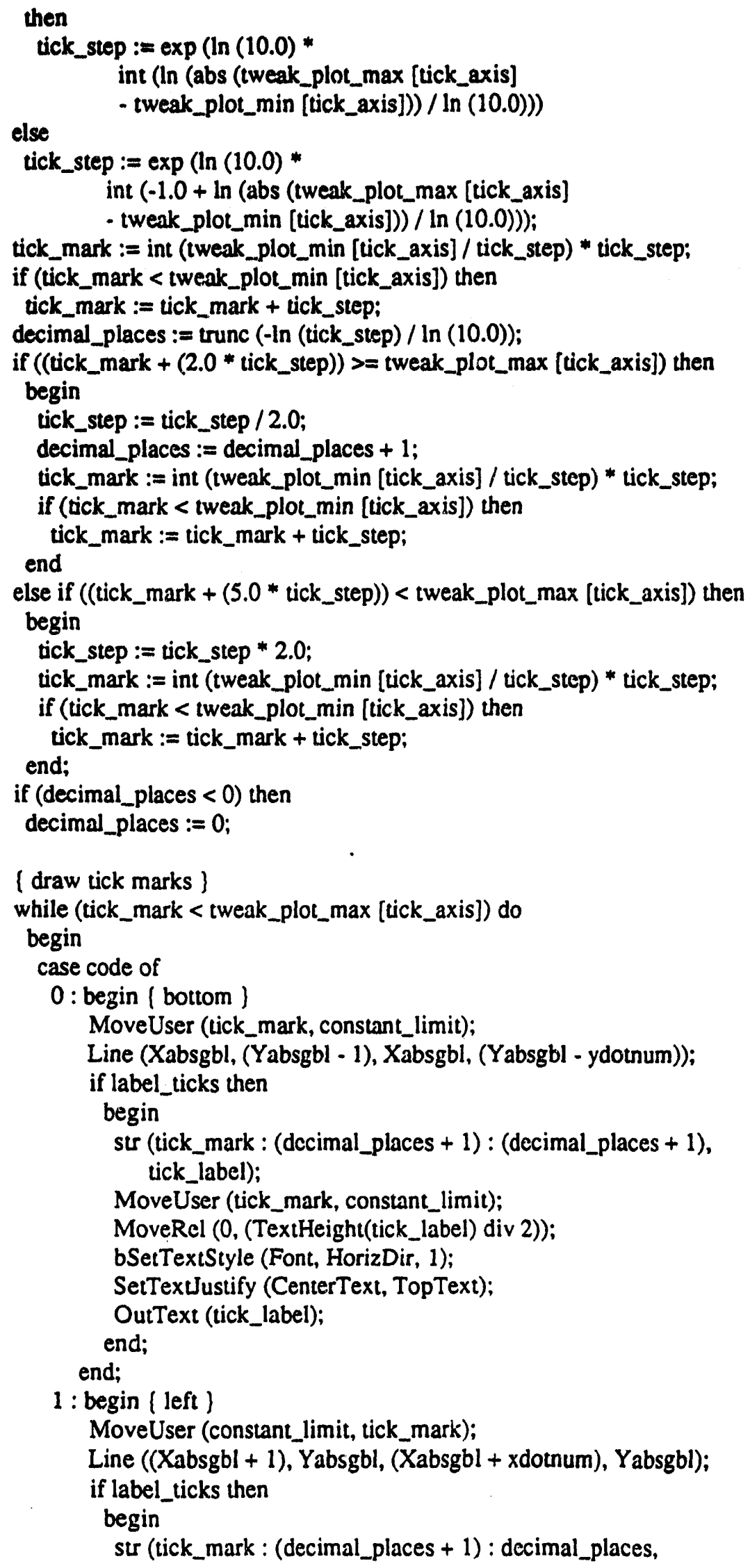




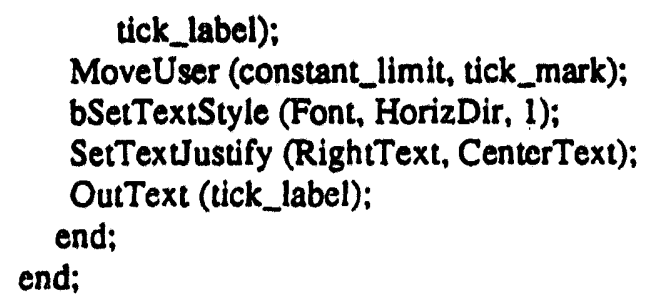




\section{SetColor (Lightoray);}

DrawLineUser (1.0, 1.0);

DrawLineUser (1.0, 0.0);

SetColor (Red):

DrawLineUser $(1.0,-1.0)$;

DrawLineUser $(0,-1.0)$;

SetColor (Cyan);

DrawLineUser $(0,1.0)$;

MoveUser (-1.0, 0.0);

DrawLineUser $(1.0,0.0)$;

txs_label_axes (detached_electron_signal);

txs_label_axes (laser_power);

txs_label_axes (dissociated_radical_signal);

txs_label_axes (normalized_signal);

end; [ procedure tweak_draw_axes ]

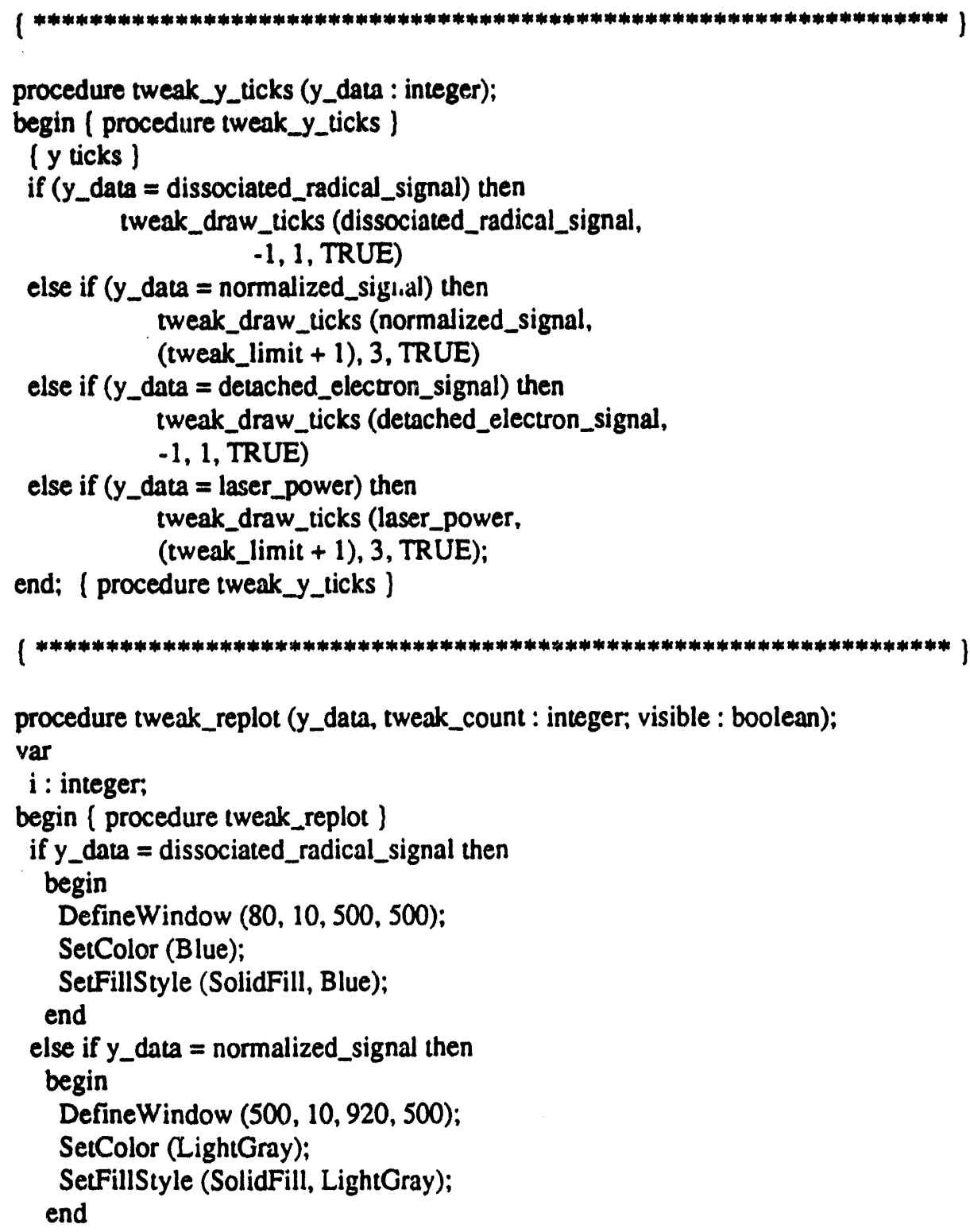




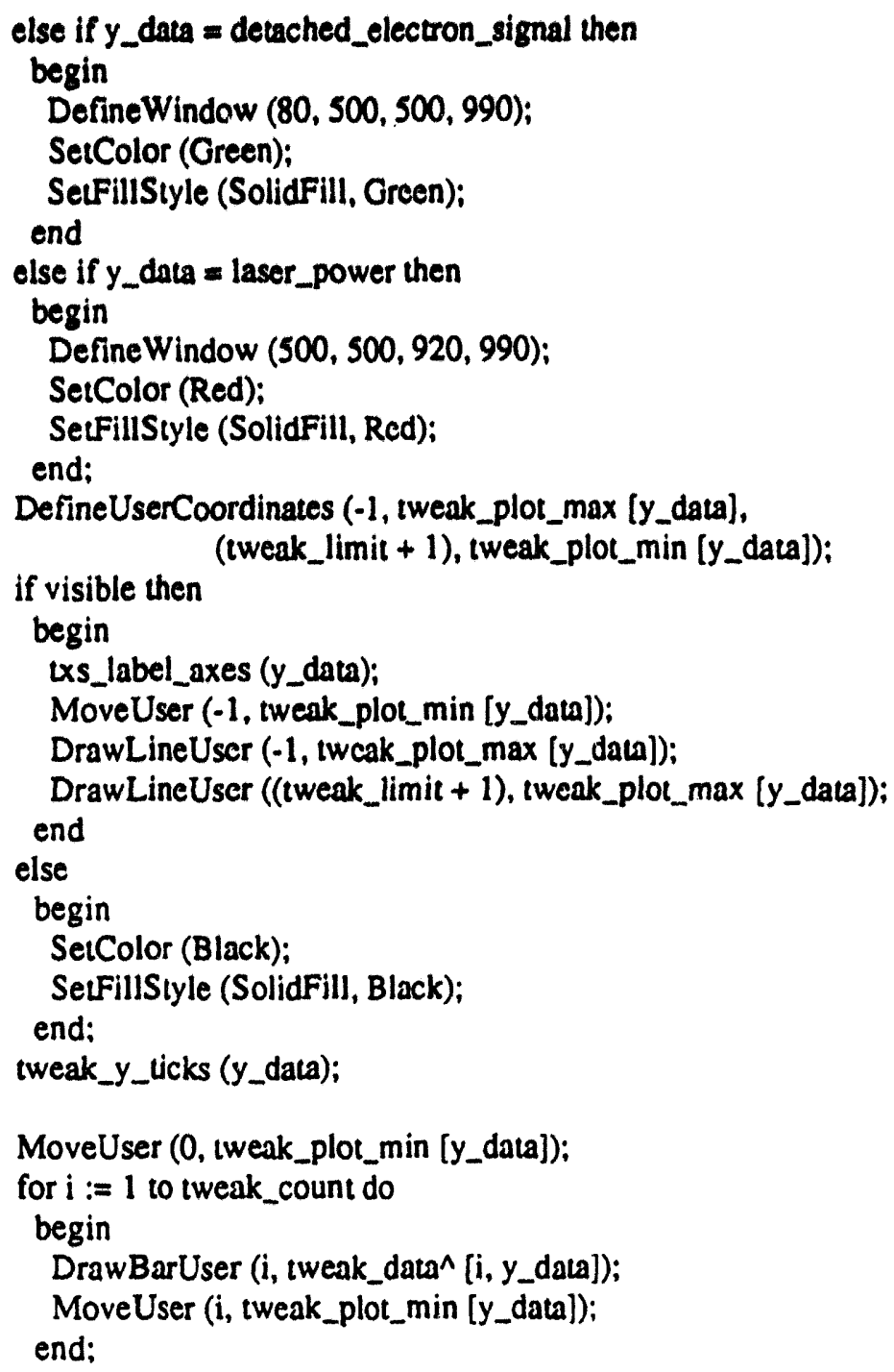




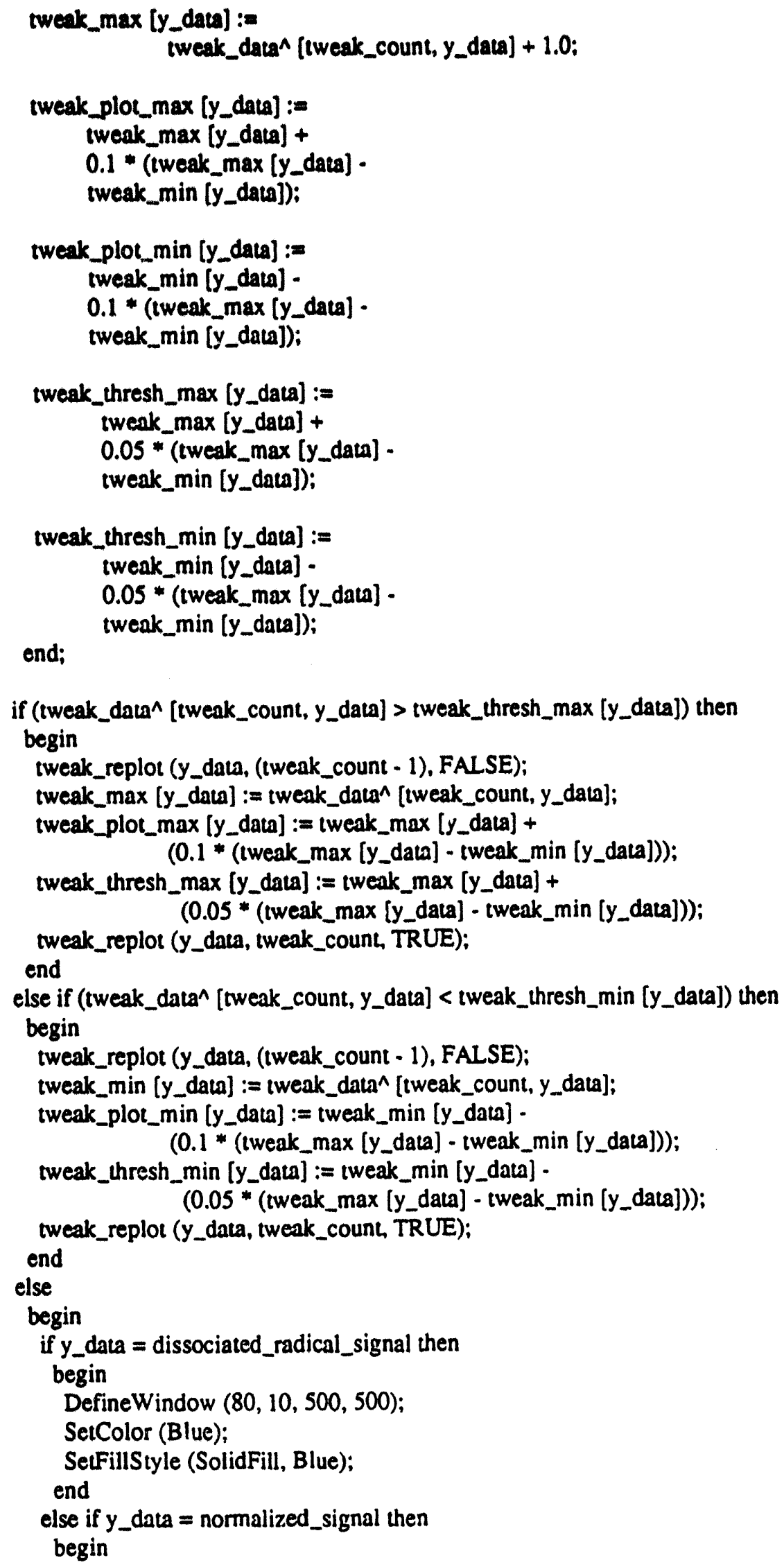

if (tweak_data^ [tweak_count, y_data] > tweak_thresh_max [y_data]) then 


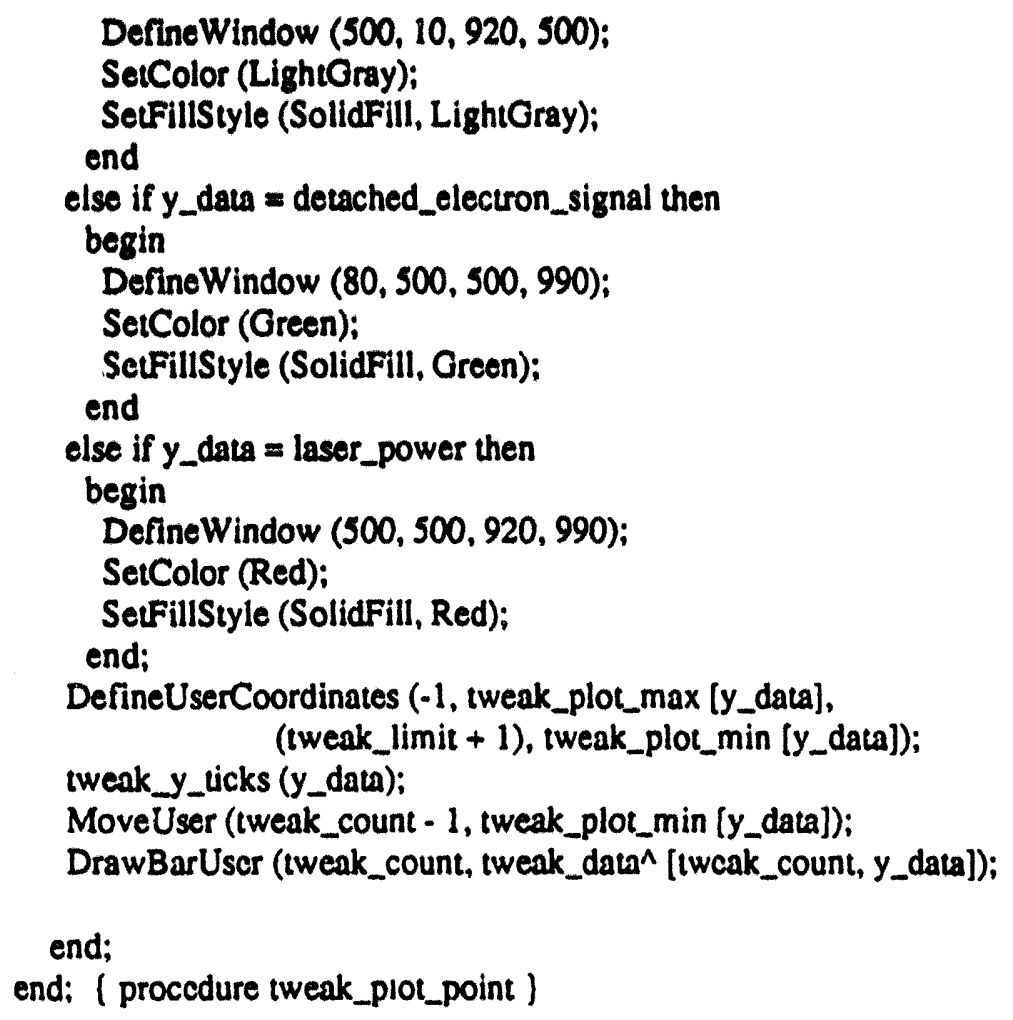

\subsection{5 txs_exp.pas}

( txs_exp.pas

l contents :

( procedure read_LeCroy_ADC (var data_point : real_column_array)

( read and average data from LeCroy ADC over 'shots_per_point' counts )

I without controlling laser

( procedure manual_experiment

i move laser from computer keyboard, a'so read LeCroy ADC

( procedure obtain_zeros

( obtain and store pedestal values of LeCroy ADC channels

( procedure save_data

( save data to individual '.***' file, (extension depends on type of \}

( experiment) append scan parameter info to one 'date.log' file \}

I per day

( function pause_menu : boolean 
I menu of things to do when experiment is paused

( function pause_experiment (var txs_count : integer) : boolean

1 pauses, cleans up, restarts experiment

I procedure initialize_for_Lxs_experiment

$f$ initializes all variables that must be fresh each time

1

( procedure run_txs_experiment

(data collection and display with computer controlled

I LeCroy ADC and laser

1

unit lxs_exp;

$(\mathrm{SO}+)$

(SF+]

interface

uses globals,txsparam,plotutil,txsplot,twkplot,dyelaser,

gpibutil,grafutil,graph,utility, tpdecl,codes,CRT,DOS;

procedure read_LeCroy_ADC (var data_point : txs_rcal_column_array);

procedure manual_experiment:

procedure obtain_zeros:

function pause_menu : boolean;

function pause_experiment (var txs_count : integer) : boolean;

procedure initialize_for_uxs_experiment;

procedure run_lxs_experiment:

(

implementation

(SF.)

[\$I camturbo.v4]

$\{\mathrm{SF}+\}$

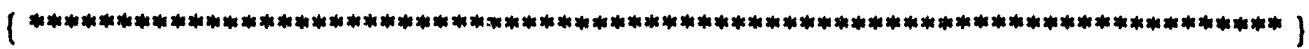

procedure read_LeCroy_ADC (var data_point : txs_real_column_array);

var

$D, Q, X$, txs_count : integer;

normalized_laser_power, normalized_detached_electron_signal : real;

begin (procedure read_LeCroy_ADC)

data_point [detached_electron_signal] $:=0.0$;

data_point [laser_power] $:=0 . \overline{0}$;

data_point [dissociated_radical_signal] $:=0.0$;

data_point [normalized_sigral] $:=0.0$; 
CAMCL (CRIACL):

CAMCL (dataway_z);

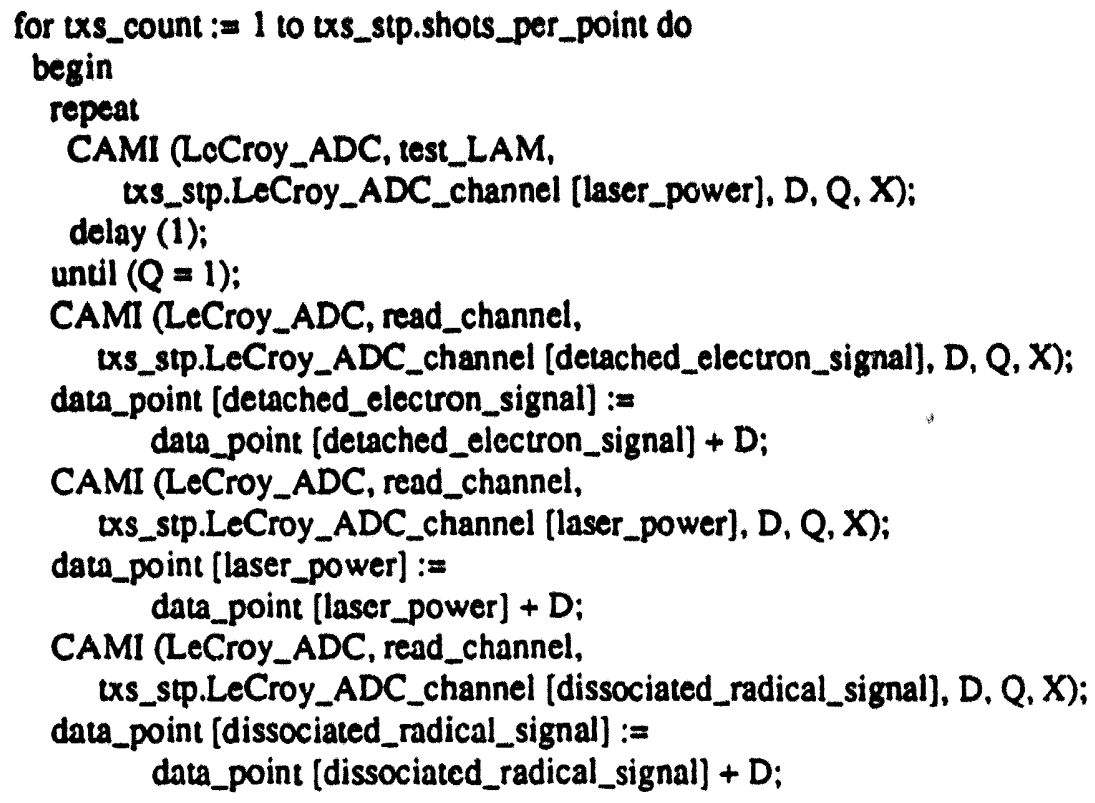

CAMCL (dataway_c);

end:

CAMCL (dataway_z);

CAMCL (dataway_i);

data_point [detached_electron_signal] :=

data_point [detached_electron_signal] / txs_stp.shots_per_point;

data_point [laser_power] :=

data_point [laser_power] / txs_stp.shots_per_point:

data_point [dissociated_radical_signal] :=

data_point [dissociated_radical_signal] / txs_stp.shots_per_point;

normalized_detached_electron_signal := data_point [detached_electron_signal]

- txs_stp.zero [detached_electron_signal];

if normalized_detached_electron_signal $<1.0$ then

normalized_detached_electron_signal := 1.0 ;

normalized_laser_power := data_point [laser_power] - txs_stp.zero [laser_power];

if normalized_laser_power $<1.0$ then normalized_laser_power $:=1.0$;

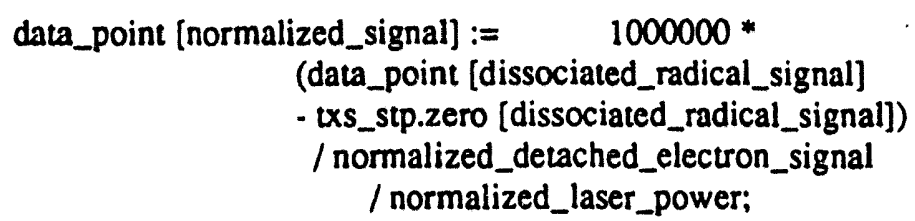




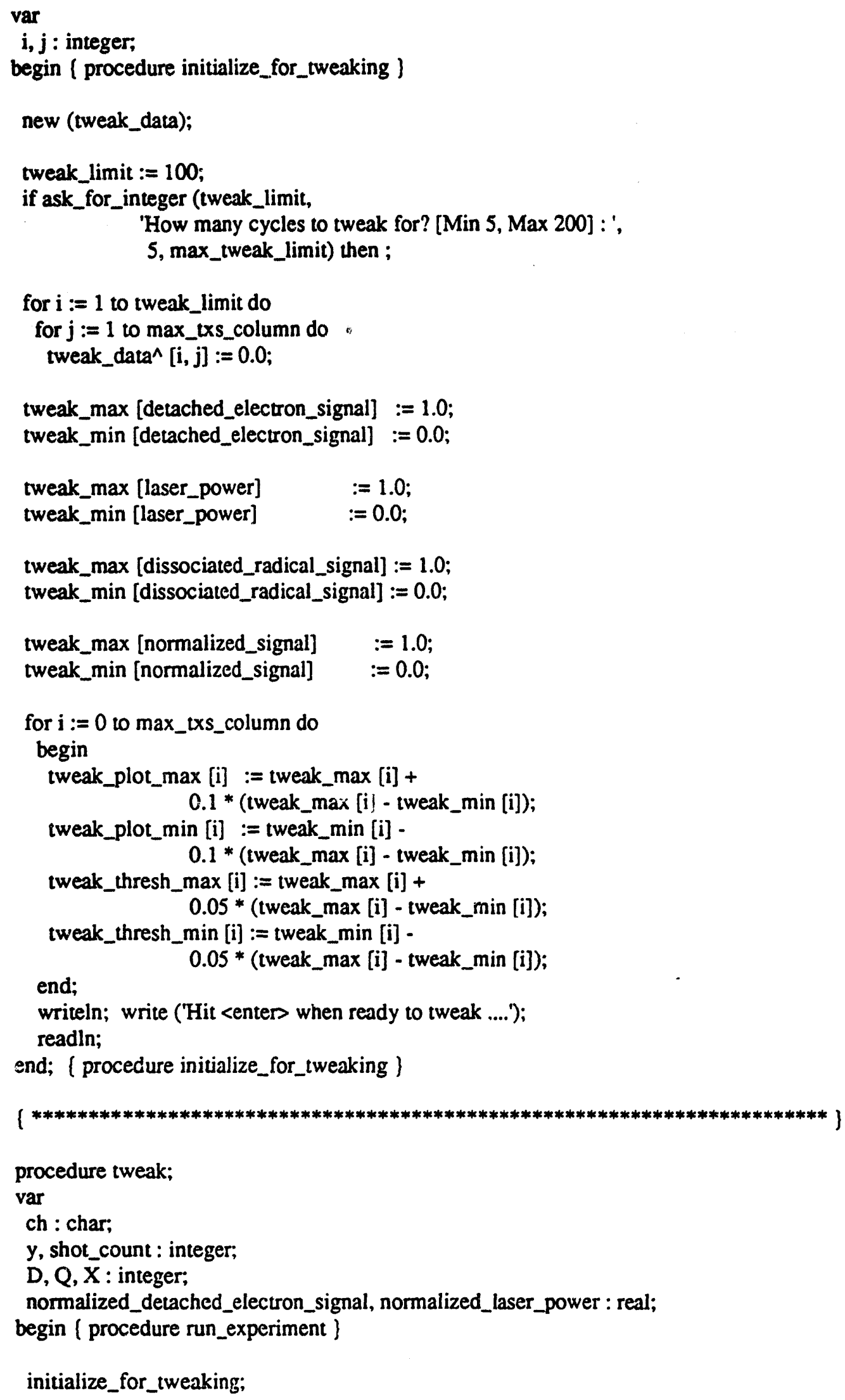




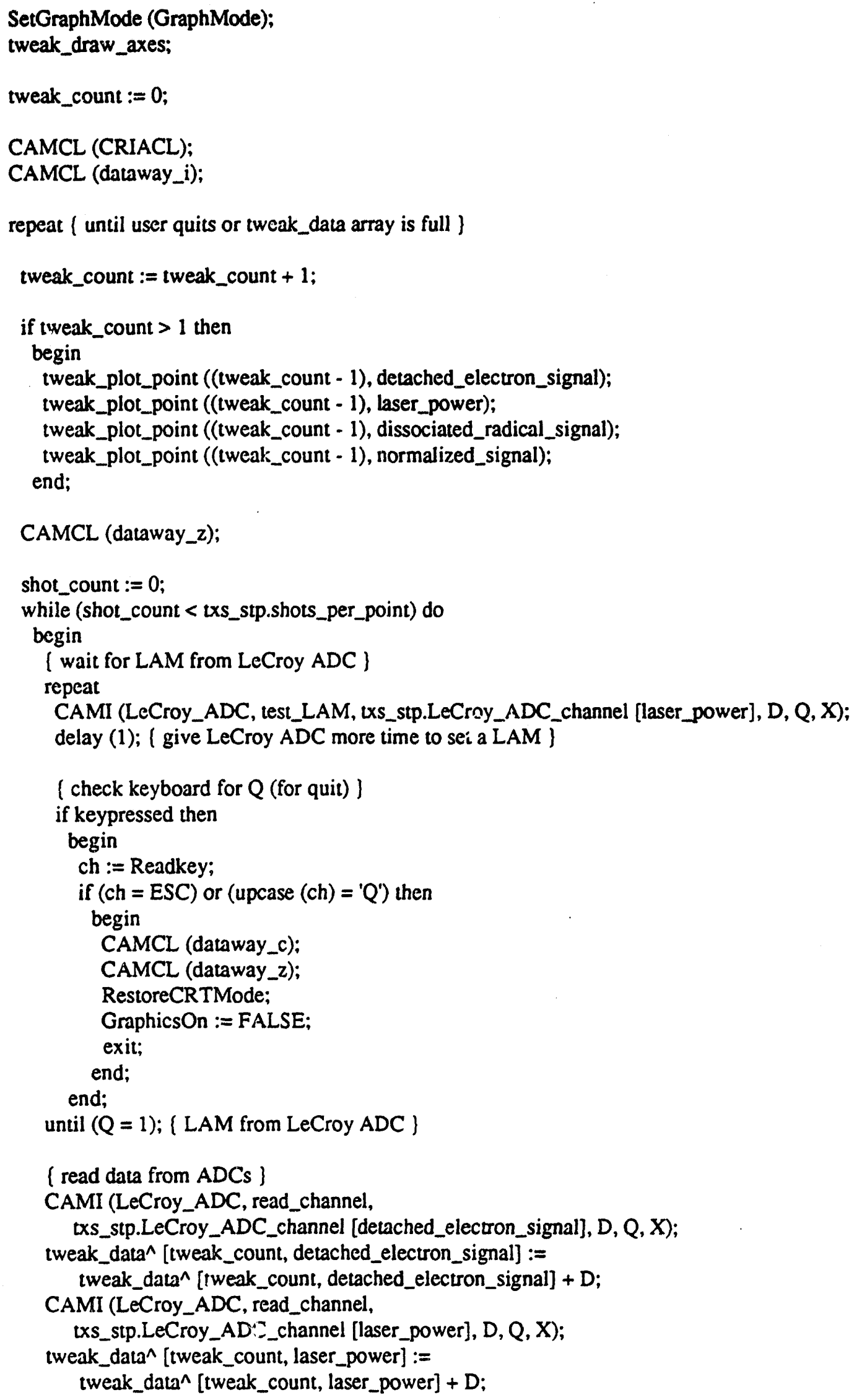


CAMI (LeCroy_ADC, read_channel,

txs_stp.LeCroy_ADC_channel [dissociated_radical_signal], $D, Q, X$ );

tweak_data^ [tweak_count, dissociated_radical_signal] :=

tweak_dati^ [tweak_count, dissociated_radical_signal] + D;

( clear all ADCs and LAM ]

CAMCL (dataway_c);

shot_count := shot_count +1 ;

end;

tweak_data^ [tweak_count, detached_electron_signal] :=

tweak_data^ [tweak_count, detached_electron_signal]

/ txs_stp.shots_per_point

- txs_stp.zero [detached_electron_signal];

tweak_data^[tweak_count, laser_power] :=

tweak_data^ [tweak_count, laser_power] / txs_stp.shots_per_point

- txs_stp.zero [laser_power];

tweak_data^ [tweak_count, dissociated_radical_signal] :=

tweak_data^ [tweak_count, dissociated_radical_signal]

/ txs_stp.shots_per_point

- txs_stp.zero [dissociated_radical_signal];

normalized_detached_electron_signal := tweak_data^ [tweak_count, detached_electron_signal];

if normalized_detached_electron_signal $<1.0$ then normalized_detached_electron_signal $:=1.0$;

normalized_laser_power := tweak_data^ [tweak_count, laser_power];

if normalized_laser_power $<1.0$ then normalized_laser_power $:=1.0$;

tweak_data^ [tweak_count, normalized_signal] := 100000 * tweak_data^ [tweak_count, dissociated_radical_signal]

/ normalized_detached_electron_signal

/ normalized_laser_power;

until tweak_count = tweak_limit;

tweak_plot_point (tweak_count, detached_electron_signal);

tweak_plot_point (tweak_count, laser_power);

tweak_plot_point (tweak_count, dissociated_radical_signal);

tweak_plot_point (tweak_count, normalized_signal);

CAMCL (dataway_z);

CAMCL (dataway_i);

set_done_flag;

ch := Readkey;

dispose (tweak_data);

RestoreCRTMode;

end: \{ procedure tweak \} 


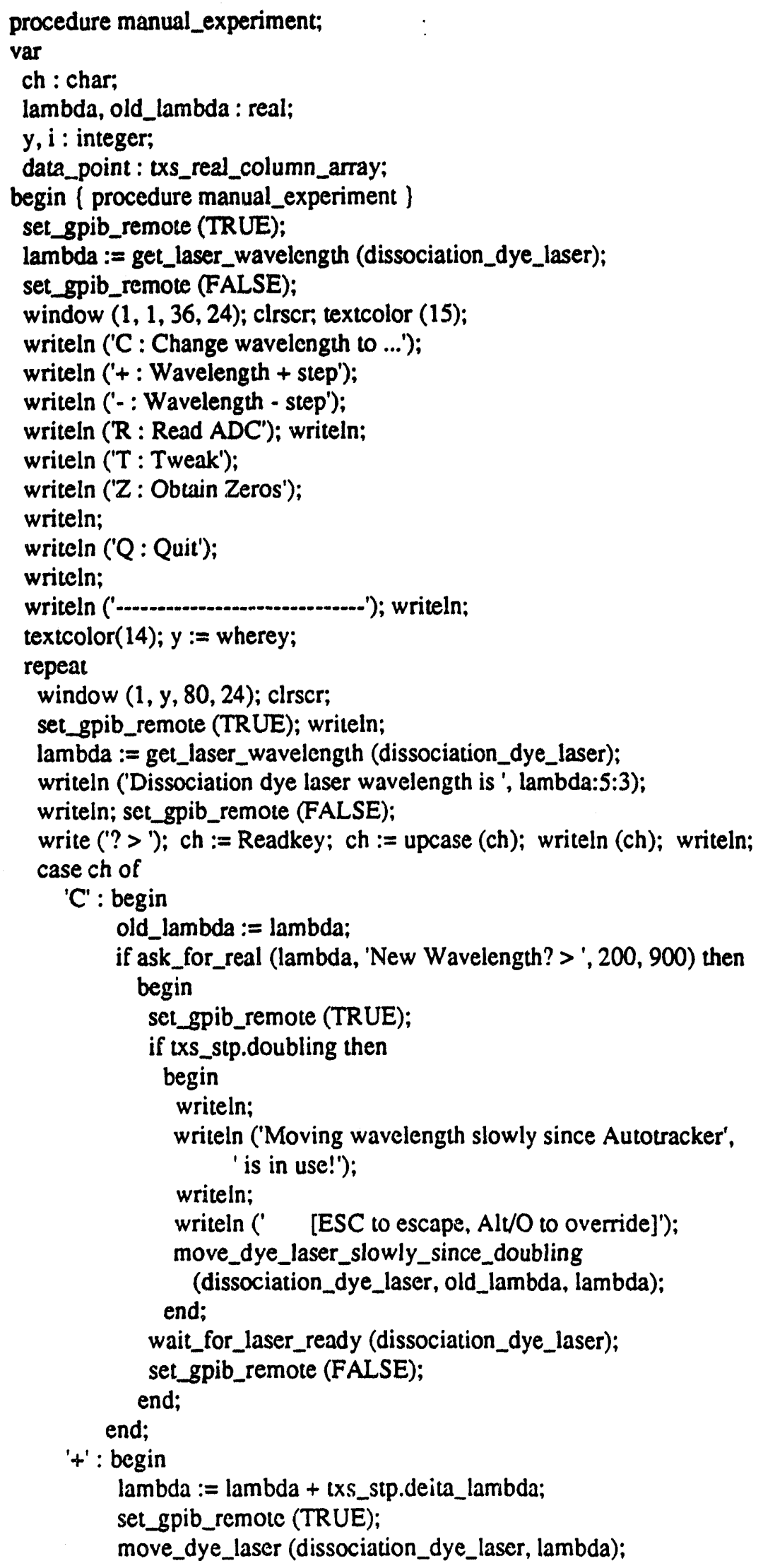




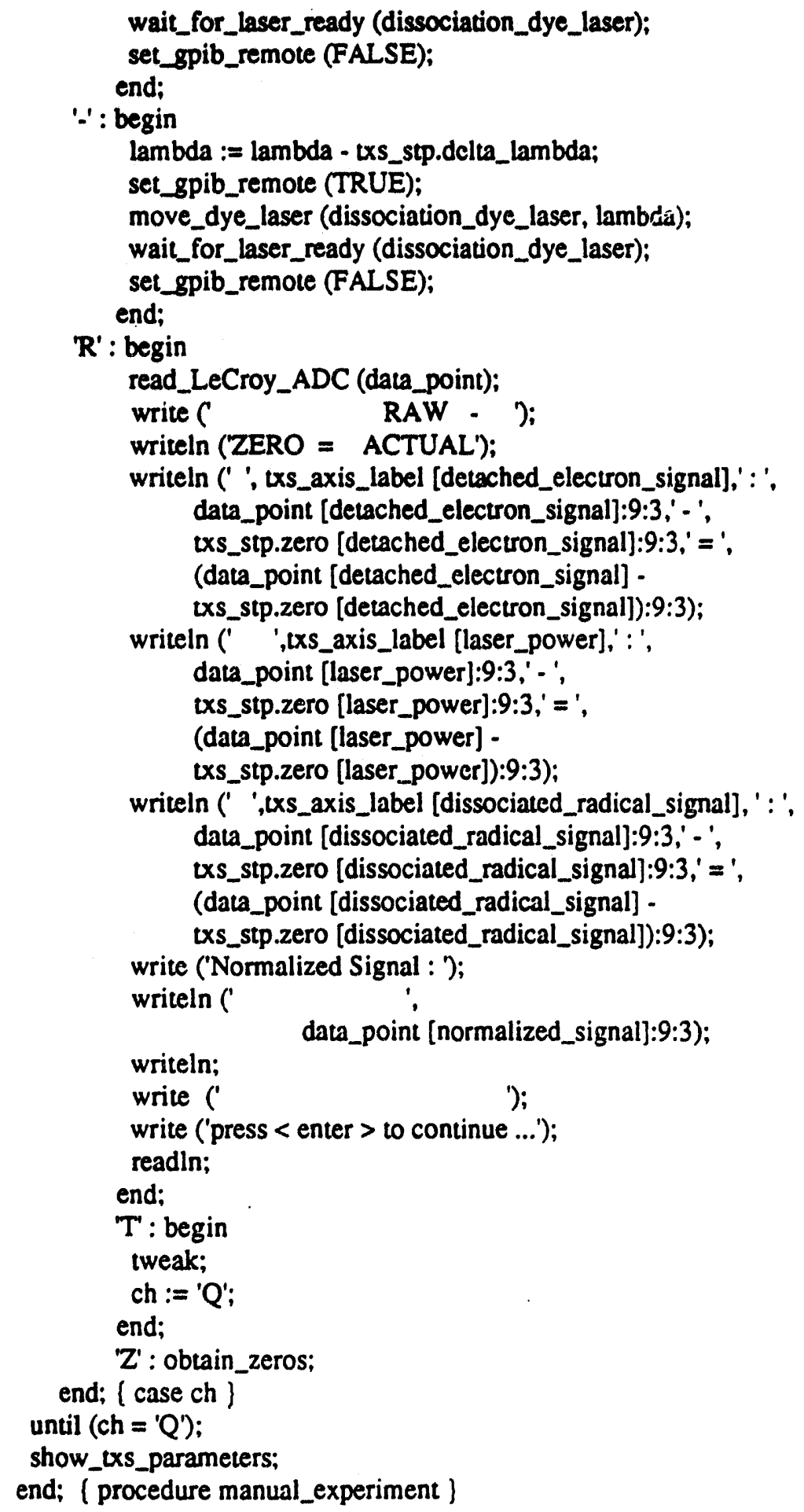




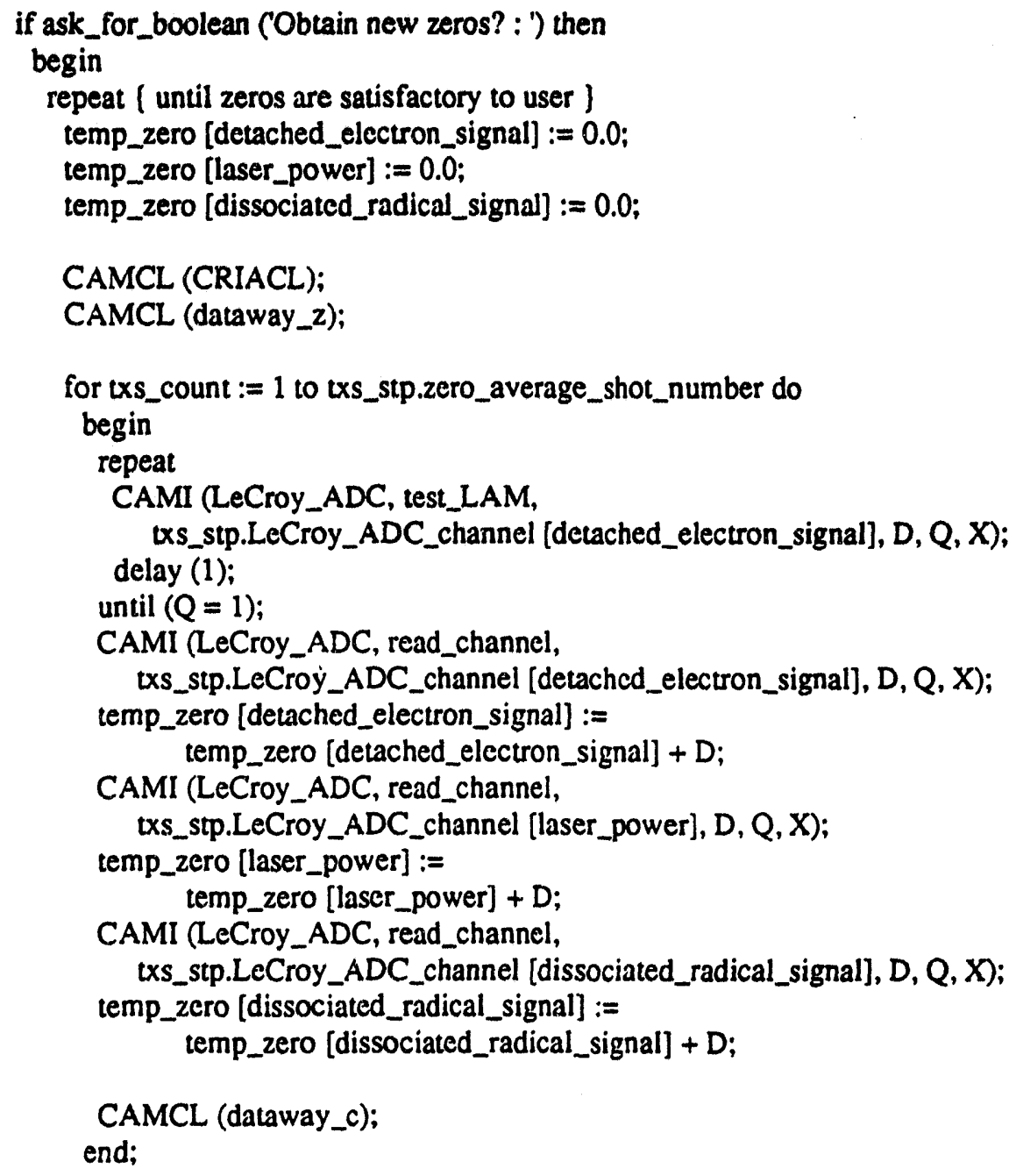

CAMCL (dataway_z);

CAMCL (dataway_i);

temp_zero [detached_electron_signal] := temp_zero [detached_electron_signal] / txs_stp.zero_average_shot_number;

temp_zero [laser_power] := temp_zero [lascr_power] / txs_stp.zero_average_shot_number;

temp_zero [dissociated_radical_signal] := temp_zero [dissociated_radical_signal] / txs_stp.zero_average_shot_number;

writeln; writeln ('New zeros:'); writeln; writeln (txs_axis_label [detached_electron_signal], ' : ', temp_zero [detached_electron_signal]:5:3); writcln (txs_axis_label [laser_power], ' :', tcmp_zero !laser_power]:5:3); writeln (txs_axis_label [dissociated_radical_signal], ' : ', temp_zero [dissociated_radical_signal]:5:3); writeln; 


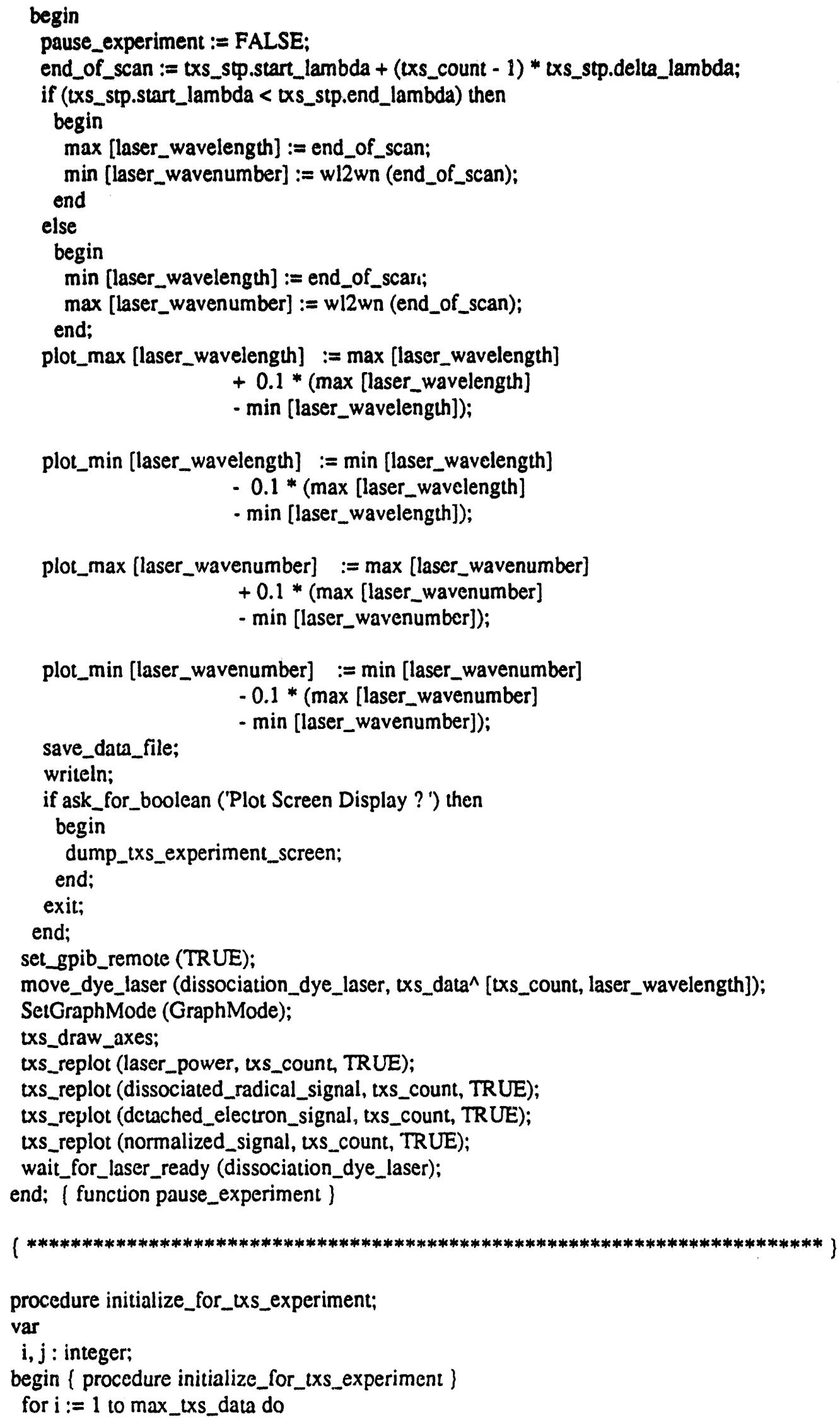




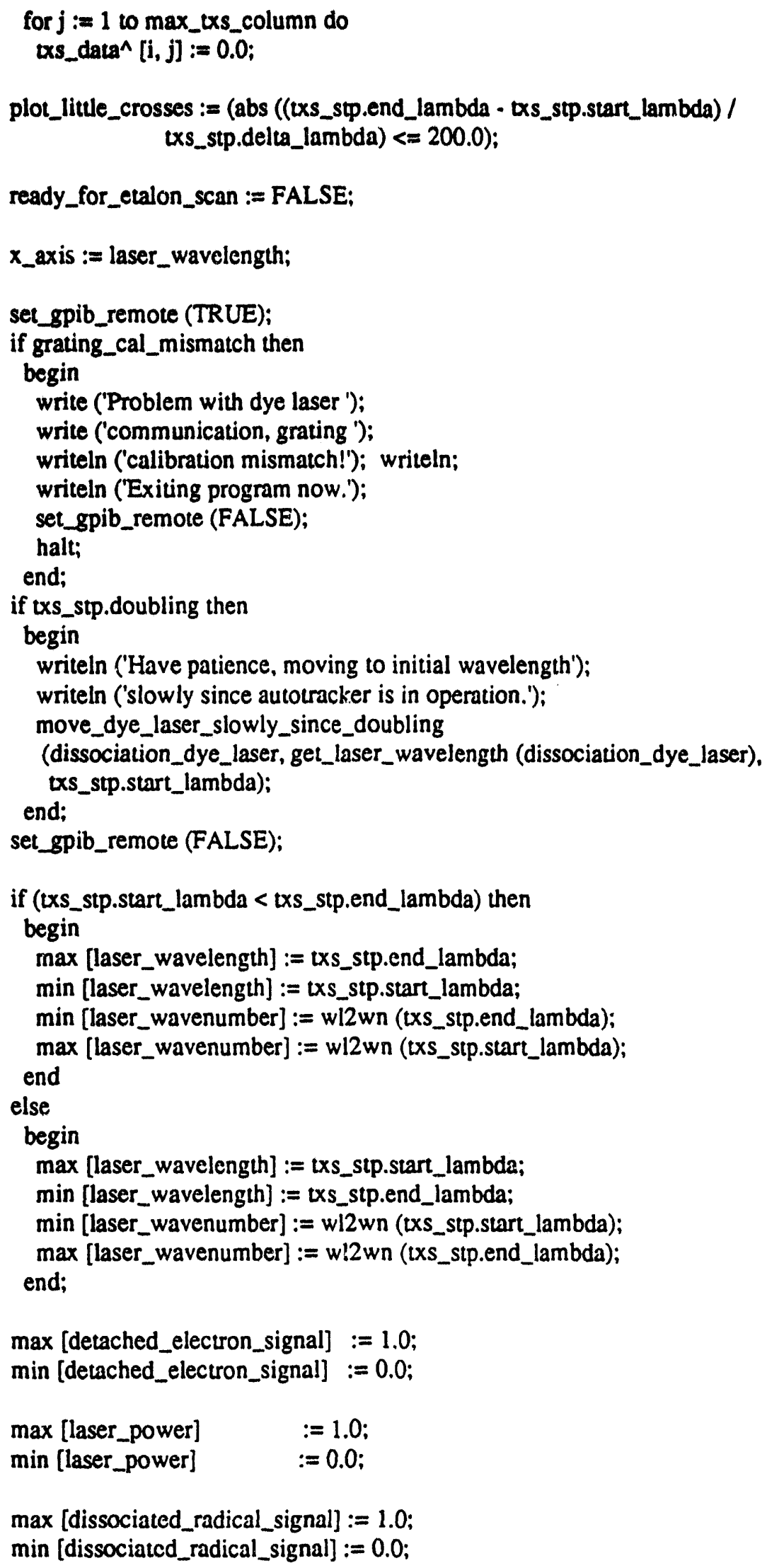




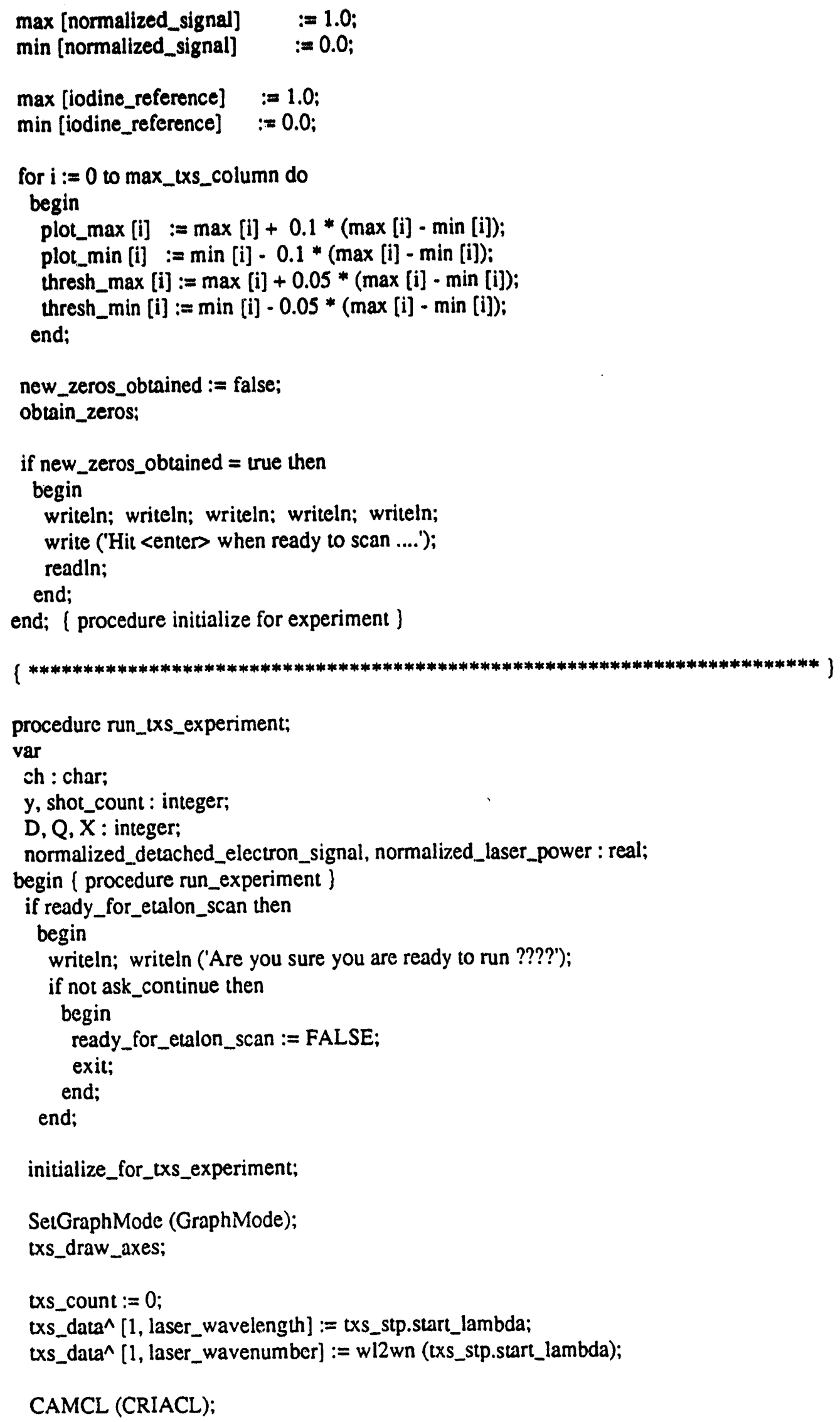


CAMCL (dataway_i);

set_spib_remote (TRUE);

repeat ( until scan is done or data array is full )

txs_count : $=$ uxs_count +1 ;

move_dye_laser (dissociation_dye_laser, txs_data^ [us_count, laser_wavelength]);

[ while laser is changing wavelength, plot last point ]

if (ux_count $>1$ ) then

begin

txs_plot_point ((txs_count - 1), detached_electron_signal);

txs_plot_point ((txs_count - 1), laser_power);

txs_plot_point ((txs_count - 1), dissociated_radical_signal);

txs_plot_point ((txs_count - 1), normalized_signal);

end;

wait_for_laser_ready (dissoniation_dye_laser);

[ask dissociation dye laser what its wavelength really is \}

txs_data^ [txs_count, laser_wavelength] := get_laser_wavelength (dissociation_dye_laser);

txs_data^ [txs_count, laser_wavenumber] := wl2wn (txs_data^ [txs_count, laser_wavelength]);

CAMCL (dataway_z);

shot_count : $=0$;

while (shot_count < txs_stp.shots_per_point) do

begin

(wait for LAM from LeCroy ADC )

repeat

CAMI (LeCroy_ADC, test_LAM, txs_stp.LeCroy_ADC_channel [laser_power], $D, Q, X$ );

delay (1); ( give LeCroy ADC morc time to set a $L .4 M$ )

[ check keyboard for $P$ (for pause)]

if keypressed then

begin

ch := Readkey;

if (upcase $(\mathrm{ch})=$ ' $\mathrm{P}$ ') then

begin

CAMCL (dataway_z); CAMCL (dataway_i); shot_count :=0;

txs_data^ [txs_count, detached_electron_signal] $:=0.0$;

Lxs_data^ [txs_count, laser_power] :=0.0;

txs_data [txs_count, dissociated_radical_signal] : $=0.0$;

if not pause_experiment (txs_count) then

begin

show_txs_parameters;

exit;

end;

CAMCL (dataway_c);

end;

end; 
until $(Q=1) ;($ LAM from LeCroy $A D C$ \}

( read data from ADCs)

CAMI (LeCroy_ADC, read_channel,

txs_stp.LeCroy_ADC_channel [detached_electron_signal], $D, Q, X)$;

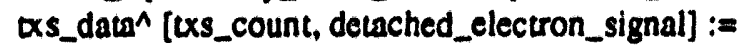

ixs_data^ [uxs_count, detached_electron_signal] + D;

CAMI (LeCroy_ADC, read_channel,

uxs_stp.LeCroy_ADC_channel [laser_power], $D, Q, X)$;

txs_data^ [txs_count, laser_power] := txs_data^ [txs_count, laser_power] $+D$;

CAMI (LeCroy_ADC, read_channel, Lxs_stp.LeCroy_ADC_channel [dissociated_radical_signal], $D, Q, X)$;

Lxs_data^ [txs_count, dissociated_radical_signal] := txs_data^ [txs_count, dissociated_radical_signal] + D;

( clear all ADCs and LAM ]

CAMCL (dataway_c);

shot_count $:=$ shot_count +1 ;

end;

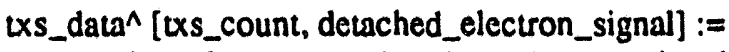

txs_data^[txs_count, detached_electron_signal] / txs_stp.shots_per_point

- txs_stp.zero [detached_electron_signal];

txs_data^ [txs_count, laser_power] :=

txs_data^ [txs_count, laser_power] / Lxs_stp.shots_per_point

- txs_stp.zero [laser_power];

Lxs_data^ [Lxs_count, dissociated_radical_signal] :=

txs_data^ [txs_count, dissociated_radical_signal] / txs_stp.shots_per_point

- txs_stp.zero [dissociated_radical_signal];

normalized_detached_electron_signal :=

txs_data^ [txs_count, detached_electron_signal];

if normalized_detached_electron_signal $<1.0$ then normalized_detached_electron_signal $:=1.0$;

normalized_laser_power :=

uxs_data^ [xx_count, laser_power];

if normalized_laser_power $<1.0$ then

normalized_laser_power $:=1.0$;

txs_data^ [txs_count, normalized_signal] := $1000000^{*}$ txs_data^ [txs_count, dissociated_radical_signal]

/ normalized_detached_electron_signal

/ normalized_laser_power;

Lxs_data^ $[($ txs_count +1$)$, laser_wavelength $]:=$ txs_stp.start_lambda + (txs_count * txs_stp.delta_lambda);

Lxs_data^ [( Lxs_count +1$)$, laser_wavenumber] := wl 2 wn (txs_data^ $[($ txs_count +1$)$, laser_wavelength $])$;

until (((txs_stp.start_lambda < txs_stp.end_lambda) and

(txs_data^ [(txs_count+1), laser_wavelength] $>$

(xs_stp.end_lambda + (txs_stp.delta_lambda / 2.0)))) or

((txs_stp.start_lambda $>$ txs_stp.end_lambda) and

(txs_data^ [ $($ txs_count +1$)$, laser_wavelength] $<$

(txs_stp.end_lambda + (txs_stp.delta_lambda / 2.0)))) or 
(txs_count = max_txs_data));

us_plot_point (us_count, detached_electron_signal);

ux_plot_point (txs_count, laser_power);

ux_plot_point (exs_count, dissociated_radical_signal);

txs_plot_point (txs_ccount, normalized_signal);

set_spib_remote (FALSE);

CAMCL (dataway_z);

CAMCL (dataway_i);

set_done_flag;

ch := Readkey;

RestoreCRTMode;

save_data_file;

writeln; writeln:

if ask_for_boolean ('Output screen display to LaserWriter? ') then begin

dump_txs_experiment_screen;

end;

show_txs_parametcrs;

end; ( procedure run_txs_experiment )

end. (unit Lxs_exp )

\subsection{6 tofms.pas}

I tofms.pas

This unit allows the main FRBM program to take mass spectra as an option and stores the mass spectra data files as .tof files with associated $\log$ files to be stored as .tof files

unit tofnis;

[SO+]

[SF+]

interface

uses

graph,graphadd, grafutil,tofplot,plotutil,globals, utility,DOS,CRT;

type

integer_24 $=a x ., y[0 . .2]$ of byte;

var

$f, a, d, x, q$, 


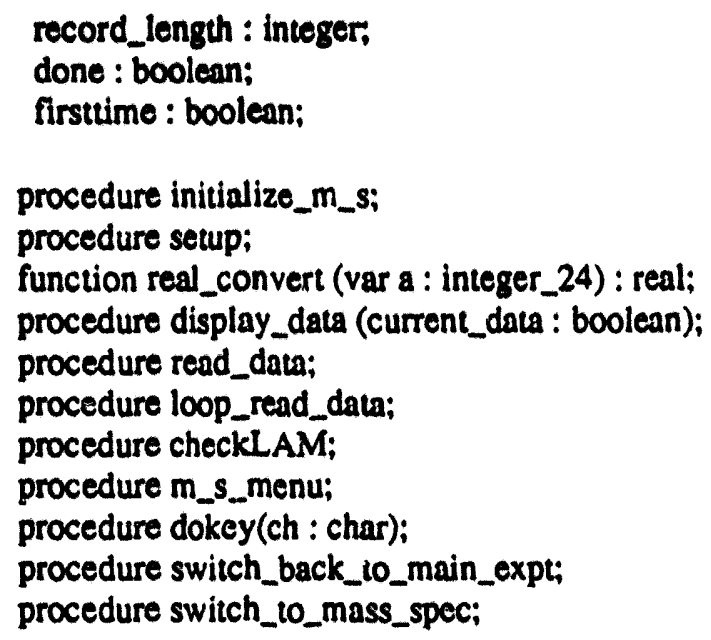

[\$I camturbo.v4]

[SF+]

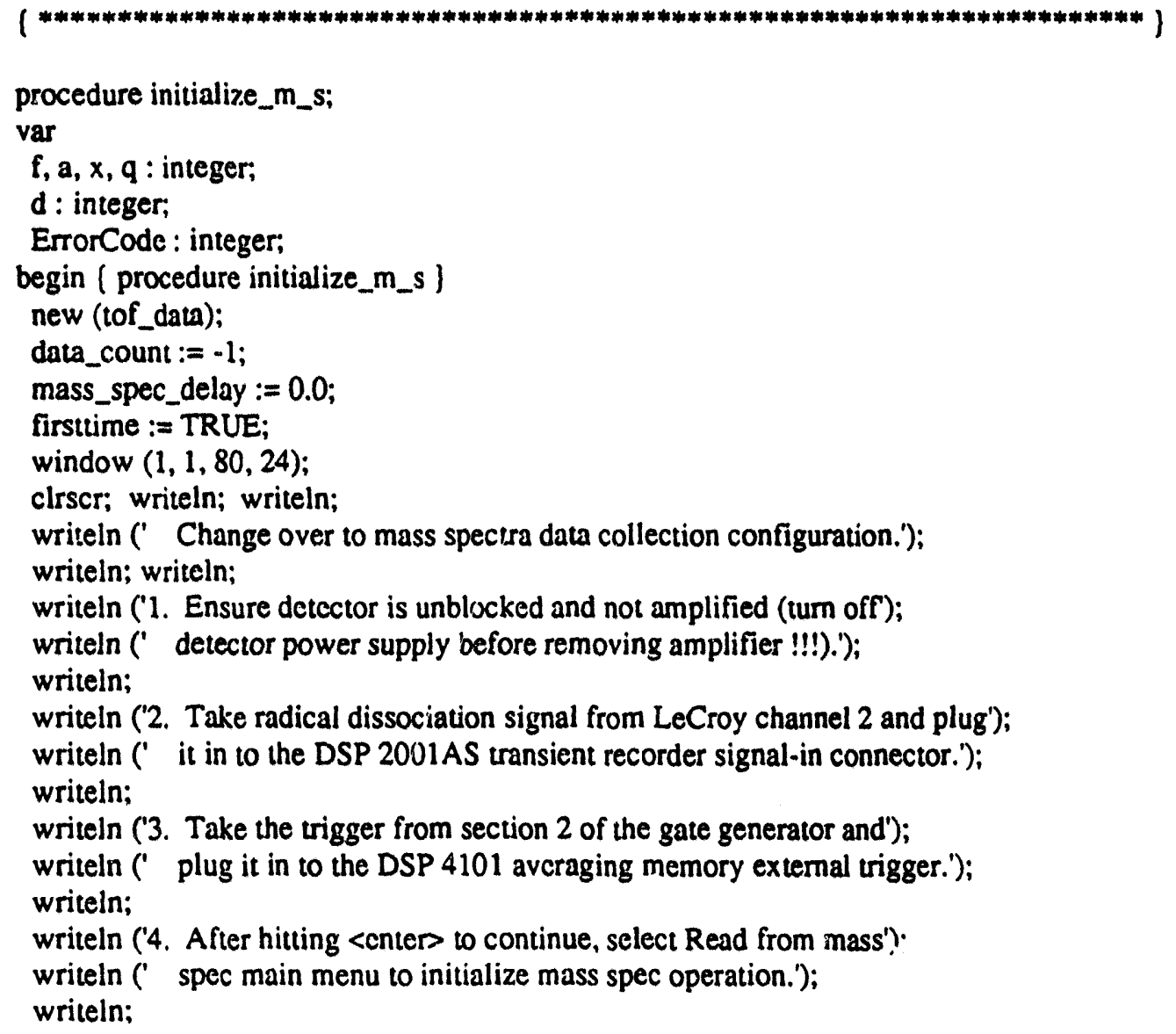


readln;

\{disable averaging \}

$f:=24 ; a:=1 ; d:=0$;

CAMO (memory, $f, a, d, x, q$ );

\{ enable LAM on CAMAC dataway \}

$\mathrm{f}:=26 ; \mathrm{a}:=0 ; \mathrm{d}:=0$;

CAMO (memory, $f, a, d, x, q$ );

\{ initialize digitizer control register for: \}

\{ 0 pretrigger samples, $1 \mathrm{k}$ record length, 10ns sampling interval \}

digitizer_control $:=\$ 0018$;

$\mathrm{f}:=16 ; \mathrm{a}:=0$;

CAMO (digitizer, $f, a$, digitizer_control, $x, q$ );

\{ initialize memory control register for : \}

( O TRAQ channels, sweep terminated after each trigger, subtraction mode, \}

\{ 2's complement arithmetic, stop averaging on overflow, \}

\{ external trigger disabled \}

memory_control $:=\$ 0060$;

$\mathrm{f}:=16 ; \mathrm{a}:=2$;

CAMO (memory, $f, a$, memory_control, $x, q$ );

\{ initialize memory record length to $1 \mathrm{k}$ \}

record_length $:=\$ 0400$;

$f:=16 ; a:=3$;

CAMO (memory, $f, a$, record_length, $x, q$ );

[ initialize memory sweeps register to 200 sweeps ]

sweeps_to_average $:=\$ 00 \mathrm{C} 8$;

$\mathrm{f}:=17 ; \mathrm{a}:=0 ; \mathrm{d}:=$ SFFFF - sweeps_to_average +1 ;

CAMO (memory, $f, a, d, x, q$ );

[ reset internal LAM \}

$\mathrm{f}:=10 ; \mathrm{a}:=0 ; \mathrm{d}:=0$;

CAMO (memory, f, a, d, x, q);

\{ reset memory and digitizer -- dataway $Z$ \}

$\mathrm{f}:=1$;

CAMCL (f);

delay ((record_length div 4000) + 1);

end; (procedure initialize_m_s

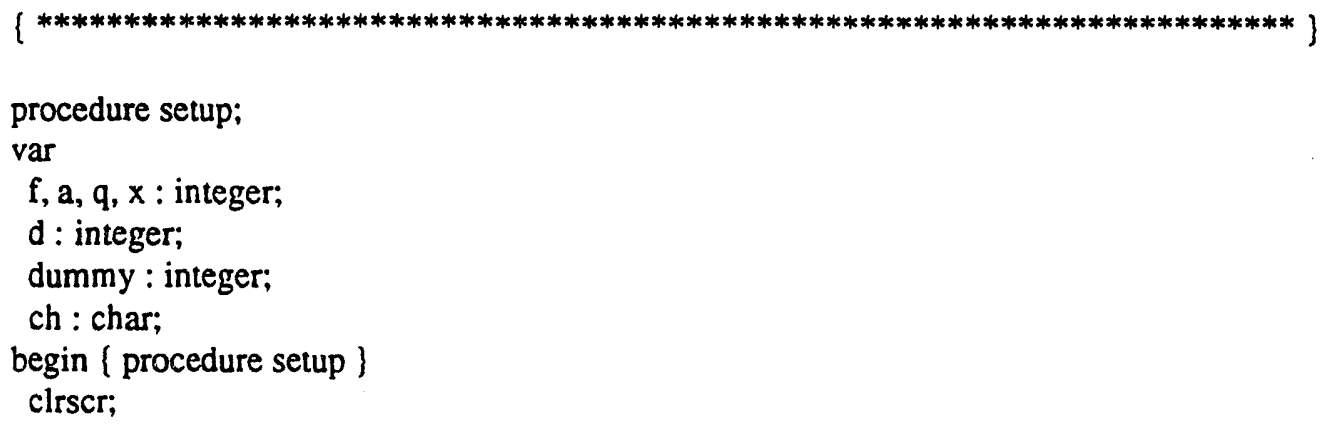




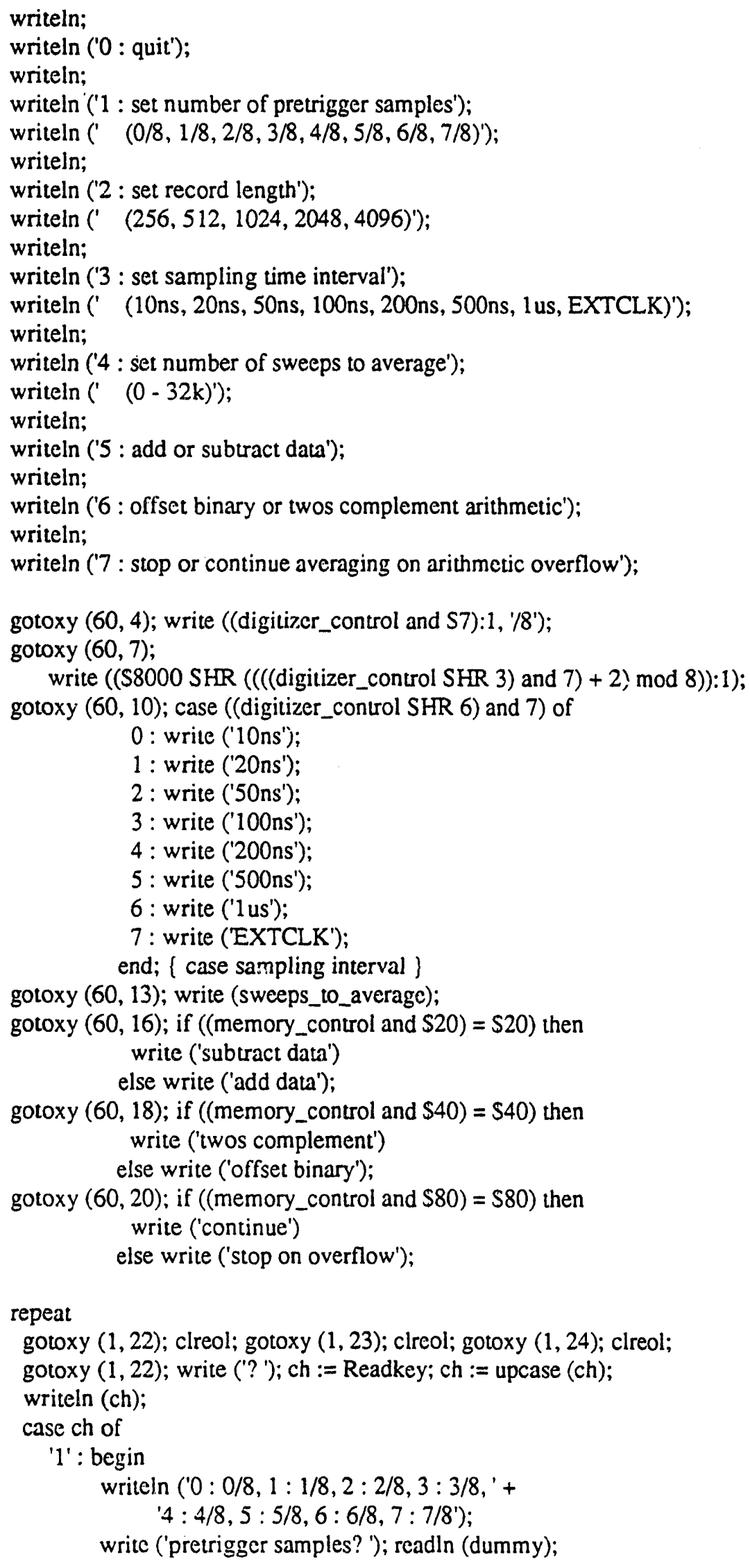




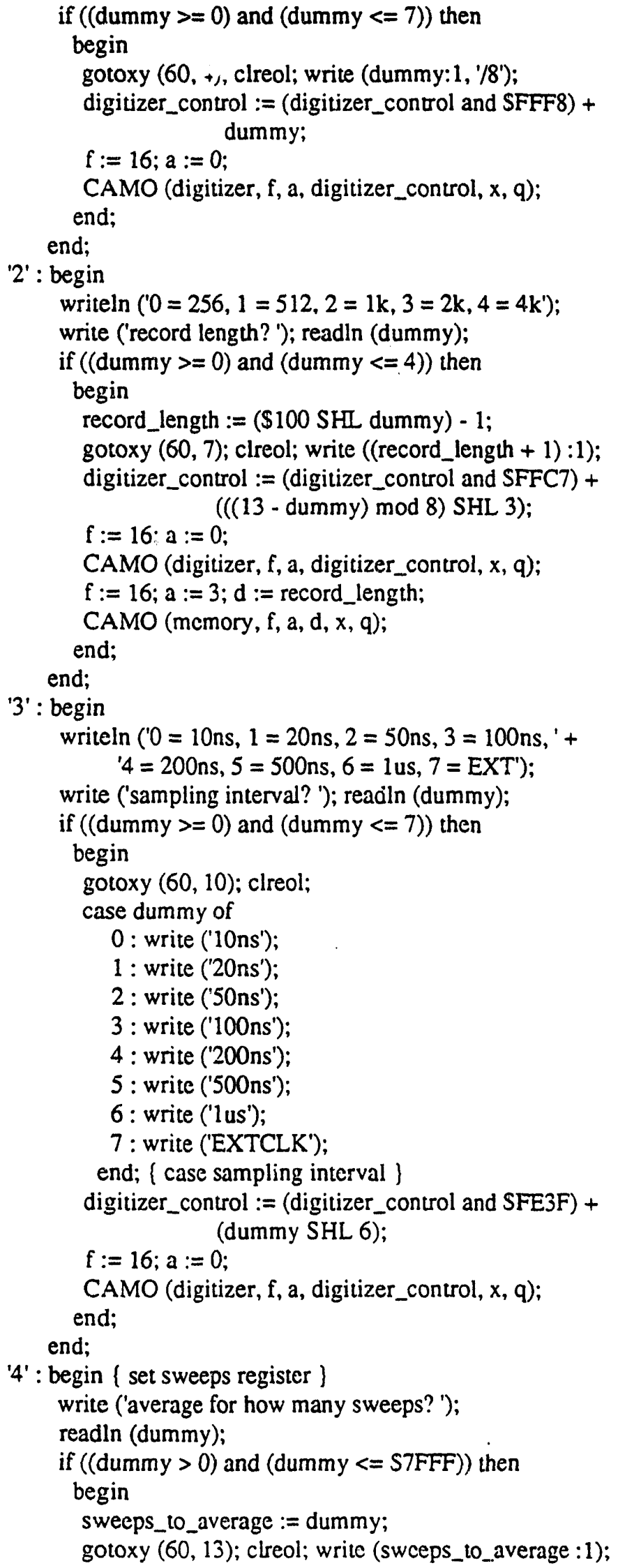




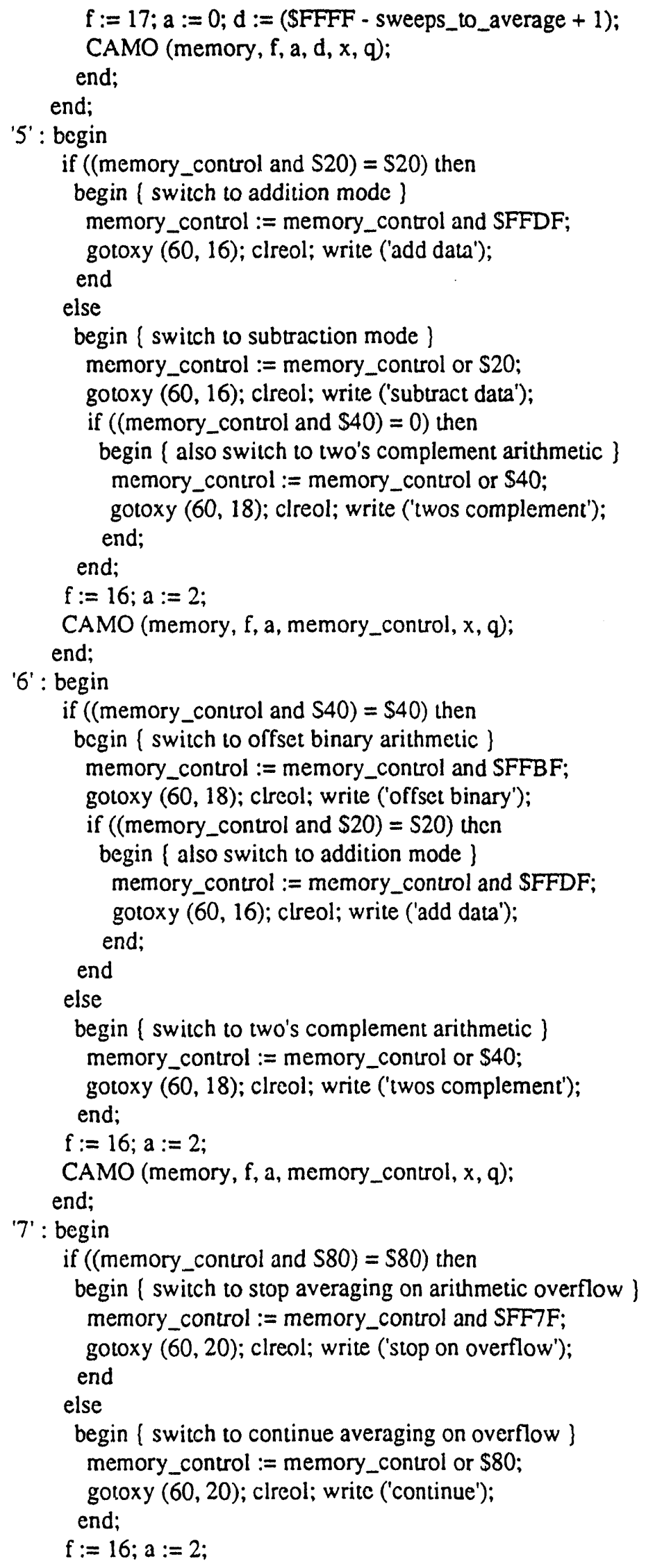


CAMO (memory, $f, a$, memory_control, $x, q$ );

end;

end; $\{$ case $\mathrm{ch}$ \}

until $\left(\mathrm{ch}=\mathrm{C}^{\prime}\right)$;

end; ( procedure setup )

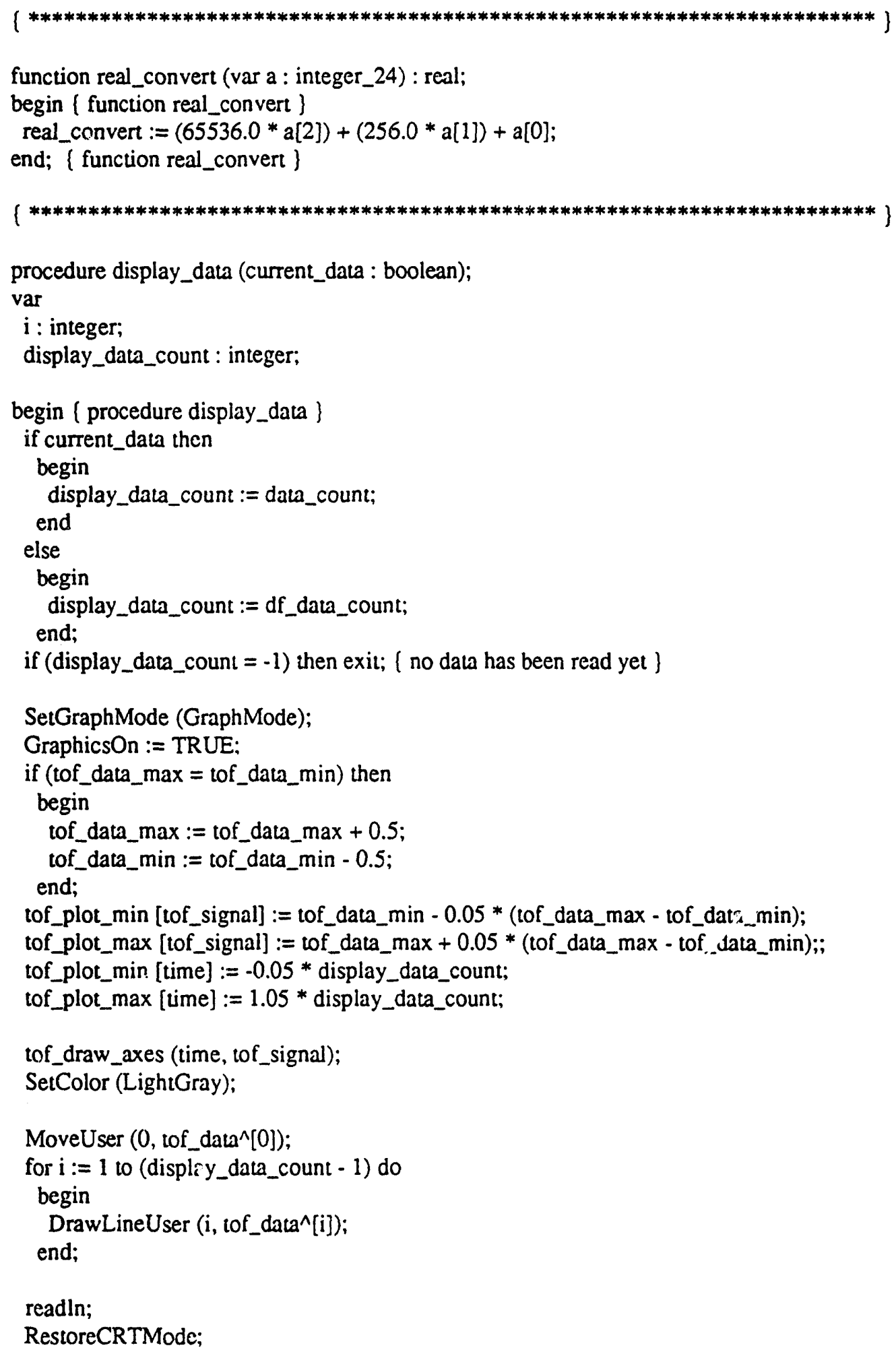




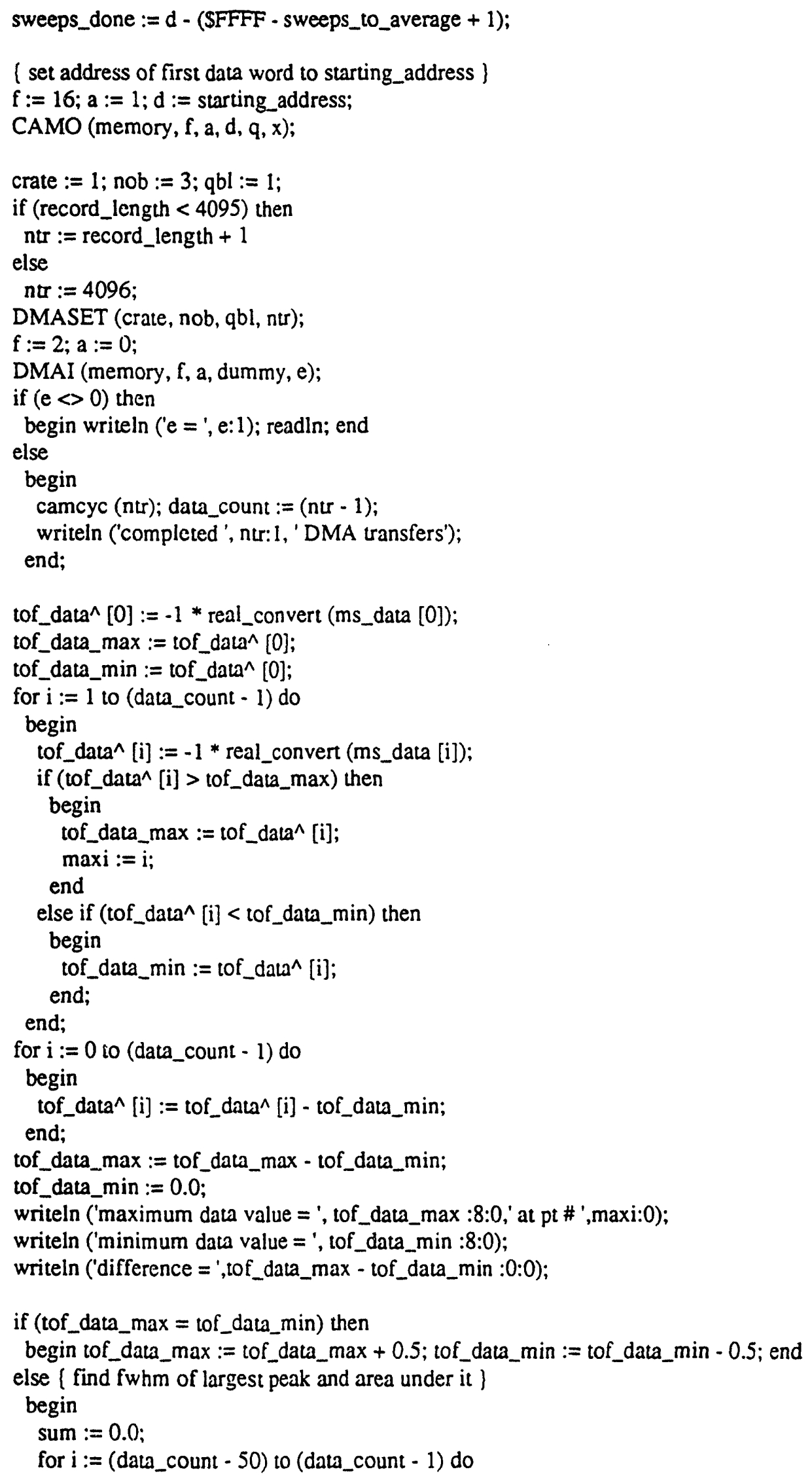




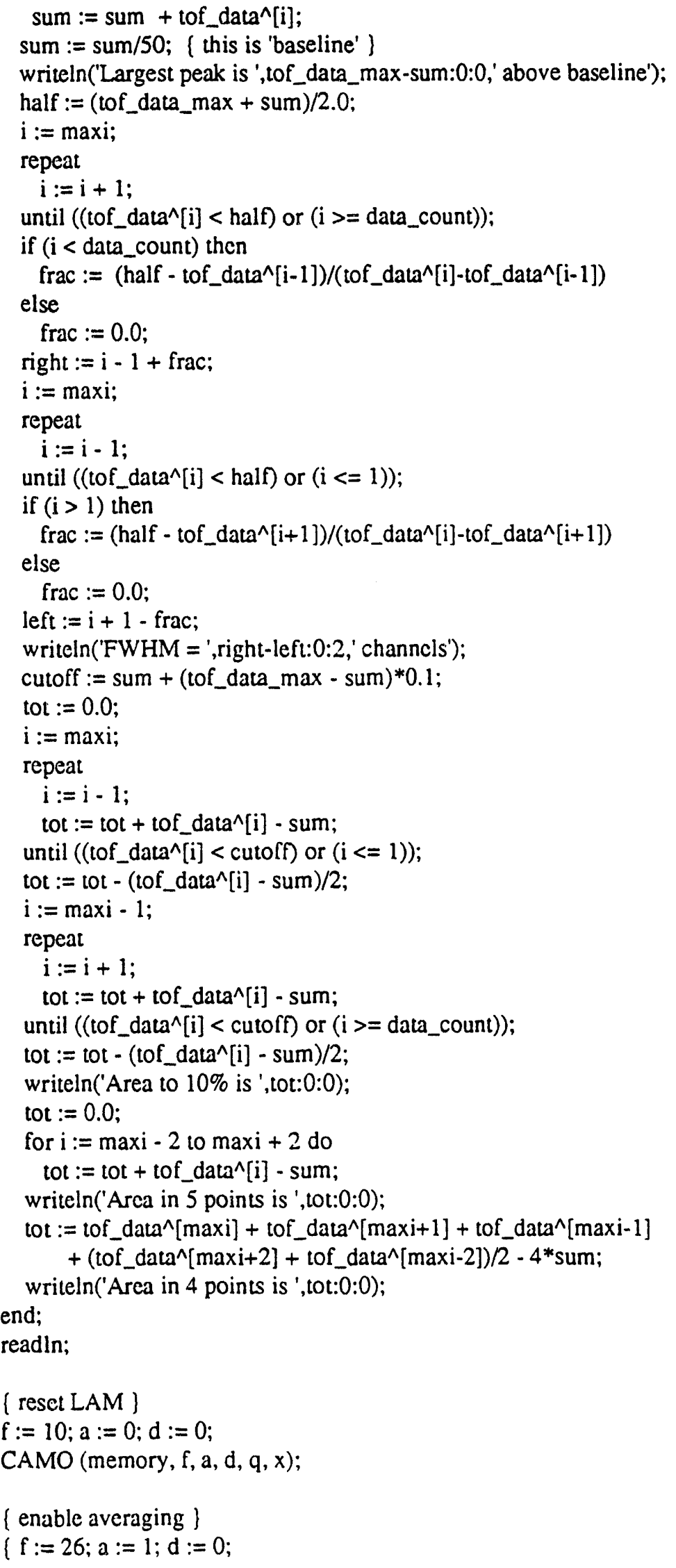


CAMO (memory, f, a, d, q, x); \}

end; (procedure read_data )

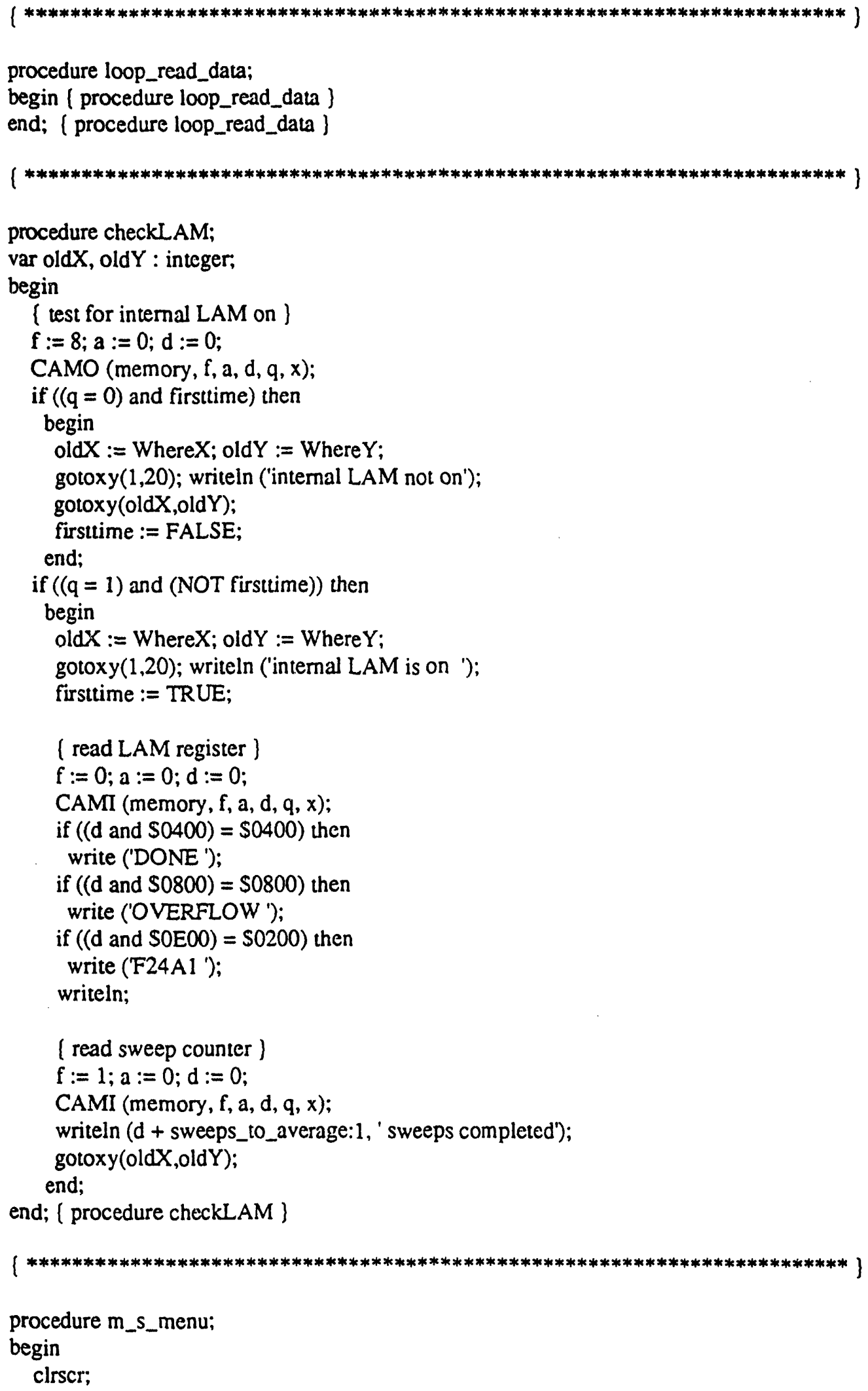


writeln ('D : disable extemal trigger');

writeln ('E : enable external trigger');

writeln (' $\mathrm{Z}$ : reset and enable external trigger');

writeln ('R : read data and display on screen');

writeln ('C : reload sweeps counter');

writeln ('S : set up control registers');

writeln ('W : write data to file');

writeln ('G : get data from file');

writeln ('Q : quit');

writeln; write ('? ');

end; ( procedure m_s_menu )

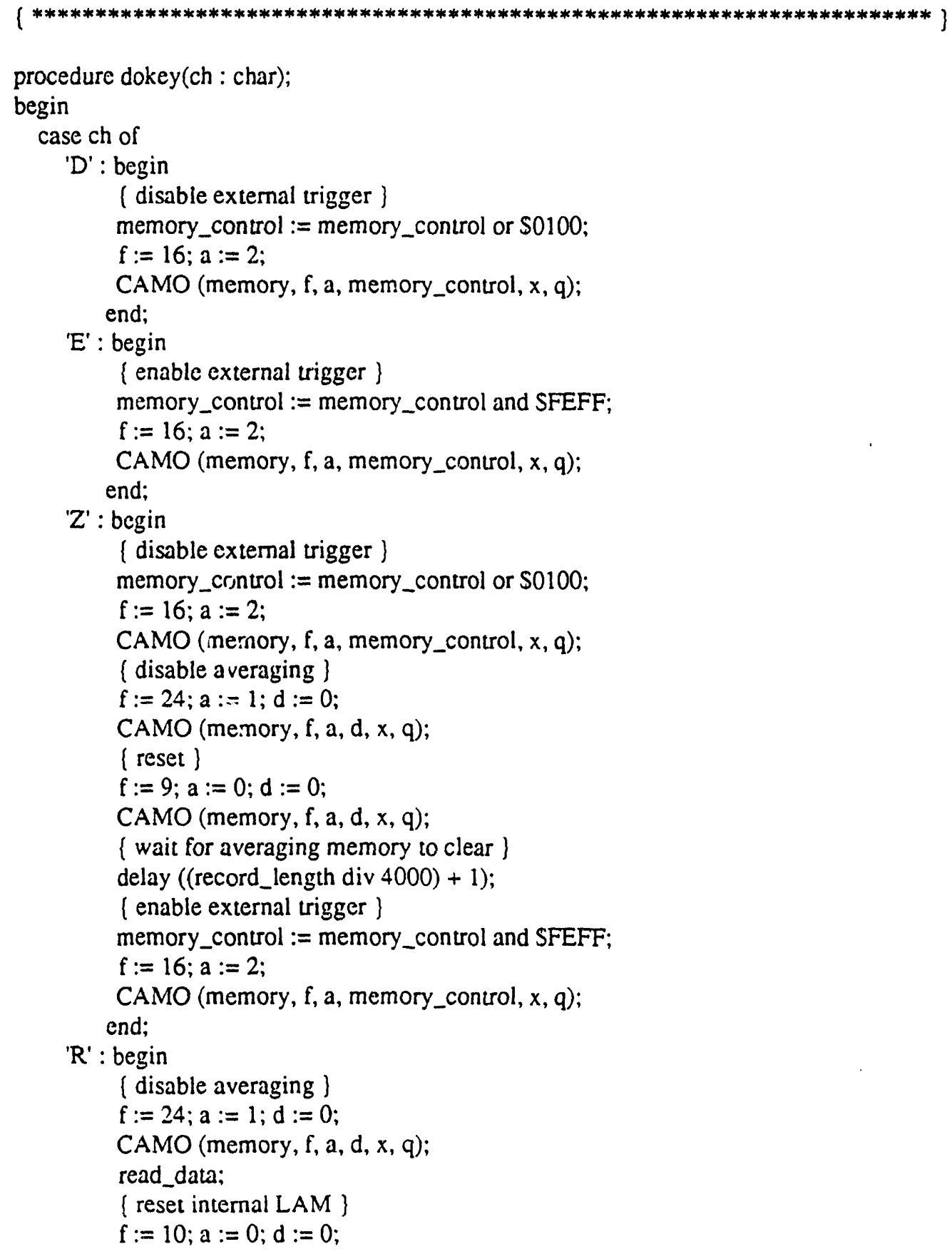


CAMO (memory, f, a, d, x, q);

[ enable averaging ]

$f:=26 ; a:=1 ; d:=0$;

CAMO (memory, f, a, d, x, q);

display_data (TRUE);

end;

' $\mathrm{C}$ ' : begin

[ disable averaging \}

$f:=24 ; a:=1 ; d:=0$;

CAMO (memory, $f, a, d, x, q$ );

\{reload sweep counter \}

$\mathrm{f}:=25 ; \mathrm{a}:=1 ; \mathrm{d}:=0$;

CAMO (memory, $f, a, d, x, q$ );

(reset internal LAM )

$\mathrm{f}:=10 ; \mathrm{a}:=0 ; \mathrm{d}:=0$;

CAMO (memory, $f, a, d, x, q)$;

( enable averaging )

$f:=26 ; a:=1 ; d:=0$;

CAMO (memory, $f, a, d, x, q$ ); end;

'S' : begin

\{disable averaging \}

$f:=24 ; a:=1$; $d:=0$;

CAMO (memory, $f, a, d, x, q$ );

setup;

( reset intemal LAM )

$f:=10 ; a:=0 ; d:=0$;

CAMO (memory, $f, a, d, x, q)$;

(enable averaging \}

$f:=26 ; a:=1 ; d:=0$;

CAMO (memory, $f, a, d, x, q$ );

end;

'W' : save_data_file (tof);

' $G$ ' : begin

read_data_file (tof);

delay (500);

display_data (FALSE);

end;

'Q' : done := TRUE;

end; $\{$ case ch \}

end; \{ procedure dokey \}

$\{* * * * * * * * * * * * * * * * * * * * * * * * * * * * * * * * * * * * * * * * * * * * * * * * * * * * * * * * * * * * * * * * * * * * * * * * *)]$

procedure switch_back_to_main_expt;

begin (procedure swilch_back_to_main_expt \}

clrscr; writeln; writeln;

writeln (' Change over to total photodissociation cross section');

writeln (' data collection configuration.');

writeln; writeln;

writeln ('1. Take radical dissociation signal from the DSP 2001AS');

writeln (' transient recorder signal-in connector and plug');

writeln (' it in to channel 2 of the LeCroy 2249SG analog to digital');

writeln (' converter.'); 


\section{writeln;}

writeln ('2. Take the trigger from the DSP 4101 averaging memory external');

writeln (' trigger and plug it in to section 2 of the gate generator.');

writeln;

write (" Hit <enter> when these steps are complete....');

readln;

end: \{ procedure switch_back_to_main_expt \}

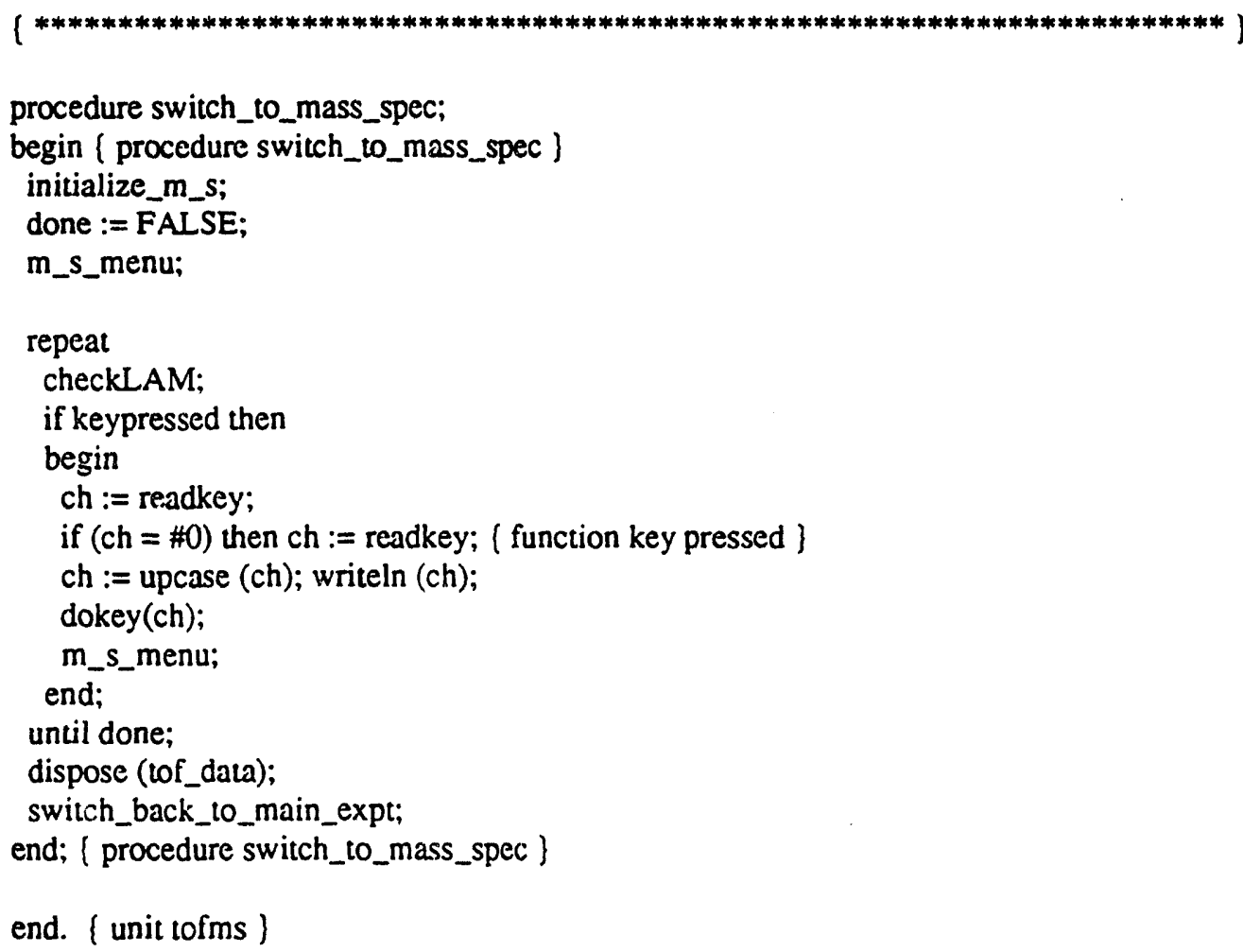

\subsection{7 gpibutil.pas}

\{ gpibutil.pas

procedures used for GPIB control

$* * * * * * * * * * * * * * * * * * * * * * * * * * * * * * * * * * * * * * * * * * * * * * * * * * * * * * * * * * * * * * * * * * * * * * * * * *)$

unit gpibutil;

\{SO+\}

$\{\mathrm{SF}+\}$

interface

uses globals,graph,utility,tpdecl,CRT;

type

string_80 = string $[80]$; 


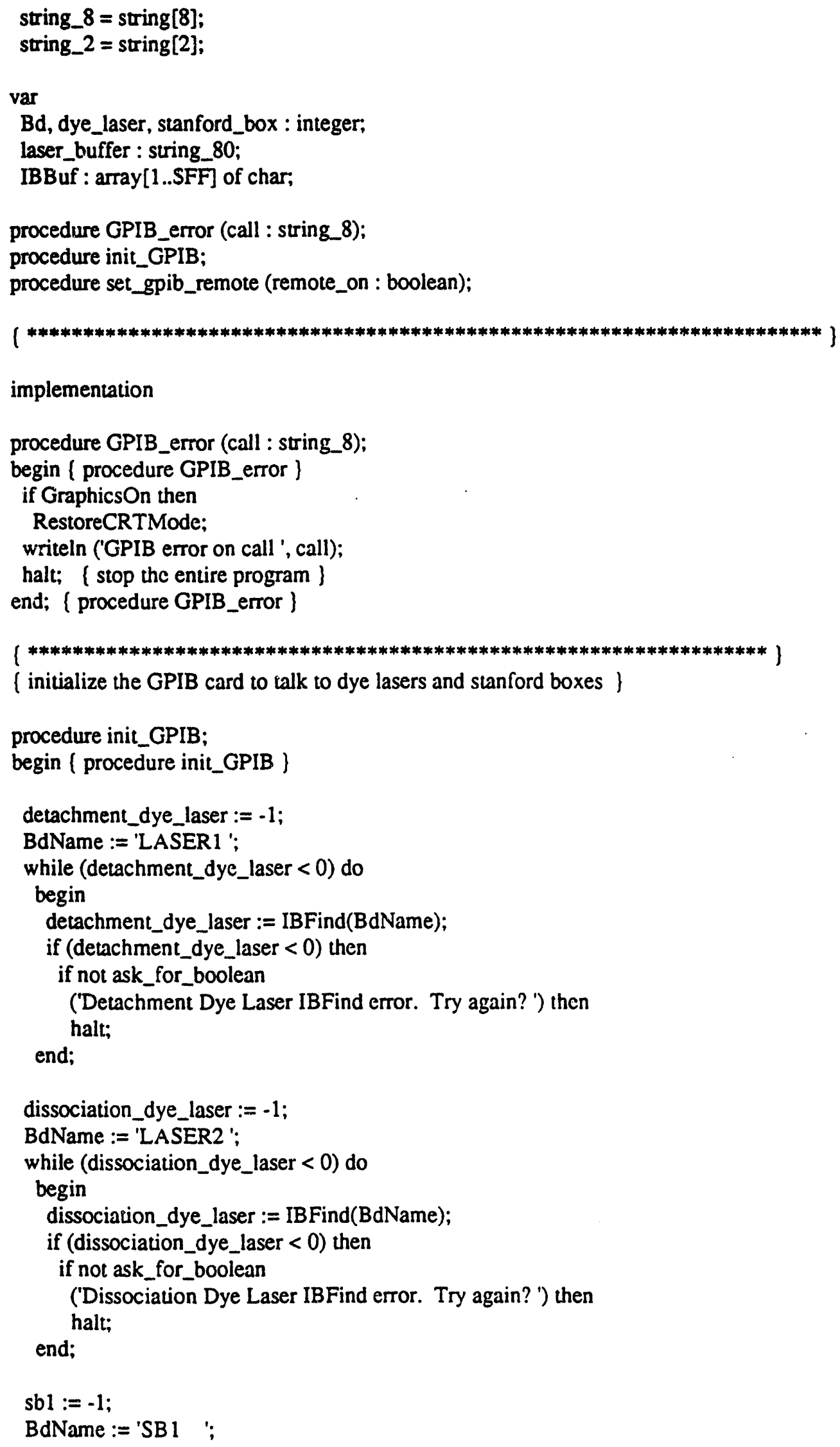




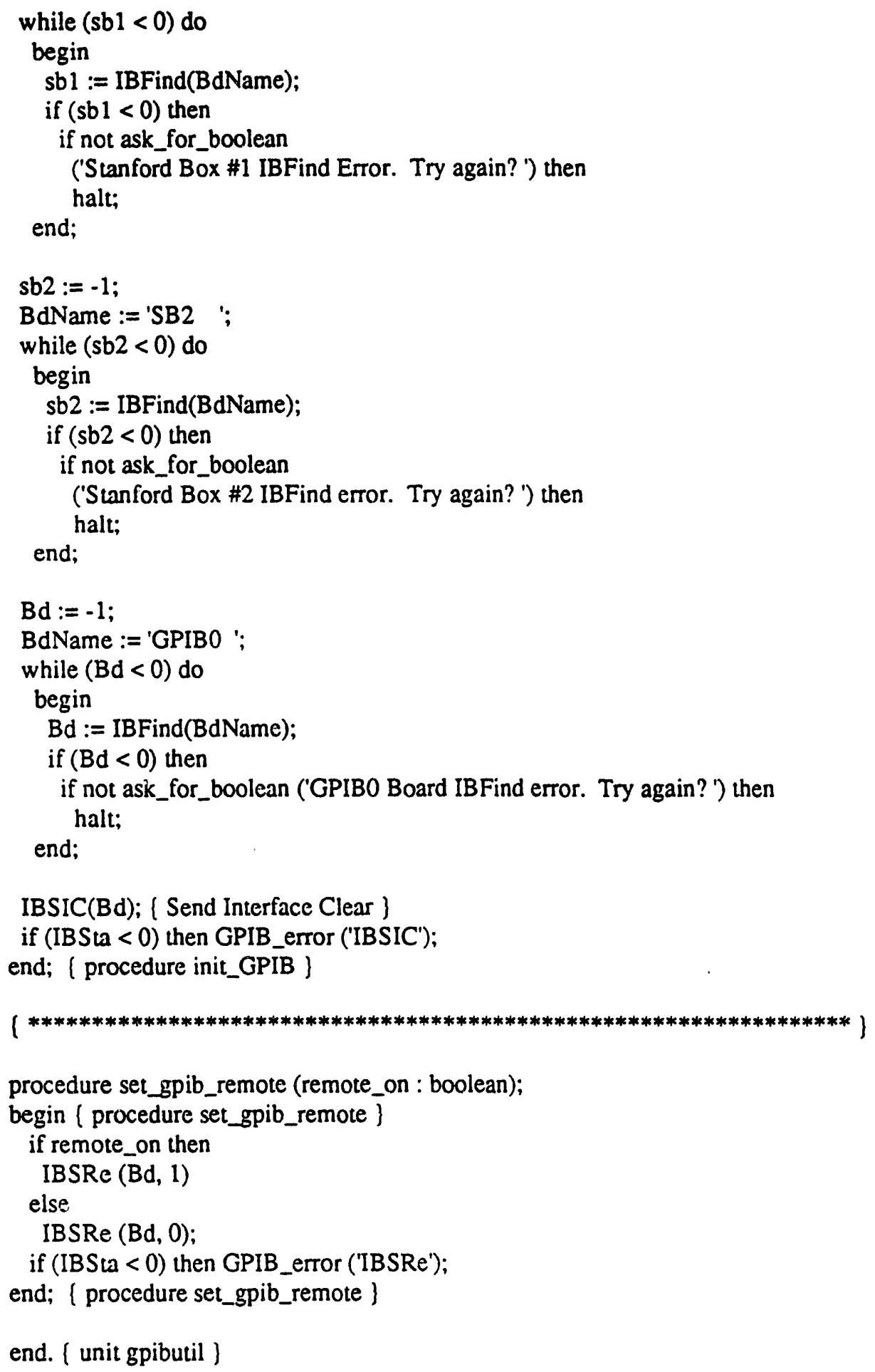

\subsection{8 tpdecl.pas}

unit tpdecl; 
[\$L wib]

interface

Const

(* GPIB Commands:

*)
$\mathrm{UNL}=\$ 3 \mathrm{f} ;$
UNT = \$5f;
GTL = \$01;
SDC $=\$ 04 ;$
$\mathrm{PPC}=\$ 05 ;$
GGET = \$08;
TCT = \$09;
LLO = \$11;
$\mathrm{DCL}=\$ 14$;
$\mathrm{PPU}=\$ 15$;
SPE $=\$ 18$
SPD $=\$ 19 ;$
$\mathrm{PPE}=\$ 60$;
$P P D=\$ 70$;

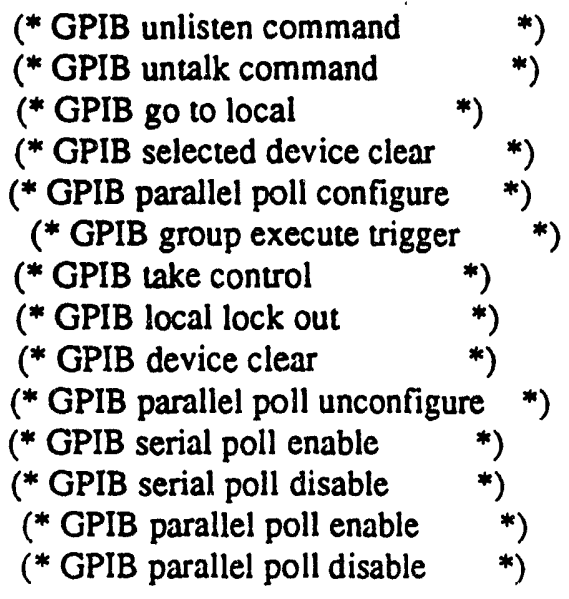

(* GPIB status bit vector:

*)
$E R R=\$ 8000 ;$
TIMO = \$4000;
UEND $=\$ 2000$;
SRQI = \$1000;
RQS = \$800;
CMPL = $\$ 100$;
LOK $=\$ 80$;
REM =\$40;
$\mathrm{CIC}=\$ 20$;
ATN =\$10;
TACS $=\$ 8 ;$
LACS $=\$ 4$;
DTAS $=\$ 2$;
DCAS $=\$ 1$;

(* Error detected

(* Timeout

(*EOI or EOS detected

(* SRQ detected by CIC

(* Device needs service

(* I/O completed

(* Local lockout state

(* Remote state

(* Controller-in-Charge

(* Attention asserted

(* Talker active

(* Listener active

(* Device trigger state

(* Device clear state
*)

*)

*)

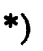

*)

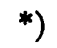

*)

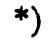

*)

*)

*)

*)

(* Error messages returned in global variable IBERR:

EDVR $=0 ;$
ECIC $=1 ;$
ENOL $=2 ;$
EADR $=3 ;$
EARG $=4 ;$
ESAC $=5 ;$
EABO $=6 ;$
ENEB $=7 ;$
EOIP $=10 ;$
ECAP $=11 ;$
EFSO $=12 ;$

(* DOS error

*)

(* Function requires GPIB board to be CIC *)

(* Write function detected no Listeners *)

(* Interface board not addressed correctly*)

(* Invalid argument to function call

*)

(* Function requires GPIB board to be SAC *)

(* I/O operation aborted

*)

(* Non-existent interface board

*)

(* 1/O operation started before previous *)

(* operation completed

*)

(* No capability for intended operation *)

(* File system operation error

*) 
EBUS = 14;
ESTB $=15 ;$
ESRQ $=16 ;$

(* EOS mode bits:

BIN $=\$ 1000 ;$

$\mathrm{XEOS}=\$ 800$;

REOS $=\$ 400$;
(* Command error during device call

(* Serial poll status byte lost

(* SRQ remains asserted
*)

*)
*)

(* Eight bit compare

(* Send EOI with EOS byte

(* Terminate read on EOS
*)

*)

(* Timeout values and meanings:

*)

TNONE $=0$;

T10us $=1$;

T30us $=2$;

T100us $=3$;

T300us = 4;

T1ms = 5;

$\mathrm{T} 3 \mathrm{~ms}=6$;

$\mathrm{T} 10 \mathrm{~ms}=7$;

$\mathrm{T} 30 \mathrm{~ms}=8$;

$\mathrm{T} 100 \mathrm{~ms}=9$;

$\mathrm{T} 300 \mathrm{~ms}=10$;

T1s = 11;

T3s = 12;

T10s = 13;

$\mathrm{T} 30 \mathrm{~s}=14$;

$\mathrm{T} 100 \mathrm{~s}=15$

$\mathrm{T} 300 \mathrm{~s}=16$;

$\mathrm{T} 1000 \mathrm{~s}=17$;
(* Infinite timeout (disabled)

(* Timeout of 10 us (ideal)

(* Timeout of 30 us (ideal)

(* Timeout of 100 us (ideal)

(* Timeout of 300 us (ideal)

(* Timeout of $1 \mathrm{~ms}$ (ideal)

(* Timeout of $3 \mathrm{~ms}$ (ideal)

(* Timeout of $10 \mathrm{~ms}$ (ideal)

(* Timeout of $30 \mathrm{~ms}$ (ideal)

(* Timeout of $100 \mathrm{~ms}$ (ideal)

(* Timeout of $300 \mathrm{~ms}$ (ideal)

(* Timeout of $1 \mathrm{~s}$ (ideal) *)

(* Timeout of $3 \mathrm{~s}$ (ideal) *)

(* Timeout of $10 \mathrm{~s}$ (ideal) *)

(* Timeout of $30 \mathrm{~s}$ (ideal) *)

(* Timeout of $100 \mathrm{~s}$ (ideal) *)

(* Timeout of $300 \mathrm{~s}$ (ideal) *)

(* Timeout of $1000 \mathrm{~s}$ (maximum)
*)

*)

*)

*)

*)

*)

*)

*)

*)

*)

(* Misccllaneous:

*)

$$
\begin{aligned}
& S=\$ 08 \\
& L F=\$ 0 A
\end{aligned}
$$

(* Parallel poll sense bit

(* ASCII linefeed character

*)

$(* * * * * * * * * * * * * * * * * * * * * * * * * * * * * * * * * * * * * * * * * * * * * * * * * * * * * * * * * * * * * * * * * * * * * * * * * * * * * * *))$

Type $\quad$ nbuf $=$ array[1..nbufsize $]$ of char; $\left({ }^{*}\right.$ device/board names $\left.{ }^{*}\right)$

flbuf $=$ array[1..flbufsize $]$ of char; $\left(*\right.$ filenames $\left.{ }^{*}\right)$

$(* * * * * * * * * * * * * * * * * * * * * * * * * * * * * * * * * * * * * * * * * * * * * * * * * * * * * * * * * * * * * * * * * * * * * * * * * * * * *)$

(* These three variables are to be accessed directly in application program. *) 


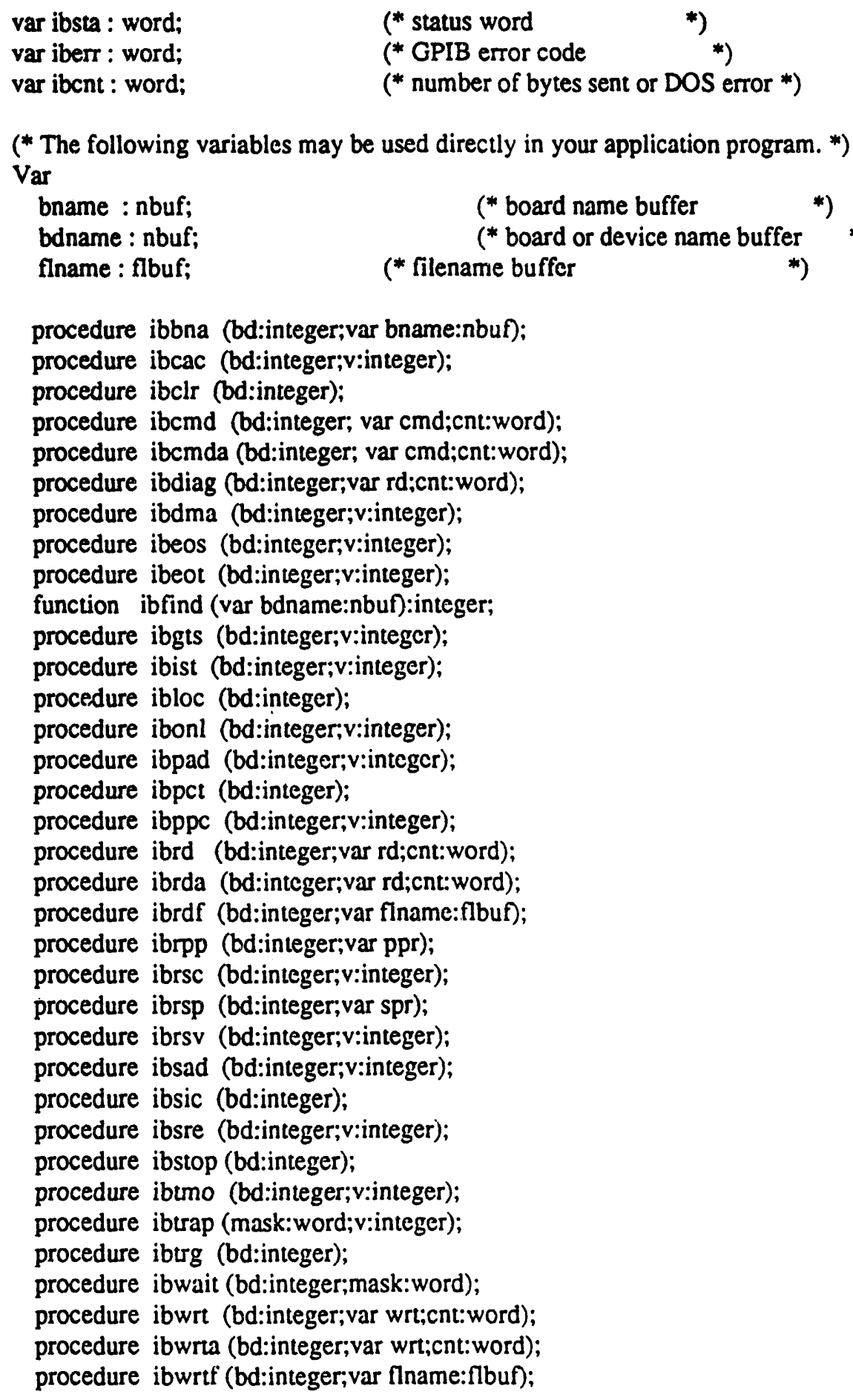

procedure ibbna (bd:integer;var bname:nbuf);

procedure ibcac (bd:integer;v:integer);

procedure ibclr (bd:integer);

procedure ibcmd (bd:integer; var cmd;cnt:word);

procedure ibcmda (bd:integer; var cmd;cnt:word);

procedure ibdiag (bd:integer;var rd:cnt:word);

procedure ibdma (bd:integer;v:integer);

procedure ibeos (bd:integer,v:integer);

procedure ibeot (bd:integer;v:integer);

function ibfind (var bdname:nbuf):integer;

procedure ibgts (bd:integer;v:integcr);

procedure ibist (bd:integer;v:integer);

procedure ibloc (bd:integer);

procedure ibonl (bd:integer;v:integer);

procedure ibpad (bd:integer;v:integcr);

procedure ibpct (bd:integer);

procedure ibppc (bd:integer;v:integer);

procedure ibrd (bd:integer;var rd;cnt:word);

procedure ibrda (bd:integer;var rd;cnt:word);

procedure ibrdf (bd:integer;var flname:flbuf);

procedure ibrpp (bd:integer;var ppr);

procedure ibrsc (bd:integer;v:integer);

procedure ibrsp (bd:integer;var spr);

procedure ibrsv (bd:integer;v:integer);

procedure ibsad (bd:integer;v:integer);

procedure ibsic (bd:integer);

procedure ibsre (bd:integer;v:integer);

procedure ibstop (bd:integer);

procedure ibtmo (bd:integer;v:integer);

procedure ibtrap (mask:word;v:integer);

procedure ibtrg (bd:integer);

procedure ibwait (bd:integer;mask:word);

procedure ibwrt (bd:integer;var wrt;cnt:word);

procedure ibwrta (bd:integer;var wrt;cnt:word);

procedure ibwrtf (bd:integer;var flname:flbuf);

implementation

var

found : integer; $\quad$ (* flag set after first successful ibfind *)

our_lcv : integer; $\quad(*$ local loop control variable *)

(* The GPIB board functions declared public by TPIB.OBJ: 
procedure ibbna (bd:integer;var bname:nbuf); external;

procedure ibcac (bd:integer;v:integer); extemal;

procedure ibclr (bd:integer); external;

procedure ibcmd (bd:integer; var cmd;cnt:word); external;

procedure ibcmda (bd:integer; var cmd;cnt:word); external;

procedure ibdiag (bd:integer; var rd;cnt:word); external;

procedure ibdma (bd:integer;v:integer); external;

procedure ibeos (bd:integer;v:integer); extemal;

procedure ibeot (bd:integer;v:integer); external;

function ibfind (var bdname:nbuf):integer; external;

procedure ibgts (bd:integer;v:integer); external;

procedure ibist (bd:integer;v:integer); external;

procedure ibloc (bd:integer); external;

procedure ibonl (bd:integer;v:integer); external;

procedure ibpad (bd:integer;v:integer); external;

procedure ibpct (bd:integer); external;

procedure ibppc (bd:integer;v:integer); external;

procedure ibrd (bd:integer;var rd;cnt:word); external;

procedure ibrda (bd:integer;var rd;cnt:word); external;

procedure ibrdf (bd:integer;var flname:flbuf); external;

procedure ibrpp (bd:integer;var ppr); external;

procedure ibrsc (bd:integer;v:integer); external;

procedure ibrsp (bd:integer;var spr); external;

procedure ibrsv (bd:integer;v:integer); external;

procedure ibsad (bd:integer;v:integer); external;

procedure ibsic (bd:integer); external;

procedure ibsre (bd:integer;v:integer); external;

procedure ibstop (bd:integer); external;

procedure ibtmo (bd:integer;v:integer); external;

procedure ibtrap (mask:word;v:integer); external;

procedure ibtrg (bd:integer); external;

procedure ibwait (bd:integer;mask:word); external;

procedure ibwrt (bd:integer;var wrt;cnt:word); external;

procedure ibwrta (bd:integer;var wrt;cnt:word); external;

procedure ibwrtf (bd:integer;var flname:flbuf); external;

begin

found: $=0 ; \quad(*$ initialize successful ibfind flag *)

ibsta: $=0 ; \quad \quad \quad(*$ initialize global status variables $*)$

iberr: $=0$;

ibent: $=0$;

for our_lcv:=1 to nbufsize do (* blank fill name buffers *)

begin

bname[our_lcv]:=' ';

end: bdname[our_lcv]:=' ';

for our_lcv:=1 to flbufsize do

flname[our_lcv]:=' ';

end. 


\section{Appendix B}

\section{TPS - The Control and Data Acquisition Program for Photodissociation Dynamics Experiments Involving Time- and Position-Sensitive Detection}

This Appendix contains TPS, the data acquisition program used to collect the relative time-of-flight and the measured charge on each of the 6 conductors of the coincidence wedge-and-strip anode used in the time- and position-sensitive detector, for each dissociation event. The program saves all the raw data for later treatment on the Sun workstations, as well as displaying the build-up of dissociation fragment signal on the detector face as the experiment progresses. Routines are included that allow the user to vary all of the critical parameters in data collection (i.e., hardware and software ADC discrimination thresholds) and display (i.e., multiplicative, additive and crosstalk coefficients in the position finding algorithm). Memory management is particularly important in this program; a large heap is desirable so that as much data can be kept in memory as possible, limiting down time that accompanies transfers to disk. Please refer to the Borland Turbo Pascal manuals for information on the use of dynamic memory.

Units that are identical in both FRBM and TPS appear in Appendix A will not be repeated here. They are insgrdrv.pas, plotutil.pas, grafutil.pas. codes.pas appears in this program courtesy of Dr. John Price.

\section{Description of Files}

tps.pas Contains a list of units to be built into the program, and calls initialization routines followed by the main menu.

inti_ovr.pas Initializes Turbo Pascal overlay manager to automatically overlay all units in tps.pas marked with the compiler directive \$O+. 
tps_init.pas Initializes all parameters and hardware (i.e., the ADC's) used by the program.

tps_mm.pas Presents the user with an array of menu items, calling the routine corresponding to the selection made by the user.

tps_glob.pas Declares all variables, constants, data types, strings etc. that are shared among units

insgrdrv.pas Installs various graphics drivers.

tps_util.pas This is a catch-all unit which includes somewhat common functions and procedures that can be called from other units. See utility.pas of Appendix A.

codes.pas A.CII codes are assigned constant designations for readability. tpsparam.pas Searches for $\mathrm{at}_{2} \mathrm{j}$ reads in a setup file containing initial parameters for experiments. If such a file is not found, it prompts the user for this information and allows such a setup file to then be written.

tps_radc.pas radc stands for 'Read ADC', and this is primarily the function of this unit. tps_exp.pas Controls the actual time and position data acquisition, storage and display.

dfplot.pas Allows an image of the detector face with the build-up of coincidence photofragment events to be displayed on the computer screen as the experiment is in progress.

tps_dcc.pas Allows the input of additive and multiplicative factors for the position algorithm.

tps_ctf.pas Allows the input of cross-talk factors for the position algorithm.

tps_lld.pas Allows the user to enter new hardware lower level discrimination settings for the ADC channels (upper level hardware discrimination is not possible). 
tps_swd.pas Allows the user to enter new lower and upper software discrimination settings to guard against spurious ADC readings.

\section{Source Code}

\section{1 tps.pas}

( tps.pas

$\{* * * * * * * * * * * * * * * * * * * * * * * * * * * * * * * * * * * * * * * * * * * * * * * * * * * * * * * * * * * * * * * * * * * * * * * *\}\}$

( main program TPS

program TPS (input, output);

$[\mathbf{S F}+]$

[SM $16000,140000,300000$ ]

uses overlay,init_ovr,tps_init;

(\$O tps_init)

[SO tps_glob]

(SO insgrdrv)

(SO tps_util)

[SO plotutil]

[SO grafutil]

[\$O codes]

[\$O tpsparam]

[SO ips_radc)

[\$O tps_exp]

(\$O dfplot)

[SO tps_dcc)

[SO tps_ctf]

(\$O tps_lld]

(SO tps_swd)

(\$O tps_mm]

begin ( main program TPS )

initialize_overlay;

initialize_tps_program;

end. (main program TPS ) 


\section{2 init_ovr.pas}

\{ init_ovr.pas
\{ contents :
$\left\{\begin{array}{l}\text { procedure initialize_overlay } \\ \text { initializes overlay }\end{array}\right\}$
$\left\{\begin{array}{l}* * * * * * * * * * * * * * * * * * * * * * * * * * * * * * * * * * * * * * * * * * * * * * * * * * * * * * * * * * * * * * * *)\end{array}\right.$

unit init_ovr;

[SF+]

interface

uses overlay;

procedure initialize_overlay;

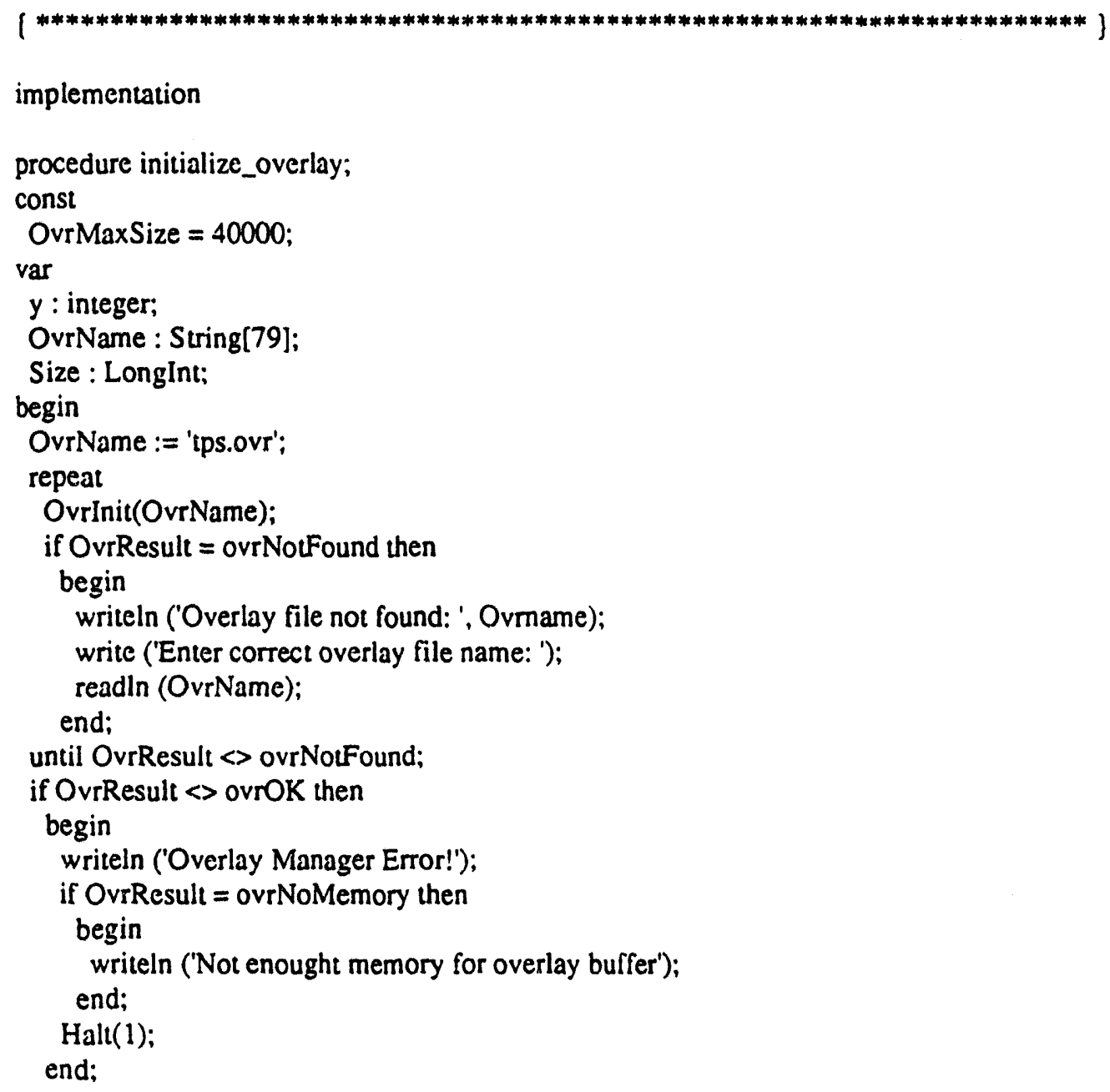




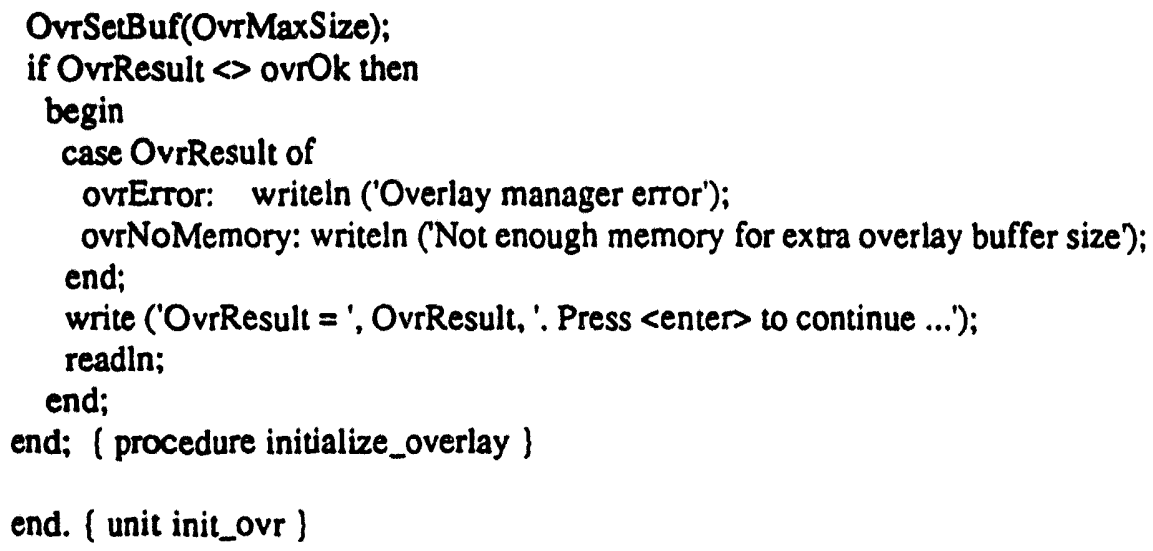

\section{3 tps_init.pas}

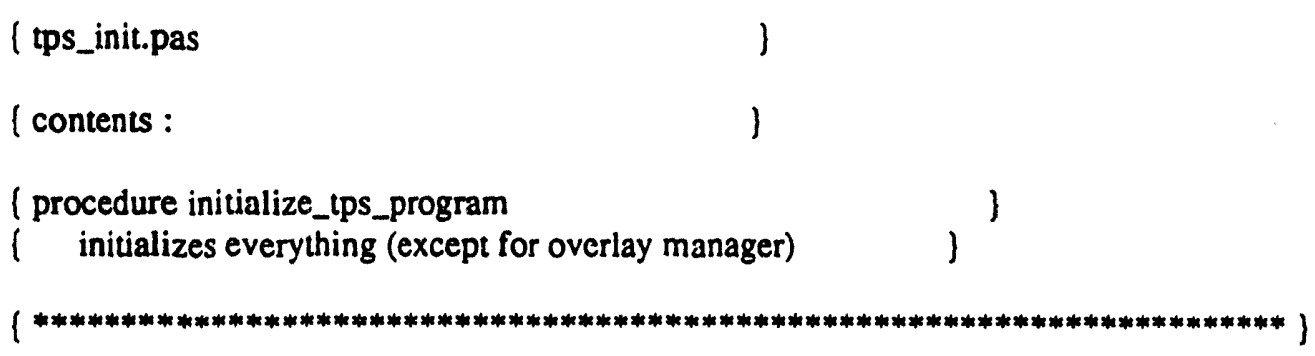

interface

uses tps_glob,tps_util,tpsparam,tps_lld,tps_dcc,insgrdrv,grafutil,tps_mm,CRT,DOS;

procedure initialize_tps_program;

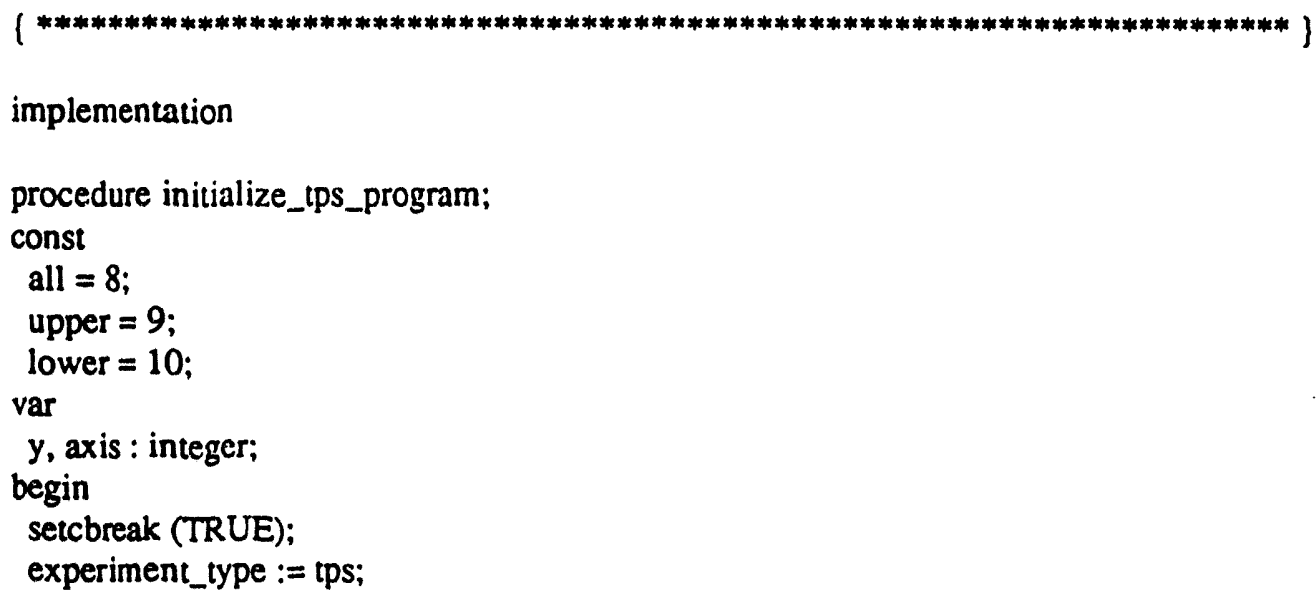


wedge_1_channel :=0;

strip_1_channel $:=1$;

2_1_channel $:=2$;

TAC_channel $:=3$;

wedge_2_channel : $=0$;

strip_2_channel $:=1$;

z_2_channel :=2;

InstallScreenGraphicsDriver;

GraphicsOn := FALSE;

window $(1,1,80,24)$; clrscr;

full_parameter_listing := FALSE;

list_gate_settings := FALSE;

initial_read := TRUE;

if not read_sct_up then

begin

with tps_stp do

begin

TAC_delay : $=250$;

blocking_strip_full_width $:=4$;

Ortec_ADC_channel [wedge_1] :=1;

Ortec_ADC_channel [strip_1] $:=2$;

Ortec_ADC_channel $\left[z_{-} 1\right] \quad:=3$;

Ortec_ADC_channel [TAC] $:=4$;

Ortec_ADC_channel [wedge_2] :=5;

Ortec_ADC_channel [strip_2] : $=6$;

Ortec_ADC_channel [2_2] :=7;

Ild_setting [wedge_1] := 500;

1ld_setting [strip_1] $:=500$;

lld_setting $\left[z_{-} 1\right] \quad:=500$;

lld_setting [TAC] := 500;

lld_setting [wedge_2] := 500;

lld_setting [strip_2] := 500;

lld_setting [z_2] $:=500$;

dmc_setting [wedge_1] :=1;

dmc_setting [strip_1] $:=1$;

dmc_setting [z_1] :=1;

dmc_setting [TAC] ::=1;

dmc_setting [wedge_2] : $=1$ :

dmc_setting [strip_2] :=1;

dmc_setting [z_2] :=1;

dac_setting [wedge_1] $:=0$;

dac_setting [strip_1] $:=0$;

dac_setting [wedge_2] $:=0$;

dac_setting [strip_2] $:=0$;

ctf_setting [upper_ctf, ws] $:=0$;

ctf_setting [upper_ctf, wz] : $=0$;

ctf_setting [upper_ctf, sz] : $=0$;

ctf_setting [lower_ctf, ws] : $=0$;

ctf_setting [lower_ctf,wz] : $=0$;

ctf_setting [lower_ctf, sz] : $=0$; 


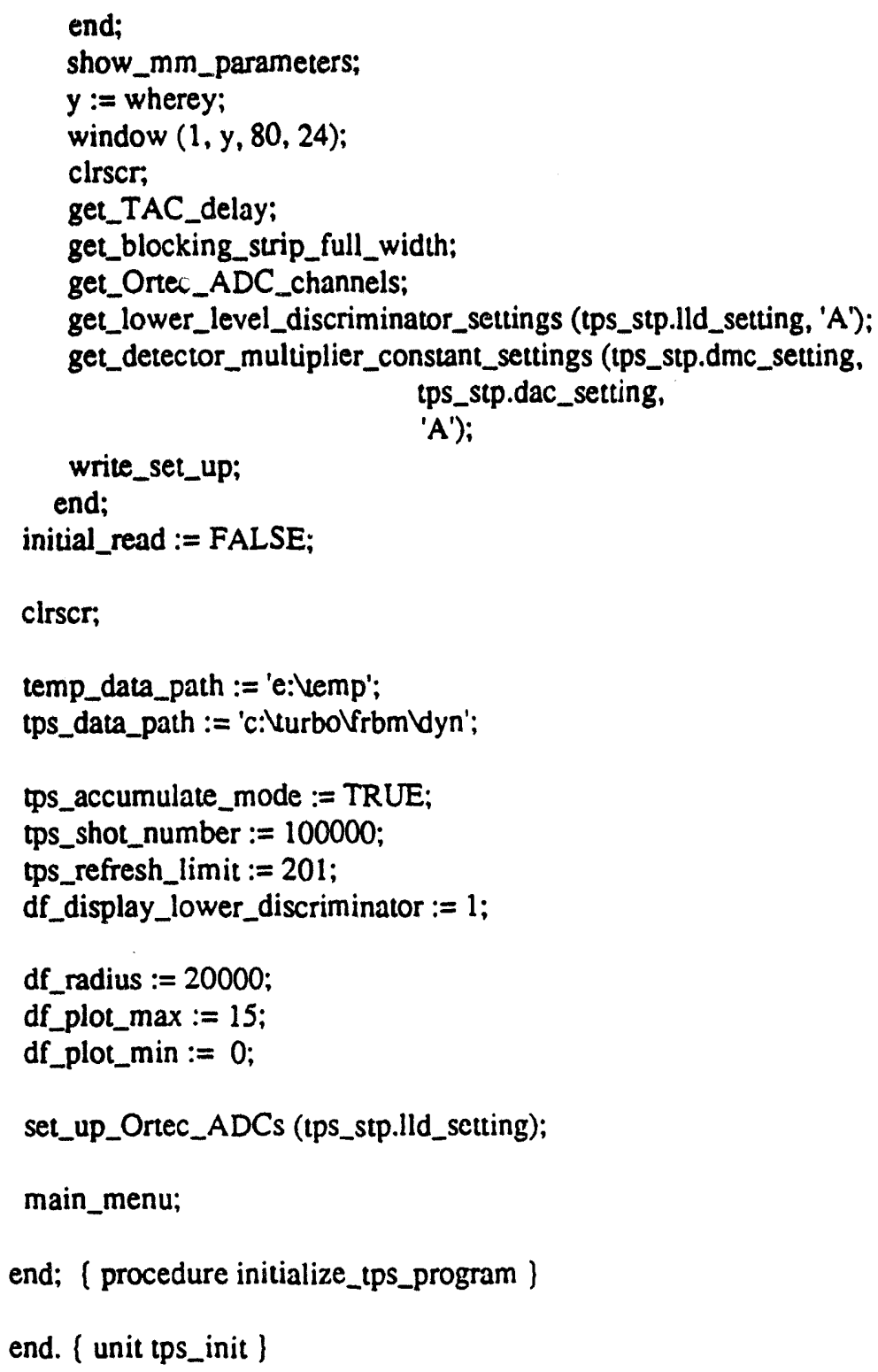

\section{4 tps_mm.pas}

\{tps_mm.pas \}

( contents :

$\left\{\begin{array}{l}\text { ( procedure main_menu } \\ \text { menu of things to do before and after dynamics experiment }\}\end{array}\right.$

$[* * * * * * * * * * * * * * * * * * * * * * * * * * * * * * * * * * * * * * * * * * * * * * * * * * * * * * * * * * * * * * * * * * * * * * * *)]$

unit tps_mm; 


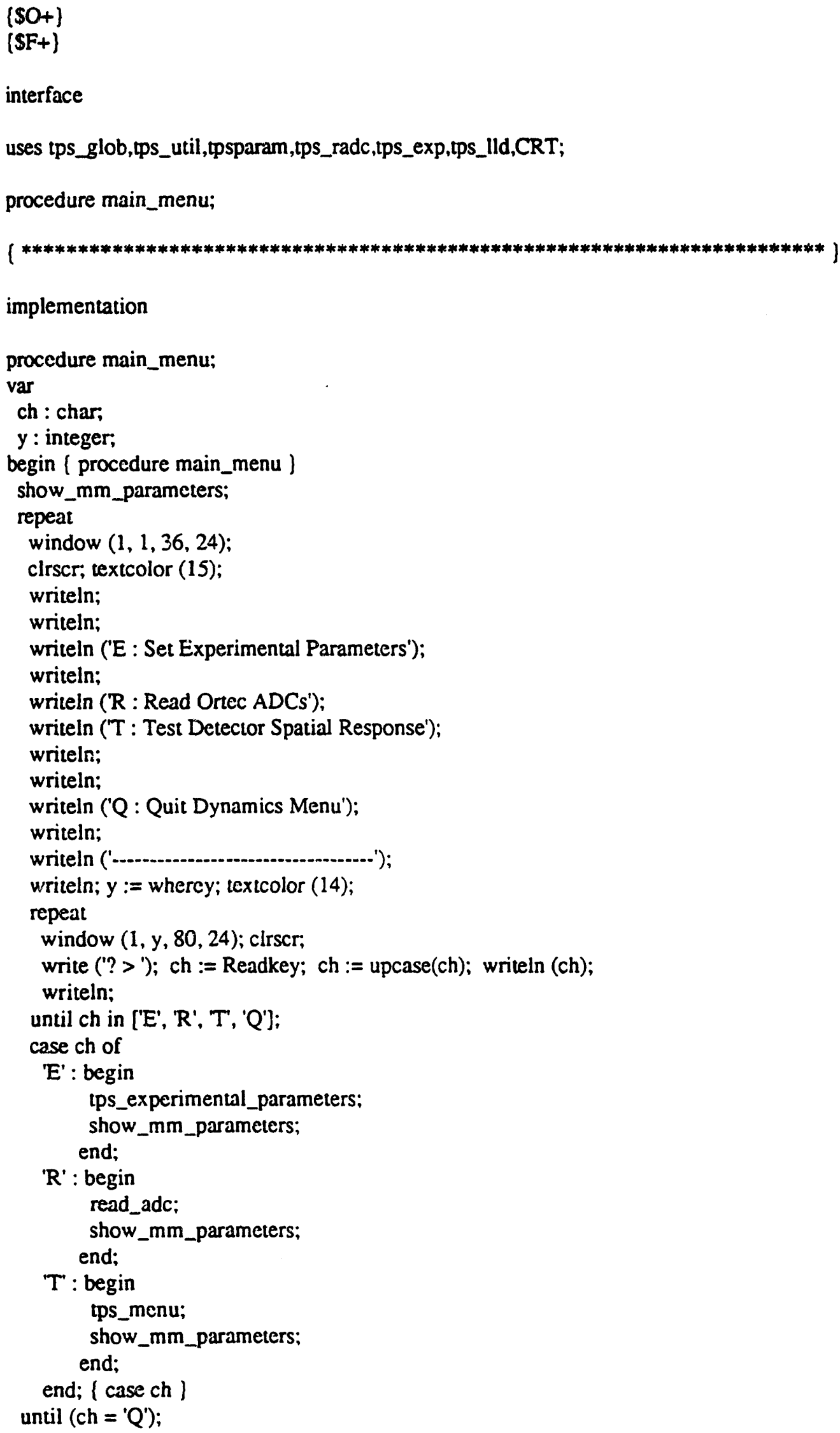




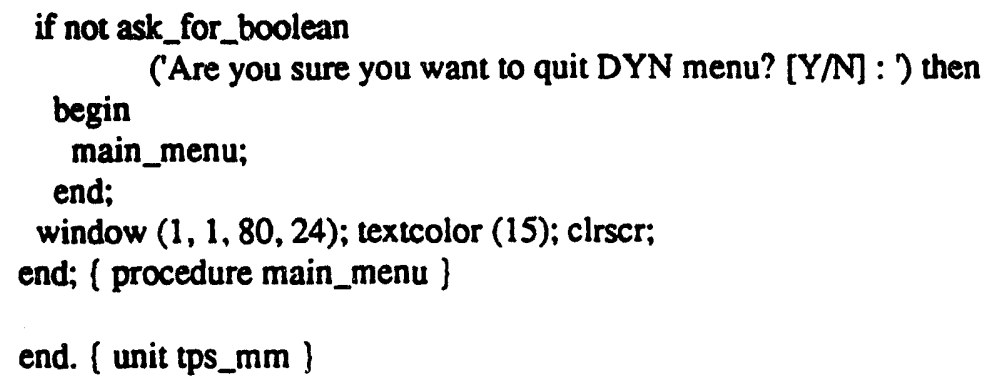

\section{5 tps_glob.pas}

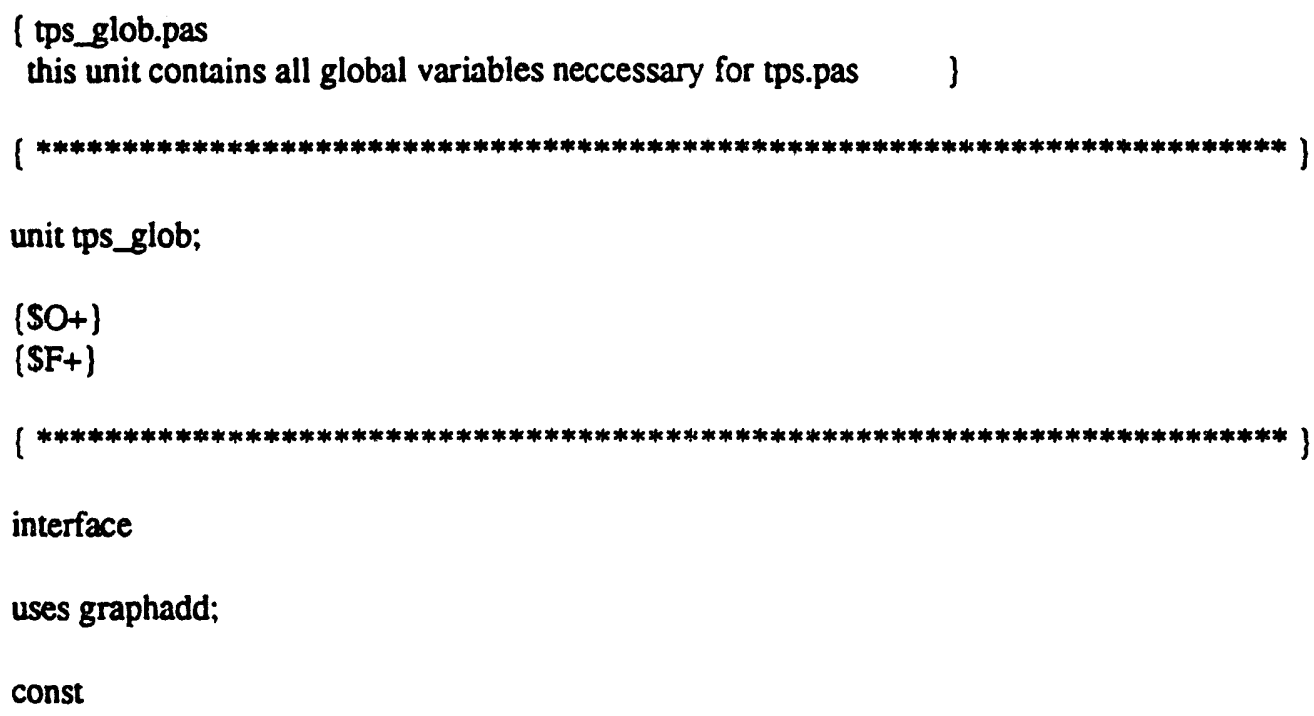




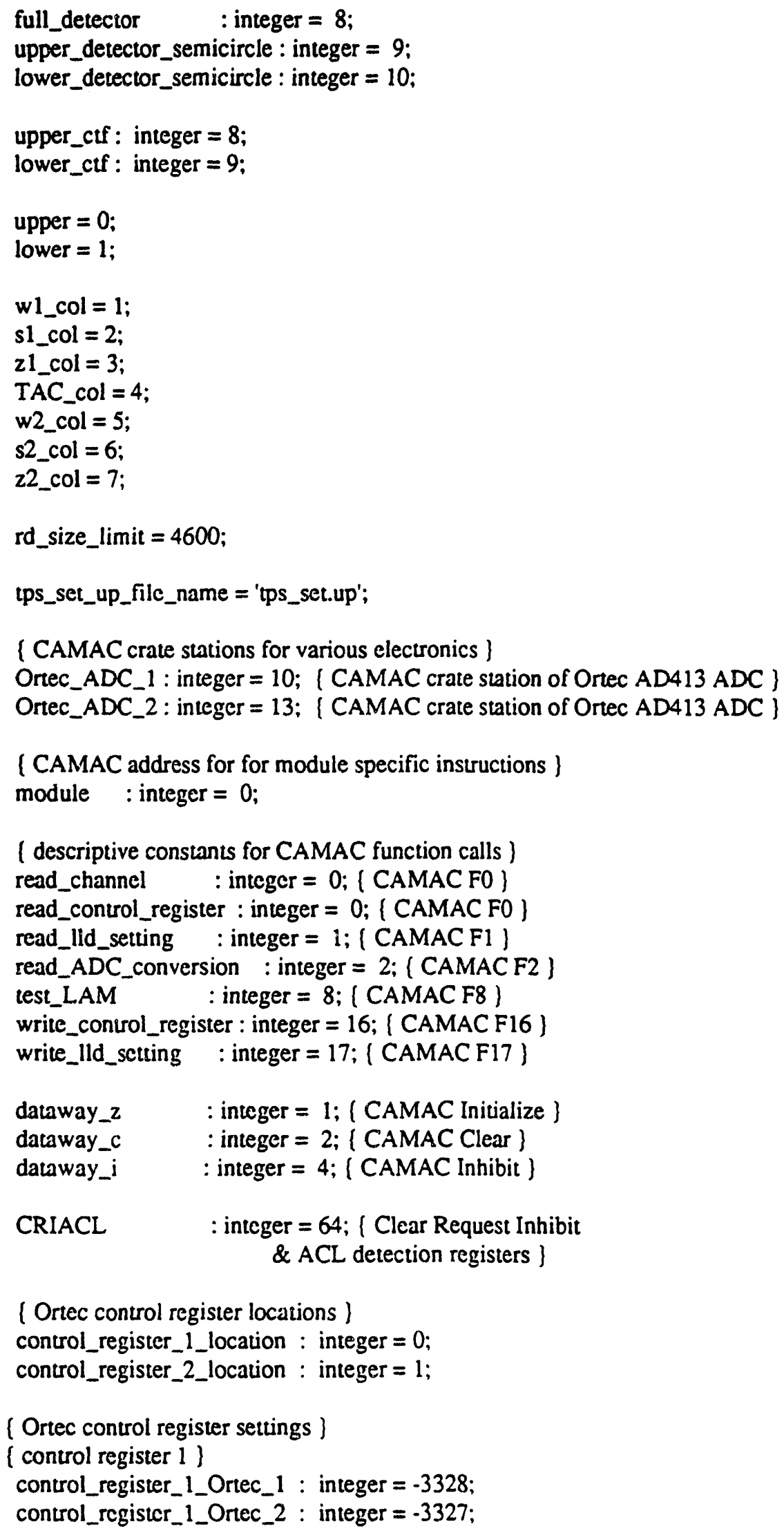


( in control register 1 the bits have the following functions :

bits 1..8: Virtual Station Number : set to 0 and 1 respectively for each ADC

(Ortec \#1 value : 0 , Ortec \#2 value : 1 )

bit 9 : Zero Suppression Enable : set to 1 for sequential or random access)

(value : 256)

bit 10 : ECL Port Enable : set to 1 to enable CAMAC readout (value : 512)

bit 11 : Not Used

bit 12 : Not Used

bit 13 : Coincidence/Singles Mode : set to 1 for singles mode (value : 4096)

bit 14 : CAMAC Random Access Enable : set to 1 for addressed readout (value : 8192)

bit 15 : CAMAC LAM Enable : sct to 1 so LAM is asserted when data is ready to be read out on the CAMAC port (value : 16384)

bit 16 : Overflow-Suppression Enable : set to 1 so all pulses above the upper level discriminator are reported with a value between 8064 and 8191

(value : makes the integer value negative if set to 1)

total integer value (excluding VSN) : -21248

PathToGraphics : string = 'c:leurbolgraphics';

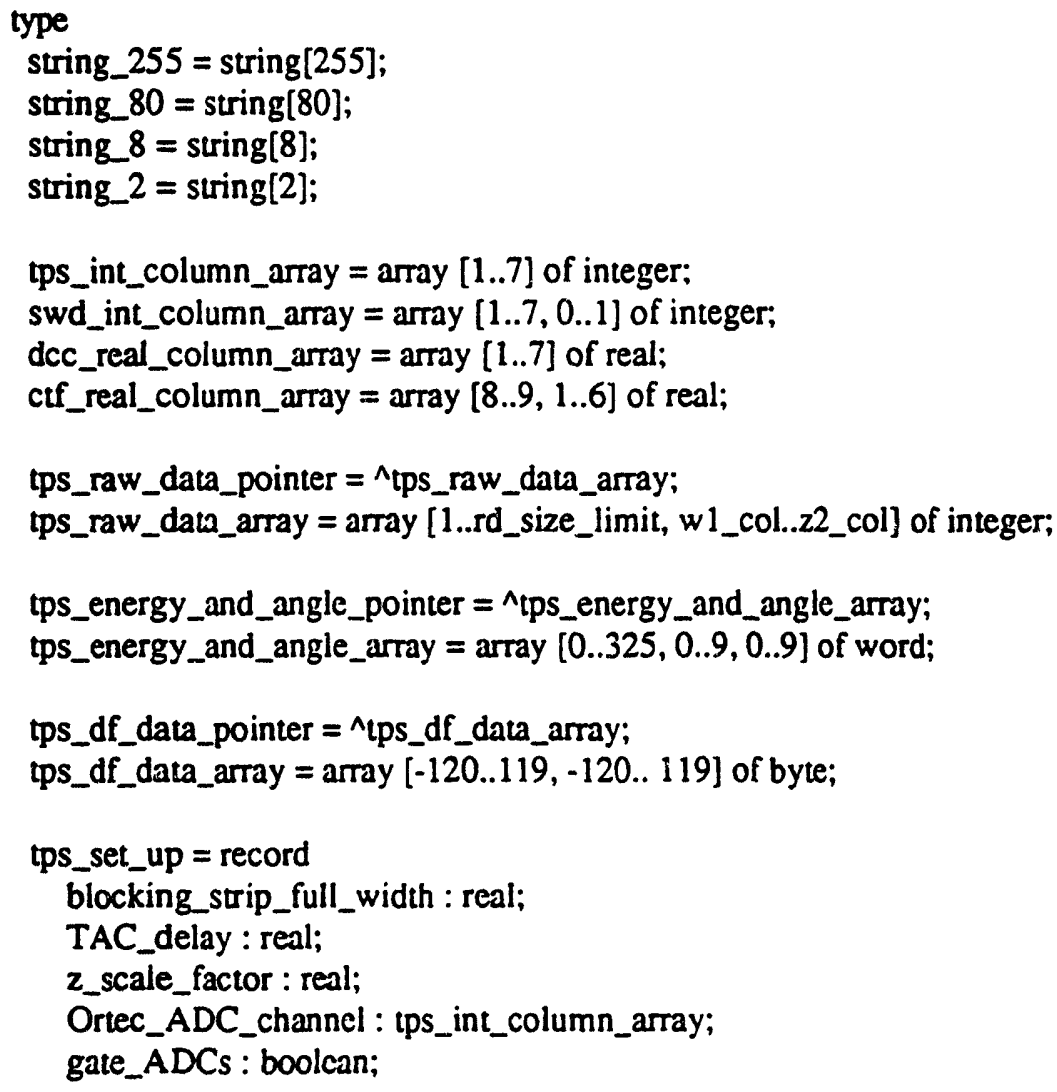


lld_setting : tps_int_column_array;

swd_setting : swd_int_column_array;

dmc_setting : dcc_real_column_array:

dac_setting : dcc_real_column_array;

ctf_setting : ctf_real_column_array;

end; ( record tps_set_up )

$\operatorname{var}$

$D, Q, X:$ integer;

wedge_1_channel : integer;

strip_1_channel : integer;

2_1 channel : integer;

TAC_channel : integer:

wedge_2_channel : integer;

strip_2_channel : integer;

z_2_channel : integer;

( control register 2 \})

control_register_2 : integer;

f in control register 2 of both ADCs bits $1 . .5$ are enable/disable (0/1)

gate 1...master gate respectively. Therefore useful settings for us are

\$1F (disable all gates) and

$\$ 10$ (disable master gate, enable individual gates

Year, Month, Day, DayofWeek, Hour, Minute, Second, Sec100 : word;

tps_data_path : string;

temp_data_path : string;

experiment_type : integer;

detector_element : integer;

tps_stp : tps_sct_up;

tps_df_data : tps_df_data_pointer;

tps_energy_and_angle_data : tps_energy_and_angle_pointer;

file_name : string_80;

upper_file_name : string_80;

lower_file_name : string_80;

comp_file_name : string_80;

data_file_name : string_80;

log_file_name : string_80;

data_file : text:

$\log$ file : text;

initial_read : boolean;

upper_left_comer_y : integer;

full_parameter_listing : boolean;

list_gate_settings : boolean; 
sw_discriminator : swd_int_column_array;

df_min, df_max : word;

df_plot_min, df_plot_max : word;

tps_accumulate_mode : boolean;

tps_shot_number: longint;

tps_data_count : longint;

tps_refresh_count : word;

tps_refresh_limit : word;

tps_data_read_cycles : longint;

rec_data_read_cycles : word;

zero_data_count, low_data_count : longint;

high_data_count, overflow_count : longint;

outside_active_area_count, non_coincidence_count : longint;

rec_zero_data_count, rec_low_data_count : word;

rec_high_data_count, rec_overflow_count : word;

rec_outside_active_area_count, rec_non_coincidence_count : word;

df_radius, df_display_lower_discriminator : integer;

IOErrorCode : integer,

active_directory : string;

bin_data_file : file;

int_data_file : file of integer,

text_data_file : text;

$h, m, s, s 100$ : word;

initial_start_time, start_time : real;

count_time, total_count_time : real;

total_exp_time : word;

count_rate, average_count_rate : real;

GraphDriver : integer; [ The Graphics device driver ]

GraphMode : integer; (The Graphics mode value )

ErrorCode : integer; (Reports any graphics errors)

MaxColor : word; (The maximum color value available ]

OldExitProc : Pointer; ( Saves exit procedure address )

GraphicsOn : Boolean; ( Saves status of CRT mode (graphics or text))

HP7470Plotter : integer;

PostScriptLaserWriterFile : integer;

status : word;

magic : ^byte;

wordptr : ^ word;

hold : byte;

(

implementation

end. ( unit tps_glob ) 


\section{6 tps_util.pas}

( tps_util.pas ) ]

( contents :

(i. math

( function int_power (base : rcal;

$\left\{\begin{array}{c}\text { power : integer) : real } \\ \text { raises any real 'base' to positive integer 'power' }\end{array}\right.$

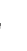

$\begin{array}{ccc}1 & 1 \\ 1 & 1\end{array}$

(ii. user input )

(ii.a. yes/no ]

( function ask_for_boolean (prompt : string_80) : boolean

( user must answer yes/no question, 'prompt'

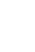

\{ii.b. continue/quit

( function ask_continue : boolean

f user must hit <enters to continue or Esc to quit

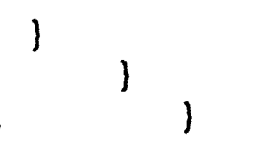

(ii.c. numbers

\}

(these functions convert strings to numbers and ignore inappropriate )

( characters -- beware of misintcrpretation of invalid input

( true if user enters 'response' between ' $m$ in' and 'max' or

( $<$ ' which sets 'response' to ' $\mathrm{min}$ ' or )

I $>$ ' which sets 'response' to 'max'

( false if user just presses 'enter'; 'response' is unchanged ]

('prompt' is repeated if user enters something else ]

$\left\{\begin{array}{l}\text { \{function ask_for_integer (var response : integer, } \\ \text { prompt : string_80; } \\ \text { min, max : integer) : boolean }\end{array}\right\}$

( function ask_for_real (var response : real; ]

( prompt: suring_80;

$\{\min , \max :$ integer) : boolean \}

(ii.d. wavelength to wavenumber conversion and vise-versa \}

\{ function w/2wn (wl : real) : real;

( function wn2wl (wn : real) : real; ) ]

[ii.e. file names ]

( function exist (file_name : string_80) : boolean ]

i true if file called 'filc_name' is present on disk )

\{ function legal_name (file_name : string_80) : boolean , \} 
( procedure get_out_file_name (var file_name : string_80;

true if user enters a new 'file_name' or wants to overwrite an

( existing file; if overwrite, sets overwrite to TRUE

( function get_in_file_name (var file_name : string_80:

( prompt : string_80) : boolean

I true if user entered 'file_name' of an existing file

I iii. save and read data

( procedure save_data (experiment_type : integer);

( save data to individual'.dat' file, append scan parameter info I

I to one 'date.log' file per day

( iv. miscellaneous

I procedure reserved;

I displays message : 'reserved for future development'

1

( function real_convert (var a : integer_24) : real;

( converts DSP output to a real number
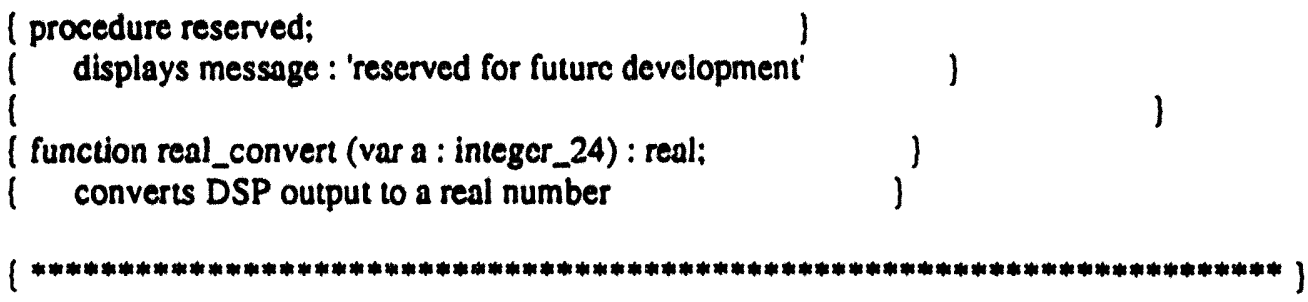

unit tps_util;

(SO+]

[SF+]

interface

uses tps_glob,codes,DOS,CRT;

function int_power (base : real; power : integer) : real;

function ask_for_boolean (prompt : string_80) : boolean;

function ask_continue : boolean;

function ask_for_byte (var response : byte; prompt : string_80;

min, $\max :$ byte $)$ : boolean;

function ask_for_integer (var response : integer; prompt : string_80;

$\min , \max :$ integer) : boolean:

function ask_for_long_integer (var response : longint; prompt : string_80;

min, max : longint) : boolean;

function ask_for_real (var response : real; prompt : suring_80; min, max : real) : boolean;

function w12wn (wl : real) : real;

function wn2wl (wn : real) : real;

function I2S (Val, Digit: : Integer) : String;

function W2S (Val, Digit: Word) : String:

function R2S (Val : real: Digit, Decimal : Integer) : Suring;

function exist (file_name : string_80) : boolean; 
function legal_name (file_name : string_80) : boolean;

procedure get_out_file_name (var file_name : string_80; var overwrite : boolean;

experiment_type : integer;

var got_good_out_file_name : boolean);

function get_in_file_name (var file_name : string_80) : boolean;

procedure form_date_and_time_strings (var date_string : string:

var time_string : string);

procedure clock_on (var start_clock : real);

function elapsed_tume (start_clock : real) : rcal;

procedure save_data_file;

procedure read_data_file:

function compress (file_name : suring_80) : boolcan:

function uncompress (comp_file_name : string_80) : boolean;

procedure reserved;

(

implementation

function int_power (base : real;

power : integer) : real;

begin ( function int_power)

if (power $=0$ ) then

int_power : $=1.0$

else

int_power := base * int_power (base, (power - 1));

end: (function int_power)

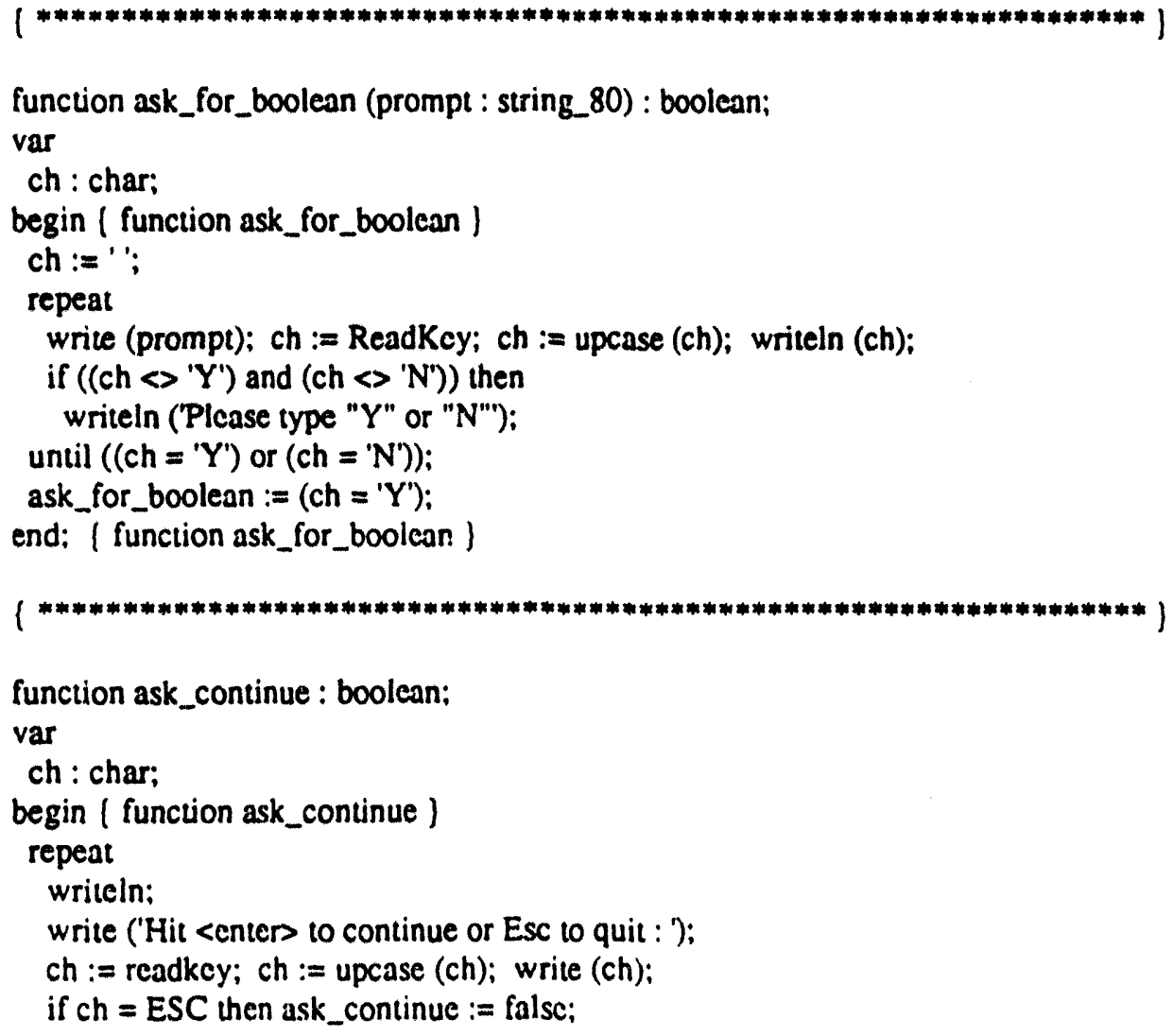

function ask_continue : boolean;

var

ch : char;

begin ( function ask_continue )

repeat

writein:

write ('Hit <enters to continue or Esc to quit : ');

ch := readkcy; ch := upcase (ch); write (ch);

if $\mathrm{ch}=\mathrm{ESC}$ then ask_continue $:=$ falsc; 
If $\mathrm{ch}=$ \#13 then ask_continue := true;

until ((ch = ESC) or (ch = \#13));

end; ( function ask_continue )

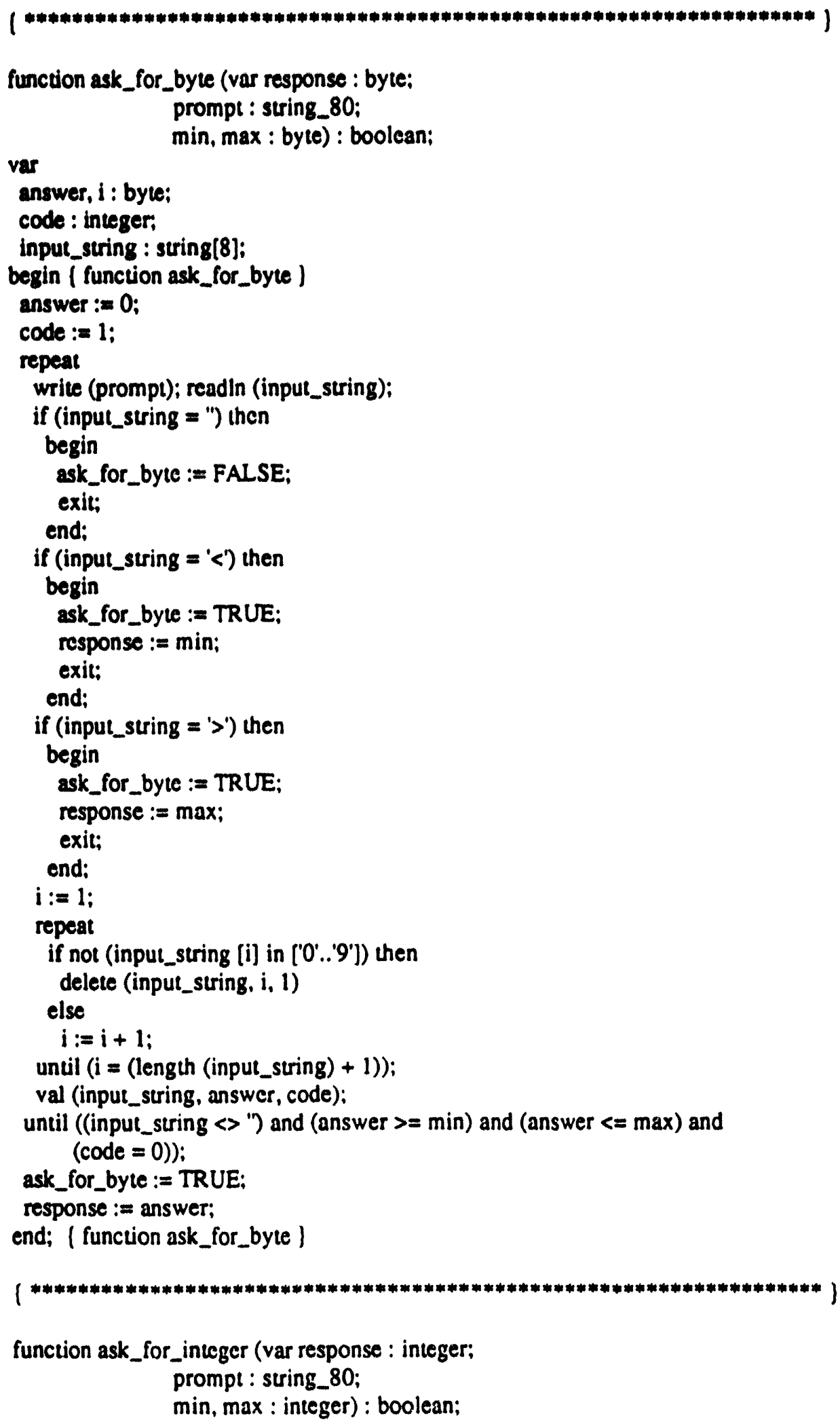




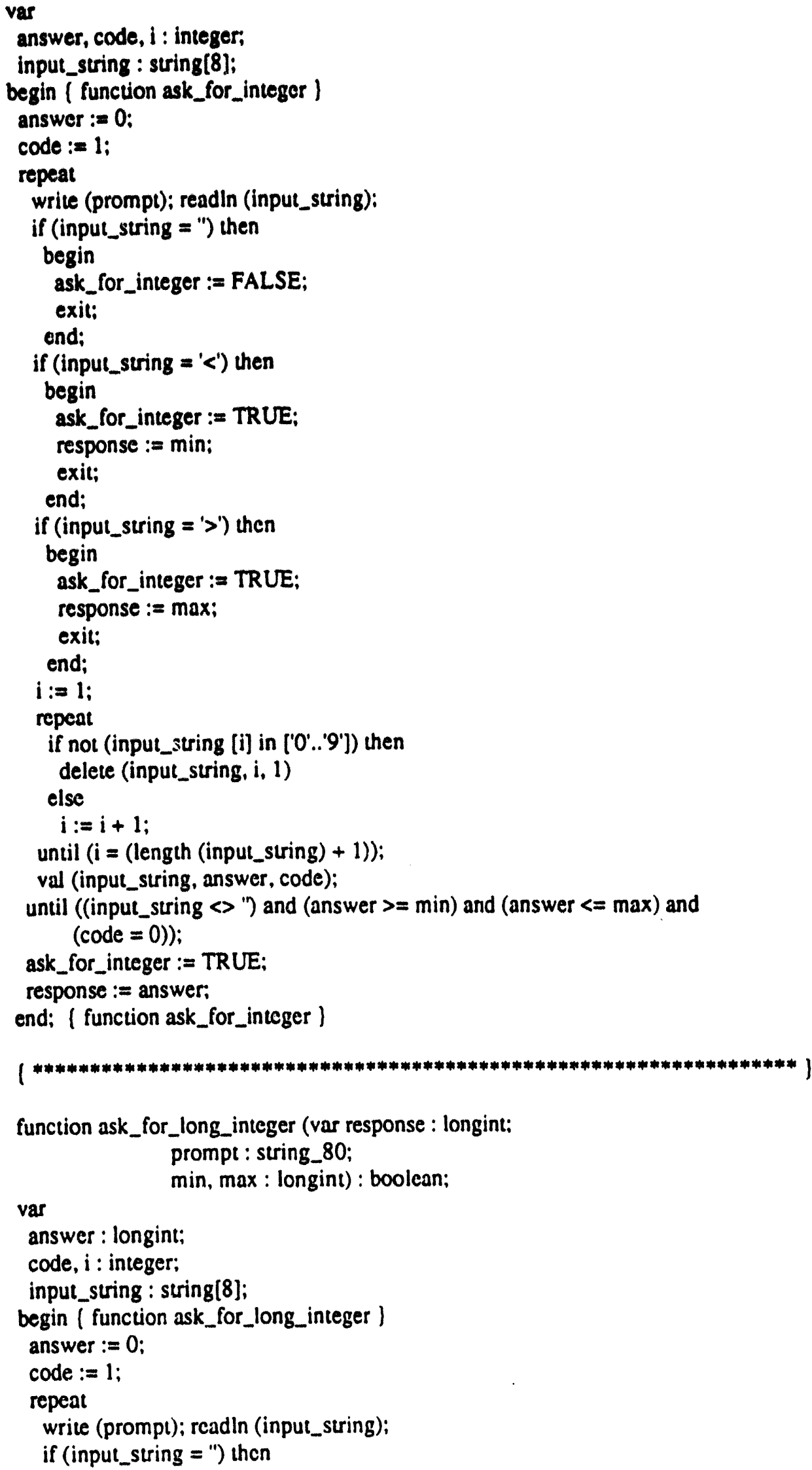




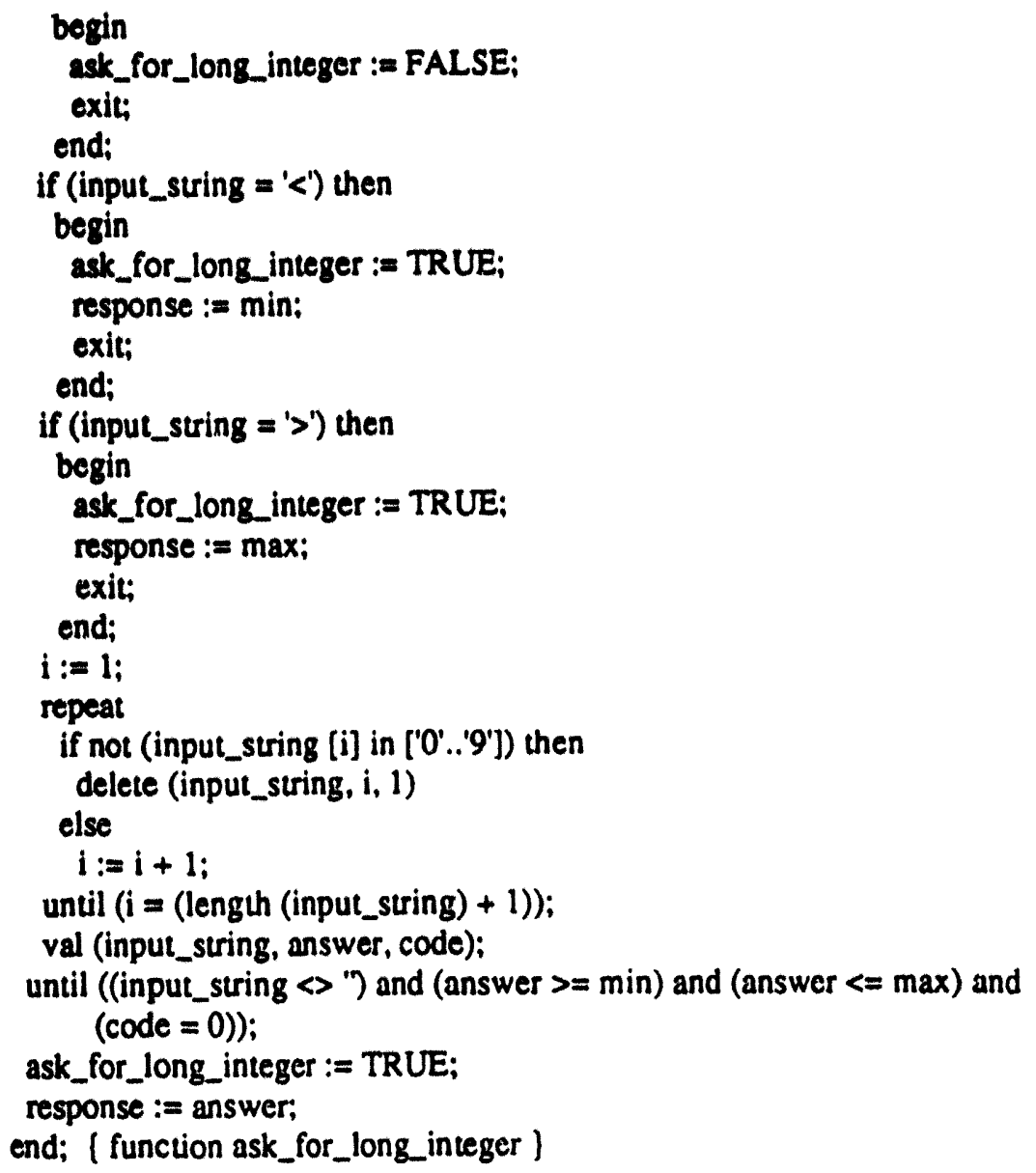

function ask_for_real (var response : real; prompt : suring_80; $\min , \max$ : real) : boolean;

var

answer : real;

code, $i$ : integer;

input_string : string[10];

begin ( function ask_for_rcal )

answer :=0.0;

code $:=1$;

repeat

write (prompt); readln (input_string);

if (input_string = ") then

begin

ask_for_real :=FALSE;

exit;

end;

if (input_string $=$ ' $<$ ') then

begin

ask_for_real := TRUE;

response := $\min$;

exit: 
end;

if (input_string = ' $>$ ') then

begin

ask_for_real := TRUE;

response := max;

exit:

end;

$i:=1$;

repeat

if not (input_string [i] in ['0'..'9', '., 'e', 'E', ' $\because$, '+']) then

delete (input_string, $i, 1$ )

else

$i:=i+1 ;$

until $(i=($ length (input_string) +1$))$;

val (input_string, answer, code);

until ((input_string $<>$ ") and (answer $>=\min$ ) and (answer $<=\max$ ) and $($ code $=0)$ );

ask_for_real := TRUE;

response := answer;

end: ( function ask_for_real )

$\{* * * * * * * * * * * * * * * * * * * * * * * * * * * * * * * * * * * * * * * * * * * * * * * * * * * * * * * * * * * * * * * * * * *)\}$

function wl2wn (wl : real) : real;

begin

wl $2 w n:=(10000000 / w l)$;

end;

$\{* * * * * * * * * * * * * * * * * * * * * * * * * * * * * * * * * * * * * * * * * * * * * * * * * * * * * * * * * * * * * * * * * * * * *)]$

function wn2wl (wn : real) : rcal;

begin

wn2wl := (10000000/wn);

end;

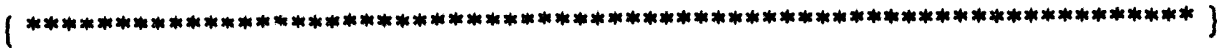

function I2S (Val, Digit : Integer) : Suring;

var

buffer : suring;

begin

str(Val:Digit, Buffer);

I2S := Buffer:

end;

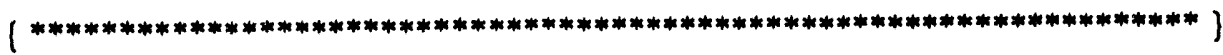

function W2S (Val, Digit: Word) : String;

var

buffer : string;

begin

str (Val:Digit, Buffer);

W2S := Buffer:

end; 
function R2S (Val : real; Digit, Decimal : Integer) : String;

var

buffer : string;

begin

str(Val:Digit:Decimal, Buffer);

R2S := Buffer;

end;

(

function exist (filc_name : string_80) : boolean;

var

test_file : file;

begin ( function exist )

assign (test_file, file_name);

[\$I-]

reset (test_file);

close (test_file);

[\$I+]

exist : $=($ IOresult $=0)$;

end: [ function exist \}

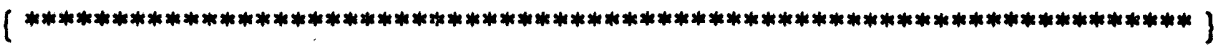

function legal_name (file_name : string_80) : boolean:

var

test_file : file;

begin (function legal_name ]

assign (test_file, file_name);

[\$I-]

rewrite (test_file);

[SI+]

legal_name $:=($ IOresult $=0)$;

close (test_file);

end; ( function legal_name ]

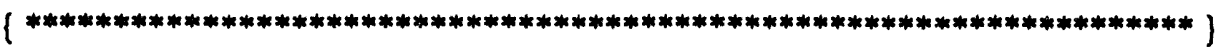

procedure get_out_file_name (var file_name : string_80;

var overwrite : boolean; experiment_type : integer;

vargot_good_out_file_name : boolean);

var

upper_extension, lower_extension, prompt : string;

$\mathrm{i}$ : integer;

begin ( function get_out_file_name ]

case experiment_type of

tps :

begin

upper_extension := '.upp';

lower_extension := 'llow';

prompt := 'Output data file name? [No extension] :' 


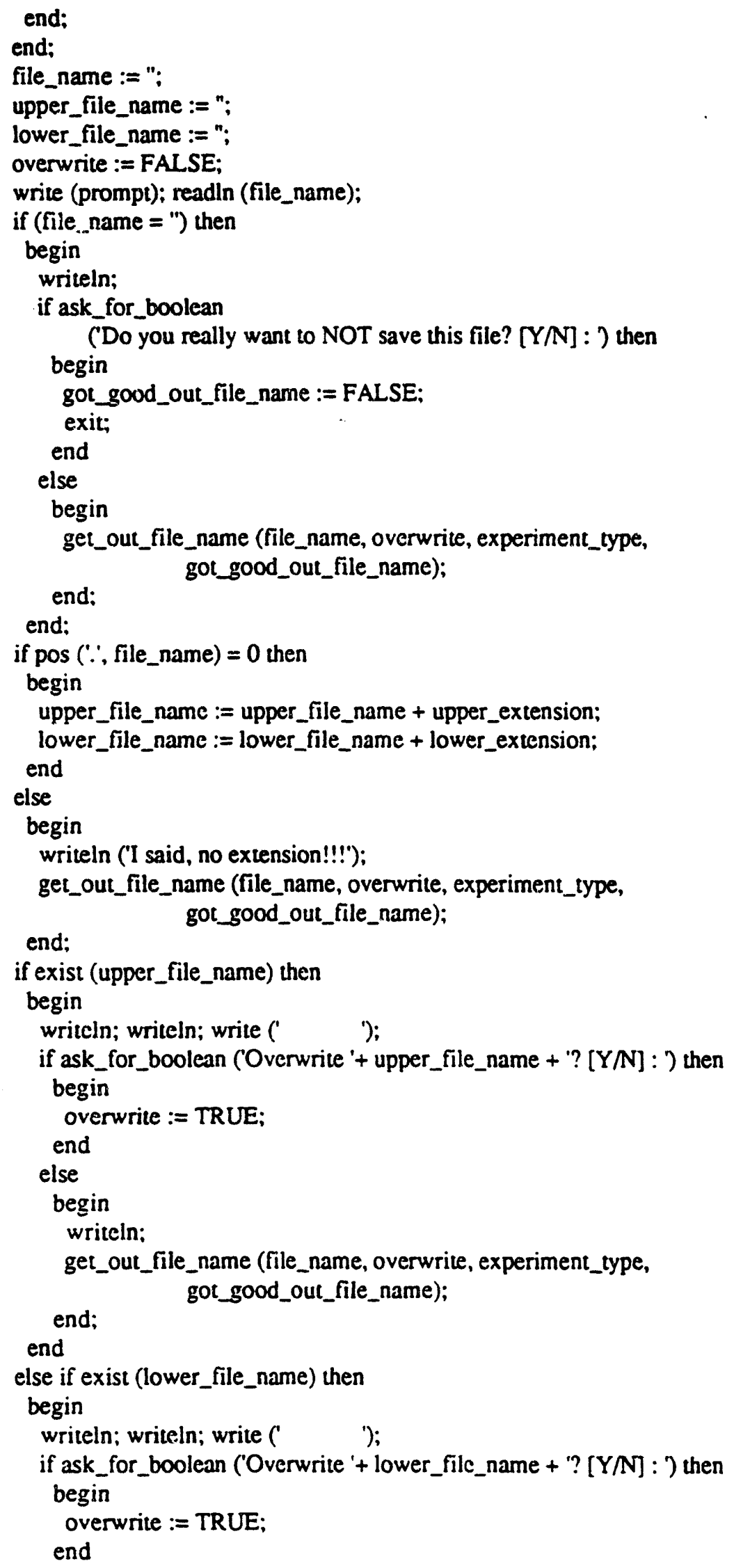




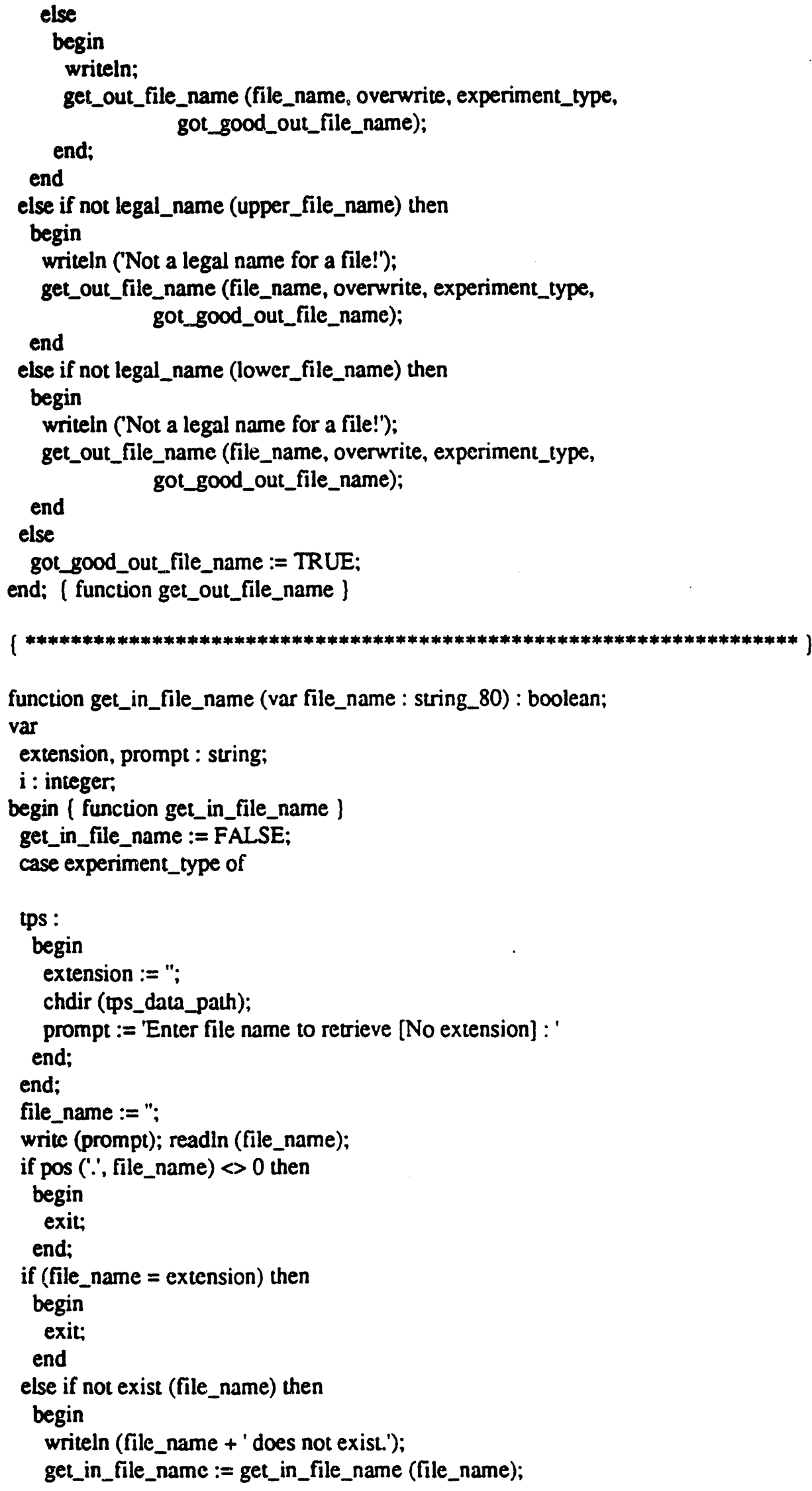




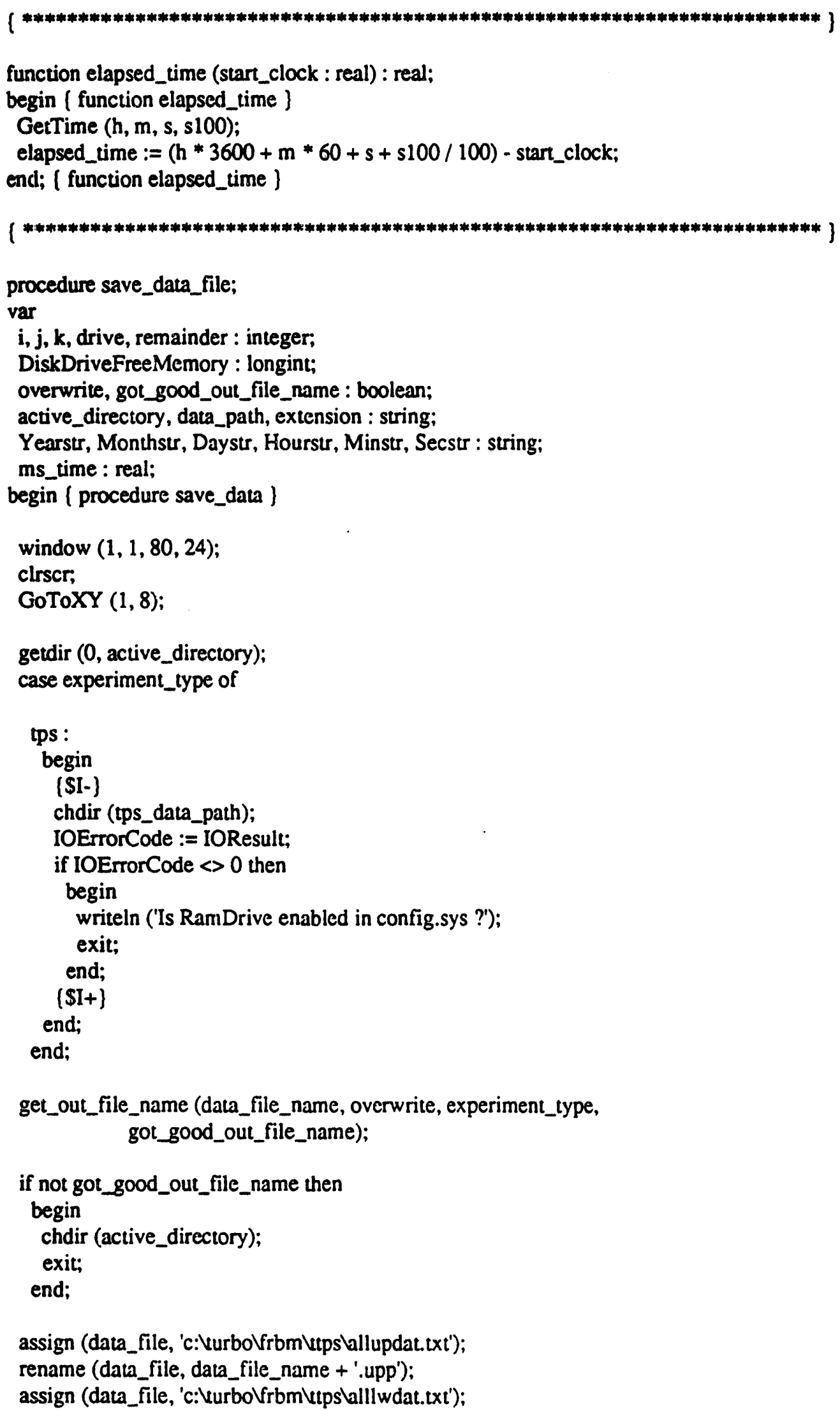


rename (data_file, data_file_name + '.low');

end; \{ procedure save_data \}

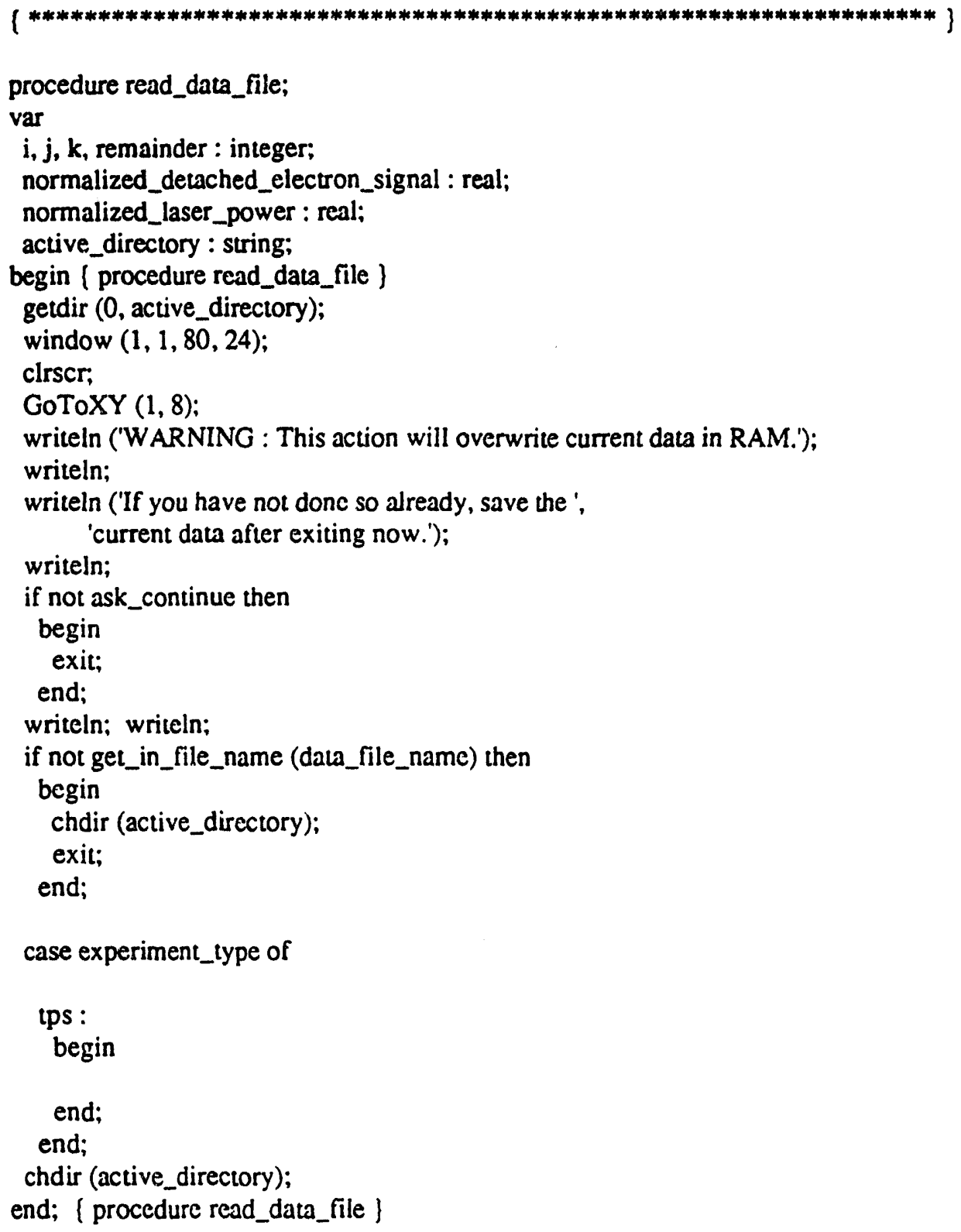




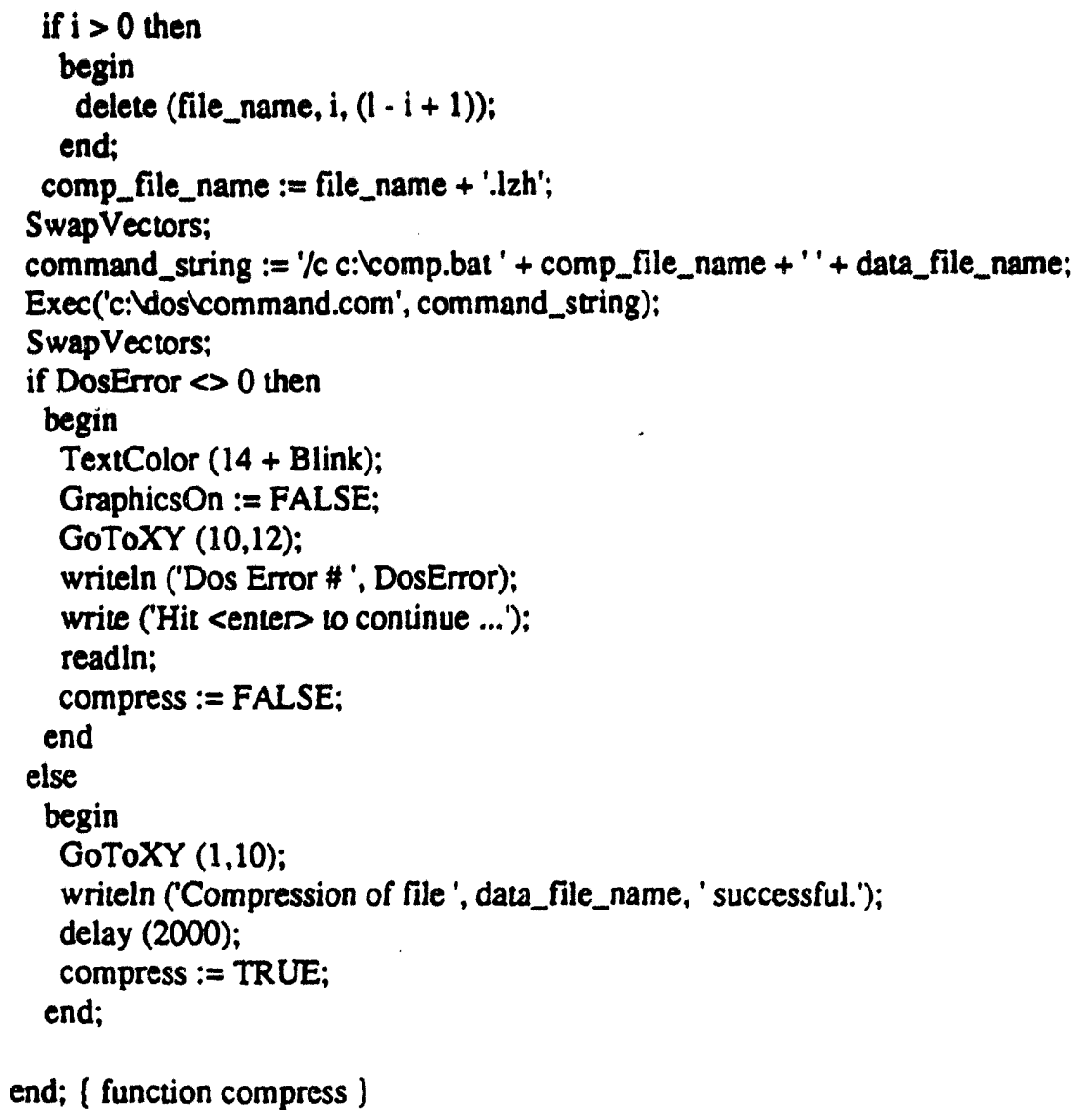

end. \{ of unit tps_util \}

\section{7 codes.pas}

\section{UNIT Codes;}

[SO+]

(SF+)

( This unit is simply a list of the ascii codes for the various editing and function keys. Any program 
employing this unit can use the constant designations in a readkey statement. -- I got sick of looking up the codes. -. JMP.]

INTERFACE

CONST

(Editing Key Codes)

$E S C=\# 27 ;$

RETURN = \#13;

UPARROW = \#72;

DOWNARROW = \#80;

LEFTARROW = \#75;

RIGHTARROW = \#77;

HOME = \#71;

PGUP $=\# 73$;

ENDKEY = \#79;

PGDN = \#81;

INS = \#82;

$\mathrm{DEL}=\# 83$;

[Function Key Codes ]

$\mathrm{F} 1=\# 59 ;$

$F 2=\# 60$;

$F 3=\# 61$;

$F 4=\# 62$;

F5 = \#63;

$\mathrm{F} 6=\# 64 ;$

$F 7=\# 65$;

$\mathrm{F} 8=\# 66$;

$F 9=\# 67$;

$F 10=\# 68$;
ALTF1 = \#104;
ALTF2 = \#105;
ALTF3 = \#106;
ALTF4 = \#107;
ALTF5 = \#108;
ALTF6 = \#109;
ALTF7 = \#110;
ALTF8 = \#111;
ALTF9 = \#112;
ALTF $10=\# 113$;
CTRLF1 = \#94;
CTRLF2 = \#95;
CTRLF3 = \#96;
CTRLF4 = \#97;
CTRLF5 = \#98;
CTRLF6 = \#99;
CTRLF7 $=\# 100$;
CTRLF8 = \#101; 


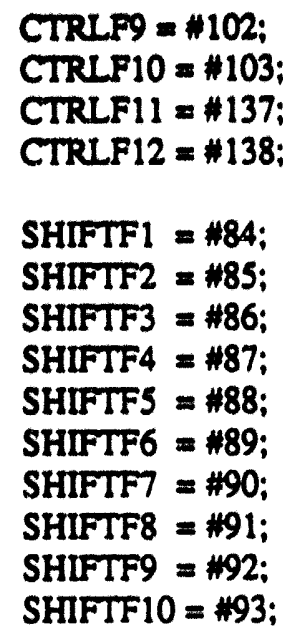

IMPLEMENTATION

END.

\section{8 tpsparam.pas}

I tpsparam.pas

1

l contents :

( procedure tps_experimental_parameters

menu for changing experimental set_up parameters

( procedure get_Ortec_ADC_channcls

1

( procedure get_lower_level_discriminator_settings

了

( function read_set_up : boolean

]

\{ initialize dynamics experimental sct-up parameters from a file \}

( procedure write_set_up

I write dynamics experimental set_up paramcters to a file

( procedure show_mm_parameters

1

( display experimental set_up parameters on right hand side of screen )

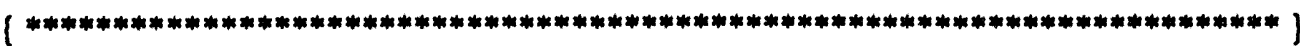

unit tpsparam;

$(\mathrm{SO}+]$

$[\mathrm{SF}+]$

interface 
uses tps_glob,tps_util,.ps_lld,tps_swd,DOS,CRT;

procedure show_mm_parameters;

procedure get_blocking_strip_full_width;

procedure get_TAC_delay;

procedure get_Ortec_ADC_channels:

procedure get_gate_ADCs:

function read_set_up : boolean;

procedure write_set_up;

procedure tps_experimental_parameters;

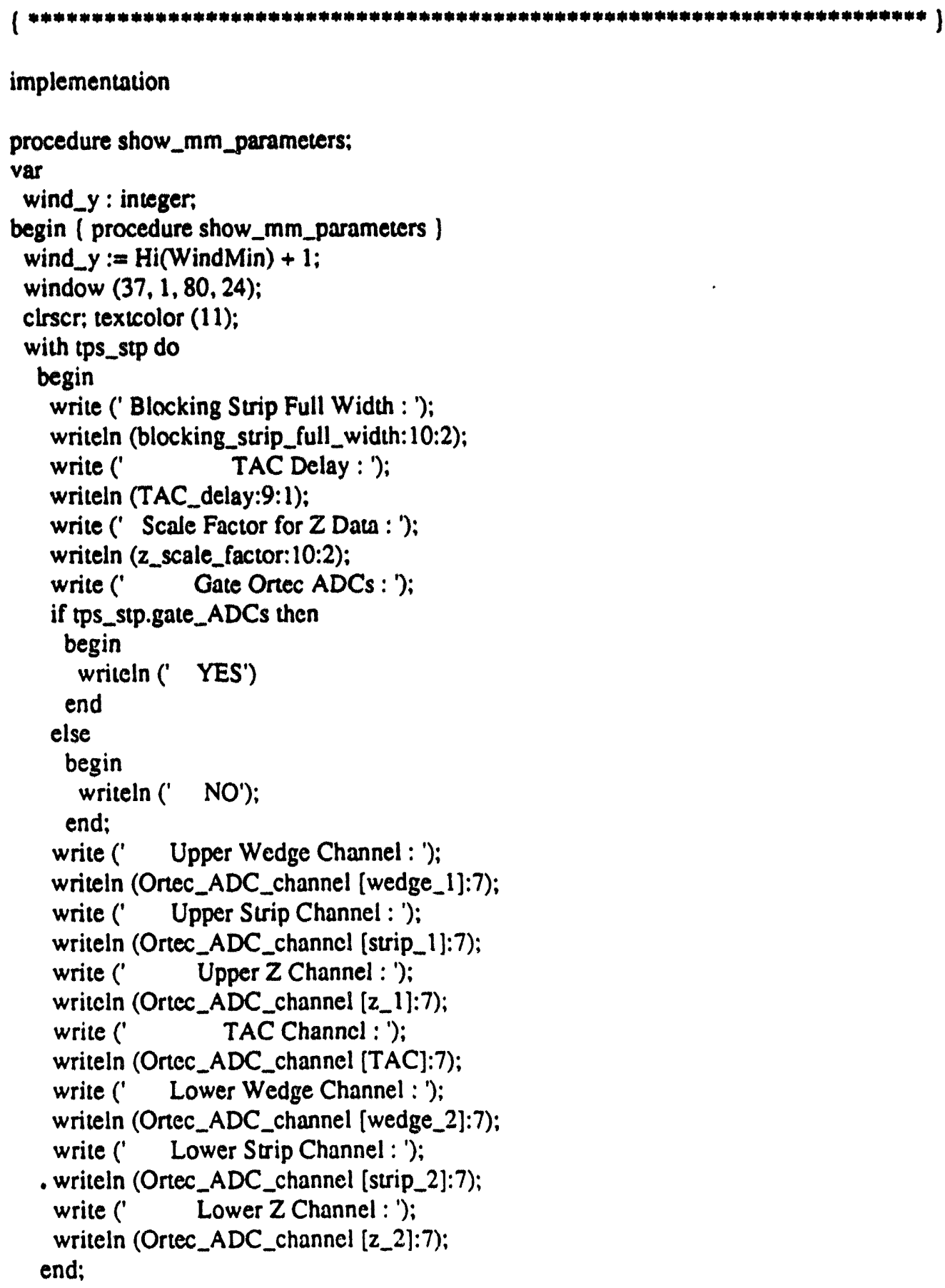


window (1, wind_y, 80, wind_y +1$)$;

textcolor (14);

end; ( procedure show_mm_parameters )

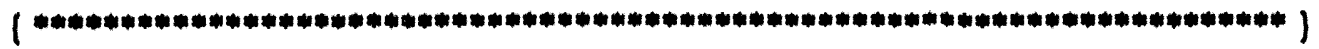

procedure get_blocking_strip_full_width;

begin ( procedure get_blocking_strip_full_width )

if ask_for_real (tps_stp.blocking_strip_full_width,

'Blocking Strip Full Width [mm] : ', 0, 10) then

show_mm_parameters:

end; ( procedure blocking_strip_full_width)

(

procedure get_TAC_delay;

begin (procedure get_TAC_delay )

if ask_for_real (tps_stp.TAC_delay, TAC Delay [ns] : ', 0, 1000) then

show_mm_parameters;

erid; ( procedure TAC_delay )

(

procedure get_2_scale_factor:

begin ( procedure get_z_scale_factor)

writeln " Z Data Scaling Factor : ', tps_stp.z_scale_factor:3:2);

if ask_for_real (t,ps_stp.z_scale_factor, 'New Z Data Scaling Factor : ',

$-10,10$, then

show_mm_füiniseters;

end; ( procedure get_z_scale_factor)

(

procedure get_Orec_ADC_channels;

var

$y:$ integer;

begin (procedure get_Ortec_ADC_channels)

$y:=$ wherey $+\mathrm{Hi}(\mathrm{Wind} \mathrm{Min})$

window $(1, y, 80,(y+1))$;

writeln (' Upper Wedge Signal in Ortec ADC Channel : ,

tps_stp.Ortec_ADC_channel [wedge_1]:1);

if ask_for_integer (tps_stp.Ortec_ADC_channcl [wedge_1],

'New Upper Wedge Signal Ortec ADC Channel : ', 1,7) then

wedge_1_channel := (wedge_1 - 1) $\bmod 4$;

show_mm_parameters:

writeln (' Upper Strip Signal in Ortec ADC Channel : ', tps_stp.Ortec_ADC_channel [strip_1]:1);

if ask_for_integer (tps_stp.Ortec_ADC_channel [strip_1],

'New Upper Strip Signal Ortec ADC Channel : ', 1,7) then strip_1_channel $:=($ surip_1 -1$) \bmod 4$;

show_mm_parameters;

writeln (' Upper Z Signal in Ortec ADC Channel : ', tps_stp.Ortec_ADC_channel [2_1]:1);

if ask_for_integer (tps_stp.Ortcc_ADC_channel [z_l], 
- New Upper Z Signal Ortec ADC Channel : $, 1,7)$ then 2_1_channel := (z_1 - 1) $\bmod 4$; show_mm_parameters;

writeln (" TAC Signal in Ortec ADC Channel : ', tps_stp.Ortec_ADC_channel [TAC]:1);

if ask_for_integer (tps_sip.Oriec_ADC_channel [TAC], New TAC Signal Orec ADC Channel : ', 1, 7) then TAC_channel : $=($ TAC -1$) \bmod 4$; show_mm_parameters;

writeln (' Lower Wedge Signal in Ortec ADC Channel : ', tps_stp.Ortec_ADC_channel [wedge_2]:1);

if ask_for_integer (tps_stp.Ortec_ADC_channel [wedge_2],

'New Lower Wedge Signal Ortec ADC Channel : $, 1,7)$ then wedge_2_channel $:=($ wedge_2 - 1) $\bmod 4$; show_mm_parameters;

writeln (' Lower Strip Signal in Ortec ADC Channel : ', tps_stp.Ortec_ADC_channel [strip_2]:1);

if ask_for_integer (tps_stp.Orrec_ADC_channel [strip_2],

New Lower Strip Signal Ortec ADC Channel : $, 1,7)$ then strip_2_channel := (surip_2 - 1) $\bmod 4$; show_mm_parameters;

writcln (' Lower Z Signal in Ortec ADC Channel : ', tps_stp.Ortec_ADC_channel $\left.\left[z_{-} 2\right\}: 1\right)$;

if ask_for_integer (tps_stp.Ortec_ADC_channcl [z_2],

- New Lower Z Signal Ortec ADC Channel : $, 1,7)$ then z_2_channel := (z_2 - 1) mod 4; show_mm_parameters:

end ( procedure get_Ortec_ADC_channels ):

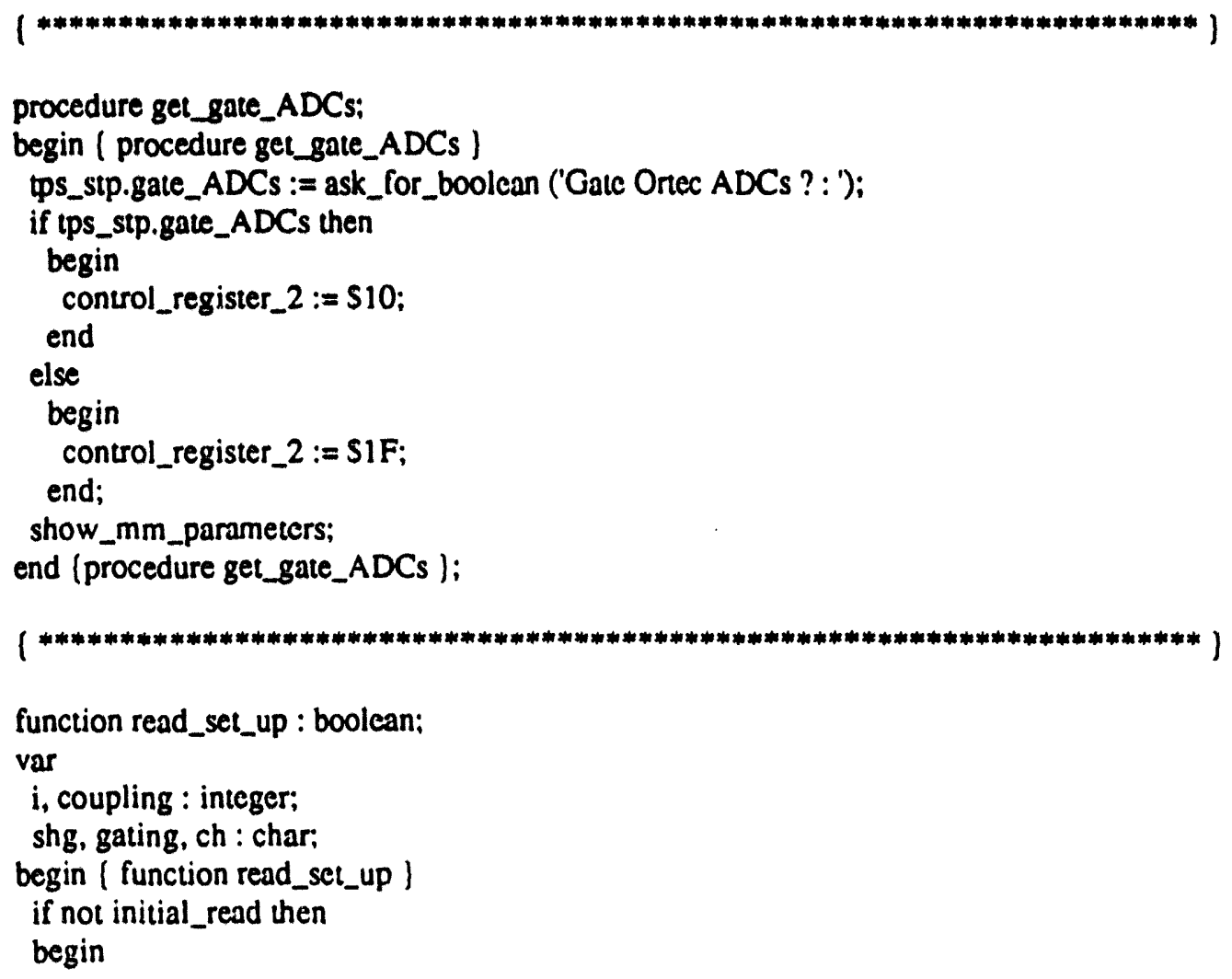




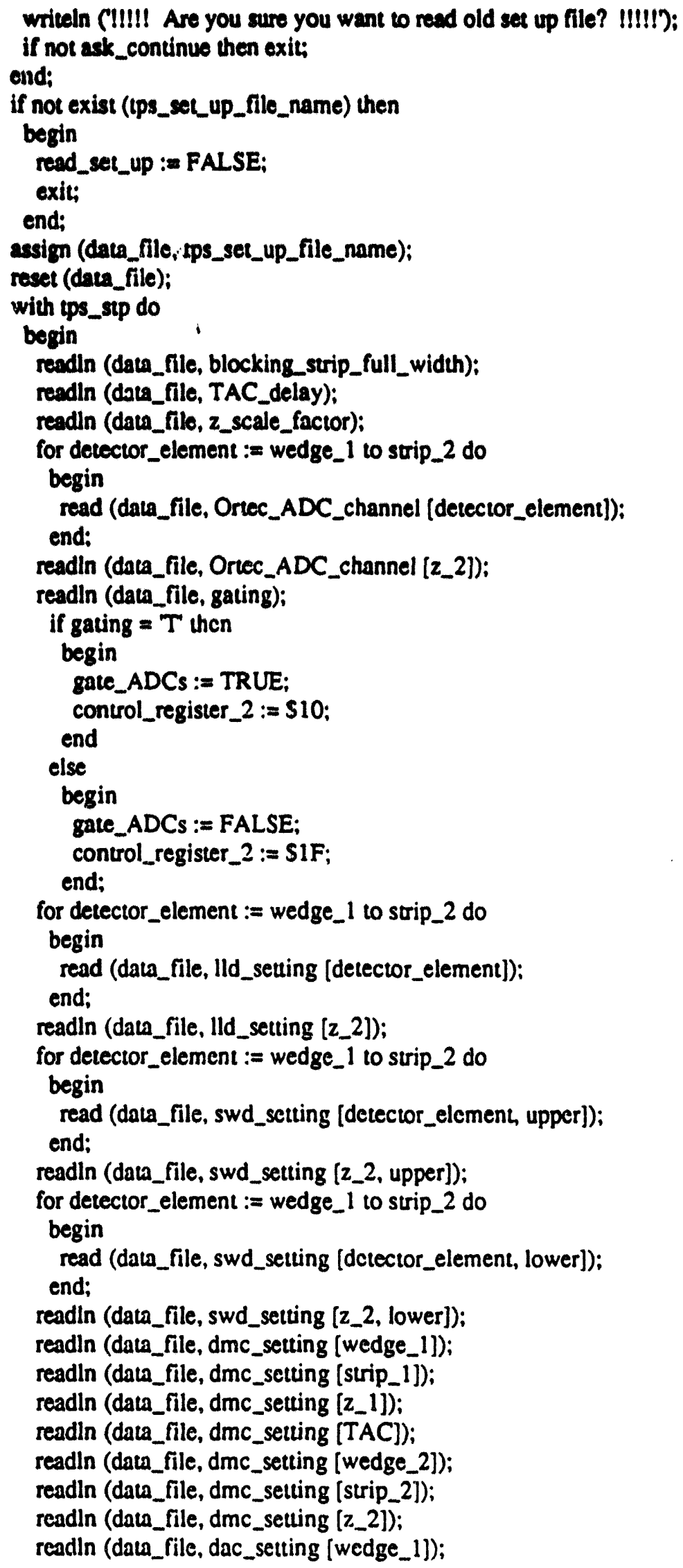




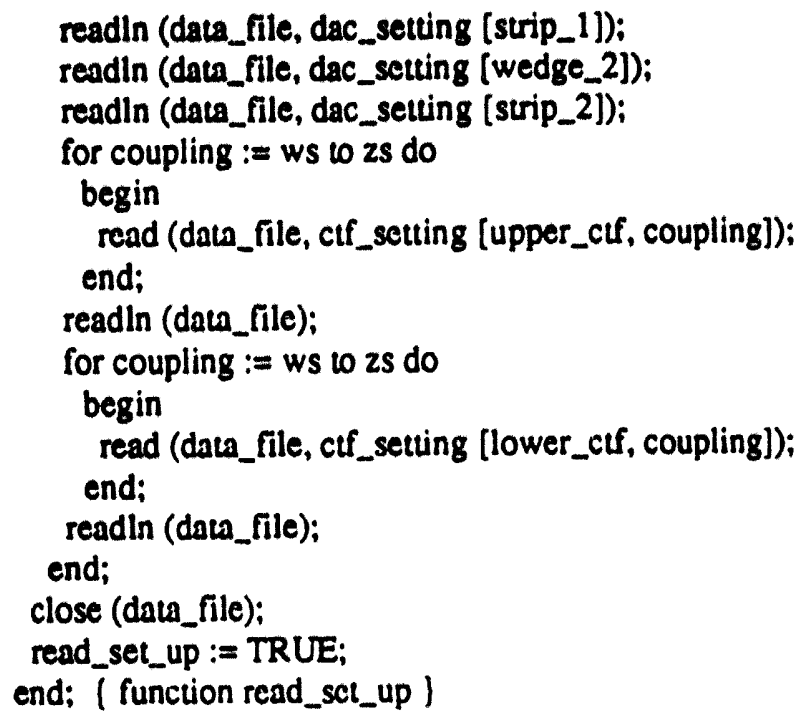




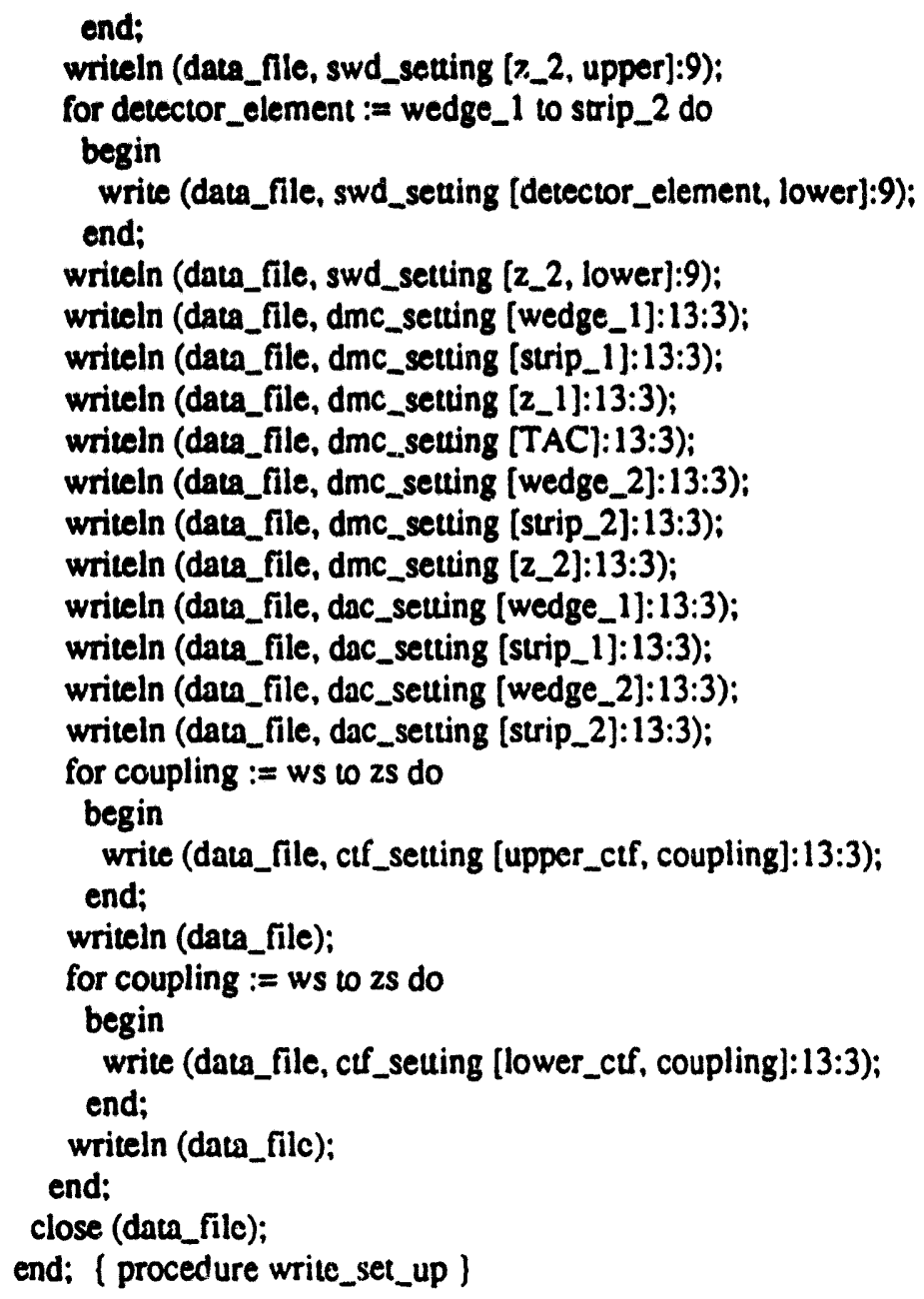




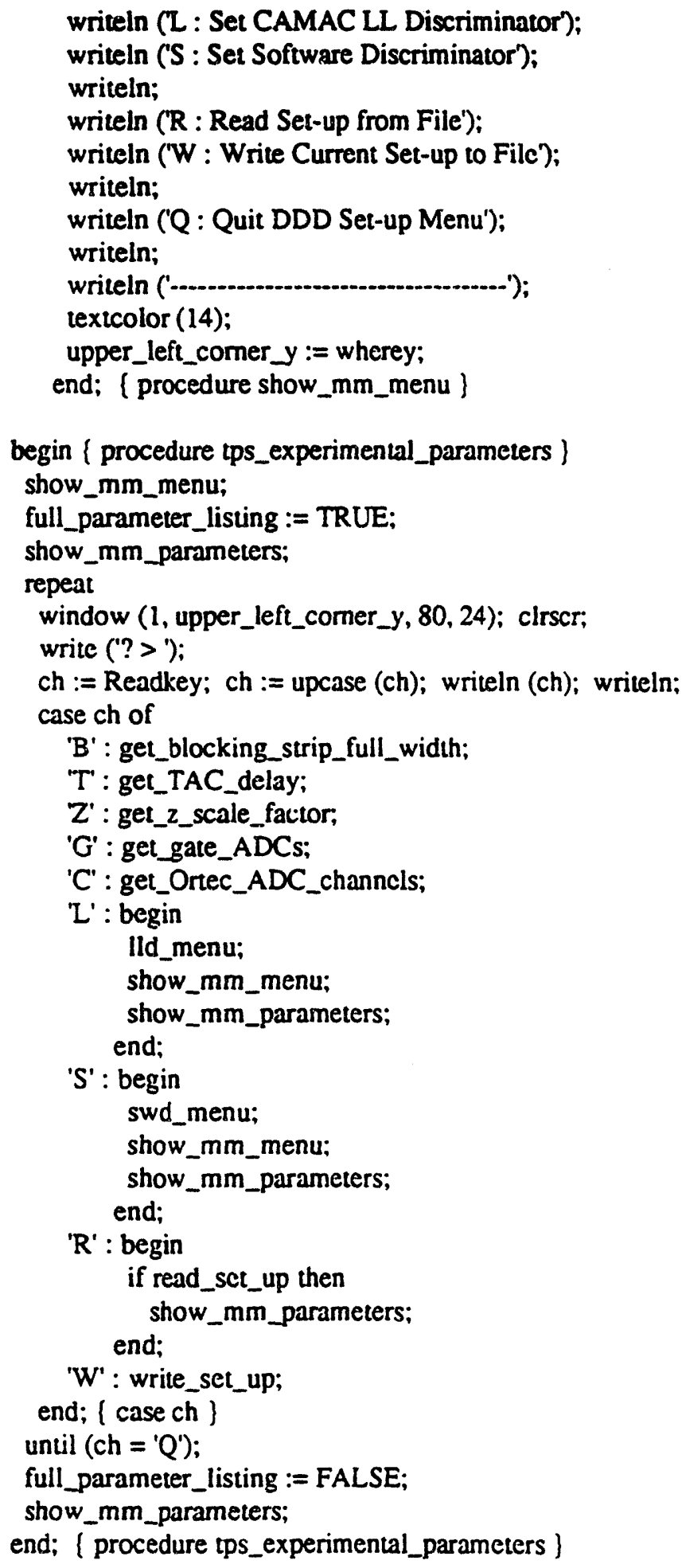

end. \{ unit tpsparam \} 


\section{9 tps_radc.pas}

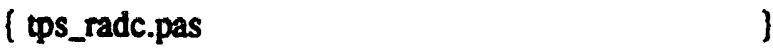

unit tps_radc;

$\{\mathrm{SO}+\}$

[SF+\}

interface

uses ps_glob,tps_util,codes,tpsparam,tps_lld,DOS,CRT;

procedure read_adc;

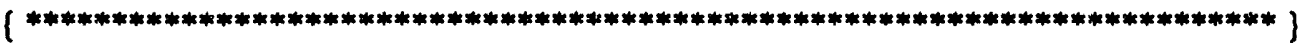

implementation

[SF-]

[\$I camturbo.v4]

$\{\mathrm{SF}+\}$

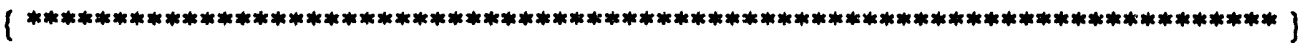

procedure read_adc:

var

ch : char,

$w, y, i, Q 1, Q 2$ : integer;

wedge_1_charge, strip_1_charge, z_1_charge : integer:

wedge_2_charge, strip_2_charge, z_2_charge : integer;

TAC_voltage : integer;

tps_delta_lambda, lambda, old_lambda : real;

begin ( procedure read_adc )

show_mm_parameters;

repeat

window $(1,1,36,24)$;

clrscr, textcolor (15);

writeln;

writeln ('R : Rcad Ortcc ADCs');

writeln;

writeln ('L : Set Lower Level Discriminator');

writeln;

writeln;

writeln;

writeln;

writeln ('Q : Quit');

writeln;

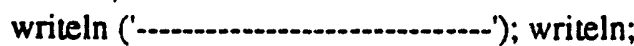




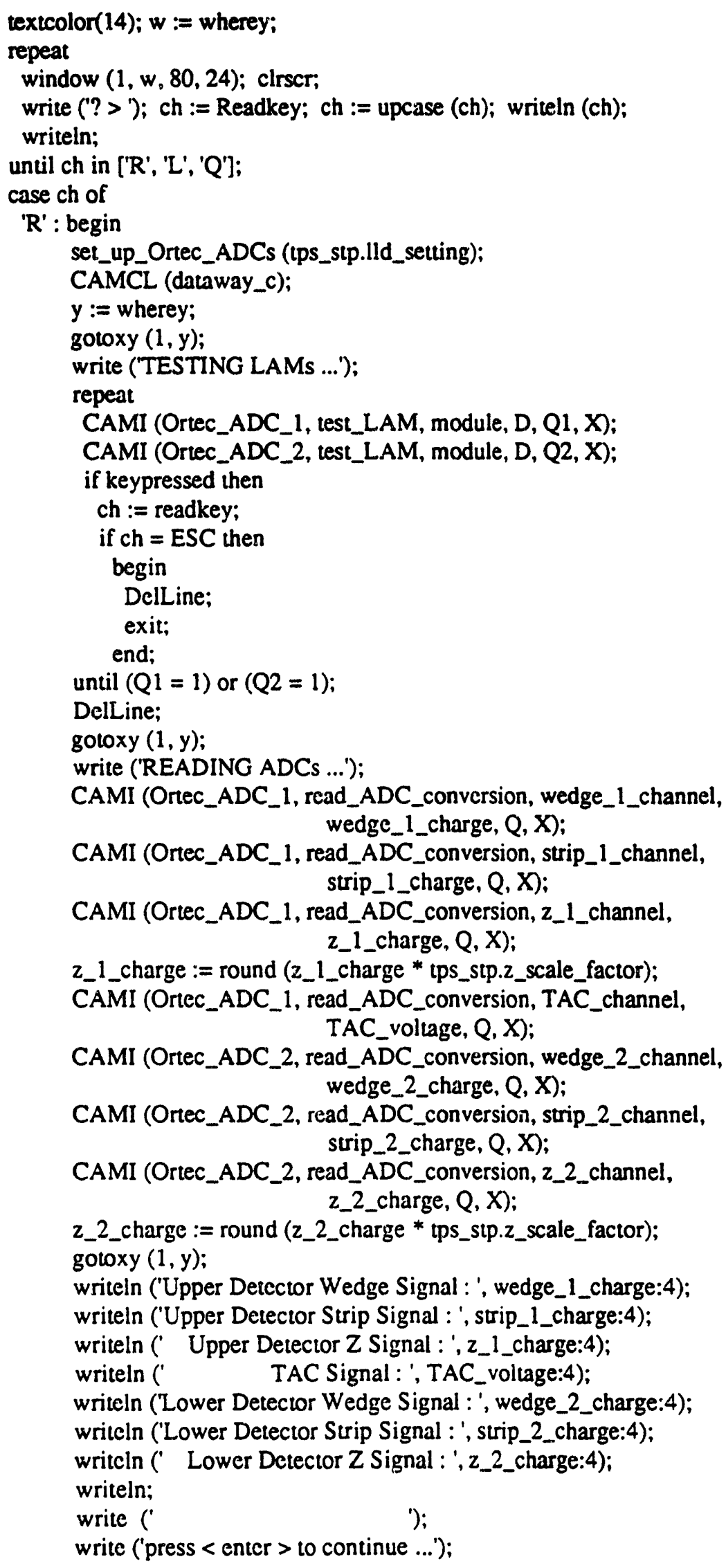


readln;

end;

'L' : begin

lld_menu;

show_mm_parameters;

end:

end; ( case ch )

until (ch = 'Q');

window $(1,1,80,24)$; textcolor (15); clrscr;

end: (procedure read_adc )

end. ( unit tps_radc )

\subsection{0 tps_exp.pas}

(tps_exp.pas ]

( contents : )

$\{* * * * * * * * * * * * * * * * * * * * * * * * * * * * * * * * * * * * * * * * * * * * * * * * * * * * * * * * * * * * * * * * * * * * * * * *)]$

unit tps_exp;

[SO+]

$[\$ F+]$

interface

uses tps_glob,tps_util,tpsparam,tps_lld,tps_dcc,tps_ctf,

dfplot,tpsplot,graph,plotutil,codes,CRT,DOS;

function test_data (half : integer; tps_data : tps_int_column_array) : boolean;

procedure obtain_tps_data;

procedure show_tps_parametcrs;

procedure tps_menu;

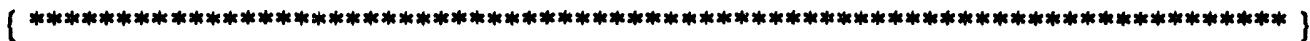

implementation

\{SF- $\}$

[\$I camturbo.v4]

$\{\mathrm{SF}+\}$

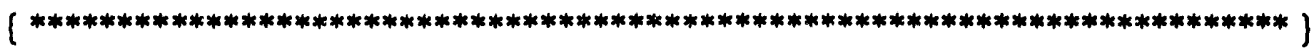

function test_data (half : integer; tps_data : tps_int_column_array) : boolean; 


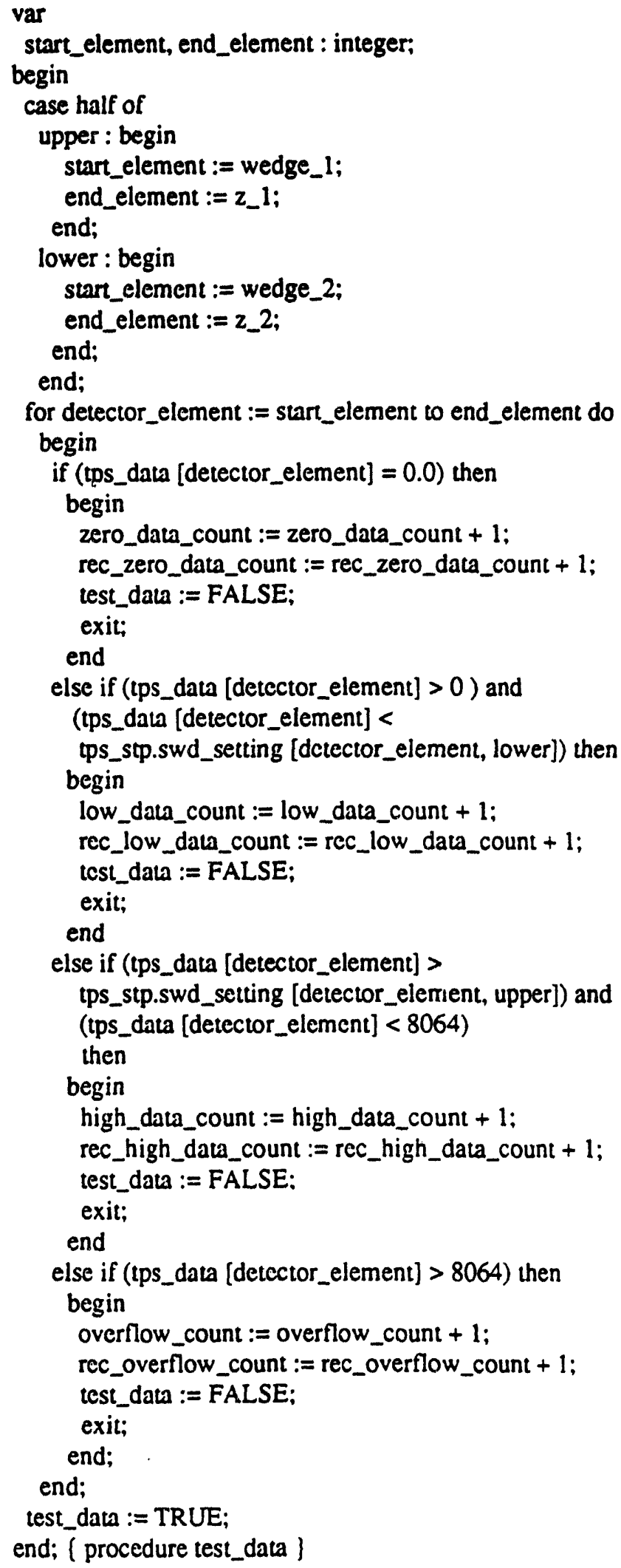




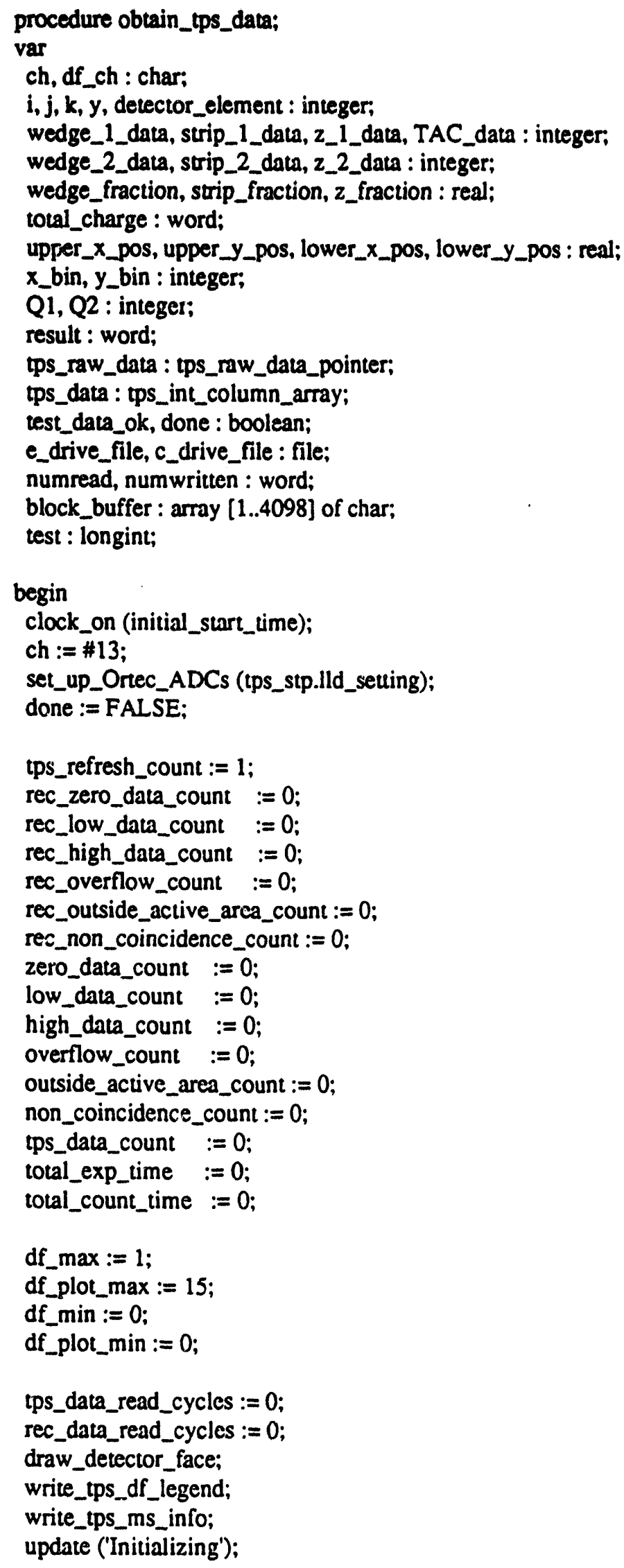




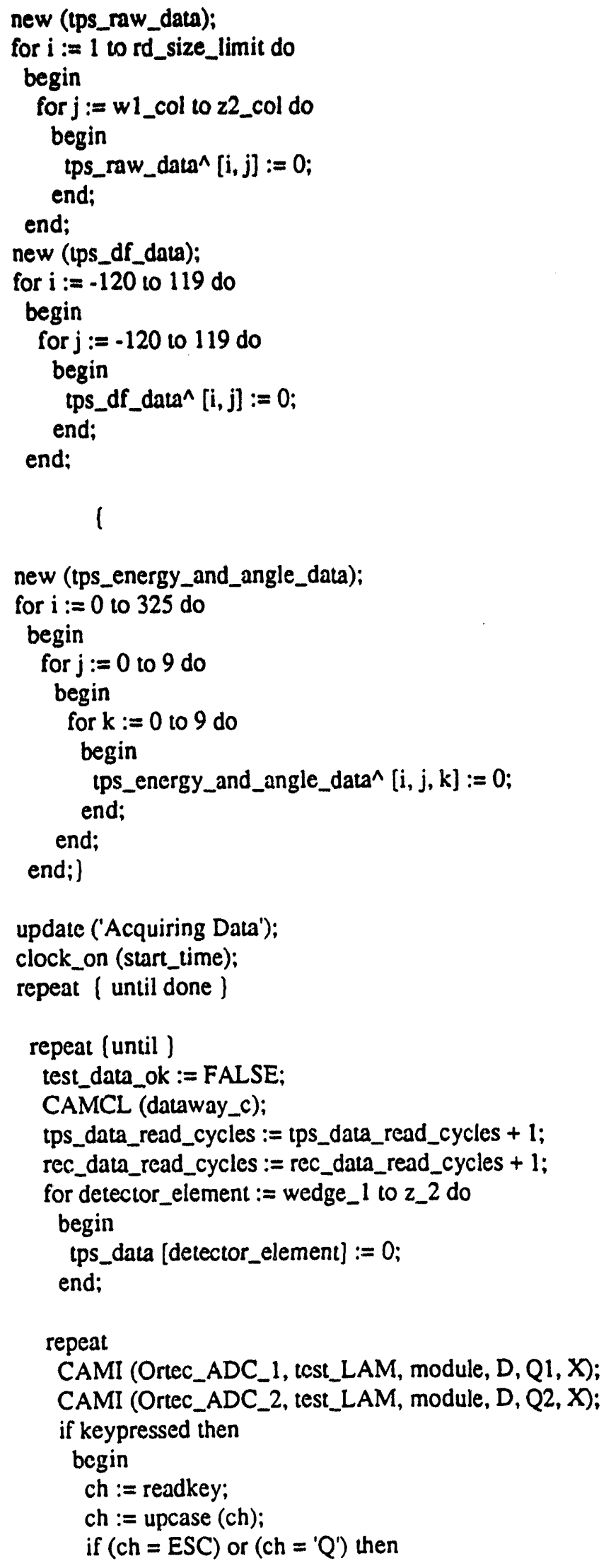




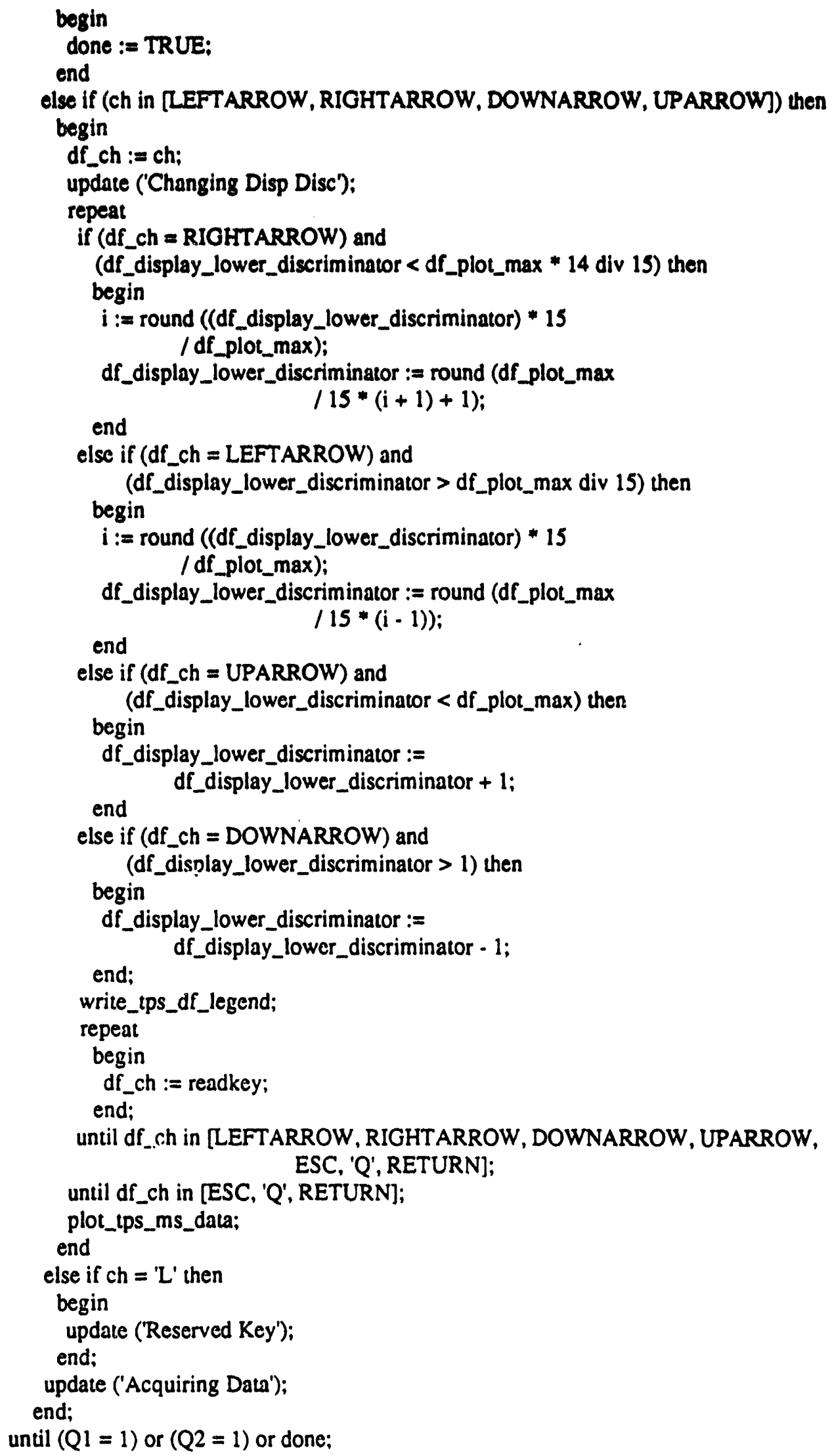




\section{if not dorie then \\ begin \\ delay (1);}

CAMI (Ortec_ADC_1, read_ADC_conversion,

TAC_channel, tps_data [TAC], $Q, X$ ):

( To run in non-coincidence mode, i.e. with no TAC 'enforcement' for flat

field acquisition, replace the following if statement with the if $(1=1)$ then statement following it which is normally commented out)

if (tps_data [TAC] $>=$ tps_stp.swd_setting [TAC, lower]) and (tps_data [TAC] $<=$ tps_stp.swd_setting [TAC, upper]) then \{if $(1=1)$ then\}

begin

CAMI (Ortec_ADC_1, read_ADC_conversion, wedge_1_channel, tps_data [wedge_1], Q, X);

CAMI (Ortec_ADC_1, read_ADC_conversion, strip_1_channel, tps_data [strip_1], Q, X);

CAMI (Ortec_ADC_1, read_ADC_conversion, 2_1_channel,

if test_data (upper, tps_data) then tps_data [z_1], Q, X);

\section{begin}

tps_data [2_1] := round (tps_data [2_1] *tps_stp.z_scale_factor); tps_raw_data [tps_refresh_count, w1_col] := tps_data [wedge_1]; tps_raw_data^ [tps_refresh_count, s1_col] := ps_data [strip_1]; tps_raw_data^ [tps_refresh_count, z1_col] := t.ps_data [z_1]; tps_raw_data^ [tps_refresh_count, TAC_col] := tps_data [TAC];

CAMI (Ortec_ADC_2, read_ADC_conversion, wedge_2_channel, tps_data [wedge_2], $Q, X$ ):

CAMI (Ortec_ADC_2, read_ADC_conversion, strip_2_channel, tps_data [strip_2], Q, X);

CAMI (Ortec_ADC..2, read_ADC_conversion, 2_2_channel,

if test_data (lower, tps_data) then tps_data [z_2], Q, X);

begin

tps_data [z_2] := round (tps_data [z_2] * tps_stp.z_scale_factor);

tps_raw_data^ [tps_refresh_count, w2_col] := tps_data [wedge_2];

tps_raw_data^ [tps_refresh_count, s2_col] := tps_data [strip_2];

tps_raw_data [tps_refresh_count, z2_col] := tps_data [z_2];

tps_refresh_count $:=$ tps_refresh_count +1 ;

tps_data_count $:=$ tps_data_count +1 ; test_data_ok := TRUE;

end;

end:

end

else

begin

non_coincidence_count $:=$ non_coincidence_count +1 ;

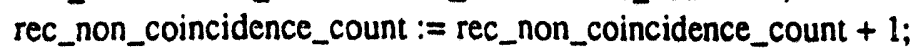
end;

end;

until test_data_ok or done;

if not done then

bcgin 


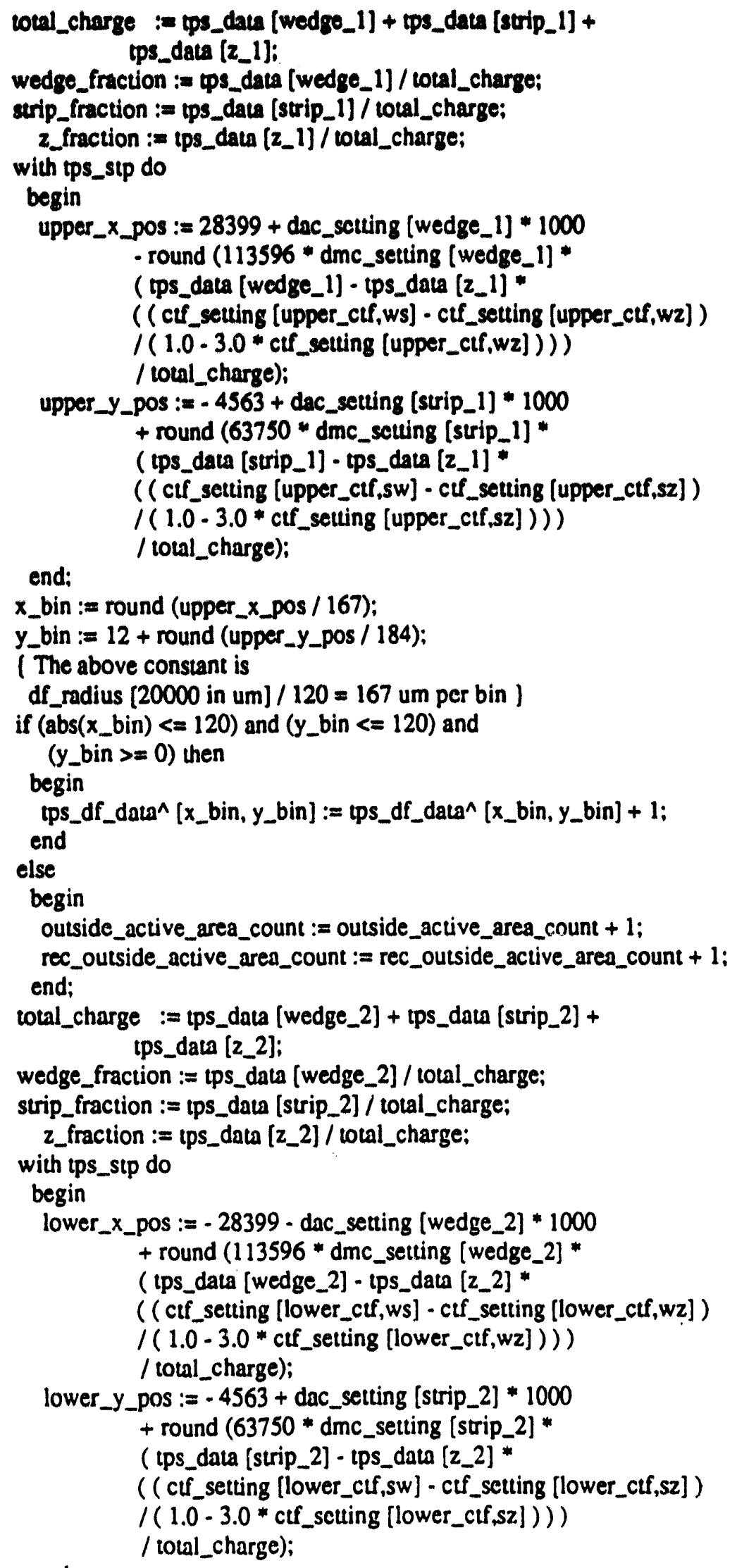




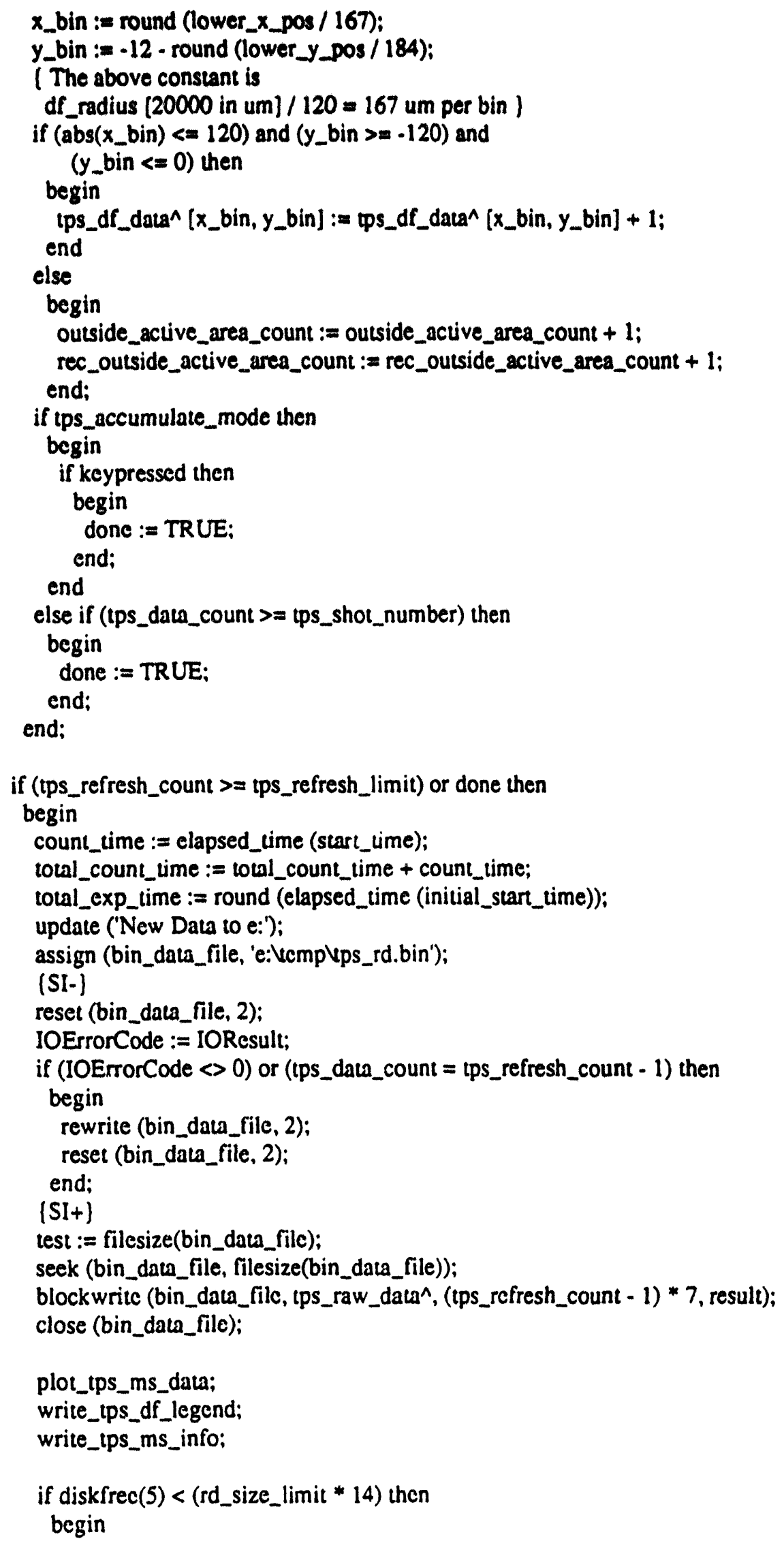




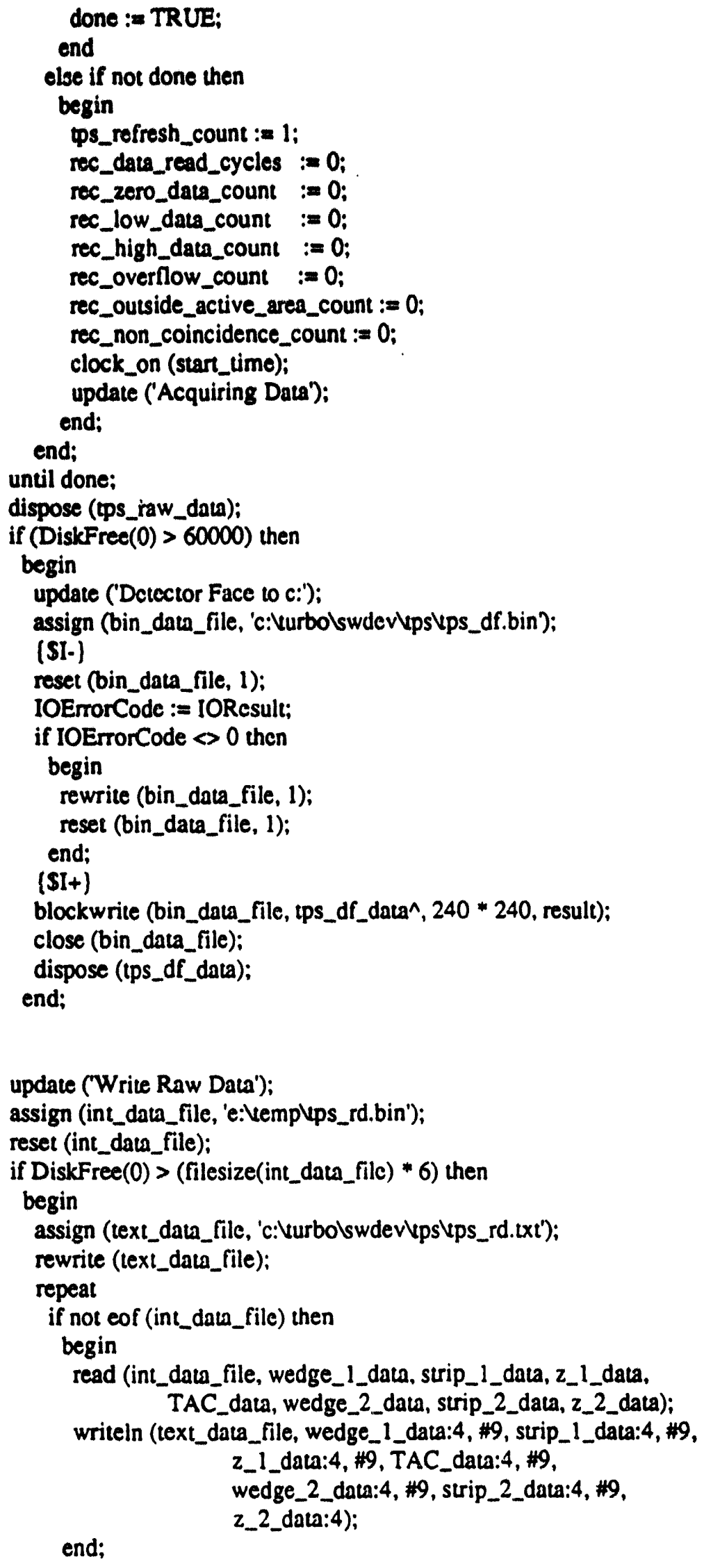




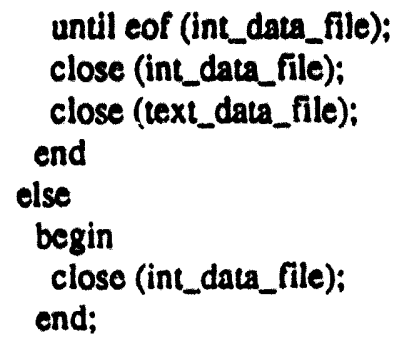

if (DiskFree $(0)>2000000)$ then

begin

update ('Write E \& A Data');

assign (text_data_file, 'c:Ufrbm Vynvps_ea.txt');

rewrite (text_data_file);

new (tps_energy_and_angle_data);

for $i:=0$ to 325 do

begin

writcln (text_daca_file, 'Encrgy = ', i:3);

for $j:=0$ to 9 do

begin

writeln (text_data_file, 'Theta $=\mathrm{j}, \mathrm{j}: 2$ );

writeln (text_data_file, ' 0.9 9-18 18-27 27-36 36-45 45-54',

- $54-63$ 63-72 72-81 81-90 ');

for $k:=0$ to 9 do

begin

write (tcxt_data_file, tps_energy_and_angle_data^ $[i, j, k]: 4)$;

end:

writeln (text_data_file);

end:

writeln (text_data_file);

end;

close (text_data_file);

dispose (tps_energy_and_angle_data);

end:

*)

update ('!!! DONE !!!');

sct_done_flag;

RestoreCRTMode:

GraphicsOn := FALSE;

end;

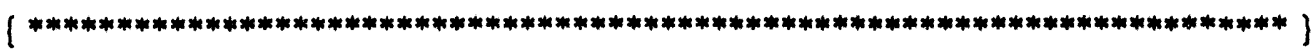

procedure get_tps_shot_number;

begin (procedure get_tps_shot_number )

if ask_for_long_integer (tps_shot_number, 'Shots per Scan : ',

$1,2000000000)$ then

show_tps_parameters:

end (procedure get_tps_shot_number ):

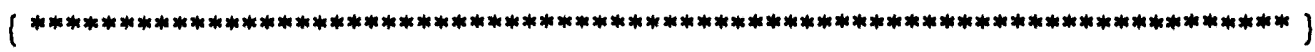




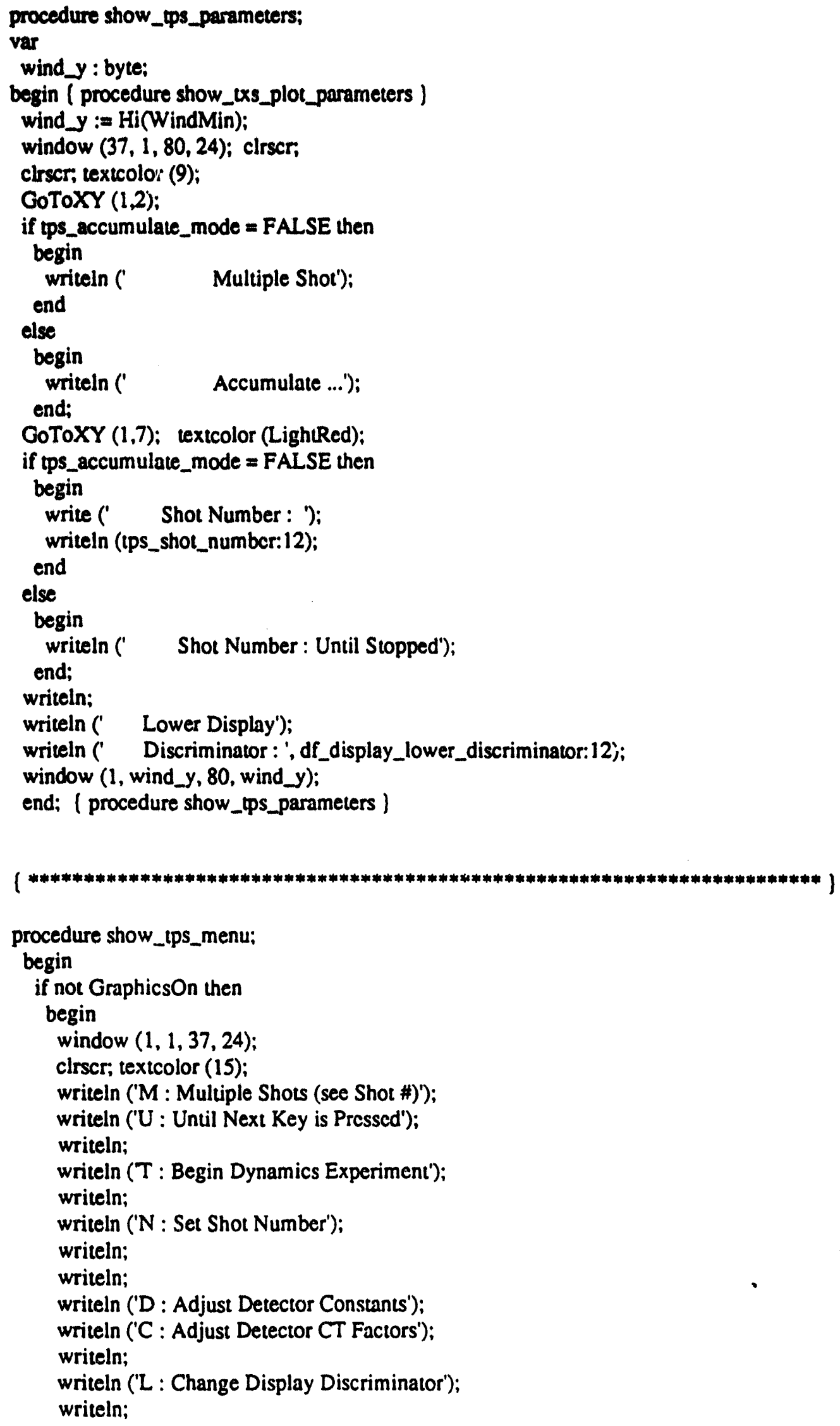




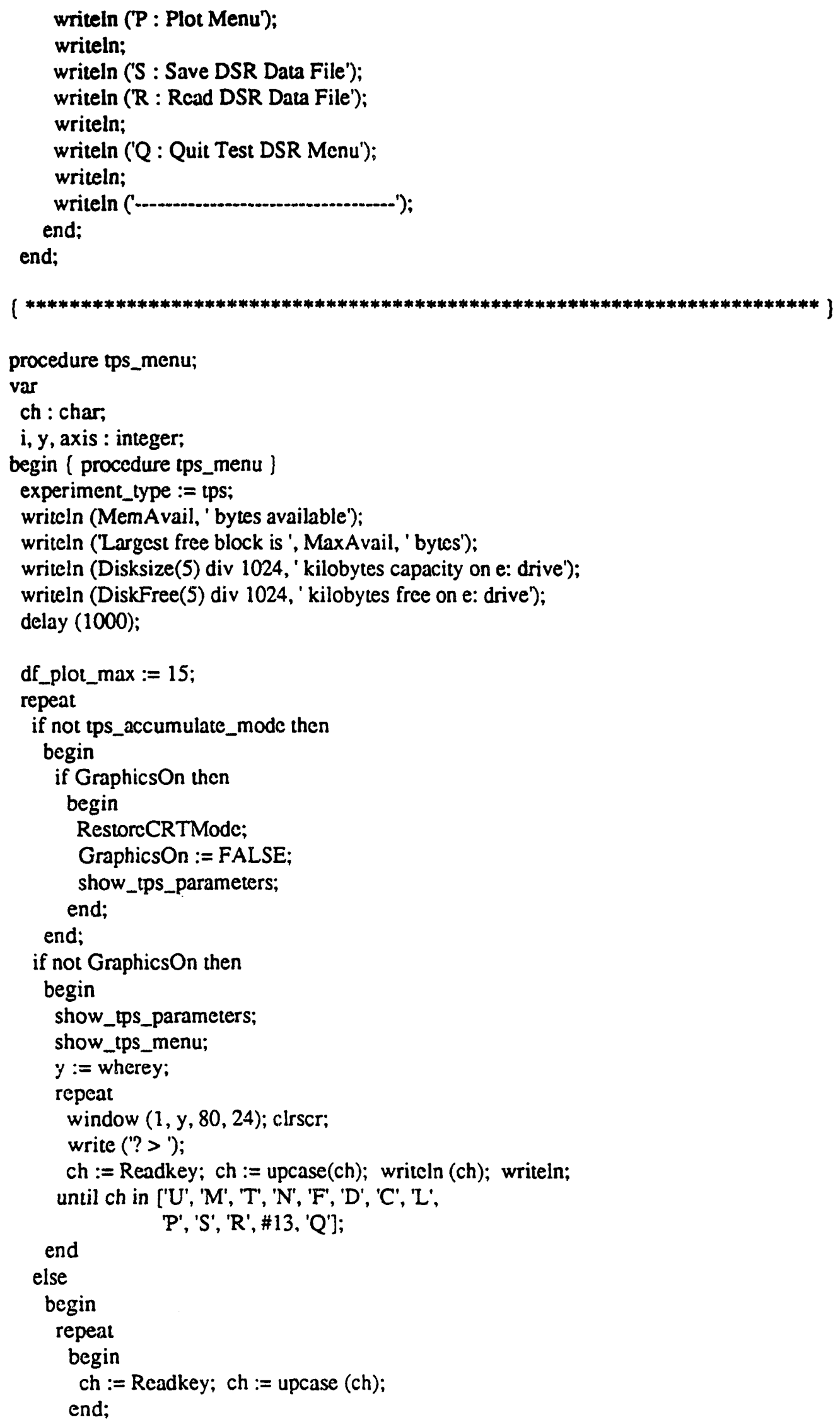




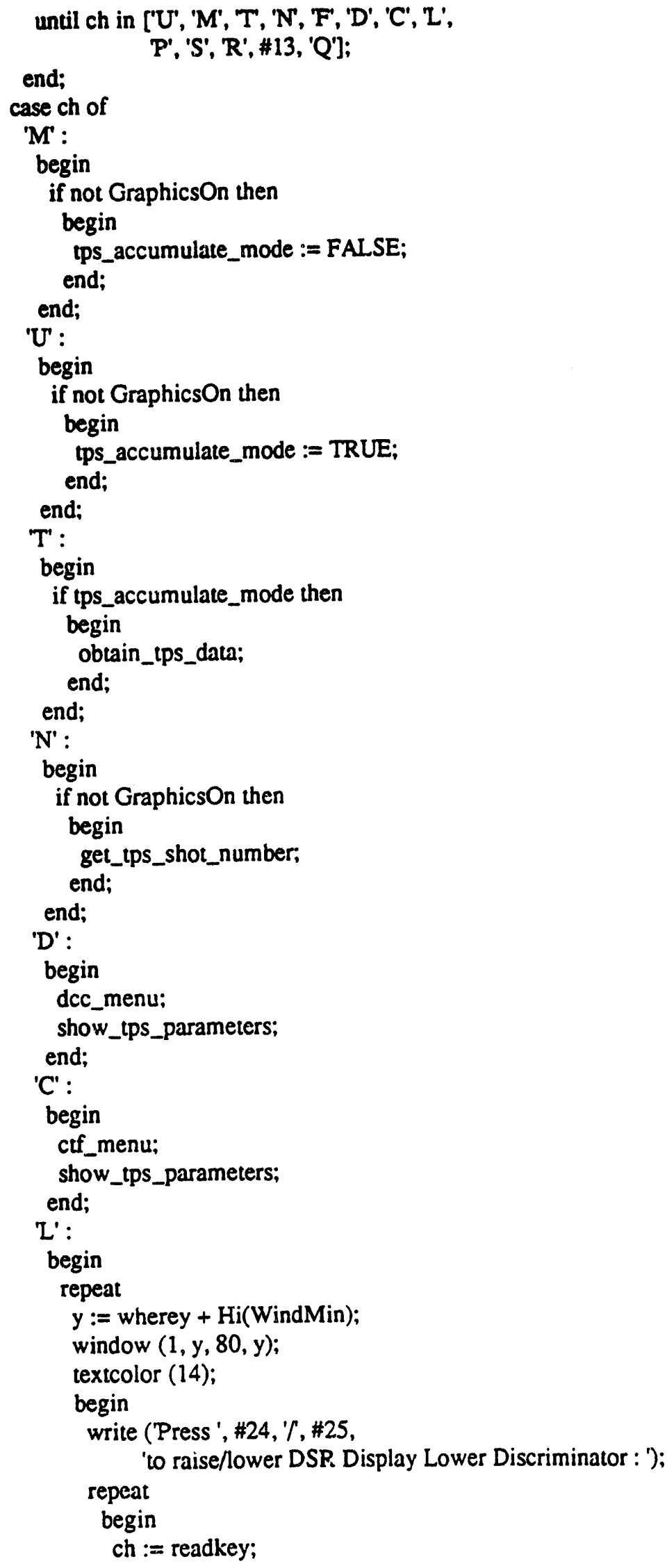




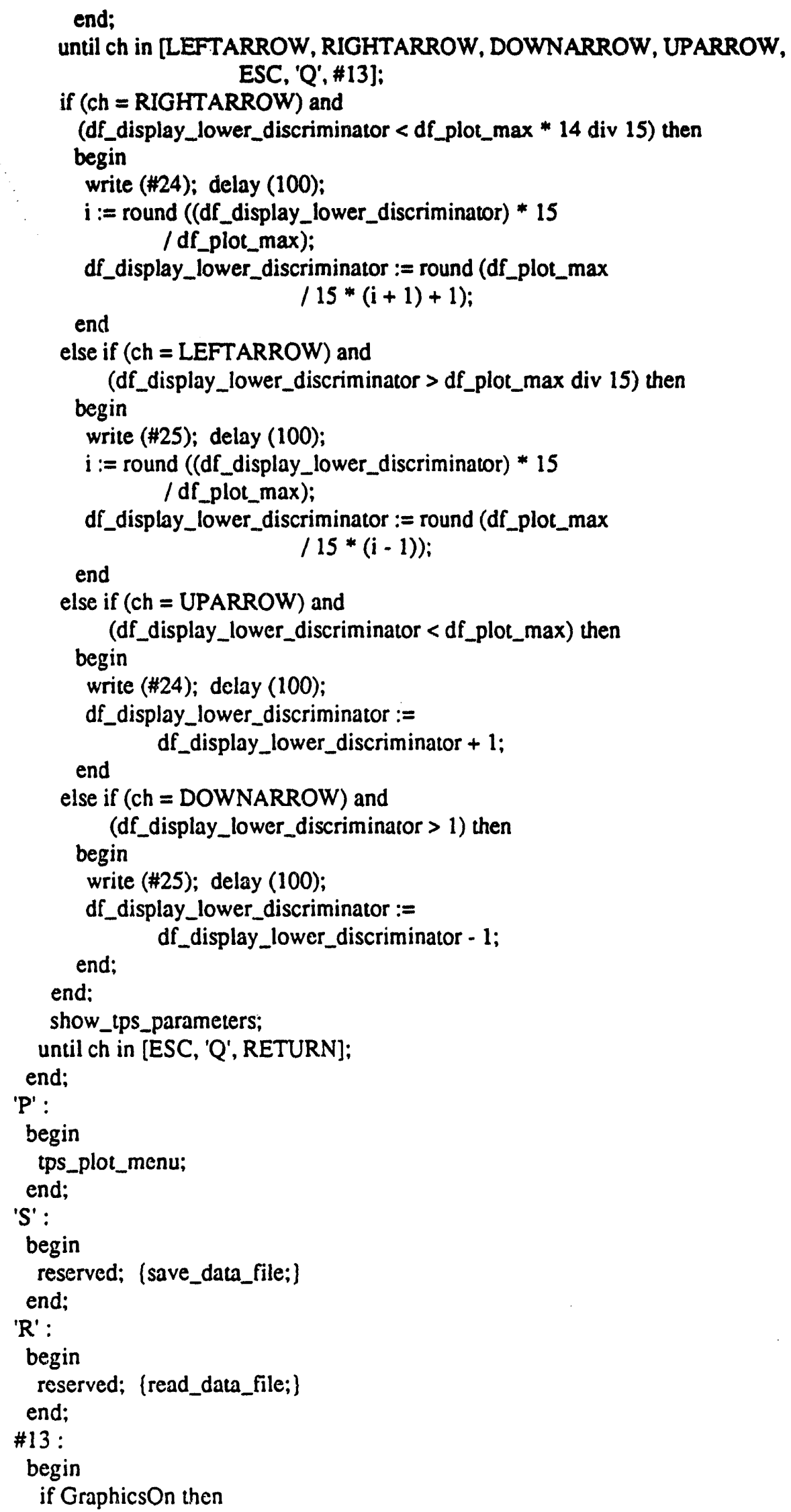




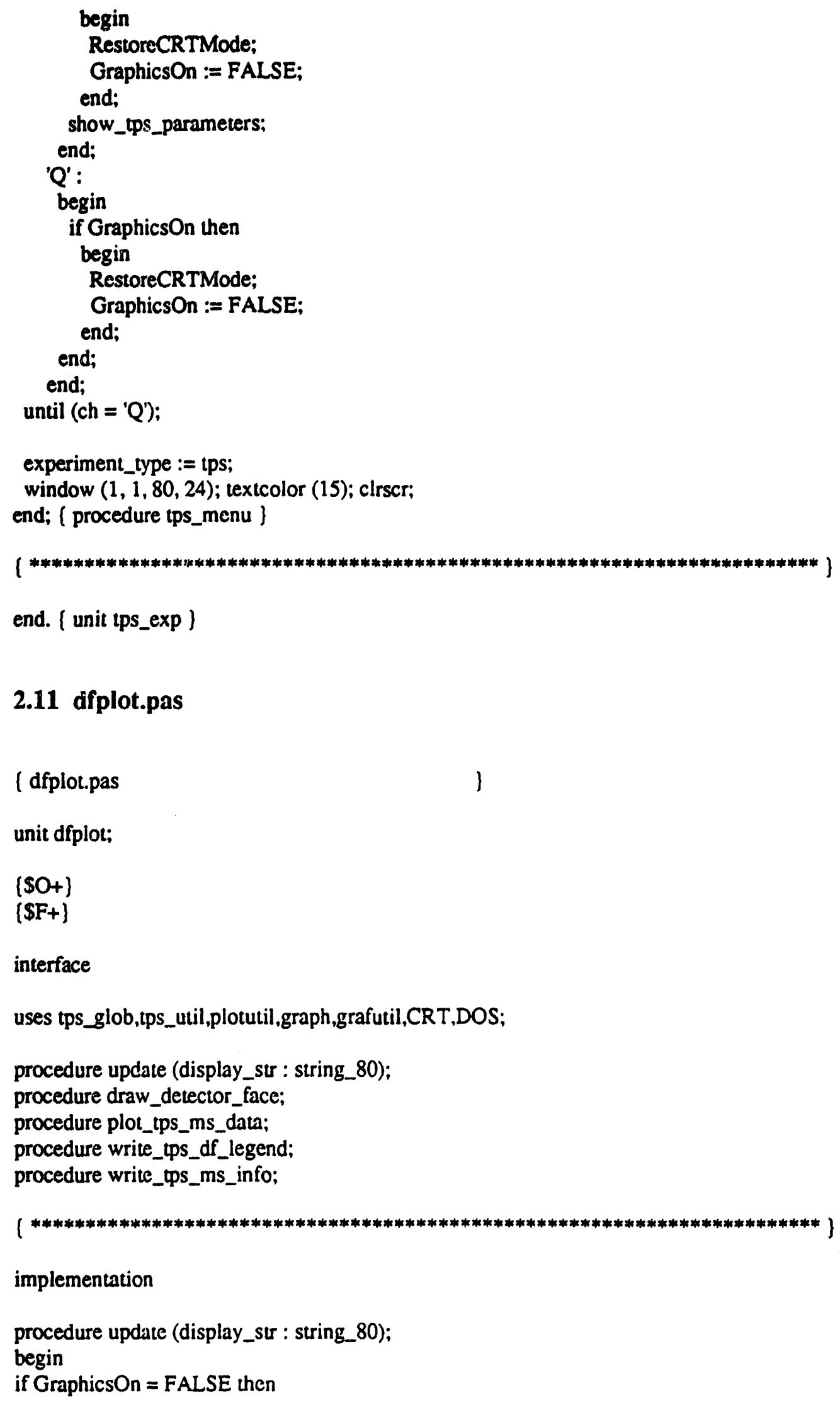

2.11 difplot.pas

\section{( dfplot.pas}

unit dfplot;

$[\mathrm{SO}+]$

$[\mathrm{SF}+]$

interface

uses tps_glob,tps_util,plotutil,graph,grafutil,CRT,DOS;

procedure update (display_str : string_80);

procedure draw_detector_face;

procedure plot_tps_ms_data;

procedure write_tps_df_legend;

procedure write_tps_ms_info; 


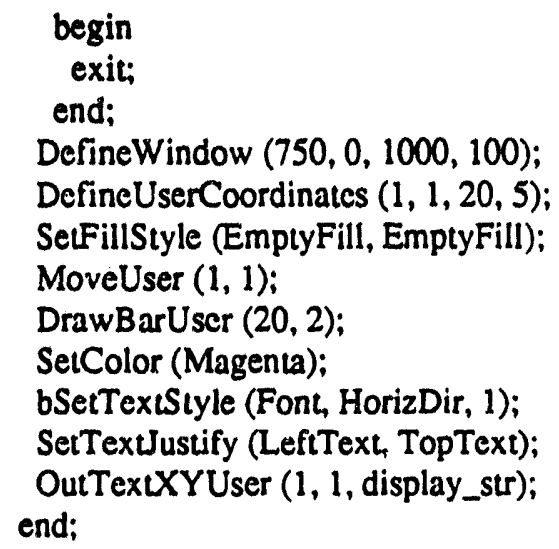




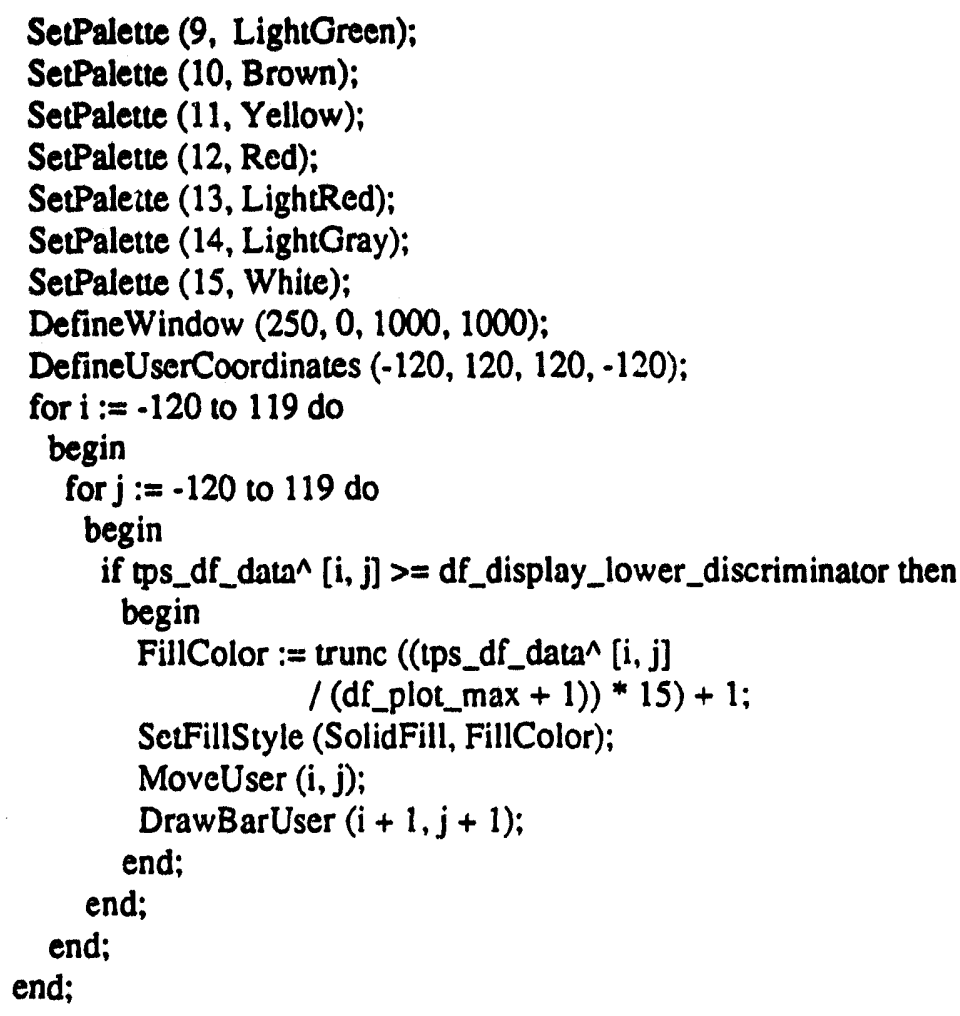




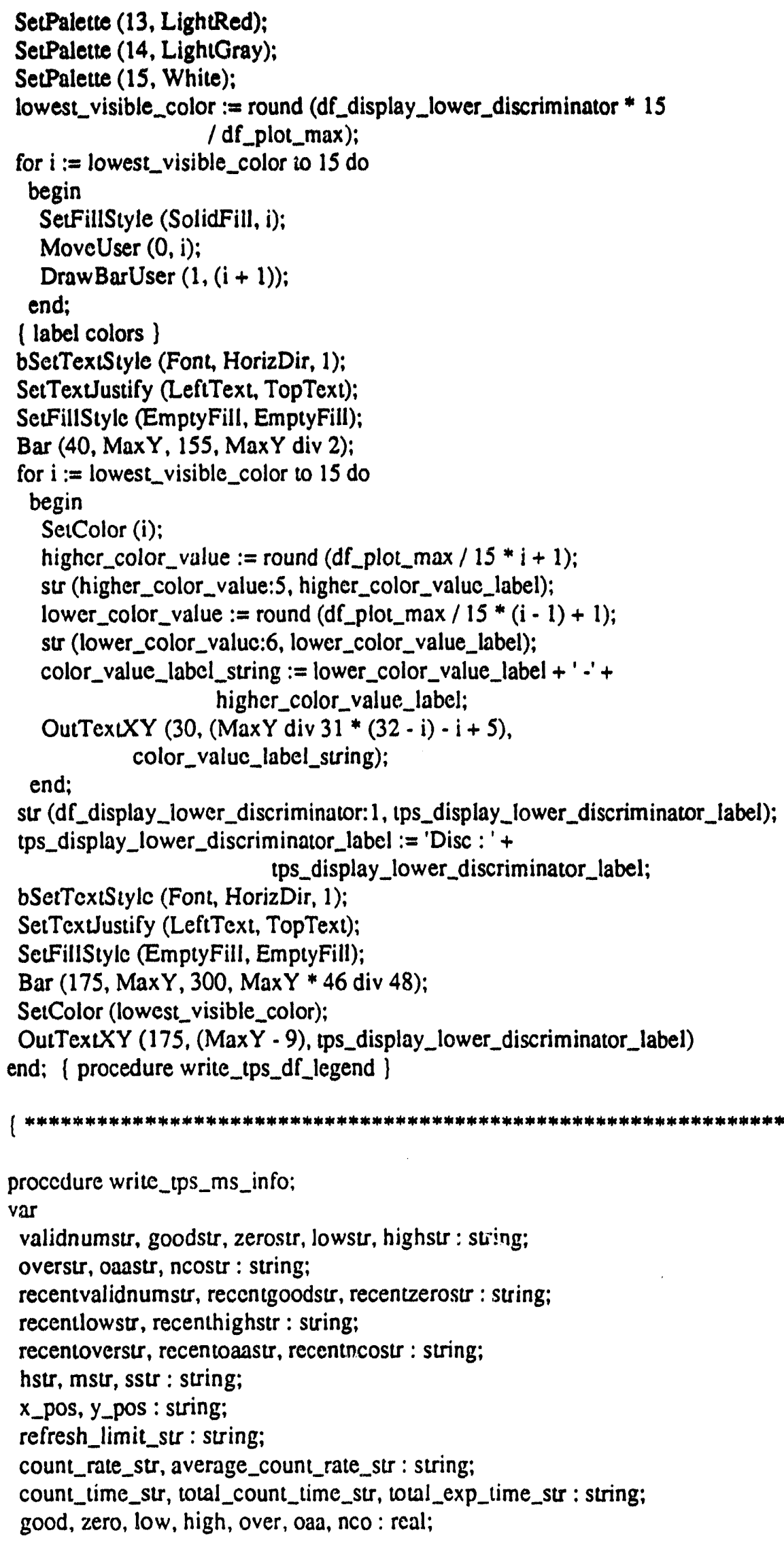


rgood, rzero, rlow, thigh, rover, roaa, mco : real;

begin (procedure write_tps_ms_info )

if GraphicsOn = FALSE then

begin

SetGraphMode (GraphMode);

GraphicsOn := TRUE;

end;

DefineWindow $(0,0,300,500)$;

DefineUserCoordinates $(1,1,24,25)$;

SetColor (LightRed);

bSetTextStyle (Font, HorizDir, 1);

SetTextJustify (LeftText, TopText);

OutTextXY (1, 1, 'Events Last[\%] Cum[\%]');

if rec_data_read_cycles $=0$ then

begin

rec_data_read_cycles := 1 ;

if tps_data_read_cycles $=0$ then

begin

tps_data_read_cycles := 1 ;

end;

end;

good := tps_data_count / tps_data_read_cycles * 100;

zero := zero_data_count / tps_data_read_cycles * 100;

low := low_data_count / tps_data_read_cycles * 100;

high := high_data_count / tps_data_read_cycles * 100;

over $:=$ overnlow_count / tps_data_read_cycles * 100;

oaa $:=$ outside_active_area_count / tps_data_read_cycles * 100 ;

nco $:=$ non_coincidence_count / tps_data_read_cycles * 100 ;

rgood $:=($ tps_refresh_count -1$) /$ rec_data_read_cycles * 100;

rzero $:=$ rec_zero_data_count $/$ rec_data_read_cycles * 100 ;

rlow := rec_low_data_count / rec_data_read_cycles * 100;

rhigh := rec_high_data_count / rec_data_read_cycles * 100 ;

rover $:=$ rec_overflow_count / rec_data_read_cycles * 100;

roaa := rec_outside_active_area_count / rec_data_read_cycles * 100 ;

mco $:=$ rec_non_coincidence_count / rec_data_read_cycles * 100;

str (good:5:1, goodstr);

str (zero:5:1, zerostr);

str (low:5:1, lowstr);

str (high:5:1, highstr);

str (over:5:1, overstr);

str (oaa:5:1, oaastr);

str (nco:5:1, ncostr);

str (rgood:5: 1, recentgoodstr);

str (rzero:5:1, recentzerostr);

str (rlow:5:1, recentlowstr);

str (rhigh:5:1, recenthighstr);

str (rover:5:1, recentoverstr);

str (roaa:5:1, recentoaastr);

str (mco:5:1, recentncostr);

goodstr := ' Valid : ' + recentgoodstr + ' ' + goodstr;

zerostr := ' Zero : ' + recentzerostr +' ' + zerostr;

lowstr := ' Low : ' + recentlowstr +' ' + lowstr; 


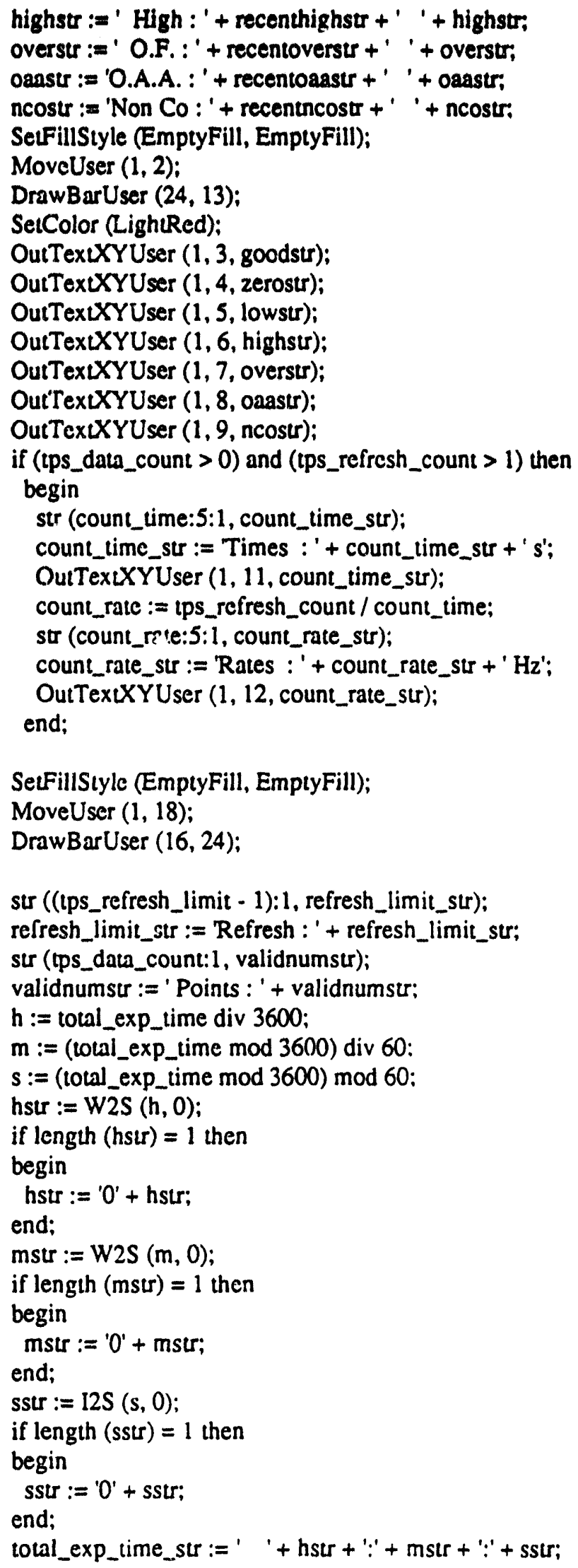


SetColor (LightOreen);

bSetTextStyle (Font, HorizDir, 1);

SetTextustify (LeftText, TopText);

OutTexLXYUser (1, 17, refresh_limit_str);

OutTextXYUser (1, 19, 'Valid Data');

OutTexiXYUser $(1,20$, validnumstr);

OutTexLXYUser (1, 22, 'Elapsed Time');

OutTexiXYUser (1, 23, total_exp_time_str);

SetColor (LightGreen);

bSetTextStyle (Font, HorizDir, 1);

SetTextJustify (LeftText, TopText);

OutTextXY (480, (MaxY - 9), 'Hit Esc to Quit ...);

end; (procedure write_tps_ms_info )

end. ( unit dfplot )

\subsection{2 tps_dcc.pas}

I tps_dcc.pas

unit tps_dcc:

[SO+]

$(\mathrm{SF}+$ )

interface

uses tps_util,tps_glob,DOS,CRT;

procedure show_dcc_parameters (dmc_setting : dcc_real_column_array; dac_setting : dcc_real_column_array);

procedur, get_detector_multiplier_constant_settings

(vardmc_setting : dcc_rcal_column_array;

dac_setting : dcc_real_column_array;

ch : char);

procedure get_detcctor_additive_constant_settings

(dmc_setting : dcc_real_column_array;

var dac_setting : dcc_real_column_array;

ch : char);

procedure dcc_menu;

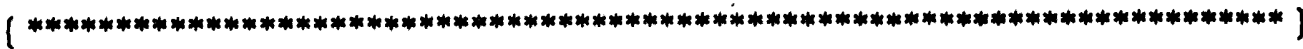

implementation

procedure show_dcc_paramcters (dmc_setting : dcc_real_column_array; dac_setting : dcc_real_column_array);

var

wind_y : byte;

begin \{ procedure show_dcc_parametcrs \} 


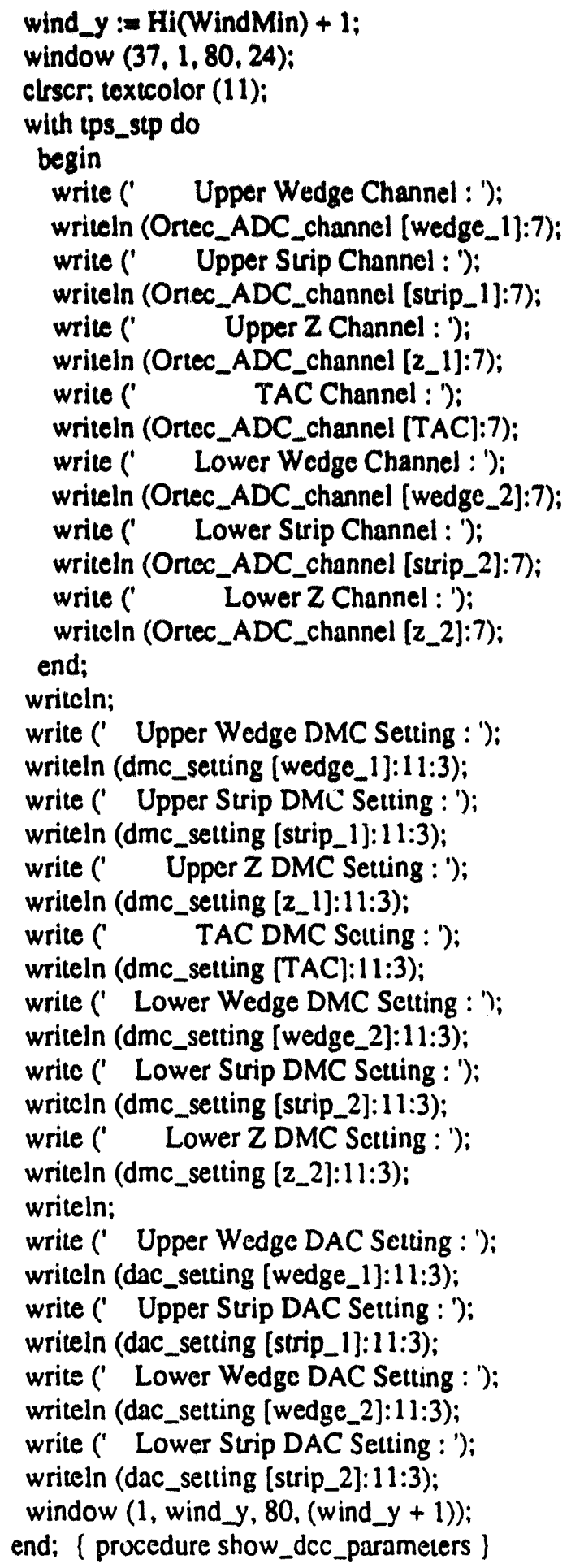




\section{$y$ : integer;}

begin ( procedure get_detector_multiplier_constant_settings )

case ch of

'A': begin

$y:=$ wherey + Hi(WindMin);

window $(1, y, 80, y)$;

if ask_for_real (dmc_setting [wedge_1],

- New Upper Wedge Detector Multiplier Constant Setting : ',0, 10) then

begin

show_dcc_parameters (dmc_setting, dac_setting);

textcolor (14);

end;

if ask_for_real (dmc_setung [strip_1],

- New Upper Strip Detector Multiplier Constant Setting : ', 0, 10) then begin

show_dcc_parameters (dmc_setting, dac_setting);

texicolor (14);

end;

if ask_for_real (dmc_seuing [2_1],

- New Upper Z Detector Multiplier Constant Sctung : ', 0, 10) then begin

show_dcc_paramcters (dmc_setting, dac_setting);

textcolor (14);

end;

if ask_for_real (dmc_setting [TAC],

New TAC Detector Multiplier Constant Setting : $, 0,10$ ) then

begin

show_dcc_parameters (dmc_setting, dac_setting);

textcolor (14);

end;

if ask_for_real (dmi_sctting [wedge_2],

- New Lower Wedge Detector Multiplicr Constant Setting : ', 0, 10) then begin

show_dcc_parameters (dmc_setting, dac_setting);

textcolor (14);

end;

if ask_for_real (dmc_setting [strip_2],

' New Lower Strip Detector Multiplier Constant Setting : ', 0, 10) then begin

show_dcc_parametcrs (dmc_setting, dac_setting);

textcolor (14);

end;

if ask_for_real (dmc_setting [z_2],

- New Lower $\bar{Z}$ Detector Multiplier Constant Setting : ', 0, 10) then begin

show_dcc_parameters (dmc_setting, dac_setting);

textcolor (14);

end;

end;

' 1 ' : begin

if ask_for_real (dmc_setting [wedge_1],

- New Upper Wedge Detector Multiplier Constant Setting : ', 0, 10) then show_dcc_parameters (dmc_setting, dac_sctuing); 


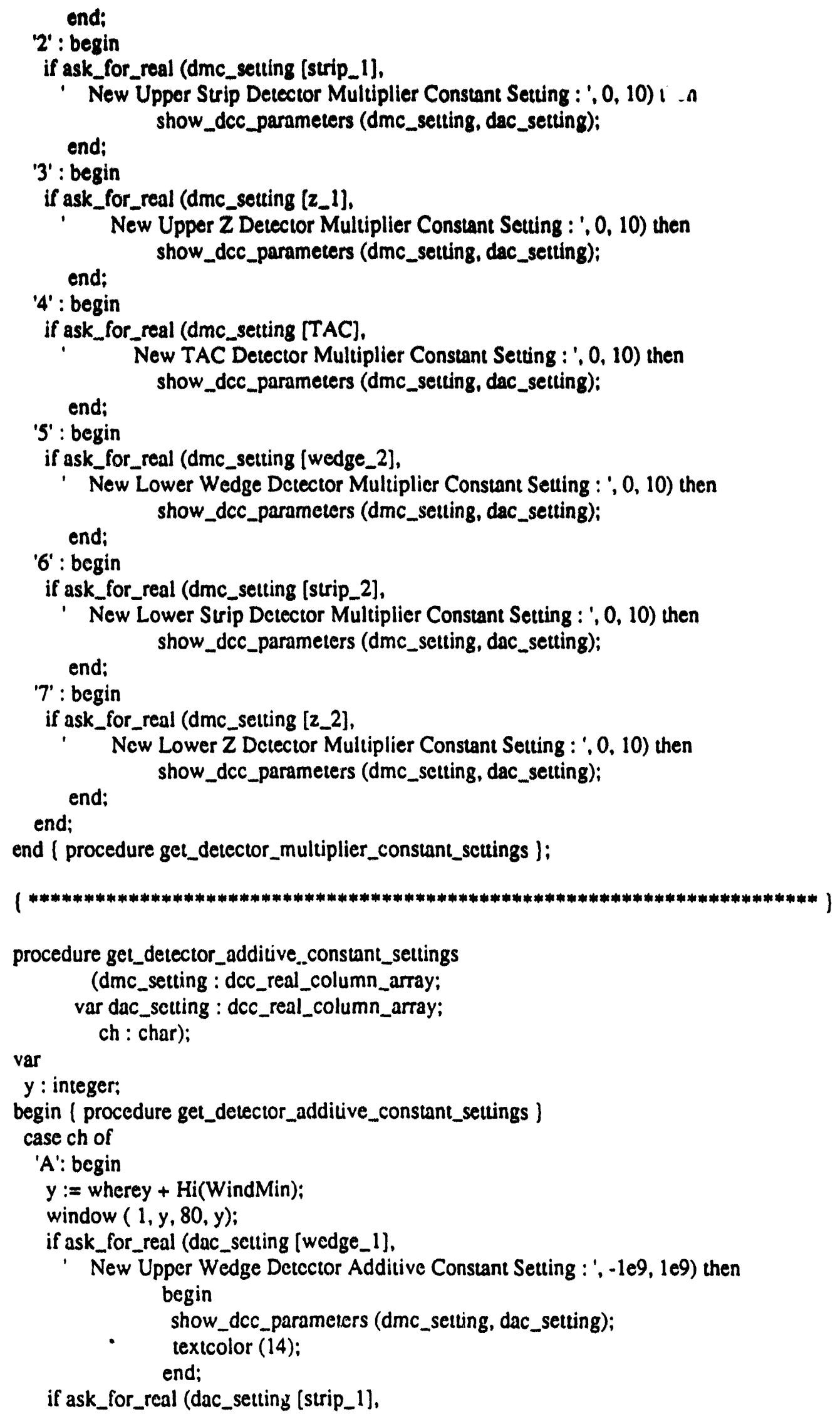


- Now Upper Strip Detector Additive Constant Seuing : ', -1e9, 1e9) then begin show_dcc_paramcters (dmc_setting, dac_setting); textcolor (14); end:

If ask_for_real (dac_setting [wedge_2],

- New Lower Wedge Detector Additive Constant Setting : ', -109, 1e9) then begin show_dcc_parameters (dmc_setting, dac_setting); textcolor (14);

end;

if ask_for_real (dac_setting [strip_2],

- New Lower Strip Detector Additive Constant Setting : ', -1e9, 1e9) then begin

show_dcc_parameters (dmc_setting, dac_setting); textcolor (14);

end; end;

' 1 ' : begin

if ask_for_real (dac_setting [wedge_1],

- New Upper Wedge Detector Additive Constant Setting : ', -1e9, 1e9) then end; show_dcc_parameters (dmc_setting, dac_setting);

'2' : begin

if ask_for_real (dac_setting [strip_1],

- New Upper Strip Detector Additive Constant Setting : ', -1e9, 1e9) then end;

' 5 ' : begin

if ask_for_real (dac_setting [wedge_2],

- New Lower Wedge Detector Additive Constant Setting : ', -1e9, 1e9) then end; show_dcc_parameters (dmc_setting, dac_setting);

'6' : begin

if ask_for_real (dac_setting [strip_2],

- New Lower Strip Detector Additive Constant Setting : ', -1e9, 1e9) then end; show_dcc_parameters (dmc_setting, dac_setting);

end;

end \{ procedure get_detector_additive_constant_settings \};

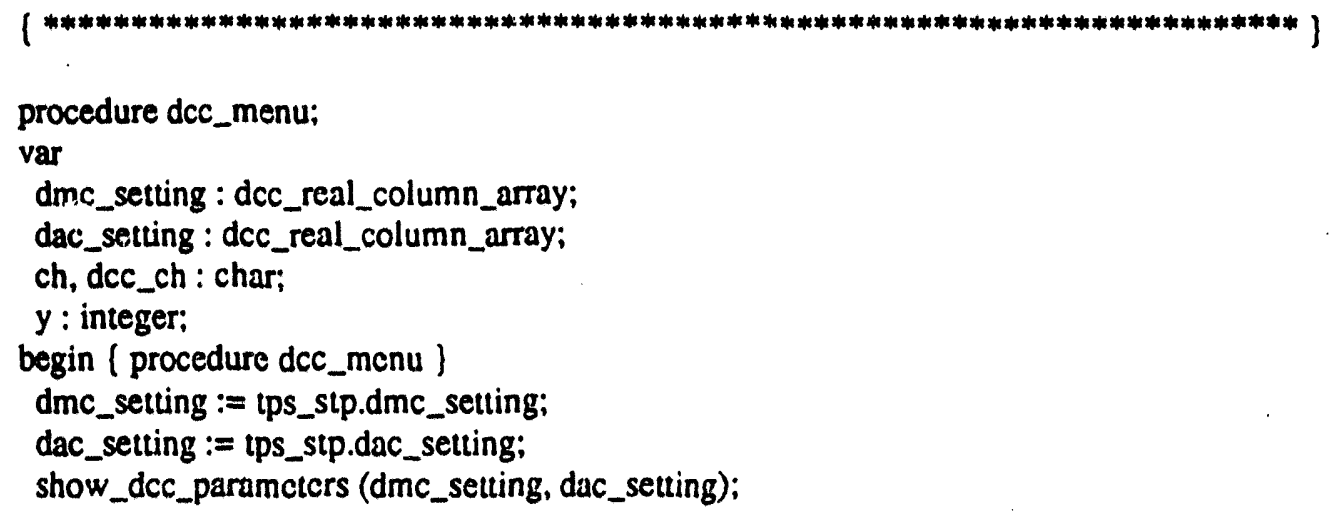




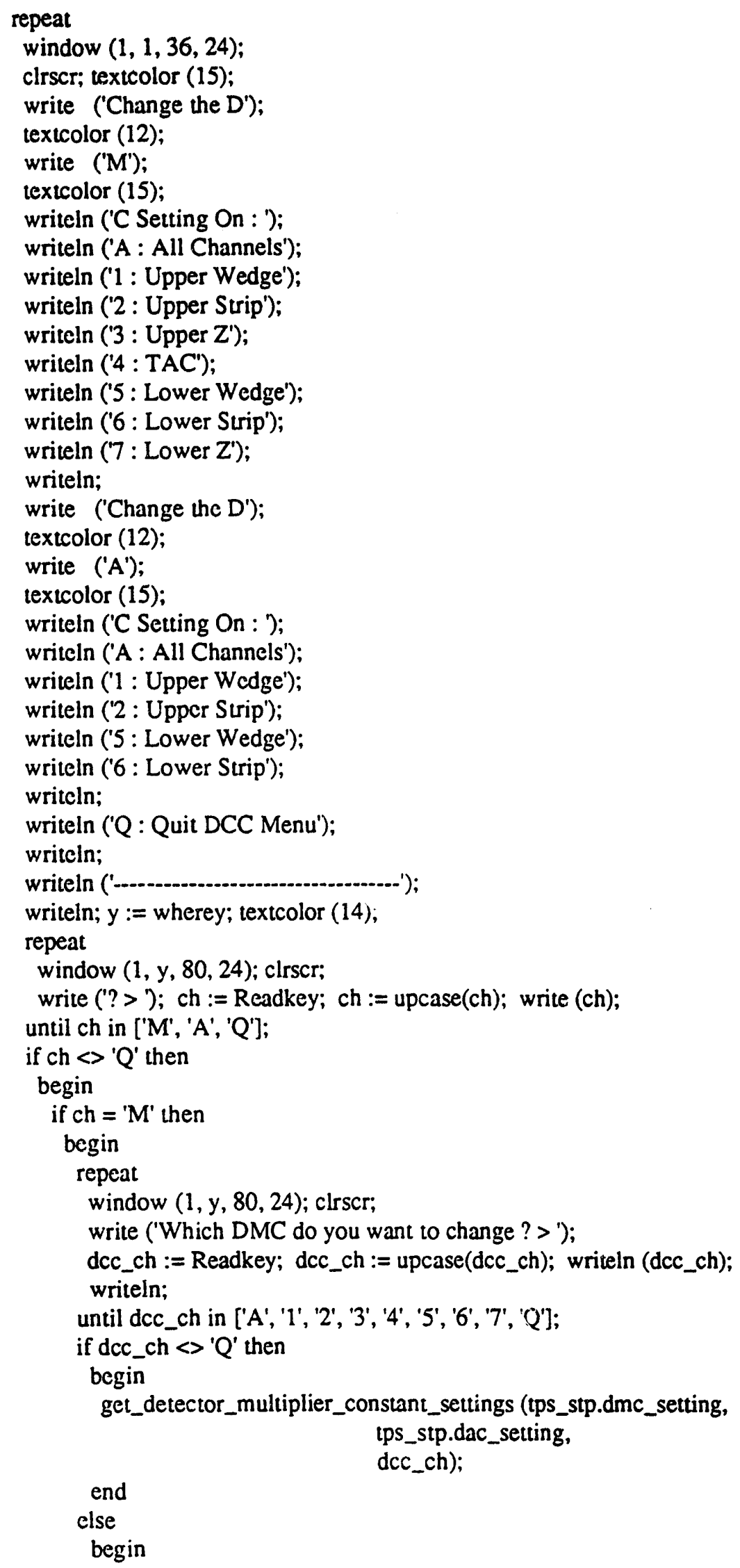




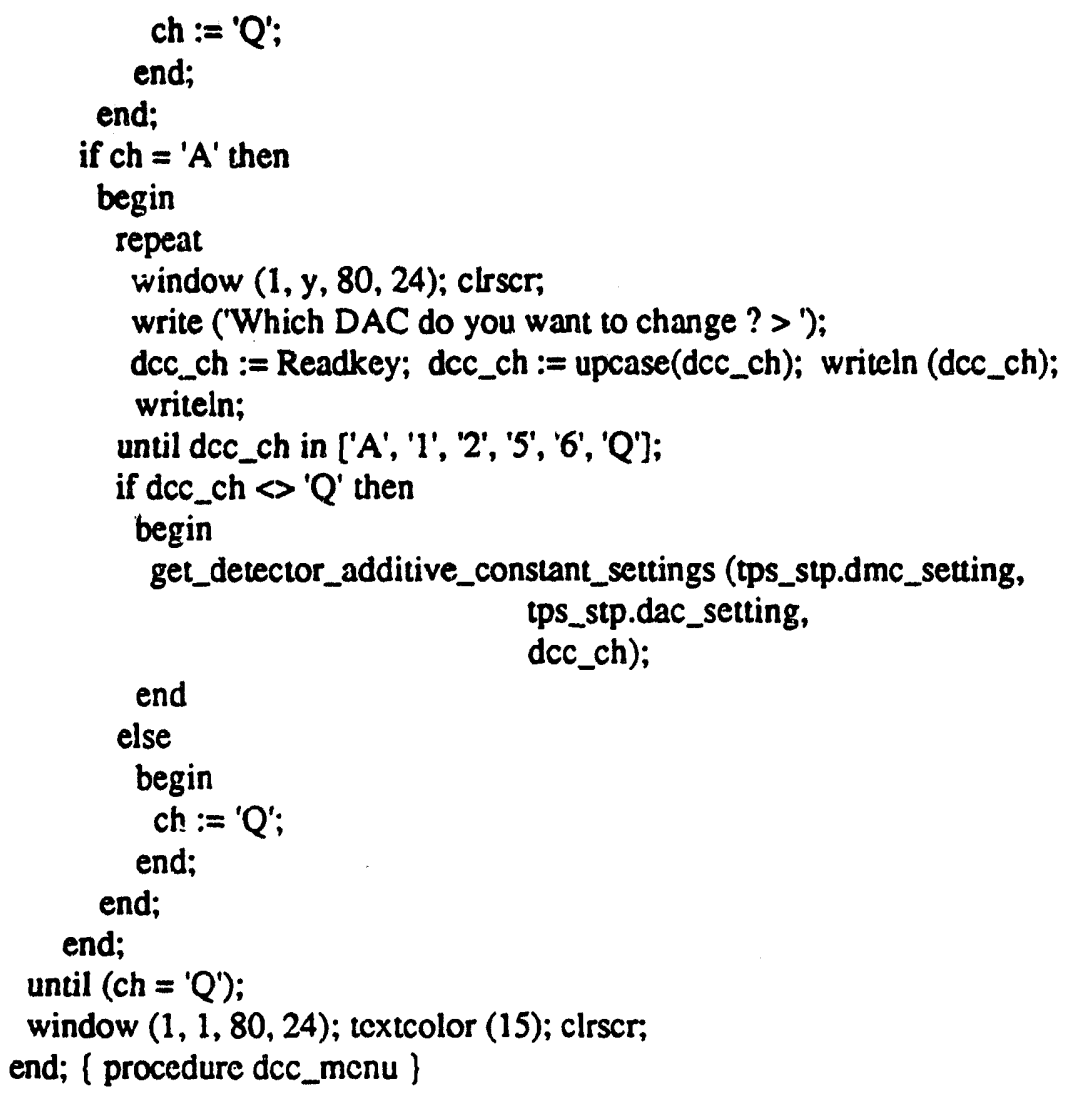

end. \{ unit tps_dcc \}

\subsection{3 tps_ctf.pas}

( tps_ctf.pas

unit tps_ctf;

[SO+]

$\{\mathrm{SF}+]$

interface

uses tps_util,tps_glob,DOS,CRT;

procedure show_ctf_parameters (ctf_setting : ctf_real_column_array);

procedure get_detector_crosstalk_factor_settings

(var ctf_setting : ctf_real_column_array;

ch : char);

procedure ctf_menu;

$\{* * * * * * * * * * * * * * * * * * * * * * * * * * * * * * * * * * * * * * * * * * * * * * * * * * * * * * * * * * * * * * * * * * * * * * * * * *\}$ 


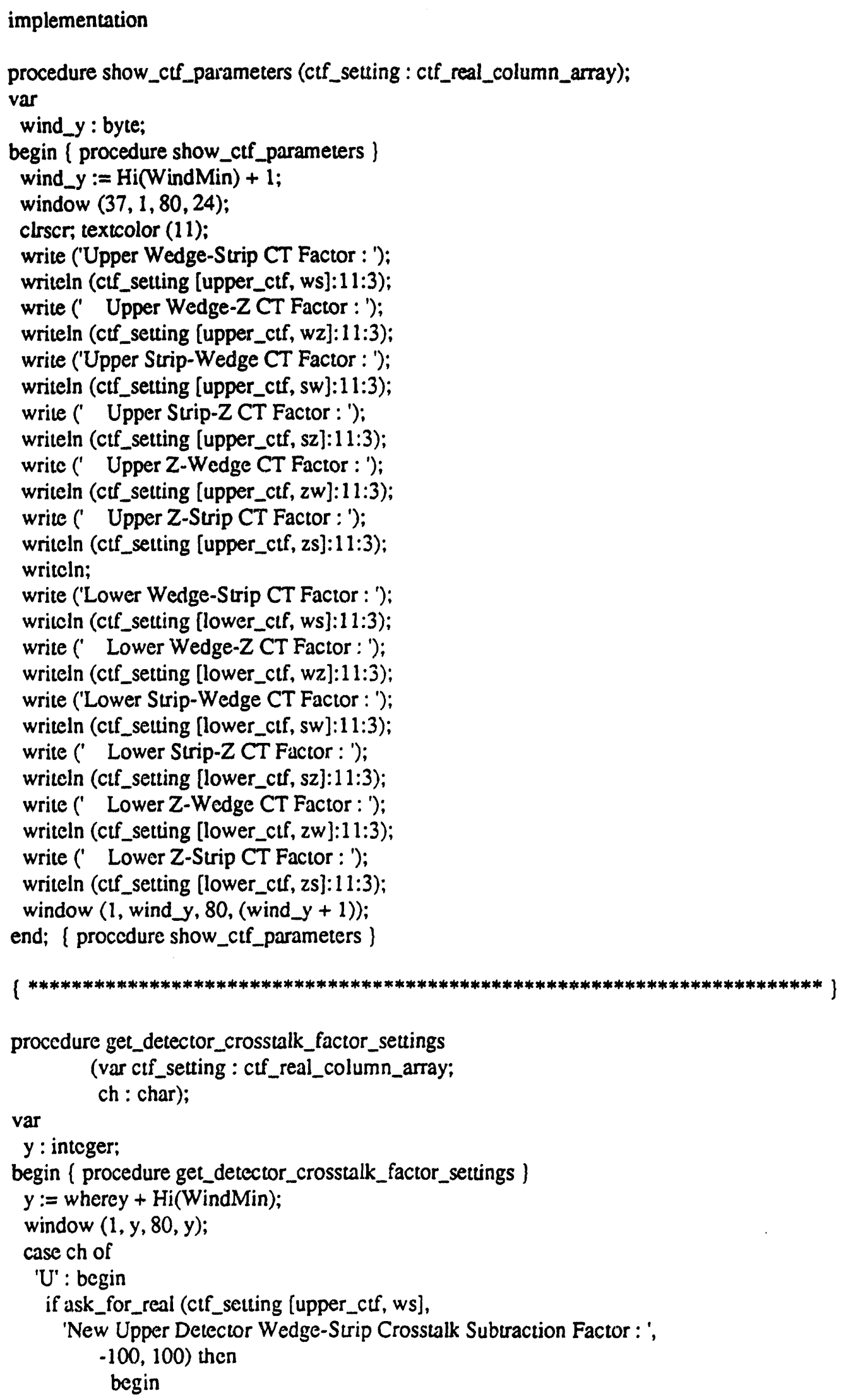


show_ctf_parameters (ctf_setting);

textcolor (14);

end;

if ask_for_real (ctf_setting [upper_ctf, wz],

- New Upper Detector Wedge-Z Crosstalk Subtraction Factor : ', $-100,100)$ then

begin

show_ctf_parameters (ctf_setting);

textcolor (14);

end;

if ask_for_real (ctf_setting [upper_ctf, sw],

'New Upper Detector Strip-Wedge Crosstalk Subtraction Factor : ', $-100,100)$ then

begin

show_ctf_parameters (ctf_setting);

textcolor (14);

end;

if ask_for_real (ctf_setting [upper_ctf, sz],

- New Upper Detector Strip-Z Crosstalk Subtraction Factor : ', $-100,100)$ then

begin

show_ctf_parameters (ctf_setting);

textcolor (14);

end;

if ask_for_real (ctf_setting [upper_ctf, zw],

- New Upper Detector Z-Wedge Crosstalk Subtraction Factor : ', $-100,100)$ then

begin

show_ctf_parameters (ctf_scting);

textcolor (14);

end;

if ask_for_real (ctf_setting [upper_ctf, zs],

- New Upper Detector Z-Strip Crosstalk Subtraction Factor : ',

$-100,100)$ then

begin

show_ctf_parametcrs (ctf_setting);

textcolor (14);

end;

end;

'L' : begin

if ask_for_real (ctf_setting [lower_ctf, ws],

'New Lower Detector Wedge-Strip Crosstalk Subtraction Factor : ', $-100,100)$ then

begin

show_ctf_parameters (ctf_setting);

textcolor (14);

end;

if ask_for_real (ctf_setting [lower_ctf, wz],

- New Lower Detector Wedge-Z Crosstalk Subtraction Factor : ', $-100,100)$ then

begin

show_ctf_parameters (ctf_setting);

textcolor (14);

end; 


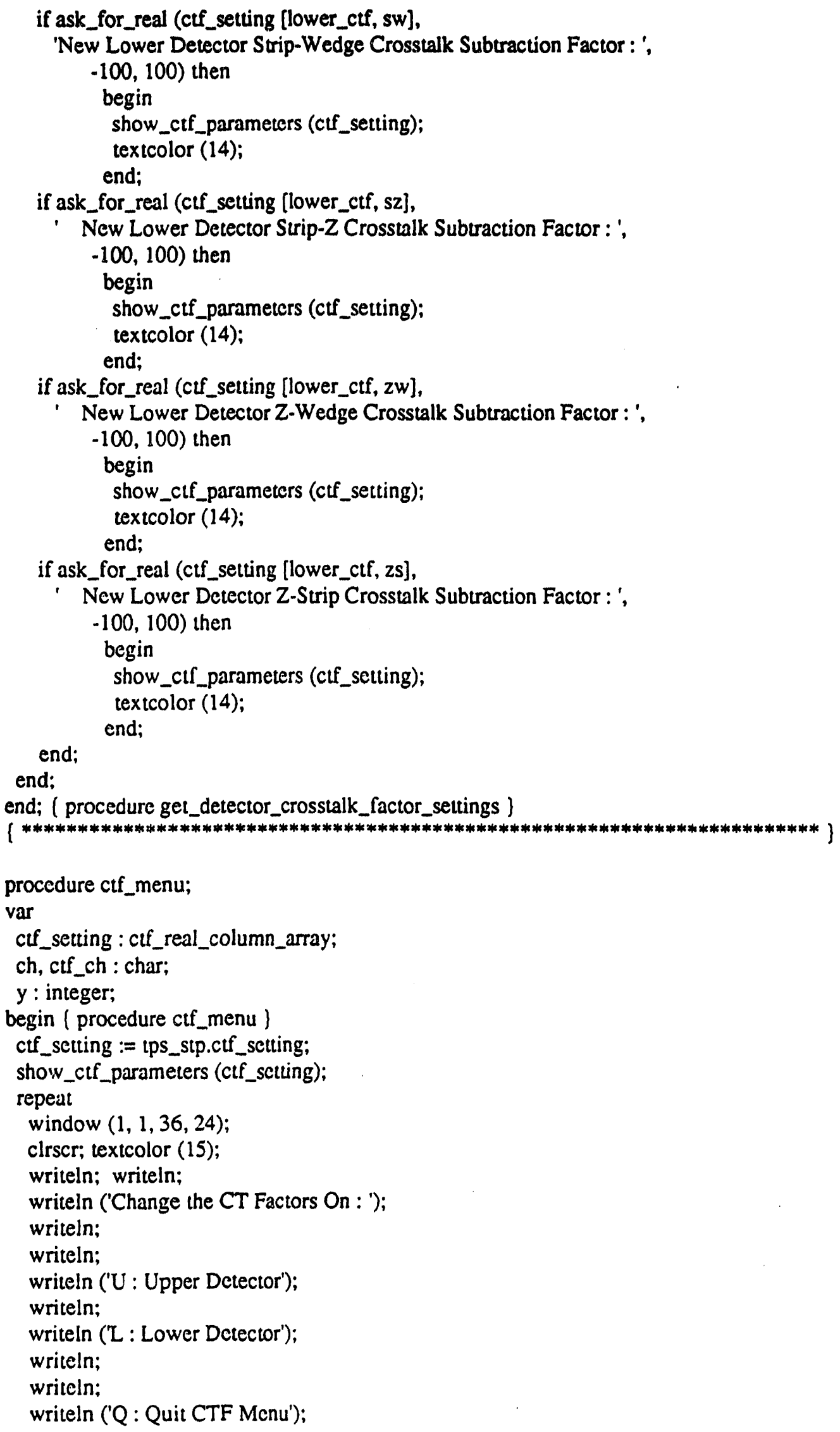




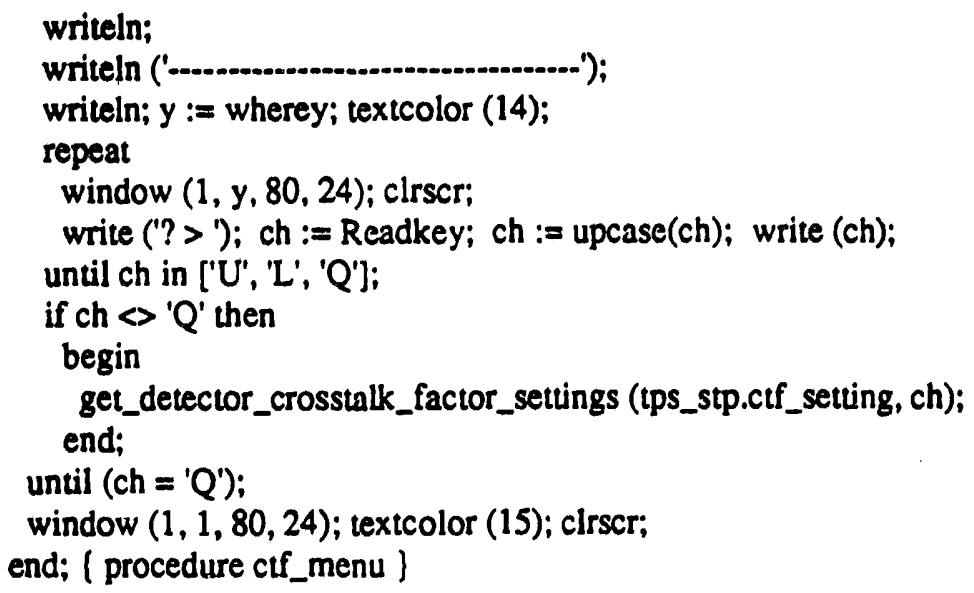

end. \{ unit tps_ctf \}

\subsection{4 tps_lld.pas}

[ tps_lld.pas

unit tps_lld;

$(\mathrm{SO}+]$

$\{\mathrm{FF}+\}$

interface

uses tps_util,tps_glob,DOS,CRT;

procedure show_lld_parameters (lld_sctting : tps_int_column_array);

procedure get_lower_level_discriminator_scttings

(var lld_setting : tps_int_column_array; ch : char);

procedure set_up_Ortec_ADCs (lld_setuing : tps_int_column_array);

procedure lld_menu;

$\{* * * * * * * * * * * * * * * * * * * * * * * * * * * * * * * * * * * * * * * * * * * * * * * * * * * * * * * * * * * * * * * * * * * * * * * *\}$

implementation

\{\$F-\}

\{\$I camturbo.v4\}

$\{\mathbf{S F}+\}$

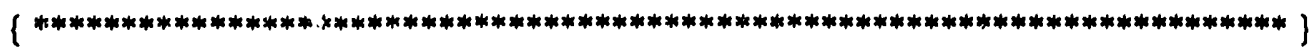

procedure show_lld_parameters (lld_setling : tps_int_column_array); 


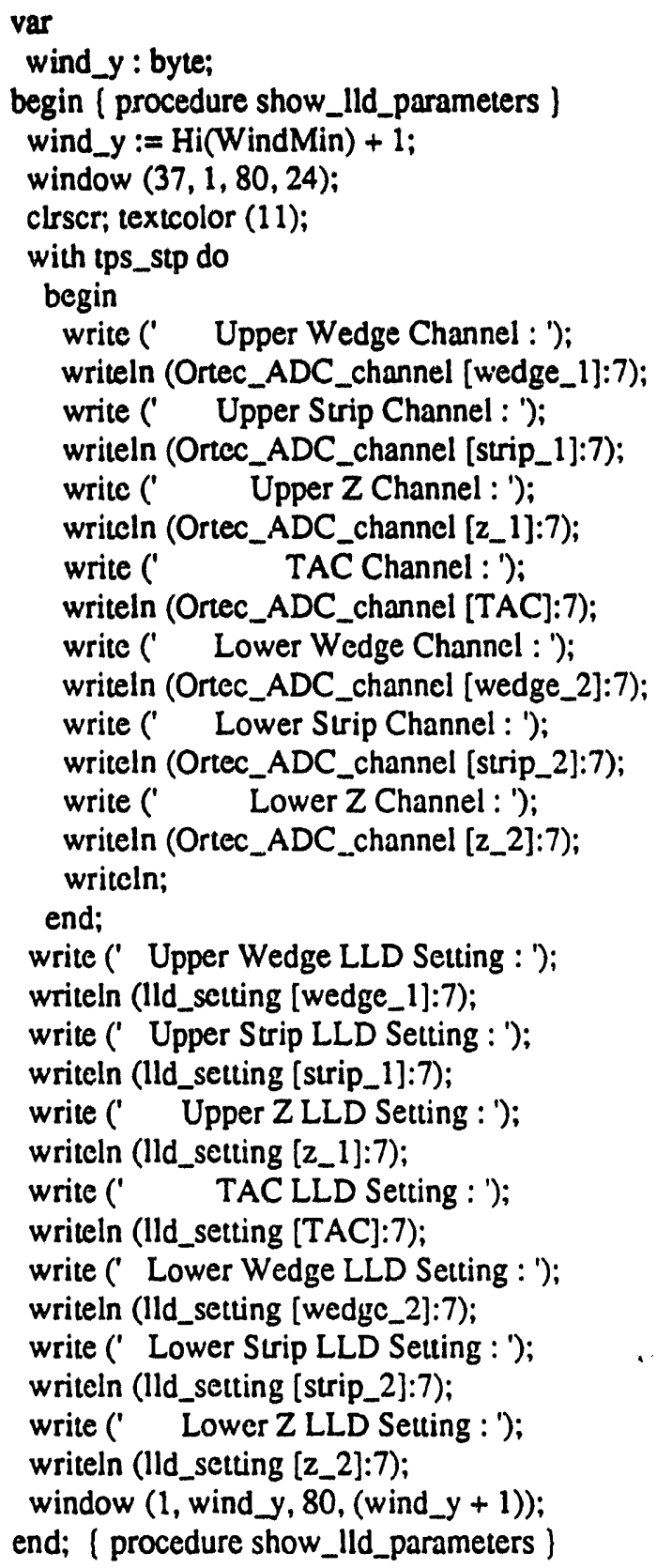




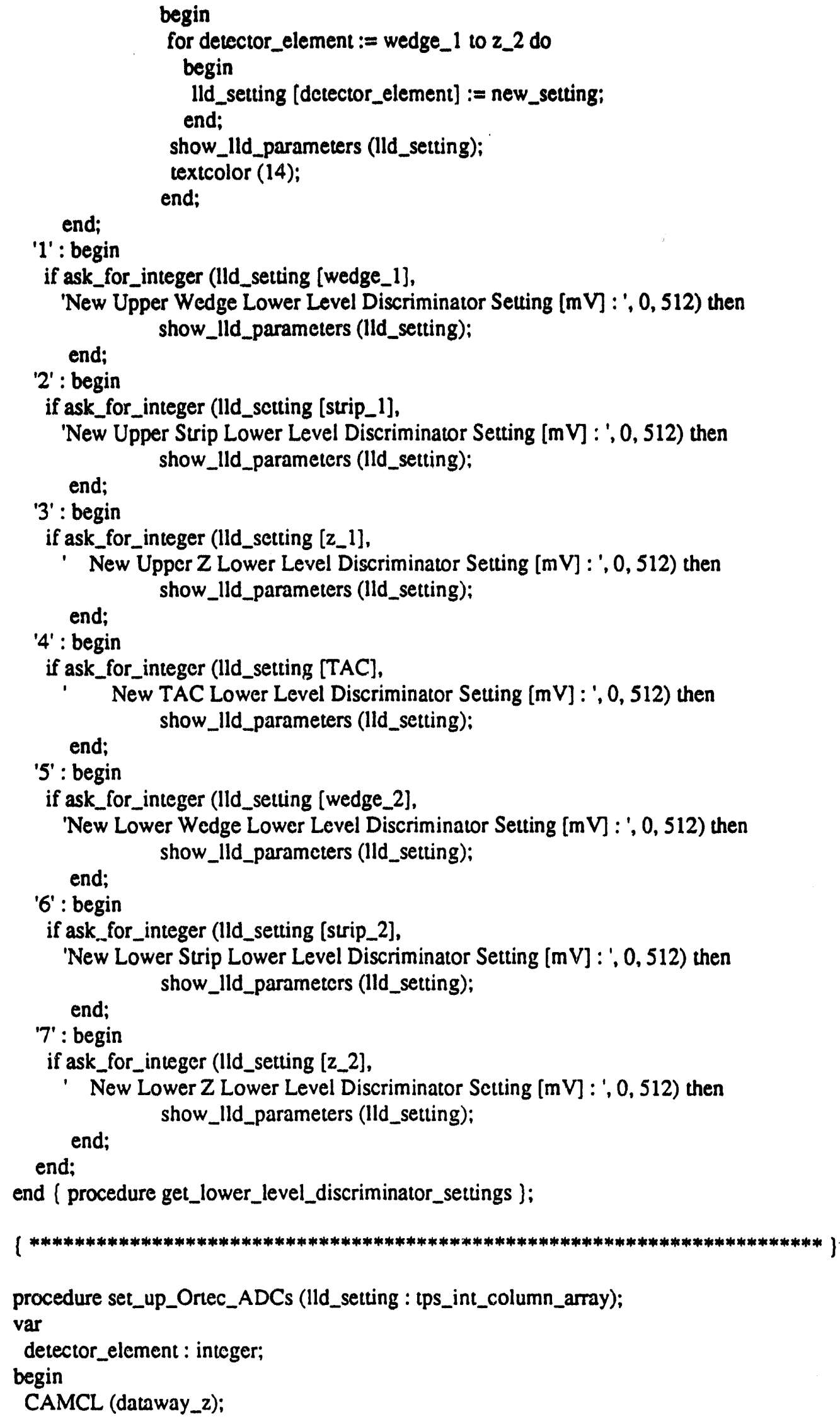


CAMCL (dataway_i);

CAMO (Ortec_ADC_1, write_control_register, control_register_1_location, control_register_1_Ortec_1, $Q, X)$;

CAMO (Ortec_ADC_1, write_control_register, control_register_2_location, control_register_2, $Q, X)$;

CAMO (Ortec_ADC_2, write_control_register, control_register_1_location, control_register_1_Ortec_2, $Q, X)$;

CAMO (Ortec_ADC_2, writc_control_register, control_register_2_location, control_register_2, $\mathrm{Q}, \mathrm{X}$ );

for detector_elcment := wedge_1 to z_2 do

begin

lld_setting [detector_element] := Ild_setting [detector_element] div 2;

end;

CAMO (Ortec_ADC_1, write_lld_setting, wedge_1_channel, lld_setting [wedge_1], $Q, X$ );

CAMO (Ortec_ADC_1, write_lld_setting, strip_1_channel, lld_setting [strip_1], $Q, X)$;

CAMO (Ortec_ADC_1, write_lld_setting, z_1_channel, lld_setting [z_1], $\mathrm{Q}, \mathrm{X})$;

CAMO (Ortec_ADC_1, write_lld_setting, TAC_channel, lld_setting [TAC], $Q, X)$;

CAMO (Ortcc_ADC_2, write_lld_setting, wedge_2_channel, Ild_setting [wedge_2], $Q, X$ );

CAMO (Ortec_ADC_2, write_lld_setting, strip_2_channel, lld_setting [strip_2], $\bar{Q}, X)$;

CAMO (Ortcc_ADC_2, write_lld_setuing, z_2_channel,

end; lld_setting [7._2], $\bar{Q}, \bar{X}$ );

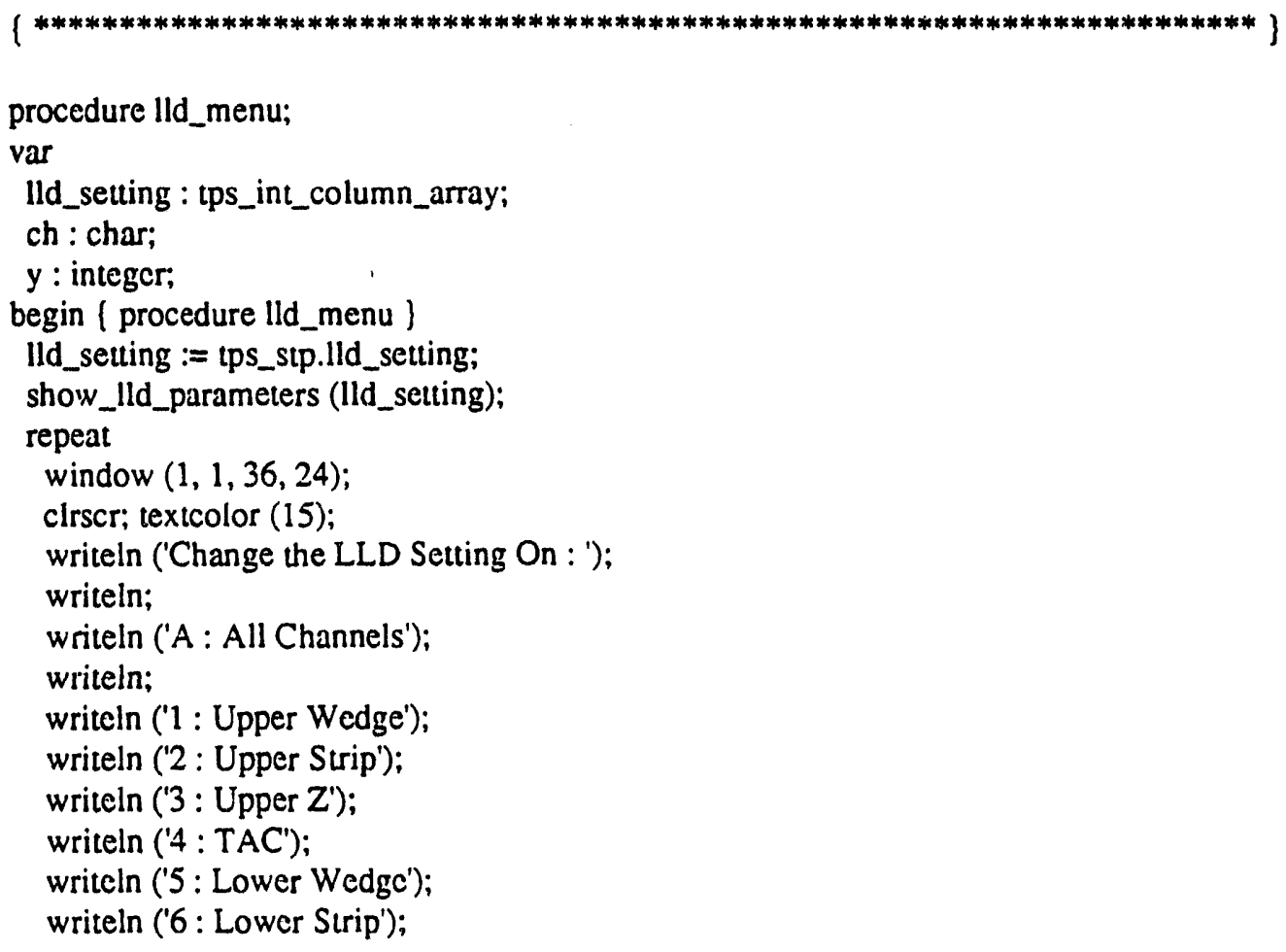




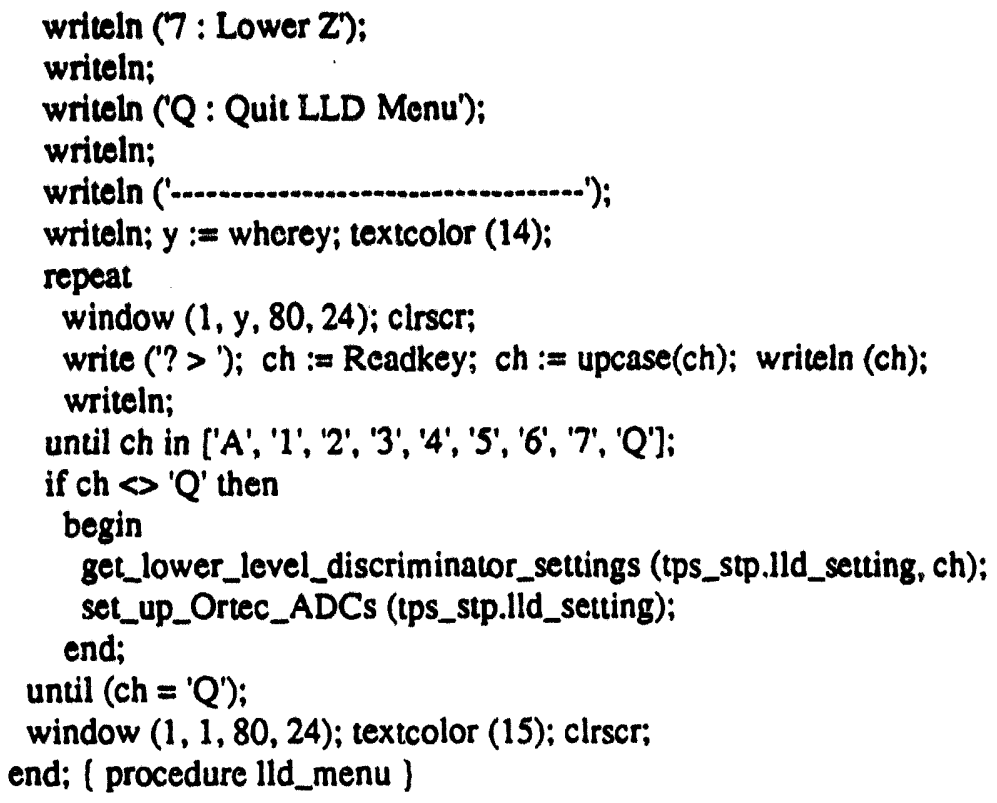

end. [ unit tps_lld \}

\subsection{5 tps_swd.pas}

(tps_swd.pas ] ]

unit tps_swd;

$\{\mathrm{SO}+\}$

$\{\mathrm{SF}+\}$

interface

uses tps_util,tps_glob,DOS,CRT;

procedure show_swd_parameters (swd_setting : swd_int_column_array);

procedure get_software_discriminator_settings

(var swd_setting : swd_int_column_array; ch : char); procedure swd_menu;

\section{\{}

implementation

\{\$F-\}

\{\$I camturbo.v4\}

$\{\$ F+\}$ 


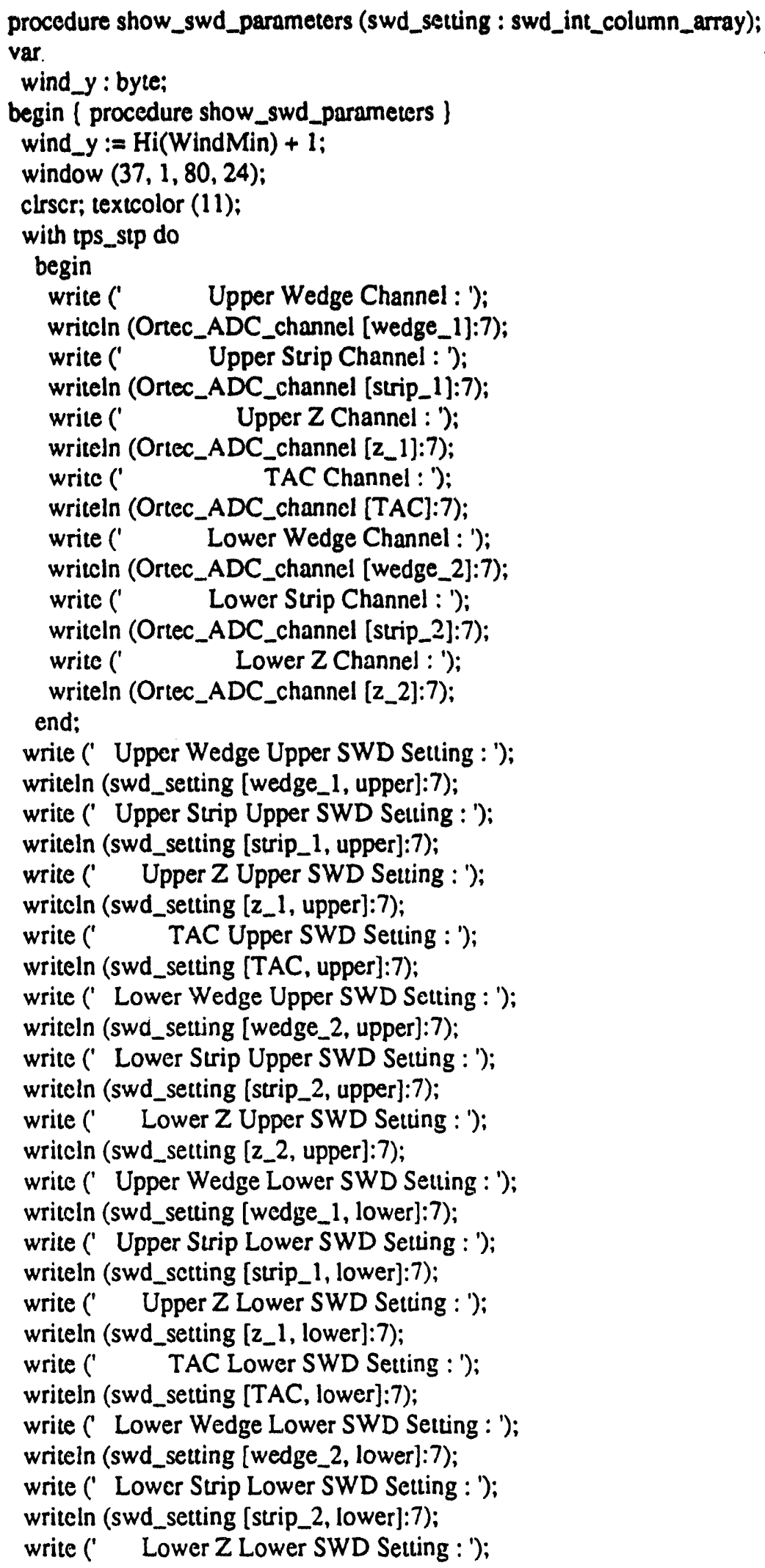


writeln (swd_setting [z_2, lower]:7);

window $(1$, wind $y, 80$, (wind $z+1)$ );

end: (procedure show_swd_parameters )

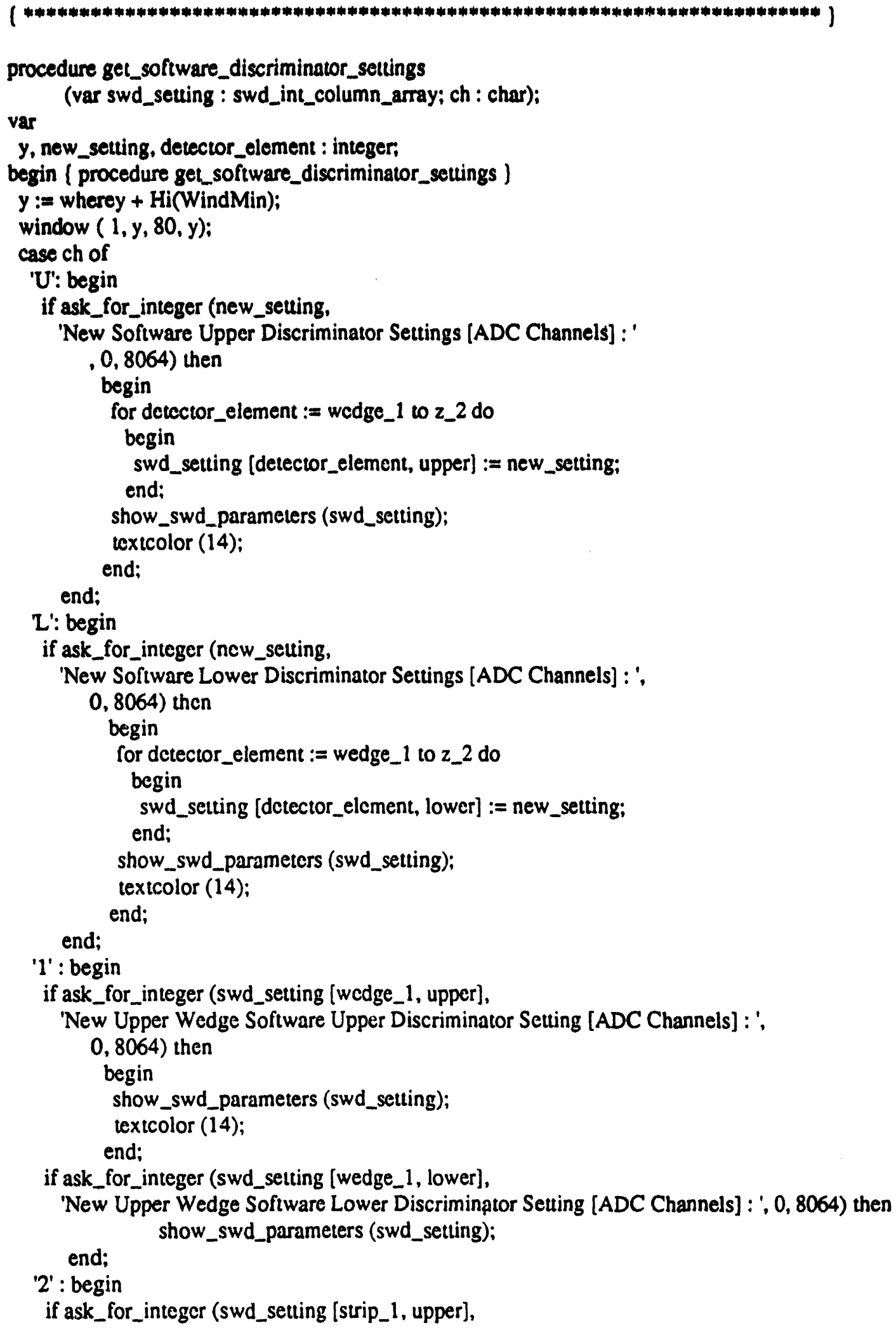




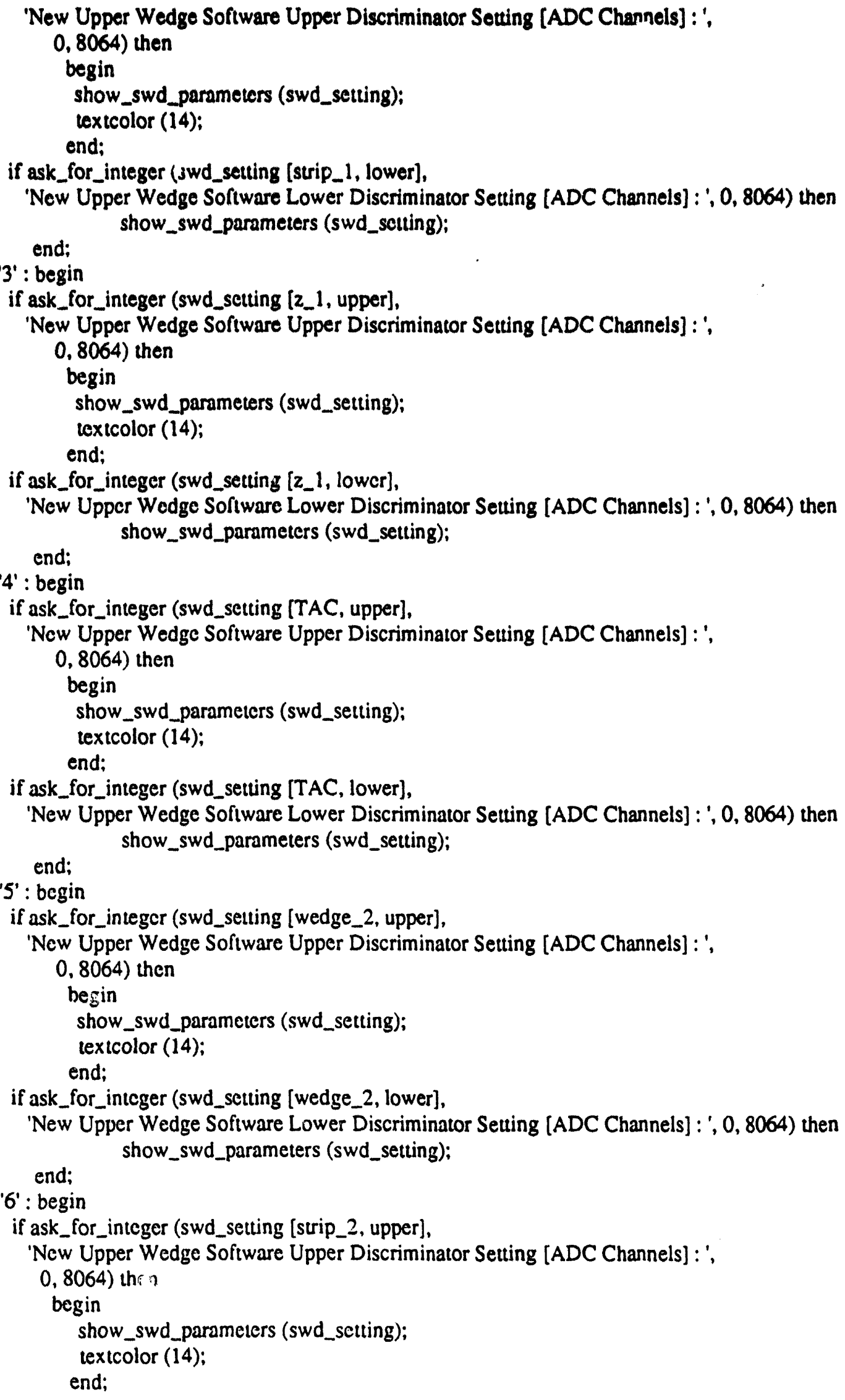




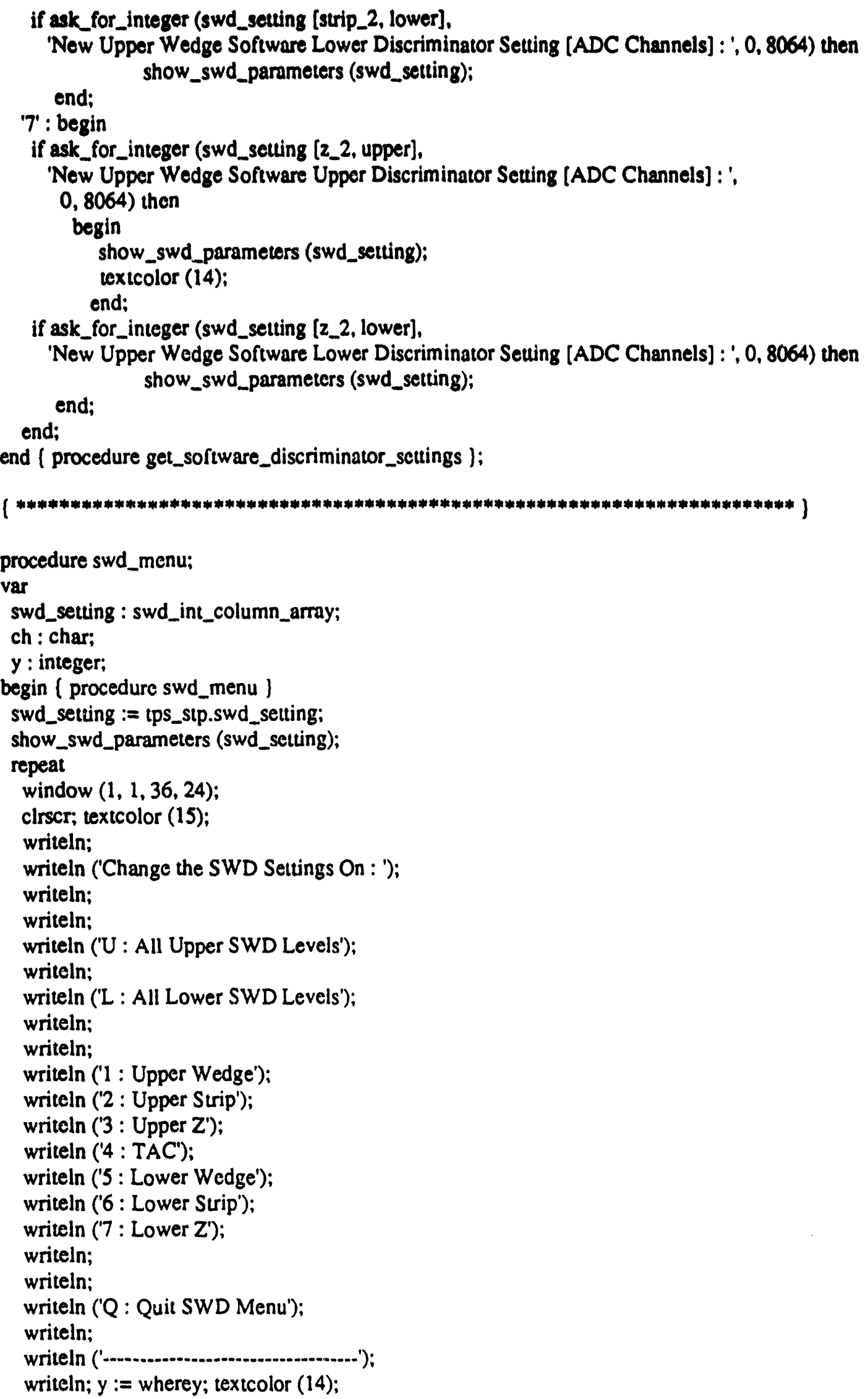




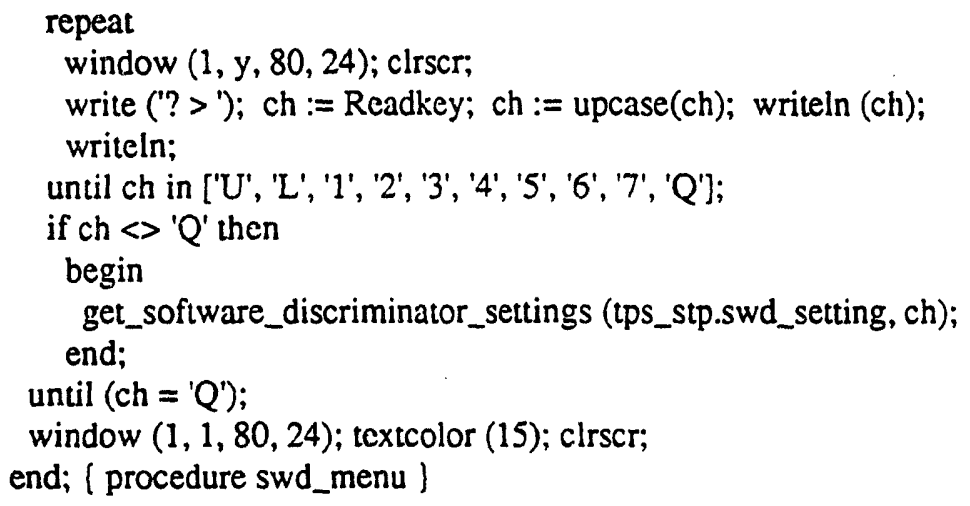

end. ( unit tps_swd \} 
